\title{
Grenzüberschreitende Kundenbindung im Einzelhandel: Das Treueverhalten deutscher und niederlandischer Konsumenten im Grenzgebiet des Nachbarlandes
}

Citation for published version (APA):

Scholz, U. (2007). Grenzüberschreitende Kundenbindung im Einzelhandel: Das Treueverhalten deutscher und niederlandischer Konsumenten im Grenzgebiet des Nachbarlandes. [Doctoral Thesis, Maastricht University]. Shaker Verlag. https://doi.org/10.26481/dis.20070905us

Document status and date:

Published: 01/01/2007

DOI:

10.26481/dis.20070905us

Document Version:

Publisher's PDF, also known as Version of record

Please check the document version of this publication:

- A submitted manuscript is the version of the article upon submission and before peer-review. There can be important differences between the submitted version and the official published version of record. People interested in the research are advised to contact the author for the final version of the publication, or visit the DOI to the publisher's website.

- The final author version and the galley proof are versions of the publication after peer review.

- The final published version features the final layout of the paper including the volume, issue and page numbers.

Link to publication

\footnotetext{
General rights rights.

- You may freely distribute the URL identifying the publication in the public portal. please follow below link for the End User Agreement:

www.umlib.nl/taverne-license

Take down policy

If you believe that this document breaches copyright please contact us at:

repository@maastrichtuniversity.nl

providing details and we will investigate your claim.
}

Copyright and moral rights for the publications made accessible in the public portal are retained by the authors and/or other copyright owners and it is a condition of accessing publications that users recognise and abide by the legal requirements associated with these

- Users may download and print one copy of any publication from the public portal for the purpose of private study or research.

- You may not further distribute the material or use it for any profit-making activity or commercial gain

If the publication is distributed under the terms of Article 25fa of the Dutch Copyright Act, indicated by the "Taverne" license above, 


\title{
Grenzüberschreitende Kundenbindung im Einzelhandel
}

Das Treueverhalten deutscher und niederländischer Konsumenten im Grenzgebiet des Nachbarlandes

\author{
Ulrich Scholz
}


Alle rechten voorbehouden. Niets van deze uitgave mag worden verveelvoudigd, opgeslagen in een geautomatiseerd gegevensbestand, of openbaar gemaakt, in einige vorm, zonder schriftelijke toestemming van de uitgever.

ISBN 978-90-423-0323-2

Shaker Publishing B.V.

St. Maartenslaan 26

5221 AX Maastricht

Tel.: $043-3500424$

Fax: 043 - 3255090

http://www.shaker.nl 


\section{Grenzüberschreitende Kundenbindung im Einzelhandel}

Das Treueverhalten deutscher und niederländischer Konsumenten im

Grenzgebiet des Nachbarlandes

\section{PROEFSCHRIFT}

Ter verkrijging van de graad van doctor aan de Universiteit Maastricht, op gezag van de Rector Magnificus, prof. mr. G.P.M.F. Mols volgens het besluit van het College van Decanen, in het openbaar te verdedigen op woensdag 5 september 2007 om 12.00 uur

door

Ulrich Scholz 


\section{Promotoren}

Prof. dr. J.D.P. Kasper

Prof. dr. B. Günter (Universität Düsseldorf)

\section{Beoordelingscommissie}

Prof. dr. W.H. Gijselaers (voorzitter)

Prof. dr. D. Standop (Universität Osnabrück)

Prof. dr. M.G.M. Wetzels 
Für Gaby und Anke

„Man gibt immer den Verhältnissen die Schuld für das, was man ist. Ich glaube nicht an die Verhältnisse. Diejenigen, die in der Welt vorankommen, gehen hin und suchen sich die Verhältnisse, die sie wollen, und wenn sie sie nicht finden können, schaffen sie sie selbst.“

George Bernhard Shaw 


\section{Vorwort}

Bei der Verfassung dieser Arbeit haben mich viele Menschen unterstützt, denen ich auf diesem Wege herzlich danken möchte.

Zuallererst danke ich meinen beiden Promotoren, Professor Dr. Hans Kasper und Professor Dr. Bernd Günter für die vielen Anregungen und die hervorragende Betreuung meiner Arbeit. Dank schulde ich beiden aber auch für die Motivation, mich an die Thematik „Das Treueverhalten deutscher und niederländischer Konsumenten im Grenzgebiet des Nachbarlandes“ heranzuführen, deren Relevanz beide bereits erkannten, bevor Schlagworte wie Kundenloyalität und Kundenbindung die Marketingtheorie und -praxis prägten. Ihre kritischen Impulse und ihr umfassender fachlicher Beistand haben maßgeblich zum Gelingen der Arbeit beigetragen. Beiden schulde ich Dank - nicht nur für ihre fachliche, sondern auch menschliche Unterstützung in allen Belangen meiner Dissertation.

Ebenso bin ich zu besonderem Dank Professor Dr. Martin Wetzels verpflichtet, der meinen Ehrgeiz geweckt hat, mich mit der Analyse von Strukturgleichungsmodellen mit Hilfe des Partial Least Squares-Verfahrens zur Bestimmung von Kausalmodellen auseinander zu setzen. Seine unzähligen hilfreichen und konstruktiven Ratschläge haben wesentlich zum Erfolg dieser Arbeit beigetragen.

Ferner bedanke ich mich bei den Mitgliedern meiner Beurteilungskommission Herrn Prof. Dr. Wim H. Gijselaers und Herrn Prof. Dr. Dirk Standop für ihre freundliche Unterstützung beim Gelingen dieser Arbeit. Beide haben viel Zeit und Mühen auf sich genommen um meine Dissertation zu lesen und zu beurteilen.

Ohne die Unterstützung meiner Frau Gaby und meiner Tochter Anke hätte ich nie eine Dissertation geschrieben. Beide standen während meiner Promotionszeit fest zu mir und gaben mir fortwährenden Rückhalt und halfen mir nicht nur durch moralische Unterstützung, sondern kämpften sich durch mehrere Fassungen des Manuskripts hindurch. Herzlichen Dank für die mir entgegengebrachte Geduld, für den Mut der mir zugesprochen wurde und für das Selbstvertrauen, das mir von beiden gegeben wurde.

Ulrich Scholz 



\section{Inhaltsverzeichnis:}

Kapitel 1: Einführung in die Untersuchungsthematik 1

1.1 Einleitung 2

1.2 Ausgangssituation der Untersuchung 2

1.3 Relevanz und Problemstellung des Themas 6

1.4 Besondere Einflüsse in der Grenzregion auf die Kundenbindung 8

1.5 Forschungsziele der Arbeit 9

Kapitel 2: Einkaufsstättenspezifische Determinanten der

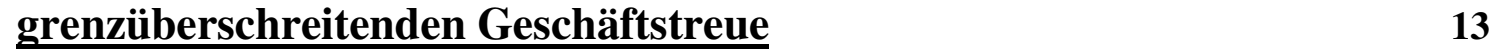

$\begin{array}{lll}2.1 & \text { Einleitung } & 14\end{array}$

2.2 Rolle der Landesgrenze in der Beziehung zwischen Anbieter und Kunde im $\begin{array}{ll}\text { deutsch/niederländischem Grenzgebiet } & 14\end{array}$

2.3 Einfluss der weiteren sozialen Umwelt auf die Kaufmerkmale $\begin{array}{ll}\text { des Konsumenten } & 23\end{array}$

2.4 Kulturelle Aspekte im grenzüberschreitenden Kaufverhalten 25

2.4.1 Eingliederung der deutsch - niederländischen Kulturen im europäischem Vergleich

2.4.2 Lebensstile und Kaufverhalten im deutsch-niederländischen Vergleich 36

2.5 Zusammenfassung des Kapitels $\quad 42$

Kapitel 3: Konzeptuelle und empirische Grundlagen der Kundenbindungsforschung $\quad 45$

$\begin{array}{lll}3.1 & \text { Einleitung } & 46\end{array}$

3.2 Konsumentenbindung als zentrales Thema im Handel 46

3.3 Entwicklung und Stand der Kundenbindungsforschung 56

3.4 Kundenloyalität als Bestimmungsfaktor der Kundenbindung 63

3.4.1 Konzeptionalisierungsvorschläge ausgewählter Kundenbindungsmodelle $\quad 64$

3.4.2 Ursachenanalyse des Zusammenhangs zwischen Kundenzufriedenheit $\begin{array}{ll}\text { und Kundenbindung } & 68\end{array}$

3.4.3 Analyse der leistungsabhängigen Determinanten der Kundenbindung 69 
iv | Inhaltsverzeichnis

3.4.4 Leistungsunabhängige Derterminanten der Kundenbindung

3.4.5 Soziale, situative und soziodemographische Determinanten der Kundenbindung

3.5 Kundenzufriedenheit und Kundenbindung als Marketingziel der Einzelhandelsunternehmen

3.6 Zusammenfassung der Ergebnisse und Ableitung von Schlussfolgerungen

Kapitel 4: Einstellungstheorie als Erklärungsansatz der Kundenbindung 85

$\begin{array}{lll}4.1 & \text { Einleitung } & 86\end{array}$

4.2 Einstellungstheorie und Kundenbindung $\quad 86$

4.3 Messung der Einstellung und der Kundenloyalität zur Erklärung der Kundenbindung $\quad 99$

4.4 Entwicklungsstand der Einstellungsforschung 105

4.5 Eignung der Einstellungsforschung als Erklärungsansatz $\begin{array}{ll}\text { der Kundenbindung } & 109\end{array}$

$\begin{array}{lll}\text { 4.6 Zusammenfassung des Kapitels } & 111\end{array}$

\section{Kapitel 5: Spezifische Struktur des grenzüberschreitenden}

$\begin{array}{ll}\text { Kundenbindungsmodells } & 113\end{array}$

$\begin{array}{lll}5.1 & \text { Einleitung } & 114\end{array}$

5.2 Kundenbindungsmodell unter Berücksichtigung der neueren

Einstellungstheorie und der Auswirkung der Grenze auf Einstellungen der Konsumenten

5.3 Einfluss der Verhaltenskontrolle und der wahrgenommenen Selbstrelevanz auf das Kundenbindungsmodell im Grenzgebiet 122

5.4 Zusammenfassung der Struktur und der Zielsetzung $\quad 125$ 
Kapitel 6: Methodik der Modellschätzung 127

$\begin{array}{lll}\text { 6.1 } & \text { Einleitung } & 128\end{array}$

6.2 Einführung in die allgemeine Modellspezifikation $\quad 128$

6.3 Konkretisierung der Fragestellungen mit Hilfe von Focus-Gruppen 132

6.4 Eignung multivariater Analysemethoden für die Modellschätzung 136

6.5 Entwicklung des Prüfschemas 136

6.6 Beurteilung des Strukturmodells 142

6.7 Datenerhebung im Befragungsgebiet 146

$\begin{array}{lll}\text { 6.8 Zusammenfassung des Kapitels } & 149\end{array}$

Kapitel 7: Überprüfung des Modells im Feld 151

$\begin{array}{lll}7.1 & \text { Einleitung } & 152\end{array}$

7.2 Statistische Analysen der Ergebnisse 152

7.2.1 Analyse der befragten niederländischen Konsumenten 153

$\begin{array}{lll}\text { 7.2.2 Analyse der befragten deutschen Konsumenten } & 157\end{array}$

7.2.3 Vergleich der befragten niederländischen und deutschen

Konsumentengruppen hinsichtlich Einstellung, Motive und

$\begin{array}{ll}\text { Bindungsverhalten } & 160\end{array}$

7.2.4 Analyse weiterer Unterschiede und Zusammenfassung 173

7.3 Überprüfung der hypothetischen Konstrukte 175

7.3.1 Reliabilitätsprüfung der Daten mit Cronbachs Alpha 175

7.3.2 Überprüfung des Modells mit Hilfe der Faktorenanalyse 177

7.3.3 Überprüfung des Modells mit Hilfe der Clusteranalyse 183

7.4 Empirische Ergebnisse und Evaluierung der Wirkungszusammenhänge $\begin{array}{ll}\text { und Modellbeurteilung } & 193\end{array}$

7.4.1 Evaluation der Wirkungszusammenhänge und Modellbeurteilung $\begin{array}{ll}\text { der Kundenbindung der niederländischen Konsumenten } & 197\end{array}$

7.4.2 Evaluation der Wirkungszusammenhänge und Modellbeurteilung $\begin{array}{ll}\text { der Kundenbindung der deutschen Konsumenten } & 201\end{array}$

7.4.3 Vergleich der Evaluation der Wirkungszusammenhänge und Modellbeurteilung der Kundenbindung zwischen niederländischen und deutschen Konsumenten $\quad 204$

7.4.4 Fazit zu den Befunden und Hypothesenüberprüfung 211

$\begin{array}{lll}7.5 & \text { Zusammenfassung des Kapitels } & 217\end{array}$ 
vi | Inhaltsverzeichnis

Kapitel 8: Empirische Ergebnisse und Schlussfolgerungen 223

$\begin{array}{lll}\text { 8.1 } & \text { Einleitung } & 224\end{array}$

8.2 Zusammenfassung der empirischen Ergebnisse der Untersuchung 224

8.2.1 Das modellierte Kundenbindungsmodelle zur Erklärung

der grenzüberschreitenden Kundenbindung unter kulturellen Aspekten

8.2.2 Identifizierung der Determinanten der Kundenbindung in ausgewählten Einzelhandelsbranchen in einer Grenzregion $\quad 229$

8.2.3 Identifizierung der Stimulanzen zum grenzüberschreitenden Einkauf und Einkaufsstättentreue

8.2.4 Identifizierung relevanter Determinanten der Kundenbindung in einer $\begin{array}{ll}\text { Grenzregion } & 231\end{array}$

8.3 Vergleich der Wirkungszusammenhänge und Modellbeurteilung 232

$\begin{array}{lll}\text { 8.4 Zusammenfassung Kapitel } & 233\end{array}$

Kapitel 9: Zusammenfassung $\quad 235$

$\begin{array}{lll}9.1 & \text { Einleitung } & 236\end{array}$

9.2 Diskussion, Legitimation und Schlussfolgerungen 236

$\begin{array}{lll}9.3 & \text { Fazit } & 244\end{array}$

$\begin{array}{ll}\text { Literaturverzeichnis } & 247\end{array}$

$\begin{array}{ll}\text { Anhang } & 265\end{array}$

Niederländische Zusammenfassung 289

$\begin{array}{ll}\text { Lebenslauf } & 295\end{array}$ 


\section{Abbildungsverzeichnis:}

Abb. $1.1 \quad$ Struktur der Studie $\quad 1$

Abb. 2.1 Beurteilung der deutschen Einkaufsstätten am linken

$\begin{array}{ll}\text { Niederrhein durch niederländische Konsumenten } & 17\end{array}$

Abb. 2.2 Bevorzugte Einkaufsstädte der Niederländer im Grenzgebiet 18

Abb. $2.3 \quad$ Beziehungen zwischen Kultur und Verhalten 26

Abb. $2.4 \quad$ Usunier's Kultur-Zonen von Europa 28

Abb. 2.5 Kasper's Cluster der europäischen Länder Kulturen 29

Abb. 3.1 Ziele der Kundenbindungsforschung 63

Abb. 3.2 Konzeptualisierung des Konstruktes Kundenbindung 67

Abb. 3.3 Der Begriff der Kundenbindung als Zustand und Tätigkeit 68

Abb. 3.4 Das C/D Paradigma 74

Abb. 3.5 „means end disconfirmation“ Modell 75

Abb. 3.6 Die Folgen des komperativen Konkurrenzvorteils 76

Abb. 3.7 Die Entwicklung von Kundenzufriedenheit -

$\begin{array}{ll}\text { Kundenbindung-Kundenloyalität bis zur Loyalty } & 77\end{array}$

Abb. 3.8 Das Evoket Set der selektiven Einkaufsstättenwahl 79

Abb. 3.9 Auswahl möglicher ökonomischer und außerökonomischer

Ziele von Kundenbindungsaktivitäten $\quad 80$

Abb. 3.10 Kundenzufriedenheit entscheidet über Markenloyalität und Händlertreue 81

Abb. 4.1 Operationalisierung der Einstellung zu einer Einkaufsstätte 101

Abb. 4.2 Zusammenhang der Messung von Kundenzufriedenheit,

$\begin{array}{ll}\text { Kundenloyalität und Kundenbindung } & 103\end{array}$

Abb. 4.3 Erweiterte Variante des Aufbaues von „shopper relationship proneness“ auf der Theorie des geplanten Verhaltens

Abb. 5.1 Bedeutung der sozialen Norm und der wahrgenommenen

Selbstrelevanz auf die grenzüberschreitende Kundenbindung 122

Abb. 6.1 Analyseschritte der Konstruktoperationalisierung 139

Abb. 6.2 Annahmen im Partial Least Squares-Modell 140

Abb. 7.1 Befunde zum Bindungsmodell der niederländischen Konsumenten 195

Abb. 7.2 Befunde zum Bindungsmodell der deutschen Konsumenten 196 



\section{Tabellenverzeichnis:}

Tab. 1.1 Vergleich des deutsch-niederländischen Handels auf $\begin{array}{ll}\text { volkswirtschaftlichem Niveau } & 3\end{array}$

Tab. 1.2 Vergleich des deutsch-niederländischen Teils der Euregio Rhein-Maas 4

Tab. 1.3 Ergebnisse der BRO Untersuchung 5

Tab. 2.1 Die Bevölkerungsanteile der Regionen in der Euregio Maas-Rhein 16

Tab. 2.2 Arbeitslosenquote in der Euregio (http.//euregio.etil.nl) 19

Tab. 2.3 Indexwerte nach Hofstede für Niederlande, Deutschland 33

Tab. 2.4 Vergleich der Anforderungen der Konsumenten an ein Handelsgeschäft in den Niederlanden und Deutschland $\quad 39$

Tab. 2.5 Unterschiede zwischen deutschen und niederländischen Konsumenten $\begin{array}{ll}\text { nach BRO Studie } & 41\end{array}$

Tab. 3.1 Einordnung der deutschen und englischen Begriffe 49

Tab. $3.2 \quad$ Wirkungseffekte der Kundenbindung 59

$\begin{array}{lll}\text { Tab. 6.1 Quellen der Messkriterien } & 132\end{array}$

Tab. 6.2 Konstrukte, Indikatoren und ihre Bedeutung im Rahmen des $\begin{array}{ll}\text { Strukturgleichungsmodells } & 142\end{array}$

$\begin{array}{lll}\text { Tab. 6.3 Gütebeurteilung des Strukturmodells } & 144\end{array}$

Tab. 6.4 Gütemaße reflektiver Messmodelle 145

Tab. 6.5 Aufteilung der Befragung nach Befragungsort und Befragungstag 147

Tab. 6.6 Aufteilung der befragten Konsumenten nach Nationalität, $\begin{array}{ll}\text { Geschlecht und Alter } & 148\end{array}$

Tab. 7.1 Die soziodemografische Verteilung der befragten niederländischen Konsumenten 153

Tab. 7.2 Verfügbares Einkommen der Einwohner des niederländischen Befragungsgebietes im Jahre 2000 im Vergleich zu 1999

Tab. 7.3 Verteilung der Personen pro Haushalt der befragten niederländischen Konsumenten sowie Verteilung der Haushaltsnettoeinkommen $\quad 154$

Tab. 7.4 Vergleich niederländischer Nettoeinkommen nach Haushaltsgröße 154

Tab. 7.5 Herkunftsort der in Deutschland befragten niederländischen Konsumenten 155

Tab. 7.6 Wohnort und Einkaufsort der niederländischen Konsumenten 156

Tab.7.7 Nutzung der Verkehrsmittel durch niederländische Konsumenten zum Einkauf im Nachbarland 
$\mathrm{x}$ | Inhaltsverzeichnis

Tab. 7.8 Die soziodemografische Verteilung der befragten deutschen

Konsumenten

Tab. 7.9 Verfügbares Einkommen der Einwohner des deutschen

Befragungsgebietes im Jahre 2002

158

Tab. 7.10 Verteilung der Personen pro Haushalt der befragten deutschen

Konsumenten sowie Verteilung der Haushaltsnettoeinkommen $\quad 158$

Tab. 7.11 Vergleich deutscher Nettoeinkommen nach Haushaltsgröße 159

Tab. 7.12 Wohnort und Einkaufsort der deutschen Konsumenten 159

Tab. 7.13 Nutzung der Verkehrsmittel durch niederländische Konsumenten

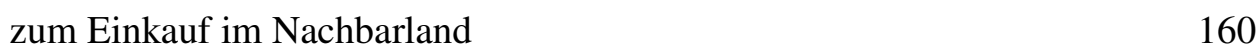

Tab. 7.14 Verteilung der Einkäufe in \% nach Probandengruppe 161

Tab. 7.15 Gründe der Probanden zum Einkauf im Nachbarland 162

$\begin{array}{lll}\text { Tab. 7.16 Bevorzugte Betriebsform der befragten Konsumenten } & 163\end{array}$

Tab. 7.17 Welche Probandengruppe kauft wo ein? 163

Tab. 7.18 Motive und Einstellungen der befragten Konsumenten zum

$\begin{array}{ll}\text { Einkauf im Nachbarland } & 165\end{array}$

Tab. 7.19 Mittelwertvergleich Werturteile der sozialen Gruppe 166

Tab. 7.20 Mittelwertvergleich der Wichtigkeit „soziale Norm“ 166

Tab. 7.21 Mittelwertvergleich der Bedeutung ,soziale Norm“ 167

Tab. 7.22 Mittelwertvergleich der Gesamtzufriedenheit der Konsumenten mit Stammgeschäft im Nachbarland

167

Tab. 7.23 Mittelwertvergleich der Gesamtzufriedenheit der Konsumenten mit $\begin{array}{ll}\text { Einkauf im Nachbarland } & 167\end{array}$

Tab. 7.24 Mittelwertvergleich ,persönliche Norm“ 168

Tab. 7.25 Mittelwertvergleich ,wahrgenommene Verhaltenskontrolle“ 169

Tab. 7.26 Mittelwertvergleich ,wahrgenommene Selbstrelevanz“ 169

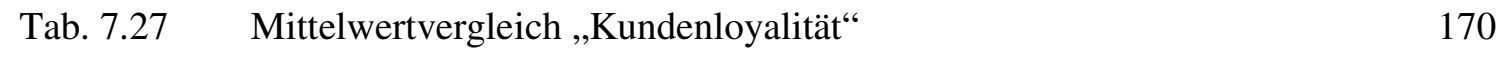

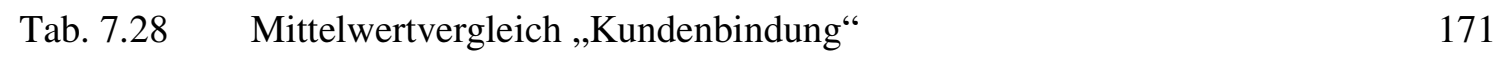

Tab. 7.29 Gegenüberstellung der Fragen nach Konsumentengruppe mit hoher Zustimmung 172

Tab. 7.30 Realibilitätskennzahlen der durchgeführten Befragung 176

$\begin{array}{lll}\text { Tab. 7.31 Eignung der Ausgangsdaten für die Faktorenanalyse } & 178\end{array}$

Tab. 7.32 Hauptachsenanalyse der niederländischen Konsumenten 179

Tab. 7.33 Hauptfaktorenanalyse der deutschen Konsumenten 181

Tab. 7.34 Vergleich der Faktoren im niederländischen und deutschen Modell 182 
Tab. 7.35 Vergleich der niederländischen und deutschen Cluster

Tab. 7.36 Quality Criteria der Daten der niederländischen Konsumenten

Tab. 7.37 Befunde des Strukturmodells

Tab. 7.38 Niederländisches Structural Model-Boot Strap

199

Tab. 7.39

Korrelation der latenten Variablen

200

Tab. 7.40 Quality Criteria der Daten der deutschen Konsumenten

201

Tab. 7.41 Befunde des deutschen Strukturmodells 202

Tab. 7.42 Deutsches Structural Model-Boot Strap 202

Tab. 7.43 Korrelation der latenten Variablen (D) 203

Tab. 7.44 Vergleich der Befunde der Höhe der Pfadkoeffizienten des Strukturmodells

Tab. 7.45 Außenladungen des PLS Modells 207

$\begin{array}{lll}\text { Tab. 7.46 Ausprägungen der Effektgröße } 208 & 208\end{array}$

Tab. 7.47 Ausprägungen der Effektgröße und ihr substanzieller Einfluss 210

Tab. 7.48 Untersuchungsergebnisse zur Überprüfung der Hypothesen 211

Tab. 7.49 Überblick über die Konstrukte der Kundenbindung für niederländische Konsumenten 214

Tab. 7.50 Überblick über die Konstrukte der Kundenbindung für deutsche Konsumenten

Tab. 7.51 Gegenüberstellung der Totaleffekte der deutschen und niederländischen Konstrukte der Kundenbindung 

Abkürzungsverzeichnis:

AGFI

C/D Paradigma

CRM

D

GKDZ SN

GLSS

GKDZ Kauf

GFI

$\mathrm{KB}$

$\mathrm{KL}$

NFI

NL

Pers. Norm

PLS

POS

S-O-R Paradigma

SN

TZSN

TZ

WRS

WSN

WVK
Adjusted Goodness of Fit Index

Confirmation/Disconfirmation Paradigma

Customer Relationship Management

Deutsche Konsumenten

Bedeutung der sozialen Norm

Gesamtzufriedenheit

Gesamtzufriedenheit Kauf

Goodness of Fit Index

Kundenbindung

Kundenloyalität

Normed Fit Index

Niederländische Konsumenten

Persönliche Norm

Least Squares-Verfahren

Point of sale

Stimulus Organismus Response

Soziale Norm

Werturteile der sozialen Gruppe

Teilzufriedenheit

Wahrgenommene Selbstrelevanz

Wichtigkeit der sozialen Norm

Wahrgenommene Verhaltenskontrolle 



\section{Anhang:}

Anhang 1 Fragebogen für niederländische Konsumenten 265

Anhang 2 Fragebogen für deutsche Konsumenten 268

Anhang 3 Einstellung der niederländischen Konsumenten zum

Einkaufsort und zur Einkaufsstätte

Anhang 4 Einstellung der deutschen Konsumenten zum

Einkaufsort und zur Einkaufsstätte

Anhang 5 Konstrukte, Indikatoren und Bedeutung im

Rahmen des Strukturgleichungsmodells $\quad 274$

Anhang $6 \quad$ Clusteranalyse der Fälle für deutsche Konsumenten 275

Anhang $7 \quad$ „Die Minimalkunden“(Cluster 1) deutsche Kunden 277

Anhang 8 „Die preisbewussten Kunden von Filialbetrieben“(Cluster 2) $\begin{array}{ll}\text { deutsche Kunden } & 278\end{array}$

Anhang 9 „Die Gelegenheitskunden“ (Cluster 3) deutsche Kunden 279

Anhang 10 „Die begeisterten Fachgeschäftskunden“ Cluster 4) deutsche Kunden 280

Anhang 11 Clusteranalyse der Fälle für niederländische Konsumenten 281

Anhang 12 „Die Minimalkunden“(Cluster 1) niederländische Kunden 283

Anhang 13 „Die Gelegenheitskäufer“ (Cluster 2) niederländische Kunden 284

Anhang 14 „Die begeisterten für Stammgeschäfte (Cluster 3) niederländische Kunden 285

Anhang 15 „Die schnellen zielgerichteten, preisorientierten Käufer“ 286

Anhang 16 Pfadkoeffizienten und Effektstärke der einzelnen Konstrukte 287 



\section{$\underline{\text { Kapitel 1: Einführung in die Untersuchungsthematik }}$}

Der Studie liegt folgende Struktur zu Grunde: Der erste Teil beschränkt sich auf die theoretischen Grundlagen. Im zweiten Teil wird das Modell in der Praxis überprüft. Zum Abschluss werden die wichtigsten Ergebnisse der Studie zusammengefasst, diskutiert und in einen theoretischen und übergreifenden Kontext gebracht.

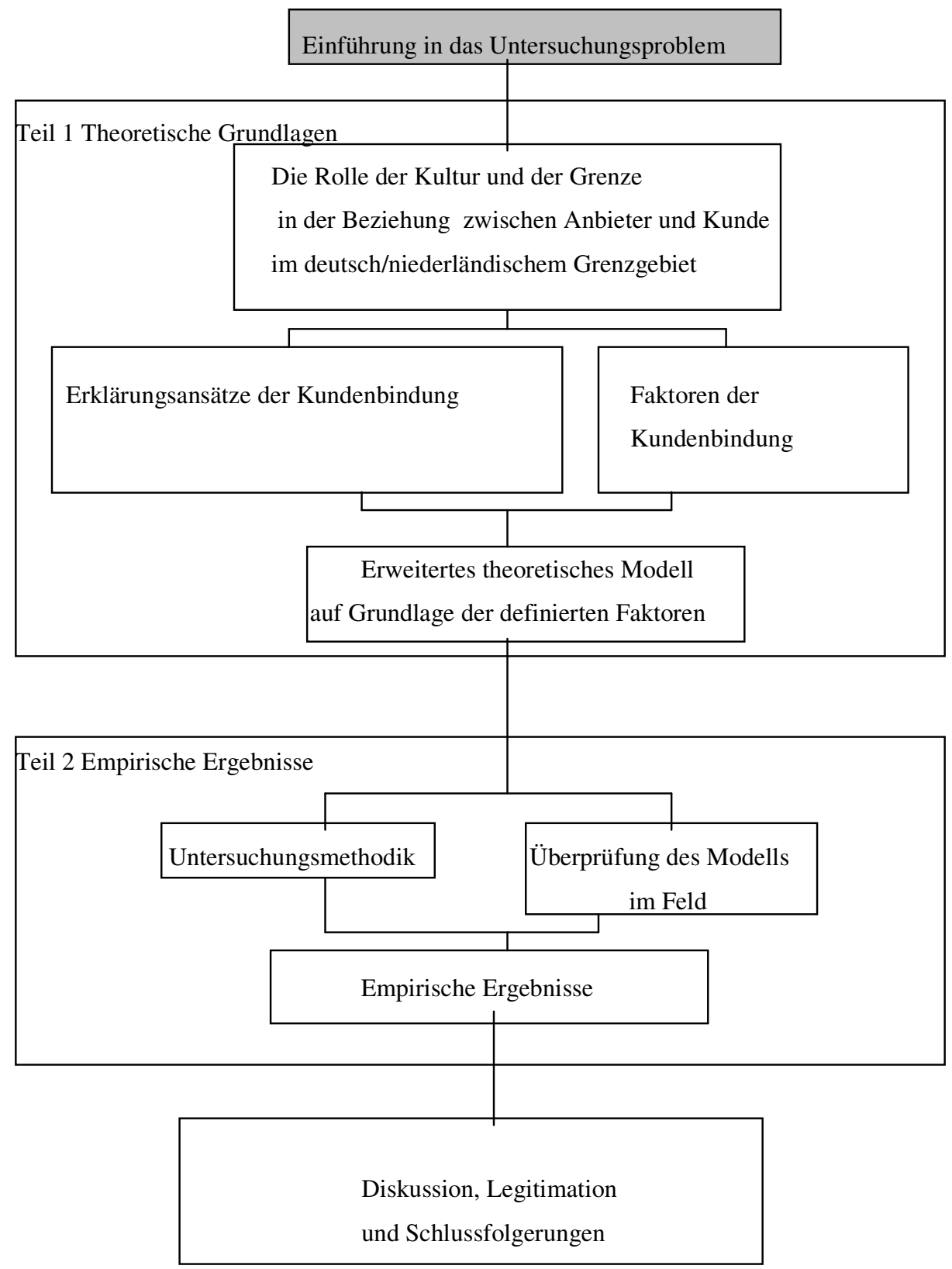

Abb. 1.1:

Struktur der Studie 


\subsection{Einleitung}

Unternehmen verfolgen in einem marktwirtschatlichen System den Zweck, sowohl eine angemessene Rendite auf das eingesetzte Kapital zu erwirtschaften, als auch ein profitables Wachstum zu erreichen. Insbesondere in einem Grenzgebiet zwischen zwei benachbarten Ländern ist dieses Ziel für den Einzelhandel nur zu erreichen, wenn ein Verständnis für das Verhalten und ein Wissen über das Verhalten des Konsumenten aus den angrenzenden Ländern in den Unternehmen vorhanden ist. Dies gilt im Besonderen für das deutschniederländische Grenzgebiet zwischen Maastricht/Aachen und Venlo/Mönchengladbach, da die Einkaufsorte auf beiden Seiten der Grenze kurze Distanzen voneinander aufweisen. Die vorliegende Arbeit setzt sich mit dieser Problematik auseinander. Zunächst wird im Folgenden die Ausgangssituation im Untersuchungsgebiet erläutert und das Forschungsanliegen beschrieben.

\subsection{Ausgangssituation der Untersuchung}

„Alles fängt an bei der Inkongruenz. Für Holländer ist Deutschland zunächst einmal ein großes, nahes und etwas unzuverlässiges Faktum. Man hat es im Auge und sich vom Leib zu halten. Für Deutsche ist Holland - abgesehen vom Reisebüroklischee des Windmühlen- und Tulpenparadies - im Grunde ein Land, in dem die Leute versehentlich holländisch sprechen statt deutsch“ (Von der Dunk, 1986). Dieses Zitat verdeutlicht, wie wichtig es ist über Klischees und Stereotypen und über negative und positive Aspekte insbesondere zwischen benachbarten Völkern nachzudenken. Klischees sagen allerdings nichts über die Realität aus. Deutschland ist der wichtigste Handelspartner der Niederlande, die Niederlande ein wichtiger Handelspartner für Deutschland. Die Niederlande und Deutschland pflegen intensive Handelsbeziehungen miteinander. Der deutsch-niederländische Außenhandel belief sich 2003 nach Zahlen des Bundesministeriums für Wirtschaft auf insgesamt 97,7 Mrd. Euro. Die Ausfuhren der Niederländer nach Deutschland betrugen dabei 56,7 Mrd. Euro; die Einfuhren dagegen 41 Mrd. Euro. Die niederländischen Ausfuhren nach Deutschland betragen diesen Angaben zufolge rund fünfundzwanzig Prozent des niederländischen Gesamtexports. Die größten Posten im bilateralen Handel sind (2002) Industrieerzeugnisse (Einfuhr in die Niederlande: 33,7 Mrd. Euro; Ausfuhr nach Deutschland: 23,29 Mrd. Euro); Mineralische Brandstoffe, Rohöl, Erdgas, Schmiermittel (Einfuhr: 1,1 Mrd. Euro; Ausfuhr 22,6 Mrd. Euro) und landwirtschaftliche Erzeugnisse (Einfuhr: 5,0 Mrd. Euro; Ausfuhr: 11,5 Mrd. Euro). Der deutsch-

niederländische Außenhandel ist damit einer der größten bilateralen Handelsströme über- 
haupt. Deutschland ist und bleibt weiterhin der wichtigste Absatzmarkt für die niederländische Wirtschaft. Beim deutschen Außenhandel liegen die Niederlande auf dem 5. Platz hinter Frankreich, den Vereinigten Staaten, Großbritannien und Italien (www.auswärtiges-amt.de; www.deutschland.delaufeinenblick/index) .

Werden die Volkswirtschaften beider Länder miteinander verglichen, zeichnet sich folgendes Bild ab:

Handelsbeziehungen Niederlande-Deutschland

Bevölkerung

Bruttoinlandsprodukt insgesamt in Euro zu jew. Marktpreisen

Bruttoinlandsprodukt pro Kopf der Bevölkerung

Reales Wachstum in \%

Ausfuhr

Einfuhr

Saldo

Einfuhr aus Nachbarland (absolut)

Einfuhr (in \%)

Ausfuhr nach Nachbarland (absolut)

Ausfuhr (in \%)

Direktinvestitionen NL-D, D-NL

Wichtigste Exportgüter

$\begin{array}{ll}\text { Niederlande } & \text { Deutschland } \\ \mathbf{2 0 0 3}^{\mathbf{1}} & \mathbf{2 0 0 4} \\ \text { 16.224.000 Mio. } & 82.500 .000 \text { Mio. } \\ \text { 454 Mrd. Euro } & 2178,20 \text { Mrd. Euro } \\ \text { 25.300 Euro } & 26400 \text { Euro } \\ -0,9 \% & +1,7 \% \\ \text { 232,4 Mrd. Euro } & 731,0 \text { Mrd. Euro } \\ \text { 204,4 Mrd. Euro } & 575,4 \text { Mrd. Euro } \\ \text { 28 Mrd. Euro } & 155,6 \text { Mrd. Euro } \\ \text { 41 Mrd. Euro } & 56,7 \text { Mrd. Euro } \\ \text { 19,8 \% } & 9,8 \% \\ \text { 56,7 Mrd. Euro } & 41 \text { Mrd. Euro } \\ \text { 24,4\% } & 5,6 \% \\ \text { 14 Mrd. Euro } & 48 \text { Mrd. Euro } \\ \text { Mineralische Brandstoffe, } & \text { Autos und Autoteile, } \\ \text { Rohöl, Erdgas, Schmier- } & \text { Maschinen, chemische } \\ \text { mittel, landwirt- } & \text { Erzeugnisse } \\ \text { schaftliche Erzeugnisse } & \end{array}$

Tab. 1.1: Vergleich des deutsch-niederländischen Handels auf volkswirtschaftlichem Niveau (www.auswärtiges-amt.de; www.deutschland.delaufeinenblick/index)

Einen Ländervergleich auf euregionaler Ebene durchzuführen ist nicht möglich. Es liegen keine Vergleichswerte zwischen den regionalen Gebieten Limburg und Nordrhein-Westfalen vor, da im Rahmen der Landesstatistiken nur die Regionalgebiete mit dem gesamten jeweiligen Nachbarland in die amtliche Statistik aufgenommen werden. Allerdings ist von Etil $(2005)^{2}$ in den Jahren 2004 und 2005 mit der Kamer van Koophandel Maastricht, Eupen, Lüttich, Hasselt und der IHK Aachen eine Untersuchung zu den Motiven der Unternehmen zum Export und Import aus dem Nachbarland durchgeführt worden. Im Rahmen dieser Untersuchung wurden die niederländisch-deutschen Beziehungen im grenznahen Bereich beider Länder analysiert. Nach dieser Studie macht der erwirtschaftete Umsatz der Unternehmen aus den Exporten innerhalb dieser Euregio insgesamt 6\% des Gesamtumsatzes aus. Insgesamt $12 \%$ des gesamten Einkaufs tätigen die Unternehmen von Unternehmen aus Nachbarländer innerhalb der Euregio. Insbesondere Unternehmen aus dem belgischen Teil der Euregio haben

\footnotetext{
${ }^{1}$ Die Zahlen für 2004 liegen noch nicht vor.

${ }^{2}$ Befragt wurden Unternehmen aus dem industriellem Bereich
} 
4 | Kapitel 1

einen hohen Anteil an den Import- und Exportaktivitäten. Dennoch erscheint für die meisten Unternehmen innerhalb des deutsch-niederländischen Grenzgebietes die Erschließung eines euregionalen Absatzgebietes schwieriger zu sein als der Import von Gütern.

Einen Unterschied aufgrund der unterschiedlichen Größe gibt es innerhalb der beiden Gebiete im erwirtschafteten Bruttoinlandsprodukt (BIP). Insgesamt wird im deutschen Teil ein gröBeres Bruttoinlandsprodukt erwirtschaftet. Wird allerdings das BIP pro Kopf in Euro miteinander verglichen, so liegt das niederländische Gebiet mit Euro 24.226,20 vor dem deutschen Gebiet mit Euro 21.112,14.

$\begin{array}{lcc}\begin{array}{l}\text { Handelsbeziehungen Niederlande-Deutschland in der Euregio } \\ \text { Rhein-Maas }\end{array} & \begin{array}{c}\text { Niederlande } \\ \text { Euregio 2002 } \\ 747.835\end{array} & \begin{array}{l}\text { Deutschland } \\ \text { Euregio 2002 } \\ \text { Bevölkerung }\end{array} \\ \begin{array}{l}\text { Bruttoinlandsprodukt insgesamt in Euro zu jeweiligen. } \\ \text { Marktpreisen }\end{array} & 15.613,20 & 26.680,40 \\ \text { Bruttoinlandsprodukt pro Kopf der Bevölkerung } & 24.226,20 & 21.112,14\end{array}$

Tab. 1.2: $\quad$ Vergleich des deutsch-niederländischen Teils der Euregio Rhein-Maas (www.euregio-maas-rhein.de )

Die Attraktivität des Kammerbezirk Aachen für niederländische Unternehmer als Importgebiet ist insbesondere in der geringen Entfernung, geringe Sprach- und Kulturunterschiede und der guten Qualität der Produkte aus dem deutschen Gebiet zu sehen. Die deutschen Unternehmen im Kammerbezirk Aachen sind stark an einer Zusammenarbeit innerhalb der Euregio interessiert. Dies belegt auch die Etil-Analyse, nach der „fast zwei Fünftel aller Betriebe im Kammerbezirk Aachen exportiert und drei Zehntel importiert.“ Ungefähr vier Prozent des Umsatzes der exportierenden Betriebe und sieben Prozent des Einkaufs dieser Betriebe stammt aus der Euregio (Etil, 2004, S.II). Auf der niederländischen Seite gibt es dagegen relativ wenig Betriebe, die innerhalb der Euregio exportieren oder importieren. Wenn allerdings in der Euregio exportiert wird, so hat dies eine hohe Bedeutung für die Unternehmen (Etil, 2005, S. XIV). Für produzierende Unternehmen liegt somit mittlerweile eine qualitative Betrachtung des Außenhandels vor.

Der europäische Einzelhandel sieht sich seit Anfang der 90er Jahre einem starken Veränderungsprozess unterworfen. Der Einzelhandel sieht sich konfrontiert mit stagnierenden und rückläufigen Bevölkerungszahlen, aber auch sich verändernden Betriebsformen, die insbesondere den klassischen Einzelhandel, aber auch kleinere und mittelgroße Städte berühren. Eine verschärfte Konkurrenzsituation zwischen den aufstrebenden Betriebsformen wie den 
Discountern und dem klassischen Einzelhandel ist die Folge. Zahlreiche Handelsunternehmen versuchen stagnierende Umsätze durch Flächenexpansion zu kompensieren, oder aber das Einzugsgebiet durch erhebliche Werbeanstrengungen zu erweitern. In grenznahen Gebieten stellt sich dabei die Frage, ob Konsumenten jenseits der Grenze von diesen grenzüberschreitenden Handelsunternehmen angesprochen werden können. Ferner, ob Kundenbindung grenzüberschreitend durch die Handelsleistung entsteht, oder ob gerade die Grenze - das Andere - eine besondere Anziehungskraft auf den Konsumenten ausübt. Sofern es den Handelsunternehmen gelänge, Unterschiede deutlich zu machen, könnte hierdurch für den Einzelhandel ein neues Kundenpotential erschlossen werden. Im Jahre 2004 ist im Auftrag der Kamer van Koophandel Zuid-Limburg, der Provincie-Limburg, sowie den Städten Sittard/Geleen, Maastricht und Heerlen eine Untersuchung zum grenzüberschreitenden Einkauf der Konsumenten in Süd-Limburg und den angrenzenden Regionen durchgeführt worden (BRO, 2005). Einige Ergebnisse dieser Untersuchung sind:

\begin{tabular}{|l|l|}
\hline $\begin{array}{l}\text { Anteil der in anderen Regionen kaufenden Konsumen- } \\
\text { ten }\end{array}$ & $87 \%$ aller Konsumenten gehen in anderen Regionen einkaufen \\
\hline $\begin{array}{l}\text { Einkaufsvolumen der Konsumenten von nicht-täglichen } \\
\text { Artikeln außerhalb der eignen Region (Niederländer, } \\
\text { Deutsche und Belgier gemeinsam) }\end{array}$ & $€ 1,26$ Miliarden \\
\hline Konsumentenausgaben & $\begin{array}{l}\text { Deutsche Konsumenten geben 2,35 mal mehr in den Niederlanden für Konsumartikel } \\
\text { aus als Niederländer in Deutschland }\end{array}$ \\
\hline $\begin{array}{l}\text { Die wichtigsten Einkaufsstädte } \\
\text { (außer Lebensmittel) }\end{array}$ & Maastricht, Aachen, Heerlen, Maasmechelen Village, Sittard, Hasselt und Roermond \\
\hline
\end{tabular}

Tab. 1.3 Ergebnisse der BRO Untersuchung (BRO, 2005)

In dieser Untersuchung sind belgische, niederländische und deutsche Konsumenten nach ihren Kaufgewohnheiten befragt und die Antworten ausgewertet worden. Die Untersuchung der BRO erläutert die in den unterschiedlichen Regionen vorhanden Kaufströme. Ferner werden die Charakteristiken der in diesen Regionen liegenden Einkaufsgebiete sowie die Konsumentenmotive beschrieben, die Einfluss auf den Einkauf haben. Konzeptionell wurde bislang allerdings der Begriff der Kundenbindung im deutsch-niederländischem Grenzgebiet nur unvollständig durchdrungen. Die zum Einkauf für die Konsumenten attraktivsten Städte befinden sich im deutsch-niederländischem Grenzgebiet. Mit einem Volumen von über einer Milliarde Euro erreicht dieser ,private“ Außenhandel eine attraktive Größe. 
6 | Kapitel 1

\subsection{Relevanz und Problemstellung des Themas}

Durch die Außenhandelsstatistiken der Niederlande und Deutschland ist sehr viel Datenmaterial über den Außenhandel der beiden Länder vorhanden. Auch für die Grenzregionen ist Zahlenmaterial zumindest ansatzweise vorhanden und selbst die Motive der Unternehmer Im- und Export-Geschäfte zu tätigen sind untersucht worden. Doch auch BRO (2005) stellt fest, dass über grenzüberschreitende Einkaufsgewohnheiten der Konsumenten in den Grenzgebieten wenig bekannt ist. Insbesondere ist noch keine Analyse der Endkonsumenten vorhanden, die ergründet, warum deutsche und niederländische Konsumenten zum Einkauf in das Nachbarland fahren, welche Motive sie haben (gleiche oder unterschiedliche) und ob sie sich an eine Einkaufsstätte im Nachbarland langfristig binden wollen, obwohl es doch einen regen Austausch von Gütern auf Import- und Exportbasis beider Länder gibt. Ferner fehlen Arbeiten, die analysieren, welche Aspekte die Einstellung der Konsumenten zum Einkauf im Nachbarland prägen. Es fehlen auch Untersuchungen, die sich mit den Motiven der Konsumenten zum Einkauf im Nachbarland auseinandersetzen, obwohl es doch eine große Anzahl von importierten Produkten in den Einkaufsstätten des jeweiligen Nachbarlandes gibt. Aus diesem Grund setzt sich diese Arbeit mit den Fragen der Kundenbindung im Einzelhandel im grenznahen Gebiet auseinander, den Motiven und Einstellungen, die Konsumenten haben, um im jeweiligen Nachbarland einzukaufen und die Produkte zu ,,importieren“, die sie für private Zwecke benötigen. Die Zielsetzung der Arbeit ist somit zu klären, ob Konsumenten aus Deutschland und aus den Niederlanden, die im Grenzgebiet zwischen Maastricht/Aachen und Mönchengladbach/Venlo einkaufen, an eine Einkaufsstätte (Textil, Schuhe, Elektro) im Nachbarland (innerhalb einer Grenzregion) gebunden werden können und welche Faktoren diese Bindung fördern (siehe auch Anhang 1 und 2, Fragebogen für Probanden).

In der Literatur findet sich eine fast unüberschaubare Anzahl von Veröffentlichungen zum Thema Kundenzufriedenheit und Kundenbindung. Die Schwerpunkte dieser Arbeiten liegen in der Beschreibung der Faktoren zur Kundenbindung in einem Ein-Land-Eine-BrancheMarkt. Kaum untersucht worden sind die Auswirkungen der Kundenbindung in einem grenzüberschreitenden Raum. Erst Odekerken-Schröder (1999, 2003) zeigt in ihrer Arbeit eine vergleichende Studie verschiedener Länder auf. Sie konzentriert sich allerdings auf einen Vergleich des niederländisch, belgischen und amerikanischen Marktes und vergleicht in diesen Ländern die Textil- und Lebensmittelbranche. Eine Analyse zwischen zwei größenmäßig sehr unterschiedlichen Ländern wie den Niederlanden und Deutschland in ihren Grenzregionen und die vergleichende Betrachtung der Faktoren der Loyalität und Bindung der Konsumenten 
zwischen großen Einzelhandelsfilialunternehmen und kleinen Einzelhandelsfachgeschäften in ausgesuchten Branchen existiert bis heute nicht. Auf Grund der zunehmenden Europäisierung und dem langfristigen Wegfall der Grenzen als (Handels-) barrieren, ist es wichtig, diese Faktoren zu kennen. Daher konzentriert sich die vorliegende Arbeit auf die Erklärung der Kundenbindung - oder (Nicht) Bindung grenzüberschreitend einkaufender Konsumenten in kleinen Einzelhandelsgeschäften und Filialunternehmen im Non-food Bereich. Die bisherigen Kundenbindungsuntersuchungen beziehen sich - sofern ein Ländervergleich stattfindet - auf einen Vergleich des gesamten Ländermarktes. Bisher wenig erforscht sind die Loyalitätseffekte in Grenzregionen. Ferner liegt wenig Wissen über die Entwicklung dieser Loyalitätseffekte vor und wie diese zu Kundenbindung führen.

Ausgangspunkt der vorliegenden Untersuchung sind die Gedanken und Ergebnisse der Untersuchung von Odekerken-Schröder aus dem Jahre 1999. Die Untersuchung beschäftigt sich mit der Rolle des Käufers im Aufbau einer Käufer-Verkäufer Beziehung. Das Ergebnis der Studie von Odekerken-Schröder ist, dass Kundenbeziehungen nicht allein vom Einzelhandelsgeschäft ausgehen, sondern dass effektive Kundenbeziehungen erst dann aufgebaut werden können, wenn auch die Kunden bereit sind, solche Beziehungen einzugehen. Die Studie zeigt ebenso, dass eine effektive Kundenbeziehung weniger von den Angeboten der Anbieter abhängig ist, als stärker von dem emotionalem Verhältnis der Konsumenten zum Anbieter. Anbieterstrategien können erfolgreich oder weniger erfolgreich sein, je nachdem, ob und wie umfassend sie zielgruppenorientiert ausgerichtet sind oder nicht. Dabei haben laut Odekerken-Schröder die ersten beiden Beziehungen nur indirekten Einfluss auf das Bindungsverhalten. Entfernung zum Einzelhandelsgeschäft, Monopolstellung des Geschäftes und persönliche Beziehungen sind kritische Größen, die das Einkaufsverhalten des Konsumenten beeinflussen. Diese direkten Einflüsse wirken sich in erster Linie auf Bekleidungsgeschäfte aus. Bei Lebensmittelgeschäften ist ihr Einfluss nicht so deutlich zu erkennen. Hier sind andere Faktoren bedeutsamer wie Entfernung/Erreichbarkeit, Preis und Übersichtlichkeit der Warenpräsentation.

Diese Zusammenhänge von Einstellung, Motiv und Bindung werden in der vorliegenden Untersuchung für alle Einzelhandelsgeschäfte (außer Lebensmittelhandel) zugrunde gelegt und der Einfluss der Landesgrenze sowie die dort relevanten Faktoren in den Focus gestellt. ${ }^{3}$

\footnotetext{
${ }^{3}$ Anmerkung des Autors: Dem Lebensmittelhandel kommt eine Sonderstellung am Markt zu, da er in erster Linie einen anderen Bedarf anspricht und der Kaufentscheidungsprozess nach anderen Kriterien abläuft.
} 
8 | Kapitel 1

\subsection{Besondere Einflüsse in der Grenzregion auf die Kundenbindung}

Das Phänomen Kundenbindung ist für jeden Anbieter unabhängig vom Wirtschaftszweig aber auch unabhängig von der Region - von betriebswirtschaftlichem Interesse. Grenzüberschreitende Wirtschaftsräume - vor allem, wenn sie sich im Strukturwandel befinden - haben dabei ihre Attraktivität dauerhaft unter Beweis zu stellen. Denn neben dem Kaufkraftabfluss aus der Region durch erhöhte Arbeitslosigkeit leiden diese Gebiete zusätzlich darunter, dass sie für Konsumenten außerhalb der Region als nicht attraktiv genug erscheinen. Die Einkaufsstätten dieser Regionen müssen daher mit weiteren Renditeeinbußen rechnen und es fehlt das notwendige Kapital für Neuinvestitionen. Doch nur durch ein attraktives Erscheinungsbild können sie Investoren für sich interessieren bzw. für Investoren interessant bleiben. Investoren, die Arbeitsplätze schaffen, ziehen Menschen an, die neben einem attraktiven Arbeitsplatz auch eine attraktive Region erwarten, die ihnen eine entsprechende Lebens- und Einkaufsqualität bietet. Zur Lebensqualität gehören aber nicht nur Freizeit-, Kultur- und Wohnangebote, sondern auch attraktive Einkaufsmöglichkeiten mit entsprechend attraktiv gestalteten Einkaufsstätten. Dies sind entscheidende Rahmenbedingungen, von denen Konsumenten ihre Bindungswilligkeit insbesondere in Grenzregionen abhängig machen und durch ihre Einkäufe zu einer Kaufkrafterhöhung in einer Region beitragen (Van Middendorp, Van der Velde und Vergossen, 1999, S. 13ff.). Um die Anforderungen der Konsumenten in Grenzregionen besser zu verstehen, ist die Entwicklung eines Modells zur Erklärung des Bindungsverhaltens erforderlich, das auch den kulturellen sowie den sozialen Aspekt der Kaufgewohnheiten berücksichtigt. Nur unter Berücksichtigung auch dieser Aspekte kann die Service- und Dienstleistungsqualität der Einzelhandelsstätten in einem Grenzgebiet anhand der Kundenwünsche erhöht werden.

Trotz der immer weiter voranschreitenden europäischen Integration weisen nationale Märkte in der Europäischen Union zum Teil erhebliche Unterschiede auf. Neben der nationalen Dimension europäischer Konsummärkte existiert im Sinne eines „Europa der Regionen“ eine regionale Dimension, die in den europäischen Grenzregionen besonders deutlich wird. So sollte man davon ausgehen, dass ein Zusammenwachsen regionaler Konsummärkte vor allem dort zu beobachten ist, wo Staaten unmittelbar aneinander grenzen. Insbesondere durch den europäischen Binnenmarkt und den hiermit verbundenen Wegfall der Grenzen müsste ein Konvergenzprozess in Gang gekommen sein, der auch die Disparitäten auf dem jeweiligen Konsumgütermärkten dies- und jenseits der ehemaligen Grenzen verringern sollte. Darüber hinaus sollte der grenzüberschreitende „Erfahrungsaustausch" der Konsumenten durch ver- 
wandschaftliche, freundschaftliche und berufliche Beziehungen zu einer Verstärkung eines Konsumtourismus führen. Insbesondere in einer Grenzregion ist es bereits historisch begründet, das die Konsumenten grenzüberschreitend einkaufen. In einer Grenzregion spielen dennoch insbesondere Fragen der unterschiedlichen Einkaufskulturen, Zielgruppen und Rahmenbedingungen eine entscheidende Rolle. Deshalb erscheint es in Bezug zur Gesamtattraktivität einer Region von Bedeutung, die besonderen Determinanten der Kundenbindung für eine Einkaufsstätte zu identifizieren, um daraus ein Modell zur Erklärung von Bindung in einem Konsumgütermarkt in Grenzregionen zu konzeptionieren und zu überprüfen, ob sie auch der Realität standhalten. Dadurch kann auch ein Beitrag zur Kaufkrafterhöhung in einer Region geleistet werden. Aus der Sicht der zunehmenden Integration der niederländischen und deutschen Märkte wächst das Interesse, Daten über Kundenströme / Kaufverhalten zu erheben sowie die Motive der Konsumenten im Nachbarland einzukaufen, zu erforschen.

\subsection{Forschungsziele der Arbeit}

Das zentrale Forschungsanliegen besteht darin, zu klären, ob sich die Einstellung der Konsumenten zum Einkauf im Nachbarland in einem Grenzgebiet im Zeitablauf angleicht und gleiche Konstrukte in gleicher Intensität für die Kundenbindung dieser Konsumenten verantwortlich sind. Sofern die Einflüsse durch die soziale Gruppe, durch die Kulturdimensionen und durch weitere Konstrukte in den beiden Ländern unterschiedlich sind, wirken auch in einer Grenzregion unterschiedliche Faktoren, mit unterschiedlicher Gewichtung auf die Einstellung der Konsumenten auf ihre Bindung zu einem Einzelhandelsgeschäft im Nachbarland ein. Das zentrale Forschungsanliegen der vorliegenden Arbeit besteht somit in der Entwicklung und empirischen Prüfung eines Modells zur Konsumententreue und -bindung an eine Einkaufsstätte, die aus Sicht des Konsumenten in einem anderen Land, aber einer gemeinsamen Grenzregion liegt. Dabei gilt es, diejenigen Bestimmungsfaktoren zu gewichten (persönliche, soziale Norm, wahrgenommene Selbstrelevanz), die einen Konsumenten veranlassen, eine bestehende Geschäftsbeziehung zu einem bestimmten, ausländischen Anbieter aufrecht zu halten oder abzubrechen bzw. erst gar nicht aufzunehmen. Die zu untersuchenden Fragen und Subfragen sind deshalb:

- Ergeben sich für deutsche und niederländische Konsumenten unterschiedliche Kundenbindungsfaktoren?

- Welche Loyalitätseffekte entwickeln sich in Grenzregionen? 
10 | Kapitel 1

- Existieren grenzüberschreitend andere Kundenbindungsfaktoren als die, die bereits erforscht wurden?

- Gibt es ein gemeinsames Kundenbindungsmodell für deutsche und niederländische Konsumenten?

- Bestätigt sich dieses neue Modell in der Praxis?

Aus diesen Fragestellungen heraus ist das grundlegende Ziel der Studie, einen verbesserten Erklärungs- und Prognoseansatz der grenzüberschreitenden Kundenbindung in ausgewählten Einzelhandelsbranchen zu erarbeiten. Die Arbeit setzt bereits untersuchte Faktoren und abgeleitete Gesetzmäßigkeiten aus der Studie von Odekerken-Schröder voraus und konzentriert sich auf folgende Teilziele, die sich aus der oben skizzierten Ausrichtung ableiten lassen:

- Identifizierung der Determinanten der Kundenbindung in ausgewählten Einzelhandelsbranchen in einer Grenzregion.

- Identifizierung relevanter Determinanten der Kundenbindung in einer Grenzregion.

- Identifizierung der Stimulanzen zum grenzüberschreitenden Einkauf und Einkaufsstättentreue.

Bereits Odekerken-Schröder (1999, S.122) weißt in ihrem Modell darauf hin, dass soziale Einflüsse, soziale Gruppen zu denen der Konsument gehört, die Freude am Einkauf und die Einstellung zum Einkauf das Einkaufs- und Bindungsverhalten des Konsumenten beeinflussen. Aus diesem Grund sollen in der vorliegenden Arbeit folgende Faktoren betrachtet wer$\operatorname{den}^{4}:$

- Soziale Norm vor kulturellem Hintergrund.

- Einstellung gegenüber Handlung.

- Wahrgenommene Selbstrelevanz.

Es werden die sozialen, psychischen und kulturellen Faktoren betrachtet, da diese insbesondere in einer Grenzregion bindungsentscheidend sein können, aber in der Literatur bisher vernachlässigt wurden. Für die vorliegende Untersuchung wird folgende Vorgehensweise gewählt: Im ersten Teil wird ein Bindungsmodell auf Basis der Einstellungstheorie sowie die

\footnotetext{
${ }^{4}$ Soziale Norm vor kulturellem Hintergrund: Einfluss des sozialen Umfeldes (Dick und Basu, 1994 und Oliver 1999).

Einstellung gegenüber Handlung: Eine als erstrebenswert erachtete Handlung (Kuhl, 1994).

Wahrgenommene Selbstrelevanz: Wertorientierte Konzeptualisierung des Involvementkonstruktes (Houston und Walker, 1996).
} 
Entwicklung eines Bindungsmodells auf Grundlage der Theorie des geplanten Verhaltens aus der Literatur abgeleitet. Daran schließt sich die Spezifizierung des Modells in einer Grenzregion sowie die Darstellung der methodischen Grundlagen zur Schätzung des Bindungsmodells an. Im zweiten Teil erfolgt die Überprüfung des Modells in der Grenzregion in ausgewählten Einzelhandelsbranchen sowie die Beschreibung der Konzeption der Studie in der Grenzregion, unter Berücksichtigung der Fachgeschäft- und Einzelhandelsfilialbetriebe. Im nächsten Schritt erfolgt die Entwicklung eines angepassten Bindungsmodells differenziert nach niederländischen und deutschen Konsumenten. Den Schlusspunkt bildet die Diskussion der Ergebnisse für Theorie und Praxis. Die Betrachtung der grenzüberschreitenden Kundenbindung wird nicht a priori auf eine bestimmte Branche des Einzelhandels beschränkt. Eine Eingrenzung auf eine bestimmte Anzahl von Einzelhandelsbranchen erfolgt im Rahmen der empirischen Analyse aus forschungsökonomischen Gründen. In der vorliegenden Arbeit sind grenznahe Discounter mit einem hohen Hardware Anteil, sowie Textil- und Schuhanbieter, Elektrogeschäfte und Gartencenter in die Analyse der Kaufgewohnheiten der Konsumenten mit einbezogen worden. 

Kapitel 2: Einkaufsstättenspezifische Determinanten der grenzüberschreitenden Geschäftstreue

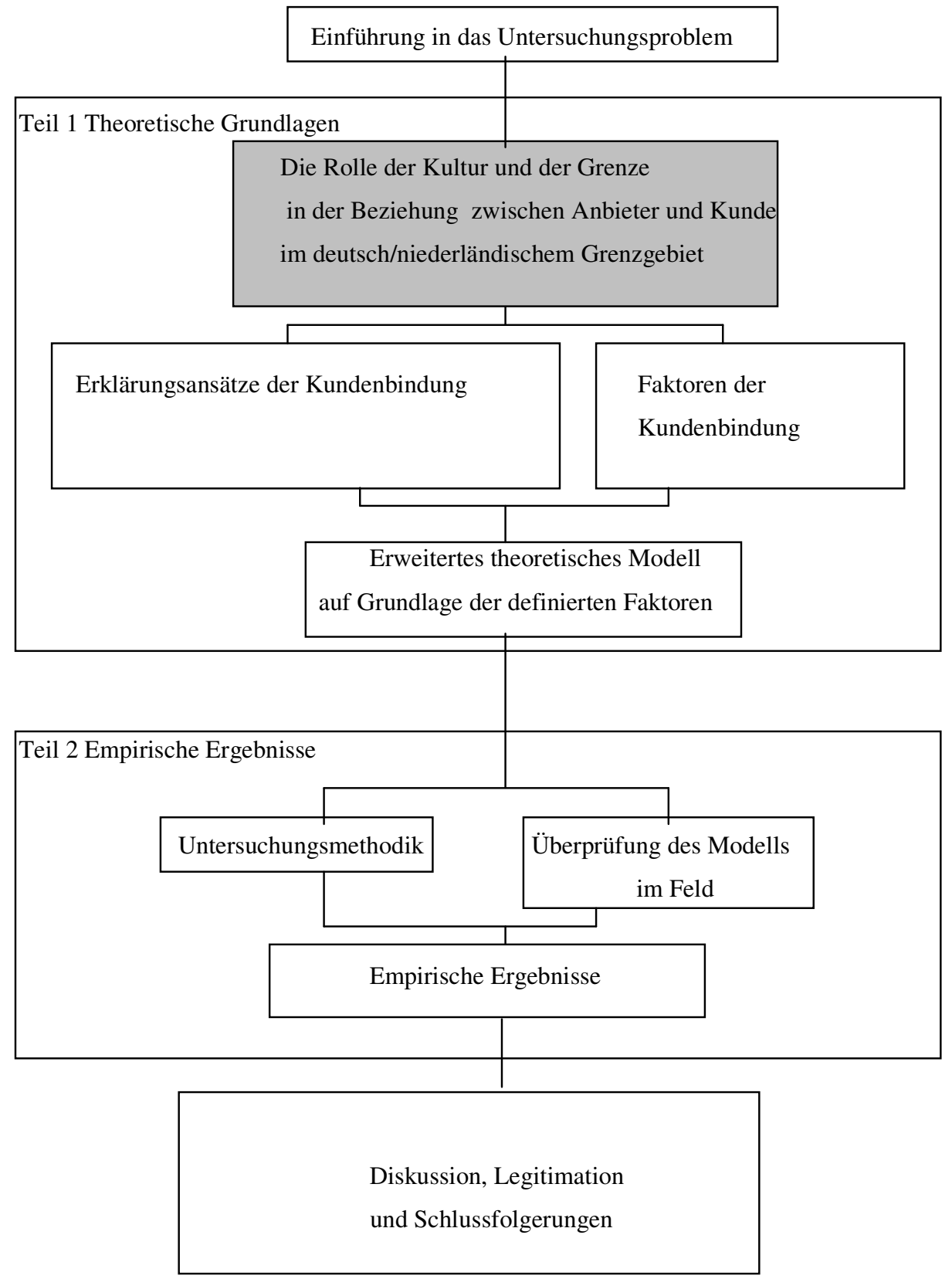


14 | Kapitel 2

\section{1 $\quad$ Einleitung}

Die grenzüberschreitende Treue der Konsumenten zu einer Einzelhandelsstätte ist abhängig von unterschiedlichen einkaufsspezifischen Determinanten. Im folgenden Kapitel sollen die Fragen untersucht werden, welche Rolle die Landesgrenze und die Kultur sowie das weitere soziale Umfeld in der Beziehung zwischen Anbieter und Kunde im deutschniederländischem Grenzgebiet spielt.

\subsection{Rolle der Landesgrenze in der Beziehung zwischen Anbieter und Kunde im deutsch/niederländischem Grenzgebiet}

Als Untersuchungsgebiet der vorliegenden Arbeit dient das deutsch-niederländische Grenzgebiet zwischen Maastricht/Aachen und Venlo/Mönchengladbach. Dieses Gebiet umfasst die zwei Euregio-Gebiete Rhein-Maas und Rhein-Maas Nord. Die Euregio Rhein-Waal ist mit in die theoretischen Überlegungen aufgenommen worden, da die beiden Euregios Rhein-MaasNord und Rhein-Waal seit 2003 eine administrative Einheit bilden. An dieser Stelle werden insbesondere die Besonderheiten des Grenzgebietes zwischen Deutschland und Niederlande aufgezeigt in Bezug gemeinsamer Verflechtungen in wirtschaftlicher, kultureller und familiärer Hinsicht.

Nachfolgend wird das Untersuchungsgebiet kurz beschrieben, um die Besonderheiten dieser Region zu dokumentieren und um im weiteren Verlauf die Bedeutung einer Landesgrenze zur Entwicklung der Kundenbindung aus Kundensicht darzustellen. Die Wahl fiel aus verschiedenen Gründen auf dieses Gebiet. So wird hier mit besonderer Anstrengung versucht, die dort lebenden Menschen einander näher zu bringen. Hinzu kommt, dass sich sehr attraktive Einkaufsstädte auf beiden Seiten der Grenze befinden und diese Region zu der am dichtesten bevölkerten Region im Grenzgebiet zwischen Deutschland und den Niederlanden gehört. Die Darstellung der Region beinhaltet die geschichtliche Entwicklung, das wirtschaftliche Potential sowie zukünftige Entwicklungsmöglichkeiten.

Eine grenzüberschreitende Region mit Menschen, die ohne Begrenzungen in einer solchen Region leben und arbeiten, ist noch kein für den Einzelnen konkret greifbares Faktum. Um eine vereinte Region zu schaffen, ist es nötig, Menschen zu überzeugen, dass diese in der Realität existiert, dass eine solche Region mehr ist als ein bloß theoretisches Konstrukt oder utilitaristisches Funktionsgefüge. Damit Menschen an ein soziales Konstrukt glauben, müssen 
sie ihre individuelle Identität durch die neuen Strukturen umfasst wissen (Böttcher, 1995, S. 32). Symbole bieten Hilfestellung an, um das persönliche Bedürfnis nach Zugehörigkeit mit der Herausforderung sozialen Wandels zu vereinbaren, indem sie einen Sinn von Identifikation mit der neuen Struktur kreieren. Das niederländisch-deutsche Grenzgebiet mit seinen Euregios ist ein solches Symbol. Nach Böttcher (1995, S. 31) machen Einheit und Vielfalt nicht nur das typisch Relevante unseres Kontinents aus, sondern auch das typisch Relevante der Euregio. Durch staatliche Grenzen wird territorialer Besitz unterteilt, die Bewohner dieser Gebiete voneinander getrennt. In welcher Stärke diese Trennung ausfällt, hängt ab von der geschichtlichen und kulturellen Entwicklung der Grenzstaaten (Von der Dunk, 1995, S. 4950). Wenn Grenzen das Resultat zufälliger Machtverhältnisse sind, dazu noch eine zeitliche Begrenztheit kommt, dann werden sie vor allem von der Grenzbevölkerung unterhöhlt. Dies wird verstärkt, wenn beide Seiten der Grenzanwohner in ähnlichen Verhältnissen leben und die Sprache eng miteinander verwandt ist.

Bezogen auf die deutsch-niederländische Grenze führt Von der Dunk (1995, S. 54ff.) aus, dass die Landschaft überall beiderseits der Grenze die gleiche ist und die Dialekte im westfälischen und niederrheinischen Raum viele Gemeinsamkeiten aufweisen. Dies verwundert insbesondere in der Provinz Limburg nicht, da Limburg erst in der zweiten Hälfte des 19. Jahrhunderts vollständig in die Niederlande aufgenommen wurde und das Rheinland, als Ganzes erst nach der napoleonischen Zeit an Preußen fiel. Insbesondere in diesem ländlich geprägten Grenzgebiet zwischen den Niederlanden und Nordrhein-Westfalen hielt die Bevölkerung an ihrer heimischen Sprache, ihrem Lebensstil und an Kontakten auf beiden Seiten der Grenze fest. Dennoch darf nicht übersehen werden, dass diese Grenze in Folge der Nationalisierung zu Unterschieden in der Mentalität geführt hat. Im Folgenden soll kurz das deutsch- niederländische Grenzgebiet dargestellt werden, auf das sich dann die weiteren Ausführungen beziehen.

Das deutsch-niederländische Grenzgebiet zu Nordrhein-Westfalen wird im Folgenden durch demografisch-ökonomische Kennzahlen charakterisiert. Das besondere Augenmerk liegt auf den Auswirkungen der Grenze auf das Kaufverhalten der Konsumenten innerhalb des definierten Gebietes. Ferner soll durch die Zusammenstellung der Daten das Gemeinsame und das Trennende der zu untersuchenden Euregio dargestellt werden, um die Rolle der geografischen Grenze transparenter zu machen. Die folgende Tabelle zeigt die Einwohnerzahlen der einzelnen Regionen der Euregio Rhein-Maas. Für die weitere Betrachtung ist allerdings nur der Bevölkerungsanteil des deutsch-niederländischen Grenzgebietes von Interesse. 


\begin{tabular}{|l|l|l|l|l|}
\hline Region Aachen D & $\begin{array}{l}\text { Provinz Limburg } \\
\text { NL }\end{array}$ & Provinz Lüttich B & $\begin{array}{l}\text { Provinz Limburg } \\
\text { B }\end{array}$ & $\begin{array}{l}\text { Deutschsprachige } \\
\text { Gemeinschaft B }\end{array}$ \\
\hline 1.245 .379 & 747.835 & 1.016 .672 & 783.927 & 70.117 \\
\hline
\end{tabular}

Tab. 2.1: $\quad$ Die Bevölkerungsanteile der Regionen in der Euregio Maas-Rhein (eigene Zusammenstellung Jahr 2004 aus Euregio)

Die Region Aachen/Maastricht besitzt für den Einzelhandel aufgrund der hohen Städtedichte eine große Bedeutung. Für die deutsch-niederländische Betrachtung dominieren die Oberzentren Maastricht und Aachen, die einen hohen Teil der Kaufkraft abschöpfen. In der Euregio Rhein-Maas leben ca. 1.900.000 Einwohner im deutsch-niederländischen Grenzgebiet. Konsumenten in diesem Wirtschaftsraum sind traditionsgemäß, auch aufgrund familiärer Verflechtungen innerhalb der Grenzregion sehr mobil. Doch auch Städte außerhalb der Euregio wie Jülich, Eschweiler und Düren profitieren von der Mobilität des EuregioKonsumenten. Diese Mobilität wird auch durch die Forschungsarbeit von Krummer (1993, S. 197-204) belegt, der bereits Anfang der 90er Jahre für diese Städte auf Grundlage einer Befragung von 2.500 Kunden einen Zufluss von niederländischen Käufern in Höhe von

- Düren 3,4\%,

- Jülich 4,0\% und

- Eschweiler 5,9\%

feststellte.

Im Frühjahr 2003 wurde eine Untersuchung zum Thema Einkaufen und die Inanspruchnahme von Dienstleistungen durch Einwohner der „Provincie Limburg“ in den Niederlanden im angrenzenden Ausland durch die Beratungsgesellschaft Etil von der Universität Maastricht durchgeführt. Ausgangspunkt dieser Untersuchung ist die Selbsteinschätzung der Konsumenten hinsichtlich ihrer wirtschaftlichen Lage am Messzeitpunkt Frühjahr 2003 und im Vergleich zu dem Jahr 2002. Ergebnis der Untersuchung ist, dass mehr als 2/3 der befragten Konsumenten regelmäßig die Grenze zum Einkaufen überschreiten. Dabei werden von den Konsumenten sowohl größere als auch kleinere Städte als Einkaufsziel angesteuert. Eingekauft werden im Gebiet des linken Niederrheins vor allem Audio Geräte und Computer und Haushaltsgeräte. Stark nachgefragt werden von Niederländern allerdings auch Bekleidung, Schuhe, Sportartikel und Accessoires. Als Argument für den Einkauf auf der deutschen Seite der Grenze gaben die Probanden in erster Linie das Preisargument an, aber auch das etwas andere Sortiment, gute Rahmenbedingungen für den Einkauf wie Parkplätze, Kundenfreundlichkeit, 
Auswahl als auch die Möglichkeit „anders“ einzukaufen als im eigenen Wohnort. Hieraus ist in der Untersuchung ein Profil erstellt worden, die eine Interpretation der Einstellung der niederländischen Konsumenten zu den Einkaufsstätten auf deutscher Seite zulässt. Die Einkaufsstätten werden von den niederländischen Konsumenten durch ein ordentliches Qualitätsangebot, niedrigen Preis, Trendy, zufrieden stellendes Parkplatzangebot und ordentliche Sortimentsstruktur positiv charakterisiert. Negativ ist für die Niederländer die Einkaufsatmosphäre in deutschen Städten, die relativ schlechte Qualität der Mitarbeiter in den Einkaufsstätten, als auch die veraltete Einrichtung vieler Einkaufsstätten. Insgesamt zeigt sich folgendes kennzeichnendes Bild (in Prozent wird die Einschätzung der niederländischen Konsumenten gegenüber deutschen Einkaufsstätten angegeben):

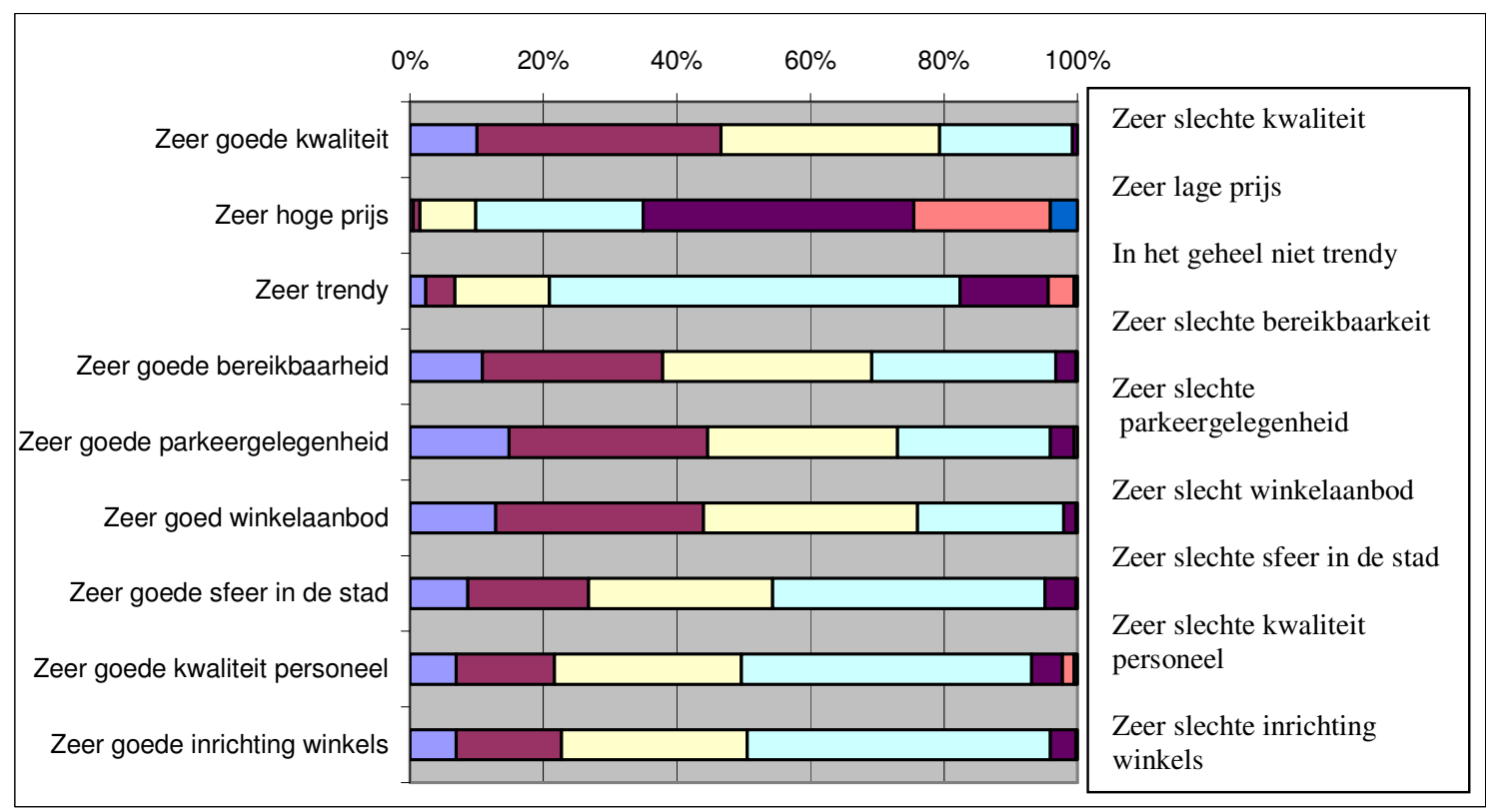

\section{Abb. 2.1: Beurteilung der deutschen Einkaufsstätten am linken Niederrhein durch nieder-} ländische Konsumenten (Etil Erhebung Frage 21c aus dem Jahr 2003) ${ }^{5}$

In dieser Untersuchung wurden die niederländischen Konsumenten in Limburg gefragt, wo sie am liebsten einkaufen würden. Es wurden die Orte in der nachfolgenden Grafik vorgegeben und die Konsumenten konnten maximal drei Orte angeben. Bei den Antworten auf die Frage, wo die Konsumenten gerne einkaufen, ist sicherlich der gute Wert den Aachen von den niederländischen Konsumenten bekommt, bemerkenswert. Es ist, wenn auch mit deutlichem Abstand, der beste Wert nach der niederländischen Stadt Maastricht. Aber auch Mön-

\footnotetext{
${ }^{5}$ Die Farbunterschiede zeigen die prozentuale Verteilung der Antworten der Probanden. So bescheinigen nur $10 \%$ der niederländischen Konsumenten deutschen Einkaufsstätten am linken Niederrhein eine sehr gute, mehr als 20\% aber eine sehr schlechte Qualität.
} 
18 | Kapitel 2

chengladbach mit $7 \%$ der Nennungen wird von den Niederländern positiv beurteilt. Erstaunlich sind sicherlich die gleichen Werte von Oberhausen und Krefeld mit $2 \%$ der Nennungen. Das CentrO Oberhausen zeigt sicherlich seinen positiven Einfluss als Anziehungspunkt für niederländische Konsumenten. Im Einzelnen sind folgende Städte genannt worden:

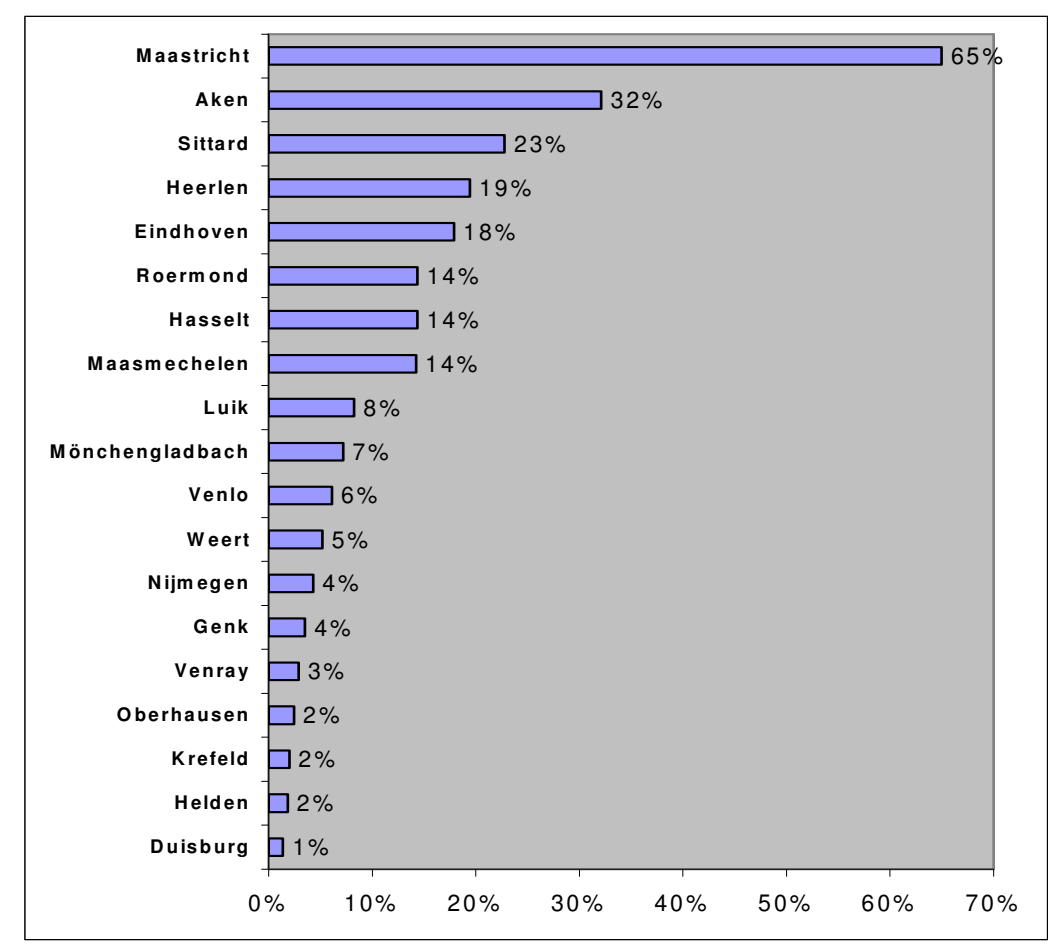

Abb. 2.2: Bevorzugte Einkaufsstädte der Niederländer im Grenzgebiet (Etil Erhebung Frage 23a, Rangfolgen nach höchsten Prozentsätze, 2003)

Zur Begründung warum die Wahl auf bestimmte Orte fiel, ergab sich, dass die Orte in der Nähe der Wohnorte der Konsumenten liegen, sie gut erreichbar sind, oder die Konsumenten einen persönlichen Bezug zu diesen Orten haben. Im Jahre 2004 ist von der BRO eine weitere Untersuchung im Einzelhandel durchgeführt worden. Diese Untersuchung zeigt ebenfalls das ökonomische Gewicht des grenzüberschreitenden Einkaufs.

Zur Euregio Maas-Rhein Nord (ehemals Grenzregio) mit einem Gebiet von 3.600 Quadratkilometern und rund 1,7 Millionen gehören auf niederländischer Seite Mittel- und Nordlimburg mit den Städten Roermond, Venlo, Venray und Weert sowie auf deutscher Seite die Städte Krefeld und Mönchengladbach sowie der südliche Teil Kreis Kleve, Viersen und Neuss. Aufgrund der kurzen und schnellen Wege zu den Industrie- und Handelsmetropolen an Rhein und Nordsee ist Mönchengladbach das wichtigste Wirtschaftszentrum am linken Niederrhein. 
Dies zeigt sich auch in der Kaufkraft. Nach Angaben der GfK liegt die Kaufkraft $€$ 15.282,pro Einwohner im Jahr. Die einzelhandelsrelevante Kaufkraft pro Einwohner lag 1997 bei umgerechnet $€ 5.299$,-. Die Kaufkraftziffer von Mönchengladbach liegt bei 103 Prozent ( www.euregio-rmn.de). Die Euregio Maas-Rhein-Nord ist mit 679 Einwohner pro qkm auf deutscher Seite und mit 316 Einwohner pro qkm auf niederländischer Seite die am dichtesten bevölkerte Grenzregion der Niederlande (KPMG, 1998, S.7). Die ökonomische Struktur der Region ist gekennzeichnet durch eine große Vielfalt von Wirtschaftsunternehmen mit Hauptschwerpunkten im industriellen Bereich, Agrarwirtschaft, Logistik und Tourismus. Die Region hat im Landesvergleich auf niederländischer Seite ein gutes Wirtschaftswachstum. Das ist vor allem dem hohen Industrieanteil zu verdanken. Dagegen ist der Handel in dieser Region geringer repräsentiert als in den sonstigen Teilen der Niederlande. Auf Grund dieser Situation nahm die Arbeitslosigkeit seit 1997 permanent ab, und die Kaufkraft stieg (KPMG, 1998, S.10). Allerdings ist die Arbeitslosigkeit in den letzten Jahren auch im niederländischem Gebiet wieder angestiegen. Bei einem Vergleich der Euregio Gebiete Euregio Maas-Rhein und Euregio Rhein-Maas-Nord im Juni des Jahres 2005 ergibt sich folgendes Bild:

\begin{tabular}{|r|c|c|}
\hline Jahr & $\begin{array}{c}\text { Mai 2004 } \\
\text { Arbeitslosenquote in \% }\end{array}$ & $\begin{array}{c}\text { Juni 2005 } \\
\text { Arbeitslosenquote in } \\
\%\end{array}$ \\
\hline Euregio Maas-Rhein & & 11,7 \\
\hline Deutsches Teilgebeit & 10,0 & 13,0 \\
\hline Niederländisches Teilgebiet & 11,7 & 10,6 \\
\hline Euregio Rhein-Maas-Nord & & 9,0 \\
\hline Deutsches Teilgebiet & 9,1 & 8,8 \\
\hline
\end{tabular}

\section{Tab.2.2: $\quad$ Arbeitslosenquote in der Euregio (http.//euregio.etil.nl)}

Auf deutscher Seite ist diese Region gekennzeichnet durch eine im Landesvergleich Nordrhein-Westfalen hohen Arbeitslosigkeit und einem geringen Haushaltseinkommen (Landesamt für Datenverarbeitung und Statistik NRW, 2004). Das Bevölkerungswachstum in der gesamten Region stagniert dagegen und die Zahl der zwischen 15- und 65- jährigen wird sich in den nächsten Jahren kaum verändern, wobei ein höherer Frauenanteil erwartet wird. Eine wesentliche wirtschaftliche Entwicklung der Region wird sich aus dem Tourismusbereich ergeben. Auch hier geht man für diese Region von einem schnellen Wachstum aus (KPMG, 1998, S. 21). Dabei ist zu berücksichtigen, dass in den Städten vor allem Kurzzeittouristen 
anzutreffen sein werden, die, wenn es zu der geplanten Liberalisierung der Ladenöffnungszeiten in den Niederlanden mit Sonntagsverkauf kommen wird, das Angebot der Städte verstärkt am Wochenende nutzen werden. Eine wichtige Entwicklung für den Handel in dieser Euregio ist das Factory-Outlet in Roermond. Diese von einem Betreiber organisierte Verkaufseinrichtung zur Direktvermarktung von Produkten, hat in der Euregio Rhein-Maas-Nord im Schnittpunkt zur Euregio Rhein Maas Konsumentenkaufströme verändert. Verbund- oder Kopplungseffekte im Sinne von Kundenzuführung werden dabei für den lokalen Einzelhandel und den gesamten Standort erzielt.

Anfang 1999 wurden die Kaufströme der Konsumenten in den Region Duisburg, Arnheim, Nijmegen und Kleve untersucht (Middendrop, Velde und Vergossen, 1999, S. Xiii - XV). Aufschlussreich bei dieser Untersuchung ist die Tatsache, dass regional starke Schwankungen in der Bindung der Kaufkraft auftreten. Verzeichnet zum Beispiel in Liemers-Achterhoek der Einzelhandel mit Bekleidung und Schuhe Branche den größten Kaufkraftabfluss, hat die Schuh- und Bekleidungsbranche in Arnheim einen hohen Kaufkraftzuwachs. Das Gleiche gilt für den Raum Nijmegen. In der Region Betuwe hat der Einzelhandel mit Schuhen dagegen kaum eine wirtschaftliche Bedeutung, genauso wenig wie in der Region Noord-Brabant \& Nord-Limburg. Auf der anderen Seite der Grenze dominieren Oberzentren wie Duisburg und Mönchengladbach, in denen die Konsumenten ihren Bedarf decken. (Middendrop, Velde und Vergossen, 1999, S. XV). Beachtlich ist dabei die Tatsache, dass Deutsche öfter zu einem Schaufensterbummel in die Niederlande fahren als umgekehrt. Laut Untersuchung kommen allein aus dem Einzugsgebiet der Euregio Rhein-Waal jährlich 1.071.000 Besucher aus Deutschland in die Niederlande. Davon besuchen 600.000 Geschäfte im niederländischen Teil der Euregio Rhein-Waal. Umgekehrt kommen nur 420.000 Niederländer aus dem Untersuchungsgebiet nach Deutschland und nur 290.000 bleiben dabei in der Euregio. Aus der betrachteten Region pendeln mehr deutsche Besucher in die Niederlande als niederländische Besucher nach Deutschland. Als Zentren, die einen höheren Zufluss an Besucherströmen als Abflüsse haben, können auf deutscher Seite Kleve, Duisburg, Mönchengladbach und Aachen identifiziert werden. Auf niederländischer Seite sind dies die Städte Nijmegen, Arnheim, Maastricht, Venlo und mit Abstrichen Sittard und Roermond ${ }^{6}$.

\footnotetext{
${ }^{6}$ Durch das FOC hat auch die City der Stadt eine höhere Frequenz von auswärtigen Konsumenten erfahren.
} 
Eine besondere Stellung nehmen in diesem Bereich großflächige Einkaufszentren wie das CentrO in Oberhausen ein. Im CentrO Oberhausen ist die Dynamik des Einzelhandels besonders deutlich zu erkennen. Da im CentrO sehr viele internationale Geschäftsketten vertreten sind, wird das CentrO insbesondere von Konsumenten besucht, die bereit sind, größere Entfernungen, selbst Landesgrenzen zu überbrücken, um dort einzukaufen. So kennen $48 \%$ der niederländischen Einwohner der Euregio Rhein-Waal das CentrO, 20\% haben es bereits besucht (Stand: Ende 1997) (Middendrop, Velde und Vergossen, 1999). Mittlerweile werden die Zahlen noch höher sein.

Aufgrund der weggefallenen Grenzkontrollen, einer verbesserten Infrastruktur in der Region und einer gemeinsamen Währung, haben sich die Rahmenbedingungen des Einzelhandels als auch das Kauf- und Mobilitätsverhalten der Konsumenten verändert. Diese Entwicklung wirkt sich auf die Einkaufsgewohnheiten in den Städten und Gemeinden aus. Konsumenten decken ihren Bedarf nicht mehr nur im eigenen Wohnort, sondern verschaffen sich einen größeren Aktionsradius, bei dem auch Ländergrenzen übersprungen werden. Wichtige Bedingung für den Konsumenten im Rahmen seiner Entscheidung, "Wo" der Einkauf getätigt wird, ist eine umfassende und kompakte Einzelhandelsstruktur, eine gute Verkehrsanbindung, eine gute Verkehrserschließung des Zentrums sowie ausreichende Parkplatzmöglichkeiten. Es stellt sich allerdings die Frage, ob Einkaufsentscheidungen grenzüberschreitend von diesen offensichtlichen Faktoren abhängen und Konsumentenmobilität allein hierdurch gefördert wird. Eine wesentliche Frage ist, ob sich im Grenzgebiet Kaufentscheidungen und Kundenbindung aufrund tiefer liegender Faktoren ableiten lassen (siehe Kapitel 4).

Ein weiterer Bereich, der einen Einfluss auf das Kundenbindungsverhalten der Konsumenten hat, ist wie der Konsument seine Einkäufe tätigen möchte. So legt der Konsument beim Einkauf im benachbartem Ausland großen Wert auf einen bequemen Aufenthalt und eine positive Ausstrahlung der Einkaufsbereiche. Attraktives Ambiente und ein abwechslungsreiches Gaststättengewerbe sowie Kulturangebot spielen für die Wahl als Einkaufsort eine entscheidende Rolle. (Middendrop, Velde und Vergossen, 1999, S. 1). Artikel des täglichen Bedarfs werden zum weitaus größten Teil von den Konsumenten in der eigenen Region gekauft. Nach einer Untersuchung der Euregio Rhein-Waal haben die Autoren festgestellt (1999, S. 16), dass etwa jeder fünfte Konsument die Artikel des nicht täglichen Bedarfs in Einkaufszentren anderer Regionen kauft. Dies bestätigen auch die Untersuchungen für die Euregio Rhein-Maas der BRO aus dem Jahre 2004, die in ihrer Untersuchung feststellen, dass 87\% der befragten Konsumenten außerhalb der eigenen Region und fast $11 \%$ alle nicht täglich notwendiges Artikel 
außerhalb der eigenen Region einkaufen. Die bedeutet ein Einkaufsvolumen von circa $€ 590$,pro Konsument und Jahr (BRO, 2005, S.7). Dies deutet darauf hin, dass auch grenzüberschreitend Kundenbindung aufgebaut wird.

Die Grenze ist somit kein trennendes Element für die Grenzbewohner der deutschniederländischen Grenze. Eher stimuliert sie Konsumenten beim „Nachbarn“ einzukaufen, da man die Besonderheiten des Anderen schätzen gelernt hat und in die Einkaufsplanung mit einbezogen hat. Dennoch wird die Grenze zwischen Deutschland und den Niederlanden immer noch als Grenze wahrgenommen und es hat sich auch trotz aller politischen Anstrengungen noch kein gemeinsames euregionales Gefühl entwickelt. Dies belegt auch eine nichtrepräsentative Studie von Anke Strüver (2004, S.34). Strüver beschreibt eine Passantenbefragung in der Innenstadt von Kleve hinsichtlich der Bekanntheit der Euregio. In dieser Befragung wurden 542 deutsche und niederländische Passanten befragt, 457 (84 \%) wussten nicht, dass sie in einer deutsch/niederländischen Euregio leben. Nur 87 Befragte (15\%) wussten, dass sie in einer Euregio leben und nur 37 Befragte oder $7 \%$ kannten auch den Namen. Interreg-Projekte ${ }^{7}$ sind nur bekannt, wenn Menschen von ihnen auch direkt profitieren. Strüver zeigt ebenfalls anhand der Verkehrsproblematik auf, dass für viele Menschen im deutschen Teil der Euregio Rhein-Waal Berlin näher liegt als Nijmegen. Belegt wird es durch die Tatsache, das es zwar einen ICE zwischen Amsterdam und Köln gibt, aber der Busverkehr innerhalb des Grenzgebietes sehr spärlich und nach 19.00 Uhr gar nicht mehr fährt (Strüver, 2004, S.34). Diese Erfahrungen lassen sich sicherlich auch auf andere deutsch - niederländische Euregio übertragen. Untersuchungen über Konsumentenmobilität in dem niederländischdeutschen Grenzgebiet haben somit eine lange Tradition und werden auch regelmäßig durchgeführt. Die Grenze hat durch die lange Tradition des grenzübergreifenden Einkaufs der im Grenzgebiet lebenden Menschen weniger etwas trennendes, sondern die Konsumenten ziehen ihren persönlichen Vorteil durch die unterschiedlichen Möglichkeiten des Einkaufs und die individuellen Kontakte zum Nachbarland. Allerdings sind die Einstellungen des Konsumenten zum Einkauf im Nachbarland bislang nicht hinreichend untersucht worden, so dass die Frage warum der Konsument im Nachbarland einkauft und was ihn positiv beeinflusst noch nicht abschließend beantwortet ist. Eine wesentliche Frage ist deshalb, welchen Einfluss die weitere soziale Umwelt und der Kulturaspekt auf die Kaufmerkmale und das Kaufverhalten des Konsumenten im Grenzgebiet haben.

\footnotetext{
${ }^{7}$ Interreg ist als Gemeinschaftsinitiative der EU-Kommission ein Förderprogramm zur Stimulierung und Unterstützung der grenzüberschreitenden Zusammenarbeit.
} 


\subsection{Einfluss der weiteren sozialen Umwelt auf die Kaufmerkmale des Konsumenten}

Nach Van Raaij (1981, S. 1-24) wird durch die Motivlage des Konsumenten und seine Erwartungen an die Zukunft das gegenwärtige Kaufverhalten beeinflusst. Entscheidend für die Motivlage des Konsumenten sind seine persönliche Einkommenssituation, seine Bildungssituation, seine Sparneigung sowie seine Zuversicht hinsichtlich seiner eigenen sowie der allgemeinen wirtschaftlichen Entwicklung. Die Zugehörigkeit zu einer bestimmten sozialen Schicht beeinflusst nicht nur den Zugang zu Bildungs- und finanziellen Ressourcen, sondern auch Geschmack und Lebensstil. Nach Solomon, Bamossy und Askegaard (2001, S. 387) besitzt jede Gesellschaft irgendeine Form von Klassenstruktur, bei "der die jeweiligen Ressourcen und der gesellschaftliche Status den Zugang zu Produkten und Dienstleistungen bestimmen." Dabei hängen die spezifischen Merkmale des Erfolgs eines Individuums in den Augen anderer in einer Gesellschaft erheblich davon ab, welche Vorstellungen von Erfolg und erfolgreichem Leben in der jeweiligen Kultur und Gesellschaft vorherrschen. Komponenten einer Gesellschaftsschicht sind dabei insbesondere berufliches Prestige, Einkommen und die Beziehung zwischen dem erzielten Einkommen und der Gesellschaftsschicht. Nach Dubois und Laurent (1993, S. 59-69) kann man, sofern Konsumentenverhalten vorhergesagt werden soll, Schlussfolgerungen zum relativen Wert der Gesellschaftsschicht, die sich ausdrückt in Wohngegend, Beruf sowie kulturellen Interessen, im Vergleich zum Einkommen ziehen. Dabei lassen sich aus den Daten der Zugehörigkeit zu der Gesellschaftsschicht vor allem solche Käufe vorhersagen, die symbolischen Wert haben, wobei die Preise der gekauften Objekte niedrig bis moderat sind. Aus den Einkommensdaten lassen sich eher Käufe vorhersagen, die weder symbolischen Wert noch Statuswert haben. Aus der Kombination von beiden Datenquellen sind Vorhersagen hinsichtlich teurer, symbolisch besetzter Produkte, wie Autos oder Luxusgüter ableitbar.

Nach Munson und Spivey (1981, S. 37-45) werden Produkte und Einkaufsstätten von den Konsumenten in Abhängigkeit nach ihrer Zugehörigkeit zu einer sozialen Schicht kategorisiert. Dabei ist das Weltbild des Konsumenten ein wesentliches Unterscheidungsmerkmal von Gesellschaftsschichten (Solomon, Bamossy und Askegaard, 2001, S. 393). Vor allem Ermessenskäufe (Käufe, die vom frei verfügbarem Einkommen getätigt werden können, jenseits der Deckung der Grundbedürfnisse) sind Indikatoren der subjektiven Einschätzung der Konsumenten bezüglich ihrer persönlichen Situation. Aus diesem Grund sind Ermessenskäufe wesentliche Indikatoren zur Bestimmung des Konsumentenverhaltens. Die Zugehörigkeit des 
Konsumenten zu einer bestimmten sozialen Schicht bestimmt dabei die Statushierarchie, nach der bestimmte Produkte eingekauft werden und auch in welchen Geschäftsstätten diese bevorzugt gekauft werden. Die Wahl von Produkten wird oft nach der Erwartungshaltung der angestrebten sozialen Schicht getroffen, oder es wird "demonstrativer Konsum betrieben", um die Zugehörigkeit zu einer angestrebten sozialen Schicht zu demonstrieren

(Solomon, Bamossy und Askegaard, 2001, S. 393). Dabei sind die Gesamtausgaben einer Konsumentenschicht in einer konkreten Produktkategorie wie zum Beispiel Bekleidung ein wesentlicher Indikator zur Wertigkeit dieser Produktkategorie in einem Land.

Kroeber-Riel und Weinberg definieren soziale Schicht mit Hilfe des Begriffs sozialer Status. Darunter verstehen die beiden Forscher die Stellung des Menschen in einem sozialen System sowohl im Sinne einer sozialen Position als auch im Sinne einer sozialen Wertschätzung. Personenmehrheiten mit gleichem sozialen Status werden dabei als soziale Schichten oder soziale Klassen bezeichnet (2003, S. 564). Als Schichtungskriterien sind dabei insbesondere

- Beruf, Ausbildung und Einkommen,

- Vermögen, Abstammung

- Macht, Interaktion sowie

- Statusinkonsistenz zu nennen.

Individuen, die zur gleichen sozialen Schicht gehören, legen dabei oft übereinstimmende Verhaltensweisen an den Tag. Das Verhalten ist dabei nicht nur eine Folge, sondern oft auch Ursache für sozialen Status, also ein statusbildendes und somit schichtbildendes Merkmal. Lebensalter des Konsumenten und auch die Epoche, in der er lebt, sind weitere Indikatoren, die das Kaufverhalten des Konsumenten erklären. Bedürfnisse und Vorlieben verändern sich im Laufe eines Konsumentenlebens, doch geht dabei die Anbindung an die eigene Generation selten verloren. Dabei haben nach Solomon, Bamossy und Askegaard (2001, S. 423) Europäer vor allem dann vieles miteinander gemein, wenn sie der gleichen Generation angehören und in derselben Region leben. Insbesondere Konsumenten über 30 teilen dabei viele kulturelle Erfahrungen, die sie im Umgang miteinander und in der gemeinsamen Region gemacht haben. Aus diesem Grund können Konsumentenentscheidungen nicht verstanden werden, „ohne dass man den kulturellen Kontext betrachtet, in dem sie getroffen werden: Kultur ist das Prisma, durch das die Menschen Produkte betrachten" (Solomon, Bamossy und Askegaard, 2001, S. 393). 


\subsection{Kulturelle Aspekte im grenzüberschreitenden Kaufverhalten}

Aufgrund der Bedeutungsvielfalt ist der Begriff Kultur schwierig prägnant zu um- und beschreiben. Nach Luhmann (1996, S. 23) wird Kultur als System gemeinsamer normativer Symbole als Voraussetzung des Entstehens von Handlungssystemen beschrieben. Unterstellt man, dass es Kulturunterschiede in einem grenzüberschreitenden Raum wie dem Grenzgebiet zwischen den Niederlanden und Deutschland gibt, kann der Kulturaspekt einen wesentlichen Einfluss auf die Kundenbindung und das Treueverhalten von ausländischen Kunden im Einzelhandel haben.

Einen vergleichbaren Standpunkt vertritt Hofstede ${ }^{8}$, wenn er sagt: „Culture is not restricted to objects in museums. Culture pervades our daily lives through the products we use, the buildings we live in, and the television programs we watch. Culture is the collective programming of the mind, the interactive aggregate of common characteristics that influence a humans group response to the environment“ (1980, S. 259). Der Begriff Kultur bezeichnet in der Ethnologie „die besonderen, historisch gewachsenen und zu einer komplexen Gestalt geronnenen Merkmale von Volksgruppen" (Kluckhohn und Strodtbeck, 1961, zitiert in: Schreyögg, 1999, S. 437). Darunter werden insbesondere Wert- und Denkmuster einschließlich der sie vermittelnden Symbolsysteme verstanden. In der angelsächsischen Diskussion um gesellschaftliche Kultur geht es um diejenigen Regeln des Alltags, die das Selbstverständliche ausmachen, das erst bemerkt wird, wenn es gestört oder nicht mehr vorhanden ist. Erst dann wird deutlich, dass eigene Selbstverständlichkeiten anderswo nicht selbstverständlich sind und dadurch das spezifisch Eigene und das Fremde erst erkennbar werden (Wagner, 1997, S. 26) ${ }^{9}$. Nach Solomon (2001, S. 461) drückt sich die Kultur einer Gesellschaft in ihren Werten, ethischen Grundsätzen und auch in den Objekten aus, die sie herstellt und mit denen sie sich umgibt. Kultur ist somit das, was Angehörige anderer Kulturen erst zu Fremden macht. Das Gleiche gilt in der Hinsicht, dass kulturelle Regeln erst das Gefühl von Vertrautheit, Sicherheit und aufgehoben sein erzeugen. Nach Aussage der Autoren umfasst Kultur sowohl abstrakte Werte und Ethik, als auch Konsumgegenstände und Dienstleistungen.

\footnotetext{
${ }^{8}$ Hofstede befasst sich mit dem Begriff Kultur nicht unter ethischen Aspekten, sondern unter dem Aspekt wie Menschen leben und handeln.

${ }^{9}$ Definition des Begriffs Kultur siehe Kapitel 3.2.
} 
Die Kultur, der eine bestimmte Konsumentengruppe angehört, bestimmt sowohl die Gesamtvorlieben dieser Gruppe als auch den Erfolg oder Misserfolg bestimmter Produkte, Dienstleistungen und Einkaufsstätten (2001, S. 432).

Kroeber-Riel und Kluckhohn bezeichnen Kultur als Übereinstimmung der Verhaltensmuster vieler Individuen (1952, S. 181). Nach Kroeber-Riel und Weinberg (2003, S. 553) ist Kultur ein Hintergrundphänomen, das unser Verhalten prägt, ohne dass wir uns dieses Einflusses bewusst sind. Deshalb haben kulturelle Verhaltensmuster sowohl Einfluss auf grundlegende Werte und Normen, als auch auf das für eine Gesellschaft wichtige Wissen und typische Handlungsmuster (Kroeber-Riel und Weinberg 2003, S. 553). Kultur (culture) wird in dieser Arbeit definiert als die Basisregeln, Erwartungen, Wünsche, die von Generation zu Generation weiter vermittelt werden. Kotler, Armstrong, Saunders und Wong haben den Begriff folgendermaßen präzisiert: „, The set of basic values, preceptions, wants and behaviours learned by a member of society from family and other important institutions“ (1999, S. 1000). Deshalb soll für diese Untersuchung folgender Zusammenhang zwischen Kultur und Konsumentenverhalten zugrunde gelegt werden:

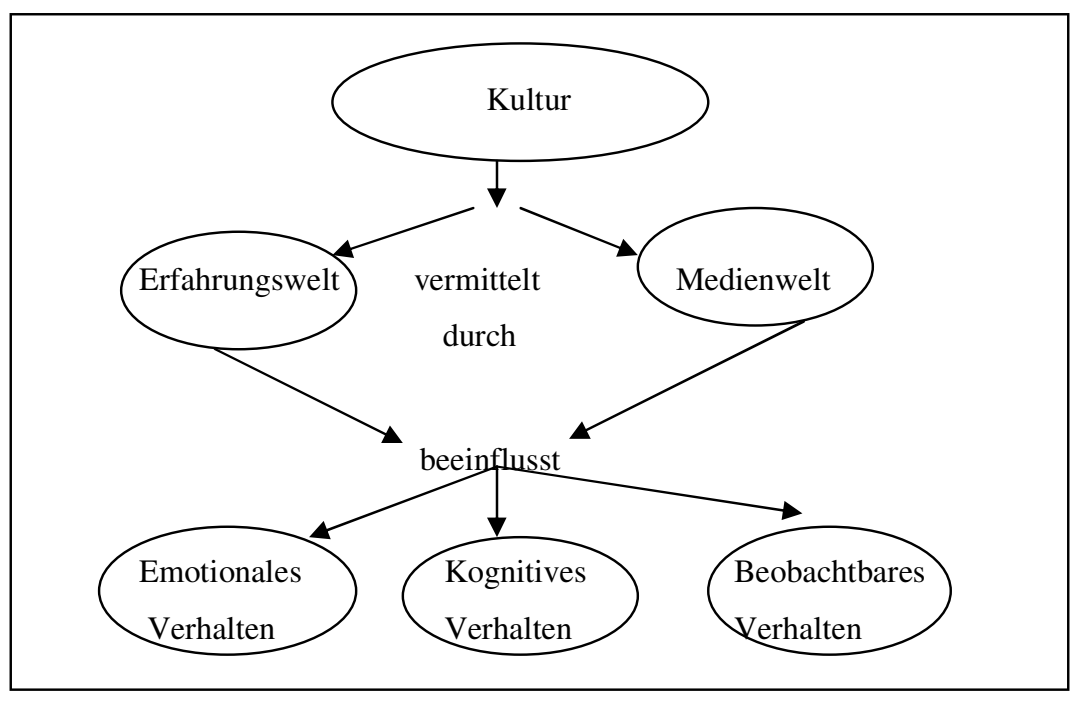

Abb. 2.3: Beziehungen zwischen Kultur und Verhalten

(Kroeber-Riel und Weinberg, 2003, S. 554)

Damit Konsumentenverhalten und Kundenbindung zu einer konkreten Einkaufsstätte erklärt werden können, sind empirische Arbeiten zu der Frage aufschlussreich, wie kulturelle und subkulturelle Werte sich im Einkauf und Konsum von Produkten niederschlagen. Um grenzüberschreitendes Konsumentenverhalten zu erklären, werden an dieser Stelle die Erklärung 
kultureller Eigenheiten wie Sprache und der Lebensstil der Konsumenten im deutschniederländischen Grenzraum herangezogen. Dabei schließt sich der Autor der Meinung von Kroeber-Riel und Weinberg an, die zu der Feststellung gelangen, dass sich kulturelle Verhaltensweisen sowohl in der Sprache als auch im Lebensstil widerspiegeln (2003, S. 556ff.). Als Lebensstil wird dabei eine Kombination typischer Verhaltensmuster einer Person oder Personengruppe im weiteren Sinne verstanden. Der Lebensstil umfasst somit sowohl Muster des beobachtbaren Verhaltens als auch von psychischen inneren Größen.

Verschiedene Autoren haben Kultur als einen bedeutenden Faktor zur Erreichung von Konsumenten identifiziert und als einen bedeutenden Faktor zur Marktsegmentierung erkannt (Helfferich, Hinfelaar und Kasper, 1997, S. 293). Der niederländische Kulturwissenschaftler Geert Hofstede gilt als Pionier dieser Untersuchungen und hat in den Jahren 1968 und 1972 im Rahmen zweier IBM-Studien in 72 Tochterunternehmen 116.000 Probanden nach ihren kulturellen Grundeinstellungen befragt. Im Anschluss daran hat Hofstede vier Dimensionen vorgeschlagen, um die Unterschiede verschiedener Kulturen $\mathrm{zu}$ verdeutlichen (Hofstede, 1984):

- Machtdistanz (PDI)

- Die Art, wie sich zwischenmenschliche Beziehungen bilden, wenn Machtunterschiede wahrgenommen werden.

- Umgehen von Unsicherheiten (UAI) - Das Ausmaß, in dem Menschen sich durch undurchschaubare Situationen bedroht fühlen.

- Maskulinität/Femininität (MAS) - Der Grad, in dem die Rollen der Geschlechter klar skizziert sind.

- Individualismus/Kollektivismus (IDV) - Das Maß, mit dem das Wohlergehen des Einzelnen gegenüber dem der Gruppe bewertet wird.

Hofstedes Ansatz, den er um eine fünfte Dimension (langfristige Orientierung versus kurzfristiger Orientierung) erweitert hat (2001, S. 19, 248) ist in der Literatur kritisiert worden, weil er die Probleme seines Kulturansatzes auf der gleichen Skala misst, wie seine Lösungen dazu. Jede Kultur, somit auch die Konsumentenkultur ist deshalb auf Basis der eigenen Prämissen zu verstehen und entsprechend zu analysieren (Solomon, Bamossy und Askegaard, 2001, S. 435).

Usunier (1993) hat Hofstedes Daten genutzt und eine hypothetische Karte der sich ähnelnden und sich weniger ähnelnden europäischen Kulturen angelegt. Helfferich, Hinfelaar und 
Kasper kommen auf dieser Grundlage zu folgender Einteilung europäischer Kulturen und schließen daraus, dass sich Internationalisierungsstrategien in sich ähnelnden Kulturen eher umsetzen lassen, als in sich unähnlichen.

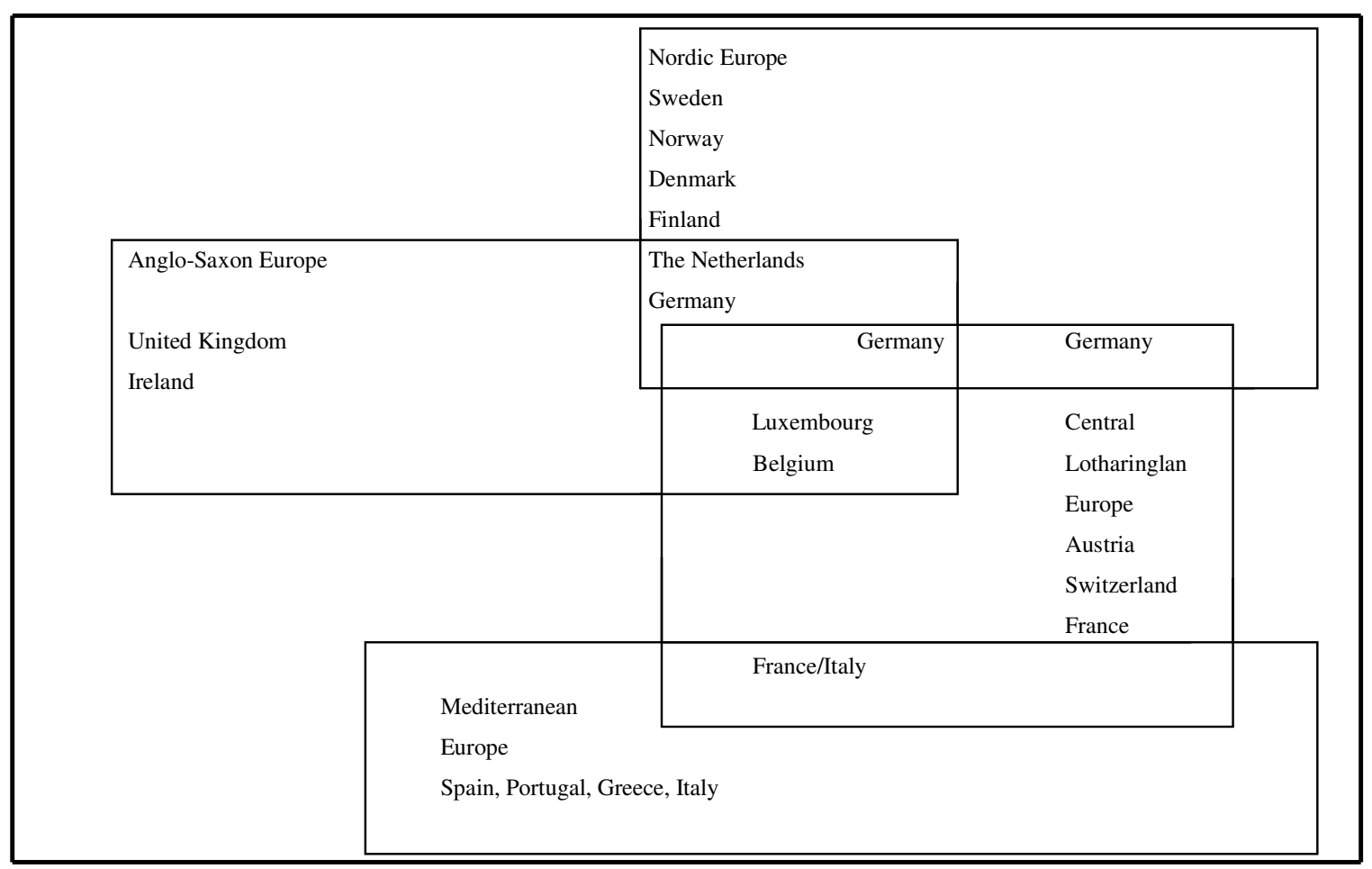

Abb. 2.4: $\quad$ Usunier`s Kultur Zonen von Europa

(Helfferich, Hinfelaar und Kasper, 1997, S.293)

Diese Zonen korrespondieren in hohem Maße mit den nationalen Kulturzonen. Kasper und Bloemer (1995, S. 1805-1815) analysierten auf Basis von siebzehn europäischen Ländern die ersten vier Dimensionen nach Hofstede und wiesen drei Cluster von europäischen Kulturen nach. Dies sind im Einzelnen:

- Das anglo-deutsche Cluster mit :

Irland, Großbritannien, Österreich, Schweiz, Deutschland und Italien

- Das niederländisch-skandinavische Cluster mit: Niederlande, Norwegen,

Schweden, Dänemark und Finnland.

- Das romanische-südeuropäische Cluster mit: Belgien, Spanien, Frankreich, Griechenland, Portugal und ehemals Jugoslawien. 


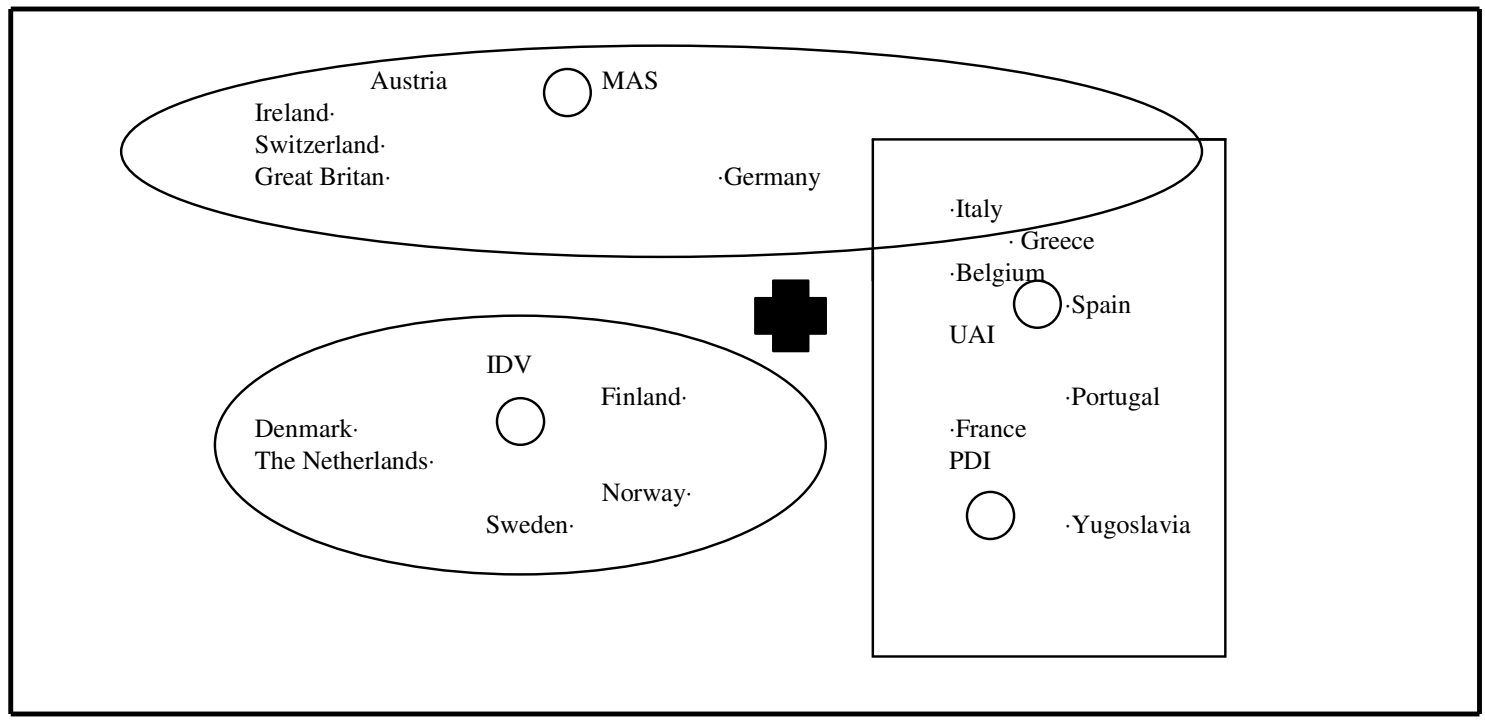

Abb. 2.5: $\quad$ Kasper`s Cluster der europäischen Länder Kulturen (Helfferich, Hinfelaar und Kasper, 1997, S. 293)

Der größte Unterschied zwischen Kasper`s und Usunier`s Clustersystem sind die Positionen verschiedener sich überlappender Länder. Besonders auffallend sind die Positionen der Niederlande und Deutschland. Beide Systeme arbeiten mit dem Datenmaterial von Hofstede ${ }^{10}$ und der Prämisse, dass Konsumenten, die dem gleichen Cluster angehören den gleichen Hintergrund bezüglich Normen und Werte haben und Konsumenten sich innerhalb dieser Cluster ähnlich verhalten. Für den weiteren Verlauf schließt sich der Autor der Meinung von Kasper an und nimmt Kasper`s Cluster der europäischen Länder Kulturen als Grundlage für weitere Untersuchungen. Die Aussage der Cluster ist, dass Konsumenten aus unterschiedlichen Clustern auch unterschiedliche Werte und Normen besitzen und sich unterschiedlich verhalten. Somit weichen auch das Einkaufs- und Bindungsverhalten beider Konsumentengruppen voneinander ab. Sollten die Verhaltensweisen der Konsumenten in der für diese Untersuchung definiertem Untersuchungsgebiet nicht voneinander abweichen, ist dies ein Indiz dafür, dass innerhalb der definierten Region aufgrund der engen nachbarschaftlichen Beziehungen besondere Einflüsse eine Rolle spielen ${ }^{11}$.

\footnotetext{
${ }^{10}$ Die Berechnung der Cluster erfolgt mit unterschiedlichen Methoden.

${ }^{11}$ Im Gegensatz zu Hofstede, der Länder miteinander vergleicht, werden in der vorliegenden Arbeit Grenzregionen miteinander verglichen. In Grenzregionen können sich wiederum Kulturen auf Grund einer gemeinsamen Tradition angleichen.
} 
Kulturelle Faktoren beeinflussen das Käuferverhalten auf grundlegende Weise. Diese Faktoren geben für die Individuen feste Größen vor, an denen sie sich im Rahmen ihrer sozialen und individuellen Faktoren orientieren können. Zwar scheint auf den ersten Blick die Kultur weniger Einfluss auf eine konkrete Kaufentscheidung eines Konsumenten zu haben als soziale und persönliche Faktoren. Dies gilt allerdings nur, solange Entscheidungen nur in einem Kulturkreis betrachtet werden. Im Umkehrschluss kann es allerdings im Bereich der intrakulturellen Betrachtung vollkommen anders aussehen. So kann es sein, dass das Ladenbaudesign einer Ladenkette in Skandinavien großen Erfolg haben kann, in Japan dagegen nicht (Nieschlag, Dichtl und Hörschgen, 2002, S. 622).

Aus der bisherigen Analyse ergibt sich das Zwischenergebnis, dass Konsumenten verschiedener Nationen oder sogar Regionen nur dann Gefallen an Marken / Produkten finden, wenn der Geschmack ihnen vertraut ist und entsprechend ihren Vorlieben produziert und vermarktet wird. Das gilt auch in Bezug auf die Werbeaussage, den Stil und die Sprache als Bestandteil der gesamten, einheitlichen Kommunikationsstrategie. Die Besonderheit des grenzüberschreitenden Marketing besteht darin, dass Entscheidungen über die Marktbearbeitung nicht mehr jeweils isoliert für einen Standort, sondern in Bezug auf mehrere regionale Märkte zu treffen sind, so dass die Aktivitäten an den verschiedenen Standorten aus einer zentralen Zielsetzung resultieren.

Die Bedeutung der Kultur im Kaufverhalten variiert von Land zu Land und von Region zu Region, da sie oftmals mit unterschiedlicher Symbolik und Sehnsucht aufgeladen ist. Gelingt es einer Marke oder einem Händler, diese Unterschiede durch einen einheitlichen Werbeauftritt zu überwinden, so kann damit eine Globalisierung eines bestimmten, in der Marke oder Einzelhandelsauftritts verankerten Lebensgefühls einhergehen (Salomon, Bamossy und Askegaard, 2001, S. 432).

Der Grundfrage, ob Konsum explizit Einfluss auf eine Globalisierung der Lebensstile nimmt, muss also insbesondere im 21. Jahrhundert, dem Zeitalter der Medialisierung, sehr differenziert nachgegangen werden. Die vorangestellten Prognosen werden von Kultur- und Wirtschaftstheoretikern wie Hofstede, Levitt und Luhmann bestätigt. Hofstede stellt beispielsweise in seiner Differenzierungsthese eindeutig die lokale Adaption der grenzüberschreitenden Werbung in den Vordergrund. Dabei betont er, dass die teilweise sehr unterschiedlichen Marktbedingungen, die im deutsch/niederländischem Grenzgebiet herrschen, die Entscheidung zugunsten der individuellen Konzipierung von grenzüberschreitender Werbung und 
Marketing beeinflussen. Es sind sowohl gesetzliche als auch psychologische Bestimmungen, die die Möglichkeiten internationaler Standardisierung von Werbung und Marketing stark einengen. Rechtliche Regelungen betreffen den Zugang zu den Medien (Beschränkungen hinsichtlich der Art des Mediums, der Sendezeiten und der Darstellungsform), die Werbeobjekte selbst (z.B. Verbot der Werbung für Tabakwaren, Arzneimittel und Spirituosen), die Art der Werbeaussage (Superlative oder vergleichende Werbung), Werbeaussagen auf Verpackungen und Produkten sowie Art und Umfang von Marketingaktivitäten. Selbst wenn die Voraussetzung homogener Zielgruppen gegeben ist, erfordern die oben genannten Unterschiede in der heutigen Mediensituation und in den rechtlichen Gegebenheiten eine differenzierte Gestaltung der einzelnen Aktivitäten für die Händler (Meffert, 2000, S.9). Neben der Anpassung an die unterschiedlichen Sprachen müssen die Werbebotschaften und die Einkaufsstätten demnach vor allem am Geschmack, an den Wünschen, Werten, Einkommen und Einstellungen der Zielgruppen unterschiedlicher Nationalität ausgerichtet sein.

Wenn man Kultur unter anderem als kollektive Programmierung des Gedankenguts bezeichnet, sozusagen als interaktive Vereinigung öffentlicher Charakteristika, die wiederum Einfluss auf die Reaktionen unterschiedlicher Lebensstilgruppen und deren Umwelt haben, wird dies deutlich. Deshalb ist der Trend zu lokalen Trends Ausdruck des globalen Wunsches auf unterschiedliche Identitäten. Eine globale Handelsgeschäftspersönlichkeit kann selbst durch die Einflusskraft von Werbung und Konsum nicht geschaffen werden. Eine Globalisierung eines bestimmten, in der Marke verankerten Lebensgefühls kann dagegen auch grenzüberschreitend Kaufimpulse auslösen. Die Annahme, dass sich die Bedürfnisse der Menschen weltweit zunehmend angleichen, konnte in der Marktforschung bisher nicht bestätigt werden. Während sich das Konsumentenverhalten in den Metropolen dieser Welt immer ähnlicher (das Warenangebot in größeren Städten wird durch das Angebot von Filialketten mit gleichem Warensortiment geprägt) wird bzw. durch den beachtlichen Einfluss von Electronic Commerce stark angleicht und eine Art Lingua franca entsteht, scheint gleichzeitig beim Konsumenten der Wunsch nach Retro, nach Tradition und Heimatgefühl zu wachsen. Kulturelle Unterschiede werden somit trotz zunehmend angestrebter wirtschaftlicher Internationalisierung und einer gemeinsamen europäischen Währung bestehen bleiben. Van Raaij (1997, S. 269) geht in seinem Artikel: „Globalisation of Marketing Communication?" sogar soweit, dass eine globale Kommunikationsstrategie für ihn nicht notwendigerweise ein hohes Maß an Standardisierung impliziert. "On the contrary, even in a global campaign, cultural differences have to be seriously taken into account. The either / or or etic / emic approach is too simple. Standardisation may take place at four levels: mission, proposition, concept, and execution. Applying stan- 
dardisation to mission and proposition does not mean that concept and execution should also be standardised". Der Prozess der Standardisierung erwächst zum größten Teil aus der Perspektive des Senders (Sender - Empfänger Modell), namentlich die Corporate Identity der Handelsunternehmen und der Hersteller. In einer postmodernen Welt, mit neuen Medien wie dem Internet, wird die Sichtweise des Empfängers als Erfolgskriterium immer gehaltvoller. Kommunikation ist nicht mehr ein bloßes Mittel der Informationsübertragung durch Medien, sondern zu jeder Zeit für den Konsumenten zugänglich, seinen Vorstellungen und Wünschen entsprechend.

Im Internet tritt der Trend zur Globalisierung am stärksten in Erscheinung. Genau dort nämlich, wo die physikalische Welt an Bedeutung verliert, suchen die Menschen geografische Orientierung. Das Heimatgefühl (gleiche Gedankenwelt, gleiche soziale Norm, Zugehörigkeitsgefühl) im „global village“ des Internets wächst als Ausdruck des Wunsches nach geo-

grafischer Identität dementsprechend im gleichen Maße, wie das Gefühl für Zeit und Raum schwindet. Unter dem Begriff Heimat wird in diesem Zusammenhang immer mehr der unmittelbare Mikrokosmos verstanden. Es stellt sich somit die Frage, ob diese Tendenz sich ebenfalls in der realen Welt des grenzüberschreitenden Einkaufs fortsetzt.

\subsubsection{Eingliederung der deutsch-niederländischen Kulturen im europäischem Vergleich}

Nach Geert Hofstede (1984) gibt es verschiedene Möglichkeiten, das Konsumentenverhalten und etablierte Lebensstile des jeweiligen Landes zu untersuchen. Er hebt zwei dominante Kriterien der Marktanalyse hervor und bezeichnet diese als vertikale Segmentierung und horizontale Segmentierung. Bei der vertikalen Segmentierung wählt er eine Methode, in der 66 Nationen in vier Dimensionen charakterisiert werden. Die Unterschiede kultureller Dimensionen zwischen Nationen werden hierbei betont und die Differenzen innerhalb einer Nation ignoriert. Da es jedoch meist große regionale kulturelle Unterschiede innerhalb eines Landes gibt, werden bei der vertikalen Segmentierung oftmals die Regionen als Grenzlinien betrachtet. Als Konsequenz dessen wird eine standardisierte Kommunikationsstrategie auf eine Region übertragen. Die vertikale Segmentierung zieht regionale Unterschiede demnach nicht in die Betrachtung ein. Das Gegenteil ist jedoch bei der so genannten horizontalen Segmentierung der Fall. Hier werden die kleinsten (sub)kulturellen Unterschiede innerhalb eines Landes und/oder einer Region beachtet. Veranschaulichende Beispiele für sich herausbildende Sub- 
kulturen in einer Gesellschaft sind die Jugendkultur oder die Bildungsklasse. Diese können weltweit mehr oder weniger ähnlich sein. In seiner Theorie der unterschiedlichen Einstufungen globaler Lebensstile setzt W. F. van Raaij voraus: ,These subgroups travel to other countries and are exposed to international media such as CNN, MTV, Time and Newsweek. Products and services geared towards these groups may be rather similar across cultures" (Van Raaij, 1997, S. 259).

Bei der vertikalen Segmentierung zeigt sich die Ambiguität des Verhaltens globaler Subkulturen. Genannte Gruppierungen reisen beispielsweise in regelmäßigen Abständen in andere Länder und Kulturen. Sie werden dort in verschiedenster Hinsicht mit fremden Traditionen und Lebensstilen konfrontiert. Gleichzeitig möchten sie insbesondere außerhalb ihres eigenen Heimatlandes ihre Gewohnheiten beibehalten, ein Stück Heimat bei sich tragen. Geert Hofstede (1984) nennt neben der strikt vertikalen bzw. horizontalen Segmentierung auch die Variante einer Kombination beider. So vertreiben beispielsweise die Unternehmen Aldi und Deichmann ihre Produkte sowohl in den Niederlanden als auch in Deutschland (vertikale Segmentierung), wobei Teile des Sortiments den regionalen Gegebenheiten angepasst und auch das Firmenlogo sowie der Werbeauftritt je nach Region verändert werden. Während diese Handelshäuser somit generell allen genannten Segmenten dieselben Produkte liefern, gibt es innerhalb der Sortimente auch Produkte, die lokal durch lokale Lieferanten adaptiert werden.

Dimension

\begin{tabular}{|l|l|l|}
\hline & Niederlande & Deutschland \\
Machtdistanz (PDI) & 38 & 35 \\
Umgehen von Unsicherheiten (UAI) & 53 & 65 \\
Maskulinität/Femininität (MAS) & 14 & 66 \\
Individualismus/Kollektivismus (IDV) & 80 & 67 \\
Langfristige Orientierung/ & & \\
Kurzfristige Orientierung & 44 & 31 \\
\hline
\end{tabular}

Tab. 2.3: $\quad$ Indexwerte nach Hofstede für Niederlande und Deutschland

(eigene Zusammenstellung aus: Hofstede, G., Lokales Denken, globales

Handeln, 2001)

Gliedert man die deutsch-niederländischen Landeskulturen in den europäischen Kontext ein, so kann dies nach der Einteilung von Hofstede geschehen. Allerdings ist zu berücksichtigen, dass die Indexwerte Länderwerte darstellen. Eine Übertragung auf das Untersuchungsgebiet 
ist 1:1 nicht unmittelbar möglich. Dennoch bieten diese Werte Anhaltspunkte um die Unterschiede zwischen Deutschen und Niederländern deutlich zu machen. Da die Detailergebnisse und die Berechnungen für die weitere Betrachtung nicht von Bedeutung sind, können die Inhalte der Untersuchung wie folgt zusammengefasst werden. Sowohl Deutsche als auch Niederländer weisen Gemeinsamkeiten im Bereich „Machtdistanz“ (PDI) auf. Allerdings haben die Niederländer nach Hofstede eine stark ausgeprägte Neigung zum Individualismus, zur Unabhängigkeit. Diese Grundeinstellung kann somit auch ein Erklärungsansatz für ein unterschiedliches Konsumverhalten gegenüber den Deutschen sein. Auffallend ist vor allem der große Unterschied zwischen dem Maskulinität/Femininität Indexwert der Niederländer und der Deutschen. Dieser Unterschied ist ebenfalls ein Ansatzpunkt zur Ansprache der Konsumenten im jeweils anderen Land. Maskulinität kennzeichnet eine Gesellschaft, in der die Rollen der Geschlechter klar gegeneinander abgegrenzt sind, es werden den Punkten Einkommen, Anerkennung, Beförderung und Herausforderung eine hohe Bedeutung zugemessen. Femininität zeichnet dagegen eine Gesellschaft aus die geprägt ist durch ein gutes Verhältnis zu anderen, Zusammenarbeit mit anderen, sich wohlfühlen in einer angenehmen Umgebung und Sicherheit. Diese Grundeinstellung der Niederländer in der Dimension Femininität und der Deutschen in der Dimension Maskulinität kann Einfluss auf das Kaufverhalten haben. Zwar befinden sich Deutsche und Niederländer im Bereich Unsicherheitsvermeidung im Mittelfeld, doch finden wir den deutschen Indexwert am oberen Ende des Mittelfeldes und den niederländischen Wert am unteren Ende. Je höher der Indexwert ist, desto stärker ist der Drang, sich an Regeln (formelle/informelle) zu halten und das Aufstellen von Regeln zu fördern. Hofstede sagt dazu, dass „Regeln in Ländern mit schwacher Unsicherheitsvermeidung zwar weniger strikt sind, aber im allgemeinen stärker beachtet werden" (2001, S. 172). Ferner kann sich das emotionale Bedürfnis nach Regeln in ein Talent für Präzision und Pünktlichkeit wandeln. Auch hierbei gibt es Ansatzpunkte, die das grenzüberschreitende Einkaufsverhalten beeinflussen. Kultur ist somit eine wesentliche Variable neben vielen anderen, die das Konsumentenverhalten erklären. Hofstede geht in seiner Interpretation sogar noch weiter. Für Hofstede passt es ins Bild, dass die deutsche Industrie insbesondere in der Schwerindustrie (dies unterstreicht die Maskulinität der Kultur) eine bedeutende internationale Rolle spielt, die niederländischen Unternehmen dagegen aufgrund des femininen Charakters ihrer Kultur im Dienstleistungssektor stärker sind.

Für die vorliegende Studie bedeutet dies, das es kulturelle Unterschiede zwischen beiden Ländern gibt. Es stellt sich allerdings die Frage, ob diese kulturellen Unterschiede ebenfalls auf die Grenzregionen zutreffen. Zweifel an diesen Unterschieden sind auch deshalb vorhan- 
den, da bereits der Clingendael Report vom März 1993 (Clingendael Report, 1993) deutliche Tendenzen aufzeigte, das das Verhältnis und die Kenntnis der deutsch-niederländischen Nachbarn im Grenzgebiet von den anderen Regionen abweicht. Zwar wurden in der Studie nur Jugendliche im Alter zwischen 15 und 19 Jahren in den Niederlanden befragt, dennoch zeichnet sich aus der Studie heraus ein deutliches Bild ab, das die Niederländer von den Deutschen haben. Das Bild besteht in dieser Untersuchung aus vier Komponenten: der Einstellung (Attitüde), den subjektiven Kenntnissen, den objektiven Kenntnissen und den Interessen. Für die vorliegende Untersuchung ist interessant, ob ein signifikanter Zusammenhang zwischen dem Interesse an Deutschland und der Häufigkeit eines Besuchs in Deutschland besteht. Aus der Clingendael Studie geht eindeutig hervor, dass je interessierter die Jugendlichen an Deutschland sind, die Zahl der Besuche dieser Gruppe in Deutschland zunimmt (Jansen, 1995, S. 190). Jansen kommt bei seiner Analyse der Studie ebenfalls zu dem Ergebnis, dass die Einstellung wesentlich durch den direkten Kontakt zu Deutschen geprägt wird. Der Autor stellt fest, dass Jugendliche mit deutschen Verwandten oder Freunden eine signifikant positivere Einstellung zu Deutschland und den Deutschen haben als andere. Auch die Höhe des Ausbildungsniveaus spielt bei der Einstellung gegenüber Deutschland eine entscheidende Rolle. Unter Schülern des VWO ist die Gruppe mit umfangreichen Kenntnissen bedeutend größer als unter allen anderen Gruppen von Schülern (1995, S. 188). Letztendlich ist die Aussage des Autors entscheidend für die vorliegende Studie, dass Jugendliche, die in der Nähe der deutschen Grenze wohnen, dem Nachbarland und dessen Bevölkerung positiver gegenüberstehen als andere. Dies ist ein Indiz dafür, das die Grenzregionen auch in der gemeinsamen kulturellen Entwicklung und der Entwicklung einer sozialen Norm sich von anderen Gebieten unterscheiden (Jansen, 1995, S. 196ff.). Insbesondere Konsumenten aus Grenzregionen sind es von Kindesalter gewohnt, im benachbartem Ausland einzukaufen. Deutsche Konsumenten aus dem Grenzgebiet kaufen Bekleidung in Roermond, Maastricht, Venlo oder Nijmegen ein und die Niederländer kommen nach Aachen oder Mönchengladbach. Diese Konsumenten kennen die im Nachbarland angebotenen Produkte und sind vertraut mit dem grenzüberschreitenden Einkauf. Für diese Konsumenten ist die Überquerung der Grenze nichts besonderes und der grenzüberschreitende Einkauf Teil ihres Alltagslebens. Dies bedeutet für Handelsunternehmen in der Grenzregion, das es ein hohes Maß an Gemeinsamkeiten der Konsumenten in Bezug auf ihre Ansprüche gegenüber dem Handel, hinsichtlich des Serviceangebots, der Sortimentsstruktur sowie der Atmosphäre in den Handelsunternehmen gibt. Diese Erkenntnis wird durch die aufgeführten Untersuchungen durch Middendorp aus dem Jahre 1999 und Etil im Jahre 2003 bestätigt. Sind diese Voraussetzungen erfüllt, ist eine grenzüberschreitende Kundenbindung möglich. Die Untersuchung der Studien ergab, dass es 
augenscheinlich relativ wenige Kulturunterschiede innerhalb des grenznahen Raumes zwischen Deutschen und Niederländern gibt. Andererseits wird aus den Studien nicht deutlich, was für Motive und Einstellungen die Konsumenten bewegen im jeweils anderen Land einzukaufen. Durch diese Motive und Einstellungen kann allerdings auch ein Kulturunterschied im grenznahem Raum erklärt werden.

\subsubsection{Lebensstile und Kaufverhalten im deutsch-niederländischen Vergleich}

Der Trend zur Expansion des heimischen Marktes oder besser gesagt, die Möglichkeit auch grenzüberschreitend Güter anzubieten, hat für Handelsfirmen unterschiedliche Auswirkungen. So wird sich ein großer Handelskonzern mit anderen Anforderungen konfrontiert sehen als ein regionaler Fachmarkt, eine kleine Einzelhandelskette wiederum mit anderen Anforderungen als ein sich grenzüberschreitend ausrichtendes Fachgeschäft. Gleichwohl sind alle Unternehmen von den neuen technologischen Entwicklungen betroffen. Im Folgenden wird versucht, die Auswirkungen auf die Handelsunternehmen darzustellen und aufzuzeigen, welche strategischen Konsequenzen sich daraus insbesondere für die niederländisch-deutsche Grenzregionen ergeben, unter Berücksichtigung der Lebensstile, der Kultur und des Kaufverhaltens deutsch-niederländischer Konsumenten.

In der Vergangenheit konnten Handelsunternehmen ihre Märkte relativ problemlos geografisch eingrenzen: Für ein Einkaufszentrum galt, dass der Einzugsradius maximal etwa $50 \mathrm{~km}$ beträgt. Damit waren - rein von der Geografie her - der Markt und das Einzugsgebiet bestimmt. Ähnliches war für nationale Handelsketten, wie zum Beispiel Ahold oder HEMA, der Fall. Auch hier konnten die Niederlande als Markt abgegrenzt werden. Diese geografische Marktdefinition verliert heute an Bedeutung. Niemand kann einen deutschen Kunden daran hindern, die Homepage der Ahold-Gruppe in den Niederlanden zu besuchen. Das Gleiche gilt natürlich auch umgekehrt für die Kunden der Ahold-Gruppe: Auch sie können problemlos auf eine deutsche, englische oder japanische Homepage zugreifen. Daraus resultiert, dass die Geografie als Marktabgrenzungskriterium nachhaltig an Wert verliert. Damit soll keinesfalls gesagt werden, dass ein homogener, globaler Markt entsteht. Vielmehr ist damit zu rechnen, dass die Marktsegmentierung über Netzwerke enorm an Bedeutung gewinnen wird, da Menschen begünstigt durch das Internet sich mit gleichgesinnten grenzüberschreitend ihre Erfahrungen austauschen können. Es wird eben nicht mehr einen nationalen Markt für Sportler geben, sondern einen weltweiten, grenzüberschreitenden Markt für Sporttaucher oder Drachen- 
flieger oder andere Segmente. Für den Handel heißt dies, dass die gesamte Marktsegmentierung überdacht werden muss. Es gilt, genau zu identifizieren, für welche spezifischen Kundengruppen welche Leistungen zu erbringen sind.

In diesem Zusammenhang sind auch andere als nur durch die Handelsleistungen gedeckte Bedürfnisse mit zu berücksichtigen. Von Bedeutung wird es demzufolge sein, Zielgruppen mit gleichen Interessenlagen zu identifizieren und zu „Segmenten" zusammenzuschließen. Mitglieder eines „Segments“ sind Konsumenten, die ihre Erfahrungen gerne mit anderen „Usern“ oder der Unternehmung teilen und bauen mit diesen über virtuelle Netze Beziehungen auf. Solche Beziehungen binden die Konsumenten weiter an die Unternehmung und fördern den Informationsaustausch innerhalb dieses Netzwerkes. Die richtige Definition und Abgrenzung derartiger „Segmente" ist von zentraler Relevanz für die zielgerichtete Ansprache auch grenzüberschreitender Konsumentengruppen. In diesem Zusammenhang kann durchaus auch in einem gewissen Umfang regionalisierten „Segmente" eine Bedeutung zukommen. Beispielsweise kann ein Handelsunternehmen definierte Konsumentengruppen in der betreffenden Region auch grenzüberschreitend zusammenfassen und auf diese Weise eine gemeinsame Erlebniswelt gestalten. Dieses „Segment“ besteht dann zwar aus Konsumentengruppen von beiden Seiten der Grenze, diese Konsumentengruppe hat aber die gleichen Interessen und spricht auf gleiche Angebote des Handelsunternehmens an.

Die Gestaltung des Leistungsprogramms ist für grenzüberschreitend tätige Handelsunternehmen von entscheidender Bedeutung. Hierzu gehören nicht nur die reinen Handelsleistungen, sondern auch Kommunikations- und Unterhaltungsleistungen sowie weitere Inhalte, die von Konsumenten beiderseits der Grenze akzeptiert und nachgefragt werden. Die Entwicklung eines solchen Leistungsprogrammes ist von zentraler Bedeutung, weil damit zu rechnen ist, dass die neuen Medien das Konsumentenverhalten nachhaltig verändern werden. Handelsunternehmen, die die diesbezüglichen Chancen erkennen und originelle Leistungssysteme entwickeln, können hier vorhandene Potenziale nutzen.

In einer Zeit des Umbruchs wird es für Handelsunternehmen schwieriger werden, Leistungen systematisch zu entwickeln und nach klassischen Methoden im Markt einzuführen. Märkte und Konsumenten erwarten eine extreme Zeitverkürzung, so dass die üblichen Entwicklungsphasen Planung, Einführung und Multiplikation stark verkürzt werden. Dies bedeutet, dass innovative Handelskonzepte, sobald deren Tauglichkeit für den grenzüberschreitenden Markt nachgewiesen ist, sofort von den Handelsunternehmen grenzüberschreitend multipliziert 
werden müssen. Aus dieser Sicht wird die grenzüberschreitende Orientierung kaum mehr eine andere Wahl lassen. Nimmt ein in der Grenzregion ansässiges Handelsunternehmen die Multiplikations-Chance nicht wahr, ist damit zu rechnen, dass seine Ideen und sein Konzept sehr rasch von Wettbewerbern aufgegriffen und von ihnen multipliziert werden.

Starke Marken stellen für Handelsunternehmen einen unschätzbaren Wert dar. Der Grund liegt darin, dass die (zumindest bis heute) anonyme Kommunikation über das Internet nur dann zum Tragen kommen kann, wo eine Vertrauensbasis besteht. Die Marke und das Image eines Handelsunternehmens sind wesentliche Bestandteile für den Aufbau einer derartigen Vertrauensbeziehung. Gelingt es einem Handelsunternehmen in der Grenzregion, neben globalen Marken auch ein eigenständiges grenzüberschreitendes Image zu etablieren, so ist dies ein nachhaltiger Wettbewerbsvorteil gegenüber anderen regionalen Anbietern. Welche Erwartungen die Konsumenten an den Handel stellen, wurde in einer Studie von Cap Gemini und Ernest\&Young (2002), in der mehr als 6.000 Konsumenten in 9 europäischen Ländern und 10.000 Konsumenten in den Vereinigten Staaten nach ihren Erwartungen an ein Handelsgeschäft gefragt wurden, untersucht. Zwar handelt es sich bei dieser Untersuchung um eine Studie, in der Inländer über den Einzelhandel ihres Landes befragt wurden, dennoch zeigten die Ergebnisse deutlich, welche Ansprüche sowohl niederländische als auch deutsche Konsumenten an den Einzelhandel stellen. Ein wesentliches Ergebnis ist, dass Konsumenten zwar auch nach guten, mit hoher Qualität versehenen Produkten, die preislich akzeptabel sind, suchen, aber durchaus auch nach geistiger Verwandtschaft, nach „human values" (Cap Gemini, Ernst \& Young, 2002, S. 6). Bei der Kaufentscheidung geht es somit verstärkt um glaubwürdige und ehrliche Beratung mit hoher Qualität kombiniert mit Vertrauen und Fairness. Dabei ist der Kunde laut dieser Studie durchaus bereit, gute Dienstleistungen entsprechend zu honorieren. Insbesondere die Gruppe der über 34-Jährigen legt Wert auf Beratung und Höflichkeit. Die Studie erbrachte folgendes Ergebnis, dass aufzeigt, welche Anforderungen die niederländischen und deutschen Konsumenten im eigenen Land an den Einzelhandel stellen. 


\begin{tabular}{|c|c|c|}
\hline \multicolumn{3}{|c|}{ Top Consumer Responses Overall } \\
\hline \multirow[t]{2}{*}{ Europe Top 5 Responses } & \multicolumn{2}{|c|}{$\%$ saying „extremely important“ } \\
\hline & NL & $\mathrm{D}$ \\
\hline The store is clean and well maintained & 68 & 64 \\
\hline Employees are courteous and respectful & 68 & 57 \\
\hline The retailer provides consistently good & & \\
\hline merchandise quality & & 57 \\
\hline The price is easily visible, the price is & & \\
\hline well marked & 68 & 55 \\
\hline You can unconditionally return & & \\
\hline merchandise with which you are unhappy & 63 & 61 \\
\hline You feel that you are getting an honest price, & & \\
\hline that the price has not been artificially & & \\
\hline increased & 64 & \\
\hline
\end{tabular}

Tab. 2.4: $\quad$ Vergleich der Anforderungen der Konsumenten an ein Handelsgeschäft in den Niederlanden und Deutschland (eigene Zusammenstellung, Zahlen aus: Cap Gemini, Ernst\&Young, 2002)

Werden die Ergebnisse miteinander verglichen, so haben Niederländer und Deutsche bei ihren fünf wichtigsten Ansprüchen gegenüber einem Einzelhandelsgeschäft vier übereinstimmende Bewertungskriterien. Daneben zählen für deutsche Konsumenten zu den fünf wichtigsten Anforderungen gegenüber einer Einkaufsstätte die Konsistenz der Qualität (dies kann ebenfalls als ein Ausdruck der Maskulinität der deutschen Kultur (siehe Kapitel 2.4.1) interpretiert werden) der angebotenen Produkte. Diese Anforderung gehört für niederländische Konsumenten nicht zu den fünf wichtigsten Anforderungen. Dagegen ist bei den Niederländern ein ehrlicher Preis ein wichtiges Argument, um sich für eine Einkaufsstätte zu entscheiden. Ein sauberes, gepflegtes Geschäft steht bei $64 \%$ der deutschen Konsumenten ganz oben auf der Wunschliste - ebenso wie beim europäischen Durchschnitt. Auf dem zweiten Platz steht mit $61 \%$ die Forderung deutscher Kunden, die Ware bei Nichtgefallen schnell und einfach umtauschen zu können. Überraschenderweise ist der Preis nicht das entscheidende Kriterium für deutsche Abnehmer. Dies liegt insbesondere am sehr ausgeprägten und gut organisierten Discountkonzept in Deutschland und den, im Vergleich zum europäischen Ausland, extrem geringen Preisunterschieden. Eine Differenzierung vom Wettbewerb gelingt dem Handel nur über andere Faktoren. In der Befragung durch Cap Gemini forderten mehr als die Hälfte der deutschen Verbraucher die Grundtugenden guter Einzelhandelsläden: konstant gute Waren- 
qualität (57\%), freundliche und hilfsbereite Mitarbeiter (57\%) sowie klare Preise $(55 \%){ }^{12}$ Besonders auffallend ist das Desinteresse der Konsumenten im Jahre 2002 ihre Einkäufe über das Internet abzuwickeln, obwohl das Internetshopping und der Aufbau einer Interneteinkaufsplattform von vielen Einzelhandelsunternehmen forciert wurde. Sowohl in Deutschland als auch im europäischen Schnitt landet dieser Aspekt auf dem letzten Rang mit acht bzw. sieben \%. Ein weiteres Ergebnis der Studie ist, dass die Hälfte der deutschen Konsumenten mindestens einmal pro Woche bei einem Discounter einkauft. Im europäischem Ausland können dies gerade $28 \%$ von sich behaupten. Dominierend auf dem deutschen Markt der Discountanbieter ist ALDI mit einem Marktanteil von beinahe $50 \%$ und mehr als 3.000 Filialen. Ein Drittel der befragten Verbraucher bezeichnet Aldi als seinen "Lieblings-Discounter", weit abgeschlagen auf Platz zwei folgt Lidl mit lediglich neun \% der Stimmen. Der große Vorsprung von ALDI liegt in günstigen Preisen, den Eigenmarken und der kundenfreundlichen Umtauschregelung begründet (Cap Gemini, Ernst\&Young, 2002). Auch im Jahr 2005 sind die Discounter bei den Konsumenten die beliebtesten Einkaufsstätten, allerdings ist ihr Wert von Oktober 2004 von 45\% auf 40\% im Februar 2005 gesunken. Im gleichen Zeitraum ist der Wert der Facheinzelhandelsgeschäfte leicht von $30 \%$ auf $33 \%$ gestiegen (www.omg-online.de/markenklima_index_q1_2005.ppt, abgefragt 26.7.2005). Zwar kann bei diesen Zahlen nicht von einer Trendwende gesprochen werden, doch die Wachstumszahlen der Discounter scheinen auch nicht weiter anzusteigen. Das Bild der Konsumenten gegenüber Supermärkten, Bekleidungs-/Schuh- und Accessoire-Geschäften sowie im Bereich Drogerie ist dagegen nur wenig differenziert. Diese Einkaufsstätten scheinen für den Konsumenten austauschbarer zu sein und kein solch klares Profil zu besitzen wie der Discounter ALDI.

Für die Niederländer ist neben der Grundforderung wie ein sauberes und gepflegtes Geschäft vor allem Ehrlichkeit und Respekt gegenüber dem Konsumenten besonders wichtig, was auch als Ausdruck der femininen niederländischen Kultur interpretiert werden kann (siehe Kapitel 2.4.1). Insbesondere der Aspekt Ehrlichkeit und Respekt vor dem Konsumenten ist bei Niederländern erheblich ausgeprägter als bei deutschen Konsumenten. Ferner wollen sie eine klare und deutliche Preisgestaltung und Auszeichnung. Dahinter folgt die Forderung nach einem guten Preis. Niederländer sind nach dieser Studie sehr preissensibel. Ferner ist den niederländischen wie den deutschen Konsumenten ein unkomplizierter Umtausch sehr wichtig. In diesem Punkt haben die niederländischen Konsumenten sogar einen höheren Anspruch als

\footnotetext{
${ }^{12}$ Die geringere Wichtigkeit hinsichtlich Übersichtlichkeit der Einzelhandelsstätte und dem Respekt der Einkaufsstättenmitarbeiter gegenüber Kunden unterstreicht ebenfalls die Unterschiede hinsichtlich maskuliner und femininer Kultur.
} 
die deutschen Konsumenten. Ferner ist der Service eines Einzelhandelsgeschäftes für die Niederländer laut dieser Studie sehr wichtig sowie ein fairer Preis, der dem Produkt angemessen ist und nicht nur als solcher vom Anbieter als Behauptung beworben wird.

Die Untersuchung der BRO (2005, S.31ff) bestätigt die Aussagen von Cap Gemini in weiten Teilen. Auch innerhalb der Euregio beurteilen die Konsumenten die im Nachbarland einkaufen das Einzelhandelsangebot nach der Qualität der Einkaufsstätte, dem angebotenen Sortiment, dem Preisniveau der Einkaufsstätte und der Qualität des Umfeldes. Dennoch unterscheiden sich Deutsche und Niederländer in ihrem Einkaufsverhalten und Einkaufsmotiven auch in dieser Studie voneinander:

\begin{tabular}{|c|c|c|}
\hline Frage: & $\mathrm{NL}$ & $\mathrm{D}$ \\
\hline Kaufe im Nachbarland ein: & $88 \%$ & $93 \%$ \\
\hline $\begin{array}{l}\text { Anteil der nicht-täglichenArtikel an } \\
\text { Gesamtausgaben im Nachbarland }\end{array}$ & $24 \%$ & $30 \%$ \\
\hline $\begin{array}{l}\text { Ausgaben im Nachbarland pro Ein- } \\
\text { kauf }\end{array}$ & $€$ 98,- & $€ 119,-$ \\
\hline Art der eingekauften Produkte & Modische Artikel & Lebensmittel, modische Artikel \\
\hline Motive & Gezielt Einkäufe tätigen & $\begin{array}{l}\text { Gaststättenbesuch, Besuch mit } \\
\text { (von) Freunden und Familie, Atmo- } \\
\text { sphäre im Nachbarland }\end{array}$ \\
\hline
\end{tabular}

Tab. 2.5: $\quad$ Unterschiede zwischen deutschen und niederländischen Konsumenten nach BRO Studie

Schaut man sich die Bevölkerungsverteilung in Deutschland und den Niederlanden an, so zeigt sich, dass der Anteil der Bevölkerung im Lebensalter 55+ in den Niederlanden eine noch größere Bedeutung hat als in Deutschland (CPB, 1997, S.114ff). Dies zeigt die genannte Studie von Cap Gemini und Ernst \& Young, die insbesondere auf die Erwartung von Respekt gegenüber ihrer Person aus Sicht dieser Gruppe (81\% der Niederländer) eingeht. Für diese Konsumentengruppe ist Respekt von Seiten der Einzelhandelsgeschäftsmitarbeiter gegenüber der Person des Kunden sehr wichtig. Ferner erwartet diese Gruppe der älteren Niederländer, dass auch der Ladenbau auf ihre Bedürfnisse (breite Laufgänge, große Übersichtlichkeit des Angebotes) abgestimmt ist. Statistisch gesehen ist allerdings die niederländische Bevölkerung relativ jung, doch auch in den Niederlanden ist mit einem Rückgang der Bevölkerung zu rechnen, nur mit einem Time-Lag gegenüber Deutschland (CPB, 1997, S. 115ff). Aufgrund den ähnlichen Einkommensverhältnissen, den ähnlichen Bevölkerungsstrukturen und auch 
den ähnlichen Auswirkungen des demografischen Faktors im Hinblick auf die zunehmende „Vergreisung" in beiden Ländern unterscheiden sich die Lebensstile der Konsumenten im deutsch-niederländischem Grenzgebiet nur marginal. Die Konsumenten haben in beiden Ländern laut dieser Studie ähnliche Werte, obwohl Deutsche und Niederländer nach Hofstede, aber auch nach Kasper und Usunier unterschiedlichen europäischen Kulturclustern angehören. Somit ergibt sich die Frage, ob die geografische Grenze oder auch vorhandene Kulturgrenzen wirklich so entscheidende Faktoren für das Treueverhalten der Konsumenten in einem so engen Wirtschaftsraum sind oder ob es nicht doch stärker die familiären Rahmenbedingungen sind, die das Kaufverhalten der grenzüberschreitenden Konsumenten in der Grenzregion beeinflussen. Ebenso ist zu klären, ob nicht auch die Anstrengungen der Annäherung auf wirtschaftlichem, politischem und kulturellem Gebiet in den vorgestellten Grenzregionen zwischen Deutschland und den Niederlanden dazu geführt haben, dass die Konsumenten auf beiden Seiten der Grenze heute viel eher bereit sind, nicht nur mobiler und flexibler ihre Einkäufe zu tätigen, sondern auch in der Lage sind, grenzüberschreitend sich loyal und treu einer Einkaufsstätte gegenüber zu zeigen. Dann würde es sich für Einzelhandelsunternehmen lohnen in Zufriedenheit und Bindung zu investieren um Konsumenten aus dem Nachbarland an sich zu binden.

\subsection{Zusammenfassung des Kapitels}

Ziel dieses Kapitels war es zu zeigen, welchen Einfluss die Landesgrenze und der Einfluss des sozialen Umfeldes auf das Kaufverhalten der Konsumenten hat. Insbesondere kulturelle Faktoren geben auch für den Konsumenten feste Größen vor, die sein Verhalten prägen und seinen Lebensstil beeinflussen. Konsumenten die im Nachbarland einkaufen suchen nicht nur nach Produkten mit hoher Qualität, die preislich akzeptabel sind, sondern sie suchen nach geistiger Verwandtschaft. Hier sind sich Niederländer und Deutsche sehr ähnlich. Dennoch herrscht eine Diskussion vor, dass beide Gruppen unterschiedlichen Kulturdimensionen angehören und auch aus diesem Grund ein unterschiedliches Kaufverhalten haben. Nach Hofstede haben Niederländer eine starke Neigung zum Individualismus, der das Verhalten steuert und einen Hang zur femininen Gesellschaft. Deutsche dagegen haben einen Hang zur maskulinen Gesellschaft. Diese Grundhaltung schlägt sich auch in den Anforderungen gegenüber einer Einkaufsstätte und im Kaufverhalten selbst nieder. Dienstleistung ist ein feminin besetztes Attribut, ebenso Ehrlichkeit und Respekt vor dem Anderen. Genau diese Anforderungen sind niederländischen Konsumenten besonders wichtig. Dagegen ist die Konsistenz der Qualität 
der angebotenen Produkte - ein maskulin besetztes Attribut - eine wesentliche Anforderung der deutschen Konsumenten. Für Hofstede ist die Charakterisierung der Kulturen mit der Charakteristik Femininität und Maskulinität eine Grundhaltung, die die Zugehörigkeit der Menschen zu einer Kultur verdeutlicht. Diese Grundhaltung beeinflusst das Handeln unbewußt. Das Kultur-Distanz Konzept von Hofstede und der angegebene Index ist ausgerichtet auf die Messung der kulturellen Unterschiede zwischen zwei Ländern, nicht zwischen individuellen Unterschieden der Menschen. Allerdings unterstellen verschiedene Autoren einen Zusammenhang zwischen den kulturellen Unterschieden von Ländern und den individuellen Auffassungen der Menschen (Earley and Mosakowsky, 2000, S. 26-49; Swift, 1999, S. 182201).

Das Unbewußte - die Einstellung zu Dingen, was für richtig und gut erachtet wird - hat somit mehr Einfluss auf das bewusste Handeln als umgekehrt. Kultur verstanden als das was unbewußt getan und geistig eingeordnet wird, entsteht zeitlich vor den Bewußtseinszuständen. Das bewusste Ich des Konsumenten hat somit wenig Einsicht in die Grundlagen seiner Wünsche und Handlungen. Kultur beeinflusst somit das Kaufverhalten auch in einer Grenzregion. Allerdings wirken dort weitere Elemente auf das Verhalten der Konsumenten ein. Dies sind einmal der traditionelle Kontakt mit dem Nachbarland, berufliche und verwandtschaftliche Kontakte. Hierdurch wird eine positive Grundtendenz zum Einkauf im Nachbarland gebildet. Dennoch wird der Konsument in seinem Kaufverhalten auch durch seine kulturelle Grundausrichtung beeinflusst und bestimmt. Auch Konsumenten die in einer engen nachbarschaftlichen Nähe miteinander wohnen, können deshalb dennoch ein unterschiedliches Kaufverhalten haben. 
44 | Kapitel 2 


\section{Kapitel 3: Konzeptuelle und empirische Grundlagen der Kundenbindungsforschung}

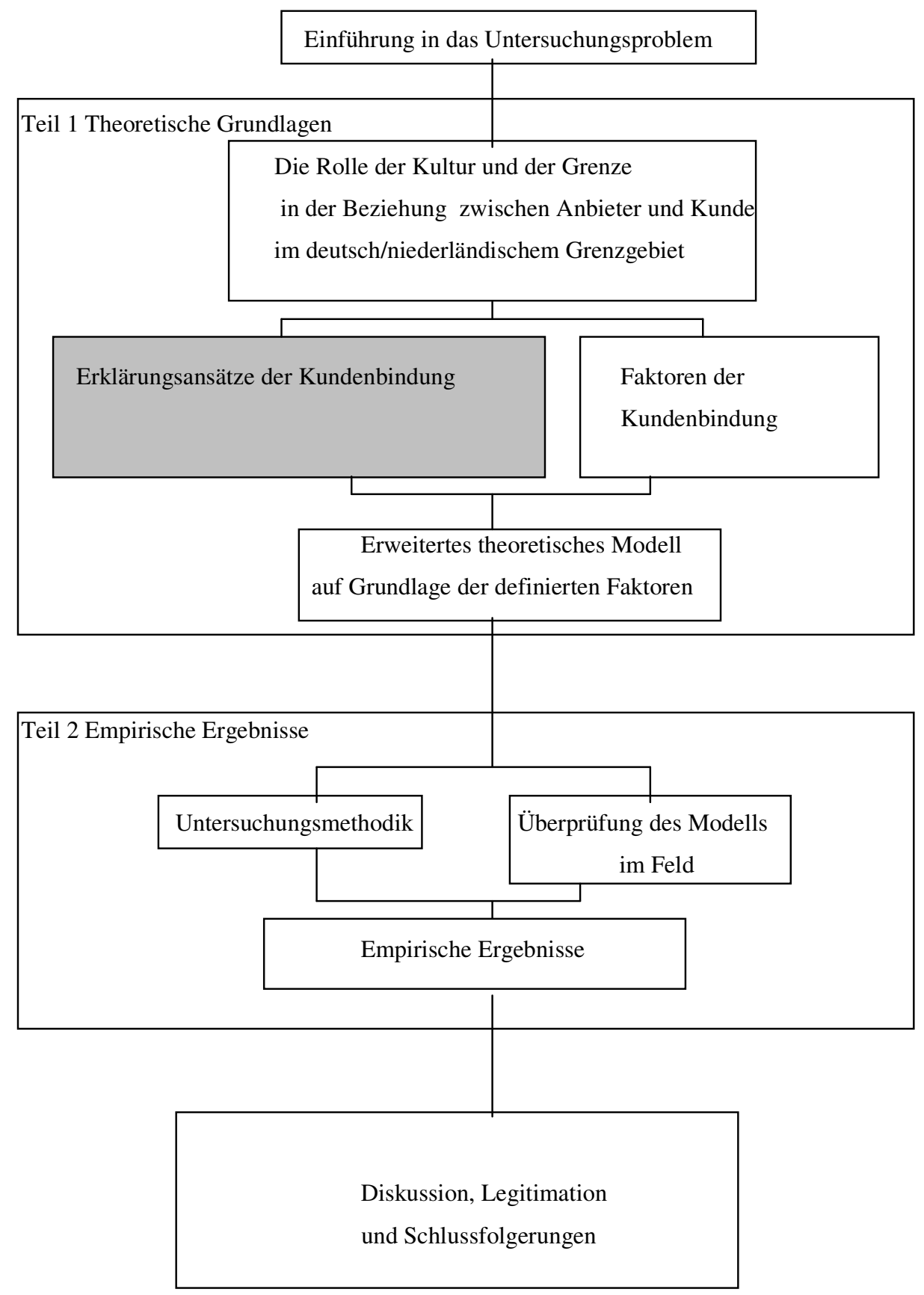


46| Kapite3

\section{1 $\quad$ Einleitung}

Das Kapitel 3 gibt einen Überblick über den Stand der Kundenbindungsforschung in der Literatur. Hierbei sollen alle wesentlichen Determinanten zur Bildung von Bindung erläutert und die begrifflichen Grundlagen der Arbeit gelegt werden. Ferner ist es Ziel dieses Kapitels Kundenzufriedenheit und Kundenbindung als Marketingziel der Einzelhandelsunternehmen darzustellen.

\subsection{Konsumentenbindung als zentrales Thema im Handel}

Für den Einzelhandel ist es standortunabhängig existentiell die Kunden an das Geschäft zu binden. Nur wenn es dem einzelnen Geschäft gelingt einen festen Kundenstamm aufzubauen, wird es zukünftig am Markt erfolgreich sein können. Aus diesem Grund kommt der Kundenbindungsforschung große Bedeutung zu. Der Begriff der Kundenbindung wird in der Literatur nicht einheitlich definiert. Eggert (1999, S.27) teilt die bestehenden Ansätze zur Definition der Kundenbindung in Anlehnung an Diller (1996, S.84) in drei Kategorien ein:

1. Kundenbindung als Bündel von Aktivitäten des Anbieters,

2. Kundenbindung als komplexe Merkmale der Kunden,

3. Kundenbindung als komplexes Merkmal der Geschäftsbeziehung zwischen Anbieter und Kunde.

Diese drei Ansatzpunkte können zu einer integrierten Definition der Kundenbindung zusammengefasst werden. Doch ist in der Praxis dieser integrierte Ansatz schwer umsetzbar, da Ausgangspunkt dieser Sichtweise die Aktivitäten des Anbieters sind. Allerdings kann auch auf Grundlage positiver Einstellungen der Kunden zum Anbieter und der Bereitschaft der Kunden zu Folgekäufen (Diller, 1996, S. 84) bereits von Kundenbindung gesprochen werden. Eggert (1999, S.78) sieht aus Sicht des Kunden bereits eine Bindung, ohne dass es zu einem wiederholten Kauf beim gleichen Anbieter gekommen ist. Kundenbindung aus Kundensicht wird als "ein psychologischer Zustand konzeptualisiert" (Eggert, 1999, S. 78). Nach Eggert liegt Kundenbindung aus Kundensicht bereits vor, wenn der Kunde eine Beziehung wahrnimmt. Der Autor differenziert dabei zwischen Verbundenheit und Gebundenheit (Eggert, 1999, S. 52 ). Verbundenheit basiert auf der Zufriedenheit des Kunden mit dem erhaltenen Nettonutzen in einer Geschäftsbeziehung. Gebundenheit beruht dagegen auf einer - negativ empfundenen Einschränkung der zukünftigen Wahlfreiheit des Kunden. Gebundenheit ist nach Eggert ein Bindungszustand, der auf ein "Nicht-Wechseln-Können" des Kunden beruht, Verbundenheit dagegen ein Bindungszustand, der ein "Nicht-Wechseln-Wollen" des Kunden beinhaltet. (Eggert, 1999, S.52-53). Odekerken-Schröder hat in diesem Zusammenhang den 
Begriff "Relationship Proneness" (Odekerken-Schröder, 1999, S. 42ff.) geprägt. Die Autorin versteht darunter die Geneigtheit des Konsumenten sich in eine engere Verbundenheit mit einem konkreten Einzelhandelsgeschäft einzulassen. In dieser Arbeit wird Kundenbindung als ein Bindungszustand der Verbundenheit betrachtet, da sich die weiteren Ausführungen auf die freiwillige Interaktion zwischen einer Ware und Dienstleistung anbietenden Einzelhandelsgeschäft und dem Endkonsumenten beziehen sollen. In vielen Fällen wird Kundenbindung mit Kundenloyalität gleichgesetzt bzw. beide Begriffe nicht eindeutig unterschieden. Deshalb werden zunächst beide Begriffe definiert und im Rahmen dieser Arbeit voneinander abgegrenzt. Im Englischen spricht man von Customer Loyalty, wobei der Begriff loyal aus dem Englischen ins Deutsche mit Treue übersetzt werden kann (Schäfer, 1979, S. 155). Eine ähnliche Definition findet sich im Oxford Dictionary (Fowler und Fowler, 1964, S. 725). Der Begriff Loyalty wird an gleicher Stelle definiert mit „loyal temper or conduct“. Loyalität wird somit als Treue betrachtet. Bloemer und De Ruyter (1998, S. 500) definieren Geschäftstreue (Kunden-Loyalität im Einzelhandel) wie folgt: „The biased (i.e. non random) behavioural response (i.e. revisit), expressed over time, by some descision making unit with respect to one store out of a set of stores, which is a function of psychological (decision making and evaluative) processes resulting in ${ }^{13}$ store commitment." Hier wird die Treue des Kunden als ein wiederkehrendes Verhalten beschrieben. Diese Einschätzung teilt auch Diller, der unter Customer Loyalty aus Sicht des Kunden: "Positive attitude towards supplier combined with a willingness to perform further transactions"(2000, S. 31) versteht. Aus Sicht des Anbieters kann man von Kundenbindung erst sprechen, wenn Kunden einer Geschäftsstätte gegenüber loyal sind und regelmäßig als Kunden wiederkehren. Die Handlungsdimension des Kaufes kommt somit hinzu. Der Begriff der Kundenbindung ist mithin umfassender und dem deutschen Begriff der Loyalität übergeordnet. Dies bestätigt auch Diller mit seiner Definition von Kundenbindung aus Sicht des Anbieters, "wenn innerhalb eines als zweckmäßig definierten Zeitraumes wiederholte Informations-, Güter- und Finanztransaktionen zwischen zwei Geschäftspartnern stattgefunden haben (ex-post Betrachtung) bzw. geplant sind (ex-ante Betrachtung)“ (1996, S. 84).

\footnotetext{
${ }^{13}$ Die Autoren nennen in ihrer Arbeit den Begriff „,brand commitment“, sie meinen aber ,store commitment“.
} 
48 | Kapitel 3

In beiden Fällen spricht Diller im Englischen jedoch von Customer Loyalty, er meint damit sowohl Treue, Loyalität als auch Kundenbindung. Er differenziert den Begriff, in dem er die Sichtweise ergänzt. Die vorliegende Arbeit konzentriert sich auf die Sichtweise des Kunden. Aus diesem Grund wird der englische Begriff Loyalty verwendet und gleichgesetzt mit dem deutschen Begriff der Kundenbindung. Für den Autor ist die Handlungsdimension des Kunden im Rahmen dieser Untersuchung jedoch sehr wichtig. Es macht aus seiner Sicht keinen Sinn, nur die Dimension der Loyalität zu betrachten, sondern es muss die Dimension des Wiederkaufs mit in die Betrachtung einbezogen werden. Ein „Kunde“ kann einem Geschäft gegenüber loyal sein, ohne dort immer wieder seine Einkäufe zu tätigen. Für den Handel ist die Handlungsdimension des Kaufes entscheidend, daher wird im Folgenden der Begriff Loyalty mit dem Begriff der Kundenbindung gleichgesetzt. Ebenso wird der Begriff der Kundenloyalität, aus den dargelegten Gründen, dem Begriff der Kundenbindung untergeordnet. Bei einer Gegenüberstellung der unterschiedlichen deutschen und englischen Begriffe ergibt sich folgendes Schema: 


\begin{tabular}{|c|c|c|c|c|c|c|}
\hline & Zufriedenheit & Kundenbindung & $\begin{array}{l}\text { Geschäftstreue } \\
\text { zur Einkaufsstätte }\end{array}$ & $\begin{array}{l}\text { Loyalität zur } \\
\text { Einkaufsstätte }\end{array}$ & $\begin{array}{l}\text { Relationship } \\
\text { Proneness }\end{array}$ & Loyality \\
\hline Definition & $\begin{array}{l}\text { Vergleich des } \\
\text { Nachfragers } \\
\text { zwischen Er- } \\
\text { wartung und } \\
\text { Resultat. (Kun- } \\
\text { denzufriedenheit } \\
\text { als Resultat eines } \\
\text { psychischen } \\
\text { Vergleichs- } \\
\text { prozesses } \\
\text { Differenzierung } \\
\text { zwischen: } \\
\text { manifeste und } \\
\text { latente Kunden- } \\
\text { zufriedenheit } \\
\text { (Bloemer und De } \\
\text { Ruyter 1998). }\end{array}$ & 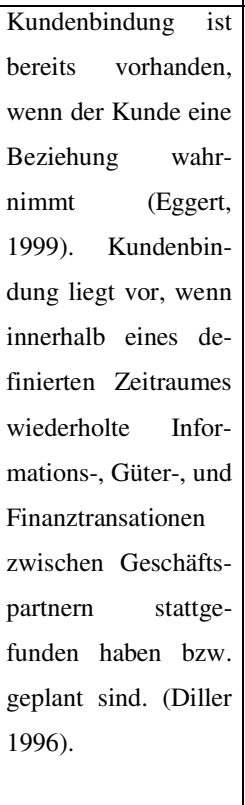 & $\begin{array}{l}\text { Treue des Konsu- } \\
\text { menten als ein } \\
\text { wiederkehrendes } \\
\text { Verhalten } \\
\text { (Bloemer und De } \\
\text { Ruyter, 1998). }\end{array}$ & $\begin{array}{l}\text { Kunde ist einer } \\
\text { Einkaufsstätte ge- } \\
\text { genüber loyal, } \\
\text { ohne wiederholte } \\
\text { Einkäufe zu } \\
\text { tätigen. } \\
\text { Wahre Loyalität: } \\
\text { Bewertungs- und } \\
\text { Entscheidungs- } \\
\text { prozess liegt der } \\
\text { Entscheidung des } \\
\text { Konsumenten zu } \\
\text { Grunde } \\
\text { Scheinloyalität: } \\
\text { Loyalität aus } \\
\text { Trägheit } \\
\text { (Bloemer und } \\
\text { Kasper 1995). }\end{array}$ & $\begin{array}{l}\text { Geneigtheit des } \\
\text { Konsumenten } \\
\text { sich in eine } \\
\text { engere Ver- } \\
\text { bundenheit mit } \\
\text { einem konkreten } \\
\text { Einzelhandelsge- } \\
\text { schäft einzulassen } \\
\text { (Odekerken- } \\
\text { Schröder, 1999). }\end{array}$ & $\begin{array}{l}\text { Positive Einstel- } \\
\text { lung des Nachfra- } \\
\text { gers kombiniert } \\
\text { mit dem Wunsch } \\
\text { auch zukünftig } \\
\text { Transaktionen mit } \\
\text { dem Anbieter } \\
\text { einzugehen } \\
\text { (Diller 2000). }\end{array}$ \\
\hline $\begin{array}{l}\text { Auswir- } \\
\text { kungen auf } \\
\text { Verhalten. }\end{array}$ & $\begin{array}{l}\text { Grundlage zum } \\
\text { Aufbau von } \\
\text { Kundenbindung. }\end{array}$ & $\begin{array}{l}\text { Kaufverhalten, } \\
\text { Weiterempfehlung, } \\
\text { Verbundenheit. }\end{array}$ & Commitment & $\begin{array}{l}\text { Wiederkauf-, } \\
\text { Weiterempfehl- } \\
\text { ungs-, Cross- } \\
\text { buying-Absicht. }\end{array}$ & Verhaltensabsicht & $\begin{array}{l}\text { Verbundenheit, } \\
\text { Weiterempfeh- } \\
\text { lung, Kaufverhal- } \\
\text { ten. }\end{array}$ \\
\hline $\begin{array}{l}\text { Einordnung } \\
\text { der Begriffe }\end{array}$ & $\begin{array}{l}\text { Ergebnis eines } \\
\text { Lernprozesses des } \\
\text { Kunden.. }\end{array}$ & $\begin{array}{l}\text { Ist dem } \text { Begriff der } \\
\text { Loyalität } \\
\text { net. } \text { übergeord- } \\
\text { entspricht } \\
\text { Englischen } \text { dem } \\
\text { Loyalty. }\end{array}$ & Teil der Loyalität. & $\begin{array}{l}\text { Teil der Kunden- } \\
\text { bindung. }\end{array}$ & $\begin{array}{l}\text { Teil der Kunden- } \\
\text { bindung. }\end{array}$ & $\begin{array}{l}\text { Entspricht dem } \\
\text { deutschen Begriff } \\
\text { der Kunden- } \\
\text { bindung. }\end{array}$ \\
\hline
\end{tabular}

Tab. 3.1 Einordnung der deutschen und englischen Begriffe (eigene Darstellung).

Kundenloyalität wächst, sie entsteht im Zeitablauf. Eine große Rolle wird dabei der Zufriedenheit des Konsumenten mit einem Einzelhandelsgeschäft beigemessen. Zufriedenheit definieren Bloemer und De Ruyter (1998, S. 501) als , The outcomes of the subjective evaluation that the chosen alternative (the store) meets or exceeds expectations“. Eine wesentliche Komponente der Zufriedenheit ist das Image des Geschäftes (store image). Das Image eines Einzelhandelsgeschäftes kann mithin wesentlichen Einfluss sowohl auf die Geschäftsloyalität des Kunden als auch auf die Zufriedenheit des Kunden mit dem Geschäftsbesuch und einkauf haben. Dieser Aspekt kann insbesondere im deutsch-niederländischen Grenzgebiet eine entscheidende Rolle spielen, wenn die Meinung der sozialen Gruppe entscheidend zum Kaufentschluß beiträgt. Bloemer und De Ruyter (1998, S. 501) definieren das Geschäftsimage wie folgt: „The complex of a consumer's perceptions of a store on different (salient) 
attributes." Das Image selbst besteht jedoch wiederum aus unterschiedlichen Komponenten, die an dieser Stelle jedoch nicht weiter betrachtet werden.

Der kausale Zusammenhang zwischen Kundenzufriedenheit und Loyalität ist in einer großen Anzahl von Studien (vgl. Anderson, Sullivan, 1993, Mittal, Ross und Baldasare 1998, Mittal, Kumar und Tsiros, 1999) bereits dargestellt worden. Insbesondere Bloemer (1993) sowie Bloemer und Kasper (1995) machen deutlich, wie komplex der Zusammenhang ist. So sehen Bloemer und Kasper in der Kundenloyalität weniger eine Komponente des Commitment, eher im Commitment eine Dimension der Kundenloyalität (Bloemer und Kasper, 1995, S. 312) und unterscheiden zwischen wahrer und Pseudoloyalität. Dabei sprechen die Autoren von wahrer Loyalität, wenn ein Bewertungs- und Entscheidungsprozess der Entscheidung des Konsumenten zu Grunde liegt, von Pseudoloyalität sprechen die Autoren, wenn die Loyalität aus der Trägheit und Untätigkeit des Konsumenten resultiert und nicht auf Commitment. Im Rahmen der Kundenzufriedenheit entwickelten Bloemer und Kasper (1995, S. 313) und Bloemer und De Ruyter (1998, S.499 ff.) einen Typologisierungsvorschlag und differenzieren zwischen der latenten und manifesten Kundenzufriedenheit. Manifeste Kundenzufriedenheit liegt dann vor, wenn die Konsumenten einen expliziten Vergleich zwischen Erwartungen und wahrgenommener Leistung vorgenommen haben, wenn dies nicht der Fall ist, liegt latente Kundenzufriedenheit vor. Dabei unterscheiden sie nach dem gedanklichen Aufwand, den ein Kunde in die Bewertung einer erbrachten Leistung setzt und dem Bewußtseinsgrad seiner Zufriedenheit hinsichtlich des Soll-Ist-Vergleiches zwischen der latenten und der manifesten Zufriedenheit (Bloemer und Kasper, 1995, S. 311 ff; Bloemer und De Ruyter, 1998, S. 499ff.). Dabei wird latente Zufriedenheit definiert als „the result of an implicit evaluation which is not elaborated upon“, manifeste Zufriedenheit dagegen als ,the result of an evaluation which is well elaborated upon“ (Bloemer und Kasper, 1995, S.315, Bloemer und Poiesz, 1989, S.43-48).

Die Begriffe Loyalität, Geschäfts- oder Einkaufsstättentreue orientierten sich lange Zeit lediglich an der behavioristischen Komponente, indem sie nur die beobachtbaren Kaufwiederholungsverhalten zum Inhalt hatten. Hierbei liegt Treue bereits vor, wenn ein Käufer dasselbe Geschäft wiederholt aufsucht bzw. Einkäufe bevorzugt in einem Geschäft tätigt ( Diller, 1992, S. 241; Jungwirth, 1997, S. 22). Es besteht aber die Notwendigkeit auf die Konsistenz der Verhaltens- und der Einstellungskomponente hinzuweisen (Day, 3/1969, S. 29), da ansonsten die Gefahr besteht, zufällige Kaufwiederholungen oder wiederholte Einkäufe im gleichen Geschäft auf Grund von nichtvorhandenen Alternativen als Geschäftstreue und Bindung zu 
bezeichnen (Jungwirth, 1997, S. 23). Nach Bloemer (1993, S. 89) ist es nicht notwendig, dass der gesamte psychologische Prozess vom Kunden vor dem Wiederkauf durchlaufen wird. Für die Autorin ist der Wiederkauf eine Form der Trägheit des Kunden, indem er das Produkt oder in der Einkaufsstätte kauft mit dem/der er bereits Erfahrungen gesammelt hat. Ferner ist ein weiterer Kaufgrund die einfache Erreichbarkeit der Einkaufsstätte und die Verfügbarkeit des Produktes. Sofern allerdings wahre Bindung vorliegt, ist für Bloemer „commitment" des Nachfragers zum Produkt oder zur Einkaufsstätte eine notwendige Voraussetzung. Jacoby bezeichnet das Wiederholungskaufverhalten als die notwendige und die positive Einstellung als die hinreichende Bedingung für das Vorliegen von Loyalty (Jacoby, 1971, S. 26; zitiert in: Jungwirth, 1997, S. 23). Im Gegensatz zum habitualisierten Einkaufen in einem Geschäft, das gewohnheitsmäßig und automatisch verläuft, entscheidet sich ein wirklich treuer Kunde auch nach kognitiv aufwändigen Informationsbeschaffungs- und verarbeitungsprozessen ganz bewusst wiederholt für die gleiche Einkaufsstätte. Dies soll aber nicht heißen, dass bei habituellen Kaufverhalten keine Geschäftstreue vorliegt. Entscheidend ist, dass auch in diesem Fall ein bewusster Entscheidungsprozess erfolgt bzw. erfolgt ist. Kundenbindung bezeichnet somit eine Form von Wiederkaufsverhalten, bei der bei mehrfachen Einkäufen im selben Geschäft/Unternehmen ein positiver psychologischer - evaluativer Entscheidungsprozess vorhergeht. Insgesamt gehen die meisten Autoren davon aus, dass der Zusammenhang zwischen diesen beiden Variablen nicht linear ist (Mittal und Kamakura, 2001). Der Zusammenhang zwischen Zufriedenheit und Loyalität ist charakterisiert durch sehr unterschiedliche Variablen wie z.B. Geschlecht, Lust auf Veränderung, soziales Umfeld und Identifikation (Anderson 1994, Mittal und Kamakura 2001, Oliva, Oliver und MacMillan 1992). Dennoch mehren sich die Zweifel an der Signifikanz der Beziehung zwischen Zufriedenheit, Loyalität und Kundenbindung und den existierenden Relationen (Braunstein, 2001, S. V), da auch zufriedene Kunden zu einem großen Anteil bei der nächsten Konsumhandlung die Einkaufsstätte oder das Produkt wechseln.

Trotz der zahlreichen Veröffentlichungen zu diesem Themenbereich kann aus heutiger Sicht nicht von einem eindeutigen Forschungsansatz ausgegangen werden, der den komplexen $\mathrm{Zu}-$ sammenhang zwischen Kundenzufriedenheit und Kundenbindung erklärt und die existierenden Relationen vollständig erläutert.

\section{'Konsument, Handel und Konsumententreue`}

Laut Nachschlagewerk (Sellien, Sellien, 1977, S. 2463) ist ein Konsument ein Bezieher und Verbraucher von Konsumwaren. Ein Konsument wird somit als ein Letztverbraucher defi- 
niert, „der ein Bedürfnis oder einen Wunsch feststellt, einen Kauf tätigt und sich schließlich des Produktes wieder entledigt" (Solomon, Bamossy und Askegaard, S. 2001, S. 23). Der Konsum verläuft nach dieser Definition in drei Schritten (1. Bedürfnis feststellen, 2. Kauf tätigen und 3. sich des Produktes wieder entledigen) ab. An diesem Prozess sind allerdings verschiedene Personen beteiligt. Dabei sind Käufer und Nutzer eines Produktes nicht immer die gleiche Person. Frauen kaufen für ihre Männer Textilien oder Schuhe ein, Eltern kaufen für ihre Kinder. Der Konsum findet dann durch die Person statt, für die gekauft wurde. So erwirbt beispielsweise eine Mutter in den meisten Fällen Produkte für ihre Kinder, die diese konsumieren. Grundsätzlich muss berücksichtigt werden, dass die Tatsache, für wen die Produkte gekauft werden, einen großen Einfluss auf den Kaufentscheidungsprozess hat (Kroeber-Riel, Weinberg, 2003, S. 446 ff.). Auch haben andere Personen Einfluss auf den Kaufprozess, indem sie Empfehlungen oder Ablehnungen zum Kauf aussprechen, ohne dass sie am Kaufprozess direkt beteiligt sind. Ferner kann es sich bei Konsumenten auch um Organisationen oder Gruppen handeln, bei denen einer die Kaufentscheidung für viele andere Personen trifft. Hierunter sind gemeinsame Einkäufe von Sport- oder Freizeitclubs zum persönlichem Gebrauch zu verstehen. Reaktionen der Konsumenten sind somit unter anderem ein Test, ob eine eingeschlagene Marketingstrategie zum Erfolg führt.

In Anlehnung an Plinke (1989, S. 305-321) kann eine Geschäftsbeziehung als eine nicht auf Zufall beruhende Folge von Markttransaktionen zwischen einem Unternehmen und dem Endverbraucher verstanden werden. Die Beziehungen sind auf beiderseitige Beweggründe zurückzuführen. Diese Beweggründe existieren sowohl auf der Anbieter als auch auf der Nachfragerseite und lassen eine planmäßige Verknüpfung zwischen Markttransaktionen sinnvoll erscheinen (Plinke, 1989, S. 307). Sie stellen die Grundlage für eine innere Bindung zwischen den Geschäftspartnern dar, die nicht notwendigerweise auf vertragliche Verpflichtungen basieren muss. Die innere Verpflichtung eines Konsumenten, zum Beispiel einer Einkaufsstätte langjährig treu zu bleiben, wird als Commitment (siehe Kapitel 3.3.5) bezeichnet (Söllner, 1999, 219-233). Commitment kann dann als Maßstab für die Qualität der KonsumentenAnbieterbeziehung herangezogen werden (Diller, 1994, S. 10). Stabile Beziehungen fördern nicht nur die Toleranz und die Treue zum Geschäftspartner, sie hat auch zur Folge, dass die Suche nach Alternativen stark eingeschränkt wird oder im Idealfall sogar vollkommen ausbleibt (Diller, Kusterer, 1988, S. 218). Aus diesem Grund wird insbesondere von der Metro 
Group die Strategie des Retail Branding ${ }^{14}$ verfolgt, in der es um die alleinige Frage geht, wie der Händler im Gedächtnis der Konsumenten bleibt.

Bereits Seyffert (Seyffert, 1972, S.1) spricht vom Handel als „das Verbindende zwischen Produktion und Konsumtion, wobei es unerheblich ist, ob er seine vermittelnde Funktion selbstständig oder angegliedert erfüllt.“ Primäre Aufgabe des Handels ist somit den Güteraustausch zwischen Produzenten und Konsumenten herbeizuführen. Konkreter wird der Katalog E des Instituts für Handelsforschung, indem zwischen Handel im funktionellen und institutionellen Sinn differenziert wird. „Handel im funktionellen Sinn liegt vor, wenn Marktteilnehmer Güter, die sie in der Regel nicht selbst be- oder verarbeiten (Handelswaren), von anderen Marktteilnehmern beschaffen und an Dritte absetzen.“ (Ausschuss für Begriffsdefinitionen, Hrsg., 1995, S.28). Der Katalog E definiert „Handel im institutionellen Sinne - auch als Handelsunternehmung, Handelsbereich oder Handlung“ als ,,jene Institutionen, deren wirtschaftliche Tätigkeit ausschließlich oder überwiegend dem Handel im funktionellen Sinne zuzurechnen ist. In der amtlichen Statistik wird eine Unternehmung oder ein Betrieb dann dem Handel zugeordnet, wenn aus der Handelstätigkeit eine größere Wertschöpfung resultiert als aus einer zweiten oder mehreren sonstigen Tätigkeiten.“ (Ausschuss für Begriffsdefinitionen, Hrsg., 1995, S.28). Der Begriff des Einzelhandels wird ebenfalls sowohl funktionell als auch institutionell verstanden. Funktionell ist Einzelhandel die wirtschaftliche Tätigkeit des Umsatzes von Gütern (Handelswaren) an Letztverbraucher (private Haushalte). Im institutionellen Sinne umfasst der Einzelhandel alle Institutionen, deren wirtschaftliche Tätigkeit ausschließlich oder doch überwiegend dem Einzelhandel im funktionellen Sinne zuzurechnen ist (Katalog E, 2. Ausgabe 1975, S.18). Den Schwerpunkt der vorliegenden Arbeit bildet die Betrachtung der Fachgeschäfte. Diese werden definiert als ein Einzelhandelsbetrieb, der Waren einer Branche (Sortiment) mit ergänzenden Dienstleistungen anbietet, wobei das Bedienungsprizip überwiegt. Diese Definition des Einzelhandelsbetriebes beinhaltet ebenso Filialbetriebe mit mindestens fünf standörtlich getrennten, aber unter einheitlicher Leitung stehenden Verkaufsstellen. (Katalog E, 2. Ausgabe 1975, S.19-20). Mit eingeschlossen in dieser Betrachtung sind Verkaufsfilialen von Herstellern.

Der europäische Einzelhandel und insbesondere der Einzelhandel in Grenzregionen sieht sich seit Beginn der 90er Jahre mit einem tief greifenden Strukturwandel und zahlreichen daraus

\footnotetext{
${ }^{14}$ Als "Unternehmensmarken" (Retail Brands) entwickeln die Vertriebslinien der Metro Grppe ihr eigenes Image für einen erfolgreichen Marktauftritt. Kunden werden individuell angesprochen und durch neue, attraktive Instrumente langfristig gebunden.
} 
resultierenden Herausforderungen konfrontiert (Jungwirth, 1997, S. 1). Einige Konsumentengruppen verhalten sich über nationale Grenzen hinweg gleich, daneben können Unterschiede im Konsumentenverhalten vielfach von Staat zu Staat, sogar von Region zu Region festgestellt werden (Solomon, Bamossy und Askegaard, 2001, S. 19). Beeinflussende Faktoren können dabei bestimmte gesellschaftliche Gruppen eines Landes oder einer Region sein, die einen starken Einfluss auf das Konsumentenverhalten ausüben oder der internationale Einfluss auf Konsumentengruppen. Insbesondere in der Grenzregion zwischen Deutschland und den Niederlanden hat der grenzüberschreitende Konsum Tradition. Auf Grund nachbarschaftlicher Verhältnisse und verwandtschaftlicher Verflechtungen ist traditionsgemäß in den Grenzregionen ein großes gegenseitiges Vertrauen auch in die Geschäftsangebote vorhanden. Dabei zeigen Studien (Dubois und Laurent, 1993, S. 58-69), dass Konsumenten, die einem Kulturwechsel offen gegenüberstehen, d.h. grenzüberschreitend konsumieren, auch diejenigen sind, die mehr Geld für Luxusgüter, aber auch für alltägliche Güter aus dem Nachbarland ausgeben und dies sogar unabhängig von ihrer demographischen und sozialen Klasse. Dies bedeutet, dass es für die Einkaufsstätten in Grenzregionen besonders wichtig ist, Kunden zu identifizieren und sie bezüglich ihrer Bedürfnisse $\mathrm{zu}$ segmentieren und Strukturen zu beschreiben. Die Zusammenhänge und Abhängigkeiten, die hierdurch bekannt werden, können genutzt werden, um Kundenbindung zu erzeugen.

Noch vor 40 Jahren waren Kundenbindungen insbesondere in der ländlich strukturierten deutsch-niederländischen Grenzregion stark in sozialen Fixpunkten wie dem Dorfladen verankert. Es wurde in mit Traditionen behafteten Bahnen konsumiert. Kundentreue wurde in der Familie ,überliefert“ und Kundenbindung war das Ergebnis gelebter sozialer Strukturen. Konsumentscheide erfolgen nach wie vor auf Grund von Fixpunkten. Aber anstatt sich an sozialen Fixpunkten zu orientieren, sind es die situativen Fragen, die der Konsument beantwortet haben möchte (Buttle, 1996, Reichheld, 1993). Die Kommunikation in der Interaktion ist geprägt durch die Erwartungen des Kaufenden, seinen Fähigkeiten Absichten zu übermitteln und der Bereitschaft sowie Fähigkeit des Verkaufenden, diese Anliegen wahrzunehmen und zu befriedigen. Die klassische Vorstellung, dass es sich im Marktgeschehen immer um eine einzige, von weiteren Aktivitäten isolierte rationale Transaktion handelt, die zwischen weitgehend anonym bleibenden Marktpartnern stattfindet, hat somit heute keine Gültigkeit mehr (Grönroos, 1994, S. 4-20, Gummesson, 1987, S. 10-20, Sheth und Parvatiyar, 1995, S. 255-272, Arndt, 1979, S. 69-75). Die Kundenbindungsforschung hat aus diesem Grund durch verhaltenstheoretische Ansätze versucht, das Konstrukt Kundenbindung zu erklären. Allerdings konzentrieren sich diese Versuche primär auf einen Ländermarkt. Weniger untersucht 
ist der Sachverhalt der grenzüberschreitenden Kundenbindung im Einzelhandel. Es wird jedoch vermutet, dass die Kundenbindung in einer Grenzregion durch weitere Faktoren, die noch nicht umfassend untersucht sind, beeinflusst wird. Solche Kriterien zu kennen ist jedoch für den Handel entscheidend.

In der vorliegenden Studie wird untersucht, ob sich verhaltenswissenschaftliche Erklärungsansätze als Nachweis der Kundenbindung in einem grenzüberschreitenden Raum eignen. Auch der Ansatz des „buyer relationship proneness“ basiert auf der behavioristischen Theorie. Die Marktpartner suchen in dieser Form der Partnerschaft nicht nur einen Austausch auf Basis einer ökonomisch begründeten Interessenpartnerschaft z.B. (Equity theory), sondern wollen auch die soziale Komponente einer geschäftlichen Partnerschaft befriedigt wissen (Adams, 1965, S. 267-299). Die Treue des Konsumenten ist der kritischste Faktor der Relationship Theorie (Odekerken-Schröder, 1999, S. 38). Aus diesem Grund wird zum Modell der Kundenorientierung und buyer relationship proneness die Theorie des geplanten Verhaltens hinzugefügt, um die Determinanten der Kundenbindung und Kundenloyalität in einem grenzüberschreitenden Wirtschaftsraum zu überprüfen. Diese Theorie eignet sich in besonderem Maße zur Erklärung der sozialen, psychischen und kulturellen Faktoren der Bindung, da sie sich mit der Übersetzung von Intentionen in Handlungen sowie mit der konkreten Ausführung von Handlungen beschäftigt. Die spezifische Struktur des Kundenbindungsmodells wird dann in einem gesonderten Kapitel dargestellt.

Konsumentenloyalität ist das zentrale Thema im Handel zum Beginn des 21. Jahrhunderts. Dennoch ist die Kenntnis über die Zusammenhänge im Relationship-Marketing noch nicht stark ausgeprägt. Es liegen kaum Erkenntnisse vor, die aufzeigen, warum bis zu $80 \%$ der Konsumenten trotz Zufriedenheit bei der nächsten Kauf- und Konsumhandlung den Anbieter wechseln (Braunstein, 2001, S.V). Zumeist stammen diese Erkenntnisse aus den USA. Eine Übertragung auf den europäischen Markt muss zumindest somit in Frage gestellt werden. Unabhängig davon würde es helfen, einen vertiefenden Einblick und Verständnis in die Abläufe zur Entscheidungsfindung des Konsumenten zu bekommen, wenn man die Hintergründe eines solchen Verhaltens betrachten würde (Singh und Sirdeshmukh, 2000, S. 150). So schreibt auch Odekerken-Schröder (1999, S. 17), dass eine unterstützende Studie vonnöten ist, die die Entscheidungsfindung der Konsumenten erklärt und abweicht von der neobehavioristischen Kundenbindungsforschung. Als Voraussetzung dafür gilt es, Kundenzufriedenheit, Kundenloyalität und Kundenbindung als Marketingziel der Einzelhandelsunternehmen in das betriebliche Zielsystem einzubinden und den derzeitigen Entwicklungsstand der Kundenbindungs- 
forschung darzustellen. Die Entscheidungsfindung des Konsumenten wird wiederum beeinflusst durch seine Lebensweise, seinen kulturellen Hintergrund, die Stärke der Ausprägung seines Heimatgefühls sowie der Vertrautheit des Konsumenten mit angebotenen Produkten, Einkaufsstätten und die Selbstverständlichkeit mit der diese aufgesucht werden. Die aufgeführten Begriffe sind inhaltlich abhängig vom näheren Umfeld des Konsumenten. Der Lebensstil (Lifestyle) wird in diesem Zusammenhang definiert als: „A person`s pattern of living as expressed in his or her activities, interests and opinions." (Kotler, Armstrong, Saunders und Wong, 1999, S. 1003). Unter Heimatgefühl soll in der vorliegenden Studie die Verbundenheit mit vorhandenen Traditionen, auch Konsumtraditionen, sowie die Identifikation mit einer Region (hier der Grenzregion) verstanden werden. Eng verbunden hiermit ist der Begriff der Vertrautheit (familiarity) definiert als ,close intercourse, intimacy with person or some subjects“ (Fowler, H.W. und Fowler, F.G., 1964, S. 436). Diese Begriffe haben insbesondere für das Untersuchungsgebiet der grenznahen Regionen eine herausragende Bedeutung, da in diesen Regionen der grenzüberschreitende Einkauf schon traditionell Bestandteil des täglichen Lebens ist.

\subsection{Entwicklung und Stand der Kundenbindungsforschung}

Bereits in den 20er Jahren beschäftigte sich die Wissenschaft damit, Ausmaß und Determinanten des Wiederkaufs von Kunden zu untersuchen. Erstmalig untersuchte wahrscheinlich Copeland (1922, S. 282-289) Fragen des Wiederkaufs von Marken. Der Fokus der Analysen zur Kundenbindung verschob sich allerdings im Zeitablauf von der Markenloyalität des Konsumenten zur Kundenloyalität. In den 70er Jahren standen die behavioristischen Modelle im Mittelpunkt, die statisches und dynamisches Wiederkaufverhalten untersuchten (Oliver, 1997). Später wechselte der Focus hin zu verhaltenswissenschaftlichen Modellen (Weinberg, 1977). Hierbei wird auf jede Berücksichtigung von Verhaltensursachen verzichtet (Cunningham 1956, Jacoby und Chestnut 1978, S. 35ff). Diese Interpretation der Kundenbindung als ein ausschließlich über das Kaufverhalten operationalisierbares Phänomen ist jedoch starker Kritik ausgesetzt (Guest, 1942, S. 180-186, Jacoby und Olsen, 1970, Newman und Werbel, 1973, S. 404). Hauptansatzpunkt dieser Kritik ist dabei, dass in Wiederholungsmustern des Kaufverhaltens auch ein gewisser Teil an „spurious loyalty“ (Day, 1969, S. 29-35) auf Grund situativer und anderer Faktoren vermutet wird, die mit tatsächlicher Kundenbindung wenig zu tun haben. 
Insbesondere beim grenzüberschreitenden Einkauf sind weitere Perspektiven zu betrachten als der reine Wiederkauf. Hinzu treten die psychologische Perspektive und die Einstellung des Kunden zu einer Einkaufsstätte. Jacoby und Kyner (1973, S. 1-9) waren die ersten, die der psychologischen Perspektive der Kundenloyalität ein wissenschaftliches und empirisches Fundament gaben. Seit dieser Zeit herrscht in der Literatur weitestgehend Übereinstimmung darüber, dass die Bindung eines Kunden neben seinem reinen Kaufverhalten auch auf irgendeine Weise die Bestimmungsfaktoren des Wiederholungskaufes erfassen sollte. Swan und Combs (1976, S. 25 -33) untersuchen in ihrer Studie den Einfluss physischer und psychologischer Dimensionen eines Produktes am Beispiel von Bekleidung. Die Autoren stellten die Hypothese auf, dass es bei der Beurteilung von Produkten durch den Konsumenten eine begrenzte Anzahl von entscheidungsrelevanten Komponenten gibt. Von diesen Attributen sind einige besonders wichtig zur Bestimmung der Zufriedenheit, andere dagegen sind nur unzufriedenheitsstiftend, ohne das sie Einfluss auf die Gesamtzufriedenheit des Kunden haben. Swan und Combs folgen dabei der Argumentation von Myers und Alpert (1968, S. 13ff), die von den „Determinant Attributes“ ausgehen, worunter beide eine begrenzte Zahl von Attributen verstehen, die für den Kunden wichtig sind und die sich bei den einzelnen Alternativen unterscheiden. Swan und Combs dehnen aber ihre Studie auch auf die Nachkaufphase aus, zudem erweitern sie das Konzept um Produktattribute, die sich bei Nichterfüllung als nicht unzufriedenheitsstiftend erweisen. Dabei differenzieren sie zwischen instrumentellen Leistungen und expressiver Leistung. Das Hauptargument der Autoren lautet, dass die Entstehung von Zufriedenheit folgenden Sachverhalt mit einschließt: Die instrumentelle Leistung entspricht oder übertrifft die Erwartung der expressiven Leistung. Oliver (1997, S. 47) beschreibt diese Sachverhalte mit anderen Termini: Instrumentelle Leistungen werden als „utilitarian“, expressive Leistungen als ,hedonistic“ bezeichnet. Zur Überprüfung der Hypothesen wurde die Critical Incident Technique angewandt, mit der bereits Herzberg arbeitete (Herzberg, Mausner und Snyderman, 1959).

In der Folgezeit wurden Aspekte der Kundenloyalität zu einem stark diskutierten Thema in der betriebswirtschaftlichen Literatur (Oliver, 1997). Viele Arbeiten richteten sich aber primär auf die Analyse der Nachfragersicht. Gleichzeitig wurden die verhaltenswissenschaftlichen Modelle und insbesondere das Konzept der Kundenloyalität vertieft und verfeinert. So identifizierten Dick und Basu (1994) drei Arten von Kundenloyalität: Echte Loyalität mit hohem Wiederkaufverhalten und positiver Einstellung, latente Loyalität bei positiver Einstellung, aber geringem Wiederkaufverhalten und unechte Loyalität bei hohem Wiederkaufverhalten aber negativer Einstellung. Der Begriff der Kundenloyalität entspricht hierbei dem der Kun- 
dentreue. Dabei erfasst der Begriff als Oberbegriff sowohl die Markenloyalität als auch die Händlerloyalität. Diese Auffassung vertreten Bauer, Huber und Bräutigam (1997, S.170f.), dagegen nimmt Weinberg eine Unterscheidung zwischen Kundenloyalität und Kundentreue vor. Weinberg sieht in der Loyalität ein inneres Commitment (Weinberg, P., 1998, S.41).

Reichheld und Sasser (1990) weisen auf die Handlungsnotwendigkeit der Unternehmen hin und haben den Begriff des Kundenbindungsmanagements ${ }^{15}$ als Aktivität eines Unternehmens geprägt. Zentral stehen die aktuellen, bereits bestehenden Kundenbeziehungen. Die Gewinnung der Neukunden sowie die Rückgewinnung von abgewanderten Kunden sind nicht Inhalt des Kundenbindungsmanagements. Die beiden Forscher belegen an Hand von Zahlen auf der Basis einer Stichprobe von 100 Unternehmen die Wirkung der dauerhaften Geschäftsbeziehungen auf den Unternehmenserfolg. Allerdings unterbleibt eine genaue Erläuterung der Vorgehensweise. Die dargelegten Resultate können damit nicht überprüft werden. Allerdings weist Reichheld (2004) darauf hin, dass wahre Kundenloyalität den Gewinn eines Unternehmens positiv beeinflusst. Ferner fördert nach Reichheld Kundentreue das wertsteigernde Wachstum eines Unternehmens, da kein Unternehmen wachsen kann, wenn der Kundenstamm abwandert (Reichheld, 2004, S.25). Kundenloyalität hilft Kundenabwanderung zu vermeiden und Kunden neigen dazu mit steigendem Einkommen mehr zu kaufen. Reichheld plädiert dafür die Kunden allein nach ihrer Weiterempfehlungsneigung zu fragen um Kundenbindung möglichst zeitnah zu messen. Diese Form der Fragestellung lässt zwar eine schnelle und einfache Messung der Kundenbindung zu, erklärt allerdings nicht das „warum“ der Kundenbindung.

Die Arbeit von Diller (Diller, 1996) setzt sich unter anderem mit dem Zusammenhang zwischen Kundenbindung und Involvement, Commitment, Vertrauen und Zufriedenheit auseinander. Diller liefert zudem einige Ideen zur Systematisierung des breiten Spektrums der Ausprägungen der Kundenbindung. Hierzu gehören nach Diller mehr Sicherheit, Wachstum und Rentabilität. Diese Hauptpunkte hat der Forscher in folgende Wirkungseffekte der Kundenbindung zusammengefügt:

\footnotetext{
${ }^{15}$ Kundenbindungsmanagement ist ,die systematische Analyse, Planung, Durchführung und Kontrolle sämtlicher auf den aktuellen Kundenstrom gewichteter Maßnahmen mit dem Ziel, dass diese Kunden auch in Zukunft die Geschäftsbeziehung aufrechterhalten oder intensiver pflegen“ (Homburg und Bruhn, 2003, S.8, S.423-450).
} 


\begin{tabular}{|c|c|c|c|}
\hline & Mehr Sicherheit & Mehr Wachstum & Mehr Gewinn/Rentabilität \\
\hline Positiv & $\begin{array}{ll}- & \text { Mehr Stabilität der Geschäfts- } \\
\text { - } & \text { beziehung } \\
\text { - } & \text { Mehr Feed-back vom Kunden } \\
\text { - } & \text { Mehr Vertrauen }\end{array}$ & $\begin{array}{ll}- & \text { Bessere Kundenpenetration } \\
\text { - } & \text { Mehr Kundenempfehlungen }\end{array}$ & $\begin{array}{ll}- & \text { Kosteneinsparungen } \\
\text { - } & \text { Erlössteigerungen }\end{array}$ \\
\hline Negativ & $\begin{array}{ll}- & \text { Commitment } \\
\text { - } & \text { Trägheit } \\
\text { - } & \text { Reaktanzgefahr }\end{array}$ & \begin{tabular}{ll} 
- & \multicolumn{2}{l}{ Einseitige Kundenstruktur } \\
$\bullet$ & Negative $\quad$ Mund-zu-Mund- \\
& Kommunikation
\end{tabular} & - $\quad$ Bindungskosten \\
\hline
\end{tabular}

Tab. 3.2: $\quad$ Wirkungseffekte der Kundenbindung (Diller, 1996, S.82)

Als Beiträge zur Operationalisierung der Kundenbindung sind insbesondere Untersuchungen aus dem Forschungsbereich der Markentreue und Loyalität zu betrachten. In diesen Beiträgen wird argumentiert, dass bei der Messung der Konstrukte Markentreue und Loyalität sowohl einstellungs- als auch verhaltenstheoretische Aspekte zu berücksichtigen sind.

Im Zuge der Operationalisierung des Konstruktes Kundennähe ${ }^{16}$ weist Homburg (1995, S. 813-834) die Leistungsfähigkeit kausalanalytischer Modelle zur Messung hypothetischer Konstrukte nach. Diller (1996, S. 84-86) unterscheidet dagegen Meßindikatoren, die auch am tatsächlich beobachtbaren Kaufverhalten und an der Absicht von Abnehmern anknüpfen. Diese Meßindikatoren beinhalten ebenso die Absicht des Konsumenten auch künftig beim gleichen Anbieter Produkte zu erwerben. Der Autor zeigt ebenfalls auf, dass die Wahl der Meßkriterien auch von dem Bezugsobjekt einer Geschäftsbeziehung abhängt. Diller fasst Kundenbindung als die Bereitschaft von Kunden zu Folgekäufen auf. Einige Autoren wie Homburg et al. (1998, S. 88) oder Johnson ( 1998, S. 41) bezeichnen die Wiederkaufabsicht als Kundenloyalität bzw. als Kundentreue. Singh u.a. (2000, S. 161) sprechen in diesem Zusammenhang von einer konativen Loyalität. Die Erklärung der konativen Kundenloyalität steht auch im Mittelpunkt der Forschungsaktivitäten, die an der nachfrageorientierten Perspektive der Kundenbindung ansetzen. Von Kundenbindung kann somit erst dann gesprochen werden, wenn es neben den anderen genannten Aspekten auch zu einem Wiederkauf gekommen ist. Braunstein (2001, S. 11) sieht inhaltlich einen Zusammenhang zwischen nachfrageorientierten Kundenbindungskonzepten. Sie unterscheidet hierbei zwischen neobehavioristischen und behavioristischen Ansätzen. Die neobehavioristischen Ansätze unterteilt sie in erstens eindimensionale und zweitens zweidimensionale Ansätze, bei denen Kundenbindung mit

\footnotetext{
${ }^{16}$ Kundennähe: Besteht aus zwei Dimensionen. 1. Dimension - Anbieter trifft mit seinem Leistungsangebot die Vorstellung der Kunden, 2. Dimension: Das Interaktionsverhalten des Anbieters gegenüber seinem Kunden.
} 
60 | Kapitel 3

Kundenloyalität gleichgesetzt wird. Im eindimensionalen Fall wird das Konstrukt direkt mit einer Gruppe von Indikatoren verbunden und im zweidimensionalen Ansatz wird das Konstrukt in eine Reihe von latenten Variablen zerlegt. $\mathrm{Zu}$ den Vertretern des eindimensionalen Ansatzes zählt Peter, die die Systematisierung Kundenbindung $=$ Kundenloyalität $=$ Wiederkaufabsicht entwickelt hat. Zu den Vertretern der zweidimensionalen Ansätze, bei denen Kundenbindung nicht mit Kundenloyalität gleichgesetzt wird, werden Homburg und Faßnacht (1998) gezählt, die Kundenloyalität mit Wiederkaufabsicht und Kundenbindung mit vergangenem Wiederkaufverhalten gleichsetzen. Auch für die Autoren Johnsen (1998) und Oliver (1999) beinhaltet die Kundenloyalität Wiederkaufabsicht und Kundenbindung mit zukünftigem Wiederkaufverhalten. Oliver unterscheidet in diesem Zusammenhang zusätzlich zwischen tatsächlichem, zukünftigem und bekundetem Treueverhalten.

Als Vertreter des behavioristischen Ansatzes sind u.a. Dekimpe et al. (1997, S. 405-420) zu nennen. Die Forscher definieren Kundenbindung als das overte vergangene Wiederkaufverhalten. Somit stehen die meisten Kundenbindungskonzepte in der Tradition der neobehavioristischen Kaufverhaltensforschung und es handelt sich um Erklärungsansätze, die dem S-O-R Paradigma $^{17}$ folgen. Die Erklärung des Phänomens Wiederkauf erfolgt im Rahmen dieses Paradigmas nicht allein auf Basis des beobachtbaren Verhaltens, sondern die Forschungsbemühungen konzentrieren sich verstärkt auf die nichtbeobachtbaren Größen, die innerhalb einer Person und deren Psyche wirksam werden (Kroeber-Riel, W. und Weinberg, P., 2000, S. 29). Dem entgegen betrachtet Odekerken-Schröder in ihren Forschungen (1999) die Bindung an ein Geschäft. Sie (1999) definiert den Begriff der „buyer relationship proneness“ und versteht darunter die Intention eines Käufers verstärkt Kontakt mit einem Anbieter oder mit einer definierten Produktkategorie zu suchen. Dabei wird das Konzept der „buyer relationship proneness" als ein individuelles Konzept dargestellt und folgendermaßen charakterisiert:

- Die Beziehungen zwischen Käufer und Verkäufer sind relativ stabil.

- Die Beziehung zwischen Käufer und Verkäufer wird von Seiten des Käufers als eine wohlüberlegte Abwägung der Alternativen verstanden.

- Die Käufer haben ein individuelles Interesse eine proaktive Beziehung zum Verkäufer aufzubauen.

- Die Käufer zeigen Interesse und identifizieren sich mit dem Handelsunternehmen und der angebotenen Produktkategorie.

\footnotetext{
${ }^{17}$ S-O-R-Paradigma (Stimulus-Organismus-Response): Neobehavioristischer Erklärungsansatz. Es wird versucht, ,die im Organismus $(\mathrm{O})$ des Menschen ablaufenden, nicht beobachtbaren Vorgänge zur Erklärung seines Verhaltens zu berücksichtigen.“(Meffert, 2000, S.99).
} 
Aus dieser Feststellung leitet Odekerken-Schröder (Odekerken 1999, S. 44f.) folgendes ab:

- Buyer relationship proneness kann als Basis zur Segmentierung für Handelsunternehmen interessanter Kundengruppen dienen.

- Auf Grund dieser Segmentierung wird Kundenbindung durch das Unternehmen verstärkt.

Der Begriff der Kundenbindung hat sich in den vergangenen Jahren kontinuierlich weiterentwickelt und ein Kundenbindungsmanagement wurde in zahlreichen Unternehmen im Zuge der Kundenorientierung implementiert. Deutlich zeigt sich auch, dass Zufriedenheit eines Kunden nicht immer dessen zukünftiges Handeln bestimmt. Vielmehr ist es notwendig, gegenwärtige und zukünftige positive Verhaltensweisen und Einstellungen - also eine stabile Kundenbindung zu generieren. Eine Notwendigkeit zu diesem Tun lässt sich allein schon aus der Tatsache ableiten, dass die Zufriedenheit mit dem Kauf, dem Service der Einkaufsstätte, für den Kunden eine Selbstverständlichkeit geworden ist, die allein den Kunden nicht zu einem Wiederholungskauf veranlasst. Doch gerade Wiederholungskäufe eines festen Kundenstamms sind die Grundlage des wirtschaftlichen Erfolges eines Einzelhandelsunternehmens. Durch die Informationen, wie es zu Wiederholungskäufen kommt, kann Wissen aufgebaut werden, um Kunden mittel- und langfristig an eine Einkaufsstätte oder einen Filialbetrieb zu binden. Der Stand der Forschungsergebnisse zeigt deutlich, dass Kundenzufriedenheit zwar positiv für das Unternehmen ist, letztlich jedoch die Loyalität der Kunden und Kundenbindung das zukünftige Ergebnis eines Unternehmens nachhaltig bestimmt (Peter, 1999, S. 4648, Braunstein, 2001, S.5, Homburg, 2000, S. 164ff.). Dass die Konzentration auf Stammkunden sich positiv auf den Erfolg des Handelsbetriebes auswirkt, belegen die Studien von Reichheld (Reichheld, 1996, S. 33ff.) sowie Reichheld und Sasser (Reichheld und Sasser, 1990, S. 105ff.). In ihren Studien kommen die Autoren zu dem Ergebnis, dass eine Erhöhung der Kundenbindung um nur 5\% zu einer Gewinnsteigerung zwischen $25 \%$ und $95 \%$ je nach Branche führt. Peter führt den positiven Effekt auf den Unternehmenserfolg darauf zurück, dass der Unternehmenserfolg durch direkte und indirekte Folgen der Kundenbindung beeinflusst wird (Peter, 1997, S. 47ff.) Unternehmen profitieren auf verschiedener Art und Weise von ihren loyalen und sich gebunden fühlenden Kunden: So zeigen die Studien von Reichheld (Reichheld, 1997, S. 65), dass eine positive Korrelation zwischen Umsatz und Geschäftsdauer der Kunden besteht. Buchanan und Gillies (1990, S. 524) weisen nach, dass gebundene Kunden häufiger die Einkaufsstätte frequentieren, sowie auch ein starkes Cross-buyingVerhalten zeigen (Eckert, S. 1994, S. 4). Stammkunden weisen auch eine geringere Preisempfindlichkeit auf (Anderson und Sullivan, 1993, S. 125f). 
Ein Ziel sollte es somit sein, durch gezielte Maßnahmen die Bereitschaft der Kunden zum Anbieterwechsel zu reduzieren und die Wiederkaufrate im eigenen Unternehmen zu erhöhen (Meffert, 1998, S. 119). Um dieses Ziel zu erreichen, bedarf es einer Kundenbindungsstrategie (Homburg und Bruhn, 1998, S. 16ff.). Unter der Kundenbindungsstrategie verstehen Homburg und Bruhn wiederum einen bedingten, langfristigen Verhaltensplan zur Erreichung der Kundenbindungsziele sowie deren Ausgestaltung in operativen Kundenbindungsmaßnahmen (Homburg und Bruhn, 1998, S. 18, Diller,1995; Homburg und Faßnacht, 1998). Dabei gehen die Autoren von den marketingpolitischen Instrumenten aus und setzen diese mit der primären Wirkung der Kundenbindung in Verbindung, um dann einzelnen Instrumentbereichen konkrete Instrumente zur Bildung von Kundenbindung zuzuordnen. Kundenbindung determiniert somit für Unternehmen eine Risikoreduzierung, da sich treue Kunden stärker resistent gegenüber akquisitorischen Bemühungen der Mitwettbewerber zeigen (Weißenberger, 1998, S. 614ff.). Die Frage nach dem ökonomischen Vorteilen gezielter Kundenbindung muss differenzierter beantwortet werden. So kann Kundenbindung nur erfolgreich sein, wenn sie gezielt auf die Kunden ausgerichtet wird. Die Implementierung einer solchen Kundenbindungsstrategie ist jedoch mit hohen Investitionen verbunden. Diese müssen berücksichtigt werden, um den ökonomischen Erfolg ermitteln zu können. Der Kerngedanke besteht in der Steigerung des Unternehmens- und Kundenwertes durch das systematische Management existierender Kunden.

Zum anderen haben die Situation der Unternehmung als solches und die Entwicklung auf dem Markt insgesamt Einfluss auf die ökonomische Bewertung. Grundsätzlich sollte für eine Bewertung berücksichtigt werden, dass sich Kundenmanagement auf wachsenden Märkten als wenig effizient erweißt, da hier abwandernde Kunden leicht durch neue ersetzt werden können (Gerpott, 2000, S. 27). Folgt man Gerpott, so ist neben der Leistungsqualität auf dynamischen Märkten keine weitere Investition in Kundenbindungsmaßnahmen notwendig. Investition in Kundenbindung bindet Ressourcen. Aus diesem Grund hat sich das Unternehmen zunächst die Frage zu stellen, ob es Sinn macht, grundsätzlich in Kundenbindungsmaßnahmen zu investieren. Ferner muss die Frage geklärt sein, welche Kunden gebunden werden sollen und durch welche konkreten Maßnahmen dies geschehen soll (O`Brien und Jones, 1995, S. 76ff.). Erst wenn diese Fragen beantwortet sind, kann eine qualifizierte ökonomischen Bewertung vorgenommen werden. Kommt eine Unternehmung zu dem Schluss, dass die Investition in Kundenbindungsmaßnahmen ökonomisch Sinn macht, muss sich das Mangement die Frage stellen, welche Kunden sich generell zur Bindung eignen und welche konkreten Aktivi- 
täten durchgeführt werden müssen. Ist das Unternehmen in einem grenznahen Gebiet beheimatet, sind die möglichen Einflüsse dieser Grenze ebenso zu berücksichtigen.

Insgesamt ergeben sich aus dem heutigen Stand der Kundenbindungsforschung folgende Ansatzpunkte zur Vorhersagbarkeit der Kundenbindung:

Ziel der Kundenbindungsforschung ist die

Umfassende Erklärung und Prognose der

Abnehmerbindung

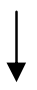

Kundenbindung

Erklärung und Prognose durch:

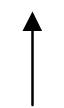

- Identifikation direkter Einflussgrößen

- Verbesserte Prädiktion der Kundenloyalität

Kundenloyalität

$\longleftarrow$ Erklärung und Prognose durch:

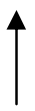

- Identifikation der relevanten Einflussgrößen

- Verbesserte Prädiktion der Kundenzufriedenheit

Kundenzufriedenheit Erklärung und Prognose durch:

- Identifikation der relevanten Einflussgrößen

Abb. 3.1: Ziele der Kundenbindungsforschung (Braunstein, 2001, S. 21)

Dennoch bleiben die Frage zu beantworten, worin der Zusammenhang zwischen Kundenzufriedenheit und Kundenbindung besteht und ferner welche Determinanten die Kundenbindung bestimmen. Dies soll im folgenden Kapitel diskutiert werden.

\subsection{Kundenloyalität als Bestimmungsfaktor der Kundenbindung}

Seit Anfang der 80-er Jahre hat sich die Erzielung einer zufriedenen Kundenbasis zu einem Schwerpunktthema sowohl in der Wissenschaft als auch in der Praxis entwickelt (Homburg, Giering und Hentschel, 1998, S. 1). Aus diesem Grund haben auch die Veröffentlichungen zum Konstrukt Kundenzufriedenheit stetig zugenommen (Day 1977, 1982 a,b, 1984, Oliver 1977, 1980, 1981, 1997, Churchill und Surprenant 1982, LaBarbera und Mazursky 1983, Oliver und DeSarbo 1988, Woodruff, Cadotte und Jenkins 1983, 1987, Fornell 1992, 
64 | Kapitel 3

Anderson, Fornell und Lehmann 1994). Kundenzufriedenheit wird als Grundvoraussetzung zum Aufbau von Kundenbindung gesehen. Dennoch erscheint es notwendig alle Bestimmungsfaktoren der Kundenbindung zu analysieren.

\subsubsection{Konzeptualisierungsvorschläge ausgewählter Kundenbindungsmodelle}

Peter (1999) teilt in ihrer empirischen Aufbereitung die in der Literatur vorliegenden Forschungsergebnisse zur Kundenbindung in drei Kategorien ein. Peter will damit aufzeigen, inwieweit sich die Kundenbindungsforschung mit der Zielsetzung der Implementierung der Kundenbindung in die Marketingstrategie der Unternehmen auseinander gesetzt hat. In der ersten Gruppe werden von der Autorin solche Ergebnisse zusammengefasst, die sich eher deskriptiv und ohne größere theoretische Fundierung mit dem Phänomen auseinandersetzen. In diesen Studien werden die Facetten und Erscheinungsformen der Kundenbindung beleuchtet und die positive Wirkung auf den Unternehmenserfolg betont. Zur zweiten Kategorie zählt die Autorin Untersuchungen, die sich schwerpunktmäßig mit der Frage der Erfassung der Kundenbindung beschäftigen und zur dritten Gruppe gehören Untersuchungen auf breiter Basis, „die in erster Linie die Identifikation und Analyse der Wirkung von Determinanten dauerhafter Geschäftsbeziehungen zum Ziel haben“ (Peter, 1999, S. 65). Im weiteren Verlauf sollen auch hier die Untersuchnungen weiter analysiert werden, die sich mit den Determinanten dauerhafter Geschäftsbeziehungen auseinandersetzen, um die grenzüberschreitende Geschäftstreue erklären zu können.

Ausgangspunkt der Kundenbindungsmodelle ist in der Literatur die Definition der Kundenloyalität. Eine Definition zur Kundenloyalität liefern Homburg, Gierung und Menon. Die Autoren beschreiben Kundenloyalität als die Intention eines Käufers die Geschäftsbeziehung zu einem Anbieter fortzusetzen und die Qualität dieser Beziehung zu verbessern (Homburg, Giering und Menon, 1999, S. 4). Die Forscher sehen in der Kundenloyalität aber auch eine Dimension der Verpflichtung des Käufers gegenüber dem Verkäufer. Diese Verpflichtung sehen sie ebenfalls als eine enge geistige Übereinstimmung zwischen beiden Parteien an.

Eine ähnliche Einstellung zur Loyalität hat Diller, obwohl Diller - ebenso wie Jones und Sasser (1995, S.85) - nicht von Loyalitäts-, sondern von Kundenbindungstypen spricht. Auch Diller bezieht neben dem Involvement das Commitment mit in seine Betrachtungen ein, um Bindungstypen und -qualitäten zu identifizieren (1996, S. 89; 2000, S. 33). Aus diesen Über- 
legungen heraus kommt Diller zu dem Schluss, dass Kundenbindung ein integraler Bestandteil des Beziehungsgeschehens zwischen Anbieter und Kunde ist. Daraus hat Diller folgende spezifischen Typologisierungsansätze der Kundenbindung zwischen Anbieter und Nachfrager formuliert:

1. Kundenbindung auf Basis von Machtpotentialen.

2. Erkaufte Kundenbindung.

3. Vertrauen in die sachliche Leistungskompetenz.

4. Exklusive Organisationsresourcen.

5. Verträge über die Art der Geschäftsentwicklung.

6. Vertrauen in die Organisationskompetenz.

7. Emotionale Macht.

8. Kumpanei.

9. Sympathie.

Ebenso erkennt Diller, wie Jones und Sasser, dass Kundenzufriedenheit ein relevantes Merkmal zur Beschreibung der Kundenloyalität ist. Doch erhalten die Autoren mit ihrer Typologisierung der Kundenloyalität auf Basis des Commitment, des Involvment oder der Kundenzufriedenheit keine weitere Zustimmung. Als Elemente der Kundenloyalität dagegen erlangen die Konstrukte in der Literatur Akzeptanz (Morgan und Hunt, 1994, S.20-38). Die Handlungsabsicht des Kunden konzentriert sich somit nicht ausschließlich auf den Wiederkauf, sondern auf sämtliche treuen Verhaltensweisen wie Weiterempfehlung oder höhere Preisbereitschaft.

Die psychischen Größen finden bei Johnson dagegen keine Aufmerksamkeit (Braunstein, 2001, S. 30). In seiner Definition der Kundenloyalität als „psychological predisposition toward repurchase“ betont Johnson den intentionalen Charakter des Phänomens (1998, S. 104). Johnson bezieht dabei nicht allein die Wiederkaufabsicht mit in die Überlegungen ein, sondern ebenso sämtliche auf Treue deutende Handlungsweisen. Johnson betrachtet Kundenloyalität somit im Gegensatz zu anderen Autoren aus einer mehrdimensionalen Sicht. Demgegenüber begnügen sich viele Autoren mit der eindimensionalen Konzeptualisierung der Kundenloyalität als Wiederkaufabsicht (Anderson und Sullivan, 1993, S. 125-143). Narayandas, der seinerseits das eindimensionale Verständnis der Kundenloyalität kritisiert, kritisiert andererseits an der Definition von Anderson und Sullivan, dass eine Trennung zwischen echter und erzwungener Loyalität sich bei dieser Sichtweise kaum vornehmen lässt (1996, S. 5). Sein Loyalitätskonzept teilt sich in sechs Subdimensionen auf. Hierzu zählt der Forscher die Wiederkaufabsicht, die Bereitschaft des Kunden auf die Lieferbereitschaft seines Lieferanten 
zu warten, die Bereitschaft zur Weiterempfehlung und die Bereitschaft einen Premiumpreis zu zahlen, Standfestigkeit gegenüber kritischer Expertenmeinung und attraktiven Konkurrenzangeboten (Narayandas, 1996, S. 5). Eine Erklärung, wodurch Treue ausgelöst wird, bleibt er allerdings schuldig. Oliver dagegen unterscheidet im Rahmen seiner Kundenloyalitätsbetrachtung zwischen der kognitiven, affektiven, konativen und aktionalen Loyalität auf Basis des psychischen Zustandes des loyalen Kunden (1996, S. 392; 1999, S. 35). Für den Autor bildet die kognitive Loyalität den Ausgangspunkt. Hier erhält der Konsument Informationen und entschließt sich auf Grund dieser Informationen zu einer Handlung. Entscheidungsgrundlage sind in diesem Fall Preis sowie Nutzen. Es liegt allerdings nur eine schwache Form der Treue vor, da die Wechselbereitschaft bei einem attraktiven anderen Angebot sehr hoch ist (Oliver, 1996, S. 392-395). Nach der kognitiven Phase schließt sich die affektive Phase an. Diese Phase schließt die Einstellungen des Konsumenten zum Anbieter vor dem Kauf mit ein. Allerdings garantiert auch diese Form der Loyalität nicht die Treue des Kunden. Echte Loyalität beruht auf weiteren Faktoren, wie beispielsweise die Verhaltensintention. Als solche sieht Oliver die Absichtserklärung. Konative Loyalität besteht für ihn aus den beiden Komponenten affektives Treueempfinden und der motivierenden Komponente. Es handelt sich hierbei um den Wunsch des Konsumenten treu $\mathrm{zu}$ sein, indem es zu einem zukünftigen Wiederkauf kommt (Oliver, 1996, S. 393). Die tatsächliche Realisierung wird nach Oliver erst in der aktionalen Phase der Loyalität realisiert. Erst in dieser Phase wird nach der - der Arbeit zugrunde liegenden Begrifflichkeit - Kundenbindung realisiert. Der Kunde hat sämtliche Hindernisse, die ihn an der Bindung hinderten, überwunden. Gegen diese Betrachtung der Loyalität lässt sich zwar einwenden, dass keine wirkliche Konzeptualisierung der Kundenloyalität vorliegt, doch ist das Stadium der aktionalen Loyalität auch noch nicht in den Focus der Forschungsbetrachtungen mit aufgenommen worden.

In vielen Konzeptualisierungsvorschlägen der Kundenloyalität wird diese als Verhaltensintention verstanden. Verhaltensintentionen werden im weiteren Verlauf in Bezug auf den Aufbau einer grenzüberschreitenden Einkaufsstättentreue untersucht. Somit kann im Rahmen dieser Untersuchung in Anlehnung an die vorliegenden Konzeptualisierungskonzepte die Betrachtung der Kundenloyalität auf drei potenzielle Dimensionen des Konstruktes reduziert werden:

- Vergangenes Verhalten (ex post)

- Verhaltensabsicht (ex ante)

- Einstellung.

Kommt zur Kundenloyalität (bestehend aus der kognitiven, affektiven, konativen und aktionalen Loyalität) noch eine Handlungsdimension hinzu, spricht man, wie bereits dargelegt, von 
Kundenbindung. Das Konstrukt der Kundenbindung besteht somit aus zwei Dimensionen: der Dimension des bisherigen Verhaltens und der Verhaltensabsicht. Der Verhaltensdimension können dabei sowohl das bisherige Kauf- wie auch das bisherige Weiterempfehlungsverhalten zugeordnet werden (Homburg, Giering und Hentschel, 1998, S. 8). Die Absichtsdimension umfasst dabei die zukünftige Wiederkauf-, Zusatzkauf- und Weiterempfehlungsabsicht eines Kunden. Zusammenfassend kann festgehalten werden, dass es bislang in der Forschungsliteratur kaum Untersuchungen gibt, die explizit den Zusammenhang zwischen Kundenloyalität und Kundenbindung betrachten. Zwar wird von den meisten Autoren (Bolton und Drew, 1991, Homburg, Giering und Henschel, 1999, Peter, 1999, Braunstein, 2001) ein positiver Zusammenhang unterstellt, doch bleiben sie eine empirische Überprüfung schuldig. Braunstein (1999, S. 32) bezeichnet aus diesem Grund die Einflußgrößen der Kundenloyalität implizit als Antezedenzien der Kundenbindung.

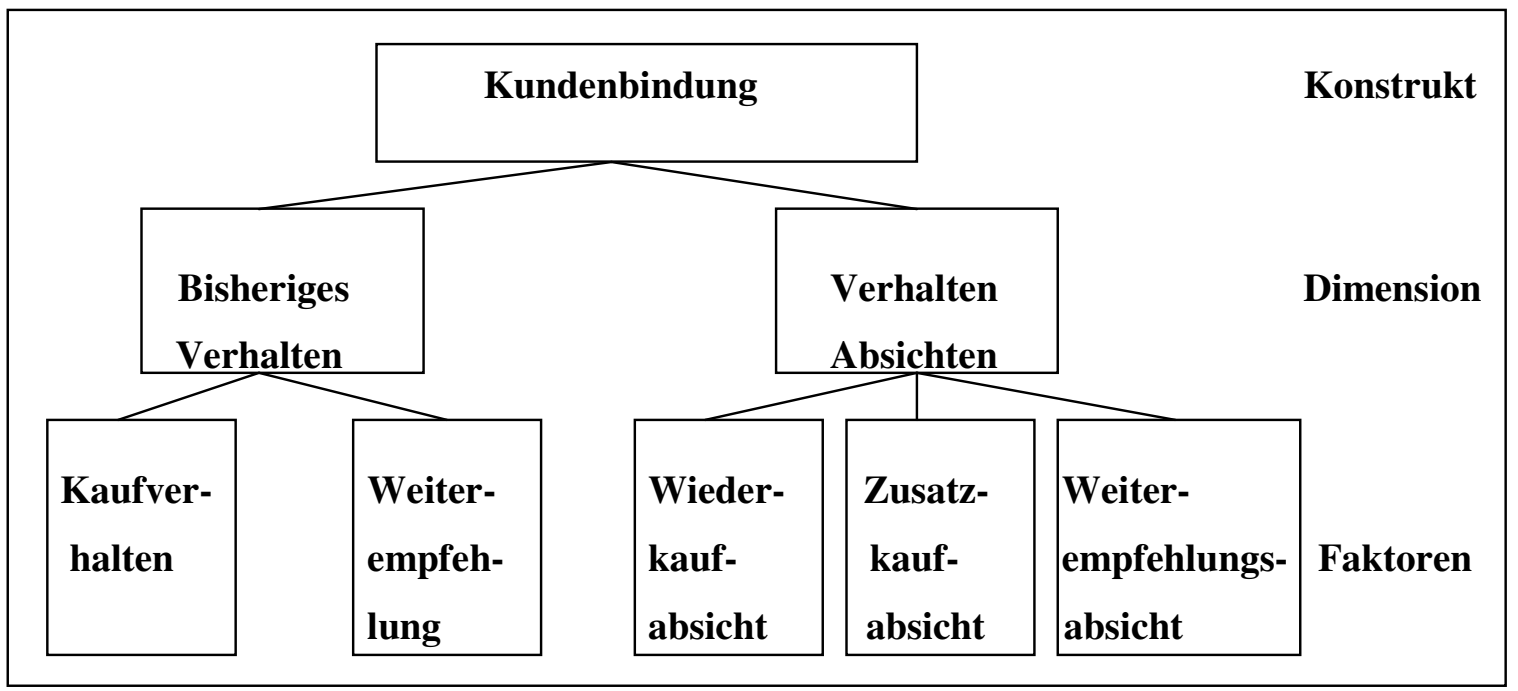

Abb. 3.2: $\quad$ Konzeptualisierung des Konstruktes Kundenbindung

(Homburg, Giering und Hentschel, 1998, S. 8)

Wenn man alle Modelle der Kundenloyalitätsbetrachtung zusammenfasst, findet man viele Begriffe im Modell von Homburg und Faßnacht (1998, S. 415f.) wieder. Man könnte es als eine Art Zusammenfassung aller Modelle bezeichnen. Hinzu kommt, dass hier auch die Handlungsdimension skizziert wird und so auch der weitergehende Begriff der Kundenbindung aufgegriffen wird. Daher wird diese Zusammenfassung aller Modelle den Überlegungen der Kundenbindung im weiteren Verlauf dieser Arbeit zu Grunde gelegt. Dies bedeutet, dass in der weiteren Untersuchung beide Dimensionen (bisheriges Verhalten, Verhaltensabsicht) der Kundenbindung betrachtet und die einzelnen Faktoren im grenzüberschreitenden Raum des definierten Untersuchungsgebietes untersucht werden. Unter dem Begriff der Kundenbindung 
68 | Kapitel 3

werden im Folgenden zum einen die Kundenbindungsmaßnahmen des Handels als auch der Zustand der Bindung des Konsumenten an eine Einkaufsstätte bezeichnet. Diese Differenzierung geht auf Mohme zurück, der Kundenbindung unterscheidet zwischen Zustand und Tätigkeit.

\begin{tabular}{|ll|} 
& \multicolumn{1}{c|}{ Kundenbindung } \\
\cline { 2 - 3 } & \\
\hline Betrifft nur die & $\underline{\text { Tätigkeit }}$ \\
gebundenen & Betrifft \\
Stammkunden & alle übrigen \\
& Kundenarten, \\
& auch die \\
\hline
\end{tabular}

Abb. 3.3: $\quad$ Der Begriff der Kundenbindung als Zustand und Tätigkeit (Mohme, 1993, S. 49)

\subsubsection{Ursachenanalyse des Zusammenhangs zwischen Kundenzufriedenheit und Kundenbindung}

Im Gegensatz zu den vielen empirischen Studien zum Thema Kundenzufriedenheit fehlt aus Sicht des Autors zur empirischen Verifikation der Kundenloyalität detailliertes Forschungsmaterial, insbesondere wenn es um des Einfluss der sozialen, psychischen Faktoren sowie um den Einfluss einer geografischen Grenze geht. Dieses Defizit wird auch in der Literatur bemängelt (Mittal, Kumar und Tsiros, 1999, S. 88). Ein Forschungsmangel besteht insbesondere in der Auseinandersetzung mit den Konsequenzen der Kundenzufriedenheit bzw. Kundenunzufriedenheit hinsichtlich Wiederkaufverhalten, Wiederkaufabsicht, Markenwechsel und Beschwerdeverhalten. In den neobehavioristischen Ansätzen dient die Lerntheorie und das Lernen nach dem Verstärkungsprinzip als interessierende Beziehung zwischen Kundenzufriedenheit und Kundenloyalität. Macht ein Kunde beim Kauf eine negative Erfahrung, so kann er dies durchaus als Bestrafung werten und wird diese Handlung zukünftig unterlassen (Wilkie, 1994, S. 270). Zufriedenheit mit einem Produkt oder Einkauf lässt sich dagegen als Belohnung interpretieren und erhöht die Wahrscheinlichkeit des Wiederkaufs (Wilkie, 1994, S. 270).

Bereits Kasper (1988, S. 387ff.) analysierte, dass zwischen der Kundenzufriedenheit und der Kundenloyalität keine direkte Beziehung im Rahmen einer eindeutigen Korrelation vorliegt. 
In ihrer empirischen Studie aus dem Jahr 1997 stellt Peter (1997, S. 114) fest, dass sich in sämtlichen zum damaligen Zeitpunkt vorliegenden Studien ein statistisch signifikanter positiver Zusammenhang zwischen Kundenzufriedenheit und Kundenloyalität nachweisen lässt. Diese Aussage gilt sowohl für den Bereich Teilleistung als auch hinsichtlich der Beurteilung der Gesamtleistung. Bloemer, Kasper und Lemmink (1990, S. 43) gehen sogar soweit zu behaupten, dass die Händlerloyalität im Automobilmarkt die Markenloyalität determiniert. Auh und Johnson (1997, S. 148f.) vermuten bei einem hohen Zufriedenheitsniveau einen asymptotischen Verlauf der Kundenloyalität. Sie gehen davon aus, dass auch zufriedene Kunden mindestens einen Grund kennen, warum sie untreu werden sollten (1997, S. 149). Aus diesen Überlegungen heraus kommen die Autoren zu dem Ergebnis, dass ein progressiver Funktionsverlauf zwischen Kundenzufriedenheit und Kundenloyalität den Zusammenhang am besten beschreibt. Für den, für diese Arbeit wesentlichen Konsumgüterbereich, identifizieren Homburg und Giering soziodemographische Variablen wie Geschlecht, Alter, Einkommen sowie leistungsabhängige Variablen wie Involvement und Variety Seeking als relevante quasi-moderierende Größen, die es weiter zu untersuchen gilt. Unter quasi-moderierenden Variablen soll dabei eine Größe verstanden werden, die sowohl einen moderierenden Einfluss als auch einen direkten Effekt auf eine Zielvariable ausübt. Die moderierende Wirkung besteht darin, dass die Variable den Zusammenhang zwischen zwei Konstrukten (Kundenzufriedenheit und Kundenloyalität) stärkt oder schwächt (Homburg, Giering und Menon, 1999, S. 6).

\subsubsection{Analyse der leistungsabhängigen Determinanten der Kundenbindung}

Kroeber-Riel und Weinberg (2000, S. 167ff.) vertreten ähnlich wie Czepiel und Rosenberg (1977, S. 93) die Meinung, dass sowohl die Kundenzufriedenheit als auch die Kundenloyalität eine bewertende Reaktion des Käufers auf die Unternehmensleistung ist. Dabei ist die Einstellung die direkte Determinante der Kundenloyalität und Kundenzufriedenheit ist eine Ausprägung der Einstellung. Als wesentliches Differenzierungsmerkmal führt Oliver das (Nicht-) Bestätigungskonzept an, das im Mittelpunkt der Kundenzufriedenheit steht. Eine positive Nichtbestätigung führt beim Kunden zu Überraschung. Kundenzufriedenheit ist nach Meinung des Autors ein kurzfristiges Konzept, Einstellung ist dagegen langfristig ausgelegt. Einstellung gegenüber einem Objekt ist im Gegensatz zur Kundenzufriedenheit ein situationsunabhängiges Konstrukt (Oliver, 1981). Für Oliver ergibt sich ein Ursache-Wirkungsgefüge, das sich aus der Einstellung an einem Zeitpunkt t1 ergibt, die in Kundenzufriedenheit mündet und sich in einer Einstellung zum Zeitpunkt t2 festigt. Kundenzufriedenheit steht in diesem 
Modell für die Gesamtheit aller Produkt/Dienstleistungserfahrungen, die ein Nachfrager mit einem Anbieter gemacht hat. In den Untersuchungen von LaBarbera und Mazursky kommen die Autoren allerdings zu dem Ergebnis, dass Kundenzufriedenheit sich ausdrückt im Wiederkaufverhalten des Kunden und nicht die Einstellung die direkte Antezedenze der Kundenloyalität repräsentiert (LaBarbera und Mazursky, 1983, S. 402). Allerdings hat dieser Ansatz nach Bloemer und Kasper (1995, S.311-329) den entscheidenden Nachteil, dass die Autoren nicht zwischen Wiederkauf und Loyalität sowie Pseudoloyalität und wahrer Loyalität unterscheiden. Ferner wird in diesen Ansätzen nicht berücksichtigt, dass zwischen unterschiedliche Formen der Zufriedenheit differenziert wird. Die Autoren kommen zu dem Ergebnis, dass es nicht nur wichtig ist zu wissen, ob ein Kunde zufrieden ist, sondern es von noch größerer Bedeutung für ein Unternehmen ist zu wissen, was einen Kunden zufrieden macht. Dabei führt die manifeste Zufriedenheit nach diesen Forschungsergebnissen zu einer direkten und deutlichen Markenloyalität, doch auch die latente Zufriedenheit führt zu Loyalität, die von Seiten des Kunden weniger ehrlich ist. Damit hat das Management eines Unternehmens dafür zu sorgen, dass der Kunde bewusst seine Entscheidung zum Kauf bei einem Unternehmen trifft. Erst dann kann von wahrer Loyalität gesprochen werden (Bloemer und Kasper, 1995, S. 325-326).

Neben der Einstellung des Nachfragers zum Produkt und zum Händler haben aber auch die Qualität der Leistung, die Attraktivität des Konkurrenzangebotes, der Beziehungsnutzen und die Wechselbarrieren Einfluss auf die Kundenloyalität. Vom Nachfrager wahrgenommene Qualität messen deshalb Henning-Tharau und Klee den größten Einfluss auf den Übergang von Kundenzufriedenheit zu Kundenloyalität zu (1997, S. 741). Den Einfluss der Attraktivität des Konkurrenzangebotes auf die Kundenloyalität findet sich bei Peter mit Hilfe der sozialpsychologischen Interaktionstheorie begründet (1997, S. 96). Braunstein differenziert den beziehungsbezogenen Nutzen in vier Dimensionen (2001, S. 79) und unterscheidet dabei zwischen dem sozialen Nutzen, dem psychischem Nutzen, dem Nutzen aus der speziellen Behandlung durch den Anbieter (ökonomischer Nutzen) und dem identitätsbezogenen Nutzen. Als Konsequenz aus der Nutzenbetrachtung kann gefolgert werden, dass die Loyalität eines Kunden letztendlich vom wahrgenommenen Nettonutzen der Beziehung abhängt. Dieser lässt sich schließlich sowohl aus dem Beziehungsnutzen als auch aus den Beziehungsbarrieren ableiten (Henning-Thurau, Gwinner und Gremler, 2000, S. 383). Das Konstrukt der Wechselbarrieren ist streng vom Konzept der Beziehungsbarrieren zu unterscheiden. Werden von Seiten der Unternehmen Wechselbarrieren aufgebaut, so beeinflussen diese das Loyalitätsverhalten der Kunden. Wechselbarrieren erschweren dem Nachfrager den Anbieterwechsel (Fornell, 
1992, S. 10). Vertrauen und Commitment, u.a. von Anderson und Weitz (1989) und von Morgan und Hunt (1994) untersucht, haben eine direkte positive Wirkung auf das Zielkonstrukt Reduzierung der Wechselbereitschaft der Konsumenten. Welche Faktoren neben der Beziehungsorientierung auf den Effekt des Vertrauens und des Commitment auf die Wiederkaufintention Einfluss nehmen, ist allerdings offen. Die Studie von Morgan und Hunt zeigt, dass die Berücksichtigung des Commitmnent und des Vertrauens neben der Kundenzufriedenheit lediglich zu einer moderaten Verbesserung der erklärten Varianz der Kundenloyalität führt (1994, S. 20ff.). Nach Bloemer und Kasper ist dies eine essenzielle Erkenntnis.

\subsubsection{Leistungsunabhängige Determinanten der Kundenbindung}

Peter zeigt in ihrer Arbeit deutlich die Relevanz der leistungsunabhängigen Determinanten zur Erklärung der Kundenloyalität auf (Peter, 1997). Den Begriff Variety Seeking hat Faison geprägt (Faison, 1977, S. 172). Der Autor versteht darunter das Bedürfnis nach Abwechslung im täglichen Leben. In der Kaufverhaltensforschung nutzen u.a. McAlister und Pessemier (Mc Alister und Pessemier, 1982, S. 311ff.) diesen Begriff. Die Forscher definieren hierbei Variety Seeking als das Bedürfnis des Konsumenten nach Abwechslung. Homburg und Giering vermuten, abgesehen von dem direkten Einfluss des Variety Seeking auf die Kundenloyalität, eine moderierende, ja sogar negative Wirkung des Konstruktes auf die Produktzufriedenheits-Kundenloyalitätsbeziehung (1999, S. 11). Eine leistungsunabhängige Determinante der Kundenloyalität erkennt Diller (2000, S. 40) im Opportunismus des Kunden. Diese liegt dann vor, wenn der Kunde sich gewillt zeigt, jede Gelegenheit wahrzunehmen, um mehr Wert für sein Geld zu erhalten. Somit gilt, je höher der Wunsch eines Individuums nach Entlastung und je geringer somit dessen Bereitschaft $\mathrm{zu}$ opportunistischem Verhalten ist, desto eher handelt es sich um einen loyalen Kunden. Eine hervorgehobene Rolle spielt in der Kaufverhaltensforschung neben dem Commitment- das Involvementkonstrukt. Eine Definition ist in diesem Zusammenhang, dass das Involvment sich auf den Grad der persönlichen Relevanz und Wichtigkeit einer spezifischen Produktkategorie bezieht (Celsi und Olson, 1988, S. 220). Die Modellierung des Einflusses des Involvement auf die Kundenloyalität nehmen Bloemer und Kasper auf zweifache Weise vor. Die Autoren beschränken sich auf eine Analyse des Kaufinvolvement und begründen diese Vorgehensweise damit, dass Produktinvolvement immer auch zu Kaufinvolvement führt (Bloemer und Kasper, 1999, S. 4). In diesem Zusammenhang postulieren sie eine direkte Wirkung. Ebenso fungiert das Involvement als moderierende Variable der Beziehung zwischen Kundenzufriedenheit und Kundenloyalität. Zur Erklärung 
dieses indirekten Effektes führen sie an, dass ein hoch involviertes Individuum die Produktbeurteilung und Kaufentscheidung bewusster und intensiver durchlebt als eine weniger involvierte Person. Je höher das Involvement des Kunden, desto stärker der Zusammenhang zwischen Kundenzufriedenheit und Kundenloyalität.

\subsubsection{Soziale, situative und soziodemographische Determinanten der Kundenbindung}

Eine weiterer leistungsunabhängiger Bestimmungsfaktor ist das Streben des Individuums nach sozialer Integration (Diller, 2000, S. 42). Je größer der Wunsch nach sozialer Integration und je geringer das Streben nach Autonomie, desto höher ist die Bereitschaft des Kunden zu Loyalität. Die Relevanz der Meinung des sozialen Umfeldes wurde u.a. von Dick und Basu (S. 1994, S. 105) berücksichtigt. Sie nahmen die situativen Faktoren zur Erklärung von loyalen Verhaltensweisen auf. Dick und Basu vertreten die Meinung, dass verschiedene situative Faktoren die Kundenloyalität bestimmen. Den kontextabhängigen Einflussfaktoren fügen sie die tatsächlich wahrgenommenen Möglichkeiten hinzu. Dazu gehört unter anderem der Vorsatz des Kunden ein beabsichtigtes Wiederkaufverhalten auch zu realisieren. Die Autoren zählen dazu den Anreiz, auf Grund von Preissenkungen oder Point of Sale (POS)- Maßnahmen der Wettbewerber den Anbieter zu wechseln. Als Begründung für die Wichtigkeit der soziodemographischen Merkmale bei der Erklärung der Kundenloyalität führen Homburg und Giering (1998, S. 22) an, dass in vielen Situationen gerade diese Charakteristika den psychischen Zustand und das Verhalten des Individuums reflektieren oder gar beeinflussen. Die Autoren unterstützen diese Aussage indem sie nachweisen, dass bei Männern eine stärkere Wirkung zwischen Produktzufriedenheit und Kundenloyalität besteht als bei Frauen. In ihrer Arbeit zeigen sie auch, dass das Alter die Stärke des positiven Zusammenhangs zwischen Kundenzufriedenheit und Kundenloyalität beeinflusst. Im Gegensatz zu den älteren Menschen fällt es jüngeren häufig schwerer, ein Qualitätsurteil zu fällen. Homburg und Giering vermuten deshalb eine stärkere Beziehung zwischen Produktzufriedenheit und Kundenloyalität bei älteren als bei jüngeren Menschen. Empirische Daten bestätigen den vermuteten moderierenden Effekt des Zusammenhangs zwischen Kauf- bzw. Nachkaufzufriedenheit und der Kundenloyalität bei älteren Menschen. Eine weitere soziodemographische Variable ist bei Homburg und Giering das Einkommen. Bereits Farley (1964, S.9ff.) argumentiert, das ein höheres Einkommen vielfach auch mit einem höheren Ausbildungsniveau einhergeht und das diese Kundengruppe sich für einen stärkeren Informationsverarbeitungsprozess engagiert. Solche Nachfrager achten insbesondere auf neue Informationen, die ihnen das Verkaufsperso- 
nal vermittelt. Der Kaufprozess selbst gestaltet sich im ersten Schritt aus der Lust zum bzw. am Einkauf (Problemerkennung). Grenzüberschreitend kommt hierbei sicherlich auch wie Kotler es ausdrückt the „evaluation of alternatives" (Kotler, Armstrong, Saunders und Wong, 1999, S. 258) hinzu, erst danach erfolgt die aktive Informationssuche, die Bewertung von Alternativen. Doch auch unerwartete situative Faktoren (Schaufenster, Sonderangebote, Einfluss anderer) haben Einfluss auf die Handlung. Erst dann kommt es zur eigentlichen Kaufentscheidung, die in der Einkaufsstätte selbst stattfindet (Kotler, Armstrong, Saunders und Wong, 1999, S. 258ff). Basierend auf diesen Überlegungen vermuten Homburg und Giering bei Kunden mit einem hohen Einkommen eine stärkere Beziehung zwischen der Kauf- und Nachkaufzufriedenheit sowie der Kundenloyalität als bei Nachfragern mit geringem Einkommen. Allerdings schränken die Forscher ihre Aussagen dahingehend ein, dass sie auf die Notwendigkeit der Validierung der Ergebnisse hinweisen (Homburg und Giering, 1999, S. 23).

\subsection{Kundenzufriedenheit und Kundenbindung als Marketingziel der Einzelhandelsunternehmen}

Seit dem Beginn der 90er Jahre haben die Einzelhandelsunternehmen die Notwendigkeit, sich verstärkt um den Aufbau und die Pflege von Beziehungen zu ihren Abnehmern zu kümmern erkannt (Peter, 1999, S. 1; Homburg und Bruhn, 1998, S. 5). Ein zentraler Faktor in der Beziehung des Einzelhandels zu seinen Kunden ist die Kundenzufriedenheit der Konsumenten zu ihrer Einkaufsstätte. Unter Kundenzufriedenheit wird dabei das Ergebnis eines komplexen psychischen Vergleichsprozesses verstanden. Der Kunde vergleicht, sofern möglich, seine wahrgenommenen Erfahrungen während des Gebrauchs eines Produktes oder nach Inanspruchnahme einer Dienstleistung (Ist-Leistung), mit seinen Erwartungen vor der Nutzung (Soll-Leistung). Zufriedenheit bzw. Unzufriedenheit entsteht durch das Maß der Abweichung von Soll und Ist. Die Erklärungsansätze zur Zufriedenheit sind in den Verhaltenswissenschaften, speziell in den Sozialwissenschaften, angesiedelt. Eine positive Einstellung zu einer Einzelhandelsverkaufsstätte ist dabei zwar eine notwendige, aber keine hinreichende Voraussetzung zur Schaffung von Kundenzufriedenheit (Oliver, 1981). Es gibt mehrere theoretische Erklärungsansätze, wobei sich diese Arbeit auf das Confirmation/ Disconfirmation Paradima ${ }^{18}$

\footnotetext{
${ }^{18}$ Ein spezieller Vergleichsstandard des Kunden wird durch Vergleich mit den tatsächlichen Erfahrungen bestätigt bzw. nicht bestätigt, was unmittelbar zur Zufriedenheit bzw. Unzufriedenheit führt. Positive und negative Disconfirmation werden unterschieden.
} 
beschränkt. In diesem Ansatz steht der Prozess der Bestätigung als vermittelnde Variable zwischen einem prekonsumptiven Vergleichsstandard sowie der wahrgenommenen Ist-Leistung und der eigentlichen Zufriedenheit (Homburg und Rudolph, 1998, S. 39.ff).

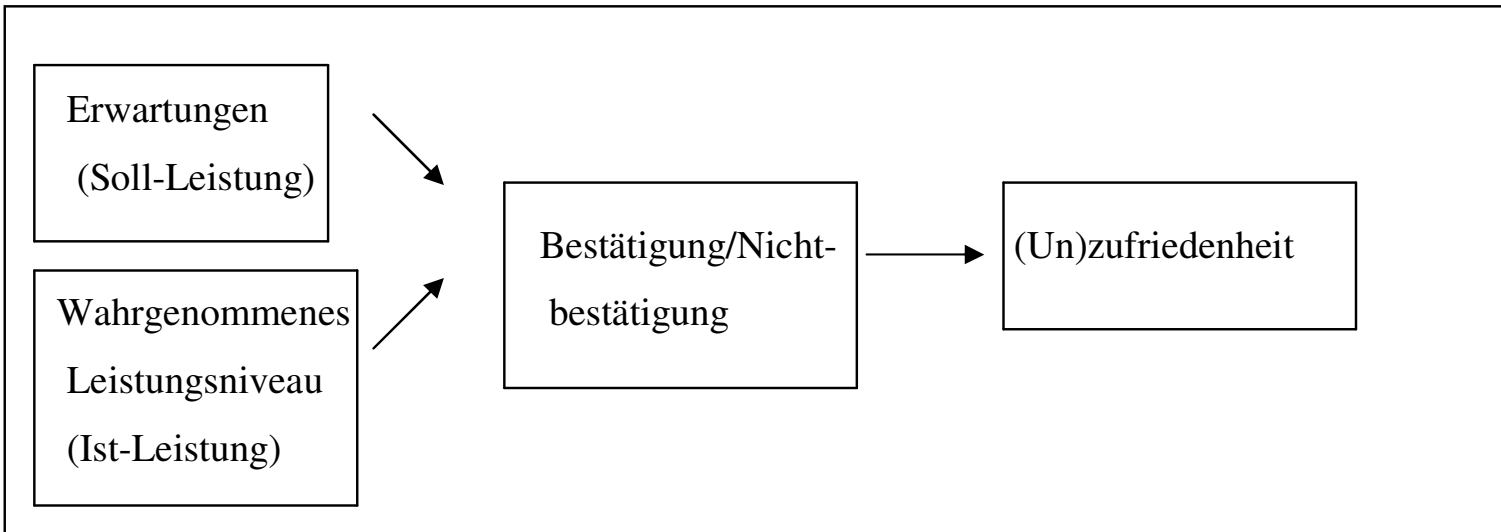

Abb. 3.4: Das C/D-Paradigma (Homburg und Rudolph, 1998, S. 39).

Als zentrale Einflussgröße der Kundenzufriedenheit erachten Clemens und Woodruff (1992, S. 413 ff.) die wertorientierte Zufriedenheit. Die Autoren streben mit dieser Vorstellung die Anpassung der Kundenzufriedenheit an die Prinzipien der Wert- und Nachfrageorientierung an. Sie verknüpfen dabei die „means end“-Theorie als Ansatz der wertorientierten Kaufverhaltensforschung mit dem C/D-Paradigma der Kundenzufriedenheitsforschung. Das daraus abgeleitete Modell „means end disconfirmation model of customer (dis-)-satisfaction“ (MED) weist gegenüber dem ,expectancy disconfirmation“-Modell folgenden Vorteil auf. Der Erwartungsdruck erfasst das Ausmaß, in dem die Leistung die Bedürfnisse und Werte eines Nachfragers erfüllt (Clemons und Woodruff, 1992, S. 414). Die Autoren gehen davon aus, dass die Konsumenten die Unternehmensleistung auf der Basis der „means-end“-Struktur beurteilen und postulieren einen höheren Motivationseffekt des nutzenbezogenen Zufriedenheitsurteils. Aus diesem Grund vermuten sie zwischen der nutzerorientierten Zufriedenheit und der Kundenloyalität einen engeren Zusammenhang als zwischen der attributbezogenen Zufriedenheit und dem Zielkonstrukt (Clemons und Woodruff, 1992, S. 416). Dies ist eine Bestätigung dafür, dass Zufriedenheit und Loyalität in einem hohen Maße von sozialen und psychischen Faktoren abhängt. Eine Aussage, die insbesondere im betrachteten Untersuchungsgebiet eine entscheidende Rolle spielt, da dies ein Erklärungsansatz für Gemeinsamkeiten und Unterschiede deutscher und niederländischer Konsumenten sein kann. Aus diesen Überlegungen wird folgendes Modell abgeleitet: 


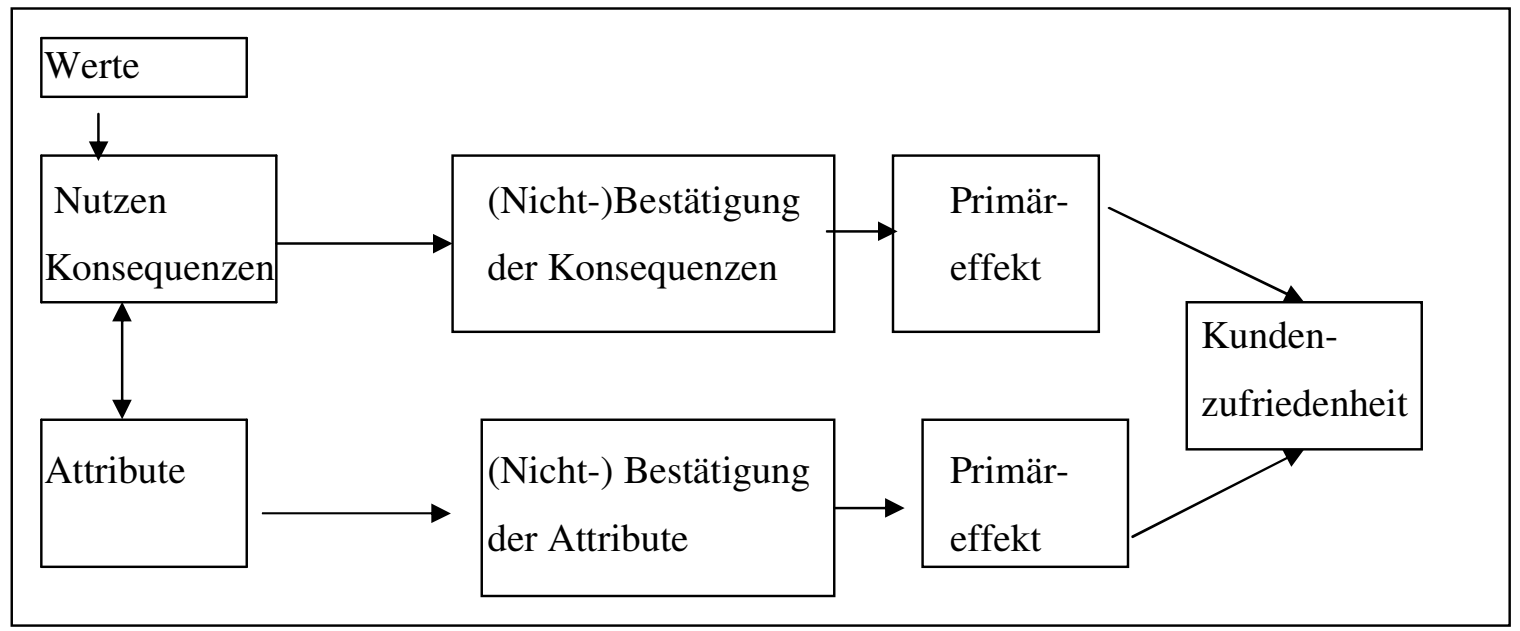

Abb. 3.5: „Means end disconfirmation“ Modell (Braunstein, 2001, S. 62, in Anlehnung an Clemon und Woodruff, 1992)

Bereits Festinger (Festinger, 1957) geht in seiner Theorie der kognitiven Dissonanz davon aus, dass Individuen ein Gleichgewicht ihres kognitiven Systems anstreben. Ein zufriedener Kunde befindet sich in einem psychischen Gleichgewicht, welches er halten möchte. Um dies zu erreichen, wird er sich gegenüber seinem Anbieter ehrlich verhalten, kognitive Dissonanzen vermeiden und bei den entsprechenden Einzelhandelsunternehmen Wiederkäufe tätigen. Individuen und somit Kunden lernen nach der Dissonanztheorie nach dem Verstärkungsprinzip. Hat der Kunde einmal positive Erfahrungen mit einem Unternehmen gemacht, so wird bei ihm eine positive Verhaltensänderung ausgelöst und die Wahrscheinlichkeit erhöht, dass es zu einem Wiederholungskauf kommt. Kunden haben allerdings auch nur ein bestimmtes Budget für den Konsum zur Verfügung. Dieses Budget wollen sie möglichst optimal und damit auch risikoarm im Hinblick auf die Erfüllung ihrer Bedürfnisse einsetzen. Aus Sicht des Kunden kann daher die Bindung an eine Einkaufsstätte auch als Risikoreduzierungsstrategie betrachtet werden. Der Kunde reduziert das Risiko der Unzufriedenheit, wenn er Wiederholungskäufe tätigt, weil er mit einer Einkaufsstätte zufrieden ist. (Simon und Homburg, 1998, S. 46-47). Solche Wiederholungskäufer können sich zu Stammkunden entwickeln, wenn sie regelmäßig in einem Geschäft einkaufen und diese nicht mehr in Frage stellen bzw. andere Geschäfte nicht mehr in die Auswahl einbeziehen. Einzelhandelsunternehmen erreichen ein solches Maß der Loyalität nur, wenn sie in den Augen der Kunden einen solche Nutzen anbieten, den die Konkurrenz nicht bietet. Die Einzelhandelsunternehmen, zu denen sich die Konsumenten hingezogen fühlen, haben sich also deutlich von der Konkurrenz abzugrenzen. Dann wird daraus langfristig ein komparativer Konkurrenzvorteil. Nach Backhaus (2003) ist 
ein komperativer Konkurrenzvorteil vorhanden, wenn das Leistungsangebot eines Anbieters von den Nachfragern in ihrer subjektiven Wahrnehmung gegenüber allen relevanten Konkurrenzangeboten als überlegen eingestuft wird und einen Ergebnisüberschuss erzeugt. Aus diesen Zusammenhängen kann man folgende Abhängigkeit ableiten, die allerdings nur Gültigkeit besitzen, sofern unterstellt wird, dass Loyalität nicht gleichzusetzen ist mit Wiederkauf und Kundenbindung:

\begin{tabular}{|l|}
\hline Kundenorientierung \\
Kundenzufriedenheit \\
Loyalität \\
Wiederholungskauf \\
Kundenbindung
\end{tabular}

Abb. 3.6: $\quad$ Die Folgen des komperativen Konkurrenzvorteils

Eine solche Abfolge ist im Unternehmensalltag nicht unproblematisch. Zwar empfiehlt sich die Integration der Kundenbindung in das Zielsystem der Einzelhandelsunternehmen, doch erscheint der Einsatz von Kundenbindungsmassnahmen nur sinnvoll, sofern diese zum Unternehmenserfolg beitragen.

Ein hohes Maß an Kundenzufriedenheit führt zunächst zu Kundenloyalität und beides zusammen dann zu Kundenbindung. Daraus resultiert, dass nur bei sehr hoher Kundenzufriedenheit und -bindung der Kunde nicht mehr beabsichtigt, “Abwechslung zu suchen”. Da dies den Schluss zulässt, dass auch ein Unternehmen mit hoher Kundenzufriedenheit eine geringe Kundenbindung erreichen kann, schlägt sich Loyalität in Kundenbindung nur nieder, wenn ein hohes Niveau an Kundenzufriedenheit zugleich mit hoher Kundenloyalität verbunden ist. Der Zusammenhang zwischen Kundenzufriedenheit/ Kundenloyalität und Kundenbindung soll in Anlehnung an Töpfer wie folgt dargestellt werden: 


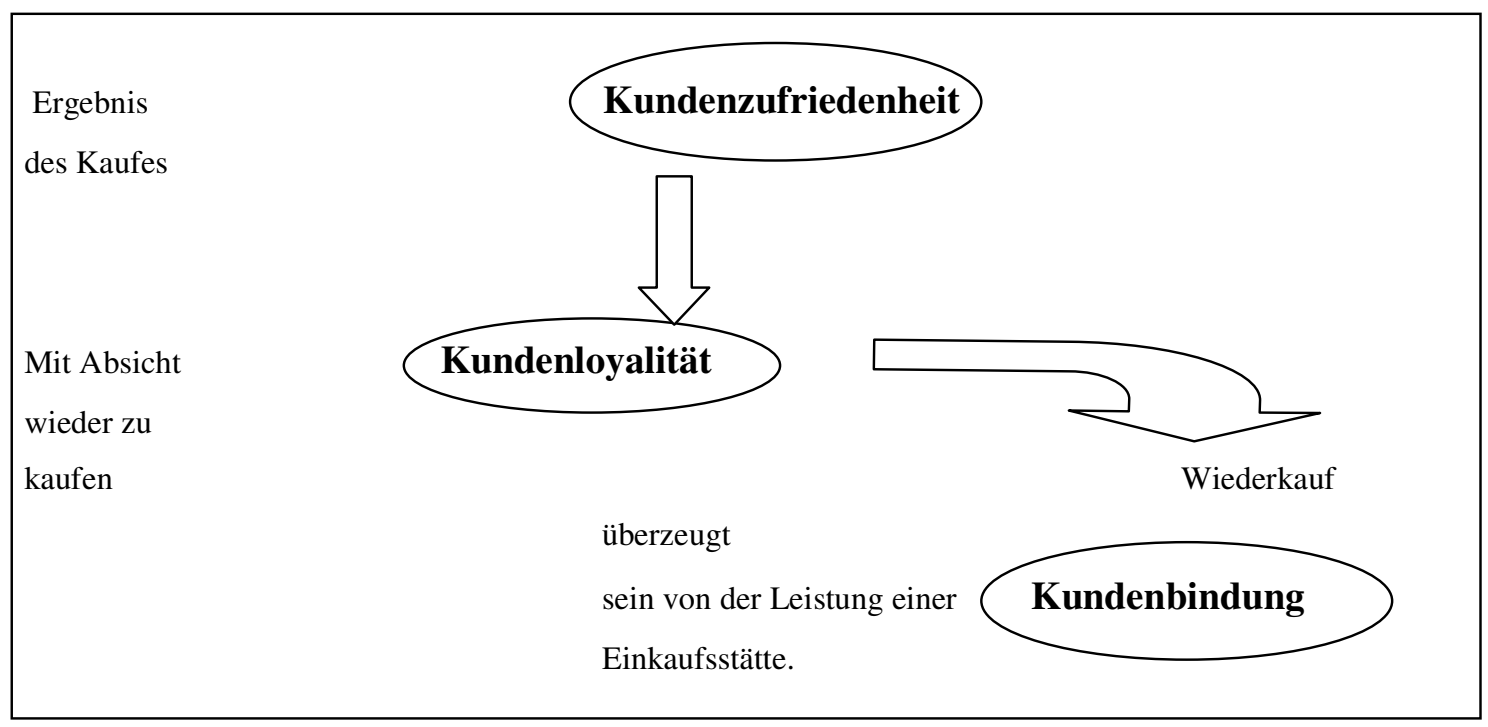

Abb. 3.7:

Die Entwicklung von Kundenzufriedenheit-KundenbindungKundenloyalität bis zur Loyalty (Töpfer, 1999, S. 341)

Kundenbindung hat direkte und indirekte Auswirkungen auf den Unternehmenserfolg. So haben Einzelhandelsunternehmen die Möglichkeit, durch verstärkte Auskunfts- und auch Beschwerdebereitschaft der Stammkunden im Rahmen eines Costumer Relationship Managements die Sortimentsstruktur zu optimieren und dadurch Lagerhaltungskosten zu reduzieren sowie Serviceverbesserungen schneller umzusetzen. Nutzt das Einzelhandelsunternehmen diese Informationen gezielt, kann es sein Leistungsprogramm erheblich besser auf die Unternehmenskunden abstimmen (Peter, 1997, S. 47). Zu den direkten Auswirkungen gehört die Verbesserung der Ertragssituation durch die Gewinnung neuer Kunden, die zu Stammkunden werden (vgl. Kapitel 3.2). Dabei ist zu berücksichtigen, dass die Geschäftstreue eines Individuums bzw. einer Familie sich nicht auf eine Einkaufsstätte beschränkt. Cunningham stellte bereits in 1962 im Rahmen einer Untersuchung im Lebensmittelhandel fest, dass die durchschnittliche amerikanische Familie nur 48,6\% ihres gesamten Lebensmittelbudgets in ihrem Lieblingsgeschäft einkauft, die Ausgaben in den drei bevorzugten Einkaufsstätten zusammen jedoch 80\% des zur Verfügung stehenden Budgets ausmachen (Cunningham, 1961, S. 129f.). Dieses Phänomen der Multistore Loyalty, der Treue zu einer kleinen Auswahl von Geschäften, ist nachweislich auch im Bekleidungseinzelhandel zu finden (Jungwirth, 1997, S. 15f). Endverbraucher scheinen auf Grund kognitiver wie psychischer Belastung die große Zahl der möglichen Geschäfte auf einige wenige zu reduzieren. Hier ist der erste Ansatzpunkt für Einzelhandelsunternehmen zu erforschen, welche Geschäfte sich in der engen Auswahl der einzelnen eigenen Kundenzielgruppen befinden, um Informationen zu erhalten, von welchem Konkurrenzunternehmen das größte Bedrohungspotential ausgeht und welche Gründe dafür vorhanden sind (Lessig, 1973, S. 72-74). 
Nach Jungwirth (1997, S. 30) lässt sich das Konzept des Evoked Set ${ }^{19}$, das ursprünglich für die selektive Markenauswahl von Konsumenten innerhalb einer Produktgruppe entwickelt wurde (Brisoux und Laroche, zitiert bei: Jungwirth, 1997, S. 30) auch für die Darstellung des Auswahlprozesses der Einkaufsstättenwahl heranziehen. Im Gegensatz zum Evoked Set gehört zum Consideration Set eine Geschäftsstätte bereits dann, wenn sie für den Konsumenten im Prozess der Bedürfnisbefriedigung bzw. Kaufentscheidung überhaupt in Erwägung gezogen wird (Boltz und Leven, 2004, S.508), Kotler, 2003, S. 79-80). Das Awareness Set beinhaltet die Gesamtheit der Einkaufsstätten, die dem Konsumenten in einer Entscheidungssituation gedanklich als Alternativen zur Verfügung stehen. Die Markenwahl findet in der Regel erst am Einkaufsort statt, die Entscheidung zur Auswahl einer Einkaufsstätte ist dieser Entscheidung vorgelagert (Jungwirth, 1997, S.30). Diese Entscheidung zur Auswahl einer Einkaufsstätte wird entweder bereits beim Erkennen des Einkaufsanlasses getroffen, spätestens aber kurz bevor das Geschäft aufgesucht wird. Ebenso ist aber auch ein signifikanter Zusammenhang zwischen dem Consideration Set von Marken und von Einkaufsstätten vorhanden, wobei Konsumenten mit einem stabilen Consideration Set von Marken i.d.R. auch über einen stabilen Evoked Set von Geschäften verfügen (Seggev, 1970, S. 22). In Anlehnung an Meffert hat Jungwirth ein Evoked Set der selektiven Einkaufsstättenwahl erstellt. Ausgehend von allen theoretisch in Frage kommenden Geschäften kommen für Konsumenten nur die Geschäfte in die engere Wahl, die sie auch kennen. Auch wenn ein Konsument ein bestimmtes Geschäft kennt, es für ihn aber ohne Einkaufsbedeutung ist, fällt es aus seiner engen Bedeutung für die Auswahl heraus. Nur Einkaufsstätten, die für den Verbraucher eine gewisse Mindestrelevanz aufweisen, haben eine Chance, einen Teil des produktspezifischen Budgets zu bekommen. In der letzten Stufe scheiden dann Unternehmen aus, in denen der Konsument in der Vergangenheit negative Erfahrungen gemacht hat. Hier spielen oft die Mitarbeiter einer Einkaufsstätte eine besondere Rolle, ob diese freundlich/kompetent bzw. unfreundlich und/oder inkompetent wirken. Ein langfristig geschäftstreues Verhalten des Konsumenten entsteht so nur bei einer kleinen Anzahl von Geschäften/Einkaufsstätten, die allen Anforderungen des Konsumenten im Wesentlichen entsprechen und als vergleichbare Alternativen betrachtet werden. Nur diesen Einkaufsstätten fließt der Großteil des Konsumentenbudgets zu. Der Rest entfällt auf Geschäfte, die möglicherweise in nur einem Selektionskriterium wie bei der Entfernung zur Einkaufsstätte oder Parkplatzangebot schlechter bewertet werden als die Alternativen (Jungwirth, 1997, S. 31). Diese beiden Selektionskriterien sind insbesondere

\footnotetext{
${ }^{19}$ Evoket set: Menge aller Marken oder Objekte, die aktiv vom Konsumenten aus dem Langzeitgedächnis abgerufen werden können und während des Entscheidungsprozesses des Konsumenten aktiv in Betracht gezogen werden.
} 
in den letzten Jahren vor dem Hintergrund der zunehmenden Mobilität wichtig geworden, da das Auto ein selbstverständliches Fortbewegungsmittel geworden ist. Hinzu kommt, dass immer mehr Verbraucher außerhalb einer Stadt oder am Stadtrand wohnen und somit auf ein Auto angewiesen sind. Sie nutzen dies somit auch beim Einkaufen. Bei der selektiven Auswahl der Geschäftsstätten werden vielfach keine objektiven Vergleichsmessungen von Seiten der Verbraucher durchgeführt, sondern es entscheiden erlebnismäßig präsent und subjektive Faktoren bei der Vorauswahl. Geschäftstreues Verhalten kann sogar aus einer bewussten Meidung bestimmter Geschäfte resultieren (Lessig, 1973, S. 72, Malhorta, 1986, S.129). Haben sich Einzelhandelsunternehmen als Marketingziel den Aufbau von Kundenzufriedenheit und Kundenbindung gesetzt, ist es Aufgabe eines jeden Unternehmens zu versuchen, in das Consideration $\mathrm{Set}^{20}$, später in das Evoket Set der unternehmensspezifischen Zielgruppe aufgenommen zu werden. Konkret kann nach Jungwirth (Jungwirth, 1997, S. 31) die Auswahl einer Einkaufsstätte selektiv folgendermaßen vorgenommen werden:

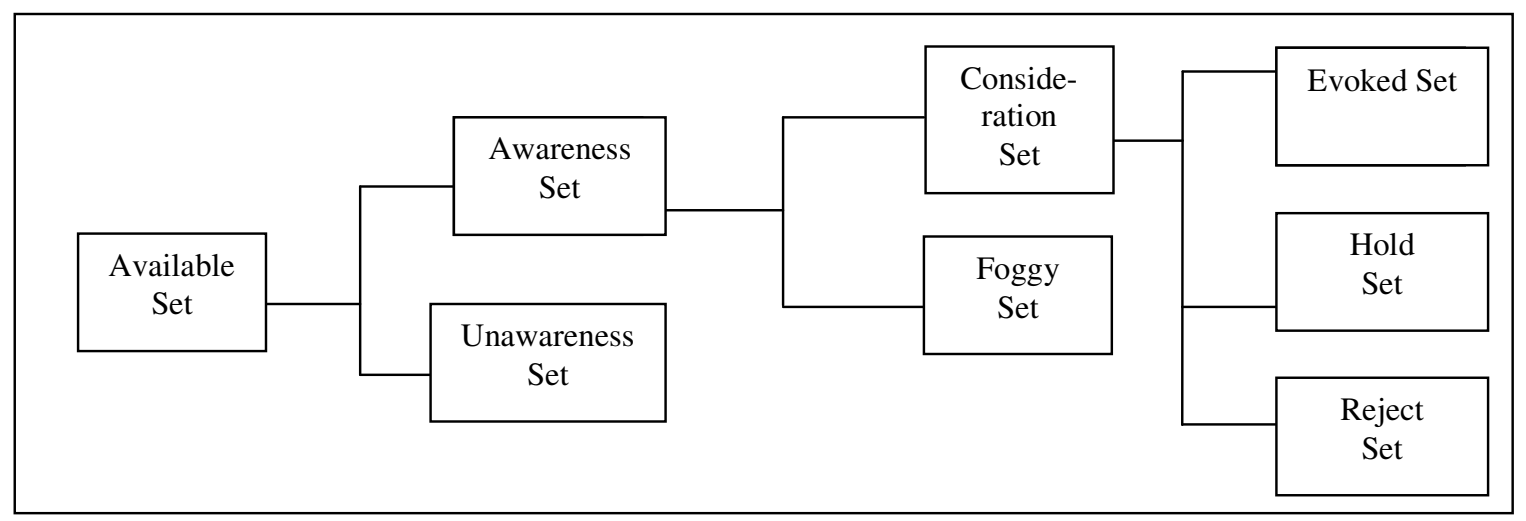

Abb. 3.8: $\quad$ Das Evoket Set der selektiven Einkaufsstättenwahl ( in Anlehnung an Jungwirth, G., 1997, S. 31)

Handelsunternehmen können Kundenbindung somit nur erreichen, wenn sie über den Consideration Set in den Evoked Set der Konsumenten gelangen, da nur hier die Einkaufsstätte aktiv aus dem Langzeitgedächnis abgerufen wird. Kundenbindung kann nicht aufgebaut werden wenn die Einkaufsstätte allein zum Awarness Set des Konsumenten gehört. Damit die Einkaufsstätte in das Evoket Set der Konsumenten gelangt, ist von Seiten der Unternehmen ein Zielsystem zu erstellen, indem sowohl ökonomische und außerökonomische Ziele durch Kundenbindungsaktivitäten verfolgt werden können. Grundsätzlich ist das Ziel aller Kundenbindungsaktivitäten von Einzelhandlesunternehmen, die Verbesserung der Bindung der Kunden an das eigene Unternehmen. Adressaten der Kundenbindungsaktivitäten sind demnach die derzeitigen Stammkunden, ehemalige Kunden der Geschäftsstätte sowie potentielle Neu-

\footnotetext{
${ }^{20}$ Jungwirth spricht in diesem Fall vom Processed Set.
} 
kunden. Im Rahmen dieser Aktivitäten werden ökonomische und außerökonomische Ziele verfolgt. Zu den ökonomischen bzw. primären Zielen gehören Aktivitäten zur Verbesserung des Umsatzes und des Marktanteils, aber auch eine Verbesserung des Deckungsbeitrages und der Gewinnsituation für das Handelsunternehmen (Schweiger, 1993, S. 16). Nicht zu den ökonomischen Kundenbindungszielen gehört die Erhöhung der Kundenfrequenz, da aus ihr kein positiver Zusammenhang zum Bindungsgrad des Konsumenten nachgewiesen werden konnte (The Coca-Cola Retailing Research Groups Europa, 1993, S. 69).

\begin{tabular}{|l|l|}
\hline \multicolumn{2}{|c|}{ Ziele von Kundenbindungsaktivitäten } \\
\hline ökonomisch & - kundennahes Unternehmensimage \\
- Absatz-, Umsatz-, Marktanteilsziele & - Erhöhung der Nachkaufzufriedenheit \\
- höhere kundenindividuelle Erträge & - positive Mundpropaganda \\
- bessere Deckungsbeitrags- und Gewinnsituation & - höhere Beschwerdezufriedenheit \\
- Kontinuität des Absatzes & - Netzwerkbildung \\
- Erhöhung des Stammkundenanteils & \\
- niedrigere Preiselastizität & \\
- Reduzierung werblicher Streuverluste & \\
- Steigerung der Wiederkaufrate & \\
\hline
\end{tabular}

Abb. 3.9:

Auswahl möglicher ökonomischer und außerökonomischer Ziele von Kundenbindungsaktivitäten (Hanse und Jeschke, 1992, S. 93)

Im Fokus der außerökonomischen Kundenbindungsziele steht die Erhöhung der Nachkaufzufriedenheit. Hier werden andere wie "positive Mundpropaganda" oder "Reduktion der Unvoiced Complaints", "Beschwerdezufriedenheit", "höhere Kundenloyalität” subsumiert. Wesentliches Ziel ist es hierbei, Meinungsführer in den Unternehmensprozess zu integrieren. Dies kann durch Instrumente wie Konsumentenbeiräte, VIP Clubs usw. geschehen. Bei dieser Strategie werden die akquisitorischen Fähigkeiten von Menschen genutzt, die außerhalb des Unternehmens stehen, eine hohe Glaubwürdigkeit in ihrem Umfeld besitzen und einen starken Einfluss auf das Kaufverhalten ihrer Mitmenschen ausüben und somit Kunden als Kunden werben (Brüne, 1989, S. 74). Für Einzelhandelsunternehmen ist es primär interessant, dass sie die angebotenen Produkte und Dienstleistungen verkaufen. Dabei ist zwischen Händlerloyali- 
tät und Markenloyalität zu differenzieren, wobei letztere als notwendige Bedingung zum Aufbau der Händlerloyalität gesehen werden kann (Burmann, 1991, S. 249). ${ }^{21}$

Auch Bloemer et al. (1990, S.43) nehmen an, dass die Händlerloyalität die Markenloyalität determiniert. Bloemer und Kasper differenzieren zwischen der Loyalität gegenüber dem Produkt und gegenüber dem Händler sowie zwischen dem Involvement bezüglich des Produktes und des Händlers (1999, S. 4ff.). Es existiert ein deutlicher Unterschied zwischen beiden Loyalitätsarten im Verhältnis zur Gesamtzufriedenheit die sich zusammensetzt aus Kauf/Produkt-/Kundendienstzufriedenheit (Burmann, 1991, S. 249; Müller und Riesenbeck, 1991, S. 69): schon bei geringem Absinken des Zufriedenheitsniveaus sind deutlich mehr Kunden bereit, den Händler zu wechseln, als der Marke untreu zu werden. Es besteht somit eine intensivere Bindung zur Marke als zu den Händlern. Dies bedeutet für die vorliegende Untersuchung, dass ein wesentlicher Schritt für die Einkaufsstätten darin liegt, Erkenntnisse über die

- Einstellung des Konsumenten zur Einkaufsstätte zu erhalten

- Den Einfluss der sozialen Norm auf die Zufriedenheit des Konsumenten zu kennen und

- Kundenbindungsaktivitäten zu entwickeln, die auf die Einstellung der Konsumenten abgestimmt sind.

Die nachfolgende Abbildung verdeutlicht noch einmal den Einfluss der Kundenzufriedenheit sowohl auf die Händlerloyalität als auch auf die Markenloyalität.

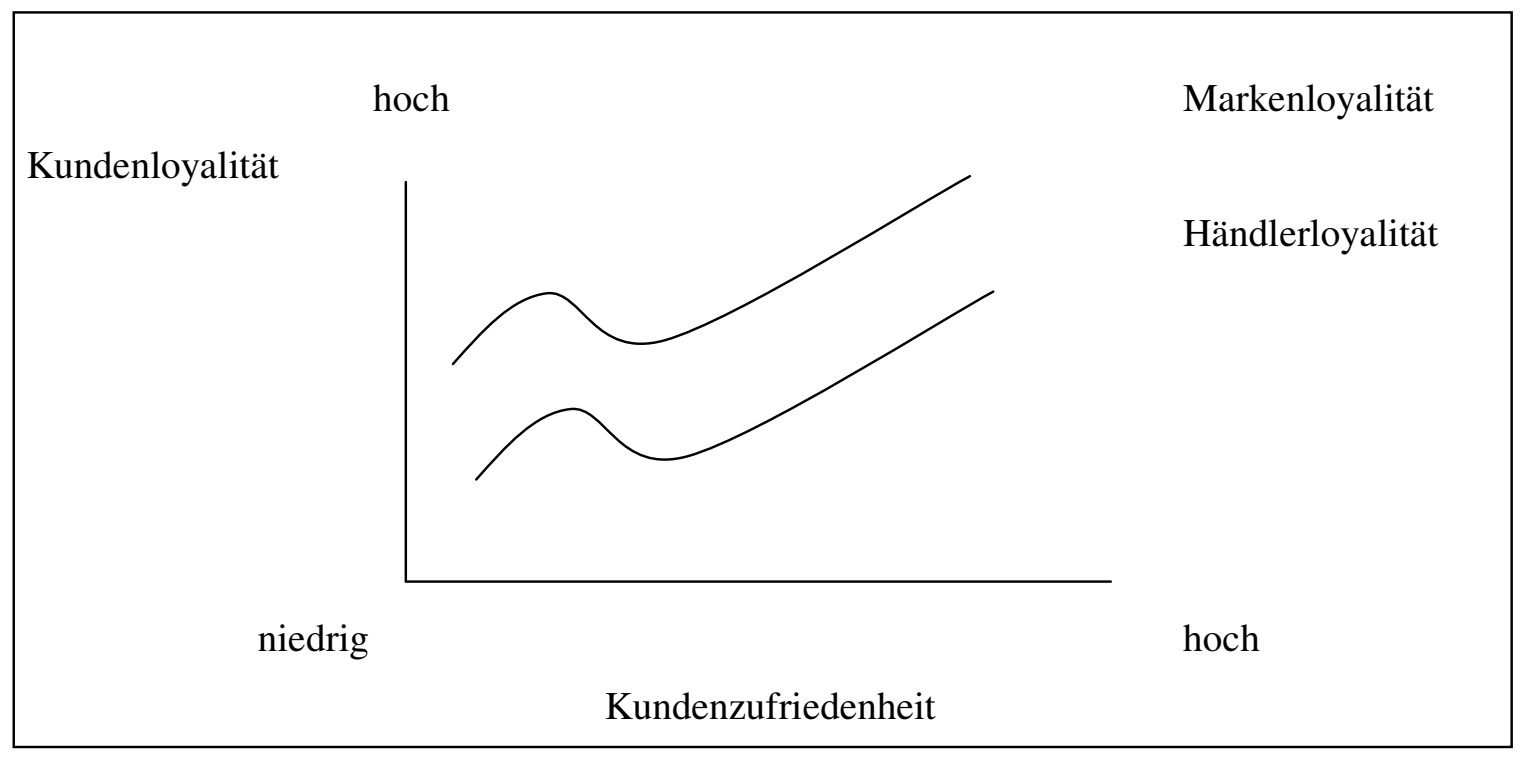

Abb. 3.10: $\quad$ Kundenzufriedenheit entscheidet über Markenloyalität und Händlertreue (Müller und Riesenbeck, 1991, S. 69)

\footnotetext{
${ }^{21}$ Burmann bezieht seine Aussagen auf die Automobilindustrie, allerdings kann zumindest angenommen werden, das es im Bekleidungshandel bei Vorliegen eines Markenbewußtseins des Konsumenten zu ähnlichen Reaktionen des Konsumenten kommt und er eher bereit ist den Händler zu wechseln als die Marke.
} 
Kundenzufriedenheit und Kundenbindung als Marketingziele bedeuten somit für Unternehmen, dass sie dafür Sorge zu tragen haben, dass eigene Kunden nicht an den Wettbewerber verloren gehen. So wird ein langfristiger Unternehmenserfolg erreicht und die Marktposition gesichert. Nicht die Maximierung einzelner Verkaufsabschlüsse steht im Vordergrund der Bemühungen der Unternehmen, sondern das Denken in langfristigen Geschäftsbeziehungen. (Peter, S., 1999, S. 1).

\subsection{Zusammenfassung der Ergebnisse und Ableitung von Schlussfolgerungen}

Wiederkauf und Weiterempfehlung bringen nach Homburg und Faßnacht (1998, S. 25 ) die Treue des Kunden zum Ausdruck. Dabei liegt nach Diller Wiederkauf erst dann vor, wenn der Kunde in zukünftigen Kaufsituationen in einer bestimmten Einkaufsstätte oder einem bestimmten Anbieter Folgekäufe tätigt (Diller, 1999, S. 85). Allerdings werden die einzelnen Begriffe in der deutschen und englischsprachigen Literatur nicht einheitlich gebraucht. Aus diesem Grund war es für die vorliegende Arbeit notwendig, eine eigene Einordnung der deutschen und englischen Begriffe vorzunehmen.

Mit der Kausalität des Zusammenhangs zwischen Kundenorientierung, Kundenzufriedenheit und Kundenbindung haben sich in der Vergangenheit viele Forschungsansätze beschäftigt. So entwickelt sich nach Odekerken-Schröder (1999, S. 72) aus der Kundenzufriedenheit des Konsumenten gegenüber dem Anbieter Treue. Kundenzufriedenheit wird beeinflusst durch die Fähigkeit des Anbieters, gezielt auf die Nachfrager einzugehen und durch „buyer relationship proneness“. Treue mündet in „relationship commitment“. Relationship commitment setzt sich neben Treue aber auch aus der Käuferzuneigung (relationship proneness) zusammen. Braunstein (2001, S. 188) fügt an dieser Stelle den Begriff der Handlungskontrolle ein. Die Autorin sieht einen Einfluss des Zustandes der Handlungskontrolle eines Individuum sowohl auf die Zufriedenheitsbeurteilungsbildung als auch auf die Loyalität des Individuums und die Kundenbindung. Der Geltungsbereich erstreckt sich von der Zufriedenheit mit dem Kauf bis zur Zufriedenheit mit der sozialen Norm (Braunstein, 2001, S. 186).

Auch Fishbein und Ajzen zeigen in ihren Arbeiten auf, dass die Einstellung gegenüber der Handlung sich als besserer Verhaltensprädikator erweist als die Einstellung gegenüber einem Objekt. Die Zufriedenheit mit dem Kauf erscheint besser geeignet, die Kundentreue vorherzusagen als die Zufriedenheit mit der Marke oder dem Anbieter. Diese Sichtweise stellt die bis- 
herige Vorgehensweise in der Kundenbindungsforschung in Frage, da die Kundenzufriedenheit als direktes Antezedens der Kundenloyalität gesehen wird und sich diese auf Czepiel und Rosenberg zurückzuführende Auffassung in der Kundenbindungsforschung durchgesetzt hat (Czepiel und Rosenberg, 1977, S. 99). Eine wesentliche Erkenntnis dieses Kapitels ist, dass eine zeitliche Aussage zwischen Kundenzufriedenheit und Kundenloyalität nicht sinnvoll ist, da sich Kundenzufriedenheit auf Erfahrungen aus der Vergangenheit bezieht, Kundentreue allerdings ein auf die Zukunft gerichtetes Phänomen ist. Aus diesem Grund wird das Konstrukt Zufriedenheit mit dem Kauf in dieser Untersuchung die Zufriedenheit mit dem Konsum implizieren. Die Untersuchungen der Literatur bestätigen einen engen Zusammenhang zwischen Kundenzufriedenheit, Treue, Kundenloyalität und Kundenbindung. Aus diesem Grund kann ausgehend von der nachfrageorientierten zweidimensionalen Konzeptionalisierung die Kundenloyalität als eine intentionale Bestimmungsgröße gesehen werden, die das Treueverhalten der Kunden beeinflusst (Braunstein, 2001, S. 27). Der Begriff der Kundenloyalität entspricht dabei dem der Kundentreue. Die Kundenloyalität beinhaltet sowohl die Markenloyalität als auch die Händlerloyalität. Für den Handel kommt als entscheidende Größe die Handlungsdimension des Kaufes hinzu. Erst wenn der Kunde sich nicht nur der Geschäftsstätte gegenüber loyal zeigt, sondern regelmäßiger Kunde der Einkaufsstätte ist, kann von Kundenbindung (Treue) ausgegangen werden (vgl. Kap. 1.1).

Für den Kaufentscheidungsprozess des Konsumenten in Grenzregionen ist nicht nur entscheidend, dass der Kunde eine manifeste Loyalität zu einem Einkaufsort und einer Einkaufsstätte aufbaut, sondern auch, dass sich der Kunde durch seine soziale und persönliche Norm mit dem Land, dem Ort und der Einkaufsstätte verbunden fühlt. Hierbei kann Loyalität einmal entstehen durch die Unterschiede des Angebotes in beiden Ländern, sei es hinsichtlich Sortiment, Preis oder Service, als auch durch die Erhöhung des Nettonutzens insgesamt für den Kunden durch den grenzüberschreitenden Einkauf. Der Nettonutzen kann dabei bereits dadurch erhöht werden, indem der Reiz des Fremden, der zugleich durch Gewohnheit vertraut ist, für den Konsumenten ein kaufentscheidendes Kriterium ist. Grenzüberschreitende Kundenloyalität ist somit nicht nur abhängig vom Leistungsangebots des Handels, sondern auch vom inner- und außerstädtischem Umfeld der Handelsbetriebe. Ferner von der Einstellung des Kunden und seines sozialen Umfeld zum grenzüberschreitenden Einkauf. Das Commitment (Verpflichtung) des Konsumenten in einer Einkaufsstätte einzukaufen ist somit eine Dimension der Kundenloyalität. Das Involvement nimmt dagegen auf zwei unterschiedliche Arten Einfluss auf Kundenloyalität. Einmal hat es eine direkte Wirkung, zum anderen ist das Invol- 
84 | Kapitel 3

vement nur eine moderierende Variable, da ein stark involvierter Konsument sowohl Produktals auch Kaufentscheidungen gründlicher überlegt als eine weniger involvierte Person. 


\section{Kapitel 4: Einstellungstheorie als Erklärungsansatz der Kundenbindung}

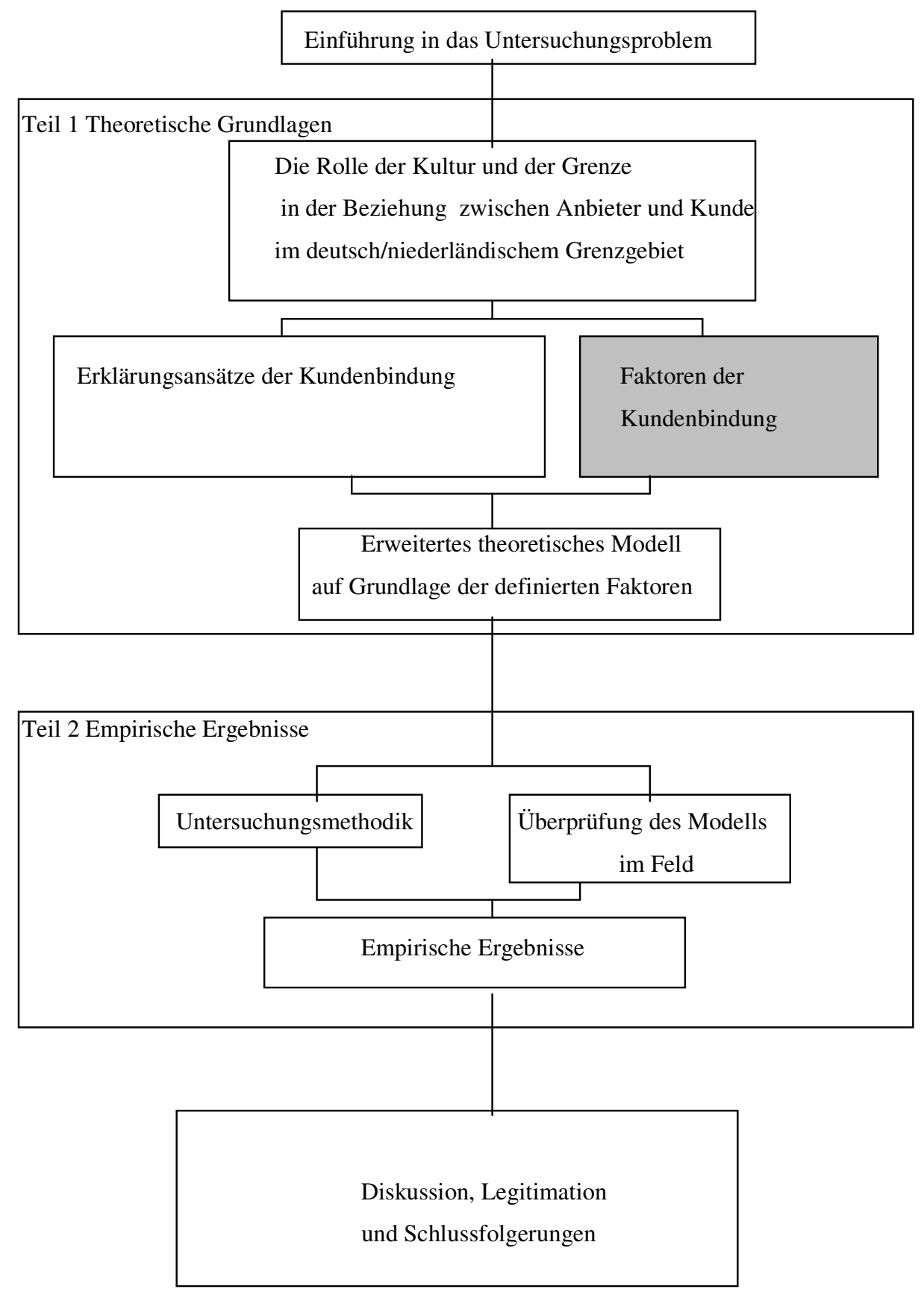




\subsection{Einleitung}

Die Ergebnisse aus Kapitel 3 zeigen, dass es zwischen Kundenzufriedenheit und Kundenbindung keine lineare Relation gibt und Kunden, obwohl sie zufrieden sind den Anbieter wechseln. In der neueren Literatur zur Bindungsforschung rufen unter anderem Oliver, Basu und Dick dazu auf, weitere Determinanten der Loyalität und Bindung des Konsumenten darzulegen. Braunstein hat auf Basis der neueren Einstellungs- und Handlungsforschung sowie den Erkenntnissen der Kundenbindungsforschung ein Ursache-Wirkungs-Gefüge rekonstruiert, um eine verbesserte Erklärung der Kundenbindung zu erreichen. Als Basis dient die Theorie des geplanten Verhaltens als zentraler Ansatz der neueren Einstellungs- und Handlungsvorschung. In diesem Kapitel wird analysiert, inwieweit diese Theorie auch zur Erklärung der Kundenbindung zu einer Einkaufsstätte im Nachbarland dienen kann.

\subsection{Einstellungstheorie und Kundenbindung}

Erfahrungen sind für Individuen in einer sich ständig verändernden Welt wesentliche Orientierungspunkte, um sich dieser Welt kontinuierlich anpassen zu können. Diese Welt, die auch als Erfahrungsfeld oder Eigenwelt bezeichnet wird, schließt Erfahrungen ein, die ein Individuum in seiner Lebensgeschichte bewusst oder unbewusst - wahrgenommen hat. Dieses Erfahrungspotenzial ist für das Individuum Realität, auf die es als Ganzes reagiert und auf das es sein zukünftiges Verhalten entsprechend ausrichtet.

Die Sozialpsychologie repräsentiert die verhaltenswissenschaftliche Variante der Soziologie (Franzoi, 1996, S. 173), die Kaufverhaltensforschung widmet sich der Erforschung individueller Verhaltensmuster im Rahmen der Marketingforschung (Braunstein, 2001, S. 95). Die Einstellung gilt allgemein als das am besten erforschte Konstrukt der Theorie des Konsumentenverhaltens (Trommsdorff, 1989, S. 123). Trommsdorff versteht unter Einstellung eine langfristig konsistent positive oder negative Bewertung. Zahlreiche Forscher setzten sich auf Grund der hohen Relevanz zur Erklärung des Verhaltens mit dem Begriff auseinander. Hierzu zählen Spencer (1862) sowie Thomas und Znaniecki (1918). Alle definieren Einstellungen als Handlungsbereitschaft (Allport, 1967, S. 8). In der Konsumentenforschung werden Einstellungen als gegenstandsbezogen, d.h. auf Sachen, Personen oder Themen gerichtet, über die ein subjektives Urteil gefällt wird, gesehen (Kroeber-Riel, 1990, S. 53f). Zur affektiven Gegenstandbeurteilung kommt hier eine kognitive Beurteilung hinzu. Kroeber-Riel definiert den Begriff Einstellung daher als subjektiv wahrgenommene Eignung eines Gegenstandes zur 
Befriedigung einer Motivation (Kroeber-Riel, 1990, S. 163). Aus der positiven oder negativen Einstellung gegenüber einem Gegenstand folgt dann im Allgemeinen eine entsprechende Verhaltensbereitschaft. Bezogen auf Loyalität bedeutet dies die Treue zu oder das Meiden einer Geschäftsstätte durch den Konsumenten. Von der Persönlichkeit, dem zweiten bestimmenden Faktor der Sozialpsychologie und Psychologie unterscheidet sich laut Ajzen (1987, S. 2) die Einstellung durch ihren situativen Charakter. Die Persönlichkeitszüge eines Menschen, definiert als langfristige Verhaltensdispositionen, beeinflussen dagegen das Verhalten über eine Vielzahl von Situationen hinweg (Ajzen, 1987, S. 2). Nach Solomon, Bamossy und Askegaard (2001, S. 154) ist eine Einstellung dauerhaft, da sie normalerweise über einen längeren Zeitraum besteht und sich im allgemeinen auf mehr bezieht, als nur auf ein vorübergehendes Ereignis. Konsumenten haben Einstellungen zu sehr produktspezifischen und zu allgemeinen konsumbezogenen Verhaltensweisen. Einstellungen beeinflussen das soziale Umfeld des Konsumenten, welche Vorlieben er hat und welchen Konsumgewohnheiten er nachgeht.

Aus der Vielzahl der Konzeptualisierungsversuche zur Einstellung haben das von Hovland und Rosenberg (Hovland und Rosenberg, 1960, S. 198ff) vorgeschlagene Drei-KomponentenModell der Einstellung sowie der eindimensionale Ansatz von Thurstone (Thurstone, 1928, S. 529ff) die größte Akzeptanz erfahren. Die Verhaltensbereitschaft eines Menschen stellt gemäß der Drei-Komponenten-Theorie neben der affektiven und der kognitiven die dritte Komponente der Einstellung dar, wobei nach Triandis Einstellungen stets durch die Konsistenz dieser drei Dimensionen des Fühlens, Denkens und Handelns gekennzeichnet sind (Triandis, 1975, S. 11). Im Einzelnen werden die drei Komponenten wie folgt definiert:

- Kognitive Komponente:

Bezeichnet das mit der Einstellung verbundene subjektive Wissen über das Einstellungsobjekt (Meffert, 1992, S. 54).

- Affektive Komponente:

Die mit der Einstellung verbundene gefühlsmäßige Einschätzung (Nutzenvorstellung) eines Objektes (Meffert, 1992, S. 56).

- Konative Komponente:

Repräsentiert die mit der Einstellung verbundene Handlungstendenz wie Kaufbereitschaft und Verhaltensabsicht (Meffert, 1992, S. 54). Nach Behrens wirkt sich die der Einstellung 
immanente Verhaltenstendenz unter gewissen Bedingungen unmittelbar auf das beobachtbare Kaufverhalten aus. Umgekehrt kann aber auch die Einstellung durch das tatsächliche Verhalten des Konsumenten beeinflusst werden (Behrens, 1991, S. 113).

Inkonsistenzen können Menschen aus ihrem psychologischen Gleichgewicht bringen und Dissonanzen verursachen. In der Regel wird versucht, diese Inkonsistenzen durch eine Änderung der Einstellungskomponente abzubauen (Jungwirth, 1997, S. 109). Dies könnte z.B. durch eine veränderte gefühlsmäßige Haltung gegenüber einer Einkaufsstätte im benachbartem Ausland (affektive Komponente) geschehen. Diese Veränderung der Einstellung führt dann ebenfalls zu einer Veränderung der Bereitschaft, diese Einkaufsstätte zukünftig verstärkt aufzusuchen (konative Komponente). Auf dieser verhaltenssteuernden Wirkung der affektivmotivationalen und der kognitiven Einstellungskomponente beruht die so genannte E-VHypothese (Einstellungs-Verhaltens-Hypothese), der zufolge Einstellungen das Verhalten bestimmen (Jungwirth, 1997, S. 109). Die Hypothese in dieser Untersuchung besagt, je stärker die positive Einstellung eines Individuums zu einem Handelsunternehmen oder einer Einkaufsstätte ist, um so höher ist die Wahrscheinlichkeit, dass es zu einem Wiederholungskauf in dem Handelsunternehmen oder der Einkaufsstätte kommt. Es kann sich dabei sowohl um die Verhaltensintention als auch um das tatsächliche Verhalten handeln (Stahlberg und Frey, 1996, S. 221f). Dennoch führt die Änderung der gefühlsmäßigen Haltung (affektive Komponente) gegenüber einem Handelsunternehmen oder Einkaufsstätte im allgemeinen ebenso zu einer Änderung der Bereitschaft (konative Komponente) in diesem Unternehmen oder Einkaufsstätte einzukaufen. Damit wird deutlich, dass Individuen durch individuelle Anpassungen permanent versuchen, sich wieder ins psychische Gleichgewicht zu setzen. Damit beeinflusst direkt oder indirekt jede Dimension die andere und wirkt sich sowohl auf das Verhalten gegenüber als auch der Treue zu einem Handelsunternehmen oder Einkaufsstätte aus.

Kritik erfährt dieses mehrdimensionale Einstellungskonzept vor allem von Fishbein. Der Autor fordert, dass Überzeugungen und Einstellungen gesondert und nach ihren eigenen Gesetzen untersucht werden sollten (Fishbein, 1967, S. 479). Ferner kritisiert er die Skalierung des dreidimensionalen Einstellungskonstruktes. In der Analyse seiner zahlreicher Studien, die jedoch bereits vor mehr als 35 Jahren erfolgte und somit ihrerseits kritisch betrachtet werden muss, stellte Fishbein fest, dass die wenigsten Studien die eingesetzten Instrumente in den drei Komponenten gleichermaßen messen (Fishbein, 1967, S. 479). 
Eine weitere wesentliche Frage ist, warum Individuen Einstellungen formen. Auf diese Frage versucht die funktionale Einstellungsforschung eine Antwort zu geben. Die funktionale Einstellungstheorie wurde von dem Psychologen Daniel Katz (1960, S. 163-204) mit dem Ziel entwickelt zu erklären, wie Einstellungen das Sozialverhalten der Individuen beeinflussen. Einstellungen formen sich nach diesem Ansatz, weil sie einem Individuum als Funktion dienen und als solche durch die Motive des Individuums gebildet werden. Individuen, die glauben, zukünftig ähnliche Entscheidungen treffen zu müssen, sammeln Informationen, um Einstellungen entsprechend aufzubauen. Dies dient dem Zweck, zukünftigen Ereignissen zuvorzukommen und aufgrund ihrer Einstellung schneller Entscheidungen treffen zu können ( Russell, Fazio, Lenn und Effrein, 1984, S. 214-234).

Einstellungen können sich, wie bereits dargelegt wurde, aus unterschiedlichen Merkmalskomponenten zusammensetzen. Ein Forschungsproblem besteht darin, dass ein Produkt oder eine Dienstleistung aus vielen Attributen oder Eigenschaften bestehen kann, die einzelnen Individuen unterschiedlich wichtig sind. Ein weiteres Phänomen ist darin begründet, dass die Entscheidung eines Individuums, ihrer Einstellung entsprechend zu handeln, von vielen Faktoren beeinflusst werden kann. So ist die Zustimmung des sozialen Umfeldes eine wesentlich die Entscheidung unterstützende Komponente. Nach Braunstein (2001, S. 99) besitzt in der sozialpsychologischen Einstellungsforschung das Erwartungs-Wert-Modell von Fishbein eine besondere Bedeutung. Die Autorin begründet diese Aussage damit, dass im Gegensatz zu den anderen Erklärungsvorschlägen das Erwartungs-Wert-Modell kausale Beziehungen zwischen den Determinanten spezifiziert. Nach dem Prinzip der kognitiven Konsistenz streben Konsumenten dagegen Harmonie in ihren Gedanken, Gefühlen und Verhaltensweisen an und wollen bei diesen Elementen Uniformität bewahren (Salomon, Bamossy und Askegaard, 2001, S. 160). Folgende Konsistenztheorien haben in der Einstellungsforschung eine Bedeutung erlangt:

\section{,Festingers Dissonanztheorie“}

Die Grundannahme von Festingers Dissonanztheorie (1957) ist, das Personen nach einem Gleichgewicht innerhalb ihres kognitiven Systems streben. Die Dissonanztheorie gehört zu den Konsistenztheorien. Kognitionen sind dabei Gedanken unterschiedlichsten Abstraktionsniveaus und unterschiedlichster Gegenstandsbereiche, die im Moment ihres Bewusstwerdens als Einheit erlebt werden, wie politische Einstellung oder das Wissen über das augenblickliche Handeln. Festingers Dissonanztheorie stellt die Beziehung zwischen kognitiven Prozessen und Verhalten her. Dissonanz ist dabei die Unfähigkeit zur Handlungskontrolle, die 
Reduktion macht Handeln erst wieder möglich. Aber die Toleranz hinsichtlich der Dissonanz ist sehr unterschiedlich, es wird in der Regel ein mittleres Aktivierungsniveau angestrebt, nicht das Fehlen von Dissonanz.

Jede Kaufentscheidung ist für den Konsumenten eine Wahl zwischen mindestens zwei Alternativen. Entscheidet sich der Konsument für eine konkrete Einkaufsstätte, so bedeutet das für ihn, dass er die Vorteile der anderen Einkaufsstätte nicht in Anspruch nimmt, die Nachteile der von ihm gewählten Einkaufsstätte allerdings akzeptiert (Festinger, 1957, S. 3). Frey und Benning (1984, S. 107) differenzieren in diesem Zusammenhang zwischen Vorkauf- und Nachkaufdissonanz, je nachdem, ob die Unvereinbarkeit der Kognition vor oder nach dem Einkauf stattfindet. Dissonanzen verursachen motivationale Spannungen, die vom Individuum als Unbehagen, schlechtes Gewissen oder Unzufriedenheit mit einer bestimmten Entscheidung einhergeht. Die Verhaltenswirksamkeit dieser Symptome ist allerdings abhängig von der individuellen Intensität dieser Dissonanz. Nach Corsten und Meier können Dissonanzen nicht nur vor und nach extensiven Entscheidungen auftreten, sondern auch nach impulsiven Entscheidungen (Corsten und Meier, 1982, S. 112-136).

Dissonante Käufer haben verschiedene Möglichkeiten, den entstandenen psychischen Spannungszustand zu beseitigen. Ein Konsument kann ein Geschäft verlassen und eine andere Einkaufsstätte aufsuchen, er kann andere für seine schlechte Entscheidung (der Rat in dieser Einkaufsstätte einzukaufen war schlecht) verantwortlich machen oder aber seine Entscheidung durch das gezielte Suchen nach Argumenten und Sachverhalten rechtfertigen bzw. gezielt nach negativen Aspekte der nicht gewählten Einkaufsstätte (man weiß ja, dass die Beratung in großen Einzelhandelsketten oft schlecht ist) suchen. In dieser Phase werden insbesondere Informationen, die die Richtigkeit der Entscheidung bestätigen (alle aus meinem Bekanntenkreis kaufen dort ein) aufgenommen, wohingegen solche, die Inkonsistenzen vergrößern, tendenziell vermieden werden (Heinemann, 1976, S. 223). Dissonanzen lassen sich somit für den Konsumenten dadurch reduzieren, indem die Attraktivität der gewählten Einkaufsstätte erhöht und die der anderen Alternativen herabgestuft werden. Eine Möglichkeit dissonanzauslösende Prozesse zu reduzieren, ergibt sich für den Konsumenten dadurch, indem er nach positiven Einkaufserfahrungen wiederholt das gleiche Geschäft aufsucht. Der Konsument bestärkt sich in seinem Einkaufsverhalten, indem er Einkaufshandlungen habitualisiert und Informationen über alternative Einkaufsstätten meidet oder verdrängt (Kroeber-Riel, 1990, S. 178). 


\section{,Attributionstheorien“}

Attributionstheorien behandeln die Problematik der Wahrnehmung von Handlungsergebnissen anderer als Ursache individueller Absichten (Jones und Davis, 1965). Attributionstheorien besagen, dass der Mensch das Handeln einer anderen Person zuerst als eine Folge ihrer Absichten (personale Attribution) deutet, dann als eine Folge der sie umgebenden situativen Bedingungen. Der Handelnde selbst orientiert sein Handeln dagegen meist am situativen Kontext (situative Attribution). Auf dieser Differenz beruht der so genannte Attributionsfehler: Er besagt, dass es für Menschen einfacher ist, Handlungen der Persönlichkeit eines Handelnden zuzuschreiben als der Situation, aus der heraus er handelt. Aus diesem Grunde werden zum Beispiel neue Gruppenmitglieder oft stark nach dem so genannten ersten Eindruck bewertet, der länger anhält als es ihr späteres Verhalten rechtfertigt. Ferner werden für Gruppen wichtige Entscheidungen oft stärker von der Einschätzung der Motivation des Führers der anderen Gruppe geprägt, als es ihnen zuträglich ist.

Die Interpretation aus attributionstheoretischer Sicht beruht auf dem Selbstwertkonzept, dass annimmt, das Individuen von ihrem Verhalten aus auf innere Zustände Rückschlüsse ziehen: Die Personen, die eine große Belohnung durch ihr Handeln erfahren, attribuieren external und behalten ihre Meinung bei. Personen mit geringer Belohnung hingegen müssen internal (z.B. auf Einstellungsänderung) attribuieren (Bem, 1972, S. 1-62). Die allgemeine attributionstheoretische Erklärung der Dissonanzphänomene ist, dass eine Handlungsweise um so eher dann internal attribuiert wird, je weniger das Verhalten mit extrinsischen Einflüssen konsistent ist, und je weniger die Person in der Lage ist, externe Faktoren für ihr Verhalten zu finden. Demnach kann es bei einer einstellungskonsistenten Handlung durch extrinsische Faktoren zu einer Abwertung der internen Überzeugungen kommen (,Korrumpierungseffekt"). Konsumenten verdrängen dabei oft den Einfluss anderer Personen und führen bei guten Erfahrungen mit einer Einkaufsstätte diesen Erfolg auf ihre eigene Entscheidungsfindung zurück. Bei schlechten Erfahrungen wird dagegen das Umfeld dafür teilweise verantwortlich gemacht.

\section{,Erwartungs-Wert-Theorie nach Fishbein und Ajzen'}

Fishbein will mit seiner Einstellungsmessung nicht nur die grundsätzliche Einstellung eines Individuums einem Untersuchungsgegenstand gegenüber ermitteln, sondern auch die Frage nach dem „Warum“ der Einstellung beantworten (Fishbein, 1967). Nach Meinung von Ajzen und Fishbein (1980) wird die Verhaltensabsicht nicht nur von der zugrunde gelegten Einstellung beeinflusst, sondern auch davon, welches Verhalten das soziale Umfeld von dem Aus- 
führenden erwartet und in wieweit der Handelnde bereit ist, diesen Ansprüchen zu entsprechen (,,subjective norm"). Die spezifischere Einstellung (z.B. Wille eine bestimmte Einkaufsstätte im Nachbarland zu besuchen) berücksichtigt implizit auch den Einfluss sozialer Normen und die budgetären Möglichkeiten eines Individuums und eignet sich zur Prognose künftigen Kaufverhaltens besser als die allgemeine Einstellung gegenüber einer Einkaufsstätte. Beide Autoren (1980, S. 5 u. S. 57) schreiben dazu: ,...individuals will intend to perform a behavior when they evaluate it positively and when they believe that important others think they should perform it." Überzeugungen üben nach Ansicht der Autoren einen hohen Einfluss auf die Verhaltensabsicht und das tatsächliche Verhalten des Individuums aus (1975, S. 14). Es hat sich allerdings gezeigt, dass der empfundene soziale Druck geringere Bedeutung besitzt als die eigentliche Verhaltenseinstellung (Ajzen und Fishbein, 1980, S. 57). Ajzen (1991) selbst spricht sich gegen die Eingliederung der „subjective beliefs", also der Meinungen über die sozialen Erwartungen in die Kaufeinstellung aus. Seiner Auffassung nach sollte die ,subjective norm“ als eigenständiges Konzept mit einer direkten Wirkung auf die Kaufabsicht erhalten bleiben. Individuen beabsichtigen ein Verhalten zu realisieren, wenn sie sowohl über eine positive Einstellung gegenüber einer Handlung verfügen, als auch der Überzeugung sind, dass ihnen wichtige Personen ihr Verhalten positiv bewerten (Frey, Stahlberg und Gollwitzer, 1993, S. 367). Die Beziehungen zwischen Überzeugungen und Einstellungen modelliert Fishbein mit Hilfe des Erwartungs-Wert-Ansatzes und legt zur theoretischen Fundierung die Lerntheorie diesem Ansatz zugrunde. Er geht davon aus, dass zwischen der Einstellung des Individuums zu einem ausgewählten Objekt (Produkt) und der kognitiven bzw. affektiven Beurteilung dieser Person ein funktionaler Zusammenhang besteht.

Ein möglicher Verhaltensprädiktor ist die Intention. Intentionen sind subjektive Wahrscheinlichkeiten bezüglich des Auftretens bestimmter Verhaltensweisen. Die Höhe der Korrelation zwischen Intention und Verhalten ist abhängig vom Grad der Übereinstimmung bezüglich der Aspekte Handlung, Ziel, Kontext, Zeit. Ein weiterer Faktor ist die Zeit, die zwischen Intentionsmessung und Ausführung des Verhaltens vergangen ist. Je länger das Intervall, um so ungenauer wird die Intention das Verhalten vorhersagen. Intentionen hängen von zwei unmittelbaren Ursachen ab:

- Einstellung zum Verhalten und

- Sozialer Norm

die sich zusammensetzt aus dem Grad der Wichtigkeit für den Konsumenten, dass Freunde und Bekannte bestimmte Kaufentscheidungen treffen und weiterempfehlen, sowie der Meinung der Freunde und Bekannte über ihre Kaufentscheidungen. 
Kritisiert wurde die Erwartungs-Wert-Theorie, indem andere Kausalbeziehungen zwischen den Variablen vorgeschlagen und ergänzende Komponenten eingefügt wurden. Ferner löst die vergleichsweise geringe Vorhersagekraft des Erwartungs-Wert-Modell sowie sein linearer Charakter Kritik aus. Seit Mitte der 70er Jahre focussiert Fishbein seine Forschungen deshalb verstärkt auf den Einstellungs-Verhaltens-Zusammenhang. In der Theorie des vernünftigen Verhaltens (theory of reasoned action, TORA) werden die kausalen Beziehungen zwischen Überzeugungen, Einstellungen und Verhalten konkretisiert (Frey, Stahlberg und Gollwitzer, 1993, S. 374). Gewohnheitsmäßiges oder automatisiertes Verhalten werden dabei ausgeschlossen und nicht als Treue bzw Loyalität interpretiert. Dem Menschen wird die Fähigkeit zugestanden, soziale Verhaltensweisen durchführen zu können. Den Willen des Individuums sich an diese sozialen Verhaltensweisen zu halten, erfasst Fishbein mit dem Konstrukt der Verhaltensabsicht.

\section{,Die soziale Norm‘}

Die soziale Norm wird hier als der wahrgenommene soziale Druck des Konsumenten verstanden und besteht aus den Komponenten: Verhaltensvorschriften wichtiger Personen und dem inneren (in welchem Ausmaß) Zwang, diesen Wünschen zu folgen (siehe Kapitel 2.1). Nach Kroeber-Riel und Weinberg muss das Individuum aus diesem Grund nicht jedes mal von neuem entscheiden, „ob das, was es vorhat oder tut, „richtig“ ist. Das an den Normen ausgerichtete Verhalten (Verhaltensmuster) ersetzt also eine Vielzahl von individuellen Entscheidungsprozessen“ (2003, S. 492). Die Einstellung zum Verhalten setzt sich zusammen aus der Erwartung von Verhaltenskonsequenzen und der Bewertung der Konsequenzen. Die relative Stärke, die durch Regressionsanalysen ermittelt werden kann, wird durch die Intentionsursachen bestimmt. Ist die Norm stärker als die Einstellung, dann hängt das Verhalten mehr von den (vermeintlichen) Ansprüchen anderer Personen ab als von der eigenen Einstellung. Allerdings ist die Abgrenzung zwischen einstellungsorientierten und normativen Überzeugungen nicht immer eindeutig. Allein die Aussage eines Probanden ,wenn ich das Produkt X in einer bestimmten Einkaufsstätte im Nachbarland kaufe, gefällt das meinem Freundeskreis" zeigt, dass eine scheinbar normative Aussage durchaus zu den einstellungsbezogenen Überzeugungen zählen kann, da sie sich auf eine Verhaltenskonsequenz bezieht. Eine normativ korrekte Formulierung wäre in diesem Fall, ,mein Freundeskreis denkt (erwartet), dass ich das Produkt $\mathrm{X}$ in einer bestimmten Einkaufsstätte im Nachbarland kaufe."

Innerhalb der Familie verliert die Zahl der gemeinsamer Entscheidungen mit fortschreitendem Familienzyklus an Bedeutung. Dies bedeutet nicht, dass die Meinungen der anderen Mitglie- 
der keine Berücksichtigung mehr finden. Vielmehr sind die für den Einkauf verantwortlichen Personen zunehmend in der Lage, allgemein akzeptierte Entscheidungen eigenständig zu fällen (Kroeber-Riel, 2003, S. 440ff). Häufig wird in diesem Zusammenhang auch von einer „gatekeeper"-Funktion der Familie gesprochen. Die Ansicht der sozialen Gruppe - das nähere Umfeld des Konsumenten, zu dem die Familie, Freunde und Berufskollegen zu zählen sind hat vor allem dann Einfluss auf das Kaufverhalten, wenn es sich um Produkte oder Einkaufsstätten handelt, über die der Käufer nur mangelnde Kenntnisse besitzt. In diesem Fall helfen Empfehlungen von Freunden häufig weiter (Kroeber-Riel und Weinberg 2003, S.457, S. 504). Grundsätzlich gehen Kroeber-Riel und Weinberg davon aus, dass Bezugsgruppen in erster Linie Anpassungsdruck auf das Individuum ausüben, der wesentlich für das konforme Verhalten verantwortlich ist (2003, S.490). Der soziale Druck kann unterschiedlich stark sein, er kann von positiven und negativen sozialen Sanktionen bis zur bloßen Empfehlung eines Verhaltens durch eine Beeinflussungsquelle reichen. Für den Einzelnen sind dabei vor allem positive oder negative Sanktionen wichtig, somit ist verständlich, dass er seine Normen in erster Linie von seinen Bezugsgruppen bezieht. Festgestellt werden kann, dass der Bezugsgruppe normative Funktion zu kommt. Sie liefert und verstärkt Normen, die zu Beweggründen des individuellen Handels werden. (Kroeber-Riel, 1992, S. 503) Genormtes Verhalten muss jedoch nicht jedes mal von neuem reglementiert werden. Genormtes Verhalten substituiert den persönlichen Einfluss der Gruppe ${ }^{22}$. Die Handlung erfolgt automatisch nach den Normen der Gruppe (Thibaut und Kelly, 1959, S. 130ff, Kroeber-Riel, 1992, S. 504). Hat sich z.B. eine Bezugsgruppe einmal gegen den Kauf in einer Einkaufsstätte im Ausland ausgesprochen („Man kauft nicht im Ausland“), werden die Mitglieder dieser Gruppe ihren Einkauf nicht in einer solche Einkaufsstätte tätigen. Ein solches Verhalten würde nicht der Werteordnung der Gesellschaft, in der sich das Individuum befindet, entsprechen.

Fricke (1996) hat im Zusammenhang mit dem Konsumentenverhalten den Begriff der „Konsumentensozialisation“ geprägt. Im Rahmen dieser Sozialisation werden in der Gesellschaft vorherrschende Werte, Normen und Verhaltensmuster vermittelt. Für die Weitergabe beziehungsweise Beeinflussung sind so genannte „Sozialisationsagenten“ verantwortlich. Hierzu zählen Individuen (Mutter, Vater, Lehrer, Kind) genauso wie einfache und komplexe Sozialsysteme (Familie, Nachbarschaft, Freundeskreis, Schule, Verein, Massenkommunikationssysteme etc.). Aus der näheren Umgebung üben vor allem Familie und „Peers“ (Gruppen oder Einzelpersonen zu denen ein Individuum einen besonders engen Kontakt unterhält) einen

\footnotetext{
${ }^{22}$ Die von der Gruppe festgelegten Normen stehen über der temporären Meinung der Gruppe. Diese kann sich ändern, Normen sind langfristige Verhaltensgrößen.
} 
langfristig prägenden Einfluss aus. Der Mensch strebt nach Ajzen nach Rationalität seines Handelns (Ajzen und Fishbein, 1980,S. 5). Fishbein und Ajzen haben das Modell folgendermaßen formalisiert ( Fishbein und Ajzen,1972, S. 487ff, zitiert bei : Mayer und Van Eimeren, S.219):

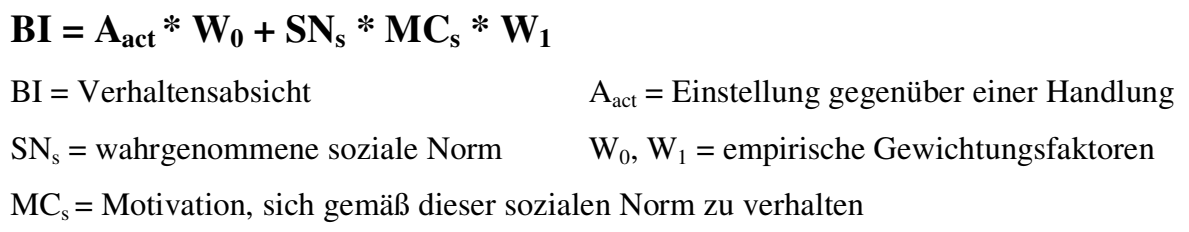

Nach Mayer und Van Eimeren wird die soziale Norm $\mathrm{SN}_{\mathrm{s}}$ durch die Frage operationalisiert, ob eine wichtige Bezugsperson zu einer konkreten Handlung rät. Die Bereitschaft dem Rat dieser Person zu folgen, wird über $\mathrm{MC}_{\mathrm{s}}$ erfasst. Auch die Gewichtungsfaktoren $\mathrm{W}_{0}$ und $\mathrm{W}_{1}$ scheinen produkt- und konsumentenunabhängig zu sein. Sie variieren in Abhängigkeit von der Art des Objektes, der jeweiligen Konsumentengruppe und der Situation, in der das Verhalten ausgeführt wird (Mayer und Van Eimeren, 1985, S. 219). Insbesondere bei für das Individuum besonders bedeutsamen Produkten bzw. Einkäufen brachte das Modell von Fishbein und Ajzen gute Vorhersageergebnisse ( Mayer und Van Eimeren, 1985, S. 220).

\section{,Die wahrgenommene Selbstrelevanz}

Nach Meinung von Ajzen (1991) erscheint es sinnvoll, einen weiteren Einflussfaktor zu berücksichtigen, der die wahrgenommene moralische Verpflichtung des Einzelnen widerspiegelt. Demnach wird Verhalten nicht nur von der Meinung anderer beeinflusst, sondern auch davon, in wie weit das betreffende Verhalten dem Ausführenden als moralisch und ethisch vertretbar beziehungsweise aus dieser Sicht erforderlich erscheint. Es wird zudem dafür plädiert, das Selbstbild des Konsumenten bei der Einkaufsentscheidung zu berücksichtigen (Shepherd und Sparks, 1994). Entscheidend ist hierbei der Grad der Ich-Beteiligung des Konsumenten, sein Involvement sich für bestimmte Sachverhalte oder Aufgaben zu interessieren oder einzusetzen (Meffert, 2000, S. 112). Die wahrgenommene Selbstrelevanz ist hierbei die wertorientierte Konzeptualisierung des Involvementkonstruktes. Darunter sollen die Werte verstanden werden, deren Realisierung ein Individuum durch den Kauf und Konsum einer Leistung anstrebt.

Die wahrgenommene Selbstrelevanz (WRS) wird verstanden als eine wertorientierte Konzeptualisierung des Involvementkonstrukts deren Realisierung ein Individuum durch den Kauf anstrebt (Houston und Walker, 1996, S. 223). Houston und Walker haben in ihren Studien zur Erfassung der wahrgenommenen Selbstrelevanz individuelle „,means-end" Leitern herangezo- 
gen. Dabei gehen die Autoren von folgendem Zusammenhang aus: Je mehr Assoziationsketten auf der Werteebene enden, desto eher empfindet der Konsument den Kauf als selbstrelevant. Würde diesem Gedankengebäude gefolgt, wäre mit jedem Probanden eine „means-end“ Analyse durchzuführen. Eine solche Vorgehensweise wäre in einem Massenmarkt allerdings nicht sinnvoll. Braunstein (2000, S. 219) spricht auf der Suche nach alternativen Erhebungsmethoden davon, das die „Anzahl der Assoziationsketten, die in einer Werthaltung münden", nichts anderes angeben als das Ausmaß, „,in dem ein Kunde eine Leistung mit diesem Wert verknüpft." Es empfiehlt sich deshalb, die von jedem Individuum wahrgenommene Wichtigkeit der Werthaltung zu überprüfen. Auch Odekerken-Schröder (1999 S. 46ff), die in diesem Zusammenhang von buyer relationship proneness spricht, sieht hierin den Schlüssel zur Ermittlung des Faktorwertes, der hohen Einfluss auf Kundentreue und Kundenbindung hat. Einfluss auf die wahrgenommene Selbstrelevanz haben sowohl die wahrgenommene Verhaltenskontrolle als auch die persönliche Norm. Je höher wiederum die wahrgenommene Selbstrelevanz bei einem Individuum ist, umso höher ist auch das Involvement des Individuums und sein Entscheidungsverhalten (Kroeber-Riel und Weinberg, 2003, S. 372, 373). Die beiden Forscher führen Involvement auf personen-, situations- und stimulusspezifische Faktoren zurück (Kroeber-Riel und Weinberg, 2003, S. 372).

\section{,Persönliche Norm'}

Das Verhalten eines Individuums in einer besonderen Situation wie z.B. der Kaufsituation wird dennoch auch von der persönlichen Norm eines Individuums beeinflusst. Die persönliche Norm ist dabei als ideale Verhaltensabsicht zu verstehen, die das Individuum realisieren würde, sofern keine Störreaktionen erkennbar sind. Cialdini, Kallgren und Reno verstehen das Konstrukt als Gesamtheit der individuellen Werte, die für das Individuum in einem bestimmten Bereich wichtig sind (1991, S. 201ff.). Die persönliche Norm ist somit eine moderierende Variable, die alle Wirkungsbeziehungen der Theorie des geplanten Verhaltens beeinflusst. Peter, Olson und Grunert vertreten dabei die Auffassung, dass das Ausmaß bis zu welchem Punkt die persönliche Norm zur Verhaltensprädikation beiträgt, von der wahrgenommenen Selbstrelevanz der Verhaltenssituation abhängt (Peter, Olson und Grunert, 1999, S. 78). Die Autoren kommen dabei auch zu dem Ergebnis, dass eine Person, sofern sie ihr Verhalten als selbstrelevant erachtet, eher bereit ist, das Verhalten auch zu realisieren.

\section{,Wahrgenommene Verhaltenskontrolle،}

Die wahrgenommene Verhaltenskontrolle des Konsumenten beeinflusst nicht nur seine Kundentreue, sondern auch die Kundenbindung selbst. Im Mittelpunkt dieser Überlegungen ste- 
hen die internen und externen Kontrollerwartungen des Individuums. Zu den Internen zählen nach Braunstein (2001, S. 173) Bequemlichkeit für den Kunden, Gewohnheiten, seine Kenntnis sowie seine finanziellen Möglichkeiten, die einem Einkauf in der gleichen Einkaufsstätte im benachbarten Ausland entgegenstehen. Die externen Kontrollerwartungen sind für den Konsumenten nicht beeinflussbare Kriterien, mit deren Hilfe es gelingt, die Realisierbarkeit des Wiederkaufs oder einer Weiterempfehlung abzuschätzen. Beispiele könnten ein Arbeitsplatz im benachbarten Ausland und die bequeme Möglichkeit sein, ein bestimmtes Produkt in einer Einkaufsstätte im benachbartem Ausland zu kaufen. Ferner kann ein Beispiel die Verfügbarkeit einer bestimmten Marke im benachbartem Ausland in einer konkreten Einkaufsstätte sein. Die wahrgenommene Verhaltenskontrolle setzt sich somit zusammen aus interner Kontrollerwartung, Bewertung der internen Kontrollerwartungen durch den Konsumenten, externe Kontrollerwartungen und Bewertung der externen Kontrollerwartungen durch den Konsumenten.

\section{,Bindungsvorsatz 6}

Neben dem, was der Konsument möchte, und dem, was andere von ihm erwarten, darf auch die Umsetzbarkeit eines Vorhabens nicht außer Acht bleiben. Die Umsetzbarkeit des Vorhabens wird durch den Bindungsvorsatz des Konsumenten ausgedrückt. Dieser wird beeinflusst durch den Willen eine konkrete Handlung auszuführen, als auch durch den Verhaltensplan (die Vorstellung des Konsumenten über den Handlungsablauf) über den ein Konsument verfügt. Die Lebensbedingungen beschränken die Wahlfreiheit der Verbraucher in materieller sowie immaterieller Weise. Ein Produkt, das der Verbraucher besitzen möchte, dessen Erwerb aber ausgesprochen schwierig erscheint, wird nicht gekauft. Ajzen (1991) führte hierfür den Begriff der „perceive" ein (Ajzen, 1991, S. 184). Ajzen spricht in diesem Zusammenhang auch von der wahrgenommenen Verhaltenskontrolle und schlägt eine direkte, den Gesamteindruck messende als auch eine indirekte auf den Erwartungs-Wert-Ansatz zurückzuführende Messung vor (Ajzen, 1991, 196ff.). Aus diesem Grund wird auf eine seperate Messung des Kundenbindungsvorsatzes in dieser Arbeit verzichtet. ${ }^{23}$

\footnotetext{
${ }^{23}$ Dieses Konzept geht auf Bandura (1982, S.122) zurück. Dieses Konstrukt umfasst bei Bandura die Möglichkeit des Individuums zu beurteilen, wie einfach es ist Handlungen durchführen, sowie ebenfalls die Möglichkeit, ein Urteil zu fällen, ob die Anstrengungen die das Individuum für diese Handlungen auf sich nehmen muss, der Situation angemessen sind. Bandura zeigt, dass das Vertrauen in die eigenen Fähigkeiten, die Vorbereitung der Handlungen, die Anstrengungen während der Realisation, die kognitiven Muster und die emotionalen Reaktionen das Verhalten beeinflussen. Ajzen berücksichtigt die Einsichten Banduras bei der Beschreibung seines Konzeptes der wahrgenommenen Verhaltenskontrolle.
} 


\section{,Weiterentwicklung der Theorie des geplanten Verhaltens'}

Insbesondere Fazio (Fazio und Zanna, 1978, 1981, Fazio, 1990) hat Kritik an dem Modell von Ajzen zur wahrgenommenen Verhaltenskontrolle geübt und daraufhin einen Ansatz entwickelt, der die Wirkungen von Moderatorvariablen auf einen gemeinsamen Nenner zurückführt: die Abrufbarkeit der Einstellung im Gedächtnis. Fazio setzt voraus, dass Einstellungen Verhalten beeinflussen können. Das Verhalten wird auch hier von Einstellungen und Normen beeinflusst. Einstellungen wirken nach Fazio auf unsere Wahrnehmung, sie färben unsere Wahrnehmung und wirken somit indirekt auf unser Verhalten ein. Der Autor definiert „Wahrnehmen" im weiteren Sinne als „Erkennen und Interpretieren". Eine aktivierte Einstellung bewirkt eine konsistente Wahrnehmung des Einstellungsobjekt und ermöglicht so Handlungen. Der Autor definiert Einstellung als Assoziation zwischen einem Erkenntnisobjekt und seiner Bewertung. Dies ergibt nach Fazio zusammen einen Knoten im Gedächtnis. ${ }^{24}$ Die Zugänglichkeit hängt demnach von der Stärke des Bewertungsknotens und der Assoziation ab (Messung durch Reaktionszeiten). Ist eine Einstellung gerade aktiviert, kann sie indirekt auf das Verhalten einwirken. Je häufiger eine Einstellung aktiviert wird, desto zugänglicher ist sie. Je zugänglicher, desto eher wird Einstellung spontan aktiviert und desto stärker ist ihre Beziehung zum Verhalten (Fazio, 1990, S. 75 ff). Doch hat dieser Ansatz auch zu Kritik geführt. Der zentrale Kritikpunkt richtet sich auf den eingeschränkten Geltungsbereich. Denn oft verlangt die Realisierung eines konkreten Handelns spezielle Fähigkeiten und Ressourcen, oder das Vorhandensein von Gelegenheiten (Fishbein und Ajzen, 1975, S. 371). Die Kontrolle seines Handelns ist dem Individuum dabei nur bedingt möglich. Fishbein und Ajzen argumentieren, dass automatisiertes Handeln ohne kognitiven Aufwand abläuft. Dies bedeutet z.B. für die grenzüberschreitende Kundenbindung, dass es einmal Kaufakte des Konsumenten gibt, die nicht hinterfragt werden (,Wenn ich in den Niederlanden bin, kaufe ich dort Kaffee und Butter ein, weil es preiswerter ist"“) und es auch auf der anderen Seite Produkte und Dienstleistungen gibt, bei dessen Kauf die Grenze eine Hürde darstellt (Elektronik, Reisen, Auto). Diese Phänomene des automatisierten Handelns werden in der Theorie des geplanten Verhaltens deshalb nicht berücksichtigt, da sie für die Sozialwissenschaften nur geringe Relevanz haben.

Die Kritikpunkte wurden in Ajzen“s „Theorie des geplanten Verhaltens “ berücksichtigt. Hier nahm er eine neue Variable, „die subjektive Kontrollierbarkeit des Verhaltens“, auf. Die sub-

\footnotetext{
${ }^{24}$ Einstellungen kann man als Knoten im semantischen Netzwerk verstehen, die durch Relationen mit anderen Knoten verbunden sind.
} 
jektive Kontrollierbarkeit entspricht den früheren Erfahrungen mit dem Verhalten und den erwarteten Behinderungen; sie ist abhängig von inneren Faktoren (Fähigkeit, Willen...) und äußeren Faktoren (Gelegenheit, Mittel, Abhängigkeit...). Die Einstellung des Individuums wird in diesem erweiterten Modell von Fishbein nicht zu einem Objekt (wie einer konkreten Einkaufsstätte), sondern zu einer Handlung, wie dem Kauf bzw. Wiederkauf in einer Einkaufsstätte gemessen. Bedeutsam ist diese Unterscheidung insbesondere deshalb, weil das Individuum durchaus eine positive Einstellung zu einer Einkaufsstätte haben kann, gleichzeitig aber auf Grund der Konsequenzen, die mit dem Einkauf verbunden sind (Entfernung, Preis, andere Sprache) eine negative Einstellung zur Kaufhandlung besitzen kann. (Mayer und Van Eimeren, 1985, S. 219). Handlungen nehmen Individuen demnach nicht gedankenlos oder unkontrolliert vor, sondern stets kontrolliert.

\subsection{Messung der Einstellung und der Kundenloyalität zur Erklärung der Kunden- bindung}

Im Folgenden sollen Ansätze zur Messung der Einstellung und der Kundenloyalität zur Erklärung der Kundenbindung dargestellt werden. Ausgangspunkt ist dabei der Ansatz von Fishbein. Doch auch die Messung der Einstellung selbst und die Frage, welche Wahrscheinlichkeit dem Vorhandensein einer Sache beigemessen werden kann, sowie welche Auswirkungen diese auf die tatsächliche Kaufentscheidung haben, sind auch im Fishbein-Modell nicht unproblematisch (Kroeber-Riel und Weinberg, 2003, S. 202). Im Fishbein-Modell wird die Gewinnung von Eindruckswerten durch Multiplikation der beiden Rating-Werte vorgenommen. Dabei ist allerdings ein Problemfeld die Ermittlung der Wissens-Elemente. So wäre es notwendig nicht nur nach der Wahrscheinlichkeit des Vorhandenseins eines Merkmals zu fragen, sondern auch die Frage nach der subjektiv empfundenen Wahrscheinlichkeit in Bezug auf das Vorhandensein einer Ausprägung zu stellen. Dies würde allerdings zu einem extrem hohen Befragungsaufwand führen. Aus diesem Grund ist es effektiver die wahrscheinlichste Ausprägung eines Produktes oder Einkaufsstätte durch eine direkte Frage zu ermitteln um so die Einstellung zum Kauf eines Produktes, die Einstellung zu einer Marke oder Einkaufsstätte zu ermitteln (Kroeber-Riel und Weinberg, 2003, S. 202). Ein weiterer Kritikpunkt zur Messung und der Verwertbarkeit der Ergebnisse ist, dass die Einstellungsmessung davon ausgeht, dass Verhalten geplant erfolgt. Impulskäufe werden darin eingeschlossen. Das Verhalten eines Individuums kann allerdings von unterschiedlichen Faktoren wie Veränderung, den Drang nach Abwechslung und sogar Wetterwechsel beeinflusst werden. Diese Elemente können die 
Validität der Messergebnisse beeinflussen. Beim Einsatz der Einstellungsmessung ist darauf zu achten, ein angemessenes Abstraktionslevel zu verwenden. Denn auch wenn Individuen eine positive Einstellung zu Produkten haben, bedeutet das nicht, dass sie dieses Produkt auch kaufen (z.B. Kauf Ferienhaus auf Mallorca, Kauf eines Porsche, Kauf einer Rolex-Uhr usw.). Hier sind nämlich der Preis des Objektes sowie das Einkommen des Individuums für einen Kauf ausschlaggebende Kriterien. Entscheidend in der Einstellungsmessung ist somit nicht nur wie gemessen, sondern auch was gemessen wird. Wesentlich bei der Einstellungsmessung ist, dass der Grad der Spezifität zwischen Einstellung und Verhaltensabsicht übereinstimmen. Dies bedeutet, dass die durch die Einstellung angegebene und auf einen konkreten Gegenstand bezogene Haltung hierdurch für eine konkrete Handlungssituation differenziert und konkretisiert wird ( Kroeber-Riel, und Weinberg, 2003, S. 54). Auch der Zeitfaktor in der Einstellungsmessung ist zu berücksichtigen. Je größer der zeitliche Abstand zwischen Einstellungsmessung und Realisierung einer Kaufabsicht ist, desto unsicherer werden die Ergebnisse (Solomon, Bamossy und Askegaard, 2001, S. 173).

Bei allen Messansätzen zur Erklärung der Kundenloyalität steht die Erklärung des Treueverhalten des Individuums im Zentrum. Dazu werden Erklärungsansätze benötigt, warum der Konsument einer Einkaufsstätte gegenüber loyal und treu ist oder nicht. Bereits Heinemann betont, dass in der Literatur eine hohe Anzahl von Messansätzen existieret, die sich sowohl am „overt behavior“ als auch am „covert behavior“ orientieren (Heinemann, 1976, S. 32). Ursprünglich handelte es sich bei den Messansätzen um Verfahren, die zur Messung der Konzentration auf eine Marke herangezogen und nur geringfügig modifiziert wurden. Diese Verfahren konzentrieren sich auf die behavioristischen Komponenten der Geschäftstreue. Gemeinsam ist diesen Verfahren, dass sie vom beobachtbarem Verhalten des Konsumenten ausgehen und nicht die neobehavioristische Komponente berücksichtigen. Zu den direkt auf einen konkreten Kaufakt oder Kunden zuzuordnenden Kennzahlen gehören insbesondere die umsatzorientierten Kundenkennzahlen (Anteil Stammkunden am Gesamtumsatz, durchschnittliche Einkaufssumme eines Stammkunden, durchschnittliche Jahresumsatz eines Stammkunden), die gewinnorientierten Kennzahlen (durchschnittlicher Deckungsbeitrag pro Stammkunde, Anteil der Stammkunden am Deckungsbeitrag), die verhaltensorientierten Kundenkennzahlen (Einkaufsfrequenz eines Stammkunden, durchschnittliche Einkaufsmenge eines Stammkunden) sowie allgemeine Kennzahlen (Stammkundenanteil, durchschnittliche Dauer einer Kundenbeziehung und das Verhältnis zwischen gewonnenen und verlorenen Kunden). Diese Kennzahlen geben allerdings keinen Aufschluss darüber, warum ein Kunde sich der Einkaufsstätte gegenüber loyal und gebunden verhält. Damit aber auch die neobeha- 
vioristischen Komponenten der Kundenloyalität einer Messung unterworfen werden können, müssen weitere Grundannahmen getroffen werden. Dazu gehören, dass sich die Zufriedenheit mit dem Kauf aus verschiedenen Teilzufriedenheiten zusammensetzt. Ferner gehört die Teilzufriedenheit mit der Qualität der Region, in der die Einkaufsstätte liegt, dazu. Ebenso gehört dazu die Teilzufriedenheit des Konsumenten mit der Qualität der Attribute der Einkaufstätte, sowie die Teilzufriedenheit mit der Qualität, die der Nutzen eines Kaufs in der Einkaufsstätte für den Konsumenten hat. Aus den Teilzufriedenheiten ergibt sich eine Gesamtzufriedenheit der Kunden. Kundenzufriedenheit ist wiederum Determinante und Prozess der Kundenloyalität zugleich (Oliver, 1993, S. 418-430). Die Frage der Einstellung und warum der Konsument zu einem Treueurteil kommt, wird in diesen Ansätzen allerdings nicht berücksichtigt. Da die Einstellung ein nicht direkt beobachtbares Konstrukt darstellt, lassen sich Einstellungen oftmals nur durch Einzelindikatoren operationalisieren. Ein Ansatz, der sich aus einer theoretischen und einer empirischen Ebene zusammensetzt, lässt sich auf Friedrichs (1973, S.164) zurückführen und ist von Kroeber-Riel und Weinberg (2003, S. 190) weiter operationalisiert worden. Für eine Einkaufsstätte, die auch oder gerade ausländische Kunden aus dem Grenzgebiet ansprechen möchte, kann die Operationalisierung der Einstellung zu dieser Einkaufsstätte folgendermaßen dargestellt werden:

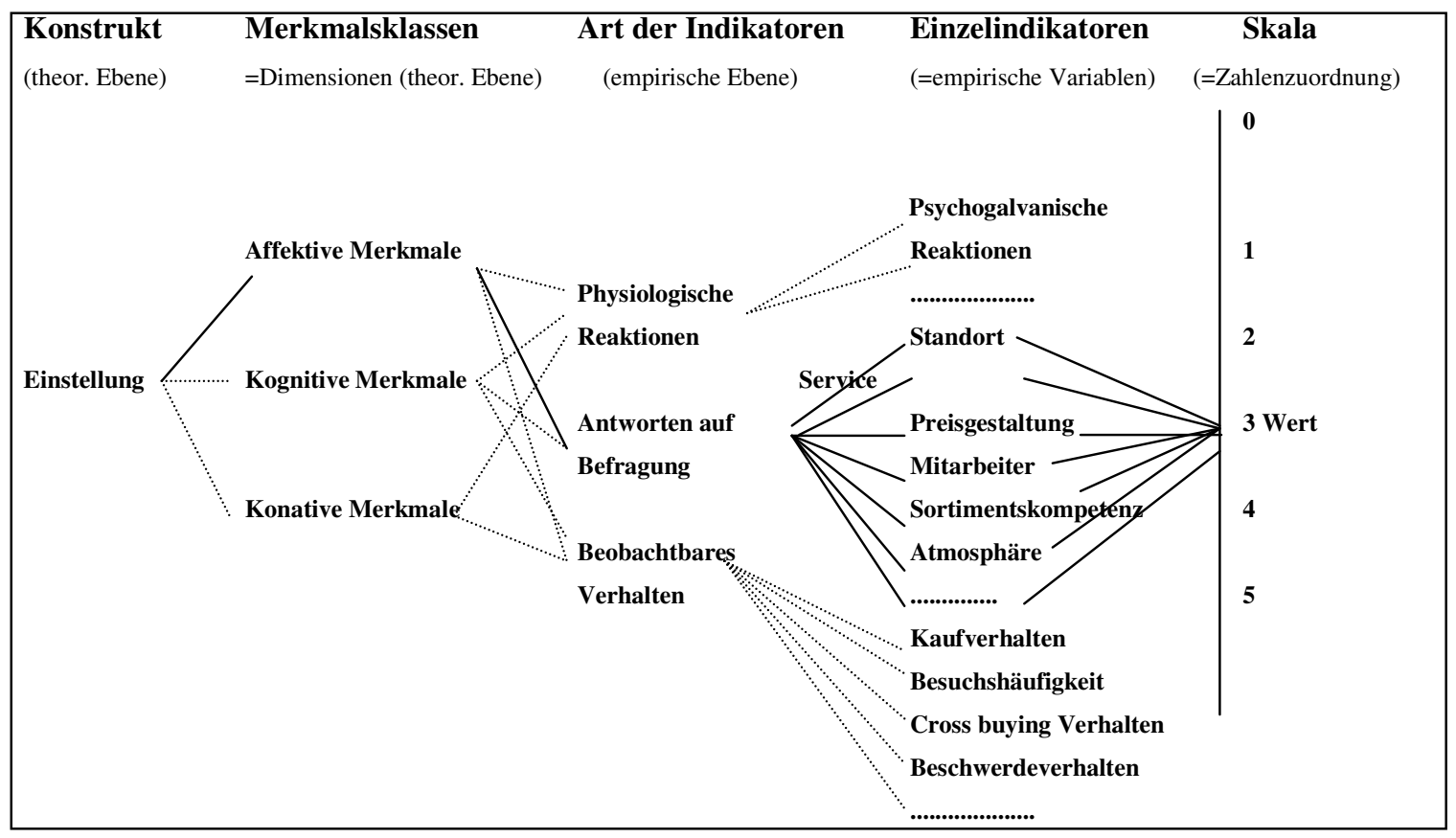

Abb.4.1: $\quad$ Operationalisierung der Einstellung zu einer Einkaufsstätte ( in Anlehnung an Jungwirth, 1997, S. 107) 
Die Kriterien, an denen dann die Einstellung der Konsumenten gegenüber einer Einkaufsstätte gemessen werden kann, lassen sich dann von den affektiven, kognitiven und konativen Merkmalen ableiten. Durch die affektive Komponente wird die gefühlsmäßige Einschätzung des Objektes durch den Konsumenten gemessen. Indikatoren wie die Einschätzung von Kundenklub, Kundenzeitung, Couponing durch den Konsumenten werden abgefragt. Es geht hierbei somit um die gefühlsmäßige emotionale Bewertung der Einkaufsstätte. Durch die kognitive Komponente wird das mit der Einstellung verbundene subjektive Wissen des Konsumenten über die Einkaufsstätte abgefragt, wogegen die konative Komponente sich auf die Handlungstendenz wie Kaufbereitschaft, Verhaltensabsicht und beobachtbares Kaufverhalten richtet (Behrens, 1991, S. 113).

Zur Messung der Kundenzufriedenheit sind verschiedene Analyseverfahren entwickelt worden. Dazu gehören die bereits oben erwähnten objektiven Verfahren. Die subjektiven Verfahren sind dagegen auf die individuelle Wahrnehmung physischer und psychischer Sachverhalte ausgerichtet. Die Messung der Kundenzufriedenheit nach subjektiven Kriterien lässt sich nach Töpfer in merkmals- oder ereignisorientierte Verfahren unterteilen. Die Unterscheidung wird hinsichtlich der Art des Untersuchungsobjektes getroffen. Nach Töpfer wird der Customer Satisfaction Index (CSI) aus der Zusammenfassung der ereignisorientierten und den mehrdimensionalen merkmalsorientierten Verfahren gebildet (Töpfer, 1999, S.301). Grundansatz der ereignisorientierten Messverfahren ist die Messung der Kundenzufriedenheit anhand von realen Erlebnissen bei der Inanspruchnahme der Dienstleistung oder des Produktes. Stauss und Henschel (1995, S. 117) bezeichnen diese Kundenerlebnisse auch als „Augenblicke der Wahrheit" und subsummieren darunter alle personal- und nicht personalbezogenen gewöhnlichen bzw. außergewöhnlichen Kontakte des Kunden mit Einkaufsstätten bzw. Unternehmen insgesamt. Dem merkmalsorientierten Ansatz liegt dagegen die Annahme zugrunde, dass die Individuen die Servicequalität als das Resultat einer subjektiven Bewertung der Einzelmerkmale der dargebotenen Leistung erfassen und die Servicequalität einen starken Einfluss auf die Gesamtzufriedenheit des Konsumenten besitzt (Stauss und Henschel, 1995, S. 116). Die Messung der Kundenzufriedenheit wird von den Autoren als eine notwendige Voraussetzung angesehen, um die Kundenbindung zu messen. Kundenbindung wird danach durch folgenden Zusammenhang gemessen: 


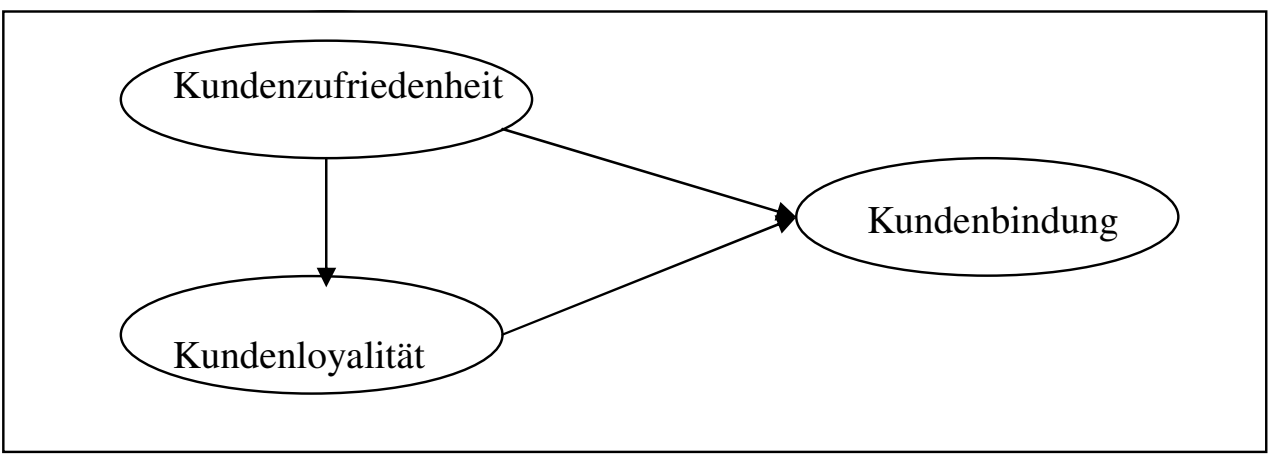

\section{Abb. 4.2: Zusammenhang der Messung von Kundenzufriedenheit, Kundenloyalität und Kundenbindung (vgl. Töpfer, 1999, S. 341)}

Auch bei der Berechnung des Kundenbindungsindexes werden quantitative Messansätze eingesetzt wie der Kundenbindungsquotient, die Netto-Kundenveränderungsrate, die Kundenabwanderungsrate, die Bruttokundenzuwanderungsrate und die Neukundenrate. Bei dieser Vorgehensweise steht wiederum der behavioristische Ansatz zentral. Diese Lücke versucht Töpfer zu schließen, indem er den Kundenbindungsindex aus zwei Perspektiven heraus misst. Zum einen aus der Sicht des Kunden, basierend auf seinen Anforderungen, zum anderen aus Unternehmenssicht. Ausgangspunkt des Autors ist die Selbsteinschätzung des Unternehmens hinsichtlich der Erfüllung der Kundenanforderungen in Bezug zum Value Marketing (Töpfer, 1999, S. 348 ff.). Töpfer`s These ist, dass von den Erfahrungen von heute auf die zukünftigen Anforderungen geschlossen werden kann. Aus diesem Grund ist der Ausgangspunkt für Töpfers Messansatz der Kundenbindung auch die Kundenzufriedenheit und das Beschwerdemanagement. Töpfer differenziert ferner zwischen der operativen Kundenbindung - die aus der Lösung von akuten Kundenproblemen resultiert - und der strategischen Kundenbindung, die die Elemente der Kundenloyalität beinhaltet (Töpfer, 1999, S. 345). Von vorhandener Kundenbindung kann nach Töpfer ausgegangen werden, wenn hohe Kundenzufriedenheit und ein hohes Kundenproblemlösungsbewusstsein im Unternehmen nachweislich vorhanden sind.

In der Literatur finden sich eine Vielzahl von Loyalitätsmaßen. Besondere Beachtung haben in der deutschen und der amerikanischen Literatur insbesondere die Messansätze von Fornell et al., Narayandas und Peter gefunden. Kundenloyalität wird in der Literatur (Anderson und Sullivan, 1993; Peter, 1999; Narayandes, 1996) durch folgende Loyalitätsmaße gekennzeichnet, die allerdings keine Aussagen zur Einstellung des Konsumenten gegenüber einer Einkaufsstätte machen:

- zukünftiger Wiederkauf 
- Wiederbeschaffung nach Diebstahl

- Bereitschaft der Mund-zu-Mund-Propaganda

- Weiterempfehlungsabsicht

- Bereitschaft einen Premiumpreis zu bezahlen

- Cross buying Bereitschaft

- Widerstand gegen Kritik an Kauf durch Dritte

- Resistenz gegen Konkurrenz.

Fornell führt die Messung der Kundenloyalität auf einer 10er Skala durch. Dabei wird die Kundenloyalität beurteilt nach der Wiederkaufwahrscheinlichkeit, der maximalen Preistoleranz sowie nach der Preisgrenze, ab der das Individuum bereit ist, die Wiederkaufhäufigkeit zu erhöhen. Parasuraman et. al. (Parasuraman, Zeithaml und Berry, 1994, S. 25-27) legen dagegen die Wechselwahrscheinlichkeit nach Erhöhung der Ausgaben sowie die Reaktion der Individuen auf interne und externe Probleme mit dem Anbieter ihren Messansätzen zugrunde. Doch auf Grund nur mäßiger Reliabilität der Loyalitätsskalen forderten die Forscher selbst, weitere Forschungsbemühungen zu starten. Peter entwickelte daraufhin eine sehr komplexe Loyalitätsskala und testete diese in der Automobilindustrie. Eine Überprüfung der Messskala in der Pharmaindustrie erbrachte dagegen ernüchternde Ergebnisse. Erwiesen sich die Indikatoren Wiederkaufabsicht, Wiederkaufabsicht bei Diebstahl, Wiederkaufwahrscheinlichkeit, Weiterempfehlungsabsicht und Cross-buying-Potenzial als aussagefähig, waren im Pharmasektor nur noch die Indikatoren Wiederkaufabsicht, Weiterempfehlungshäufigkeit, Erhöhung des Einkaufsvolumens und Cross-buying-Potenzial relevant. Auch die Studien von Odekerken-Schröder zeigen zwar auf, wie buyer relationship proneness entsteht, allerdings lassen auch hier die Indikatoren keinen optimalen Messansatz der Kundenloyalität zu. Auch Krafft kommt in seiner Analyse der Skalen zur Erfassung der Loyalität zu diesem Ergebnis (Krafft, 1999, S. 522 zitiert bei Braunstein, 2001, S. 200). Boulding et al. schlagen in diesem Zusammenhang als Messgröße eine Zwei-Items-Skala vor, die aus den Konstrukten der Wiederkauf- und der Weiterempfehlungsabsicht besteht (Boulding, Kalra, Staelin und Zeithaml, 1993, S. 20ff). Dabei kommen allerdings Zweifel auf, ob sich eine Konzentration auf zwei Items gegenüber einer umfassenderen Itemskala als vorteilhafter erweist, da auch zur Messung der Kundenbindung die Items Cross-buying und Premium-Preis herangezogen werden. Aus diesem Grund kann bei Reduzierung der Items bei der Messung der Kundenloyalität die Aussagefähigkeit der Ergebnisse leiden. Ferner bedarf es eines zeitlichen Bezuges, da ansonsten die Einstellungs- und Verhaltensintentionen der Individuen keine Berücksichtigung finden. Ferner würde dann auch die Verbindung zum Kundenbindungskonstrukt fehlen, denn 
dieses umfasst ebenfalls das overte Wiederkauf und Weiterempfehlungsverhalten innerhalb eines konkreten Zeitraums (Braunstein, 2001, S. 201).

Die Item-Skala ist somit abhängig davon, was gemessen werden soll. Hier stehen die Handlung, das Ziel und die Zeit der Messung zentral. Insgesamt kann allerdings festgehalten werden, dass in der Literatur bislang kein optimaler Messansatz der Kundenloyalität existiert. Übereinstimmung existiert lediglich in der Aussage, dass die Wiederkauf- und die Weiterempfehlungsabsicht sich als Messgrößen der Kundenloyalität empfehlen.

\subsection{Entwicklungsstand der Einstellungsforschung}

Die Annahme Fishbeins und Ajzens, dass das Individuum nur für die Handlungen eine Intention bildet, deren Realisation im Rahmen der Möglichkeiten und Fähigkeiten der Individuen liegen und dadurch kognitiv kontrollierbar sind, ist auf starke Kritik gestoßen. Aus diesem Grund konzentrierte Ajzen seine weiteren Forschungsaktivitäten auf die IntentionsVerhaltens-Beziehung. Diese Größen sind in der Handlungsforschung zu finden. Auf diesem Forschungsgebiet werden die Prozesse analysiert, die für die Umsetzung der Intention in eine Handlung verantwortlich sind (Kuhl, 1991). Historisch wird bei Ach, Michotte, Lewin und Prüm der Wille als abschließender Vorgang der Intentionsbildung und Mittler zwischen Handlungsintendenz und Handlungsausführung im Zentrum der Überlegungen gestellt. Aus diesem Grund wird dieser Forschungsansatz als Willenstheorie bezeichnet. Nach Heckhausen (Heckhausen, 1983, S. 189) konzentrieren sich aktuelle Forschungsbemühungen auf die Initiierung, Ausführung, Aufrechterhaltung und Kontrolle der Handlung. Die Handlungstheorie löst den Terminus Willenstheorie ab. Darauf aufbauend ist von Ajzen sowie Ajzen und Madden die Theorie des geplanten Verhaltens entwickelt worden. Dabei unterliegt eine Handlung nur dann einer vollständigen willentlichen Kontrolle, wenn allein der Handelnde entscheidet, ob er das Verhalten durchführen möchte oder nicht ( Ajzen und Madden, 1986, S. 455). Im Ursprung entspricht das Modell des geplanten Verhaltens der Vorstellung, dass die Intention die Wirkung der wahrgenommenen Verhaltenskontrolle auf das Verhalten vollständig erklärt und die alleinige unmittelbare Determinante des Verhaltens repräsentiert (Ajzen und Madden, 1986, S. 458). Allerdings beeinflusst Gewohnheit in einem hohen Maße das Verhalten des Individuums. Werden aber von einem Individuum Käufe gewohnheitsmäßig durchgeführt, dann können auch Käufe durchgeführt werden, die eigentlich nicht beabsichtigt werden und das Individuum verfügt nicht über die willentliche Kontrolle seines Handelns. 
Auf Grund dieses Rückschlusses erscheint eine Erweiterung der Theorie des geplanten Verhaltens überflüssig. In Forschungsansätzen (Bamberg, 1995; Ouellett und Wood, 1998) wird darauf verwiesen, dass Gewohnheit über die Häufigkeit mit der das Individuum eine Tätigkeit ausführt, gemessen und dennoch nur als erklärende Variable im Rahmen der Theorie dieses geplanten Verhaltens konzeptualisiert wird, wenn die Unabhängigkeit dieser Größe auf das Verhalten nachgewiesen ist.

\section{,Der Aspekt der Selbstidentität in der Einstellungsforschung ${ }^{6}$}

In den Ansätzen von Charng, Piliavin und Callero (1988) wird der Einfluss der Selbstidentität verstärkt betrachtet. Nach Sparks und Guthrie (1998, S. 1393-1410) handelt es sich bei der Selbstidentität um das von einem Individuum wahrgenommene Selbstkonzept und umfasst den Teil des Selbst, der in einer spezifischen Handlungssituation relevant ist. Die Vorstellung, dass das Selbst aus einer Vielzahl von situationsspezifischen Selbstidentitäten besteht, ist in allen Forschungsansätzen unumstritten. Forschungsstudien zur Überprüfung der Relevanz der Selbstidentität auf das geplante Verhalten eines Individuums sind in unterschiedlichen Verhaltensbereichen wie Ökologie, Sport oder Diätverhalten durchgeführt worden. Allerdings gelangen die Forscher dabei zu dem Ergebnis, dass die Wirkung der Selbstidentität auf das tatsächliche Verhalten sehr gering ist, da sich die Selbstidentität unter dem Konstrukt der persönlichen Norm subsumieren läßt (Sparks, Shepard, Wieringa und Zimmermann, 1995; Conner und Armitage, 1998; Theodorakis, 1994). Herkner (1991) prägte den Begriff Selbstüberwachung als eine Komponente, die neben der Selbstidentität eine relevante Komponente für das tatsächliche Verhalten des Individuums spielen kann. In der englischsprachigen Literatur wird hierfür der Begriff des ,self-monitoring" gebraucht. Nach Snyder (Snyder, 1974, S. 526ff.; Snyder und Swann, 1976, S. 1034ff) wird der moderierende Effekt der Selbstbeobachtung - in der die Person sich und ihre Wirkung auf andere beobachtet - auf die Einstellungs-Verhaltens-Beziehung bestätigt. Dabei ist zu differenzieren zwischen Individuen, die einer hohen Selbstbeobachtung unterliegen, und Individuen mit einer niedrigen Selbstbeobachtung. Personen mit einer niedrigen Selbstbeobachtung verhalten sich eher konsistent zu ihren Einstellungen. Diese Personen realisieren ihre Verhaltensabsicht weniger stark als Personen mit hoher Selbstbeobachtung (Braunstein, 2001, S. 145).

Je stärker ein Individuum von dem Bedürfnis geprägt ist, sich zu einer Sache oder Zustand hingezogen zu fühlen, desto höher ist das Involvement des Individuums, das als inneres Engagement beschrieben werden kann, mit dem sich ein Individuum einer Aktivität zuwendet (Kroeber-Riel und Weinberg, 2003, S. 370ff.). Die wahrgenommene Selbstrelevanz erscheint 
somit als sinnvolle Erweiterung der Theorie des geplanten Verhaltens. Nach Braunstein eignet sich die Theorie des geplanten Verhaltens aber nicht für alle Verhaltensweisen als Erklärungsansatz. Als Beispiel führt die Autorin das Verhalten eines gelangweilten Fernsehzuschauers an, der sich trotz eines schlechten Fernsehprogramms nicht entschließen kann, das Fernsehen auszuschalten, um zum Beispiel ein Buch zu lesen (Braunstein, 2001, S. 152). In Bezug zu den Einkaufsstätten bedeutet dies, dass unzufriedene Konsumenten eine Einkaufsstätte auch deshalb nicht wechseln, weil sie auf Grund fehlender Verkehrsmittel, Bequemlichkeit oder körperlicher Gebrechen gar nicht in der Lage sind im Nachbarland einzukaufen und deshalb ihrer Einkaufsstätte vor Ort verbunden bleiben. Um dieses Verhalten zu erläutern, ist der Begriff der Selbststeuerung in die Diskussion einzuführen. Unter diesem Begriff versteht Kuhl alle Funktionen, die psychische Prozesse so miteinander kombinieren, dass die Umsetzung einer Absicht optimal verläuft (Kuhl, 1995, S. 2).

Ein weiterführender Ansatz um eine Intention in die Tat umzusetzen, ist der Verhaltensvorsatz. Diese Fragestellung der Verhaltenstheorie geht auf Ach zurück, der sich bereits im Jahre 1910 mit dieser Thematik auseinander setzte (Ach, 1910). Gollwitzer und Malzacker griffen diesen Ansatz im Jahr 1996 wieder auf und haben das Handlungsphasenmodell von Heckhausen, welches zwischen Intentionsbildung und Handlungsinitiierung unterscheidet, in diesen Ansatz integriert. Die Autoren verknüpfen dabei das gegenständliche und das aktuelle Moment des Willensaktes, so wie es bereits bei Ach zu finden ist, miteinander. Gollwitzer und Malzacher kommen dabei zu der Erkenntnis, dass die Zielintention und die Selbstverpflichtung eines Individuums die wesentlichen Triebfedern sind, um Schwierigkeiten zu überwinden und eine Handlung durchzuführen (Gollwitzer und Malzacher, 1996, S. 458). Nach dieser Theorie fühlt sich ein Individuum in bestimmten Situationen verpflichtet, ein vorher von ihm festgelegtes Verhalten auch wirklich durchzuführen. Der einmal gefasste Vorsatz sorgt hierbei für eine Handlungsrealisation bei Vorliegen bzw. Eintreten einer bestimmten Gelegenheit. Nach Braunstein sollte deshalb das Vorsatzkonstrukt (= Intention) mit in die Theorie des geplanten Verhaltens aufgenommen werden (Braunstein, 2001, S. 161).

Die Einstellung, die ein Individuum gegenüber einem Objekt hat, ist in hohem Maße abhängig von den vorhandenen Wertvorstellungen des Individuums und wird geprägt durch das kulturelle Umfeld. Darauf geht auch Bamberg (Bamberg, 1996, S. 50ff) ein. Individuelle Wertvorstellungen haben einen hohen Einfluss auf das reale Verhalten eines Individuums und einen hohen Effekt auf das Ursache - Wirkungsgefüge. Diese werden noch weiter verstärkt durch den Einfluss der Wertvorstellungen eines Individuums gegenüber einem bestimmten 
Objekt. Nach Schulze (2000; zitiert bei Kroeber-Riel und Weinberg, 2003, S. 114) sehen viele Sozialforscher in der zunehmenden Erlebnisorientierung einen grundlegenden Wertewandel in der heutigen Gesellschaft. Der bei Kroeber-Riel und Weinberg beschriebene Konsument lebt nicht mehr primär für die Zukunft, sondern in der Gegenwart. Hier wird auch die Individualität ausgedrückt. Diese emotional erlebte Individualität spiegelt sich in allen Lebensbereichen wider. Wertorientierungen lassen sich als Indikatoren eines übergeordneten Trends zur Verwirklichung eines unabhängigen und selbstständigen Lebensstils auffassen (2003, S.114f.). Diese Auffassung der Wertvorstellungen spiegelt sich auch im Kaufentscheidungsprozess wider. Je stärker der Konsument einen Bezug zum Produkt aufgebaut hat, desto intensiver ist sein Entscheidungsprozess (Kotler, Armstrong, Saunders und Wong, 1999, S.251). Diese Grundeinstellungen zum Produkt haben ebenfalls Einfluss auf die Vorstellung des Konsumenten, welche Ausstattung eine das Produkt anbietende Einkaufsstätte haben sollte und wie wichtig dem Konsumenten die von der Einkaufsstätte ausgehende Kompetenz sein sollte. Die Wertvorstellungen des Konsumenten haben somit einen hohen Einfluss auf die Art und Weise der durch die Einkaufsstätte vermittelten spezifischen und emotionalen Produkt und Markenerlebnissen. Da Wertvorstellungen eines Konsumenten über einen längeren Zeitraum stabil bleiben, erklärt sich auch der Zusammenhang zum „shopper relationship proneness“, das Odekerken-Schröder als die relative stabile Einstellung des Konsumenten zu einem Verkäufer hinsichtlich einer konkreten Produktkategorie definiert hat (Odekerken-Schröder, 1999, S. 42). Nach der Autorin zeigt sich diese Einstellung des Konsumenten insbesondere dadurch, dass sie über einen relativ langen Zeitraum stabil ist. Die Konsequenz dieses psychologischen Prozesses ist, dass der Konsument sich eine aktive Zusammenarbeit mit der Einkaufsstätte wünscht, sich auf eine Einkaufsstätte konzentriert und der Käufer dem Verkäufer eine hohe Kompetenz unterstellt. Insgesamt ergibt sich daraus folgende Variante der ,shopper relationship proneness“, aufbauend auf der Theorie des geplanten Verhaltens, die die Grundlage eines erweiterten Modells zur Kundenloyalität bildet: 


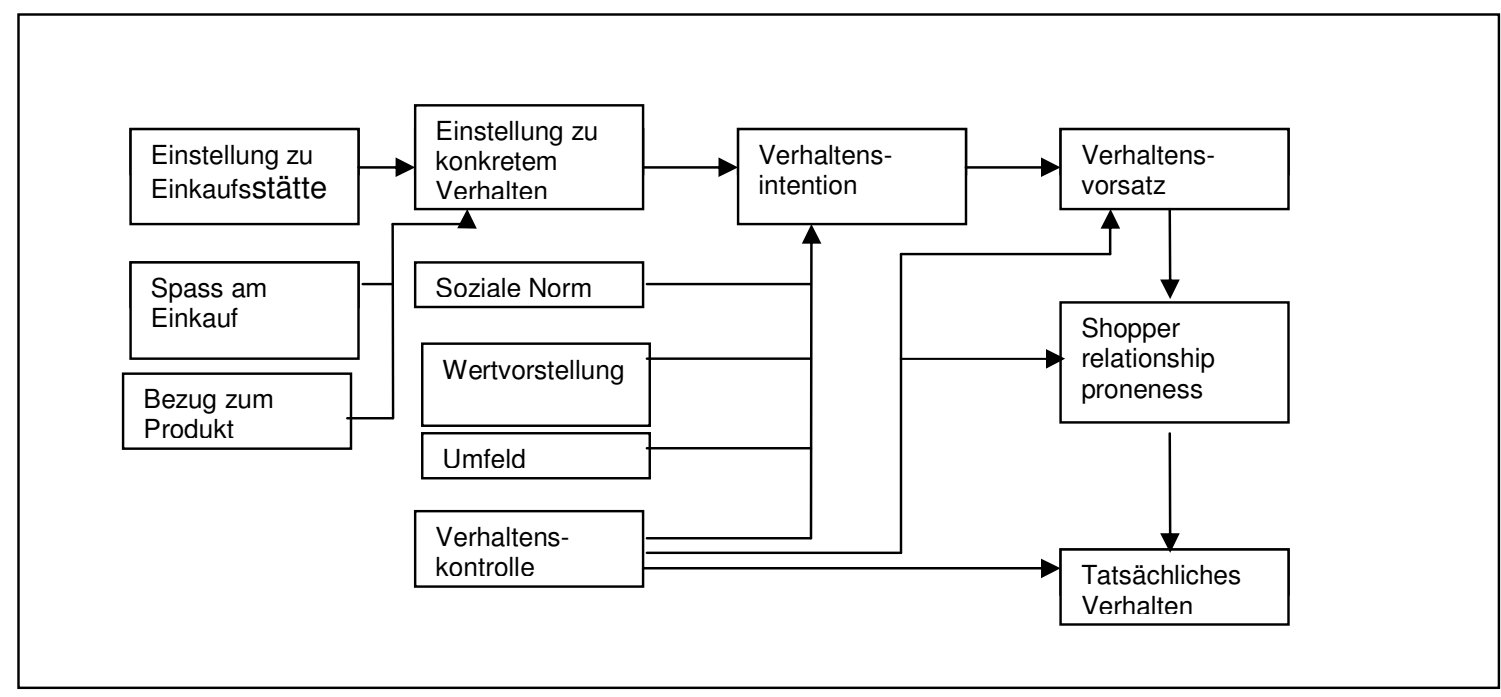

Abb. 4.3: Erweiterte Variante des Aufbaues von „shopper relationship proneness“ auf der Theorie des geplanten Verhaltens (in Anlehnung an Odekerken-Schröder, 1999, S.72)

\subsection{Eignung der Einstellungsforschung als Erklärungsansatz der Kundenbindung}

Die Einstellungsforschung wird in der Literatur als ein dauerhaftes System dreier Komponenten aufgefasst, zu denen die Aspekte Wissen, Emotion und Handeln gehören. Der Loyalitätsansatz ist ebenfalls geprägt durch die Erfahrungen des Individuums aus der Vergangenheit, seinem Involvement zu einem Produkt bzw. einer Einkaufsstätte und dem sich daraus ableitenden Handeln. Ferner werden im Rahmen der mehrdimensionalen Skalierungsverfahren neben der emotionalen Komponente auch die kognitiven Elemente angesprochen. Mit Hilfe der Einstellungsforschung wird versucht, die Struktur des Konsumentenbewusstseins zu ergründen. Insbesondere in der Loyalitätsforschung soll die Struktur des Kaufentscheidungsprozesses offen gelegt werden. Voraussetzung für diese Erklärungsstruktur ist die Existenz bestimmter psychischer Strukturen. Mentale Prozesse werden in Phasen eingeteilt, die durch Faktoren wie Einstellungen, Produkterfahrungen und Kaufabsicht charakterisiert sind. Im Gedankengang, den Trommsdorff und Schuster (1981, S. 721) einbringen, wird ein Individuum, das eine Einkaufsstätte positiv kennen gelernt hat, diese in der Zukunft eher aufsuchen als ein Individuum, das negative Erfahrungen mit einer Einkaufsstätte gemacht hat. Dies sind aber genau die Instrumente, die die Loyalitätsforschung als Erklärungsansatz zur Konsumentenloyalität heranzieht. Kroeber-Riel und Weinberg verstärken diese Meinung in der Hinsicht, indem sie im dissonanzreduzierenden Prozess eine Selbststabilisierung des Wiederholungs- 
kaufverhaltens sehen, die präferenzsteigernde Wirkung für die gewählte Einkaufsstätte und schließlich dauerhafte Einkaufsstättentreue zur Folge haben kann (Kroeber Riel, Weinberg, 2003, S.182 ff.).

Auch Kotler (1995, S. 280ff; Kotler, Armstrong, Saunders und Wong, 1999, S. 251ff) ${ }^{25}$, der vier Arten des Kaufverhaltens unterscheidet, sieht den Einfluss der Einstellungen auf den Kauf eines Produktes bzw. die Wahl einer Einkaufsstätte und somit auch auf die Kundenloyalität. Allerdings differenziert er den Einfluss der Einstellungen nach Art der Beschäftigung mit dem Kauf. Nur bei der intensiven Beschäftigung mit dem Kauf kommt es zur Bildung von Einstellungen. Je geringer die Beschäftigung mit dem Kauf ist, desto geringer der Einfluss der Einstellungen. So führt Kotler (1995, S.305) die vermeintliche Kundenloyalität beim habituellen Kaufverhalten nicht auf die Bildung von Einstellungen zurück. Häufige Wiederholungen führen hier zu einer Vertrautheit mit dem Produkt bzw. der Einkaufsstätte. Nach Kotler (1995, S. 307) können Marktführer, wie grenzüberschreitend in den Einkaufszonen der Städte präsente Filialisten, das Gewohnheitsverhalten der Konsumenten fördern. Neben der visuellen Präsenz in den Städten erfolgt dies durch eine auf die Zielgruppe abgestimmte Sortimentsstrategie sowie durch Werbung. Der Konsument muss jedoch keinesfalls von dem Produkt bzw. der Einkaufsstätte überzeugt sein. Er übernimmt passiv Informationen z.B. aus der Werbung und durchläuft nicht die übliche Abfolge: Herausbildung von Ansichten, Einstellungen und Handlungsakten. Beim komplexen Kaufverhalten (Kotler, 1995, S. 304) beschäftigt sich der Konsument persönlich intensiv mit einer Anschaffung. Hier durchläuft der Käufer einen Lernprozess, in dessen Verlauf er zunächst Ansichten, dann Einstellungen über das Produkt entwickelt und schließlich eine überlegte Kaufentscheidung trifft. Hier hat die Einstellung sehr wohl Einfluss auf den Kauf bzw. die Wahl der Einkaufsstätte und mithin auf die Kundenloyalität, die sich erst einstellt, wenn der Kunde mit dem Kauf zufrieden ist. Dies findet sich ebenso beim dissonanzmindernden Kaufverhalten. Im Prozess der intensiven Beschäftigung mit dem Kaufobjekt haben Einstellungen Einfluss auf die Kaufentscheidungen. Sie werden gebildet und sind dem Kaufakt vorgelagert (Kotler, 1995, S. 300f.).

Insbesondere die Überlegungen zur Theorie des vernünftigen und des geplanten Verhaltens liefern Erklärungsansätze wie die Entscheidungen eines Individuums sich entwickeln bzw.

\footnotetext{
${ }^{25}$ Kotler unterscheidet zwischen den Dimensionen High involvement und Low involvement und hohem Unterschied zwischen Marken und geringen Unterschied zwischen Marken. Diesen Dimensionen ordnet Kotler das komplexe Kaufverhalten, das Dissonanzreduzierende Kaufverhalten, das Abwechslung suchende Kaufverhalten und das habituelle Kaufverhalten zu.
} 
warum sich ein Individuum in konkreten Kaufsituationen auf eine bestimmte Art und Weise verhält. Eagly und Chaiken vertreten die Ansicht, dass sich die Einstellung gegenüber dem Objekt als relevante Determinante des Verhaltens identifizieren lässt (Eagly und Chaiken, 1993, S. 204). Vor allem Handelsunternehmen haben ein hohes Interesse daran, Einfluss auf das Treueverhalten der Konsumenten zu nehmen. Erst wenn die Unternehmen Kenntnis darüber besitzen, warum ein Kunde sich entschließt, einer Einkaufsstätte oder einem Handelsunternehmen gegenüber treu zu bleiben, kann von Seiten des Handels ein geeignetes Kundenbindungsmanagement aufgebaut und gepflegt werden. Die Theorie des geplanten Verhaltens - dies hat Braunstein in ihrer Studie nachgewiesen - ermöglicht es zu erklären, wie es zu solchen Entscheidungen beim Konsumenten kommt und wie sie umgesetzt werden. Die Einstellung gegenüber dem Verhalten wird nach Braunstein allerdings auch wieder stark von der Gesamtzufriedenheit mit dem Kauf geprägt (Braunstein, 2001, S. 179ff). In einer Erweiterung der Forschungsansätze von Braunstein und Odekerken-Schröder ist nun zu überprüfen, welche Rolle eine Landesgrenze innerhalb dieses Ansatzes bei direkt benachbarten Ländern spielt.

Darauf aufbauend gilt es dann, die Anwendbarkeit der erweiterten Theorie des geplanten Verhaltens zur Prognose der Kundenbindung zu untersuchen. Dazu ist es notwendig zu erkennen und zu erklären, inwieweit die einzelnen Modellkomponenten sich zur Vorhersage der Kundenbindung eignen. Aus diesem Grund bietet sich neben der Untersuchung der Faktoren Grenze und Kultur die Verknüpfung der einstellungstheoretischen Komponenten mit dem Erkenntnisstand der Kundenbindung an. Ziel ist es dann, ein Kundenbindungsmodell zu entwickeln, das eine verbesserte Vorhersage der Kundenbindung in einer grenzüberschreitenden Region zulässt.

\subsection{Zusammenfassung des Kapitels}

Insbesondere die Einstellungstheorie leistet einen Beitrag zur Prognose und Erklärung der Kundenbindung. Deutlich wird, dass um die Gesamtzufriedenheit mit dem Kauf zu erklären, das Modell von Fishbein und Ajzen nicht ausreicht. Damit die Theorie des geplanten Verhaltens zur Erklärung der Kundenbindung herangezogen werden kann muss auch sie angepasst werden. Dies trifft insbesondere für die Einstellung des Konsumenten zu einer Einkaufsstätte zu. In Anlehnung an Braunstein wird deshalb die Einstellung gegenüber dem Verhalten ersetzt durch die Gesamtzufriedenheit mit dem Kauf. Erst hierdurch ist es möglich den Einfluss 
der Handlungskontrolle und die wahrgenommene Selbstrelevanz mit in die Überlegungen einzubeziehen. Doch insbesondere in einem grenznahem Gebiet wie dem Untersuchungsgebiet ist es notwendig in diese Überlegungen die Aspekte Kultur und Grenze mit in die Überlegungen einzubeziehen. Damit stellt sich die Frage, welchen Einfluß die soziale Norm, die persönliche Norm und insbesondere die wahrgenommene Selbstrelevanz zur Erklärung des Einkaufsverhaltens haben. Diese Normen nehmen Einfluss auf das Kaufverhalten und auf die Anforderungen, die die Konsumenten an eine Einkaufsstätte stellen. Feminine Kulturen legen andere Schwerpunkte und erachten andere Dinge als wichtig als maskuline Kulturen. Hierdurch leiten sich nicht nur wie dargestellt Verhaltensweisen ab, sondern auch der Einfluss der sozialen Gruppe variiert in diesen beiden unterschiedlichen Kulturausprägungen. Die Werturteile der jeweiligen sozialen Gruppe beeinflussen somit die Bedeutung der sozialen Norm. Insbesondere in einer Region wie dem deutsch/niederländischen Grenzgebiet können Normen unterschiedlichen Einfluss auf das jeweilige Kaufverhalten nehmen. Dabei ist ebenso die Frage zu klären, ob diese Normen gleichermaßen das Kaufverhalten der deutschen und niederländischen Konsumenten beeinflussen, oder ob die Stärke des Einflusses dieser Normen unterschiedlich bei der jeweiligen Konsumentengruppe ist. Der Aspekt der Selbstidentität ist ebenso ein entscheidender Aspekt beim Einkauf im unmittelbarem Nachbarland. In diesem Aspekt kommt die Bedeutung der sozialen Norm zum Ausdruck. Letztendlich führt dies zur individuellen Wertvorstellung des Konsumenten und die individuelle Wertvorstellung hat wiederum einen starken Einfluss auf das reale Kaufverhalten. 
Kapitel 5: Spezifische Struktur des grenzüberschreitenden Kundenbindungsmodells

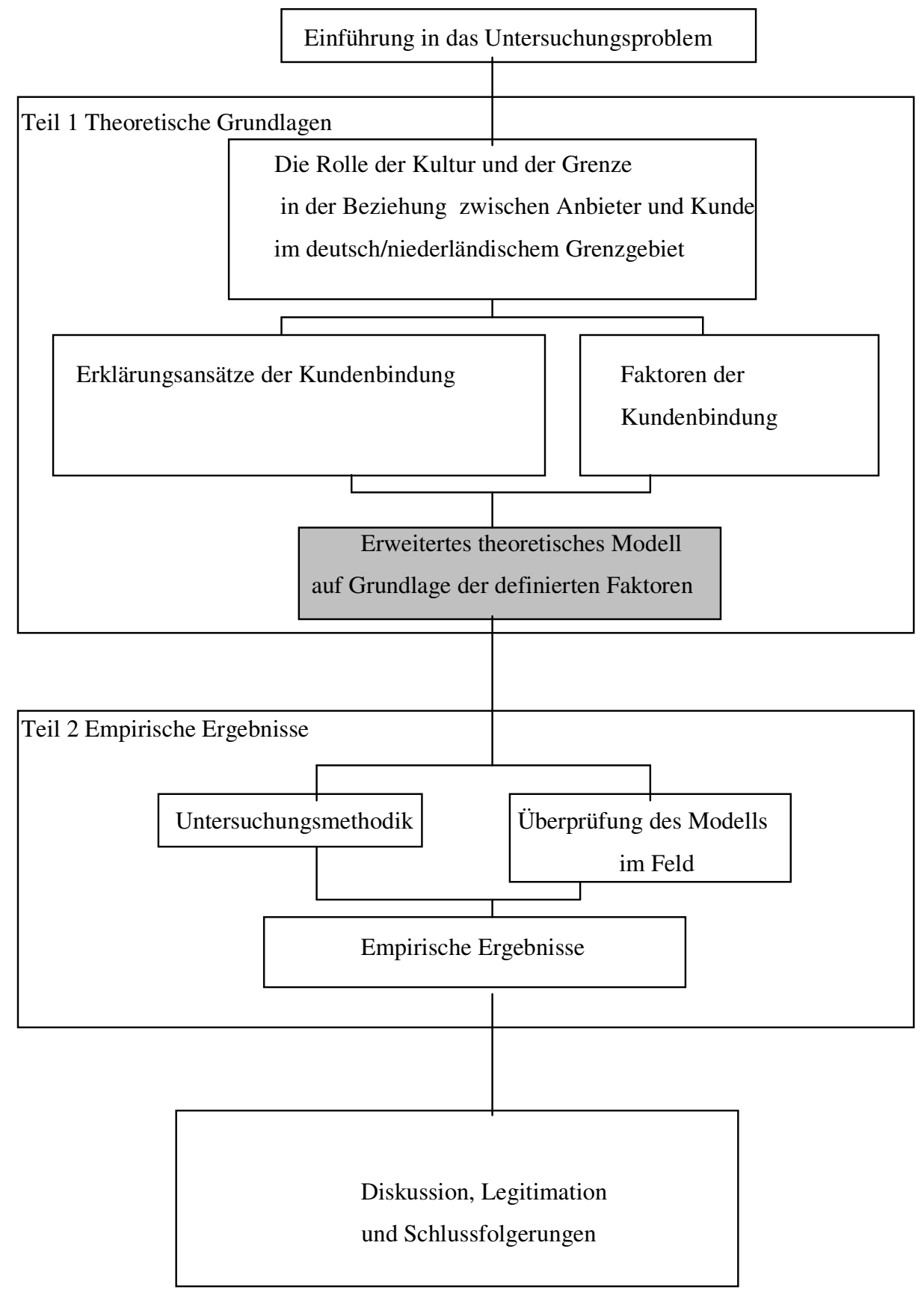




\subsection{Einleitung}

In diesem Kapitel wird das zu untersuchende Kundenbindungsmodell dargestellt und die $\mathrm{zu}$ überprüfenden Hypothesen formuliert. Anschließend werden die erwarteten Haupteffekte und Nebeneffekte des aufgestellten grenzüberschreitenden Kundenbindungsmodells erläutert. Es werden die methodischen Grundlagen zur Konzeptualisierung gelegt und die hypothetischen Konstrukte erläutert. In diesem Teil der Arbeit werden die Informationen zu den einzelnen Fragen dargestellt und den einzelnen Hypothesen zugeordnet. Zum Zweck der Überprüfung der Hypothesen werden folgende Fragen aus der Befragung weiter analysiert:

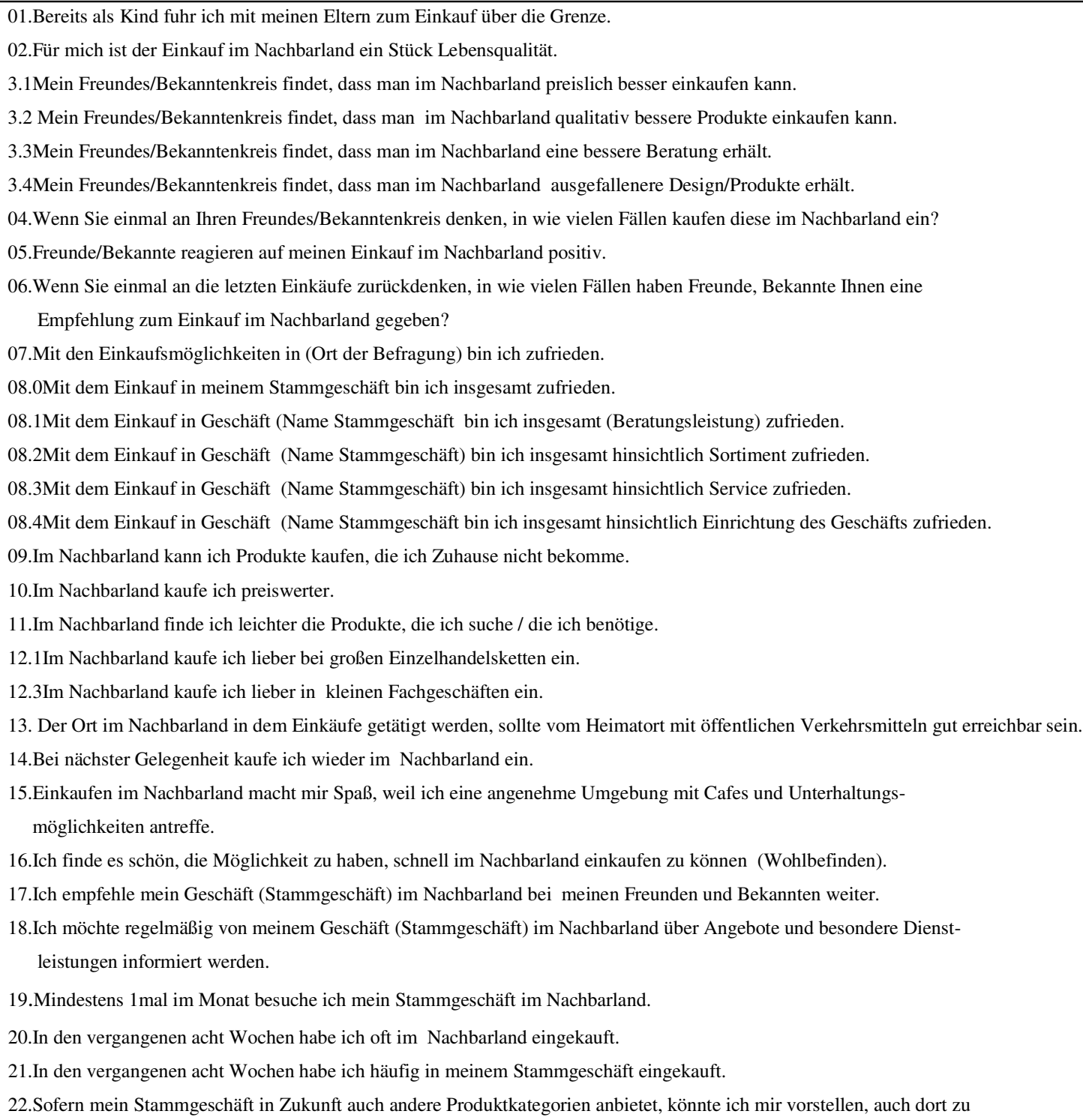




\subsection{Kundenbindungsmodell unter Berücksichtigung der Einstellungstheorie und der Auswirkung der Grenze auf Einstellungen der Konsumenten}

Im Gegensatz zu den bisher in der Literatur diskutierten Kundenbindungsmodellen liegt bei dem dieser Arbeit zu Grunde gelegten Modell, der Untersuchungsschwerpunkt auf dem Einfluss der sozialen und der persönlichen Norm sowie der wahrgenommenen Selbstrelevanz auf die grenzüberschreitende Bindung der Konsumenten an eine Einkaufsstätte. Dabei wird untersucht, was die soziale Norm bestimmt und welchen Einfluss sie auf die Gesamtzufriedenheit des Konsumenten mit dem grenzüberschreitenden Einkauf hat. Zufriedenheit mit dem Kauf bzw. mit der Einkaufsstätte ist allerdings auch abhängig von den Werten, deren Realisierung ein Individuum durch den Kauf und Konsum einer Leistung anstrebt. Dies ist die wertorientierte Konzeptualisierung des Involvementkonstruktes, die wahrgenommene Selbstrelevanz des Konsumenten. Indem der Einfluss der sozialen und persönlichen Norm sowie der wahrgenommenen Selbstrelevanz in der Kundenbindung nachgewiesen wird, kann eine sicherere Prognose zum Bindungsverhalten der Konsumenten im bevorstehenden Einkaufsprozess erstellt werden (siehe auch Anhang 3 und 4).

Die Bemühungen zur Konzeptualisierung der Kundenbindung unter Berücksichtigung der Einstellungstheorien führten zu der Einsicht, dass der Zustand des Konsumenten hinsichtlich der Handlungskontrolle seines Tuns einen Einfluss sowohl auf die Zufriedenheitsurteilsbildung als auch auf seine Kundenloyalität und Kundenbindung hat. Diese Tatsache gilt für die Zufriedenheit mit dem Kauf in einer Einkaufsstätte und für die Zufriedenheit mit der subjektiven Norm. Die Gesamteinfluß der sozialen Norm auf Einstellung und Handlung zum Einkauf im Nachbarland setzt sich aus den Teilkriterien zusammen, die die jeweilige soziale Gruppe als wesentliche Kriterien der Zugehörigkeit zu der Gruppe aufgestellt hat und die Wichtigkeit der Norm. Die Wichtigkeit der sozialen Norm wird dadurch ausgedrückt inwiefern das Verhalten (Kauf, Weiterempfehlung) des sozialen Umfeldes Einfluss auf die eigene Einstellung oder das eigene Verhalten des Konsumenten hat. Doch auch die unterschiedliche Gewichtung hinsichtlich ihrer Wichtigkeit durch das Individuum hat Einfluss auf die Gesamtzufriedenheit mit der sozialen Norm. (Vor-) Erfahrungen des Konsumenten mit einer Einkaufsstätte hinsichtlich Warenangebot, Service prägen sein Urteilsvermögen und Einschätzung hinsichtlich des Gesamtleistungsangebotes einer Einkaufssätte. Die Handlungskontrolle determiniert somit das Ausmaß der Zusammenhänge. Sie fungiert hier als Gruppenvariable. Eine weitere Segmentierungsgröße ist die vom Kunden wahrgenommene Selbstrelevanz der Kaufentscheidung. Die wahrgenommene Selbstrelevanz determiniert das Ursache- 
Wirkungsgefüge in der Theorie des geplanten Verhaltens. Es gilt hierbei wieder zu überprüfen, ob das Konstrukt auch auf die Zufriedenheitsgrößen, die durch die Einstellung und die subjektive Norm ausgelöst werden, einen Einfluss ausübt. In der bisherigen Zufriedenheitsforschung findet das Konstrukt der wahrgenommenen Selbstrelevanz weniger Beachtung als das Konstrukt des Involvement. Bloemer und Kasper (1995, S. 312f) weisen den quasi moderierenden Effekt der Größe auf die Beziehung zwischen Kundenzufriedenheit und Kundenloyalität nach. Insbesondere Diller betont den Zusammenhang zwischen Involvement und Kundenbindung (Diller, 2000, S. 29-48). Die wahrgenommene Selbstrelevanz hat wiederum Einfluss auf die Kundentreue und der Bindung.

Insgesamt wird die Kundenzufriedenheit der im Nachbarland kaufenden Konsumenten mit einer Einkaufsstätte im benachbartem Ausland von der Größe des Einflusses der sozialen Norm auf die Kaufentscheidung beeinflusst. Aus den dargelegten Ausführungen ergeben sich folgende Hypothesen:

Ausgehend von dem in Kapitel 2.3 zugrunde gelegten Kulturbegriff beeinflusst die Kultur, in der ein Konsument lebt, sowohl seine individuellen als auch seine gesellschaftlichen Werte Daraus ergeben sich folgende Hypothesen (siehe auch Anhang 5 und 6):

H1: Die Stärke der Werturteile der Gruppe (sozialen Norm), zu der der Konsument gehört, beeinflußt die Bedeutung der sozialen Norm der im Nachbarland kaufenden Konsumenten .

Aus dieser Hypothese leiten sich folgende Fragestellungen ${ }^{26}$ ab, durch die das Ergebnis der Hypothese $\mathrm{H} 1$ negativ oder positiv beeinflusst werden kann:

\footnotetext{
${ }^{26}$ Diese Fragestellungen beziehen sich jeweils auf die Teilzufriedenheiten mit der sozialen Norm. Diese leiten sich ab aus den Fragestellungen 3.2,3.3 und 3.4 des Fragebogens. Die Wichtigkeit der sozialen Norm wurde aus den Fragen 4 und 6 abgeleitet. Die Gesamtzufriedenheit mit der sozialen Norm drückt die Zufriedenheit des Konsumenten mit der Reaktion des sozialen Umfeldes aus.
} 
- Sind die Werturteile der sozialen Gruppe relevant für die Kaufentscheidungen der Konsumenten und lässt sich ihre Bedeutung nachweisen?

- Unterscheiden sich Konsumenten aus unterschiedlichen Kulturdimensionen signifikant voneinander hinsichtlich der sozialen Anerkennung, die ein Kauf in einer bestimmten Einkaufsstätte im sozialen Umfeld hervorruft (gibt es Unterschiede durch die Meinung und Handlung beeinflusst werden)?

- Unterscheiden sich Konsumenten aus unterschiedlichen Kulturdimensionen signifikant hinsichtlich der Freude am Einkauf voneinander?

Diese Fragestellungen geben Auskunft über die Zufriedenheit der im Nachbarland kaufenden Konsumenten hinsichtlich der sozialen Norm. Ferner über die Zufriedenheit der Konsumenten mit einer Einkaufsstätte in Bezug zu ihren kulturellen Erwartungen, dem Image der Einkaufsstätte im Wohnland der Konsumenten, sowie das Verhältnis der Konsumenten (soziale Norm) zur Art der Einkaufsstätte (ob es sich um ein kleines unabhängiges Einzelhandelsgeschäft oder um eine grenzüberschreitend tätige Ladenkette handelt).

Ein weiteres Kriterium ist, ob die soziale Norm für die Entscheidung des Konsumenten überhaupt eine Rolle spielt und wie wichtig ihr Einfluss ${ }^{27}$ ist. Dies lässt sich aus folgender Hypothese ableiten:

H 2: Die Stärke der Bedeutung der sozialen Norm ist abhängig von der Wichtigkeit der sozialen Norm der im Nachbarland kaufenden Konsumenten für ihren Einkauf.

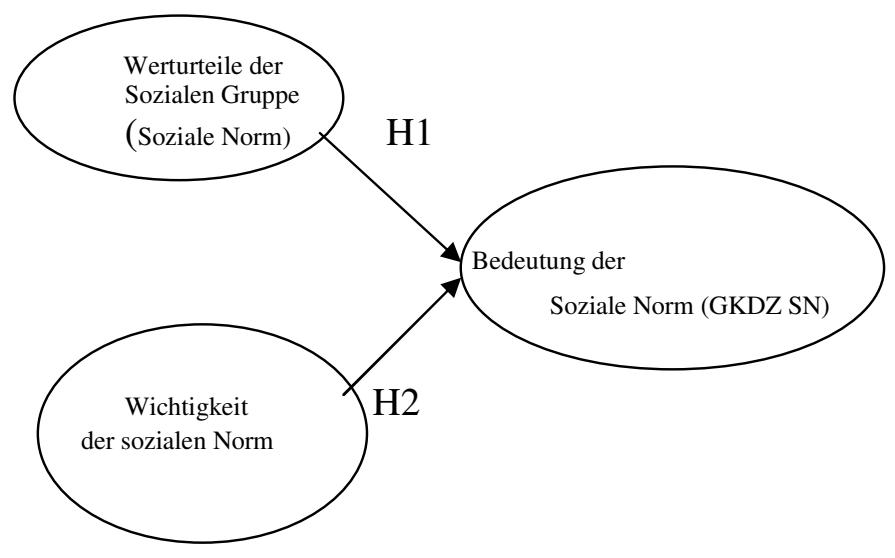

\footnotetext{
${ }^{27}$ Wichtigkeit der sozialen Norm: Einfluss der Einstellung und des Verhaltens des Umfeldes.
} 


\section{H3: Die Stärke der Bedeutung der sozialen Norm für die im Nachbarland kaufenden Konsumenten beeinflusst die Gesamtzufriedenheit mit dem Kauf in einer Ein- kaufsstätte im benachbartem Ausland positiv.}

H4: Je größer die Gesamtzufriedenheit mit dem Kauf im benachbartem Ausland ist, um so größer ist die Gesamtzufriedenheit des Kunden insgesamt.

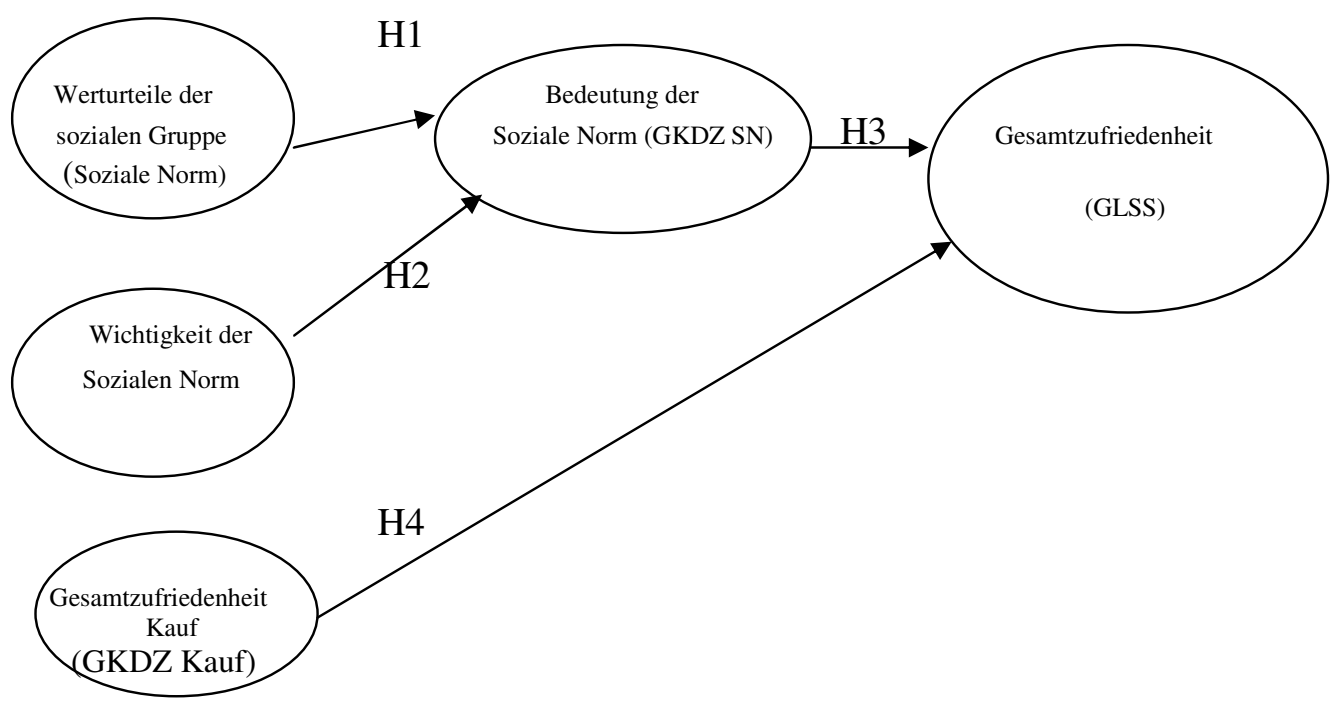

Hypothese H4 gibt Aufschluss über die Gesamtzufriedenheit des Konsumenten mit dem Kauf in einer Einkaufsstätte. Sofern die Konsumenten die Möglichkeit haben auch im benachbarten Ausland Einkäufe zu tätigen, hat sowohl die Bedeutung der Reaktion des sozialen Umfeldes Einfluss auf den grenzüberschreitenden Kaufakt, als auch die Gesamtzufriedenheit mit den Erfahrungen, die der Konsument beim grenzüberschreitenden Einkauf gesammelt hat.Wenn die Erwartung des Individuums hinsichtlich des Nutzens seiner Handlung bekannt ist, können Rückschlüsse auf die Einstellung des Individuums gezogen werden. Der Nutzen ergibt sich aus einer Handlung und der Wichtigkeit der Handlung für das Individuum selbst. Erwartung und Nutzen ergeben den Wert der Einstellung gegenüber der Handlung. Dieser Wert beeinflusst wiederum den Wert der Gesamtzufriedenheit mit dem Kauf (Peter, 1999, S. 120ff., Braunstein, 2001, S. 188ff., Odekerken-Schröder, 1999, S. 71ff). Der Einfluss der Gesamtzufriedenheit mit dem Kauf selbst soll aber in dieser Studie nicht weiter untersucht werden. Hypothese H1, H2, H3 und H4 geben zusammen Aufschluss über die Gesamtzufriedenheit des Konsumenten mit dem Kauf in einer Einkaufsstätte im benachbartem Ausland. Allerdings 
lassen die Hypothesen noch keinen Rückschluss zu, ob sich Einkäufe in der Einkaufsstätte wiederholen. Aus dieser Erkenntnis heraus wird folgende Hypothese aufgestellt:

\section{H5: Je größer die Gesamtzufriedenheit des Kunden mit dem Kauf in einer Einkaufs- stätte im benachbartem Ausland ist, umso größer ist die Kundenloyalität.}

Der Aspekt der Gesamtzufriedenheit mit dem Kauf gibt Aufschluss darüber, wie sich die Gesamtzufriedenheit mit dem Kauf selbst und den Umständen des Kaufes zusammensetzen. Dabei wird unterstellt, dass je höher das Ausmaß der Bedeutung der sozialen Norm beim Kauf im benachbartem Ausland und die Gesamtzufriedenheit mit dem Kauf selbst ist, umso geringer ist der Einflusses der geografischen Grenze auf die Kundenloyalität (Treueverhalten) der Konsumenten. Ferner wird unterstellt, dass je größer das Ausmaß der Gesamtzufriedenheit mit dem Kauf im benachbartem Ausland ist, je geringer ist der Einfluss der geografischen Grenze auf das Kaufverhalten des Konsumenten. Auf der anderen Seite wird der Bindungsvorsatz eines Konsumenten geprägt durch seine persönliche Norm (Schwartz und Tessler, 1972, S. 225ff; Kasihima und Kashima, 1988, S. 711ff), verstanden als ideale Verhaltensabsicht, die das Individuum unter bestmöglichen Umständen realisieren würde.

Sämtliche Kundenbindungsmodelle scheinen dem Einfluss des Involvement und seiner wertorientierten Konzeptualisierung als wahrgenommene Selbstrelevanz zu unterliegen. Deshalb soll die wahrgenommene Selbstrelevanz als moderierende Variable überprüft werden. Die wahrgenommene Selbstrelevanz wird beeinflußt durch die persönliche Norm des Konsumenten und seiner wahrgenommenen Verhaltenskontrolle. Sowohl die Gesamtzufriedenheit des Konsumenten mit dem Kauf in einer Einkaufsstätte im benachbarten Ausland als auch die Werte, deren Realisierung ein Individuum durch den Kauf und Konsum einer Leistung anstrebt, beeinflussen seine Kundenloyalität zu einer Einkaufsstätte. Beide Elemente werden zusammengefügt in der wahrgenommenen Selbstrelevanz.

H6: Je größer das Ausmaß der wahrgenommenen Selbstrelevanz ist, umso größer ist die Kundenloyalität des Konsumenten. 


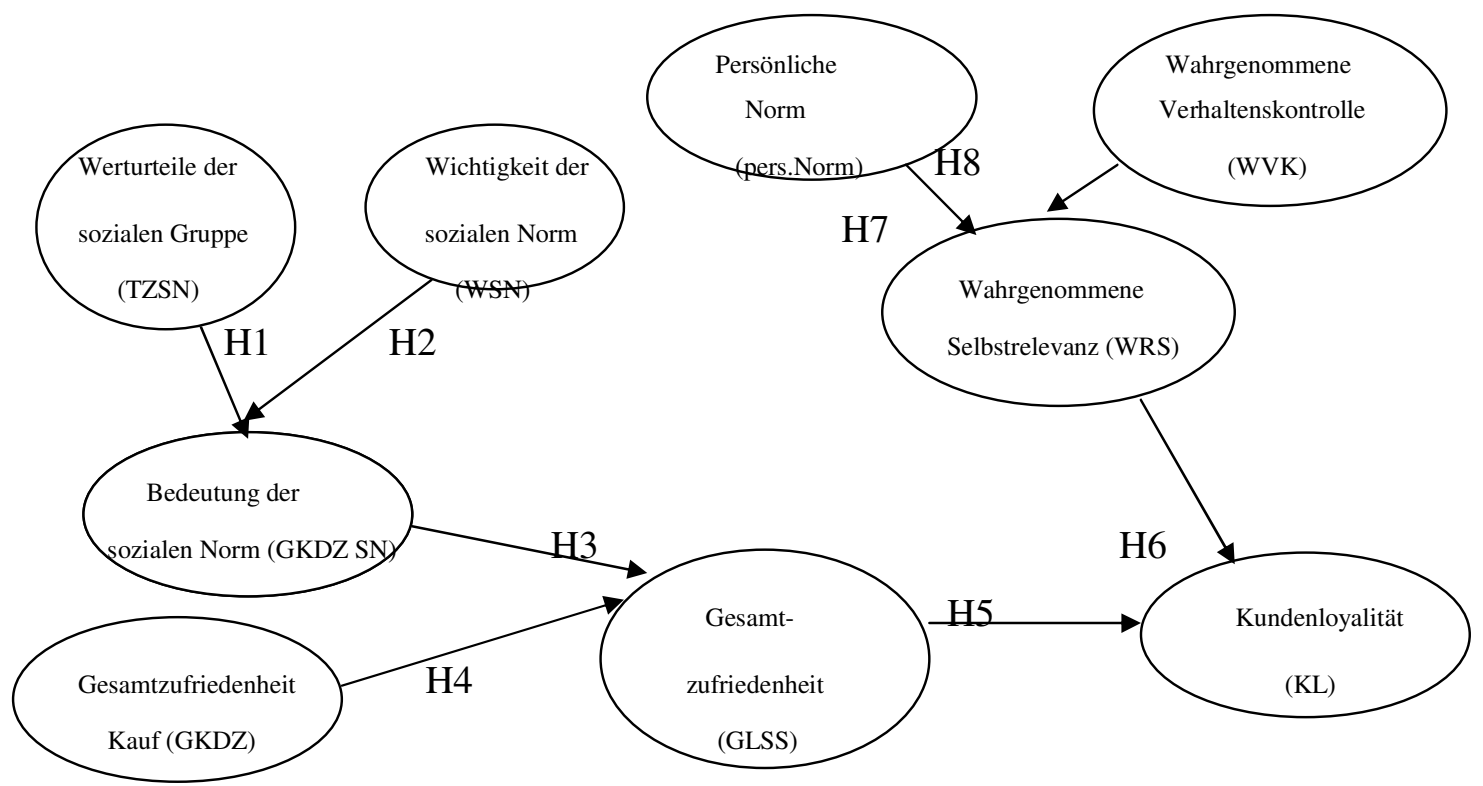

In Kapitel 4.2 wurde der Zusammenhang zwischen wahrgenommener Selbstrelevanz, wahrgenommener Verhaltenskontrolle und persönlicher Norm ausführlich dargestellt. Damit nachgewiesen werden kann, inwiefern Faktoren auf die wahrgenommene Selbstrelevanz Einfluss nehmen und sie Einfluss auf die Loyalität haben, werden die Hypothesen H 7 und H8 gebildet.

H7

Je größer der Einfluß der persönlichen Norm eines Individuums ist, umso positiver ist der Einfluß auf die wahrgenommene Selbstrelevanz.

H8

Je größer der Einfluß der wahrgenommenen Verhaltenskontrolle eines Individuums ist, umso positiver ist der Einfluss auf die wahrgenommene Selbstrelevanz.

Die wahrgenommene Selbstrelevanz ist insbesondere für die grenzüberschreitende Betrachtung des Einkaufs von entscheidender Bedeutung, da sich mit einer hohen wahrgenommenen Selbstrelevanz des Individuums auch das Involvement des Individuums erhöht. Das Involvement ist in diesem Fall das innere Engagement, mit dem sich das Individuum der Aktivität des Einkaufs im Nachbarland zuwendet. Hierdurch verstärkt (oder vermindert) dieses Konstrukt die Kundenbindung. Die Werte, die ein Individuum durch den Konsum bzw. Kauf einer Leistung anstrebt, haben entscheidenden Einfluss auf seine Loyalität und seine Kundenbindung. Da der Kunde bereits die Unternehmensleistungen erfahren hat, macht die Berücksich- 
tigung von Kundenerwartungen nach dem Erwartungs-Wert-Modell von Fishbein deshalb wenig Sinn. Die wahrgenommene Selbstrelevanz ist das entscheidende Moment, das Einfluss auf die Kundenloyalität und die Kundenbindung selbst nimmt. Hier bildet sich die wertorientierte Konzeptualisierung des Involvementkonstruktes.

Auf das Konstrukt der Kundenbindung wirken drei Faktoren direkt und indirekt ein. Dies ist die Kundenloyalität, aber auch die Gesamtzufriedenheit des Individuums mit dem Kauf im Ausland, ferner die wahrgenommene Selbstrelevanz. Trommsdorff (1993,S.291f) spricht in diesem Zusammenhang davon, dass es von der Dynamik des Marktes und der Länge des Kauf/Besuchszyklus abhängt, ob eine Wiederkauf/Wiederbesuchsentscheidung habituelle Züge annimmt, oder der Kunde vor dem Wiederkauf neue Informationen sucht und unter Bewertung der Alternativen sich für einen anderen Einkaufsort und/oder Einkaufsstätte entscheidet.

H9: Je größer die Kundenloyalität ist, umso größer ist die Bindung der Kon-
sumenten an eine Einkaufsstätte.

Kundenzufriedenheit und Treue eines Kunden zu einer Einkaufsstätte entwickeln sich relativ kurzfristig, doch wahre Bindung bedarf eines langen Zeitraumes und ist insbesondere auf die Zukunft gerichtet (Odekerken-Schröder,1999, S. 78 ; Geyskens, 1998, S. 99). Erst, wenn der Kunde wirklich zur Loyalität gegenüber einer Einkaufsstätte (vgl. Kap. 3 und 4) bereit ist und eine lange, vertrauensvolle Beziehung zu einer Einkaufsstätte besteht, kann von wirklicher, ehrlicher und freiwilliger Kundenbindung gesprochen werden. Kundenzufriedenheit mit dem grenzüberschreitenden Einkauf, die wahrgenommene Selbstrelevanz und Kundentreue haben allerdings unterschiedlichen Einfluss auf die Kundenbindung zu einer Einkaufsstätte im benachbarten Ausland. Sofern die geografische Grenze ein Faktor ist, der Kundenbindung zu einer Einkaufsstätte positiv oder negativ beeinflusst, so wird dieser Einfluss durch die das Ausmaß der wahrgenommenen Selbstrelevanz dargestellt. Daraus ergibt sich folgendes aus der Literatur abgeleitete Modell mit den aus den aufgestellten Hypothesen sich ergebenden Zusammenhängen: 


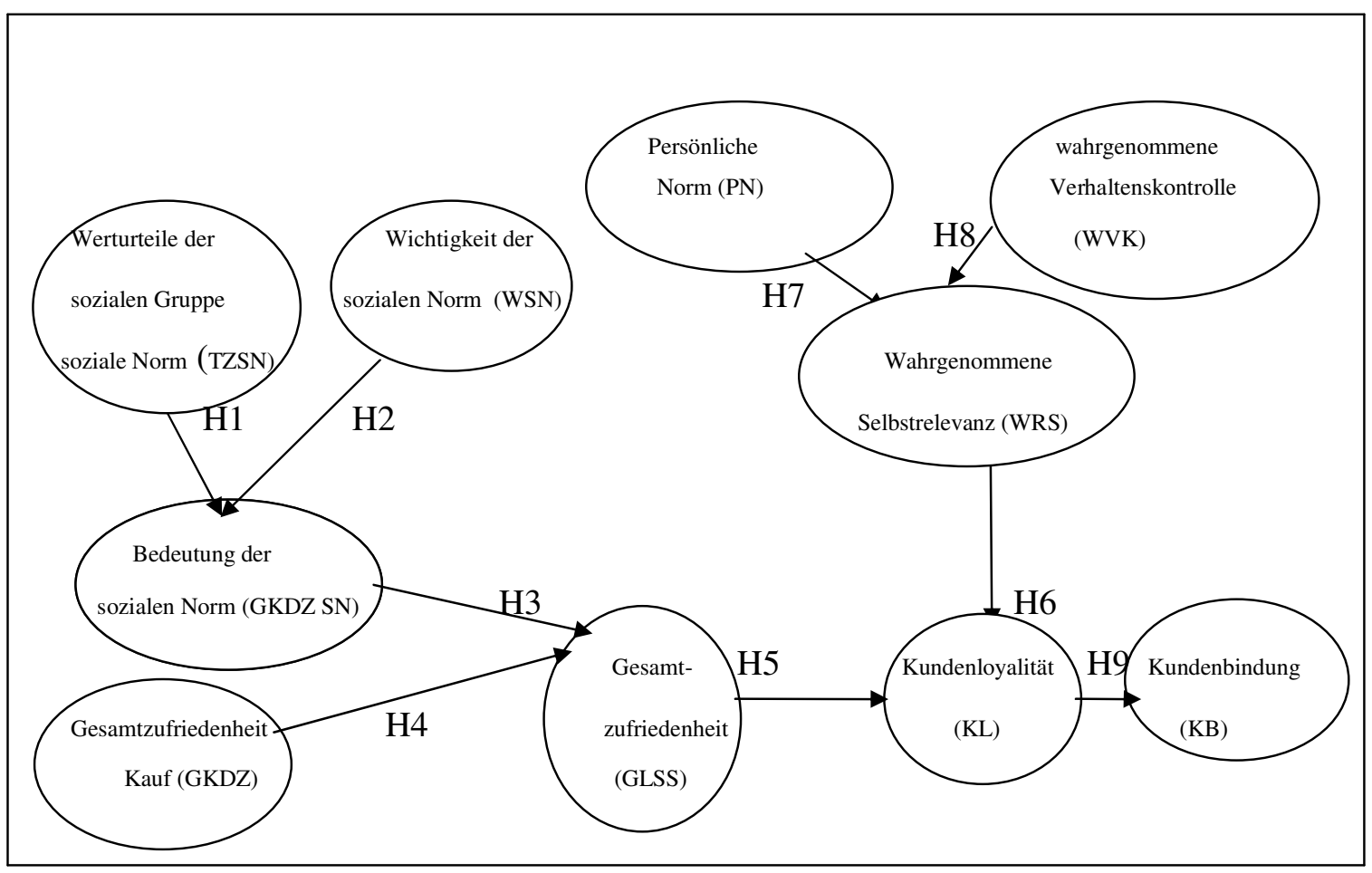

Abb. 5.1: $\quad$ Bedeutung der sozialen Norm und der wahrgenommenen Selbstrelevanz auf die grenzüberschreitende Kundenbindung

\subsection{Einfluss der Verhaltenskontrolle und der wahrgenommenen Selbstrelevanz auf das Kundenbindungsmodell im Grenzgebiet}

Kundenbindung ist nach dem vorliegenden Modell sowohl abhängig von der sozialen Norm der Probanden, der Reaktion des sozialen Umfeldes auf das Verhalten, sowie von der Gesamtzufriedenheit mit dem Kauf an sich und den Umständen unter denen der Kauf stattfindet. Die Gesamtzufriedenheit mit der Reaktion des sozialen Umfeldes, als auch die Gesamtzufriedenheit mit dem Kauf selbst, bilden die Gesamtzufriedenheit mit dem Einkauf im Nachbarland ab. Die wahrgenommene Selbstrelevanz hat wiederum einen unmittelbaren Einfluss auf die Kundenloyalität und die Kundenbindung selbst. Aus diesem Grund bilden die Hypothesen H1, H2, H3 und H4 die Erklärung der Kundenzufriedenheit in einem grenzüberschreitenden Raum.

Durch den Vergleich der Bedeutung der sozialen Norm bei deutschen und niederländischen Konsumenten lässt sich mit der Hypothese H3 die Frage beantworten, ob in einer Grenzregion 
kulturelle Unterschiede, die das Einkaufsverhalten prägen, so ausgestaltet sind, das von einem unterschiedlichen Einkaufsverhalten beider Nationalitäten gesprochen werden kann, oder ob sich in einer Grenzregion die kulturellen Einstellungen angleichen. Auch sollten hier Indizien zur Femininität oder Maskulinität der Kulturen gefunden werden. Schlussfolgerung wäre bei Ablehnung der Hypothese 3, dass auch wenn Länder unterschiedlichen Kulturregionen angehören, die Grenzregionen eine Ausnahme bilden. Auf der anderen Seite ist durch die Hypothesen H 5,6,7,8 die Frage zu beantworten, ob und wie im Nachbarland einkaufende Konsumenten anders angesprochen werden wollen, einen bestimmten Einkaufsstättentyp bevorzugen und warum sie ihn bevorzugen. Sofern es Unterschiede im Verhalten zum grenzüberschreitenden Einkauf zwischen Niederländern und Deutschen gibt, zeigen sich diese in unterschiedlichen Werten der Kundenbindung. Besondere Bedeutung kommt hierbei den Hypothesen H5, H6 und H9 zu, da diese nach dem Modell direkten Einfluss auf die Kundenbindung nehmen. Die Theorie des geplanten Verhaltens setzt einen Informationsverarbeitungsprozess nur bei der erstmaligen (Kauf-) Entscheidung voraus. Dabei bilden sich beim Erstkauf laut den Autoren Ajzen und Fishbein (1980, S. 245) die Konstrukte der Einstellung, der Gesamtzufriedenheit mit der sozialen Norm und der wahrgenommenen Verhaltenskontrolle durch die Bewertung durch den Konsumenten. Beim Wiederholungskauf greift der Konsument auf Erfahrungen zurück. Die Aktivierung der Einstellung, der sozialen Norm und der wahrgenommenen Verhaltenskontrolle erfolgt hier automatisch. Aus diesem Grund umfasst die Theorie des geplanten Verhaltens nach Braunstein (2001, S. 190) auch „habitualisierte Wiederkaufentscheidungen“. Allerdings stellt Braunstein ebenfalls fest (2001, S. 190f.), dass die Bindung der Konsumenten mit dem Modell des geplanten Verhaltens nur vorhergesagt werden kann, wenn der Konsument im Rahmen seiner Entscheidung einen intensiven Informationsverarbeitungsprozess durchlaufen hat. Auch diese Aussage unterstützt die Hypothese, dass sowohl die soziale Norm als auch die Einstellung gegenüber einer Handlung nicht nur einen wesentlichen Einfluss auf die Gesamtzufriedenheit mit dem Kauf, sondern auch auf das Bindungsverhalten haben. Die Gültigkeit des vorgestellten Kundenbindungsmodells ist somit in erster Linie abhängig von der zu Grunde liegenden Theorie des geplanten Verhaltens. Erst dann können die im Modell vorgestellten Haupthypothesen H1, H2, H3, H4, H6, H7, H8, H9 bestätigt oder widerlegt werden.

Die Einkaufsstätten haben auf Grund dieser Erkenntnisse konkretere Möglichkeiten „customer orientation“ für diese spezielle Zielgruppe der ausländischen Konsumenten unter Beweis zu stellen. Allerdings macht es für eine Einkaufsstätte oft keinen Sinn, alle grenzüberschreitend einkaufenden Konsumenten anzusprechen. Erfolgversprechender ist die Ansprache im 
Rahmen einer Kundenbindungsstrategie der Konsumenten, die einen hohen Wert in den Hypothesen H3, H4 erreichen. Erst dann kann die Einkaufsstätte davon ausgehen, dass die Gesamtzufriedenheit des Konsumenten mit dem Einkauf in einer Einkaufsstätte im benachbartem Ausland so hoch ist, dass ein direkter Rückschluss auf die Vorhersage des Wiederkaufs (H9) getroffen werden kann.

So werden die Einkaufsstätten im benachbartem Ausland motiviert, auf die kulturellen Belange der ausländischen Konsumenten verstärkt einzugehen und kleinere Fachhandelsgeschäfte können eine Strategie für eine definierte Zielgruppe ausländischer Konsumenten entwickeln. Dabei wird auch berücksichtigt, dass nicht alle Konsumententypen willens sind, sich auf eine Bindung mit einer konkreten Einkaufsstätte einzulassen. Ein weiterer wesentlicher Effekt zeichnet sich in dem Erwartungswert des Nutzens des Kaufs eines konkreten Produktes in einer Einkaufsstätte im benachbartem Ausland ab. Je größer das Produktinvolvement des Konsumenten aus dem benachbartem Ausland ist, umso größer sind seine Erwartungen (H6 und H5). Für die Einkaufsstätten im benachbartem Ausland bedeutet dies, dass der ausländische Konsument, sofern er „high involvement“ bezüglich einer Produktkategorie besitzt, einen entsprechenden Ansprechpartner erwartet, mit dem er sich auf gleichem Niveau auseinandersetzen kann. Daraus ergibt sich ein weiterer Nebeneffekt der Einkaufsstätte hinsichtlich des Profils der Mitarbeiter am ,point of sales“.

Diese Hypothesen stehen im Einklang mit Eggert, der das Phänomen der Kundenbindung aus Kundensicht durch folgende Bindungszustände repräsentiert sieht:

1. „einen inneren Zustand des Kunden, der auf affektiven und normativen Bindungsmotiven beruht, und

2. einen inneren Zustand des Kunden, der auf kognitiven Bindungsmotiven beruht" (Eggert, A., 1999, S.130).

Für den grenzüberschreitenden Einkauf von Konsumgütern bedeutet dies, dass die Konsumenten eine innere Bereitschaft zum grenzüberschreitenden Einkauf auf Grund ihrer Vorerfahrungen aus dem Freundes, Bekanntenkreis oder Elternhaus mitbringen. Daraus formt sich auch die eigene Einstellung gegenüber Risikobereitschaft, Neugier und die Verbundenheit mit Bewährtem. Dies führt zu einer Verbundenheit mit den Einkaufsmöglichkeiten des grenznahen Auslands. Verbundenheit als innerer Zustand kann danach auch losgelöst von der Verhaltensabsicht des Wiederkaufs bestehen. Somit kann die grenzüberschreitende Kundenbindung aus Kundensicht wie folgt präzisiert werden: 
Grenzüberschreitende Kundenbindung im Einzelhandel aus Kundensicht liegt dann vor, wenn der Kunde eine Affinität und somit eine Bindung zu grenznahen Orten wahrnimmt (H1, H2, H3), sowie die Gesamtzufriedenheit mit der Einkaufsstätte (Produkt, Dienstleistung, Ladenbaugestaltung) hoch ist (H4). Der innere Zustand des Kunden ist dadurch gekennzeichnet, dass er sich zu bestimmten Einzelhandelsformen und/oder Geschäften verbunden fühlt (H5, H6) und geneigt ist den Einkaufsort und auch die Einkaufsstätte wiederholt mit Kaufabsicht aufzusuchen (H9).

Für den grenzüberschreitenden Einzelhandel ist dieses zustandsbezogene Begriffsverständnis der Kundenbindung zum Aufbau einer Marketingstrategie nur dann relevant, wenn folgende Bedingungen erfüllt sind:

1. Die soziale Norm und die wahrgenommene Selbstrelevanz beeinflussen das Verhalten des grenzüberschreitenden Konsumenten.

2. Die Bindungszustände der grenzüberschreitenden Konsumenten als generische Bindungszustände erzeugen eine unterschiedliche verhaltenssteuernde Wirkung.

Ob diese Bedingungen auch in einem grenzüberschreitenden Raum erfüllt werden können, soll in den folgenden Kapiteln untersucht werden.

\subsection{Zusammenfassung der Struktur und der Zielsetzung}

In diesem Kapitel sind die Hypothesen zu einem Kundenbindungsmodell formuliert worden, durch das in einem Grenzgebiet zweier größenmäßig sehr unterschiedlichen Länder der Einfluss der sozialen und persönlichen Norm sowie die Bedeutung der wahrgenommenen Selbstrelevanz zur Einkaufsstättenbindung im Nachbarland analysiert werden soll. Dabei werden die hypothetischen Konstrukte als eigenständige Forschungsobjekte betrachtet.

Damit soll nicht nur die zentrale Frage der Arbeit geklärt werden, ob Konsumenten aus einem Nachbarland (innerhalb einer Grenzregion) an eine ausländische Einkaufsstätte gebunden werden können, sondern auch die Forschungsfragen nach den relevanten Determinanten der Kundenzufriedenheit, Kundenbindung und Loyalität beantwortet werden. Da im letzten Schritt die Frage der Unterscheidung der generischen Bindungszustände im Hinblick auf ihre verhaltenssteuernde Wirkung gestellt wird, kann auch die letzte Frage nach den Möglichkei- 
126 | Kapitel 5

ten der Förderung des grenzüberschreitenden Einkaufs durch den Einzelhandel beantwortet werden. 


\section{Kapitel 6: Methodik der Modellschätzung}

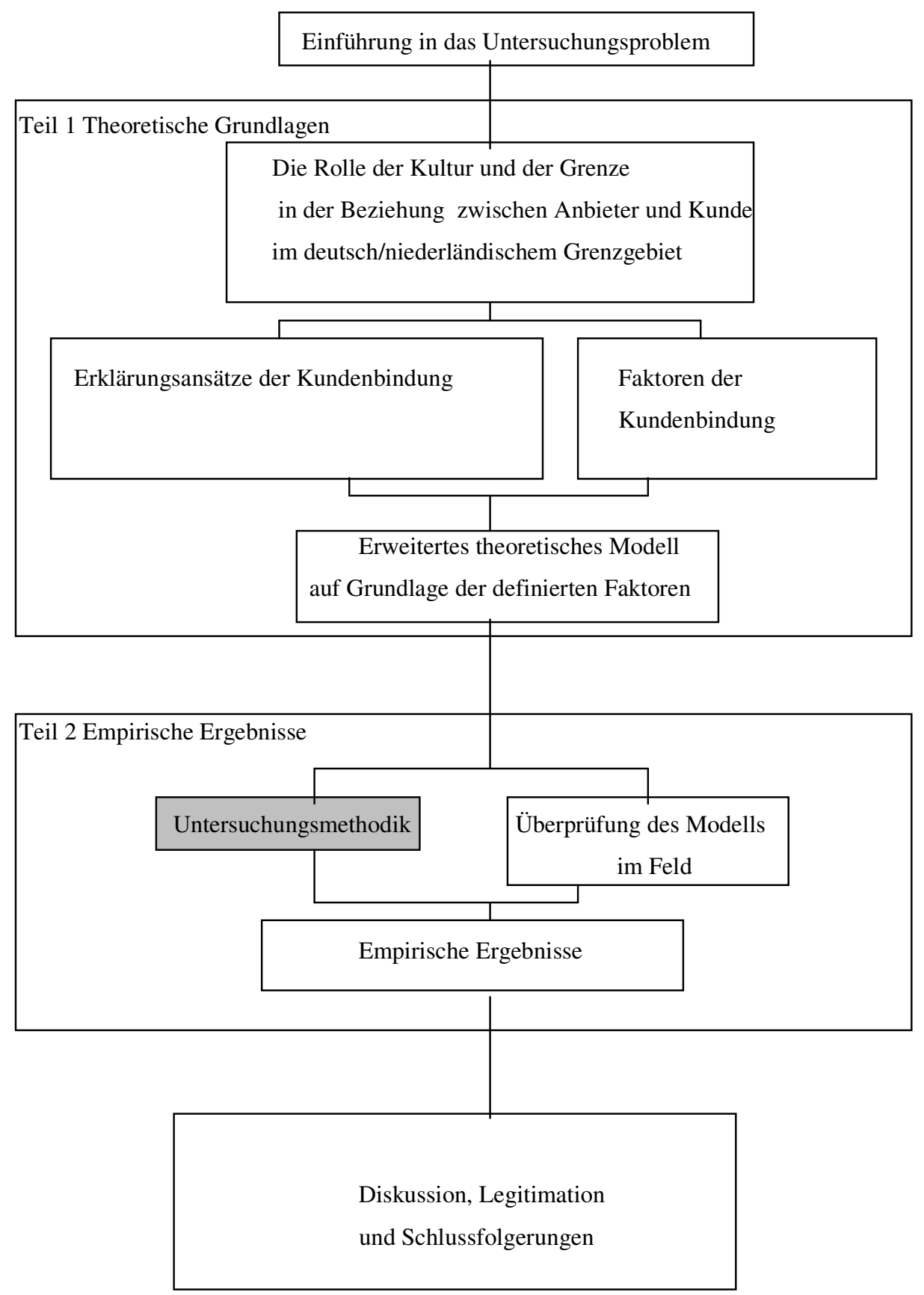




\subsection{Einleitung}

In diesem Kapitel wird die Methodik der Modellschätzung erläutert. Die Entscheidung für eine Erhebungsmethode richtet sich nach dem jeweiligen Erkenntnisziel. Deshalb wird im Folgenden nach der Einführung in die allgemeine Modellspezifikation die Konkretisierung der Fragestellungen mit Hilfe der Focus-Gruppen erläutert, sowie die Einsatztauglichkeit der multivariaten Analysemethoden für die Modellschätzung diskutiert. Anschließend wird das Prüfschema erläutert.

\subsection{Einführung in die allgemeine Modellspezifikation}

Bei den Komponenten eines Kundenbindungsmodells handelt es sich um hypothetische GröBen, die einer direkten Messung nicht zugänglich sind (Braunstein, 2001, S. 193; KroeberRiel, Weinberg, 2000, S. 28; Peter, 1997, S. 128; Hildebrandt, 1999, S. 47). Dies bedeutet, dass entsprechende Indikatoren zu finden sind, die die Faktoren des Ansatzes repräsentieren. Bei den beobachtbaren Indikatoren handelt es sich um messbare Sachverhalte, die das Vorliegen der gemeinten, aber nicht direkt messbare Phänomene widerspiegeln (Kroeber-Riel, Weinberg, 2003, S. 31). Nachdem die Spezifizierung der Konstrukte erfolgt ist, kann die Übersetzung der theoretischen Konzeption in eine Messvorschrift realisiert werden (Hildebrandt, 2000, S. 40). Nach Hildebrandt stellt das Ergebnis des Konzeptualisierungsaufwandes das Konzept dar. Dieses legt fest, was unter einem definiertem Phänomen zu verstehen ist (Hildebrandt, 2000, S. 38). Dabei sieht er die Übergänge zwischen Konzepten und Messvorschriften als fließend an. Die Messansätze der Konstrukte stellen dann das Ergebnis der Spezifierung dar. Im Rahmen der Operationalisierung der Konstrukte wird die Eignung der, auf Basis der Spezifizierungsbemühungen entwickelten, Skala zur Erfassung der Konstrukte überprüft. Dabei stellt das Ergebnis der Operationalisierungsmaßnahmen eine reliable und valide Skala dar. Die Unterscheidung zwischen Validität und Reliabilität ist in der Literatur sehr unterschiedlich. Für die vorliegende Arbeit hat sich der Autor für folgende Abgrenzung entschieden, da sie in der testtheoretischen Literatur die größte Zustimmung erfahren hat (Hossinger, 1982, S. 21). In dieser Arbeit wird zwischen Inhaltsvalidität, Konstruktvalidität und kriterienbezogene Validität unterschieden. Dabei ist die inhaltliche Validität gegeben, wenn gewährleistet ist, dass die Items eine repräsentative Stichprobe darstellen (Litwin, 1995, S. 35). Konstruktvalidität ist vorhanden, wenn ein Test das abstrakte, psychologische Gedankengerüst wiedergibt auf dem es basieren soll (Schelten, 1980, S. 99). Die kriterienbezogene Validität stellt die bedeutendste Form dar (Bortz, 1985, S. 138). Es wird 
zwischen externer und interner Validierung unterschieden, wobei die externe darin besteht, den Test anhand eines sinnvollen Kriteriums zu überprüfen, während bei der internen Validierung anhand bereits bestehender anderer als valide angesehene Tests validiert wird (Crocker und Algina, 1986, S. 225). In dieser Studie ist die externe Validierung von besonderem Interesse und ein wichtiges additionelles Evaluationskriterium, da allgemein gültige Ergebnisse für den definierten Einzelhandel gefunden werden sollen. Unter Realibilität wird das Aus$\mathrm{maß}$ bezeichnet, in dem die Skalierungsergebnisse frei von Experimentfehlern sind. In diesem Zusammenhang befasst man sich mit der Konsistenz der Testergebnisse zwischen verschiedenen Personengruppen oder Zeitpunkten beim gleichen Individuum (Green und Tull, 1982, S. 185). Damit das Kundenbindungsmodell eine hohe Aussagekraft hat, sollen zur Erfassung der Konstrukte nur die Skalen herangezogen werden, die eine hohe Reliabilität und Validität besitzen. Reliabilität und Validität sind somit Gütekriterien der Messung erzeugter Daten (Herrmann und Homburg, 2000, S. 23 f). Dies bedeutet, dass Skalen verbessert werden, sobald die vorhandenen Indikatorentests nur befriedigende Ergebnisse liefern. Eine weitere Konsequenz ist die Erweiterung des Messansatzes oder der Austausch von Items.

Zur Messung der Kundenbindung im definierten grenzüberschreitenden Raum wird in dieser Studie die breite Befragung zu einem bestimmten Zeitpunkt bevorzugt. Hierbei wird sich der Meinung von Malhotra (1996, S. 92) angeschlossen, dass eine cross-sektorale Marktforschung alle relevanten Informationen der interessierenden Gesamtheit der Konsumenten beinhaltet und valide Ergebnisse liefert. Zwar werden die Vorteile einer über einen langen Zeitraum begleitenden Marktforschung im Rahmen eines Konsumentenpanels erkannt, insbesondere wenn es um vertiefende Aussagen der Individuen geht oder um Verhaltensänderungen über einen längeren Zeitraum, die für die Erklärung der Kundenbindung sicherlich wesentliches Zahlenmaterial liefert (Green, Tull, Albaum, 1988; Malhorta, 1996) würden. Auf der anderen Seite sind die Probleme einer längeren Marktforschung mit einer festen Gruppe von Individuen im Rahmen eines Panels aber auch nicht zu übersehen. Dies sind insbesondere der im Zeitablauf geringer werdende Response und somit die natürliche Sterblichkeit innerhalb eines Panels. Ferner die Motivation der Panelmitglieder sowie der Zwang des Marktforschers im Rahmen der Validität, das Panel innerhalb der laufenden Forschung ständig mit neuen Panelmitgliedern anfüllen zu müssen. Diese neuen Panelmitglieder bergen dann auch die Gefahr, durch ihre Antworten die Verhaltensweisen des ursprünglichen Panels zu verfälschen. (Churchill, 1995). Zuletzt ist es auch das Ziel dieser Studie aufzuzeigen, wodurch grenzüberschreitende Konsumenten sich heute an im benachbartem Ausland befindlichen Einkaufsstät- 
ten gebunden fühlen. Aus diesem Grund erfolgt die Entscheidung zu Gunsten einer cross sektoralen Erhebung.

Im Wege der Kommunikation stehen mehrere Medien zur Verfügung, um Informationen von Auskunftspersonen zu gewinnen (Green und Tull, 1982, S. 137ff.). Für diese Studie erscheint die Befragung als die geeignete Methode, um zu einer Erklärung der Kundenbindung zu kommen. Der Vorteil der Befragung liegt insbesondere darin, dass mit Hilfe der Befragung strukturell Informationen unter gleichen Bedingungen von den Probanden abgefragt werden können. Des Weiteren ist im Rahmen der Befragung zwischen persönlicher-, schriftlicher-, telefonischer Befragung, Befragung über Mail Adressen und einer Internet Befragung zu differenzieren. Im Rahmen der persönlichen Befragung kann der Konsument zuhause oder direkt während seiner Einkaufstätigkeit in der Nähe der Einkaufsstätte befragt werden. Der Autor hat eine persönliche Befragung der Konsumenten in der Nähe der Einkaufsstätten mit Hilfe eines strukturierten Fragebogens vorgenommen. Der Vorteil der persönlichen Befragung liegt insbesondere darin, dass der Konsument unmittelbar in der Konsumsituation befragt wird und autenthische Antworten erwartet werden können. Dabei werden niederländische Konsumenten in Deutschland befragt und deutsche Konsumenten in den Niederlanden. Es ergibt sich folgende Vorgehensweise: Um die für diese Studie relevanten Daten zu erheben, wird eine nichtexperimentelle Forschung durchgeführt und die Befragung mit Hilfe einer persönlichen Befragung der Konsumenten in der Nähe der Einkaufsstätten im Rahmen einer crosssektoralen Befragung realisiert.

Des Weiteren gehört zur Modellspezifikation die Begründung, warum im Rahmen der Befragung die ausgewählten Fragen gestellt werden. Ferner gehört zur Spezifikation die Entwicklung des Fragebogens und eines Befragungstests, mit dessen Hilfe alle definierten Elemente auch begründet werden können. Auch hier wird auf eine Übersicht von Odekerken-Schröder zurückgegriffen, die auf den in der Forschung akzeptierten Methoden zum Aufbau eines Fragebogens basiert (Churchill, 1979 und De Vellis, 1991).

Daraus ergibt sich folgende Vorgehensweise: Aus der Literaturdatenbank zum Thema Kundenbindung und angrenzender Fachbereiche wurde Literatur zum Thema Konsumentenverhalten, Psychologie, Kultur, Handelsmarketing, Kundenzufriedenheit, Kundentreue bzw. Bindungsvorsatz und Kundenbindung gesucht. Diese Literatur wurde gefiltert hinsichtlich für diese Studie relevanter Elemente. Diese Literaturrecherche bildet die Basis zur Entwicklung einer relevanten Befragung und wird hinsichtlich der Brauchbarkeit der Erklärung der Kun- 
denbindung zu einer sich im Nachbarland befindlichen Einkaufsstätte - bestehend aus Gesamtzufriedenheit mit dem Kauf, wahrgenommener Selbstrelevanz, Kundenloyalität, Bindungsvorsatz und Kundenbindung überprüft. Ergänzt wird diese Vorgehensweise durch Gespräche mit einem grenzüberschreitend tätigen Handelsunternehmen aus dem Bereich Textil/Schuhe sowie durch ein Gespräch mit einer Einkaufsgenossenschaft, dessen klein- und mittelständischen Mitglieder in den Niederlanden als auch in Deutschland Einzelhandelsgeschäfte führen. Dabei waren Inhalte des Gespräches, sowohl der Einfluss der Kundenbindung auf die Geschäftsentwicklung, als auch der Einkaufstourismus der Deutschen und Niederländer in der Grenzregion. Im Rahmen dieser Gespräche wurden Brainstorming Techniken angewendet, um möglichst viele Ideen zu generieren.

Aus der Literaturanalyse heraus wurden dann Fragenschwerpunkte gebildet, die dazu beitragen sollen, das Erklärungsmodell der grenzüberschreitenden Kundenbindung zu validieren. Um eine entsprechende Vorgehensweise zu rechtfertigen wird sowohl auf die Erkenntnisse der relevanten Marktforschungsliteratur (Green und Tull, 1982, Herrmann und Homburg, 1999) als auch auf Studien, die sich mit dem Konstrukt der Kundenbindung auseinander setzen (Henning-Thurau und Hansen, 2000, Peter, 1999, Herrmann, 1996, Sauerwein, 2000, Odekerken-Schröder, 1999, Braunstein, 2001) zurückgegriffen. Zielsetzung ist dabei einmal die Anzahl der Fragen zu reduzieren, andererseits die Anzahl der Fragen zum jeweiligen Forschungsschwerpunkt zu gewichten. 


\begin{tabular}{|c|c|c|}
\hline Konstrukt & $\begin{array}{l}\text { Anzahl der } \\
\text { Items }\end{array}$ & Quelle \\
\hline $\begin{array}{l}\text { Soziale Norm vor kulturellem } \\
\text { Hintergrund }\end{array}$ & 6 & $\begin{array}{l}\text { Oliver, (1996); Ajzen und Fishbein (1980); Ajzen } \\
\text { und Madden (1986) }\end{array}$ \\
\hline Einstellung gegenüber Handlung & 5 & Manstead und Parker, (1995), Oliver, (1996) \\
\hline Relationship satisfaction & 2 & $\begin{array}{l}\text { Oliver, (1989); Bittner und Hubbert, (1994); Ode- } \\
\text { kerken-Schröder, (1999); Braunstein (2001) }\end{array}$ \\
\hline Wahrgenommene Selbstrelevanz & 2 & $\begin{array}{l}\text { Houston und Walker (1996);Bagozzi. (1994); } \\
\text { Hofstede, Audenaert, Steenkamp und Wedel, (1998) }\end{array}$ \\
\hline Loyalität/Treue & 3 & $\begin{array}{l}\text { Peter, 1999; Anderson und Sullivan (1993); } \\
\text { Parasuraman, Berry und Zeithaml (1991); Narayan- } \\
\text { das (1996) LaBarbera und Mazursky (1983); Parasu- } \\
\text { raman, Zeithaml und Berry (1994); Fornell, Johnson, } \\
\text { Anderson, Cha und Bryanr (1996); Narayandes } \\
\text { (1997) }\end{array}$ \\
\hline Bindungsvorsatz & 2 & Gollwitzer und Malzacher (1996) \\
\hline Kundenbindung & 3 & $\begin{array}{l}\text { Homburg und Faßnacht (1998); Diller (1996); } \\
\text { Hausknecht (1990); Ajzen (1991); Fishbein (1967) }\end{array}$ \\
\hline Total & 23 & \\
\hline
\end{tabular}

Tab. 6.1: Quellen der Messkriterien (eigene Zusammenstellung)

\subsection{Konkretisierung der Fragestellungen mit Hilfe von Focus-Gruppen}

Die Fragenschwerpunkte wurden mit einer Focus-Gruppe diskutiert. Focus-Gruppen werden im Allgemeinen als eine additionelle Quelle zur Generierung von Fragen angesehen. Nach Parasuraman (1991, S. 256) schließt eine Focus-Gruppen-Diskussion ein, dass ein Diskussionsleiter oder Moderator diese Gruppe bei der Diskussion eines vorher definierten Themengebietes begleitet. Focus Gruppen Diskussionen sind der erste wesentliche Schritt, um einen zielgruppenadäquaten Fragebogen zu entwickeln (Churchill, 1995). Ziel der Arbeit mit den Focusgruppen war neben der Generierung von Fragen vor allem die Reflexion der Deutlichkeit der formulierten Fragen. Ebenso sollte mit den Gruppen gemeinsam deutliche Fragestellungen in Bezug zur sozialen Norm, der Einstellung zur Handlung, der Gesamtzufriedenheit und der wahrgenommenen Selbstrelevanz formuliert werden. Auf Grund ihres explorativen Charakters ist eine Voruntersuchung insbesondere bei größeren Befragungen angebracht 
(Kotler, Armstrong, Saunders und Wong, 1999, S. 329f.) Um die vorgestellten Frageninhalte zu überprüfen, wurden insgesamt sechs unterschiedliche Focus-Gruppen mit deutschen und niederländischen Konsumenten zusammengestellt.

Über die Größe der Focusgruppe gibt es in der wissenschaftlichen Literatur zwar keine einheitliche Meinung, doch wird im Allgemeinen von einer Größe von sechs bis zwölf Personen ausgegangen (Atteslander, 1993, S. 169; Odekerken-Schröder, 1999, S. 89; Kotler, Armstrong, Saunders und Wong, 1999, S. 329f.). Nach Kotler (1995, S. 196f) müssen die Focus-Gruppen so homogen wie möglich hinsichtlich demografischem Charakter und sozioökonomischer Elemente sein. Die Focus-Gruppe soll die Konsumenten in dem definierten Untersuchungsgebiet widerspiegeln. Aus diesem Grund wurden soziodemografische Daten des Erhebungsgebietes berücksichtigt und drei Focus-Gruppen gebildet. Die Verteilung innerhalb der Gruppen ist mit 60\% Frauen und 40\% Männer festgelegt worden. Hierbei hat sich der Autor der Argumentation von Odekerken-Schröder (1999, S. 90) angeschlossen, die die Zusammenstellung der Gruppen auf Grund des höheren Involvement der weiblichen Konsumentinnen im Bereich Bekleidung begründete. Alle sechs Gruppen bestanden deshalb aus sechs Personen, von denen jeweils vier weiblich und jeweils zwei männlich waren. Damit das Kaufverhalten der Konsumenten in der Untersuchung über alle Altersklassen abgebildet werden kann, wurde die Fassung des Familienzyklus von Gilly und Enis (1982, S.272) der eigenen Untersuchung zu Grunde gelegt. Die Forscher nehmen in ihren Untersuchungen eine Unterteilung in die Altergruppen unter 35 Jahre, zwischen 35 und 64 Jahre und über 64 Jahre vor. Ferner unterteilen sie in Haushalte mit einem Erwachsenen, Haushalte mit zwei Erwachsenen, Haushalte mit zwei Erwachsenen und Kindern und Haushalte mit einem Erwachsenen und Kindern.

Alle Personen innerhalb der unterschiedlichen Focus-Gruppen hatten bereits Erfahrungen im grenzüberschreitenden Einkauf gesammelt und sowohl Erfahrung im Kauf von Produkten im definiertem Produktbereich als auch Erfahrungen im Einkauf in den zu untersuchenden Einkaufsstätten. Aus diesem Grund wurden die Probanden in folgende Gruppen eingeteilt: 


\begin{tabular}{|c|c|c|c|c|c|}
\hline unter 35 & & & & & \\
\hline Anzahl & Nationalität & fam. Stand & weiblich & männlich & Name \\
\hline 2 & $\bar{D}$ & Single & 1 & 1 & \\
\hline 2 & $\mathrm{NL}$ & Single & 1 & 1 & \\
\hline 1 & $\mathrm{D}$ & verh.+Kind & 1 & & \\
\hline 1 & $\mathrm{D}$ & verh. o. Kind & 1 & & \\
\hline 1 & $\mathrm{NL}$ & verh. o. Kind & & 1 & \\
\hline 1 & $\mathrm{NL}$ & verh. o. Kind & 1 & & \\
\hline \multicolumn{6}{|l|}{35 bis 64} \\
\hline Anzahl & Nationalität & fam. Stand & weiblich & männlich & Name \\
\hline 1 & $\bar{D}$ & Single & 1 & & \\
\hline 1 & NL & Single & 1 & & \\
\hline 2 & $\mathrm{D}$ & verh.+Kind & 2 & & \\
\hline 2 & $\mathrm{NL}$ & verh.+Kind & 1 & 1 & \\
\hline 1 & $\mathrm{D}$ & verh. o. Kind & & 1 & \\
\hline 1 & $\mathrm{NL}$ & verh. o. Kind & 1 & & \\
\hline \multicolumn{6}{|l|}{ über 64} \\
\hline Anzahl & Nationalität & fam. Stand & weiblich & männlich & Name \\
\hline 1 & $\bar{D}$ & Single & & 1 & \\
\hline 1 & NL & Single & 1 & & \\
\hline 3 & NL & verh. & 2 & 1 & \\
\hline 3 & $\mathrm{D}$ & verh. & 2 & 1 & \\
\hline
\end{tabular}

Odekerken-Schröder (1999, S.90) schließt sich der Meinung von Churchill (1995) und Parasuraman (1991) an, nachdem ein Gespräch mit der Focus-Gruppe zwischen neunzig und einhundertzwanzig Minuten lang sein soll. Dies deckt sich mit der Länge der durchgeführten Gespräche hinsichtlich des Untersuchungsgegenstandes. Der Moderator war mit allen Gruppenteilnehmern ins Gespräch gekommen, die Gespräche sind in einer freundlichen und positiven Atmosphäre geführt worden. Die Probanden sollten erst einmal frei über ihr grenzüberschreitendes Einkaufsverhalten in Verbindung mit den Produktgruppen Textil inklusive Schuhe sowie Elektroartikel berichten. Hierbei wurde differenziert zwischen Einkaufserlebnissen in Filialketten und Fachgeschäften. Anschließend wurde auf die Rahmenbedingungen eingegangen, die zu einem Wiederkauf in einer, im Nachbarland liegenden, Einkaufsstätte geführt haben. Hierbei wurde insbesondere die Reaktion des Umfeldes auf den Kauf, Informationsaustausch mit Freunden und Bekannten, die allgemeine Freude am Einkauf, das Einkaufserlebnis in einer Einkaufsstätte sowie der Bezug zum Produkt selbst hinterfragt. Ferner wurden besonders angenehme Kauferlebnisse der Focus-Gruppenmitglieder diskutiert. Anschließend wurden die Ergebnisse der Diskussion in die Kategorien der Items zusammengefasst und zum Schluss der jeweiligen Gruppe ein aus dem Gespräch entwickelter Fragebogen vorgelegt. Die Gespräche der Focus-Gruppen wurden mit Einverständnis der Gruppenmit- 
glieder aufgezeichnet. Der endgültige Fragebogen selbst ist aus den größtmöglichen Gemeinsamkeiten der sechs Gruppendiskussionen entwickelt und in einem Pretest getestet worden.

Die wesentlichsten Erkenntnisse dieser Focusgruppengespräche waren:

- Inhaltlich:

- Alle Mitglieder der unterschiedlichen Focuss-Gruppen bestätigten, dass sie grenzüberschreitend einkaufen (wenn auch mit unterschiedlicher Intensität).

- Es gibt Unterschiede in der Einflussnahme des sozialen Umfeldes hinsichtlich der Einstellung zum grenzüberschreitenden Einkauf zwischen deutschen und niederländischen Konsumenten.

- Die persönliche Norm nimmt sowohl bei niederländischen als auch bei deutschen Konsumenten auf Einstellung zum Einkauf im Nachbarland Einfluss.

- Das Ausmaß der wahrgenommenen Selbstrelevanz beeinflusst Loyalität und Kundenbindung an eine Einkaufsstätte.

- Methologisch:

- Die Modellvariablen verschließen sich einer direkten Messung.

- Die Modellvariablen können durch eine Unterteilung in Dimensionen durch beobachtbare Indikatoren gemessen werden.

- Die identifizierten Indikatoren der hypothetischen Konstrukte eignen sich zur Klärung der Kundenbindung an einer Einkaufsstätte im Nachbarland.

Nachdem die Erfahrungen mit den Focus-Gruppen verarbeitet waren, wurde ein erster Fragebogen erstellt. Dieser Fragebogen wurde in niederländischer und in deutscher Sprache erstellt und wiederum einem Pretest unterworfen. Der Pretest wurde sowohl in Deutschland als auch in den Niederlanden durchgeführt, um sicherzustellen, das in der cross-kulturellen Untersuchung die Fragen (weitestgehend) auf die gleiche Art und Weise von den Probanden interpretiert werden. Nach dem Pretest wurden letzte Veränderungen an dem Fragebogen vorgenommen und wiederum in beide Sprachen übersetzt. Um sicherzustellen, dass auch durch den Interviewer keine sprachlichen Probleme in der Befragung entstehen, bekamen deutsche und 
niederländische Studenten ${ }^{28}$ im Rahmen eines gemeinsamen Seminars zur grenzüberschreitenden Marktforschung die Aufgabe die Interviews im jeweiligen Nachbarland durchzuführen. Damit wurde sichergestellt, dass die Interviews von Muttersprachlern geführt wurden.

\subsection{Eignung multivariater Analysemethoden für die Modellschätzung}

Die hypothetischen Größen des Kundenbindungsmodells können nicht direkt gemessen werden. Aus diesem Grund sind die entsprechenden Indikatoren über beobachtbare Größen zu erfassen. Im Mittelpunkt der Modellbildung steht das Ziel, generalisierbares Wissen zu generieren und signifikante Phänomene des grenzüberschreitenden Konsumentenverhaltens zu erklären (Hildebrandt, 1999, S. 35). Durch die Grobkonzeptualisierung der Konstrukte erfolgt die Übersetzung der theoretischen Konzeption in eine Messvorschrift. Dabei stellt dieses Konzept nach Hildebrandt das Ergebnis des Konzeptualierungskonzeptes dar und legt unter Verwendung sprachlicher Terme fest, was unter einem Phänomen zu verstehen ist (Hildebrandt, 1999, S. 38). Hildebrandt (1999, S. 40) betont in diesem Zusammenhang, dass die Übergänge zwischen Konzepten und Messvorschriften fließend sind. In der vorliegenden Arbeit finden für den Begriff Messansatz die Synonyme Inventar, Skala, Itemset oder Messmodell Verwendung.

Das vorliegende Kundenbindungsmodell beinhaltet latente Variablen des Modells der GröBen, die bereits in anderen Arbeiten und Zusammenhängen empirisch erhoben wurden. Es sind damit bereits Messansätze vorhanden, derer man sich bedienen kann. Im Rahmen der Operationalisierung der Konstrukte wird die Eignung der, auf der Basis der Spezifizierungsbemühungen entwickelten, Skala zur Erfassung der Konstrukte überprüft. Das Ergebnis der Operationalisierungsmaßnahmen stellt eine reliable und valide Skala dar. Verschiedentlich liegen für die Operationalisierung der Konstrukte mehrere Varianten vor. Dabei sollen zur Erfassung der Konstrukte des vorliegenden grenzüberschreitenden Kundenbindungsmodells Skalen herangezogen werden, die sich durch eine hohe Validität, Reliabilität und Objektivität auszeichnen. Diese drei Komponenten der Messung sind Gütekriterien der Messung erzeugter Daten (Herrmann, Homburg, 1999, S. 23). Sofern die definierten Indikatorensets nur befriedigende Validität, Reliabilität und Objektivitätsdaten liefern, wird eine Verbesserung der Skala angestrebt. Dabei wird in Kauf genommen, dass dies einhergeht mit der Erweiterung des Messansatzes oder mit einem eventuellen Austausch von Items.

\footnotetext{
${ }^{28}$ Im Rahmen eines Seminars erhielten die Studenten der Fontys Internationale Hogeschool Economie die Aufgabe Konsumenten aus dem Nachbarland nach ihren Einkaufsgewohnheiten zu befragen.
} 
Nach Bagozzi und Fornell (1982, S.24) wird ein hypothetisches Konstrukt als „an abstract entity which represents the true, nonobservable state or nature of a phenomenon" definiert. Nach Ohlwein muss die Überprüfung eines Beziehungsgeflechtes nichtbeobachtbarer Variablen, von denen man in einem Kundenbindungsmodell ausgehen kann, durch ein Analyseverfahren erfolgen, das folgende Kriterien erfüllt (Ohlwein, 1999, S.220):

1. Es muss sichergestellt sein, dass kausale Zusammenhänge zwischen hypothetischen Konstrukten berücksichtigt werden.

2. Messfehler müssen im Analyseverfahren explizit berücksichtigt werden können.

3. Im Analyseverfahren müssen Beziehungen zwischen den Variablen abgebildet werden können, die in der Lage sind, zur Erklärung des im Mittelpunkt stehenden Konstruktes beizutragen.

4. Eine simultane Überprüfung der postulierten Hypothesen muss möglich sein (Braunstein, 2001, S. 223).

\subsection{Entwicklung des Prüfschemas}

Der Ablauf der Prüfung des Modells erfolgt in Anlehnung an Eggert, wobei darauf geachtet wird, dass die Ergebnisse der Operationalisierung das Fundament für die sich anschließende Schätzung der Strukturgleichungen bildet. Zur Überprüfung der Reliabilität und der Validität des Messmodells sollen die Methoden der ersten und der zweiten Generation miteinander verbunden werden. Dabei wird im ersten Schritt das Explorationssample mit Hilfe der exploratorischen Faktorenanalyse überprüft. Dies gilt als erster Hinweis auf die bestehende Faktorstruktur. Diese Untersuchungsstufe wird auch nur für die Konstrukte durchgeführt, für die keine hypothetische Faktorenstruktur erkennbar ist. Untersucht wird das hypothetische Konstrukt. Im zweiten Schritt erfolgt die Analyse der hypothetischen Faktoren und Konstrukte. Hierbei wird differenziert zwischen einem mehrfaktoriellen und einem einfaktoriellen Fall. Wird bereits im ersten Prüfschritt eine mehrfaktorielle Struktur vermutet, dann bezieht sich der zweite Prüfschritt auf die identifizierten Faktoren. Die Betrachtung des hypothetischen Konstruktes oder Faktors steht hierbei im Mittelpunkt der Betrachtung. Im dritten Schritt wird 
die Überprüfung der Reliabilität und der Validität durch die Berechnung von Cronbachs Alpha $^{29}$ durchgeführt. Churchill (1979, S.65) definiert Reliabilität als dann gegeben, wenn das Messergebnis frei von zufälligen oder systematischen Fehlern ist. Dabei wird bei zu kleinen Werten der Indikator mit der geringsten Item to Total Korrelation eliminiert. Dabei soll der ermittelte Faktor mindestens 50\% der Indikatorvarianz erklären und keinen Wert unter 0,4 annehmen. Alle Indikatoren mit einer zu kleinen Faktorladung werden eleminiert. Abgeschlossen wird dieser dritte Schritt mit dem Einatz der konfirmatorischen Faktoranalyse inklusive eines Chiquadrattestes. Untersucht wird hierbei das gesamte Messmodell.

Auf der vierten und letzten Stufe des Prüfschemas wird das gesamte Prüfmodell betrachtet. Die verbleibenden Indikatoren werden durch eine exploratorische Faktorenanalyse überprüft und die in den vorgestellten Schritten ermittelte Faktorenstruktur soll bestätigt werden. Durch eine erneute konfirmatorische Faktorenanalyse wird die Konsistenz des Messmodells überprüft. Dies wird durch einen Chiquadrat Test erreicht.

Zwar sollte optimalerweise das Validierungssample mit einer zweiten Befragung einer anderen Zielgruppe wiederholt werden, dies wird aber durch die „known group" Validierung obsolet. Zur Kritik an diesen Validierungsansatz siehe auch Ohlwein (Ohlwein, 1999, S.226). Eine „known group" Validierung (Braunstein, 2001, S. 233; Müller, 1991, S.218 ff.) wird dann durchgeführt, wenn die Gesamtstichprobe aus mehr als 400 Probanden besteht. In dieser Untersuchung gehen mehr als 490 Endkonsumenten in die Befragung ein, die den Bedingungen der Befragung genügen, insgesamt sind mehr als 900 Endkonsumenten befragt worden. Eine Gesamtstichprobe von mehr als 400 ist allerdings auch unerlässlich, da sonst die konfirmatorische Schätzung des Faktormodells keine präzisen Ergebnisse liefert (Braunstein, 2001, S.233). Als untere Grenze der Indikatorreabilität ist ein Anspruchsniveau von 0,4 festgelegt worden.

Hieraus ergibt sich folgende schematische Vorgehensweise:

\footnotetext{
${ }^{29}$ Das Cronbachsche Alpha misst die interne Konsistenz der Indikatoren eines Faktors. Die Größe verkörpert den Mittelwert aller Korrelationen, die sich ergeben, wenn die dem Faktor zugeordneten Indikatoren auf alle möglichen Arten in zwei Hälften geteilt und die Summe der Messwerte der jeweils resultierenden Hälften miteinander korreliert werden (Peter, 1999, S.178).
} 


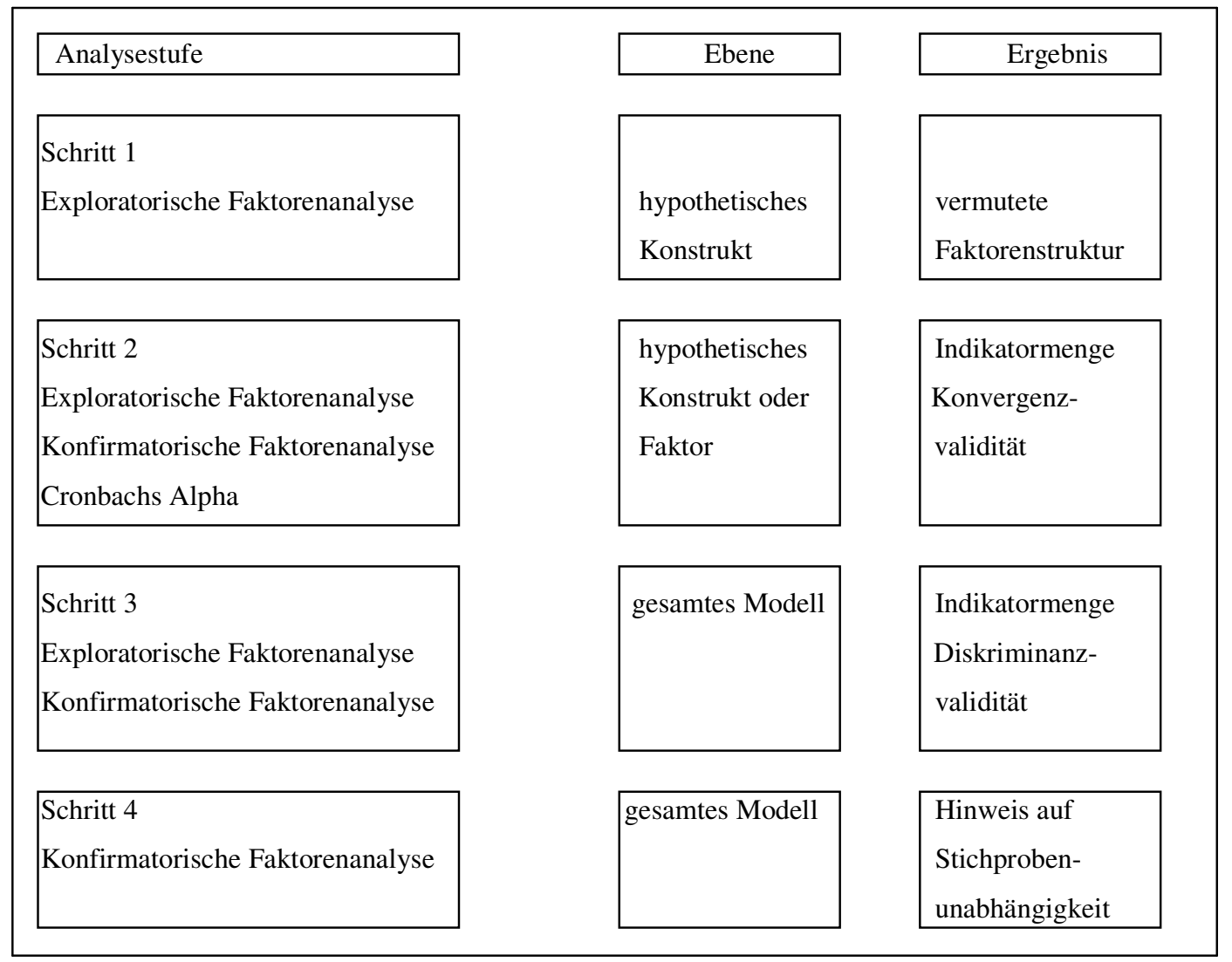

Abb. 6.1: Analyseschritte der Konstruktoperationalisierung (in Anlehnung an Eggert, 1999, S. 113 und Braunstein, 2001, S.234)

\section{Der PLS-Ansatz}

Zur Messung von Kausalmodellen existiert neben der Kovarianzstrukturanalyse mit dem Partial Least Squares-Verfahren (PLS) ein weiteres Verfahren. Die Analyse von Strukturgleichungsmodellen mit Hilfe des PLS-Ansatzes geht auf den schwedischen Ökonometriker Herman Wold zurück (Wold, 1980, S. 47-74). Das Partial Least Sqaures-Verfahren ist ein nicht-parametrisches Testverfahren, für das im Gegensatz zur Kovarianzstrukturanalyse deutlich weniger Restriktionen gelten (Ringle, 2004, S. 1). Beide Ansätze stimmen hinsichtlich formaler Überlegungen zum Strukturmodell überein. Der Unterschied zwischen den beiden Analyseformen liegt in den Schätzmethoden und den anwendbaren Messmodellen für latente exogene Variablen (Ringle, 2004, S.5). Formal setzt sich das PLS-Verfahren aus zwei linearen Gleichungssystemen zusammen. Das Strukturmodell misst die theoretisch vermuteten Beziehungen zwischen latenten Variablen, das Messmodell die Beziehungen zwischen den latenten Variablen und ihren Indikatoren (Greve, 2006, S. 124). Inwieweit die getroffenen 
Annahmen geeignet sind, die Datenlage zu beschreiben, ist die zentrale Bedeutung der Modellschätzung. Allerdings ist aufgrund fehlender empirischer Verteilungsannahmen im Vergleich zur Kovarianzanalyse die Anzahl möglicher Gütemaße wesentlich geringer (Ringle, 2004, S. 13). Die Partial Least Squares Verfahren können nicht über Beurteilungsmaße zur Bestimmung der Anpassungsgüte von Kovarianzen evaluiert werden, es müssen vielmehr schätzungsorientierte Gütemaße, die nicht-parametrisch sind, herangezogen werden. Als Beispiel für solche Maße zur Ergebnisbeurteilung nennt Ringle (2004, S.13) das Bestimmtheitsmaß latenter endogener Variablen, den Stone-Geisser Test zur Bestimmung der Schätzrelevanz sowie die auf Fornell und Larcker zurückgehende faktoranalytische Bestimmung der durchschnittlich extrahierten Varianz. Die Stabilität der Schätzung lässt sich durch das Verfahren Bootstrapping zur systematischen Veränderung der empirisch erhobenen Daten überprüfen (Gefen, Straub, und Boudreau, 2000, S. 1-78). Allerdings wird in der Literatur kein einheitliches systematisches Vorgehen zur Modellbeurteilung empfohlen. Aus diesem Grund schließt sich der Autor der Empfehlung von Ringle (2004, S. 14) an und geht folgendermaßen vor:

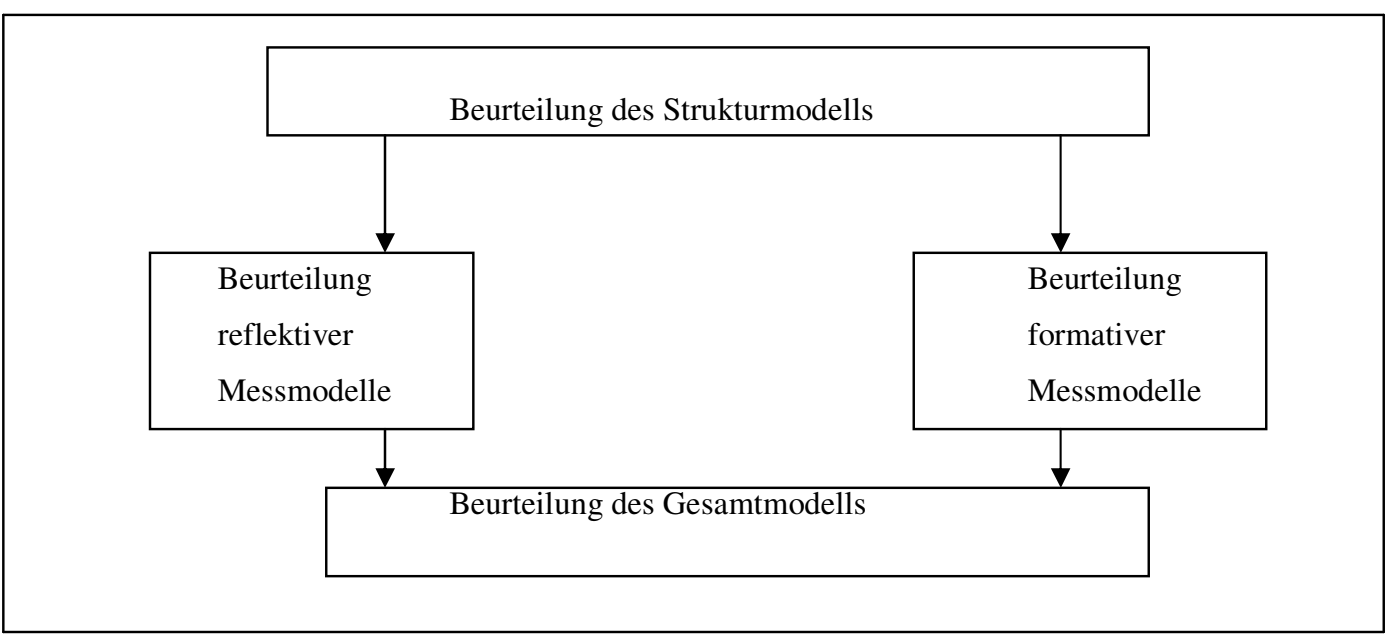

Abb. 6.2: $\quad$ Annahmen im Partial Least Squares-Modell (Ringle, 2000, S.14)

In einem reflektiven Messmodell verursacht die latente Variable ihre zugeordneten Indikatoren. Als Beispiel für reflektive Messmodelle werden in der Literatur insbesondere verhaltensbasierte Zusammenhänge wie „Einstellung“ oder „Persönlichkeit“ angegeben (Diamantopoulos und Winklhofer, 2001, S. 270). In einem formativen Messmodell verursachen die zugeordneten Indikatoren die latente Variable (Diamantopoulos und Winklhofer, 2001, S. 269f). Als Beispiel eines formativen Messmodells geben die Autoren den Marketing Mix bestehend aus Produkt-, Preis-, Distributions- und Kommunikationspolitik an. In einem 
PLS-Modell können die Beziehungen zwischen latenten Variablen und ihren Indikatoren formativ oder reflektiv modelliert werden. In einem reflektiven Messmodell verursacht die latente Variable ihre zugeordneten Indikatoren (Greve, 2006, S.126). Die Pfeilrichtung zwischen latenten Variablen und Indikatoren verläuft von Variable zu Indikator. Wird der Wert der latenten Variablen verändert, ändern sich alle ihre Indikatoren in die gleiche Richtung. Dagegen verursachen in einem formativen Messmodell die zugeordneten Indikatoren die latente Variable. Die Pfeilrichtung zwischen latenten Variablen und Indikatoren verläuft von den Indikatoren zu latenten Variablen. Aus diesem Grund messen Indikatoren formativer Messmodelle nicht den gleichen Sachverhalt, sondern unterschiedliche Facetten der latenten Variablen (Greve, 2006, S.128). Die Validität formativer Messmodelle werden beurteilt ausschließlich unter dem Gesichtpunkt der Inhaltsvalidität, die Konstruktvalidität spielt eine untergeordnete Rolle (Diamantopoulos, Winkelhofer, 2001, S.300)

Mit Hilfe des Partial Least Squares-Ansatzes können latente Variablen als eine exakte lineare Kombination ihrer Indikatoren gemessen werden. Dies geschieht mit dem Ziel, die erklärenden Varianzen der Indikatoren und der latenten Variablen zu maximieren (Chin, Marcolin, und Newsted, 1996, S. 21.ff). Durch die partielle Schätzung einzelner Elemente des Kausalmodells werden für die Ermittlung verlässlicher Ergebnisse mit dem Partial Least SquaresVerfahren weniger empirisch erhobene Fälle benötigt als für die Kovarianzstrukturanalyse (Chin, und Newsted, 1999, S. 314 und 326). Durch den Gruppenvergleich kann der Einfluss von Variablen wie Geschlecht, Alter oder auch Herkunftsland untersucht werden. Aus diesem Grund wird dieses Programm auch in der vorliegenden Untersuchung Anwendung finden. Durch dieses Vorgehen soll sichergestellt werden, dass ein Erklärungsmodell entsteht, welches die Realität widerspiegelt. Dazu werden den einzelnen Konstrukten folgende Indikatoren zugeordnet: 


\begin{tabular}{|c|c|c|}
\hline Konstrukt & Indikatoren & Bedeutung \\
\hline TZSN & vr032 vr033 vr034 & Werturteile der sozialen Gruppe \\
\hline WSN & vr04 vr06 & Wichtigkeit der sozialen Norm, d.h. Freunde und Bekannte kaufen viel im Nachbarland und empfehlen es oft \\
\hline GKDZ SN & Vr05 vr10.1 & Bedeutung der sozialen Norm für den Konsumenten \\
\hline $\begin{array}{l}\text { GKDZ } \\
\text { Kauf }\end{array}$ & vr081 vr082 vr083 & Gesamtkundenzufriedenheit mit Stammgeschäft im Nachbarland \\
\hline GLSS & Vr07 vr08 & Gesamtzufriedenheit mit dem Einkauf im Nachbarland \\
\hline $\mathrm{PN}$ & vr091 vr111 & Persönliche Norm \\
\hline WVK & vr131 & Wahrgenommene Verhaltenskontrolle \\
\hline WRS & vr141 vr151 vr161 & Wahrgenommene Selbstrelevanz (Lust auf Einkaufen im Nachbarland) \\
\hline KL & vr17 vr18 vr22 & Kundenloyalität (Treue zum Stammgeschäft) \\
\hline BINDUNG & vr19 vr20 vr21 & Kundenbindung (häufiges Einkaufen im Nachbarland in ener Einkaufsstätte) \\
\hline
\end{tabular}

Tab. 6.2: $\quad$ Konstrukte, Indikatoren und ihre Bedeutung im Rahmen des Strukturgleichungsmodells

\subsection{Beurteilung des Strukturmodells}

Chin (1998, S. 316ff.) stellt einen sehr umfangreichen Beurteilungskatalog für Partial Least Squares-Modelle vor. Mit Hilfe der statistischen Software SmartPLS können die generierten Ergebnisse berechnet werden. So lässt sich für das Strukturmodell mittels PLS geschätzten Kausalmodells das Bestimmtheitsmaß $\mathrm{R}^{2}$ ermitteln. Dazu sagen Chin und Newsted (1999, S.316), "This is obtained because the case values of latent variables are determined by the weight relations." Ringle (2004, S.15) ergänzt dazu: "Die latente endogene Variable ist in einem solchen multiplen linearen Regressionsmodell die abhängige Variable (Regressand, während die latenten exogenen Variablen die unabhängigen Variablen (Regressoren) darstellen und das Bestimmtheitsmaß den über die lineare Regressionsgleichung bestimmten Anteil der erklärten Varianz an der Gesamtvarianz angibt." Sowohl für die Beurteilung des Be- 
stimmtheitsmaßes $\mathrm{R}^{2}$ als auch für die geschätzten Werte der Regressionskoeffizienten liegen unterschiedliche Empfehlungen vor. Chin (1998, S. 323) gibt folgende Richtwerte vor:

$\mathrm{R}^{2} \quad 0,67 \quad$ „substantiell“

0,33 „durchschnittlich“

0,19 „schwach“

Ein weiteres Beurteilungsmaß des Modells ist die Effektstärke $f^{2}$. Mit Hilfe der Effektstärke lässt sich untersuchen, ob eine unabhängige (exogene) latente Variable einen substantiellen Einfluß auf abhängige (endogene) latente Variablen ausübt. Entsprechend der für die multiple Regressionsanalyse operationalisierten Definition von Cohen (1988, S. 412 ff) kann folgende Unterteilung vorgenommen werden:

$f^{2}$ Werte

0,02 geringer Einfluß

0,15 mittlerer Einfluß

0,35 großer Einfluß

Die einzelnen Pfadkoeffizienten des Strukturmodells können wie standardisierte BetaKoeffienten interpretiert werden. In der Literatur wird eine Höhe der Pfadkoeffizienten von 0.1 gefordert (Hulland, 1999, S. 198). Die Reliabilität bezüglich der Parameterschätzungen wird anhand der t-Statistiken und der entsprechenden Signifikanzen überprüft, die mit Hilfe des Bootstapping gewonnen werden können. Ferner hat neben einer Überprüfung der latenten Konstrukte im Strukturmodell eine Überprüfung zu erfolgen, ob es sich bei deren zueinander bestehenden gerichteten Beziehungen und den dafür berechneten Gewichten um statistisch signifikante Ergebnisse handelt. Dazu kommt das Bootstapping, ein nicht-parametrisches Verfahren zum Einsatz, mit dem sich die Qualität von Partial Least SquaresSchätzergebnissen beurteilen lässt, ohne bestimmte Verteilungsannahmen zu treffen (Ringle, 2004, S.18). Über alle Subsamples lassen sich dann Erwartungswerte und Standardabweichungen eines jeden Modellparameters schätzen, wodurch für die Beurteilung des vollständigen Partial Least Squares-Modells robuste Konfidenzintervalle ermittelt sowie mittels eines tTests die jeweilige Signifikanz der Schätzergebnisse bestimmt werden können.

Nach Greve (2006, S. 134) kann die Prognoserelevanz des PLS-Modells anhand einer Kreuzvalidierung überprüft werden. Die Stichprobe wird in ein Schätzsample und ein Validierungssample geteilt. Das PLS-Modell wird anhand des durch eine Zufallsauswahl gezogenen Schätzsamples erneut geschätzt. Danach werden die Schätzparameter mit t-Werten kleiner eins eliminiert, da sie keinen weiteren Beitrag zur Prognose leisten. „Die Prognosevalidität 
wird über die Korrelation zwischen den errechneten Werten der latenten endogenen Variablen des Schätzsamples und den beobachteten Werten dieser Variablen das Validierungssamples beurteilt" (Greve, G.,2006, S. 134). Nach Chin und Todd sollte der quadrierte Korrelationskoeffizient $\mathrm{r}^{2}$ nicht wesentlich vom $\mathrm{R}^{2}$-Wert der latenten endogenen Variablen des Schätzsamples abweichen. Je geringer die Abweichung von $\mathrm{r}^{2}$ und $\mathrm{R}^{2}$, umso höher die Prognosekraft des Modells (Chin, W. und Todd, P., 1995, S. 238).

Insgesamt ergibt sich folgende Gütebeurteilung des Strukturmodells:

\begin{tabular}{|c|c|c|}
\hline Gütemaß & Definition & Kritischer Wertebereich \\
\hline Bestimmtheitsmaß $\mathrm{R}^{2}$ & $\begin{array}{l}\text { Anteil der erklärten Varianz der endogenen } \\
\text { Variablen }\end{array}$ & $\begin{array}{ll}\text { Werte von } & \\
0,67 \quad \text { „substantiell“ } \\
0,33 \quad \text { „durchschnittlich“ } \\
0,19 \quad \text { „schwach“ }\end{array}$ \\
\hline Effektstärke & $\begin{array}{l}\text { Substanzieller Einfluss der exogenen Vari- } \\
\text { ablen auf die endogenen Variablen }\end{array}$ & \begin{tabular}{ll}
\multicolumn{2}{l}{ Werte von } \\
$0,02 \quad$ geringer Einfluß \\
$0,15 \quad$ mittlerer Einfluß \\
$0,35 \quad$ großer Einfluß
\end{tabular} \\
\hline 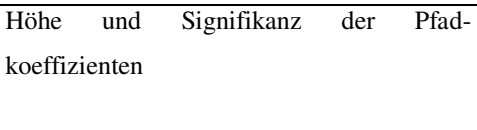 & $\begin{array}{l}\text { Stärke der Wirkungsbeziehungen zwischen } \\
\text { den Konstrukten }\end{array}$ & $\begin{array}{l}\text { Pfadkoeffizient }>0,1 \\
\text { Beurteilung der Reliabilität anhand der t- } \\
\text { Statistik }\end{array}$ \\
\hline Prognosegüte & $\begin{array}{l}\text { Anpassung des Modells an die empirischen } \\
\text { Daten }\end{array}$ & $\begin{array}{l}\text { Kreuzvalidierung, Quadrierter Korrelati- } \\
\text { onskoeffizient des Validierungssamples } \mathrm{r}^{2} \\
\text { möglichst gleich } \mathrm{R}^{2} \text { der endogenen Variab- } \\
\text { len des Schätzsamples }\end{array}$ \\
\hline Communality-Q ${ }^{2}$ (Stone-Geisser-Test) & $\begin{array}{l}\text { Vorhersagegüte tatsächlich beobachtbarer } \\
\text { Werte ist von wesentlich höherer Relevanz } \\
\text { wie die die Schätzung artifizieller Kon- } \\
\text { struktparameter }\end{array}$ & $\mathrm{Q}^{2}>0$ \\
\hline
\end{tabular}

Tab. 6.3: $\quad$ Gütebeurteilung des Strukturmodells (Greve, G., 2006, S.135)

\section{Evaluierung reflektiver Messmodelle}

Die latente Variable verursacht in einem reflektiven Messmodell ihre zugeordneten Indikatoren. Die Pfeilrichtung zwischen latenten Variablen und Indikatoren verläuft von der Variable zu den Indikatoren. Zur Beurteilung der reflektiven Messmodelle werden die Maße zur Beurteilung der Indikator- bzw. Faktorreliabilität sowie der durchschnittlich erfassten Varianz herangezogen (Völckner, 2003, S.166 ff). Im Vergleich zu Cronbachs Alpha gelten für die Faktorreliablität nach Chin (1998, S. 320) folgende Implikationen: „,.....this measure does not assume tau equivalency among the measures with ist assumption that all indicators are equally weighted. Therefore, alpha tends to be lower bound estimate of reliability, whereas Pc is a 
closer approximation under the assumption that the parameter estimates are accurate." Die Faktorreabilität sollte nach Homburg und Baumgartner ( 1998, S.69) den Wert 0,6 nicht unterschreiten.

Ein weiteres Verfahren ist die von Fornell und Larcker (1981, S. 45) zur Beurteilung geschätzter reflektiver Modelle vorgeschlagene Bestimmung der durchschnittlich erfassten Varianz (AVE). Dieses Maß sollte einen Wert von 0,5 nicht wesentlich unterschreiten (Homburg und Baumgartner, 1998, S. 361).

Das Verfahren Bootstrapping liefert wiederum Erwartungswerte und Standardabweichungen für die einzelnen Faktorladungen im reflektiven Messmodell. Es lassen sich somit für diese Ergebnisse robuste Konfidenzintervalle ermitteln und mittels eines t-Tests die jeweilige Signifikanz der Schätzergebnisse bestimmen (Ringle, 2004, S. 21). Zur Beurteilung des Gesamtmodells existiert bisher allerdings noch kein globales Gütemaß, das sich gemäß eines „Goodness of Fit Index“ (GFI) für die Überprüfung des Modells insgesamt einsetzen lässt. In Anlehung an Greve werden folgende Gütemaße reflektiver Messmodelle festgelegt:

\begin{tabular}{|l|l|l|l|}
\hline Güteart & Gütemaß & Bezug & Anspruchsniveau \\
\hline Inhaldsvalidität & $\begin{array}{l}\text { Einfakturelle } \\
\text { Faktorstruktur }\end{array}$ & Faktor & $\begin{array}{l}\text { Explorative Faktoranalyse zur Untersuchung } \\
\text { der Dimensionen }\end{array}$ \\
\hline Indikatorreliabilität & $\begin{array}{l}\text { Erklärter } \\
\text { Varianzanteil }\end{array}$ & Faktor & $>50 \%$, d.h. Faktorladungen $\lambda>0,7$ \\
\hline Indikatorreliabilität & $\begin{array}{l}\text { Faktorladungs- } \\
\text { struktur }\end{array}$ & Faktor & $\lambda>0,4$, mit deutlich niedrigeren Querladungen \\
\hline Konstruktvalidität & Cronbachs Alpha & Faktor & $\alpha>0,7$, für erstmalig verwendete Skalen $\alpha>0,5$ \\
\hline Konstruktvalidität & Interne Konsistenz & Messmodell & $>0,6$ \\
\hline Diskriminanzvalidität & $\begin{array}{l}\text { Durchschnittlich } \\
\text { erfasste Varianz }\end{array}$ & Messmodell & $\begin{array}{l}\text { Durchschnittlich erfasste Varianz }>\text { quadrierte } \\
\text { Korrelationen aller latenten Variablen }\end{array}$ \\
\hline Reliabilität & Cronbachs Alpha & Faktor & $\alpha>0,7$ \\
\hline Reliabilität & $\begin{array}{l}\text { Item-to-total- } \\
\text { Korrelation }\end{array}$ & Indikator & Möglichst hohe Korrelationen \\
\hline
\end{tabular}

Tab. 6.4: $\quad$ Gütemaße reflektiver Messmodelle (Greve, G., 2006, S.140)

\section{Evaluierung formativer Messmodelle}

In einem formativen Messmodell verursachen dagegen die zugeordneten Indikatoren die latente Variable. Die Pfeilrichtung verläuft von den Indikatoren zur latenten Variable 
(Diamantopoulos und Winkelhofer, 2001, S.269). Die Indikatoren eines formativen Messmodells bilden die inhaltlichen Dimensionen der latenten Variablen ab und sind im Gegesatz zu reflektiven Indikatoren nicht austauschbar (Greve,G., 2006, S. 141). Aus diesem Grund lassen sich die vorgestellten Verfahren zur Gütebeurteilung reflektiver Messmodelle nicht auf formative übertragen. In der vorliegenden Arbeit werden die Indikatoren reflektiv gemessen. Deshalb wird auf die Evaluierung formativer Messmodelle nicht weiter eingegangen.

\subsection{Datenerhebung im Befragungsgebiet}

Im Rahmen der quantitativen Datenerhebung werden Endkonsumenten in mehreren grenznahen Städten befragt. Hierbei sind bewusst sowohl große als auch kleine Städte mit in die Datenerhebung einbezogen worden. Die Befragung wurde zudem auf zwei Einkaufstage konzentriert, und zwar auf den Donnerstag und den Samstag. Beide Tage gelten im Einzelhandel als besonders umsatzstark. Diese Tage wurden in der Befragung aufgenommen, da an diesen Tagen eine hohe Innenstadtfrequenz erwartet wird. In allen Orten sollten an jedem festgelegten Tag mindestens 20 Interviews durchgeführt werden. Hieraus ergeben sich mindestens 200 Interviews in niederländischen Städten und mindestens 200 Interviews in deutschen Städten. Die Befragung wurde durchgeführt in der Zeit von September bis November 2004. Für die Befragung ergibt sich folgende Aufteilung der Befragungsorte: 


\begin{tabular}{|c|c|c|c|c|c|}
\hline $\begin{array}{l}\text { Befragungsort } \\
\text { Niederlande }\end{array}$ & Befragungstag & $\begin{array}{l}\text { Anzahl } \\
\text { Befragungen }\end{array}$ & $\begin{array}{l}\text { Befragungsort } \\
\text { Deutschland }\end{array}$ & Befragungstag & $\begin{array}{l}\text { Anzahl } \\
\text { Befragungen }\end{array}$ \\
\hline \multirow[t]{2}{*}{ Maastricht } & Donnerstag & 20 & \multirow[t]{2}{*}{ Aachen } & Donnerstag & 20 \\
\hline & Samstag & 20 & & Samstag & 20 \\
\hline \multirow[t]{2}{*}{ Heerlen } & Donnerstag & 20 & \multirow[t]{2}{*}{ Heinsberg } & Donnerstag & 20 \\
\hline & Samstag & 20 & & Samstag & 20 \\
\hline \multirow[t]{2}{*}{ Sittard } & Donnerstag & 20 & \multirow[t]{2}{*}{ Viersen } & Donnerstag & 20 \\
\hline & Samstag & 20 & & Samstag & 20 \\
\hline \multirow[t]{2}{*}{ Roermond } & Donnerstag & 20 & \multirow[t]{2}{*}{ Kaldenkirchen } & Donnerstag & 20 \\
\hline & Samstag & 20 & & Samstag & 20 \\
\hline \multirow[t]{2}{*}{ Venlo } & Donnerstag & 20 & \multirow[t]{2}{*}{ Mönchengladbach } & Donnerstag & 20 \\
\hline & Samstag & 20 & & Samstag & 20 \\
\hline Total & & 200 & & & 200 \\
\hline
\end{tabular}

Tab. 6.5: $\quad$ Aufteilung der Befragung nach Befragungsort und Befragungstag (eigene Darstellung).

Durch diese Aufteilung wird sichergestellt, dass gleichgewichtig Fragebögen von befragten Probanden in die Auswertung eingehen, die sowohl als Einkaufstouristen in das jeweilige Land gekommen sind, als auch wiederholt im gleichen Einzelhandelsgeschäft einkaufen. Gleichzeitig wird durch diese Vorgehensweise sichergestellt, dass Konsumenten aus einem großen regionalen Gebiet des deutsch-niederländischen Grenzgebietes befragt wurden. Ebenfalls wurde durch diese Vorgehensweise berücksichtigt, dass sowohl Konsumenten in kleinen als auch in größeren grenznahen Städten befragt wurden und dadurch auch unterschiedliche Gewohnheiten der Konsumenten in diesen Einkaufsstätten untersucht werden konnten. Insgesamt wurden 973 Konsumenten befragt. Auf Grund der zwei Filterfragen

Frage 1 Sind Sie zum Einkauf nach Deutschland/in die Niederlande gekommen? Frage 2 Kaufen Sie öfter in (Ort der Befragung) im gleichen Geschäft ein?

wurde sichergestellt, dass nur Käufer in die Befragung eingehen, die mehrmals in der gleichen Einkaufsstätte eingekauft haben. Durch diese Entscheidungsfragen reduzieren sich die zur Verfügung stehenden Fragebögen auf 504. Ferner wurden deutsche Konsumenten in den niederländischen Städten befragt und niederländische Konsumenten in den deutschen Städten. In die Befragung gingen allerdings auch Konsumenten ein, die nicht niederländischer oder 
deutscher Nationalität waren. Diese wurden ebenfalls aus der Befragung genommen, sodass sich die Menge der Fragebögen auf 498 reduzierte. Ferner ist durch den Mix von größeren und kleineren Städten gewährleistet, dass unterschiedliche Loyalitätsverhalten der Konsumenten überprüft werden können. Für das Explorationsexample wurden die Endkonsumenten nach dem Zufallsprinzip an unterschiedlichen Haupteinkaufsstraßen der verschiedenen Orte befragt. Insgesamt waren das 245 niederländische Konsumenten in deutschen Städten und 253 deutsche Konsumenten in niederländischen Städten.

\begin{tabular}{|l|l|l|l|}
\hline & Niederländer & Deutsche & Total \\
\hline männlich & $\mathbf{8 1}$ & $\mathbf{1 0 2}$ & $\mathbf{1 8 3}$ \\
\hline Bis 35 & 32 & 42 & 74 \\
\hline $35-64$ & 30 & 45 & 75 \\
\hline Über 64 & 19 & 15 & 34 \\
\hline weiblich & $\mathbf{1 6 4}$ & $\mathbf{1 5 1}$ & $\mathbf{3 1 5}$ \\
\hline Bis 35 & 79 & 62 & 141 \\
\hline $35-64$ & 71 & 72 & 143 \\
\hline Über 64 & 14 & 17 & 31 \\
\hline Total & 245 & 253 & 498 \\
\hline
\end{tabular}

Tab. 6.6: $\quad$ Aufteilung der befragten Konsumenten nach Nationalität, Geschlecht und Alter (eigene Darstellung)

Im Rahmen der Auswertung wurde mit der Ratio-Skala gearbeitet. Die Endkonsumenten wurden gebeten, das Ausmaß ihrer Zustimmung oder Ablehnung zu einer Reihe von vorgegebenen Aussagen auf einer Skala von eins - Stimme ich voll zu - bis fünf - stimme ich gar nicht zu - anzugeben. Als sechste Skala wurde noch die Antwort - trifft nicht zu - eingefügt. Diese Vorgehensweise wurde gewählt, um das Diskriminierungsvermögen der Probanden nicht zu überfordern. 


\subsection{Zusammenfassung des Kapitels}

Im Rahmen der durchgeführten Untersuchung wurden zunächst mit Hilfe strukturierter Interviews (mit Hilfe von Focus-Gruppen) die für die Untersuchung relevanten Fragestellungen konkretisiert. Im Anschluß daran ist ein Fragebogen erstellt und in einem Pretest überprüft worden. Nach Überarbeitung des Fragebogens wurden 245 niederländische Konsumenten in Deutschland und 253 deutsche Konsumenten in den Niederlanden befragt. Die Interviews wurden in Deutschland von niederländischen und in den Niederlanden von deutschen Interviewern durchgeführt. Damit wurden sprachliche Probleme vermieden. In der Befragung wurden 153 männliche Probanden und 315 weibliche Probanden befragt. Das Verhältnis der Geschlechter spiegelt das reale Kaufverhalten wider. Ferner wurden alle Altersgruppen in die Befragung aufgenommen, wobei auch hier Wert auf die Proportionalität gelegt wurde. Aus diesem Grund setzte sich die Probandengruppe wie folgt zusammen:

- 215 Probanden jünger als 35 Jahre,

- 218 Probanden zwischen 35 und 64 Jahre und

- 65 Probanden über 65 Jahre.

Die Befragung wurde an zwei unterschiedlichen Wochentagen durchgeführt. Der Donnerstag und der Samstag wurden deshalb genommen, da diese Tage zu den umsatzstärksten Wochentagen des Einzelhandels zählen. Die Befragung wurde im Grenzgebiet zwischen Maastricht und Aachen sowie zwischen Venlo und Mönchengladbch sowohl in kleinen Grenzstädten als auch in Oberzentren durchgeführt.

In der empirischen Wissenschaft sind reliable und valide Messungen eine Grundvoraussetzung. Deshalb war es notwendig, Gütekriterien und Realibilitätskoeffizienten für das Prüfschema festzulegen. Allerdings bestehen keine allgemein gültigen, konkrete Grenzwerte. Auch lässt ein als gut klassifizierter Fit noch keine konkreten Aussagen über die Qualität der einzeln geschätzten Modellparameter zu. Aus diesem Grund ist es Ziel in der im nächsten Kapitel beschriebenen Überprüfung des Modells im Feld, möglichst wenige Anspruchsniveaus der Gütekriterien zu verletzen und ein hohes Anspruchsniveau der Gütekriterien zu erreichen.

Die Messung der Kausalmodelle erfolgt mit dem Partial-Least Squares-Verfahren (PLS), da hiermit latente Variablen als eine exakte lineare Kombination ihrer Indikatoren gemessen werden können. Durch den Gruppenvergleich kann der Einfluß von Variablen wie Geschlecht oder Herkunftsland untersucht werden. 
$150 \mid$ Kapitel 6 


\section{Kapitel 7: $\quad$ Überprüfung des Modells im Feld}

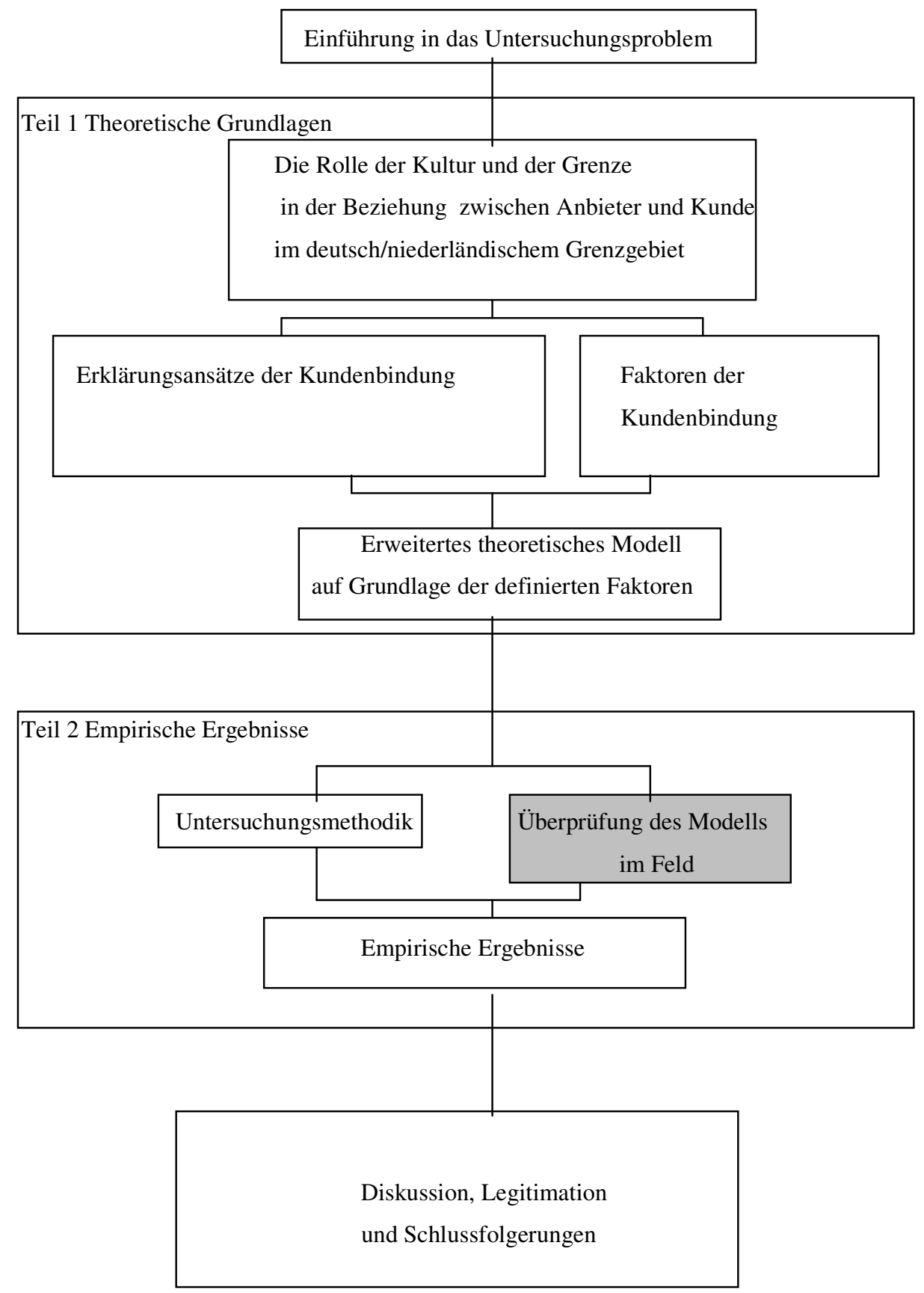




\section{1 $\quad$ Einleitung}

In Kapitel 7 werden die statistischen Analysen zur Verifizierung und Falsifizierung des aufgestellten Modells in der Praxis vorgenommen. Dazu werden die Daten mit Hilfe des Programms SPSS auf ihre Reliabilität überprüft und im Rahmen der deskriptiven Statistik durch eine Faktoren- und einer Clusteranalyse untersucht. Im zweiten Teil des Kapitels werden mit Hilfe des Programms PLS die unterschiedlichen Probandengruppen mit Hilfe von Strukturgleichungsmodellen analysiert. Um die Aussagen der Analyse deutlich und anschaulich darzustellen, werden zunächst die Ergebnisse der niederländischen Probanden dargestellt, anschließend die der deutschen Probanden. Dazu werden die einzelnen Nationalitäten hinsichtlich der Verteilung soziodemographischer Merkmale analysiert. Die Analyse erfolgt im Rahmen der deskriptiven Statistik und der relationalen Statistik ${ }^{30}$. Auch im Rahmen der Datenanalyse mit Hilfe des Programms PLS werden zunächst beide Probandengruppen getrennt betrachtet, anschließend miteinander verglichen.

\subsection{Statistische Analysen der Ergebnisse}

Bevor das aufgestellte Modell zur Erklärung des grenzüberschreitenden Einkaufverhaltens im deutsch-niederländischen Raums analysiert wird, soll untersucht werden, ob die bei der Befragung von deutschen und niederländischen Konsumenten aufgetretenen Mittelwertunterschiede sich mit zufälligen Schwankungen erklären lassen. Anschließend werden die Häufigkeitsverteilungen und die in der deskriptiven Statistik beobachteten Verhaltensweisen analysiert. Im Rahmen der Überprüfung inwieweit sich beide Konsumentengruppen signifikant voneinander unterscheiden, kann durch den t-Test festgestellt werden, dass die Verhaltensunterschiede der beiden Gruppen auf nationale Unterschiede und der Zugehörigkeit zu unterschiedlichen Nationalitäten zurückzuführen sind.

\footnotetext{
${ }^{30}$ Es wird unterstellt, dass die Ausgangsdaten intervallskalliert sind. Ziel der Untersuchung ist es den innerlichen Zweck zu erfahren, der den beobachteten Variablen zugrunde liegt (Green und Tull, 1982, S. 392). Multivariaten Verfahren wird in der Literatur eine hohe Bedeutung zugeordnet). Die Kreuztabellierung ist die einfachste Form der Analyse von Zusammenhängen (Green und Tull, S. 253-254).
} 


\subsubsection{Analyse der befragten niederländischen Konsumenten}

Die insgesamt 245 niederländische Probanden werden zunächst untersucht hinsichtlich ihrer soziodemographischen Aufteilung nach Geschlecht, Alter, Haushaltsgröße und Nettoeinkommen. Aus dieser Aufteilung ergibt sich folgendes Bild:

\begin{tabular}{|c|c|c|c|c|c|c|c|c|c|c|c|}
\hline \multirow[t]{2}{*}{ Nationalität } & \multicolumn{2}{|c|}{ Geschlecht } & \multirow{2}{*}{$\begin{array}{l}\text { Alter } \\
\text { bis } 34\end{array}$} & \multirow[b]{2}{*}{$35-64$} & \multirow[b]{2}{*}{65 u.ä. } & \multicolumn{3}{|c|}{ Personen pro Haushalt } & \multicolumn{3}{|c|}{ Netto Einkommen in $€$} \\
\hline & $\mathbf{M}$ & $\mathbf{W}$ & & & & \multicolumn{3}{|c|}{ und mehr } & $>2000$ & $>3500$ & $<3500$ \\
\hline NL & 32,9 & 67,1 & 45,3 & 41,2 & 13,6 & 20,0 & 43,3 & 36,7 & 50,2 & 32,7 & 17,1 \\
\hline
\end{tabular}

Tab. 7.1: $\quad$ Die soziodemografische Verteilung der befragten niederländischen Konsumenten

Bei den befragten Niederländern ist die Gruppe der jungen Konsumenten bis 34 Jahre die altersmäßig stärkste Gruppe. Besonders stark vertreten sind auch die zwei Personen Haushalte. Werden die Einkommensverhältnisse in Limburg generell betrachtet, so ergeben sich folgende Zahlen:

\begin{tabular}{lll|}
\hline Ort & $\mathbf{1 9 9 9}$ & $\mathbf{2 0 0 0}$ \\
Brunsum & $9.700,-$ & $10.200,-$ \\
Heerlen & $10.000,-$ & $10.500,-$ \\
Kerkrade & $10.100,-$ & $10.700,-$ \\
Maastricht & $10.100,-$ & $10.600,-$ \\
Roermond & $10.200,-$ & $10.800,-$ \\
Sittard-Geleen & $10.200,-$ & $10.800,-$ \\
Venlo & $10.100,-$ & $10.500,-$ \\
Venray & $9.600,-$ & $10.400,-$ \\
Weert & $10.300,-$ & $10.700,-$ \\
Limburg & $\mathbf{1 0 . 1 0 0 , -}$ & $\mathbf{1 0 . 3 0 0 , -}$ \\
\hline
\end{tabular}

Tab. 7.2: $\quad$ Verfügbares Einkommen der Einwohner des niederländischen Befragungsgebietes im Jahre 2000 im Vergleich zu 1999, umgerechnet in Euro (CBS, bewerking E,til BV, 2002)

Im Rahmen der Befragung wurden die Probanden nach ihrer Haushaltsgröße, sowie nach ihren Einkommensverhältnissen befragt. Insbesondere die Befragung nach dem Haushaltsnetto- 
einkommen ergab eine Übereinstimmung mit den allgemeinen Zahlen über das verfügbare Einkommen der Konsumenten aus der niederländischen Grenzregion aus dem Jahre 2000.

\begin{tabular}{|c|c|c|c|}
\hline & Ihl Personen im Haushalt & & \\
\hline \multirow{4}{*}{ Gültig } & 1 Person Haushalt & Häufigkeit & Prozent \\
\hline & 2 Personen Haushalt & 106 & 43,3 \\
\hline & 3 Personen und mehr Haushalt & 90 & 36,7 \\
\hline & Gesamt & 245 & 100,0 \\
\hline \multirow{6}{*}{ Gültig } & haltsnettoeinkommen pro Mo & & \\
\hline & & Häufigkeit & Prozent \\
\hline & bIs € 2000 & 123 & 50,2 \\
\hline & bis $€ 3500$ & 80 & 32,7 \\
\hline & über $€ 3500$ & 42 & 17,1 \\
\hline & Gesamt & 245 & 100,0 \\
\hline
\end{tabular}

Tab. 7.3: $\quad$ Verteilung der Personen pro Haushalt der befragten niederländischen Konsumenten sowie Verteilung der Haushaltsnettoeinkommen

Von den befragten niederländischen Konsumenten leben 20,0\% in Singlehaushalten, 43,3\% in Zweipersonenhaushalte und 36,7\% in Haushalten mit drei und mehr Mitgliedern. Das durchschnittliche Haushaltsnettoeinkommen liegt bei 50,2\% der Befragten unter $€$ 2000,- pro Monat. Nur $17,1 \%$ können zu den besser verdienenden mit über $€ 3500$,- Haushaltsnettoeinkommen gezählt werden.

\begin{tabular}{|c|c|c|c|c|c|}
\hline \multicolumn{2}{|c|}{ Niederländische Konsumenten } & \multicolumn{3}{|c|}{ Haushaltsnettoeinkommen } & \multirow[t]{2}{*}{ Gesamt } \\
\hline Anzahl & & bis $€ 2000$ & bis $€ 3500$ & $\begin{array}{l}\text { mehr als } \\
€ 3500\end{array}$ & \\
\hline \multirow{3}{*}{$\begin{array}{l}\text { Anzahl Personen im } \\
\text { Haushalt }\end{array}$} & & 34 & 8 & 7 & 49 \\
\hline & 2 & 59 & 31 & 16 & 106 \\
\hline & mehr als 3 & 30 & 41 & 19 & 90 \\
\hline \multicolumn{2}{|l|}{ Gesamt } & 123 & 80 & 42 & 245 \\
\hline
\end{tabular}

Tab. 7.4: $\quad$ Vergleich niederländischer Nettoeinkommen nach Haushaltsgröße

Nach dem Herkunftsort gefragt, ergibt sich für die niederländischen Probanden folgendes Bild: 


\begin{tabular}{|l|r|r|}
\hline Herkunftsort der Konsumenten & Häufigkeit der Nennung & Anzahl in Prozent \\
\hline Almere & 5 & 2,0 \\
Brunssum & 7 & 2,9 \\
Deurne & 1 & 0,4 \\
Eindhoven & 1 & 0,4 \\
Heerlen & 27 & 11,0 \\
Helmond & 1 & 0,4 \\
Hertogenbosch & 1 & 0,4 \\
Katwijk & 1 & 0,4 \\
Maarssen & 1 & 0,4 \\
Maastricht & 10 & 4,1 \\
Naaldwijk & 3 & 1,2 \\
Roermond & 20 & 8,2 \\
Rotterdam & 3 & 1,2 \\
Schiedam & 2 & 0,8 \\
Sittard & 16 & 6,5 \\
Veldhoven & 3 & 1,2 \\
Venlo & 82 & 33,5 \\
Venray & 24 & 9,8 \\
Weert & 37 & 15,1 \\
\hline Gesamt & 245 & 100,0 \\
\hline
\end{tabular}

Tab. 7.5: $\quad$ Herkunftsort der in Deutschland befragten niederländischen Konsumenten

Die meisten grenzüberschreitend einkaufenden niederländischen Konsumenten kommen aus Venlo, gefolgt von Weert, Heerlen, Venray und Roermond. Bei den Niederländern findet grenzüberschreitender Einkauf aus sehr grenznahen Gebieten statt. Interessant ist nun die Frage wo die Konsumenten einkaufen gehen. Darauf gibt folgende Übersicht Auskunft: 


\begin{tabular}{|l|r|r|r|r|r|r|r|r|r|r|r|}
\hline & \multicolumn{10}{|c|}{ Einkaufsort der niederländischen Konsumenten } & \\
\hline & \multicolumn{1}{|c|}{ MG } & MG & AC & AC & HS & HS & Vie & Vie & Kald & Kald. & \\
Wohnort & Do. & Sa. & Do. & Sa. & Do. & Sa. & Do. & Sa. & Do. & Sa. & \\
\hline Almere & 0 & 1 & 0 & 0 & 0 & 0 & 0 & 0 & 1 & 3 & 5 \\
Katwijk & 0 & 0 & 1 & 0 & 0 & 0 & 0 & 0 & 0 & 0 & 1 \\
Naldwijk & 0 & 1 & 0 & 0 & 0 & 0 & 0 & 0 & 1 & 1 & 3 \\
Rotterdam & 0 & 0 & 3 & 0 & 0 & 0 & 0 & 0 & 0 & 0 & 3 \\
Schiedam & 0 & 0 & 0 & 0 & 0 & 0 & 0 & 0 & 1 & 1 & 2 \\
Maarssen & 0 & 0 & 1 & 0 & 0 & 0 & 0 & 0 & 0 & 0 & 1 \\
Hertogenbosch & 0 & 0 & 0 & 0 & 0 & 0 & 0 & 0 & 0 & 1 & 1 \\
Veldhoven & 2 & 0 & 0 & 0 & 0 & 0 & 1 & 0 & 0 & 0 & 3 \\
Eindhoven & 1 & 0 & 0 & 0 & 0 & 0 & 0 & 0 & 0 & 0 & 1 \\
Helmond & 0 & 0 & 0 & 0 & 0 & 0 & 0 & 0 & 1 & 0 & 1 \\
Deurne & 0 & 0 & 0 & 0 & 0 & 0 & 0 & 0 & 1 & 0 & 1 \\
Venray & 9 & 1 & 0 & 1 & 0 & 0 & 7 & 3 & 2 & 1 & 24 \\
Venlo & 8 & 13 & 5 & 8 & 3 & 0 & 8 & 13 & 11 & 13 & 82 \\
Weert & 0 & 4 & 2 & 13 & 4 & 5 & 4 & 5 & 0 & 0 & 37 \\
Roermond & 0 & 3 & 1 & 6 & 1 & 4 & 2 & 0 & 2 & 1 & 20 \\
Sittard & 0 & 0 & 2 & 10 & 3 & 1 & 0 & 0 & 0 & 0 & 16 \\
Maastricht & 0 & 0 & 3 & 7 & 0 & 0 & 0 & 0 & 0 & 0 & 10 \\
Heerlen & 0 & 0 & 10 & 16 & 0 & 0 & 0 & 0 & 0 & 1 & 27 \\
Brunssum & 0 & 0 & 2 & 5 & 0 & 0 & 0 & 0 & 0 & 0 & 7 \\
\hline Gesamt & 20 & 23 & 30 & 66 & 11 & 10 & 22 & 21 & 20 & 22 & 245 \\
\hline
\end{tabular}

Tab. 7.6: $\quad$ Wohnort und Einkaufsort der niederländischen Konsumenten

Deutlich erkennbar ist die besondere Rolle der Stadt Aachen für den grenzüberschreitenden Einkauf. Diese Stadt hat ein sehr großes Einzugsgebiet auf der niederländischen Seite und ist insbesondere für Konsumenten aus Heerlen attraktiv. Interessant ist auch die sehr hohe Zahl von niederländischen Konsumenten aus Venlo und Venray Richtung Viersen und Mönchengladbach. Sehr stark ausgeprägt ist der grenznahe Einkauf von Konsumenten aus Venlo nach Kaldenkirchen. Hier werden insbesondere im Discountbereich die Einkäufe für den täglichen Bedarf getätigt.

Die Erreichbarkeit der Städte im Nachbarland kann ein entscheidendes Kriterium dafür sein, ob der Wille zur Bindung an eine Einkaufsstätte im Nachbarland auch wirklich realisiert werden kann. Aus diesem Grund wurde untersucht, mit welchen Verkehrsmitteln die Konsumenten ihre Einkäufe im Nachbarland tätigen. Darauf gibt folgende Tabelle Antwort: 


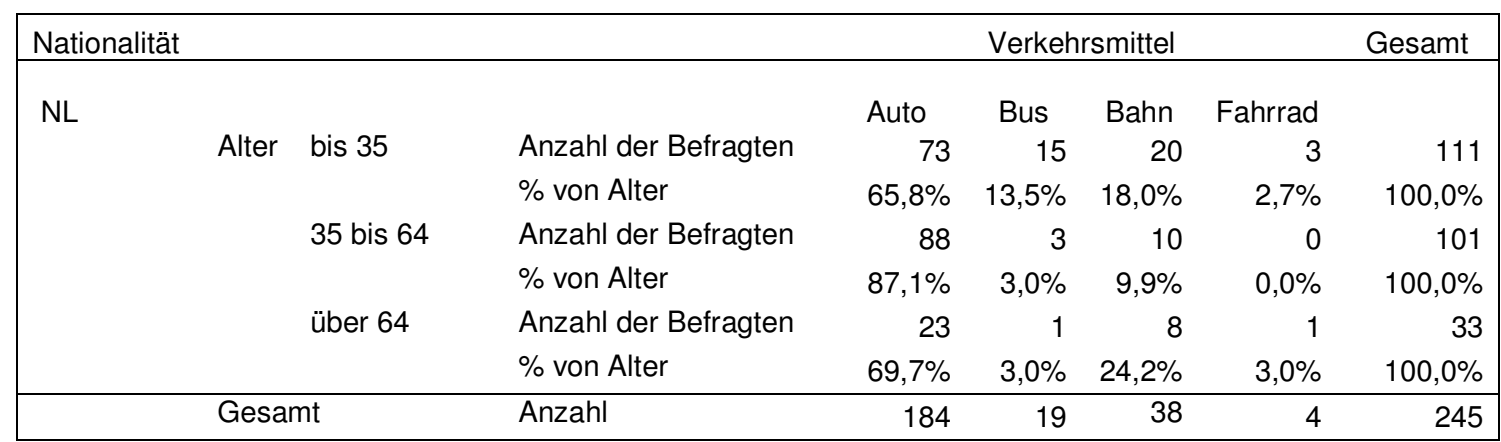

Tab.7.7: $\quad$ Nutzung der Verkehrsmittel durch niederländische Konsumenten zum Einkauf im Nachbarland

Zum Einkauf im Nachbarland wird unabhängig vom Alter von niederländischen Konsumenten am häufigsten das Auto genutzt. Allerdings nutzen die Konsumenten bis zu einem Alter von 35 Jahren zu 13,5\% auch den Bus und zu 18,0\% die Bahn zum Einkauf. Auch die Konsumenten über 64 Jahre nutzen relativ häufig die Bahn (24,2\%) zum Einkauf im Nachbarland. Selbst das Fahrrad dient den niederländischen Konsumenten als Verkehrsmittel zum Einkauf.

\subsubsection{Analyse der befragten deutschen Konsumenten}

Für die befragten deutschen Probanden ergibt sich bei der prozentualen Aufteilung in soziodemografische Merkmale folgende Verteilung:

\begin{tabular}{|c|c|c|c|c|c|c|c|c|c|c|c|}
\hline \multirow[t]{2}{*}{ Nationalität } & \multicolumn{2}{|c|}{ Geschlecht } & \multirow{2}{*}{$\begin{array}{l}\text { Alter } \\
\text { bis } 34\end{array}$} & \multirow[b]{2}{*}{$35-64$} & \multirow[b]{2}{*}{65 u.ä. } & \multicolumn{3}{|c|}{ Personen pro Haushalt } & \multicolumn{3}{|c|}{ Netto Einkommen in $€$} \\
\hline & $\mathbf{M}$ & $\mathbf{W}$ & & & & \multicolumn{3}{|c|}{ und mehr } & $>2000$ & $>3500$ & $<3500$ \\
\hline $\mathrm{D}$ & 40,3 & 59,7 & 41,1 & 46,2 & 12,6 & 17,0 & 44,3 & 38,7 & 42,7 & 37,9 & 19,4 \\
\hline
\end{tabular}

Tab. 7.8: $\quad$ Die soziodemografische Verteilung der befragten deutschen Konsumenten

Die Altersgruppe der 35 bis zu 64jährigen Konsumenten ist bei den deutschen Probanden die stärkste Gruppe der im Nachbarland kaufenden Konsumenten. Vornehmlich leben die deutschen Probanden in zwei Personenhaushalte, doch auch der Anteil der drei und mehr Personenhaushalte ist stark vertreten. Stärkste Gruppe ist die Gruppe der Konsumenten mit einem Haushaltsnettoeinkommen von bis zu $€ 2000$,- pro Monat. Auch die Zahlen des Landesamtes 
für Datenverarbeitung und Statistik Nordrhein-Westfalen bestätigen diese Ergebnisse, die den Rückschluss zulassen, dass auf der deutschen Seite in der Region zwischen Aachen und Mönchengladbach relativ viele Konsumenten mit unter dem statistischen Durchschnitt liegenden Einkommen wohnen. Im Untersuchungsgebiet ist der Kreis Heinsberg der einkommensschwächste Kreis,

\begin{tabular}{|lc|}
\hline Stadt oder Kreis & Verfügbares Einkommen je Einwohner in EUR im Jahr 2002 \\
\hline Mönchengladbach, Kreisfreie Stadt & $17.955,00$ \\
Viersen, Kreis & $17.555,00$ \\
Aachen, Kreisfreie Stadt & $16.556,00$ \\
Aachen, Kreis & $15.643,00$ \\
Heinsberg, Kreis & $15.341,00$ \\
\hline
\end{tabular}

Tab. 7.9: Verfügbares Einkommen der Einwohner des deutschen Befragungsgebietes im Jahre 2002 (Landesamt:Volkswirtschaftliche Gesamtrechnungen NordrheinWestfalen 2004)

gefolgt vom Kreis Aachen und der Stadt Aachen. Die Befragung zeigt folgende Verteilung der Haushalts- und Einkommensgrößen.

\begin{tabular}{|c|c|c|c|}
\hline \multirow{5}{*}{ Gültig } & & Häufigkeit & Prozent \\
\hline & 1 Personen Haushalt & 43 & 17,0 \\
\hline & 2 Personen Haushalt & 112 & 44,3 \\
\hline & 3 Personen Haushalt & 98 & 38,7 \\
\hline & Gesamt & 253 & 100,0 \\
\hline \multirow[t]{4}{*}{ Gültig } & Bis $€ 2000$,- & 108 & 42,7 \\
\hline & Bis $€ 3500,-$ & 96 & 37,9 \\
\hline & über $€ 3500,-$ & 49 & 19,4 \\
\hline & Gesamt & 253 & 100,0 \\
\hline
\end{tabular}

Tab. 7.10: $\quad$ Verteilung der Personen pro Haushalt der befragten deutschen Konsumenten sowie Verteilung der Haushaltsnettoeinkommen

Die befragten deutschen Konsumenten leben zu 17,0\% in Singlehaushalten, 44,3\% in zwei Personenhaushalte und zu 38,7\% in Haushalten mit drei und mehr Mitgliedern. Das durchschnittliche Haushaltsnettoeinkommen liegt bei $42,7 \%$ bei bis zu $€ 2000$,-, bei 37,9 \% bei bis zu $€ 3.500$ und bei $19,4 \%$ bei über $€ 3.500,-$. 


\begin{tabular}{|c|c|c|c|c|c|}
\hline \multicolumn{2}{|c|}{ Deutsche Konsumenten } & \multicolumn{3}{|c|}{ Haushaltsnettoeinkommen } & \multirow[t]{2}{*}{ Gesamt } \\
\hline Anzahl & & bis $€ 2000$ & bis $€ 3500$ & über €3500 & \\
\hline \multirow{3}{*}{$\begin{array}{l}\text { Anzahl Personen im } \\
\text { Haushalt }\end{array}$} & 1 & 36 & 6 & 1 & 43 \\
\hline & 2 & 43 & 53 & 16 & 112 \\
\hline & über 3 & 29 & 37 & 32 & 98 \\
\hline Gesamt & & 108 & 96 & 49 & 253 \\
\hline
\end{tabular}

\section{Tab. 7.11: $\quad$ Vergleich deutscher Nettoeinkommen nach Haushaltsgröße}

Werden die Haushaltsnettoeinkommen mit der Haushaltsgröße verglichen, liegt der Schwerpunkt der Haushaltsnettoeinkommen der Singlehaushalte bei unter $€ 2000$,- und die der Zweipersonenhaushalte bei bis zu $€ 3.500,-$. Bei den Haushalten mit drei und mehr Personen verteilen sich die Einkommensgruppen fast gleichmäßig auf die drei vorgegebenen Einkommensgruppen.

Auf die Frage, woher die deutschen befragten Konsumenten kommen, die in den Niederlanden einkaufen, gibt folgende Tabelle Auskunft. Die befragten Konsumenten kommen Schwerpunktmäßig aus den Postleitzahlgebieten 52 (Aachen), 41 (Mönchengladbach) und 47 (Duisburg).Die Verteilung sieht folgendermaßen aus:

\begin{tabular}{|c|c|c|c|c|c|c|c|c|c|c|c|c|}
\hline & & \multicolumn{10}{|c|}{ Einkaufsort der deutschen Konsumenten } & \multirow[t]{2}{*}{$\begin{array}{c}\text { Ge- } \\
\text { samt }\end{array}$} \\
\hline \multicolumn{2}{|c|}{$\begin{array}{l}\text { PLZ } \\
\text { Gebiet } \\
\end{array}$} & $\begin{array}{c}\text { Venlo } \\
\text { Do. } \\
\end{array}$ & $\begin{array}{c}\text { Venlo } \\
\text { Sa. } \\
\end{array}$ & $\begin{array}{c}\text { Roer- } \\
\text { mond } \\
\text { Do. }\end{array}$ & $\begin{array}{c}\text { Roer- } \\
\text { mond } \\
\text { Sa. }\end{array}$ & $\begin{array}{c}\text { Sittard } \\
\text { Do. } \\
\end{array}$ & $\begin{array}{c}\text { Sittard } \\
\text { Sa. }\end{array}$ & $\begin{array}{c}\text { Maas } \\
\text { tricht } \\
\text { Do. }\end{array}$ & $\begin{array}{c}\text { Maas } \\
\text { tricht } \\
\text { Sa. }\end{array}$ & $\begin{array}{c}\text { Heerlen } \\
\text { Do. }\end{array}$ & $\begin{array}{c}\text { Heerlen } \\
\text { Sa. }\end{array}$ & \\
\hline 40 & Düsseldorf & 1 & & 4 & 2 & & & & & & & 7 \\
\hline 41 & Mönchengaldb & 12 & 14 & 14 & 3 & 4 & 10 & & & & 4 & 61 \\
\hline 42 & Wuppertal & & & 4 & & & & & & & & 4 \\
\hline 44 & Dortmund & & & 1 & & & & & & & & 1 \\
\hline 45 & Essen & 5 & & 2 & & & & & & & 1 & 8 \\
\hline 46 & Oberhausen & & & 2 & & & & & & & & 2 \\
\hline 47 & Duisburg & 7 & 5 & 1 & 12 & & 1 & & & & & 26 \\
\hline 48 & Münster & & & & 3 & & & & & & & 3 \\
\hline 49 & Osnabrück & 1 & & & & & & & & & & 1 \\
\hline 50 & Köln & & & & & & & 1 & & & & 1 \\
\hline 51 & Köln-Ost & & 1 & & & & 3 & 1 & 3 & & 1 & 9 \\
\hline 52 & Aachen & & & 5 & 2 & 24 & 8 & 16 & 16 & 36 & 13 & 120 \\
\hline 53 & Bonn & & & & & & & 2 & & 1 & & 3 \\
\hline 54 & Trier & & & & & & & 2 & 1 & 1 & & 4 \\
\hline 59 & Hamm & 2 & & & & & 1 & & & & & 3 \\
\hline Ges & & 28 & 20 & 33 & 22 & 28 & 23 & 22 & 20 & 38 & 19 & 253 \\
\hline
\end{tabular}

\section{Tab. 7.12: $\quad$ Wohnort und Einkaufsort der deutschen Konsumenten}


Hoch ist die Zahl der deutschen Konsumenten aus dem Postleitzahlgebiet 47 (40\% von allen Befragten aus Krefeld) in Roermond am Samstag. Da diese Konsumenten aus dem Großraum Krefeld bis Ruhrgebiet zum Einkaufen nach Roermond kommen, wird die Anziehungskraft des Factory Outlet Center in Roermond deutlich. Diese Konsumenten nehmen auch größere Strecken zum Einkauf in Kauf. Ebenfalls ist auch für deutsche Konsumenten die Erreichbarkeit der Einkaufsmöglichkeiten im Nachbarland ein wesentliches Kriterium. Auch für deutsche Konsumenten ist für den Einkauf im Nachbarland das Auto das hauptsächlich genutzte Verkehrsmittel. Allerdings nutzen deutsche Konsumenten bis 35 Jahre das Auto erheblich mehr zum Einkauf als niederländische Konsumenten. Hoch ist allerdings der Anteil der deutschen Bahnreisenden über 64 Jahre mit 31,3\% der Befragten.

\begin{tabular}{|c|c|c|c|c|c|c|c|c|}
\hline \multirow[t]{2}{*}{ Nationalität } & & & & \multicolumn{4}{|c|}{ Verkehrsmittel } & \multirow[t]{2}{*}{ Gesamt } \\
\hline & & & & Auto & Bus & Bahn & Fahrrad & \\
\hline \multirow[t]{8}{*}{ D } & Alter & bis 35 & Anzahl & 94 & 4 & 5 & 1 & 104 \\
\hline & & & $\%$ von Alter & $90,4 \%$ & $3,8 \%$ & $4,8 \%$ & $1,0 \%$ & $100,0 \%$ \\
\hline & & 35 bis 64 & Anzahl & 108 & 4 & 5 & 0 & 117 \\
\hline & & & $\%$ von Alter & $92,3 \%$ & $3,4 \%$ & $4,3 \%$ & $0,0 \%$ & $100,0 \%$ \\
\hline & & über 64 & Anzahl & 20 & 2 & 10 & 0 & 32 \\
\hline & & & $\%$ von Alter & $62,5 \%$ & $6,3 \%$ & $31,3 \%$ & $0,0 \%$ & $100,0 \%$ \\
\hline & Gesal & & Anzahl & 222 & 10 & 20 & 1 & 253 \\
\hline & & & $\%$ von Alter & $87,7 \%$ & $4,0 \%$ & $7,9 \%$ & $0,4 \%$ & $100,0 \%$ \\
\hline
\end{tabular}

Tab.7.13: $\quad$ Nutzung der Verkehrsmittel durch deutsche Konsumenten zum Einkauf im Nachbarland

\subsubsection{Vergleich der befragten niederländischen und deutschen Konsumentengruppen hinsichtlich Einstellung, Motive und Bindungsverhalten}

Im Rahmen der Überprüfung inwieweit sich die beiden Konsumentengruppen signifikant voneinander unterscheiden, kann durch den t-Test festgestellt werden, dass die Verhaltensunterschiede der beiden Gruppen auf nationale Unterschiede und der Zugehörigkeit zu unterschiedlichen Nationalitäten zurückzuführen sind. Aus den in Kapitel 7.2.1 und 7.2.2 genannten Statistiken ergeben sich Gemeinsamkeiten und Unterschiede in den Lebensumständen der beiden Nationalitäten. Sowohl deutsche als auch niederländische Konsumenten kamen bei der Befragung aus unmittelbarer Grenznähe. Dies wird aus einem Vergleich der Postleitzahlen deutlich. Fahrtstrecken von mehr als 50 km Anfahrt zu Einkaufsort und Einkaufsstätte bildeten die Ausnahme. Eine große Anzahl deutscher Konsumenten, die im nördlichen Befra- 
gungsgebiet von Roermond und Venlo einkaufen, kommen aus dem Postleitzahlgebiet 41 (Raum Mönchengladbach). Deutsche Konsumenten, die nach Maastricht und Heerlen zum Einkaufen fahren, kommen schwerpunktmäßig aus dem Postleitzahlgebiet 52 (Raum Aachen). Die Stadt Sittard profitiert allerdings von beiden Einzugsgebieten gleichermaßen. Sowohl deutsche $(87,7 \%)$ als auch niederländische $(75,3 \%)$ Konsumenten tätigen ihre Einkäufe hauptsächlich mit dem Auto, die anderen Verkehrsmittel haben für den grenzüberschreitenden Einkauf keine Bedeutung. Die niederländischen Konsumenten kommen ebenfalls aus dem grenznahem Raum. Auf die Frage was Niederländer und Deutsche im Nachbarland einkaufen, ergeben sich sowohl Gemeinsamkeiten als auch Unterschiede bei den befragten Konsumenten. Während deutsche Konsumenten neben Bekleidung und Schuhe häufig in den Niederlanden Gartenartikel kaufen, kaufen die niederländischen Konsumenten in Deutschland neben Bekleidung und Schuhe mehr Elektroartikel. In der nachfolgenden Tabelle werden die unterschiedlichen von den befragten Probanden gekauften Produkte nach ihrem prozentualem Anteil an die insgesamt gekauften Produkte im Nachbarland aufgeführt. Die Probanden machten folgende Angaben ${ }^{31}$ :

\begin{tabular}{|l|c|c|}
\hline & $\begin{array}{l}\text { Anteil an Einkäufe der } \\
\text { niederländische Probanden in \% }\end{array}$ & $\begin{array}{l}\text { Anteil an Einkäufe der } \\
\text { deutsche Probanden in \% }\end{array}$ \\
\hline Bekleidung, Schuhe & 49,0 & 49,4 \\
\hline Gartenartikel & 1,6 & 22,5 \\
\hline Elektro & 20,5 & 7,1 \\
\hline Sonstiges inklusive Lebensmittel & 28,9 & 21,0 \\
\hline
\end{tabular}

Tab. 7.14: $\quad$ Verteilung der Einkäufe in \% nach Probandengruppe

\footnotetext{
${ }^{31}$ Für die folgenden Tabellen (Tabelle 7.14 -7.17 wurde ein Chi-Quadrat Test durchgeführt. Ein signifikanter Zusammenhang wurde nicht festgestellt. Das Konfidenzniveau mit 0.15-0.25 sehr schwach. Es ist ein Unterschied zwischen den beiden Befragungsgruppen vorhanden, allerdings nur sehr schwach ausgeprägt. Begründet ist dies auch durch die kleine Anzahl der Antworten der Probanden. Zur besseren Analyse der vorliegenden Antworten wird aus diesem Grund im späteren Verlauf der Arbeit das Partial Least Squares (PLS) zur weiteren Analyse verwendet und nicht LISREL.
} 
Nach den Gründen für den Einkauf im Nachbarland gefragt, gaben die Probanden folgende Antworten:

\begin{tabular}{|l|c|c|}
\hline & $\begin{array}{c}\text { Antworten niederländi- } \\
\text { scher Probanden in } \%\end{array}$ & $\begin{array}{c}\text { Antworten deutscher } \\
\text { Probanden in } \%\end{array}$ \\
\hline Preis & 38,8 & 37,9 \\
\hline Auswahl im Geschäft & 10,5 & 13,6 \\
\hline Größe des Geschäftes & 12,1 & 2,8 \\
\hline Parkplätze & 8,9 & 9,2 \\
\hline Beratung & 0,4 & 5,6 \\
\hline Erreichbarkeit mit öffentlichen Ver- & 5,0 & 14,0 \\
kehrsmitteln & & 12,2 \\
\hline Service & 12,0 & 100 \\
\hline sonstiges & 12,3 & 100 \\
\hline Total & 100 & \\
\hline
\end{tabular}

Tab. 7.15: $\quad$ Gründe der Probanden zum Einkauf im Nachbarland

Die Gründe für den Einkauf im Nachbarland sind bei beiden Probandengruppen in einigen Punkten ähnlich. Beide Gruppen nennen als Gründe für den Einkauf im Nachbarland den vorteilhaften Preis, sowie die größere Auswahl, als auch den besseren Service in der gewählten Einkaufsstätte. Dennoch sind Unterschiede erkennbar. Die Unterschiede liegen insbesondere in der Auswahl des Geschäftes nach Größe, der Verkaufsfläche, der Beratung und der Erreichbarkeit der Einkaufsstätten mit öffentlichen Verkehrsmitteln. Niederländerländische Probanden nennen als Grund für den Einkauf im Nachbarland großflächige Einzelhandelsstätten, deutschen Konsumenten die Beratung als Grund für einen Einkauf im Nachbarland. Differenzierter sind die Antworten bei der Frage nach dem Betriebstyp. Hierbei ergibt sich folgende Verteilung:

Frage: Wenn Sie an die nächsten Einkäufe denken, in welchem Geschäftstyp kaufen Sie im Nachbarland in erster Linie ein? 


\begin{tabular}{|l|c|c|}
\hline & $\begin{array}{c}\text { Angaben niederländi- } \\
\text { scher Probanden in } \\
\%\end{array}$ & $\begin{array}{c}\text { Antworten deutscher } \\
\text { Probanden in \% }\end{array}$ \\
\hline Discounter & 25,9 & 4,3 \\
\hline Kleines Fachgeschäft mit Bedienung & 20,6 & 45,5 \\
\hline Filialisten, die ich aus meinem Heimatland kenne & 18,5 & 14,6 \\
\hline Warenhäuser & 13,6 & 4,0 \\
\hline Filialisten, die ich aus mehreren Einkaufsorten im Nachbar- & 10,7 & 28,9 \\
land kenne & & 2,0 \\
\hline Verbrauchermärkte & 7,8 & 0,7 \\
\hline Sonstige & 2,9 & $\mathbf{1 0 0 , 0}$ \\
\hline Total & $\mathbf{1 0 0 , 0}$ & \\
\hline
\end{tabular}

\section{Tabb. 7.16: Bevorzugte Betriebsform der befragten Konsumenten ${ }^{32}$}

Wird dieses Ergebnis noch einmal nach Altersklassen sortiert und erweitert um die Frage „Welche Probandengruppe kauft wo ein?“ ergibt sich folgendes Bild der Betriebsformen nach der Höhe der prozentualen Nennung durch die befragten Probanden:

\begin{tabular}{|c|c|c|c|c|}
\hline & & Niederländische Probanden kaufen & \multicolumn{2}{|c|}{ Deutsche Probanden kaufen } \\
\hline \multicolumn{5}{|c|}{ In der Altersgruppe bis 35} \\
\hline männlich & $37,5 \%$ & bei Filialisten, die aus dem eigenen Land bekannt sind. & $50 \%$ & im Fachgeschäft \\
\hline weiblich & $30,8 \%$ & bei Filialisten, die aus dem eigenen Land bekannt sind. & $45,2 \%$ & im Fachgeschäft \\
\hline \multicolumn{5}{|c|}{ In der Altersgruppe 35 bis 64} \\
\hline männlich & $44,8 \%$ & bei Discountern & & $\begin{array}{l}\text { bei Filialisten, die man aus } \\
\text { dem Nachbarland kennt. }\end{array}$ \\
\hline weiblich & $38 \%$ & bei Discountern & $50 \%$ & im Fachgeschäft \\
\hline \multicolumn{5}{|c|}{ In der Altersgruppe über 64} \\
\hline männlich & $31,6 \%$ & im Fachgeschäft & $46,7 \%$ & $\begin{array}{l}\text { bei Filialialisten, die man aus } \\
\text { dem Nachbarland kennt. }\end{array}$ \\
\hline weiblich & $57,1 \%$ & im Fachgeschäft & $47,1 \%$ & im Fachgeschäft \\
\hline
\end{tabular}

Abb. 7.17:

Welche Probandengruppe kauft wo ein?

Werden die Merkmale „Wo wird eingekauft“, „Was wird eingekauft“ und „Warum wird eingekauft" miteinander verglichen, zeigen alle Merkmale eine hohe Signifikanz nach dem ChiQuadrattest nach Pearson auf. Der Kontingenzkoeffizient von 0.427 deutet auf einen engen

\footnotetext{
${ }^{32}$ Die Voraussetzungen sind nicht ganz erfüllt. Eine Warnung nach dem Chi-Quadrat-Test weist aus, dass $25 \%$ der Felder eine erwartete Häufigkeit kleiner 5 haben. Da aber die Obergrenze von 20\% nur knapp überschritten ist und die betreffenden Felder auf Grund ihrer recht geringen standardisierten Residuen ohnehin nur einen unwesentlichen Beitrag zum Chi-Quadrat-Wert leisten, kann die Störung der Voraussetzung als unwesentlich gelten.
} 
Zusammenhang zwischen der Nationalität und der Wahl der Betriebsform hin. Doch auch die Merkmale „Was wird im benachbartem Ausland eingekauft“ mit einem Kontingenzkoeffizienten von 0.285 und das Merkmal „Warum wird im Nachbarland eingekauft“ mit einem Kontingenzkoeffizienten von 0.233 weisen auf einen Einfluß der Nationalität auf das Merkmal hin. Der Vergleich der beiden Probandengruppen zeigt, das deutsche Konsumenten bei ihren grenzüberschreitenden Einkäufen mehr zum Einkauf im Fachgeschäft und zum Einkauf in Filialunternehmen aus dem Nachbarland neigen. Niederländische Konsumenten präferieren dagegen Filialisten, die sie aus dem eigenen Land kennen und Discounter. Ältere niederländische Konsumenten kaufen dagegen im Fachgeschäft. Im Folgenden werden die Unterschiede der Motive zum Einkauf im Nachbarland untersucht. Dazu werden folgende Fragen aus den Fragebogen zu Grunde gelegt:

- Im Nachbarland kaufe ich preiswerter.

- Im Nachbarland finde ich leichter die Produkte, die ich suche/benötige.

- Einkaufen im Nachbarland macht mit Spaß, weil ich eine angenehme Umgebung mit Cafés und Unterhaltungsmöglichkeiten antreffe.

- Ich finde es schön, die Möglichkeit zu haben, schnell im Nachbarland einkaufen zu können.

Um zu untersuchen, ob Unterschiede zwischen Niederländern und Deutschen hinsichtlich ihrer Motive zum grenzüberschreitenden Einkauf bestehen, wird ein Vergleich der beiden unabhängigen Stichproben vorgenommen und einem t-Test unterzogen. Zusammenfassend ergeben sich folgende Ergebnisse für die oben gestellten Fragen, wobei die Skalierung der Fragen mit $1=$ stimme ich voll $\mathrm{zu}, 5=$ stimme ich nicht $\mathrm{zu}$, zugrunde gelegt wurde. 


\begin{tabular}{|l|l|l|l|c|c|}
\hline & Nationalität & $\mathrm{N}$ & Mittelwert & Standardabweichung $^{33}$ & T-Test $^{34}$ \\
\hline Im Nachbarland kaufe ich preiswerter & $\mathrm{NL}$ & 245 & 2,46 & 1,088 & $-2,241$ \\
\hline & $\mathrm{D}$ & 253 & 2,69 & 0,990 & $-2,242$ \\
\hline Im Nachbarland finde ich Produkte leichter & $\mathrm{NL}$ & 245 & 2,43 & 1,101 & 5,201 \\
\hline & $\mathrm{D}$ & 253 & 1,92 & 1,093 & 5,201 \\
\hline Einkaufen im Ausland macht Spass & $\mathrm{NL}$ & 245 & 2,00 & 0,887 & 4,075 \\
\hline & $\mathrm{D}$ & 253 & 1,68 & 0,866 & 4,073 \\
\hline Einkaufen im Ausland Wohlbefinden & $\mathrm{NL}$ & 245 & 2,43 & 1,064 & 6,568 \\
\hline & $\mathrm{D}$ & 253 & 1,86 & 0,884 & 6,549 \\
\hline
\end{tabular}

Tab. 7.18: $\quad$ Motive und Einstellungen der befragten Konsumenten zum Einkauf im Nachbarland

Auch hier werden Unterschiede deutlich. Bedeutung erlangen diese Unterschiede, wenn sie unter Einbeziehung der Wahl der Betriebsform betrachtet werden. Insbesondere hinsichtlich Spass beim Einkauf im Nachbarland sind die deutschen Konsumenten zufriedener beim Einkauf im Nachbarland als niederländische Konsumenten, gefolgt vom Gefühl des Wohlbefindens beim Einkauf. Wohlbefinden beschreibt dabei die ganze Atmosphäre einer Einkaufsstadt und der bevorzugten Einkaufsstätte. Niederländische und deutsche Konsumenten haben dagegen unterschiedliche Motive zum Einkauf im Nachbarland hinsichtlich des Wunsches günstiger einzukaufen und gewünschte Produkte schneller im Nachbarland zu finden. Wenn somit die meisten Konsumenten aus dem grenznahen Raum auch grenzüberschreitend einkaufen, ist es interessant zu wissen, welchen Einfluss die Werturteile der sozialen Gruppe auf diese Entscheidung haben. Die Fragen „Mein Freundes und Bekanntenkreis findet...“(v031- v034) spiegeln die Werturteile der sozialen Gruppe wider.

\footnotetext{
${ }^{33}$ Die Standardabweichung ist in der Stochastik ein Maß für die Streuung der Werte einer Zufallsvariable um ihren Mittelwert.

${ }^{34}$ Der t-Test kann eingesetzt werden, wenn die zu untersuchende abhängige Variable mindestens (mehr oder weniger) intervallskaliert ist. Außerdem sollte das Merkmal in den untersuchten Populationen normalverteilt sein. Vor allem bei großen Stichproben und nicht zu unterschiedlichem Umfang der beiden Gruppen ist das Verfahren relativ robust und wird deshalb auch in der vorliegenden Untersuchung verwendet. Negative t-Werte können wie positive Werte interpretiert werden. Der t-Wert sollte - sofern signifikant - einen Wert von größer 2 aufweisen. Bortz, 1985, S.197ff.
} 


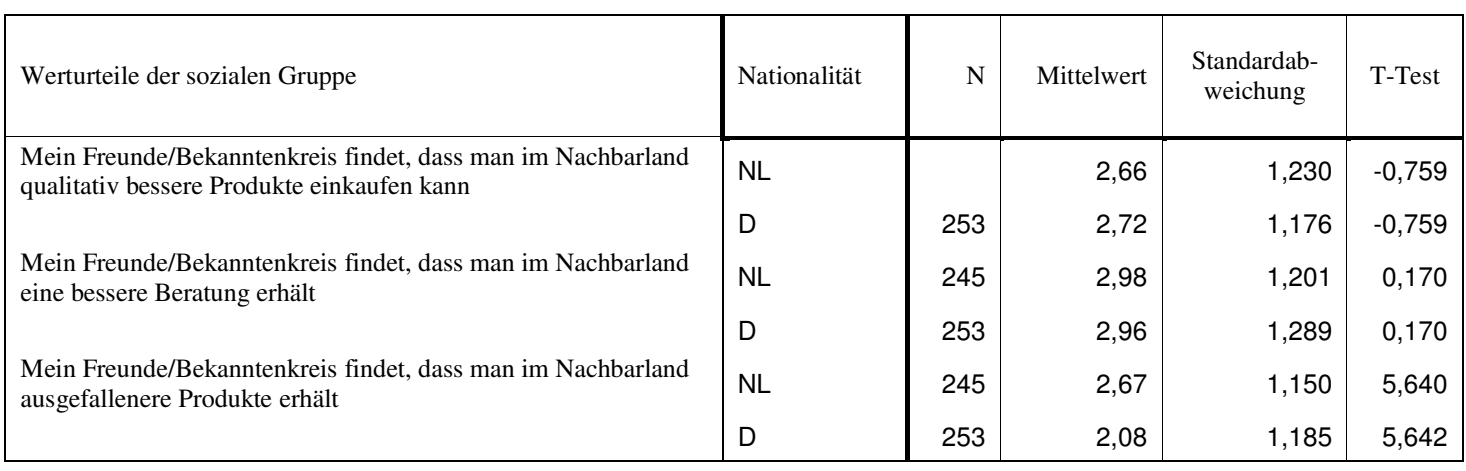

Tab. 7.19: $\quad$ Mittelwertvergleich Werturteile der sozialen Gruppe

Die Wichtigkeit der sozialen Norm, dass heißt auch Freunde und Bekannte kaufen im Nachbarland ein und empfehlen den Einkauf, wird durch die Fragen „Wenn Sie einmal an Ihren Freundes/Bekanntenkreis denken, in wie vielen Fällen kaufen diese im Nachbarland ein“ und „Wenn Sie einmal an die letzten Einkäufe zurückdenken, in wie vielen Fällen haben Freunde, Bekannte Ihnen eine Empfehlung zum Einkauf im Nachbarland gegeben?" ausgedrückt. Bei diesen Fragen sind die Unterschiede zwischen deutschen und niederländischen Probanden gering. Doch über das Gefühl, dass Freunde und Bekannte es positiv sehen, wenn im Nachbarland eingekauft wird, gibt es Unterschiede zwischen Niederländern und Deutschen. Ein weiterer Unterschied besteht in der Empfehlung des Einkaufs im Nachbarland durch Freunde und Bekannte. Hier spricht das soziale Umfeld der deutschen Konsumenten stärker Empfehlungen aus als das Umfeld der niederländischen Konsumenten. Die Wichtigkeit der soziale Norm zeigt Unterschiede bei den beiden Konsumentengruppen auf. Insgesamt ergibt sich im Mittelwertvergleich folgendes Bild:

\begin{tabular}{|l|l|l|l|r|r|}
\hline Wichtigkeit der sozialen Norm & Nationalität & N & Mittelwert & Standardabweichung & T-Test \\
\hline In wie vielen Fällen kaufen Freunde und Bekannte im & NL & 245 & 2,47 & 0,674 & 2,169 \\
Nachbarland ein & D & 253 & 2,33 & 0,696 & 2,170 \\
Wie oft haben Freunde Einkauf im Nachbarland empfoh- & NL & 245 & 2,40 & 0,679 & 2,915 \\
len? & D & 253 & 2,22 & 0,687 & 2,915 \\
\hline
\end{tabular}

Tab. 7.20: $\quad$ Mittelwertvergleich der Wichtigkeit „, soziale Norm“

Werden die Mittelwerte der Bedeutung der sozialen Norm miteinander verglichen, so ergibt sich folgendes Bild: 


\begin{tabular}{|l|l|r|r|r|r|}
\hline Bedeutung der sozialen Norm & Nationalität & N & Mittelwert & $\begin{array}{c}\text { Standardab- } \\
\text { weichung }\end{array}$ & T-Test \\
\hline Bereits als Kind fuhr ich mit meinen Eltern zum Einkauf & NL & 245 & 2,66 & 1,243 & 1,757 \\
über die Grenze. & D & 253 & 2,45 & 1,423 & 1,761 \\
Im Nachbarland kaufe ich preiswerter. & NL & 245 & 2,46 & 1,088 & $-2,241$ \\
& D & 253 & 2,69 & 0,990 & $-2,242$ \\
\hline
\end{tabular}

Tab. 7.21: Mittelwertvergleich der Bedeutung „, soziale Norm“

Zufriedenheit ist ein wesentlicher Punkt, um weiter in einem Ort oder einer Einkaufsstätte einzukaufen. Hier ergeben sich im Vergleich zwischen Deutschen und Niederländern folgende Werte:

\begin{tabular}{|l|l|l|l|l|r|}
\hline Gesamtzufriedenheit mit Stammgeschäft im Nachbarland & Nationalität & N & Mittelwert & $\begin{array}{c}\text { Standardab- } \\
\text { weichung }\end{array}$ & T-Test \\
\hline Zufriedenheit Stammgeschäft hinsichtlich Sortiment & NL & 245 & 1,91 & 1,039 & 3,254 \\
Service & D & 253 & 1,65 & 0,738 & 3,237 \\
& NL & 245 & 2,37 & 1,172 & 1,262 \\
Beratung & D & 253 & 2,25 & 0,987 & 1,259 \\
& NL & 245 & 2,46 & 1,199 & 0,969 \\
\hline
\end{tabular}

\section{Tab. 7.22: $\quad$ Mittelwertvergleich der Gesamtzufriedenheit der Konsumenten mit Stammgeschäft im Nachbarland}

Unterschiede sind insbesondere in der Zufriedenheit der Probandengruppe mit dem Stammgeschäft im Nachbarland zu sehen. Hier gibt es signifikante Unterschiede zwischen beiden Kundengruppen.

\begin{tabular}{|l|c|c|c|c|c|}
\hline $\begin{array}{l}\text { Zufriedenheit der Konsumenten mit Kauf im Nachbar- } \\
\text { land }\end{array}$ & Nationalität & N & Mittelwert & $\begin{array}{c}\text { Standardab- } \\
\text { weichung }\end{array}$ & T-Test \\
\hline $\begin{array}{l}\text { Mit den Einkaufsmöglichkeiten im Ort der Befragung } \\
\text { bin ich zufrieden. }\end{array}$ & NL & 245 & 1,82 & 0,692 & $-1,495$ \\
& D & 253 & 1,92 & 0,805 & $-1,498$ \\
$\begin{array}{l}\text { Mit dem Einkauf in meinem Stammgeschäft bin ich } \\
\text { zufrieden. }\end{array}$ & NL & 245 & 2,04 & 1,041 & 3,926 \\
& D & 253 & 1,74 & 0,631 & 3,897 \\
\hline
\end{tabular}

Tab. 7.23: Mittelwertvergleich der Gesamtzufriedenheit der Konsumenten mit Einkauf im Nachbarland 
Beide Konsumentengruppen haben eine hohe Zufriedenheit mit dem Einkaufsort im Nachbarland. Doch in der Beurteilung der Leistungen des Stammgeschäfts weicht die Zufriedenheit der beiden Probandengruppen voneinander ab. Wird die Standardabweichung betrachtet, zeigt die Auswertung eine hohe Homogenität der Aussagen in beiden Gruppen. Bei der Beurteilung der Einkaufsstätte selbst gehen die Aussagen der niederländischen Konsumenten stärker auseinander als bei deutschen Konsumenten. Die deutschen Konsumenten haben eine einheitlichere Meinung gegenüber Einkaufsort und Einkaufsstätte. In beiden Gruppen erhält die Zufriedenheit mit der Beratung und dem Service in den Einkaufsstätten im Nachbarland schlechtere Werte als die Zufriedenheit mit dem Sortiment der Einkaufsstätte. Das Sortiment der niederländischen Geschäfte wird durch deutsche Konsumenten dennoch positiver beurteilt als umgekehrt. Die befragten niederländischen Probanden sind mit der Einrichtung der deutschen Geschäfte weniger zufrieden als deutsche Probanden mit der Einrichtung niederländischer Einkaufsstätten.

Beide Probandengruppen beurteilen das Sortiment der Einkaufsstätten im Nachbarland als für sie attraktiv. Unterschiedliche Beurteilungen der Nationalitäten finden sich im Bereich Einrichtung. Hier beurteilen die deutschen Probanden die Einrichtung der niederländischen Geschäfte deutlich besser als die niederländischen Probanden die deutschen Geschäfte.

\begin{tabular}{|l|c|c|c|c|c|}
\hline Persönliche Norm & Nationalität & $\mathrm{N}$ & Mittelwert & $\begin{array}{c}\text { Standardab- } \\
\text { weichung }\end{array}$ & $\mathrm{t}$-Test \\
\hline $\begin{array}{l}\text { Im Nachbarland kann ich Produkte kaufen, die ich } \\
\text { Zuhause nicht bekomme. }\end{array}$ & $\mathrm{NL}$ & 245 & 2,43 & 1,101 & 5,201 \\
\hline $\begin{array}{l}\text { Im Nachbarland finde ich leichter die Produkte, die } \\
\text { ich suche/benötige. }\end{array}$ & $\mathrm{NL}$ & 245 & 2,68 & 1,112 & 2,077 \\
\hline
\end{tabular}

\section{Tab. 7.24: Mittelwertvergleich „persönliche Norm“}

Die Analyse der Mittelwerte zeigt deutliche Unterschiede zwischen den beiden Probandengruppen auf. Starke Unterschiede zwischen den beiden Gruppen gibt es bei der Frage „Im Nachbarland kann ich Produkte kaufen, die ich Zuhause nicht bekomme" sowie bei der Frage „Im Nachbarland kaufe ich lieber in kleinen Fachgeschäften“. In diesen Bereichen liegen Unterschiede in der persönlichen Norm der betrachteten Probanden vor. Deutsche Probanden kaufen im Nachbarland, weil sie ganz konkrete Produkte suchen, die im Heimatland so nicht 
erhältlich sind und sie kaufen lieber in kleinen Fachgeschäften ein. Umgekehrt gilt diese Aussage bei großen Einzelhandelsketten. Da die Standardabweichungen bei beiden Probandengruppen ähnliche Werte aufzeigen, zeigen die Antworten der Probanden doch eine starke Streuung der Antworten in den einzelnen Gruppen auf.

Niederländer und Deutsche zeigen erhebliche Unterschiede im Bereich der wahrgenommenen Verhaltenskontrolle, wenn es darum geht, über den nächsten Einkauf im Nachbarland nachzudenken. In diesem Fall sind deutsche Konsumenten positiver gegenüber dem nächsten Einkauf im Nachbarland eingestellt als Niederländer. Diese Werte sind höchst signifikant. Insbesondere der Vorsatz den nächsten Einkauf wieder im Nachbarland zu tätigen erhält bei den deutschen Konsumenten eine höhere Zustimmung, im Gegensatz zu den niederländischen Konsumenten. Diese Einstellung zum Vorsatz hat wahrscheinlich Einfluss auf das Bindungsverhalten der jeweiligen Konsumentengruppe.

\begin{tabular}{|c|c|c|c|c|c|}
\hline Wahrgenommene Verhaltenskontrolle & Nationalität & $\mathrm{N}$ & Mittelwert & $\begin{array}{l}\text { Standardab- } \\
\text { weichung }\end{array}$ & T-Test \\
\hline \multirow[t]{2}{*}{$\begin{array}{l}\text { Der Ort im Nachbarland in dem Einkäufe getätigt werden, } \\
\text { sollte vom Heimatort mit öffentlichen Verkehrsmitteln gut } \\
\text { erreichbar sein }\end{array}$} & NL & 245 & 2,96 & 1,469 & 2,352 \\
\hline & $\mathrm{D}$ & 253 & 2,65 & 1,482 & 2,352 \\
\hline
\end{tabular}

Tab. 7.25: Mittelwertvergleich „wahrgenommene Verhaltenskontrolle“

$\mathrm{Zu}$ der wertorientierten Konzeptualisierung des Involvementkonstruktes gehören die Werte, die ein Individuum durch den Kauf in einer Einkaufsstätte anstrebt. Dazu werden folgende Items einem t-Test unterzogen:

\begin{tabular}{|c|c|c|c|c|c|}
\hline wahrgenommene Selbstrelevanz & Nationalität & $\mathrm{N}$ & Mittelwert & $\begin{array}{l}\text { Standard- } \\
\text { abweichung }\end{array}$ & $\mathrm{t}$-Test \\
\hline \multirow[t]{2}{*}{ Bei nächster Gelegenheit kaufe ich wieder im Nachbarland ein. } & NL & 245 & 2,68 & 1,250 & 7,795 \\
\hline & D & 253 & 1,88 & 1,021 & 7,770 \\
\hline $\begin{array}{l}\text { Einkaufen im Ausland macht Spass, weil ich eine angenehme } \\
\text { Umgebung mit Cafes und Unterhaltungsmöglichkeiten antreffe, } \\
\text { Zustimmung. }\end{array}$ & $\mathrm{D}$ & 253 & 1,68 & 0,866 & 4,073 \\
\hline $\begin{array}{l}\text { Ich finde es schön, die Möglichkeit zu haben, schnell im Nach- } \\
\text { barland einkaufen zu können (Wohlbefinden), Zustimmung. }\end{array}$ & $\mathrm{D}$ & 253 & 1,86 & 0,884 & 6,549 \\
\hline
\end{tabular}

Tab. 7.26: Mittelwertvergleich „wahrgenommene Selbstrelevanz““ 
Die aufgeführten Fragen sind der Gruppierungsvariable der wahrgenommenen Selbstrelevanz zuzuordnen. Dies bedeutet, je höher die Zustimmung ausfällt, desto höher ist die Bindung des Konsumenten zu einem Einkaufsort oder Einkaufsstätte. Die wahrgenommene Selbstrelevanz zeigt deutliche Unterschiede zwischen Deutschen und Niederländer. Grenzüberschreitender Einkauf ist bei deutschen Konsumenten sehr stark mit Spass, angenehmer Einkaufsatmosphäre mit Cafès und Verweilmöglichkeiten verbunden. Unterstützt wird dies durch die Aussage, dass insbesondere bei Deutschen der grenzüberschreitende Einkauf Wohlbefinden auslöst. Die Unterschiede sind signifikant.

Hinsichtlich ihres Loyalitätsverhaltens unterscheiden sich deutsche und niederländische Konsumenten voneinander, indem deutsche Konsumenten weitere angebotene Produkte ihrer favorisierten Einkaufsstätte stärker beim Folgekauf berücksichtigen würden als niederländische Konsumenten. Deutsche Konsumenten stehen dem cross-buying Verhalten somit positiv gegenüber. Im Weiterempfehlungsverhalten unterscheiden sich beide Gruppen dagegen nicht voneinander.

\begin{tabular}{|c|c|c|c|c|c|}
\hline Kundenloyalität & Nationalität & $\mathrm{N}$ & Mittelwert & $\begin{array}{c}\text { Standard- } \\
\text { abweichung }\end{array}$ & $\mathrm{t}$-Test \\
\hline \multirow{2}{*}{$\begin{array}{l}\text { Ich empfehle mein Stammgeschäft im Nachbarland bei meinen } \\
\text { Freunden und Bekannten weiter }\end{array}$} & NL & 245 & 2,22 & 1,074 & 1,881 \\
\hline & D & 253 & 2,04 & 1,070 & 1,881 \\
\hline \multirow{2}{*}{$\begin{array}{l}\text { Ich möchte regelmäßig von meinem Stammgeschäft im Nachbar- } \\
\text { land über Angebote und besondere Dienstleistungen informiert } \\
\text { werden }\end{array}$} & NL & 245 & 2,81 & 1,425 & 1,231 \\
\hline & D & 253 & 2,66 & 1,404 & 1,231 \\
\hline \multirow{2}{*}{$\begin{array}{l}\text { Sofern mein Stammgeschäft in Zukunft auch andere Produktka- } \\
\text { tegorien anbietet, könnte ich mir vorstellen diese auch dort zu } \\
\text { kaufen }\end{array}$} & NL & 245 & 2,55 & 1,304 & 3,573 \\
\hline & D & 253 & 2,15 & 1,172 & 3,567 \\
\hline
\end{tabular}

Tab. 7.27: Mittelwertvergleich „Kundenloyalität“

Werden die Items der Kundenbindung analysiert, zeichnen sich deutliche Unterschiede zwischen den deutschen und niederländischen Konsumenten hinsichtlich des Bindungsverhaltens ab. Niederländische Konsumenten sind weniger bindungsbereit als deutsche Konsumenten. Sowohl im Einkaufsverhalten im Nachbarland selbst, als auch im Einkauf in der favorisierten Einkaufsstätte unterscheiden sich die beiden Konsumentengruppen voneinander. Die befragten deutschen Konsumenten fahren mehr ins Nachbarland und besuchen auch das Stammgeschäft deutlich häufiger. 


\begin{tabular}{|c|c|c|c|c|c|}
\hline Kundenbindung & Nationalität & $\mathrm{N}$ & Mittelwert & $\begin{array}{l}\text { Standard- } \\
\text { abweichung }\end{array}$ & t-Test \\
\hline $\begin{array}{l}\text { Mindestens } 1 \text { mal im Monat besuche ich mein Stammgeschäft im } \\
\text { Nachbarland. }\end{array}$ & NL & $\begin{array}{l}245 \\
253\end{array}$ & $\begin{array}{l}2,93 \\
2,81\end{array}$ & $\begin{array}{l}1,431 \\
1,377\end{array}$ & $\begin{array}{l}0,988 \\
0,987\end{array}$ \\
\hline $\begin{array}{l}\text { In den vergangenen acht Wochen habe ich oft im Nachbarland } \\
\text { eingekauft. }\end{array}$ & $\begin{array}{c}\text { NL } \\
\text { D }\end{array}$ & $\begin{array}{l}245 \\
253\end{array}$ & $\begin{array}{l}2,96 \\
2,48\end{array}$ & $\begin{array}{l}1,386 \\
1,283\end{array}$ & $\begin{array}{l}4,054 \\
4,049\end{array}$ \\
\hline $\begin{array}{l}\text { In den vergangenen acht Wochen habe ich häufig im Stammge- } \\
\text { schäft eingekauft. }\end{array}$ & $\begin{array}{c}\text { NL } \\
\text { D }\end{array}$ & $\begin{array}{l}245 \\
253\end{array}$ & $\begin{array}{l}3,09 \\
2,65\end{array}$ & $\begin{array}{l}1,406 \\
1,348\end{array}$ & $\begin{array}{l}3,579 \\
3,577\end{array}$ \\
\hline
\end{tabular}

Tab. 7.28: Mittelwertvergleich „Kundenbindung“

Im Folgenden werden die Fragen genauer betrachtet, die von den befragten Konsumenten durchschnittlich mit einem Mittelwert von unter 2,3 beantwortet wurden und somit mit einer hohen Zustimmung bewertet wurden. Die Betrachtungsgrenze ist bei einem Mittelwert von 2,3 gezogen worden, da bei der Befragung eine Fünferskala eingesetzt wurde. Die Bewertung mit 1 steht für eine hohe Zustimmung, eine 5 für keine Zustimmung. Werden dabei die Antworten der deutschen und niederländischen Konsumenten verglichen, so trifft dies auf folgende Fragen zu: 


\begin{tabular}{|c|c|c|c|c|c|c|}
\hline Fragen mit hoher Zustimmung & NL & $\mathrm{D}$ & $\begin{array}{l}\text { Mitt } \\
\text { NL }\end{array}$ & Wert & $\begin{array}{l}\text { T-Tes } \\
\text { NL }\end{array}$ & \\
\hline $\begin{array}{l}\text { Mein Freundes-/Bekanntenkreis findet, dass man im Nachbarland preislich besser (günstiger) ein- } \\
\text { kaufen kann. }\end{array}$ & $\mathrm{X}$ & & 2,29 & 2,54 & $-2,534$ & $-2,532$ \\
\hline $\begin{array}{l}\text { Mein Freundes-/Bekanntenkreis findet, dass man im Nachbarland ausgefallenere (Design) Produkte } \\
\text { erhält. }\end{array}$ & & $\mathrm{X}$ & 2,67 & 2,08 & 5,640 & 5,642 \\
\hline Freunde und Bekannte kaufen häufig im Nachbarland ein. & & $\mathrm{X}$ & 2,47 & 2,33 & 2,169 & 2,170 \\
\hline Freunde und Bekannte reagieren auf meinen Einkauf im Nachbarland positiv. & & $\mathrm{X}$ & 2,46 & 2,06 & 4,356 & 4,349 \\
\hline $\begin{array}{l}\text { Wenn Sie einmal an die letzten Einkäufe zurückdenken, in wie vielen Fällen haben Freunde, Be- } \\
\text { kannte Ihnen eine Empfehlung zum Einkauf im Nachbarland gegeben? }\end{array}$ & & $\mathrm{X}$ & 2,40 & 2,22 & 2,915 & 2,915 \\
\hline Mit den Einkaufsmöglichkeiten in (Ort der Befragung) bin ich zufrieden. & $\mathrm{X}$ & $\mathrm{X}$ & 1,82 & 1,92 & $-1,495$ & $-1,498$ \\
\hline Mit dem Einkauf im Stammgeschäft bin ich insgesamt zufrieden. & $\mathrm{X}$ & $\mathrm{X}$ & 2,04 & 1,74 & 3,926 & 3,897 \\
\hline Mit dem Einkauf im Stammgeschäft bin ich hinsichtlich Sortiment zufrieden. & $\mathrm{X}$ & $\mathrm{X}$ & 1,91 & 1,65 & 3,254 & 3,237 \\
\hline Mit dem Einkauf im Stammgeschäft bin ich hinsichtlich Service zufrieden. & & $\mathrm{X}$ & 2,37 & 2,25 & 1,262 & 1,259 \\
\hline Mit dem Einkauf im Stammgeschäft bin ich hinsichtlich Einrichtung zufrieden. & & $\mathrm{X}$ & 2,39 & 2,01 & 4,031 & 4,019 \\
\hline Im Nachbarland kann ich Produkte kaufen, die ich zuhause nicht bekomme. & & $\mathrm{X}$ & 2,43 & 1,92 & 5,201 & 5,201 \\
\hline Im Nachbarland kaufe ich lieber in kleinen Fachgeschäften ein. & & $\mathrm{X}$ & 3,12 & 2,28 & 7,915 & 7,910 \\
\hline Bei nächster Gelegenheit kaufe ich wieder im Nachbarland ein. & & $\mathrm{X}$ & 2,43 & 1,86 & 6,568 & 6,549 \\
\hline $\begin{array}{l}\text { Einkaufen im Nachbarland macht Spass, weil ich eine angenehme Umgebung mit Cafes und Unter- } \\
\text { haltungsmöglichkeiten antreffe. }\end{array}$ & & $\mathrm{X}$ & 2,68 & 1,88 & 7,795 & 7,770 \\
\hline $\begin{array}{l}\text { Ich finde es schön, die Möglichkeit zu haben, schnell im Ausland einkaufen zu können (Wohlbefin- } \\
\text { den). }\end{array}$ & $\mathrm{X}$ & $\mathrm{X}$ & 2,00 & 1,68 & 4,075 & 4,073 \\
\hline Ich empfehle mein Stammgeschäft im Nachbarland bei meinen Freunden und Bekannten weiter. & $\mathrm{X}$ & $\mathrm{X}$ & 2,22 & 2,04 & 1,881 & 1,881 \\
\hline $\begin{array}{l}\text { Sofern mein Stammgeschäft in Zukunft auch andere Produkte anbietet, könnte ich mir vorstellen, } \\
\text { diese auch dort zu kaufen. }\end{array}$ & & $X$ & 2,55 & 2,15 & 3,573 & 3,567 \\
\hline
\end{tabular}

Tab. 7.29: Gegenüberstellung der Fragen nach Konsumentengruppe mit hoher Zustimmung

Die Gegenüberstellung der Fragen mit hoher Zustimmung der Probanden zeigt, dass deutsche Konsumenten eine positivere Grundeinstellung gegenüber dem Einkauf im Nachbarland und auch gegenüber der Bindungsbereitschaft zu einer Einkaufsstätte im Nachbarland haben als niederländische Konsumenten. Werden die oben genannten Fragen dazu genutzt um, etwas über die Einstellung der Konsumenten zum Einkaufsort und zur Einkaufsstätte im Nachbarland zu erfahren, sollten folgende Fragen näher untersucht und einem Mittelwertvergleich unterzogen werden. Der Signifikanztest zeigt, dass Unterschiede in der Beurteilung der einzelnen Items bei den untersuchten Gruppen vorhanden sind. Allein in der Beurteilung des Einkaufsortes, der Beurteilung des Service und der Weiterempfehlungsbereitschaft unterscheiden sich die beiden Gruppen nicht voneinander. Der Vergleich der beiden Gruppen zeigt, dass deutsche Probanden durch ihre Freunde und Bekannten nach ihrer Einschätzung eine positive Reaktion auf ihren Einkauf im Nachbarland erhalten. Ferner glaubt auch der Freundes- und Bekanntenkreis der Probanden im Nachbarland ausgefallenere Produkte kaufen zu 
können. Auch hinsichtlich der Einschätzung „Einkaufen im Nachbarland macht Spass“ und „Ich finde es schön die Möglichkeit zu haben, schnell im Ausland einkaufen zu können“ bewerten deutsche Probanden positiver als niederländische Probanden. Hieraus erklärt sich auch die positivere Einschätzung zur Empfehlungsbereitschaft und auch die Absicht im Stammgeschäft auch andere Produkte zu kaufen, sofern sie dort angeboten werden. Die Einstellung der deutschen Probanden zum Einkauf im Nachbarland ist somit positiver als die Einstellung der niederländischen Probanden zum Einkauf in Deutschland.

\subsubsection{Analyse weiterer Unterschiede und Zusammenfassung}

Die befragten niederländischen Konsumenten in der Altersgruppe über 64 sind mit dem Angebot ihrem Stammgeschäft in Deutschland insbesondere mit der Beratungsleistung, aber auch mit der Einrichtung der Einkaufsstätte zufrieden. Zufrieden sind sie mit der Einkaufsstätte insbesondere deshalb, weil sie dort Produkte einkaufen können, die sie zuhause nicht kaufen können. Diese Konsumentengruppe fühlt sich auch an diese Einkaufsstätte gebunden und möchte bei nächster Gelegenheit wieder dort einkaufen. Gar nicht zufrieden mit den Einkaufsstätten in Deutschland sind die jungen niederländischen Konsumenten bis 35 Jahre. Unzufrieden ist aber auch die Gruppe der zwischen 35 und 64 jährigen, obwohl sie eine positive Grundeinstellung zum grenzüberschreitenden Einkauf hat. Die Einkaufsorte in Deutschland werden im Gegensatz zu den Einkaufsstätten positiver beurteilt.

Die deutschen Konsumenten erzielen bei der Betrachtung ihrer Einstellung zum Nachbarland positivere Werte als die niederländischen Konsumenten. Die bindungswilligste Gruppe bei dieser Betrachtung ist die Gruppe der 35- bis 64-jährigen deutschen Frauen. Diese sind sowohl sehr zufrieden mit dem Einkaufsort als auch mit der Qualität der angebotenen Produkte in ihrem Stammgeschäft. Diese Konsumentengruppe gibt in der Befragung auch die größte Zustimmung dafür an, dass es für sie Spaß macht, in dem Einkaufsort im Nachbarland einzukaufen. Diese Gruppe empfindet eine positive Atmosphäre am Einkaufsort, aber auch in der von ihnen favorisierten Einkaufsstätte. Dies schlägt sich auf das Einkaufsverhalten positiv nieder. Bei den männlichen deutschen Konsumenten ist die bindungswilligste Gruppe in der Altersgruppe über 64 Jahren zu finden. Diese Gruppe ist mit dem Stammgeschäft sehr zufrieden, aber auch mit dem Einkaufsort insgesamt. Sie haben ebenso wie die Gruppe der 35-64jährigen Frauen eine große Bereitschaft wiederzukommen, da sie sich in ihrem Stammgeschäft, aber auch in der Umgebung des Einkaufsortes wohlfühlen. 
Dagegen steht bei den niederländischen Konsumenten der Preis im Vordergrund. Dies zeigt ihr besonderes Interesse an dem Discountangebot in Deutschland, als auch in ihrem Weiterempfehlungsverhalten, welches sich auf die Preisoptik konzentriert. Die Bewertung des Einkaufsortes spiegelt sich ebenfalls in dem preisaggressivem Angebot der Anbieter wieder. Als bindungswillige Gruppe sind die niederländischen Konsumenten über 64 Jahre zu sehen. Sowohl die männlichen als auch die weiblichen Konsumenten sehen ihre favorisierte Einkaufsstätte positiv und sind bereit, eine längerfristige Bindung aufzubauen. Den jüngeren niederländischen Konsumenten ist es wie den deutschen Konsumenten wichtig, das Einkaufen Spaß macht, die Innenstädte zum Verbleiben einladen und das man sich in dem Einkaufsort und der Einkaufsstätte wohlfühlt. Allerdings sind alle Werte deutlich unter denen der deutschen Probanden. Allein diese Analyse zeigt Unterschiede in den beiden Erhebungsgruppen auf. Innerhalb der Altersgruppen sind ebenfalls Unterschiede festzustellen.

Deutsche Konsumenten bauen Bindung zu einer niederländischen Einkaufsstätte insbesondere dann auf, wenn sie sowohl mit dem Kauf selbst, aber auch mit der Reaktion ihrer Umwelt auf den Kauf zufrieden sind. Dieser Faktor (Abhängigkeit, ob Kundenbindung aufgebaut wird oder nicht) ist aber auch abhängig von den persönlichen Lebensumständen der Konsumenten. Das Haushaltseinkommen der Konsumenten, das Geschlecht und ob die individuellen Wünsche der Konsumenten mit den Gegebenheiten der Einkaufsstätte übereinstimmen sind dabei wesentliche Komponenten. Eine besondere Komponente ist dabei auch die Beeinflussbarkeit der deutschen Konsumenten von der sozialen Norm. Wird diese Erkenntnis in Verhältnis gesetzt zu den Beziehungen zwischen Kultur und Verhalten nach Kroeber-Riel und Weinberg (siehe Kapitel 2.3), so werden deutsche Konsumenten beeinflusst durch ihre Erfahrungswelt, wodurch emotionales, kognitives und beobachtbares Verhalten dieser Konsumentengruppe ausgelöst wird. Die Gesamtzufriedenheit mit der Reaktion der Umwelt auf den Kauf und die Zufriedenheit hinsichtlich Produktqualität und Qualität der Einkaufsstätte hat einen entscheidenden Einfluss auf die Bindung der deutschen Konsumenten an die Einkaufsstätte. Die Faktoren Loyalität, persönliche Norm und Betriebstyp verstärken dagegen die Kundenbindung zur Einkaufsstätte.

Inwieweit Kundenbindung bei den niederländischen Probanden entsteht, ist abhängig von dem was gekauft wird, was die Einkaufsstätte zu bieten hat und dem Betriebstyp, der das jeweilige Produkt anbietet. Kundenbindung entsteht auch bei dieser Probandengruppe durch die Zufriedenheit mit der Einkaufsstätte, der Einstellung zum grenzüberschreitenden Einkauf und der Loyalität zur Einkaufsstätte. Ferner ist entscheidend die soziale Norm der Gruppe nebst 
eigener persönlicher Norm. Indem der Preis eine wesentliche Bedeutung hat, bestärkt dieser Faktor die Loyalität zur Einkaufsstätte oder schwächt sie ab. Das Verhalten der niederländischen Konsumenten ist im Gegensatz zu den deutschen Konsumenten stärker geprägt durch die von Hofstede definierte Dimension „Umgehen von Unsicherheiten" und „Maskulinität/Femininität", da die getroffenen Entscheidungen stark vom einfachen Kriterium Preis abhängig gemacht werden und es keine Dominanz der Geschlechter gibt - im Gegensatz zu deutschen Konsumenten. Dort unterscheiden sich sowohl die Geschlechter hinsichtlich ihrer Bereitschaft zur Kundenbindung zu einer Einkaufsstätte, als auch die sozialen Gruppen. Niederländische und deutsche Konsumenten legen ihren Kaufentscheidungen unterschiedliche Normen, Werte und Begrifflichkeiten zugrunde. Dies kann als ein Indiz dafür gewertet werden, dass beide Gruppen zu unterschiedlichen Kulturclustern gehören und Kundenbindung zu einer im Ausland befindlichen Einkaufstätte unterschiedlich aufgebaut wird.

\section{3 Überprüfung der hypothetischen Konstrukte}

Im Rahmen der quantitativen Analyse werden die hypothetischen Konstrukte einem Prüfschema unterzogen, mit dessen Hilfe die Güte der Konzeptualisierung und Operationalisierung beurteilt werden kann. Hierzu gehören insbesondere die Prüfung der Reliabilität der hypothetischen Konstrukte über die interne Konsistens der zugeordneten Indikatoren mit Hilfe des Cronbachs Alpha, als auch die Überprüfung der Konstrukte mit Hilfe der Faktorenanalyse und der Einsatz der Clusteranalyse.

\subsubsection{Reliabilitätsprüfung der Daten mit Cronbachs Alpha}

Die Messung von Wahrnehmungen, Präferenzen und Motivationen steckt voller Schwierigkeiten. Probleme die sich ergeben können, sind Zweifel, ob die Skalen wirklich das messen, was $\mathrm{zu}$ messen versucht wird. Reliabilität ist eine Vorbedingung für Validität. Aus diesem Grund kann eine Skala, die nicht reliabel ist auch nicht valide sein (Green und Tull, 1982, S.185). Die Herstellung einer Skalenreliabilität hängt stark davon ab, wie gleichartig eine Gruppe von Personen hinsichtlich der zu untersuchenden Eigenschaft ist.

Mit Hilfe des Statistikprogramms SPSS wurde die Reliabilitätsprüfung durchgeführt. Dabei sind die Fragen den einzelnen ursprünglichen Teilbereichen der Befragung zugeordnet und der Realibilitätsprüfung unterzogen worden. Um eine möglichst hohe Reliabilität zu erreichen 
wird eine Optimierung des Fragebogens durchgeführt. Sofern Cronbachs Alpha einen Wert von 0,7 und besser annimmt, kann davon ausgegangen werden, dass die Realibilität der Befragung sichergestellt ist und mit den Werten weitergearbeitet werden kann. Sofern eine Überprüfung mit Cronbachs Alpha Werte unterhalb von 0.7 ergibt, wird bis zu einem Wert von 0.5 eine Einzelfallüberprüfung erfolgen. Ergeben sich Werte unter 0.4, so muss die Aussagefähigkeit des theoretischen Modells in der Praxis in Zweifel gezogen werden und eine Modellanpassung erfolgen (Nunnally, 1978, S.52).

Für das entwickelte Modell der grenzüberschreitenden Kundenbindung ergeben sich nach der Überprüfung durch Cronbachs Alpha folgende Werte:

\begin{tabular}{|c|c|c|}
\hline Reliabilität mit Cronbachs Alpha & Nach Land & Total \\
\hline Wichtigkeit soziale Norm (WSN) & & 0,706 \\
\hline Deutsche & 0,733 & \\
\hline Niederländer & 0,730 & \\
\hline Werturteile der sozialen Gruppe (TZSN) & & 0,679 \\
\hline Deutsche & 0,641 & \\
\hline Niederländer & 0,735 & \\
\hline Bedeutung der sozialen Norm (GKDZ SN) & & 0,714 \\
\hline Deutsche & 0,733 & \\
\hline Niederländer & 0,729 & \\
\hline Gesamtzufriedenheit Kauf (GKDZ Kauf) & & 0,690 \\
\hline Deutsche & 0,722 & \\
\hline Niederländer & 0,659 & \\
\hline Gesamtzufriedenheit (GLSS) & & 0,767 \\
\hline Deutsche & 0,798 & \\
\hline Niederländer & 0,744 & \\
\hline Persönliche Norm & & 0,441 \\
\hline Deutsche & 0,487 & \\
\hline Niederländer & 0,414 & \\
\hline Wahrgenommene Selbstrelevanz (WRS) & & 0,487 \\
\hline Deutsche & 0,513 & \\
\hline Niederländer & 0,313 & \\
\hline Kundenloyalität (KL) & & 0,573 \\
\hline Deutsche & 0,624 & \\
\hline Niederländer & 0,506 & \\
\hline Kundenbindung (KB) & & 0,816 \\
\hline Deutsche & 0,822 & \\
\hline Niederländer & 0,807 & \\
\hline
\end{tabular}

\section{Tab. 7.30: $\quad$ Realibilitätskennzahlen der durchgeführten Befragung}

Die Überprüfung durch Cronbachs Alpha zeigt, dass die verwendeten Variablen im Modell eine gute Erklärung der grenzüberschreitenden Kundenbindung, der sozialen Norm, der Zufriedenheit mit dem gekauften Produkt und der Einkaufsstätte, ferner der Gesamtzufriedenheit des Konsumenten mit dem grenzüberschreitenden Kauf liefert. Doch lassen die schlechteren Werte der persönlichen Norm und der wahrgenommenen Selbstrelevanz (unter 0,5) darauf schließen, dass im grenzüberschreitenden Raum der niederländisch-deutschen Grenze weitere Kräfte auf die Motivation und der Entscheidung grenzüberschreitend einzukaufen einwirken. Ferner lassen diese Werte den Schluss zu, dass sich deutsche und niederländische Konsumen- 
ten hinsichtlich ihres Kaufverhaltens erheblich voneinander unterscheiden. Die wahrgenommene Verhaltenskontrolle ist aus der weiteren Untersuchung herausgenommen worden ${ }^{35}$. Für das aus der Literatur entwickelte Modell der Kundenbindung bedeutet dies, dass das Modell nicht in allen Bereichen den Praxistest bestanden hat. Für die soziale Norm, der Gesamtzufriedenheit mit dem Kauf in der Einkaufsstätte, der Gesamtzufriedenheit mit dem Kauf inclusive der Reaktion des Umfeldes und der Kundenbindung konnten sowohl für niederländische als auch für deutsche Konsumenten akzeptable bis sehr gute Werte der Reliabilität erzielt werden. Doch die Werte der persönliche Norm, der wahrgenommenen Selbstrelevanz und der Kundenloyalität erreichen nur Werte von 0,4 bis 0,5. Diese Ergebnisse lassen den Schluss zu, das das betrachtete Modell noch nicht perfekt ist. Im nächsten Schritt wird deshalb eine Faktorenanalyse durchgeführt, um zu sehen, welche übergeordneten Faktoren durch die Variablen erklärt werden. Die vorliegenden Daten werden somit einer weiteren Prüfung unterzogen.

\subsection{2 Überprüfung des Modells mit Hilfe der Faktorenanalyse}

Die Faktorenanalyse findet dann Anwendung, ,, wenn im Rahmen einer Erhebung eine Vielzahl von Variablen erhoben wurden und der Anwender an einer Reduzierung der Variablen interessiert ist“" (Backhaus, Erichson, Plinke und Weiber, 2003, S. 12; Bühl und Zöfel; 2002, S. 465). Es wird zwischen einer konfirmatorischen und einer explorativen Faktoranalyse unterschieden. Eine konfirmatorische Faktorenanalyse wird gewählt, wenn der Anwender die Beziehungen zwischen beobachteten Variablen und Faktoren aufgrund theoretischer Überlegungen vor Anwendung der Faktorenanalyse festlegt. Wird die Faktorenanalyse als Hypothesengenerierungsinstrument genutzt, dann wird von einer explorativen Faktoranalyse gesprochen. In diesem Fall dient die Faktorenanalyse als ein Analyseinstrument zur Aufdeckung unbekannter Strukturen (Backhaus, Erichson, Plinke und Weiber, 2003, S. 330). Im Folgenden wird eine explorative Faktorenanalyse durchgeführt, da später mit Hilfe von PLS die Ergebnisse weiter untersucht werden. Das Kaiser Kriterium wird angewendet, da hiermit die erklärte Varianz überprüft wird. Die Eignung der Ausgangsdaten wird mit Hilfe des Kaiser-

\footnotetext{
${ }^{35}$ Der berechnete Wert für Cronbachs Alpha entsprach nicht den Gütekriterien. Eine weitere Betrachtung innerhalb der Untersuchung ist nicht möglich.
} 
Meyer-Olkin-Kriterium überprüft ${ }^{36}$. Es wird der Weg begangen, dass aus den gegebenen Items Faktoren gebildet werden, die Zusammenhänge erklären. Die vorhandenen Variablen werden mit dem Ziel, möglichst viele Informationen aus dem ursprünglichen Variablensatz zu erklären, zu einer kleineren Anzahl von Linearkombinationen zusammengefasst (Green und Tull, 1982, S.391). Im Folgenden wird eine Hauptachsenanalyse durchgeführt. ${ }^{37}$ Die Analyse mit Hilfe der Hauptachsenanalyse wurde deshalb gewählt, da hierdurch die Erklärung der Varianz der Variablen durch hypothetische Faktoren erreicht wird. In der Hauptachsenanalyse wird die Frage gestellt, wie die Ursachen bezeichnet werden können, die für die hohen Ladungen der Variablen auf diesen Faktor verantwortlich sind. Die Hauptachsenanalyse wird mit Hilfe der Varimax mit Kaiser-Normalisierung Rotationsmethode durchgeführt. Es handelt sich somit um ein orthogonales Verfahren, das darauf abzielt, auf jeden Faktor einige hohe und einige Ladungen nahe Null zu erzeugen. Generell gibt es zur Bestimmung der Faktorzahl keine eindeutigen Vorschriften. Es ist somit ein subjektiver Eingriff des Anwenders notwendig. Wird das Kaiser-Kriterium angewendet ist die Zahl der extrahierenden „Faktoren gleich der Zahl der Faktoren mit Eigenwerten größer eins“ (Backhaus, Erichson, Plinke und Weiber, 2003, S. 295).

\begin{tabular}{|c|c|c|c|}
\hline \multicolumn{2}{|c|}{ KMO- und Bartlett-Test } & D & $\mathrm{NI}$ \\
\hline \multicolumn{2}{|c|}{ Maß der Stichprobeneignung nach Kaiser-Meyer-Olkin. } & & \\
\hline \multirow[t]{3}{*}{ Bartlett-Test auf Sphärizität } & Ungefähres Chi-Quadrat & 2276,246 & 2068,430 \\
\hline & df & 435 & 435 \\
\hline & Signifikanz nach Bartlett &, 000 & 000 \\
\hline
\end{tabular}

Tab. 7.31: $\quad$ Eignung der Ausgangsdaten für die Faktorenanalyse

Nach der Prüfgröße „measureof sampling adequacy“(MSA) von Kaiser, Meyer und Olkin eignen sich die Ausgangsdaten für eine Faktorenanalyse, da sie den Grenzwert MSA $<0,5$ übersteigen. Nach Kaiser und Rice werden Werte gleich und größer 0,7 als ziemlich gut eingestuft und Werte gleich und größer als 0,8 als verdienstvoll angesehen (Backhaus, Erichson, Plinke und Weiber, 2003, S. 276). Eine Hauptachsenanalyse wird durchgeführt. Es werden

\footnotetext{
${ }^{36}$ In der Literatur wird das Kaiser-Meyer-Olkin-Kriterium als das beste zur Verfügung stehende Verfahren zur Prüfung der Korrelationsmatrix angesehen, weshalb die Anwendung des Verfahrens vor der Durchführung einer Faktorenanalyse durchzuführen ist. Varimax mit Kaiser Normalisierung: Bei dieser Methode handelt es sich um eine orthogonale Rotation (Backhaus, Erichson, Plinke und Weiber, 2003, S. 276, S. 318).

${ }^{37}$ Die Hauptachsenanalyse unterstellt, dass sich die Varianz einer Variable immer in die Komponenten Kommunalität und Einzelrestvarianz aufteilt. Zur Interpretation der Faktoren wird im Anschluß an die Hauptachsenanalyse die Rotation der Faktoren mit Hilfe der Methode Varimax mit Kaiser Normierung durchgeführt.
} 
sieben Faktoren nach dem Kaiser Kriterium für niederländische Probanden extrahiert, acht für deutsche Probanden. Mit dieser Vorgehensweise wird eine erklärte Gesamtvarianz für niederländische Konsumenten von 52,8\% und für deutsche Konsumenten von 57,7\% erreicht. Auch durch die Erhöhung auf neun oder zehn Faktoren wird die Varianz nicht nennenswert erhöht. Mit Hilfe der rotierten Komponentenmatrix werden die Variablen den Hauptkomponenten zugeordnet. Durch diese Zuordnung ergibt sich unter der Prämisse der Unterdrückung der absoluten Werte kleiner 0,4 folgendes Bild der Zuordnung der einzelnen Variablen zu den Faktoren $^{38}$ :

\begin{tabular}{|c|c|c|}
\hline Faktor & Faktorname & Item \\
\hline Faktor 1 & $\begin{array}{l}\text { Soziale } \\
\text { Norm NL }\end{array}$ & $\begin{array}{l}\text { 3.2 Mein Freundes/Bekanntenkreis findet, dass man im Nachbarland qualitativ bessere Produkte einkaufen kann. } \\
\text { 3.3Mein Freundes/Bekanntenkreis findet, dass man im Nachbarland eine bessere Beratung erhält. } \\
\text { 3.4Mein Freundes/Bekanntenkreis findet, dass man im Nachbarland ausgefallenere (Design) Produkte erhält. } \\
\text { 04.Wenn Sie einmal an Ihren Freunde /Bekanntenkreis denken, in wie vielen Fällen kaufen diese im Nachbarland ein? } \\
\text { 05.Freunde/Bekannte reagieren auf meinen Einkauf im Nachbarland positiv. } \\
\text { 11.Im Nachbarland finde ich leichter die Produkte, die ich suche / die ich benötige. }\end{array}$ \\
\hline Faktor 2 & $\begin{array}{l}\text { Einstellung } \\
\text { zum Einkauf } \\
\text { im Nachbar- } \\
\text { land NL }\end{array}$ & $\begin{array}{l}\text { 07.Mit den Einkaufsmöglichkeiten in (Ort der Befragung) bin ich zufrieden. } \\
\text { 08.0Mit dem Einkauf in Geschäft (Name Stammgeschäft) bin ich insgesamt zufrieden. } \\
\text { 08.1 Mit dem Einkauf in Geschäft (Name Stammgeschäft bin ich insgesamt (Beratungsleistung) zufrieden. } \\
\text { 14. Bei nächster Gelegenheit kaufe ich wieder im Nachbarland ein. } \\
\text { 16. Ich finde es schön, die Möglichkeit zu haben, schnell im Nachbarland einkaufen z u können (Wohlbefinden). } \\
\text { 17. Ich empfehle mein Geschäft (Stammgeschäft) im Nachbarland bei meinen Freunden und Bekannten weiter. }\end{array}$ \\
\hline Faktor 3 & $\begin{array}{l}\text { Echte Kunden- } \\
\text { bindung NL }\end{array}$ & $\begin{array}{l}\text { 19. Mindestens } 1 \text { mal im Monat besuche ich mein Stammgeschäft im Nachbarland. } \\
\text { 20. In den vergangenen acht Wochen habe ich oft im Nachbarland eingekauft. } \\
\text { 21. In den vergangenen acht Wochen habe ich häufig in meinem Stammgeschäft eingekauft. } \\
\text { 22. Sofern mein Stammgeschäft in Zukunft auch andere Produktkategorien anbietet, könnte ich mir vorstellen, auch dort zu } \\
\text { kaufen. }\end{array}$ \\
\hline Faktor 4 & $\begin{array}{l}\text { Gesamtzufrie- } \\
\text { denheit mit } \\
\text { Einkaufsstätte } \\
\text { NL }\end{array}$ & $\begin{array}{l}\text { 8.2 Mit dem Einkauf in Geschäft (Name Stammgeschäft) bin ich insgesamt hinsichtlich Sortiment zufrieden. } \\
\text { 8.3 Mit dem Einkauf in Geschäft (Name Stammgeschäft) bin ich insgesamt hinsichtlich Service zufrieden. } \\
\text { 8.4 Mit dem Einkauf in Geschäft (Name Stammgeschäft bin ich insgesamt hinsichtlich Einrichtung des Geschäfts } \\
\text { zufrieden. }\end{array}$ \\
\hline Faktor 5 & $\begin{array}{l}\text { Einstellung } \\
\text { zum Nachbar- } \\
\text { land NL }\end{array}$ & $\begin{array}{l}\text { 02.Für mich ist der Einkauf im Nachbarland ein Stück Lebensqualität. } \\
\text { 13.Der Ort im Nachbarland in dem Einkäufe getätigt werden, sollte vom Heimatort mit öffentlichem Verkehrsmitteln gut } \\
\text { erreichbar sein. } \\
\text { 15. Einkaufen im Nachbarland macht mir Spaß, weil ich eine angenehme Umgebung mit Cafes und Unterhaltungs- } \\
\text { möglichkeiten antreffe. }\end{array}$ \\
\hline Faktor 6 & $\begin{array}{l}\text { Persönliche } \\
\text { Norm NL }\end{array}$ & $\begin{array}{l}\text { 03.1Mein Freundes/Bekanntenkreis findet, dass man im Nachbarland preislich besser einkaufen kann. } \\
\text { 10.Im Nachbarland kaufe ich preiswerter. }\end{array}$ \\
\hline Faktor 7 & $\begin{array}{l}\text { Wichtigkeit } \\
\text { der sozialen } \\
\text { Norm NL }\end{array}$ & $\begin{array}{l}\text { 01.Bereits als Kind fuhr ich mit meinen Eltern zum Einkauf über die Grenze. } \\
\text { 06.Wenn Sie einmal an die letzten Einkäufe zurückdenken, in wie vielen Fällen haben Freunde, Bekannte Ihnen eine } \\
\text { Empfehlung zum Einkauf im Nachbarland gegeben? }\end{array}$ \\
\hline
\end{tabular}

Tab. 7.32: $\quad$ Hauptachsenanalyse der niederländischen Konsumenten

\footnotetext{
${ }^{38}$ Die Faktorwerte besitzen einen Mittelwert von 0 und eine Varianz von 1. Für die Interpretation der Faktorwerte gilt folgendes: negativer Wert Objekt ist im Vergleich zu allen anderen betrachteten Objekten in Bezug auf diesen Faktor unterdurchschnittlich ausgeprägt, Faktor wert von 0 besagt, das ein Objekt in Bezug auf diesen Faktor eine durchschnittliche Ausprägung besitzt und ein positiver Faktorwert besagt, dass das Objekt in Bezug auf diesen Faktor im Vergleich zu den anderen betrachteten Objekten überdurchschnittlich ausgeprägt ist (Backhaus, Erichson, Plinke und Weiber, 2003, S. 323).
} 
Werden diese Faktoren mit dem aus der Literatur abgeleiteten Modell verglichen, so ergeben sich für die niederländischen Konsumenten erhebliche Veränderungen zum Ursprungsmodell (siehe Tabelle 6.2). In der Hauptfaktorenanalyse fehlen unabhängige Faktoren die die Gesamtzufriedenheit mit dem Kauf im Nachbarland erklären, ferner die Erklärung der Loyalität und die Werturteile der sozialen Gruppe. Die persönliche Norm besteht in der vorliegenden Faktoranalyse der niederländischen Konsumenten aus der Einstellung des Individuums zum Preis selbst, aber auch die Werturteile der sozialen Gruppe zum Preis prägen die persönliche Norm. Der Preis wurde bereits beim Vergleich der Mittelwerte als für niederländische Konsumenten besonders wichtiger Faktor der Motivation zum Einkauf im Nachbarland identifiziert. Ferner ergibt sich ein Faktor zur Einstellung zum Einkauf im Nachbarland und ein Faktor zur Einstellung zum Nachbarland selbst.

Für die deutschen Konsumenten werden acht Faktoren benötigt um Kundenbindung aus den vorliegenden Daten zu erklären. Die folgenden Items können den acht Faktoren zugeordnet werden: 


\begin{tabular}{|c|c|c|}
\hline Faktor & Faktorname & Item \\
\hline aktor 1 & $\begin{array}{l}\text { Echte Kunden- } \\
\text { bindung D }\end{array}$ & $\begin{array}{l}\text { 11. Im Nachbarland finde ich leichter die Produkte, die ich suche / die ich benötige. } \\
\text { 19. Mindestens } 1 \text { mal im Monat besuche ich mein Stammgeschäft im Nachbarland. } \\
\text { 20. In den vergangenen acht Wochen habe ich oft im Nachbarland eingekauft. } \\
\text { 21. In den vergangenen acht Wochen habe ich häufig in meinem Stammgeschäft eingekauft. }\end{array}$ \\
\hline Faktor 2 & $\begin{array}{l}\text { Gesamtufrieden- } \\
\text { heit mit Ein- } \\
\text { kaufsstätte D }\end{array}$ & $\begin{array}{l}\text { 3.3 Mein Freundes/Bekanntenkreis findet, dass man im Nachbarland eine bessere Beratung erhält. } \\
\text { 8.2 Mit dem Einkauf in Geschäft (Name Stammgeschäft) bin ich insgesamt hinsichtlich Sortiment zufrieden. } \\
\text { 8.3 Mit dem Einkauf in Geschäft (Name Stammgeschäft) bin ich insgesamt hinsichtlich Service zufrieden. } \\
\text { 8.4 Mit dem Einkauf in Geschäft (Name Stammgeschäft bin ich insgesamt hinsichtlich Einrichtung des Geschäfts } \\
\text { zufrieden. } \\
\text { 12.3Im Nachbarland kaufe ich lieber in kleinen Fachgeschäften ein. }\end{array}$ \\
\hline Faktor 3 & $\begin{array}{l}\text { Gesamtzufrie- } \\
\text { denheit mit } \\
\text { Einkauf im } \\
\text { Nachbarland D }\end{array}$ & $\begin{array}{l}\text { 8.1 Mit dem Einkauf in Geschäft (Name Stammgeschäft bin ich insgesamt (Beratungsleistung) zufrieden. } \\
\text { 7. Mit den Einkaufsmöglichkeiten in (Ort der Befragung) bin ich zufrieden. }\end{array}$ \\
\hline Faktor 4 & $\begin{array}{l}\text { Persönliche } \\
\text { Norm D }\end{array}$ & $\begin{array}{l}\text { 3.1 Mein Freundes/Bekanntenkreis findet, dass man im Nachbarland preislich besser einkaufen kann. } \\
\text { 3.2 Mein Freundes/Bekanntenkreis findet, dass man im Nachbarland qualitativ bessere Produkte einkaufen kann. } \\
\text { 10. Im Nachbarland kaufe ich preiswerter. } \\
\text { 12. Im Nachbarland kaufe ich lieber bei großen Einzelhandelsketten ein. }\end{array}$ \\
\hline Faktor 5 & $\begin{array}{l}\text { Wichtigkeit der } \\
\text { sozialen Norm D }\end{array}$ & $\begin{array}{l}\text { 04.Wenn Sie einmal an Ihren Freunde/Bekanntenkreis denken, in wie vielen Fällen kaufen diese im Nachbarland ein? } \\
\text { 06.Wenn Sie einmal an die letzten Einkäufe zurückdenken, in wie vielen Fällen haben Freunde, Bekannte Ihnen eine } \\
\text { Empfehlung zum Einkauf im Nachbarland gegeben? }\end{array}$ \\
\hline Faktor 6 & $\begin{array}{l}\text { Loyalität zur } \\
\text { Einkaufsstätte D }\end{array}$ & $\begin{array}{l}\text { 17. Ich empfehle mein Geschäft (Stammgeschäft) im Nachbarland bei meinen Freunden und Bekannten weiter. } \\
\text { 18. Ich möchte regelmäßig von meinem Geschäft (Stammgeschäft) im Nachbarland über Angebote und besondere Dienst- } \\
\text { leistungen informiert werden. } \\
\text { 22. Sofern mein Stammgeschäft in Zukunft auch andere Produktkategorien anbietet, könnte ich mir vorstellen, auch dort zu } \\
\text { kaufen. }\end{array}$ \\
\hline Faktor 7 & $\begin{array}{lr}\text { Einstellung } & \text { zum } \\
\text { Einkauf im } & \text { im } \\
\text { Nachbarland D }\end{array}$ & $\begin{array}{l}\text { 14. Bei nächster Gelegenheit kaufe ich wieder im Nachbarland ein. } \\
\text { 15.Einkaufen im Nachbarland macht mir Spaß, weil ich eine angenehme Umgebung mit Cafes und Unterhaltungs- } \\
\text { möglichkeiten antreffe. }\end{array}$ \\
\hline Faktor 8 & Soziale Norm D & $\begin{array}{l}\text { 01. Bereits als Kind fuhr ich mit meinen Eltern zum Einkauf über die Grenze. } \\
\text { 02. Für mich ist der Einkauf im Nachbarland ein Stück Lebensqualität. } \\
\text { 05. Freunde/Bekannte reagieren auf meinen Einkauf im Nachbarland positiv. } \\
\text { 16. Ich finde es schön, die Möglichkeit zu haben, schnell im Nachbarland einkaufen z u können (Wohlbefinden). }\end{array}$ \\
\hline
\end{tabular}

\section{Tab. 7.33: $\quad$ Hauptachsenanalyse der deutschen Konsumenten}

Vergleicht man wiederum diese Faktoren mit dem ursprünglichem Modell, so ergeben sich für die deutschen Konsumenten auch Veränderungen zum Ursprungsmodell (siehe Tab. 6.2). Das neue Modell erklärt, wie das neue niederländische Modell, die Einstellung zum Einkauf im Nachbarland sowie die Gesamtzufriedenheit mit dem Einkauf im Nachbarland. Allerdings sind bei den deutschen Konsumenten die Faktoren zur wahrgenommenen Verhaltenskontrolle und zu den Werturteilen der sozialen Gruppe nicht als eigenständige Faktoren wieder zu finden. Ferner werden die einzelnen Faktoren durch andere Items erklärt als im ursprünglichen aus der Literatur abgeleiteten Modell. 
Somit ergeben sich sowohl für die befragten niederländischen, als auch für die befragten deutschen Konsumenten, ein vom Ursprungsmodell modifiziertes Modell. Werden nun die die Kundenbindung erklärenden Faktoren zwischen deutschen und niederländischen Konsumenten verglichen, ergeben sich folgende Unterschiede und Gemeinsamkeiten:

\begin{tabular}{|c|c|c|c|c|c|c|c|c|c|}
\hline Modell & Faktor 1 & Faktor 2 & Faktor 3 & Faktor 4 & Faktor 5 & Faktor 6 & Faktor 7 & Faktor 8 & Faktor 9 \\
\hline NL & $\begin{array}{l}\text { Soziale } \\
\text { Norm NL }\end{array}$ & $\begin{array}{l}\text { Wichtigkeit } \\
\text { der sozialen } \\
\text { Norm NL }\end{array}$ & $\begin{array}{l}\text { Einstellung zum } \\
\text { Einkauf im } \\
\text { Nachbarland NL }\end{array}$ & $\begin{array}{l}\text { Gesamtzu- } \\
\text { friedenheit } \\
\text { mit Ein- } \\
\text { kaufsstätte } \\
\mathrm{NL}\end{array}$ & & $\begin{array}{l}\text { Einstel- } \\
\text { lung zum } \\
\text { Nachbar- } \\
\text { land NL }\end{array}$ & $\begin{array}{l}\text { Persönli- } \\
\text { che Norm } \\
\text { NL }\end{array}$ & & $\begin{array}{l}\text { Echte Kun- } \\
\text { denbindung } \\
\text { NL }\end{array}$ \\
\hline D & $\begin{array}{l}\text { Soziale } \\
\text { Norm D }\end{array}$ & $\begin{array}{l}\text { Wichtigkeit } \\
\text { der sozialen } \\
\text { Norm D }\end{array}$ & $\begin{array}{l}\text { Einstellung zum } \\
\text { Einkauf im } \\
\text { Nachbarland D }\end{array}$ & $\begin{array}{l}\text { Gesamtzu- } \\
\text { friedenheit } \\
\text { mit Ein- } \\
\text { kaufsstätte } \\
\text { D }\end{array}$ & $\begin{array}{l}\text { Gesamtzu- } \\
\text { friedenheit } \\
\text { mit Einkauf } \\
\text { im Nachbar- } \\
\text { land D }\end{array}$ & & $\begin{array}{l}\text { Persönli- } \\
\text { che Norm } \\
\text { D }\end{array}$ & $\begin{array}{l}\text { Loyalität } \\
\text { zur Ein- } \\
\text { kaufsstätte } \\
\text { D }\end{array}$ & $\begin{array}{l}\text { Echte Kun- } \\
\text { denbindung } \\
\text { D }\end{array}$ \\
\hline
\end{tabular}

Tab. 7.34: $\quad$ Vergleich der Faktoren im niederländischen und deutschen Modell

Das Ergebnis der Faktorenanalyse sind zwei unterschiedliche Modelle zur Erklärung der Kundenbindung beim Einkauf im Nachbarland. Allerdings weisen diese Modelle auch Gemeinsamkeiten auf. Beide Modelle beinhalten folgende Faktoren: Soziale Norm, Wichtigkeit der sozialen Norm, Einstellung zum Einkauf im Nachbarland, Gesamtzufriedenheit mit der Einkaufsstätte, persönliche Norm und echte Kundenbindung. Erklärende Faktoren sind für die niederländischen Konsumenten aber auch die Einstellung zum Nachbarland. Für die deutschen Konsumenten kommen dagegen die Faktoren Gesamtzufriedenheit mit dem Einkauf im Nachbarland und die Loyalität zur Einkaufsstätte hinzu. Die Struktur der beiden Modelle ist somit verschieden. Die bedeutet, dass beide Gruppen sich voneinander unterscheiden. Beide Gruppen entscheiden sich somit aus unterschiedlichen Gründen für den Einkauf im Nachbarland und begründen ihre Kundenbindung zu einer Einkaufsstätte im Nachbarland auch aus unterschiedlichen Gründen. Damit ist nachgewiesen, dass beide Konsumentengruppen sich hinsichtlich ihres Bindungsverhaltens voneinander unterscheiden. Allerdings können mit Hilfe der aus der Faktorenanalyse abgeleiteten Bindungsmodelle die in Kapitel 5 aufgestellten Hypothesen weder verifiziert noch falsifiziert werden. Aus diesem Grund werden in einem Zwischenschritt die beiden Kundengruppen einer Clusteranalyse unterzogen, um schließlich dann mit Hilfe des Least Squares-Verfahren die aufgestellten Hypothesen zu überprüfen. 


\subsection{3 Überprüfung des Modells mit Hilfe der Clusteranalyse}

Die Clusteranalyse wird durchgeführt, um ergänzend zu der Partial Least Squares Analyse eine Charakterisierung der im Nachbarland einkaufenden Konsumenten zu geben. Mit Hilfe der Clusteranalyse wird eine Menge von Objekten so in Gruppen aufgeteilt, dass sich die Gruppen möglichst ähnlich, die Gruppen untereinander aber möglichst unähnlich sind. Im Idealfall sind die Gruppen somit möglichst homogen und im Vergleich zueinander möglichst heterogen (Büschken und von Thaden, 1999, S. 339). Die Clusteranalyse wird oft verwendet, um Kundentypologien zu entwickeln. Für die durchgeführte Clusteranalyse wurden die Fragen 1 bis 22 außer Frage vier aus der Befragung der deutschen und niederländischen Konsumenten zu Grunde gelegt.

Ein wesentliches Merkmal der Clusteranalyse ist „die gleichzeitige Heranziehung aller vorliegenden Eigenschaften zur Gruppenbildung“ (Backhaus, Erichson, Plinke und Weiber, 2003, S. 480). Im Folgenden soll diese Methode verwendet werden, um die einzelnen Variablen einzelnen Gruppen zuzuordnen. Dazu wird das Ward Verfahren gewählt, da dieses Verfahren zu homogenen Gruppen führt. ${ }^{39}$ Das Ward Verfahren wird deshalb gewählt, weil mit Hilfe des Ward-Verfahrens die Gruppen vereinigt werden, die die Varianz möglichst wenig erhöhen. Dadurch werden möglichst homogene Cluster gebildet (Backhaus, Erichson, Plinke und Weiber, 2003, S. 511). Im Rahmen der gewählten Clusteranalyse wird nach folgenden Ablaufschritten vorgegangen:

- Bestimmung der Ähnlichkeiten

- Auswahl des Fusionierungsalgorithmus

- Bestimmung der Clusterzahl (Backhaus, Erichson, Plinke und Weiber, 2003, S. 481ff.).

Da keine sachlogisch begründete Vorstellungen zur Gruppierung der Untersuchungsobjekte vorliegen, soll die Festlegung der Clusterzahl sich an statistischen Kriterien orientieren. Zur Unterstützung der Entscheidung wie viele Cluster bestimmt werden soll die Entwicklung des Heterogenitätsmaßes dienen. Die Zuordnungsübersicht des Ward-Verfahrens zeigt auf, welcher Fusionsstufe welche Fälle bzw. Cluster bei welchem Heterogenitätsmaß zusammengefasst wurden (Backhaus, Erichson, Plinke und Weiber, 2003, S. 522). Zunächst wird ein Mittelwertvergleich der deutschen und niederländischen Cluster durchgeführt. Aus diesem Ver-

\footnotetext{
${ }^{39}$ Für das Ward Verfahren hat sich der Autor deshalb entschieden, da in diesem Verfahren nicht diejenigen Gruppen zusammengefasst werden, die die geringste Distanz aufweisen, sondern es werden die Gruppen vereint, die ein vorgegebenes Heteroginitätsmaß am wenigsten vergrößern. Dabei dient dem Ward-Verfahren als Heterogenitätsmaß die Fehlerquadratsumme. (Backhaus, Erichson, Plinke und Weiber, 2003, S. 511).
} 
gleich lassen sich bereits Unterschiede der vier Cluster erläutern. Dabei wird der Vergleich folgendermaßen durchgeführt:

1. Mit welchen Aussagen stimmt das Cluster am wenigsten zu.

2. Mit welchen Aussagen stimmt das Cluster am meisten zu.

3. In welchen Aussagen hat das Cluster die größte/geringste Übereinstimmung

4. In welchen Aussagen haben die Cluster im Vergleich untereinander die geringste/größte/meisten Übereinstimmungen.

Bei dieser Vorgehensweise ergeben sich folgende speziellen Charakteristika der einzelnen Cluster der deutschen Konsumenten:

Die Minimalkunden:

\begin{tabular}{|lc|}
\hline Frage & Mittelwert \\
\hline 3.2 Mein Freundes/Bekanntenkreis findet, dass man im Nachbarland qualitativ bessere Produkte einkaufen kann. & 3,94 \\
3.3Mein Freundes/Bekanntenkreis findet, dass man im Nachbarland eine bessere Beratung erhält. & 3,68 \\
3.4Mein Freundes/Bekanntenkreis findet, dass man im Nachbarland ausgefallenere Design/Produkte erhält. & 3,34 \\
10.Im Nachbarland kaufe ich preiswerter. & 3,36 \\
11.Im Nachbarland finde ich leichter die Produkte, die ich suche/ die ich benötige. & 3,19 \\
12.1Im Nachbarland kaufe ich lieber bei großen Einzelhandelsketten ein. & 3,28 \\
20.In den vergangenen acht Wochen habe ich oft im Nachbarland eingekauft. & 3,38 \\
21.In den vergangenen acht Wochen habe ich häufig in meinem Stammgeschäft eingekauft. & 3,79 \\
\hline
\end{tabular}

Im nächsten Schritt wird das Cluster, wie nachfolgend alle weiteren Cluster, auch hinsichtlich der soziodemographischen Inhalte überprüft (Tabelle für alle Cluster siehe Anlage 9) und abschließend beschrieben.

In dem Cluster der Minimalkunden sind Männer und Frauen gleichermaßen vertreten. Auch die Altersgruppe ist gleichmäßig verteilt auf die der bis zu 35jährigen und bis zu 64jährigen Konsumenten. Die Haushaltsgröße der zwei Personen Haushalte stellt den größten Teil und das Haushaltseinkommen liegt bei bis zu $€ 2000$,-. Diese Gruppe benutzt beinahe ausschließlich das Auto zum Einkauf im Nachbarland. In diesem Cluster befinden sich Konsumenten, deren Umgebung dem Einkauf im Nachbarland nicht positiv gegenüberstehen und die selbst auch keinen großen Vorteil im Einkauf im Nachbarland sehen. Dies wird deutlich, indem die Fragen in Bezug zum sozialen Umfeld, sowie der eigenen Meinung analysiert werden. Insgesamt kann die Gruppe folgendermaßen charakterisiert werden: 
- Die Gruppe besteht aus Konsumenten, deren Freunde und Bekannten keinen großen Vorteil im Einkauf im Nachbarland sehen.

- Die Gruppe besteht aus Konsumenten, die selbst keinen großen Vorteil im Einkauf im Nachbarland sehen.

- Die Gruppe besteht aus Konsumenten, die nicht regelmäßig zum Einkauf ins Nachbarland fahren.

Die preisbewussten Kunden von Filialunternehmen

\begin{tabular}{|lc|}
\hline Frage & Mittelwert \\
\hline 01.Bereits als Kind fuhr ich mit meinen Eltern zum Einkauf über die Grenze. & 2,93 \\
3.1Mein Freundes/Bekanntenkreis findet, dass man im Nachbarland preislich besser einkaufen kann. & 2,11 \\
07.Mit den Einkaufsmöglichkeiten in (Ort der Befragung) bin ich zufrieden. & 1,68 \\
10.Im Nachbarland kaufe ich preiswerter. & 2,39 \\
12.1Im Nachbarland kaufe ich lieber bei großen Einzelhandelsketten ein. & 2,43
\end{tabular}

In dem Cluster der preisbewussten Kunden der Filialunternehmen sind verstärkt Frauen $(57,1 \%)$ vertreten. Die größte Altersgruppe ist die der 36 bis 64jährigen. Diese Gruppe lebt in Haushalten mit mehr als drei Personen, hat allerdings nur ein Haushaltseinkommen von bis zu $€$ 2.000,-. Als Verkehrsmittel wird das Auto, die Bahn und in kleinem Umfang der Bus zum Einkauf im Nachbarland genutzt. Die Gruppe besteht aus preisbewussten Konsumenten, die die Preisunterschiede zwischen den Ländern gerne nutzen. Diese Konsumenten bilden ihre Einstellung zum Einkauf im Nachbarland aus folgenden Punkten:

- Die Konsumenten haben bereits als Kind wenig Erfahrung mit dem Einkauf im Nachbarland gemacht.

- Das soziale Umfeld der Konsumenten ist der Meinung, dass im Nachbarland preiswerter eingekauft werden kann.

- Eine positive Grundeinstimmung der Konsumenten zum Einkauf im Nachbarland ist vorhanden.

- Die Konsumenten erledigen gerne ihre Einkäufe in Einzelhandelsketten und sind zufrieden mit dem Einkauf.

- Konsumenten haben eine hohe Zufriedenheit mit dem Einkaufsort im Nachbarland. 
Die Gelegenheitskunden

\begin{tabular}{lc} 
Frage & Mittelwert \\
\hline 02.Für mich ist der Einkauf im Nachbarland ein Stück Lebensqualität. & 2,74 \\
13.Der Ort im Nachbarland in dem Einkäufe getätigt werden, sollte vom Heimatort mit öffentlichen Verkehrsmitteln & 2,16 \\
$\quad$ gut erreichbar sein. & 1,72 \\
14.Bei nächster Gelegenheit kaufe ich wieder im Nachbarland ein. & 3,48 \\
19.Mindestens 1mal im Monat besuche ich mein Stammgeschäft im Nachbarland. & 2,63 \\
22.Sofern mein Stammgeschäft in Zukunft auch andere Produktkategorien anbietet, könnte ich mir vorstellen, auch dort zu \\
$\quad$ kaufen.
\end{tabular}

$\mathrm{Zu}$ den Gelegenheitskunden gehören Männer (45,7\%) und Frauen $(54,3 \%)$ gleichermaßen. Das Alter beträgt bis zu 35 Jahren und die Konsumenten leben hauptsächlich in zwei Personen Haushalte. Das Haushaltsnettoeinkommen dieser Gruppe liegt im mittleren Bereich (43,6\% bis zu $€ 2.000$,- und 40,6\% bis zu $€ 3.500,-)$. Einkäufe werden mit dem Auto, aber auch zu 12\% mit der Bahn getätigt. Das Cluster besteht aus Konsumenten, die nur gelegentlich im Nachbarland einkaufen. Diese Gruppe bildet ihre Einstellung zum Einkauf im Nachbarland aus folgenden Punkten:

- Das soziale Umfeld des Konsumenten steht dem Einkauf im Nachbarland nicht sehr positiv gegenüber.

- Einkaufen im Nachbarland erhöht aus Sicht des Konsumenten nicht die Lebensqualität.

- Mit den Einkaufsmöglichkeiten im Nachbarland ist der Konsument nicht zufrieden.

- Das Nachbarland ist verkehrsmäßig aus Sicht des Konsumenten nicht gut erreichbar .

- Der Konsument hat kein Interesse an Einkaufsinformationen aus dem Nachbarland.

Die begeisterten Fachgeschäftskunden

\begin{tabular}{|lc|}
\hline Frage & Mittelwert \\
\hline 3.4Mein Freundes/Bekanntenkreis findet, dass man im Nachbarland ausgefallenere Design/Produkte erhält. & 1,34 \\
08.0Mit dem Einkauf in meinem Stammgeschäft bin ich insgesamt zufrieden. & 1,46 \\
08.1Mit dem Einkauf in Geschäft (Name Stammgeschäft bin ich insgesamt (Beratungsleistung) zufrieden. & 1,42 \\
08.2Mit dem Einkauf in Geschäft (Name Stammgeschäft) bin ich insgesamt hinsichtlich Sortiment zufrieden. & 1,36 \\
08.4Mit dem Einkauf in Geschäft (Name Stammgeschäft bin ich insgesamt hinsichtlich Einrichtung des Geschäfts zufrieden. & 1,46 \\
09.Im Nachbarland kann ich Produkte kaufen, die ich Zuhause nicht bekomme. & 1,34 \\
12.3Im Nachbarland kaufe ich lieber in kleinen Fachgeschäften ein. & 1,44 \\
15.Einkaufen im Nachbarland macht mir Spass, weil ich eine angenehme Umgebung mit Cafes und Unterhaltungs- & 1,18 \\
möglichkeiten antreffe. & 1,28 \\
16.Ich finde es schön, die Möglichkeit zu haben, schnell im Nachbarland einkaufen z u können (Wohlbefinden). & 1,40 \\
21.In den vergangenen acht Wochen habe ich häufig in meinem Stammgeschäft eingekauft. & \\
\hline
\end{tabular}


Die begeisterten Fachgeschäftskunden sind zu einem sehr großen Anteil Frauen. Diese sind zwischen 35 und 64 Jahre alt und leben in Haushalten mit überwiegend drei und mehr Haushaltsmitgliedern $(48,2 \%)$. Allerdings leben auch viele in einem Haushalt mit zwei Haushaltsmitgliedern. Singelhaushalte sind in dieser Gruppe sehr gering vertreten. Das Haushaltsnettoeinkommen liegt bis $€ 3.500$,- (42\%), allerdings ist die Gruppe der Einkommen über $€$ 3.500,in diesem Cluster mit 30\% besonders stark vertreten. In diesem Cluster wird das Auto, aber auch die Bahn zum Einkauf im Nachbarland genutzt. Die Gruppe besteht aus Konsumenten, die sehr enthusiastisch und begeistert dem Einkauf im Nachbarland gegenüberstehen. Insgesamt kann die Gruppe charkteriesiert werden als eine Gruppe von Konsumenten, die

- Einkaufen im Nachbarland mit Spaß verbinden.

- Gute Möglichkeiten haben im Nachbarland einzukaufen.

- Ein soziales Umfeld haben, das dem Einkauf im Nachbarland positiv gegenübersteht.

- Der Meinung sind, dass sie im Nachbarland Produkte angeboten bekommen, die sie in ihrem eigenen Land nicht bekommen.

- Eine hohe Zufriedenheit mit dem Einkauf im Stammgeschäft haben und hier insbesondere das angebotenen Sortiment, die Beratung, die Atmosphäre und die Einrichtung schätzen.

- Gerne in kleinen Einzelhandelsgeschäften einkauft.

- Regelmäßig das Nachbarland und das Stammgeschäft zum Einkauf aufsuchen.

Werden die vier Cluster gegenübergestellt, so sind die Gruppe der Minimalkunden und die Gruppe der begeisterten Fachgeschäftskunden sehr konträr zueinander. Die Minimalkunden sehen keinen großen Vorteil im Einkauf im Nachbarland und auch deren soziales Umfeld ist dem Einkauf im Nachbarland gegenüber negativ eingestellt. Die Konsumenten haben ein im Verhältnis zu der Gruppe der begeisterten Fachgeschäftskunden niedriges Haushaltseinkommen und fahren auch seltener ins Nachbarland. Die Gruppe der begeisterten Fachgeschäftskunden besteht dagegen aus Konsumenten, die dem Einkauf im Nachbarland sehr positiv gegenüberstehen und im Nachbarland gezielt Produkte suchen, die sie im Heimatland nicht bekommen. Das von dieser Gruppe bevorzugte Stammgeschäft bietet aber das gesuchte Sortiment und verfügt über die relevanten Zufriedenheitstreiber dieser Gruppe wie Beratung, Atmosphäre und Einrichtung. Die preisbewussten Kunden der Filialbetriebe und die Gelegenheitskunden befinden sich zwischen diesen beiden Extremen. Allerdings setzen die Konsumenten in diesen Clustern jeweils andere Akzente, wobei die Einstellung zum Einkauf im Nachbarland der preisbewussten Kunden von Filialbetrieben positiver ist als die Einstellung der Gelegenheitskäufer. 
Aus der Übersicht der Clusteranalyse (siehe Anhang 6-10) wird deutlich, dass die größte Gruppe der deutschen Konsumenten zwar sehr zufrieden mit ihrem grenzüberschreitenden Einkauf ist, allerdings ihre Meinung zum Nachbarland von ihrer sozialen Gruppe geprägt ist. Diese Gruppe der „Gelegenheitskunden“ ist zwar zufrieden mit dem Einkaufsort, aber nur mäßig zufrieden mit der bevorzugten Einkaufsstätte. Diese Gruppe ist immer auf der Suche nach neuen Kauferlebnissen. Dennoch steigern die Einkaufsmöglichkeiten im Nachbarland aus Sicht dieser Gruppe nicht die Lebensqualität. Diese Gruppe legt Wert auf die Anbindung mit öffentlichen Verkehrsmitteln und die Erreichbarkeit des Nachbarlandes ist aus Sicht dieser Gruppe mit öffentlichen Verkehrsmitteln schlecht. Anders die Gruppe der „preisbewussten Kunden von Filialbetrieben“. Auch sie werden von der sozialen Gruppe geprägt, haben das Nachbarland aber nicht bereits als Kind als Einkaufsalternative kennen gelernt. Allerdings haben sie eigene Erfahrungen mit dem Einkauf im Nachbarland gemacht und sind neutral dem Nachbarland und der Einkaufsstätte gegenüber eingestellt. Diese Gruppe kauft auch im Nachbarland gerne in den Einkaufsstätten ein, die vom Heimatland oder aus anderen Einkaufsgelegenheiten bekannt sind. Die Gruppe der deutschen Konsumenten, die als „,begeisterte Fachgeschäftskunden " bezeichnet werden kann, sind als überzeugte Käufer stark von ihrer sozialen Gruppe geprägt und als an eine Einkaufsstätte im niederländischen Nachbarland gebundene Konsumenten einzustufen. Als eine weitere Gruppe können „die Minimalkunden“ bezeichnet werden. Diese Gruppe ist durch ihre soziale Gruppe nicht positiv gegenüber dem Einkauf im Nachbarland geprägt, hat keine positive Einstellung zum Einkauf im Nachbarland und ist auch an keine Einkaufsstätte gebunden.

Für die niederländischen Konsumenten wird nun ebenfalls eine Clusteranalyse unterteilt nach Fällen durchgeführt. Dabei wird im Rahmen der Analyse vorgegangen wie bei den deutschen Konsumenten. Es ergeben sich vier Cluster (siehe Anhang 11-15), die sich wie folgt charakterisieren und beschreiben lassen:

Die Minimalkunden

02.Für mich ist der Einkauf im Nachbarland ein Stück Lebensqualität.

3.2 Mein Freundes/Bekanntenkreis findet, dass man im Nachbarland qualitativ bessere Produkte einkaufen kann.

3.3Mein Freundes/Bekanntenkreis findet, dass man im Nachbarland eine bessere Beratung erhält.

3.4Mein Freundes/Bekanntenkreis findet, dass man im Nachbarland ausgefallenere Design/Produkte erhält.

11.Im Nachbarland finde ich leichter die Produkte, die ich suche / die ich benötige.

18.Ich möchte regelmäßig von meinem Geschäft (Stammgeschäft) im Nachbarland über Angebote und besondere Dienstleistungen informiert werden. 
Die niederländischen Minimalkunden bestehen aus einem sehr hohen Anteil an weiblichen Konsumenten $(83,3 \%)$ und haben ein Lebensalter von bis zu 35 Jahren(58,3\%). Sie kommen überwiegend aus Haushalten mit drei und mehr Haushaltsmitgliedern $(61,1 \%)$ und haben ein Haushaltsnettoeinkommen von bis zu $€ 2.000$,- (55,6\%). Diese Gruppe fährt zum Einkauf ins Nachbarland mit dem Auto (72,2\%) oder mit der Bahn (27,8\%). Die Antworten dieser Konsumentengruppe zum Einkauf im Nachbarland sind meist negativ. Auch die soziale Gruppe dieser Konsumenten ist dem Einkauf im Nachbarland nicht positiv gegenüber eingestellt. Diese Gruppe kauft selten im Nachbarland ein und sieht im Einkauf im Nachbarland keine Verbesserung der Lebensqualität. Ferner glaubt diese Gruppe auch nicht, dass der Einkauf im Nachbarland Vorteile für sie bringt. Insgesamt kann diese Gruppe charakterisiert werden als Gruppe:

- Die selten im Nachbarland einkauft .

- Die dem Einkauf im Nachbarland nicht positiv gegenüber eingestellt ist.

- Die im Einkauf im Nachbarland keine Verbesserung der Lebensqualität sieht

Die Gelegenheitskäufer Frage Mittelwert

07.Mit den Einkaufsmöglichkeiten in (Ort der Befragung) bin ich zufrieden.

Unter den Gelegenheitskäufern sind männliche Konsumenten mit 38,1\% und weibliche Konsumenten mit 61,9\% vertreten. Die stärkste Altersgruppe ist die Gruppe der bis zu 35jährigen, wobei in dieser Gruppe auch die anderen Altersgruppen vertreten sind. Dieses Cluster verfügt über ein -etwas höheres Einkommen, wobei auch hier die Gruppe mit einem Haushaltsnettoeinkommen von bis zu $€ 2.000$,- die stärkste Gruppe stellt. Es handelt sich zu einem hohen Anteil aus zwei Personenhaushalte (43,2\%), die alle Verkehrsmittel zum Einkauf im Nachbarland nutzt, wobei auch hier der Schwerpunkt auf dem Verkehrsmittel Auto (81,4\%) liegt. Diese Gruppe hat folgende Prioritäten beim Einkauf im Nachbarland:

- Die Gruppe hat einen Lieblingsort zum Einkauf im Nachbarland.

- Die Gruppe kauft gerne in Filialbetrieben ein. 
Die Begeisterten für Stammgeschäfte Frage Mittelwert

3.2 Mein Freundes/Bekanntenkreis findet, dass man im Nachbarland qualitativ bessere Produkte einkaufen kann.

3.3Mein Freundes/Bekanntenkreis findet, dass man im Nachbarland eine bessere Beratung erhält.

3.4Mein Freundes/Bekanntenkreis findet, dass man im Nachbarland ausgefallenere Design/Produkte erhält.

07.Mit den Einkaufsmöglichkeiten in (Ort der Befragung) bin ich zufrieden.

08.0Mit dem Einkauf in meinem Stammgeschäft bin ich insgesamt zufrieden.

08.1Mit dem Einkauf in Geschäft (Name Stammgeschäft bin ich insgesamt (Beratungsleistung) zufrieden.

In der Gruppe der Begeisterten für Stammgeschäfte befinden sich überwiegend weibliche Konsumenten (74\%). Die Altersgruppen bis 35 Jahre (42\%) und zwischen 35 und 64 Jahre (38\%) ist fast gleich stark vertreten. Die Konsumenten leben überwiegend in zwei Personenhaushalte in denen alle Einkommensgruppen (Schwerpunktmäßig aber das Haushaltsnettoeinkommen von bis zu $€ 2.000,-(46,0 \%))$ vertreten sind. Als Verkehrsmittel wir das Auto (44\%) und die Bahn (42\%) fast gleichmäßig für den Einkauf im Nachbarland genutzt. Auch der Bus (12\%) ist hier ein Verkehrsmittel das zum Einkauf genutzt wird. Das soziale Umfeld ist dem Einkauf im Nachbarland positiv gegenüber eingestellt und die Konsumenten selbst haben eine hohe Zufriedenheit gegenüber dem Einkaufsort, als auch gegenüber der Einkaufsstätte im Nachbarland. Diese Konsumenten kaufen sowohl in kleinen Fachhandelsgeschäfte ein, als auch in großen Filialgeschäften. Zusammenfassend lässt sich die Gruppe folgendermaßen charakterisieren:

- Es wird in bevorzugten Einkaufsstätten eingekauft (sowohl in kleinen Fachgeschäften als auch in Filialbetrieben).

- Die Gruppe ist insgesamt mit dem Einkauf im Nachbarland zufrieden.

- Die soziale Gruppe der Konsumenten ist dem Einkauf im Nachbarland gegenüber positiv eingestellt. 
Die schnellen zielgerichteten, preisorientierten Käufer

\begin{tabular}{|c|c|}
\hline Frage & Mittelwert \\
\hline 02.Für mich ist der Einkauf im Nachbarland ein Stück Lebensqualität. & 3,37 \\
\hline 3.1Mein Freundes/Bekanntenkreis findet, dass man im Nachbarland preislich besser einkaufen kann. & 1,63 \\
\hline 3.3Mein Freundes/Bekanntenkreis findet, dass man im Nachbarland eine bessere Beratung erhält. & 3,68 \\
\hline 08.1Mit dem Einkauf in Geschäft (Name Stammgeschäft bin ich insgesamt (Beratungsleistung) zufrieden. & 3,17 \\
\hline 09.Im Nachbarland kann ich Produkte kaufen, die ich Zuhause nicht bekomme. & 1,83 \\
\hline 12.3Im Nachbarland kaufe ich lieber in kleinen Fachgeschäften ein. & 3,56 \\
\hline \multicolumn{2}{|l|}{ 13. Der Ort im Nachbarland in dem Einkäufe getätigt werden, sollte vom Heimatort mit öffentlichen } \\
\hline Verkehrsmitteln gut erreichbar sein. & 4,66 \\
\hline 14.Bei nächster Gelegenheit kaufe ich wieder im Nachbarland ein. & 1,76 \\
\hline \multicolumn{2}{|l|}{ 15.Einkaufen im Nachbarland macht mir Spass, weil ich eine angenehme Umgebung mit Cafes und Unterhaltungs- } \\
\hline möglichkeiten antreffe. & 3,76 \\
\hline 16.Ich finde es schön, die Möglichkeit zu haben, schnell im Nachbarland einkaufen z u können (Wohlbefinden). & 1,41 \\
\hline 17.Ich empfehle mein Geschäft (Stammgeschäft) im Nachbarland bei meinen Freunden und Bekannten weiter. & 1,54 \\
\hline \multicolumn{2}{|l|}{ 18.Ich möchte regelmäßig von meinem Geschäft (Stammgeschäft) im Nachbarland über Angebote und besondere Dienst- } \\
\hline leistungen informiert werden. & 1,59 \\
\hline 20.In den vergangenen acht Wochen habe ich oft im Nachbarland eingekauft. & 2,39 \\
\hline 21.In den vergangenen acht Wochen habe ich häufig in meinem Stammgeschäft eingekauft. & 2,15 \\
\hline \multicolumn{2}{|l|}{ 22.Sofern mein Stammgeschäft in Zukunft auch andere Produktkategorien anbietet, könnte ich mir vorstellen, auch dort zu } \\
\hline kaufen. & 1,76 \\
\hline
\end{tabular}

$\mathrm{Zu}$ den schnellen zielgerichteten, preisorientierten Käufern gehören zu 41,5\% männliche und zu 58,5\% weibliche Konsumenten. Die stärkste Gruppe sind die zwischen 35 und 64jährigen, ältere Konsumenten (über 64 Jahre) sind in dieser Gruppe nicht vertreten. Gleichverteilt leben diese Konsumenten in zwei (46,3\%) und Mehrpersonenhaushalte (53,7\%). Das Haushaltsnettoeinkommen liegt bei $70,7 \%$ bei bis zu $€ 2.000$,- und die Konsumenten bevorzugen zum Einkauf im Nachbarland das Auto (97,6\%) als Verkehrsmittel. Allerdings ist die Einstellung dieser Gruppe nicht durchgängig. Dies ist auch in der sozialen Gruppe dieser Konsumenten so. Einerseits herrscht die Meinung vor, dass man im Nachbarland beim Einkauf Preisvorteile hat, auf der anderen Seite spielt Beratung keine große Rolle. Dennoch will man regelmäßig Informationen über das Stammgeschäft im Nachbarland und findet es schön die Möglichkeit zu haben schnell im Nachbarland einzukaufen, auf der anderen Seite macht der Einkauf im Nachbarland keinen Spaß. Gerne würde man aber auch andere Produkte im Stammgeschäft einkaufen - sofern sie angeboten werden. Negativ ist diese Gruppe gegenüber kleinen Einzelhandelsgeschäften gegenüber eingestellt. Es handelt sich in dieser Gruppe um Konsumenten, die ihre Einkäufe schnell und unkompliziert erledigen möchten und im Einkauf im Nachbarland einen sehr pragmatischen Vorteil suchen. Zusammenfassend findet man in dieser Gruppe Konsumenten mit der Einstellung: 
- Einkaufen im Nachbarland ist nicht mit Spass verbunden.

- Der preisliche Aspekt ist wichtig.

- Einkaufen muss schnell und unkompliziert sein.

- Positive Erfahrungen werden im sozialen Umfeld weitergegeben.

Insgesamt sind bei den niederländischen Konsumenten eine große Gruppe ( Die Gelegenheitskäufer, 118 Probanden) zu erkennen, die eine neutrale Position bezieht und keine konkreten Vorlieben hat. Nur eine kleine Gruppe (Die Minimalkunden, 36 Probanden) ist dem Einkauf im Nachbarland wirklich negativ gegenüber eingestellt. Eine weitere kleine Gruppe (Die schnellen zielgerichteten preisorientierten Käufer, 41 Probanden) kann als zielorientierte, preislastige Schnellkäufer bezeichnet werden. Für sie ist der Einkauf im Nachbarland eine Pflichtübung um persönliche Vorteile zu nutzen. Die letzte Gruppe sind die Begeisterten (Die Begeisterten für Fachgeschäfte, 50 Probanden), die sowohl in Fachgeschäften als auch in Filialbetrieben einkaufen.

Die Gruppe der „Minimalkunden“ geht sehr unregelmäßig im Nachbarland einkaufen und hat eine reservierte Haltung zum Einkauf im Nachbarland. Auch die soziale Gruppe dieser Käufer hat gegenüber dem Einkauf im Nachbarland eine negative Meinung. Die Gruppe der „Gelegenheitskäufer" sieht den Einkauf im Nachbarland als regionale Möglichkeit die Grenznähe zu nutzen, um auch in Orten im Nachbarland ihre Einkäufe tätigen zu können. Allerdings kaufen sie im Nachbarland nur unregelmäßig ein und fühlen sich weder an einem Ort noch an eine Einkaufsstätte im Nachbarland gebunden. Dagegen grenzt sich die Gruppe „der Begeisterten für Stammgeschäfte" dahingehend von den anderen Gruppen ab, indem sie positiv von ihrer sozialen Gruppe zum Einkauf im Nachbarland beeinflusst werden, eine positive Einstellung zum Einkauf im Nachbarland hat, sich aber nicht binden lassen möchte. Die Konsumenten, die der Gruppe „der pragmatischen Käufer“ zugeordnet werden können, haben eine positive Grundeinstellung zum Einkauf im Nachbarland, sind über die Einkaufsmöglichkeiten im Nachbarland gut informiert und geben dieses Wissen auch an Freunde und Bekannte weiter. Allerdings ist diese Gruppe stark preisfixiert und macht seine Bindung von diesem Kaufvorteil abhängig. 


\begin{tabular}{|l|l|l|l|l|l|}
\hline \multicolumn{2}{|l|}{ Niederlande } & \multicolumn{2}{l|}{ Deutschland } & Anteil in \% \\
\hline $\mathrm{n}$ & Name & Anteil in \% & $\mathrm{n}$ & Name & 21,0 \\
\hline 36 & Die Minimalkunden & 14,7 & 53 & Die Minimalkunden & 37,2 \\
\hline 118 & Die Gelegenheitskäufer & 48,2 & 56 & Die Gelegenheitskäufer & 19,8 \\
\hline 50 & $\begin{array}{l}\text { Die Begeisterten für Stamm- } \\
\text { geschäfte }\end{array}$ & 20,4 & 94 & Die begeisterten Fachgeschäftskunden & 12,1 \\
\hline 41 & $\begin{array}{l}\text { Die schnellen zielgerichteten, } \\
\text { preisorientierten Käufer }\end{array}$ & 16,7 & 50 & $\begin{array}{l}\text { Die preisbewussten Kunden von Fili- } \\
\text { alunternehmen }\end{array}$ & 22 \\
\hline
\end{tabular}

Tab. 7.35: $\quad$ Vergleich der niederländischen und deutschen Cluster

In beiden Ländern können somit die befragten Konsumenten in vier Konsumentencluster eingeteilt werden. In beiden Ländern gibt es „Minimalkunden“, „Gelegenheitskäufer“ und eine Gruppe, die gerne im Nachbarland einkauft, die Gruppe der „Begeisterten“.

\subsection{Empirische Ergebnisse und Evaluierung der Wirkungszusammenhänge und Modellbeurteilung}

Bisher wurde das Kaufverhalten der befragten deutschen und niederländischen Konsumenten mit Hilfe der deskriptiven Statistik und der Faktoren- und Clusteranalyse analysiert. Allerdings geht es bei den in dieser Arbeit aufgestellten Hypothesen um die Formulierung und Überprüfung von Modellen, die Zusammenhänge zwischen latenten Variablen erklären sollen. Es soll insbesondere untersucht werden, inwieweit die soziale Norm, die persönliche Norm und die wahrgenommene Selbstrelevanz Einfluss auf die Kaufgewohnheiten sowie auf die Kundenzufriedenheit und -bindung im deutsch-niederländischen Grenzgebiet haben. Es handelt sich somit um die Analyse von Strukturgleichungsmodellen. In diesem Fall sind einer Analyse mit Hilfe von Faktor- oder Clusteranalysen Grenzen gesetzt. Zwar stellen auch Faktoren latente Größen dar - allerdings erlaubt die klassische (explorative) Faktorenanalyse, wie sie in SPSS implementiert ist, nicht die Berechnung von Gütemaßen zur Beurteilung des Ausmaßes der Übereinstimmung zwischen denjenigen Strukturen, die innerhalb des vorliegenden Datenmaterials beobachtet werden können und denjenigen Strukturen, die durch die aufgestellten Hypothesen, d.h. durch das erstellte Modell spezifiziert werden. Es werden somit im Folgenden kausale Abhängigkeiten zwischen Merkmalen untersucht. Die Kausalanalyse besitzt konfirmatorischen Charakter und ist den hypothesenprüfenden statistischen Verfahren zuzurechnen (Backhaus, Erichson, Plinke und Weiber, 2003, S.334). Zunächst werden die abhängigen latenten Variablen als endogene Größen und die unabhängigen latenten Variablen 
als exogene Größen festgelegt, in einem nächsten Schritt ein Meßmodell für die latenten endogenen Variablen und ein Meßmodell für die latenten exogenen Variablen formuliert. Die Ablaufschritte einer Kausalanalyse können nach Backhaus et.al. (2003, S. 352) in einem Ablaufdiagramm wie folgt zusammengefasst werden:

1. Hypothesenbildung

2. Pfaddiagramm und Modellspezifikation

3. Identifikation der Modellstruktur

4. Parameterschätzungen

5. Beurteilung der Schätzergebnisse

6. Modifikation der Modellstruktur

Die Beurteilung der Gesamtstruktur liefert ein Maß für die Anpassungsgüte der theoretischen Modellstruktur an die empirischen Daten. Entsprechend den Ausführungen in Kapitel 5 dieser Arbeit setzt sich das Kundenbindungsmodell aus mittelbaren und unmittelbaren Größen zusammen. Kundenbindung entsteht nur, wenn der Erfolg der Kundenbindung durch Indikatoren für die differenzierten Kundenbedürfnisse, - erwartungen und - verhaltensweisen vorhanden sind. Diller (2001, S. 865) fordert in diesem Zusammenhang die Erfassung des Konstruktes Kundenbindung durch die Indikatoren relative Wiederbesuchshäufigkeit, relative Weiterempfehlungshäufigkeit und Kundenzufriedenheit. Bruhn (2001, S.46) fordert weiterhin die Kauffrequenz, das Integrationsverhalten und oder die Verwendung des Kommunikationsverhaltens. Diese Arbeit folgt weitestgehend den Forderungen und Diller (2001) und Bruhn (2001) und misst den Einfluss der neun Indikatoren auf das Ausmaß der Kundenbindung. Die Messung der Gesamtzufriedenheit mit dem Einkauf im Nachbarland insgesamt erfolgt durch die Gesamtzufriedenheit mit dem Kauf in der Einkaufsstätte selbst, sowie der Bedeutung der sozialen Norm für den einkaufenden Konsumenten. Die Bedeutung der sozialen Norm wird wiederum durch die Werturteile der sozialen Gruppe sowie der Wichtigkeit der sozialen Norm für den einkaufenden Konsumenten gemessen. Die individuelle Lust auf das Einkaufen im Nachbarland (wahrgenommene Selbstrelevanz) wird wiederum gemessen duch die persönliche Norm des Konsumenten, sowie seine Selbstreflexion den Vorsatz auch in die Tat umzusetzen (wahrgenommene Verhaltenskontrolle). Der wahrgenommenen Selbstrelevanz wird wiederum ein direkter Einfluss auf die Kundenloyalität unterstellt. Zur Messung der Kundenbindung soll auf bereits etablierte Messgrößen zurückgegriffen werden. In der Literatur wird zwischen subjektiven und objektiven Messgrößen unterschieden. Objektive Messgrößen werden aus überprüfbaren Zahlen (Wiederkaufrate, Häufigkeit des Einkaufsstättenbesuchs, Einkaufsbetrag) gebildet, subjektive Messgrößen dagegen beruhen auf dem Urteil der Probanden und deren Einstellung. Die Kundenbindung wird im vorliegendem Fall subjektiv durch die 
Konstrukte Bedeutung der sozialen Norm für den Konsumenten, Gesamtzufriedenheit mit dem Einkauf, und Kundenloyalität gemessen.

Die Schätzung des Bindungsmodells erfolgt mit Hilfe des in Abschnitt 6.6 dargestellten Verfahrens der Software SmartPLS. Die folgenden beiden Abbildungen zeigen die Bindungsmodelle im Überblick:



Abb.7.1 : $\quad$ Befunde zum Bindungsmodell der niederländischen Konsumenten 
196 | Kapitel 7

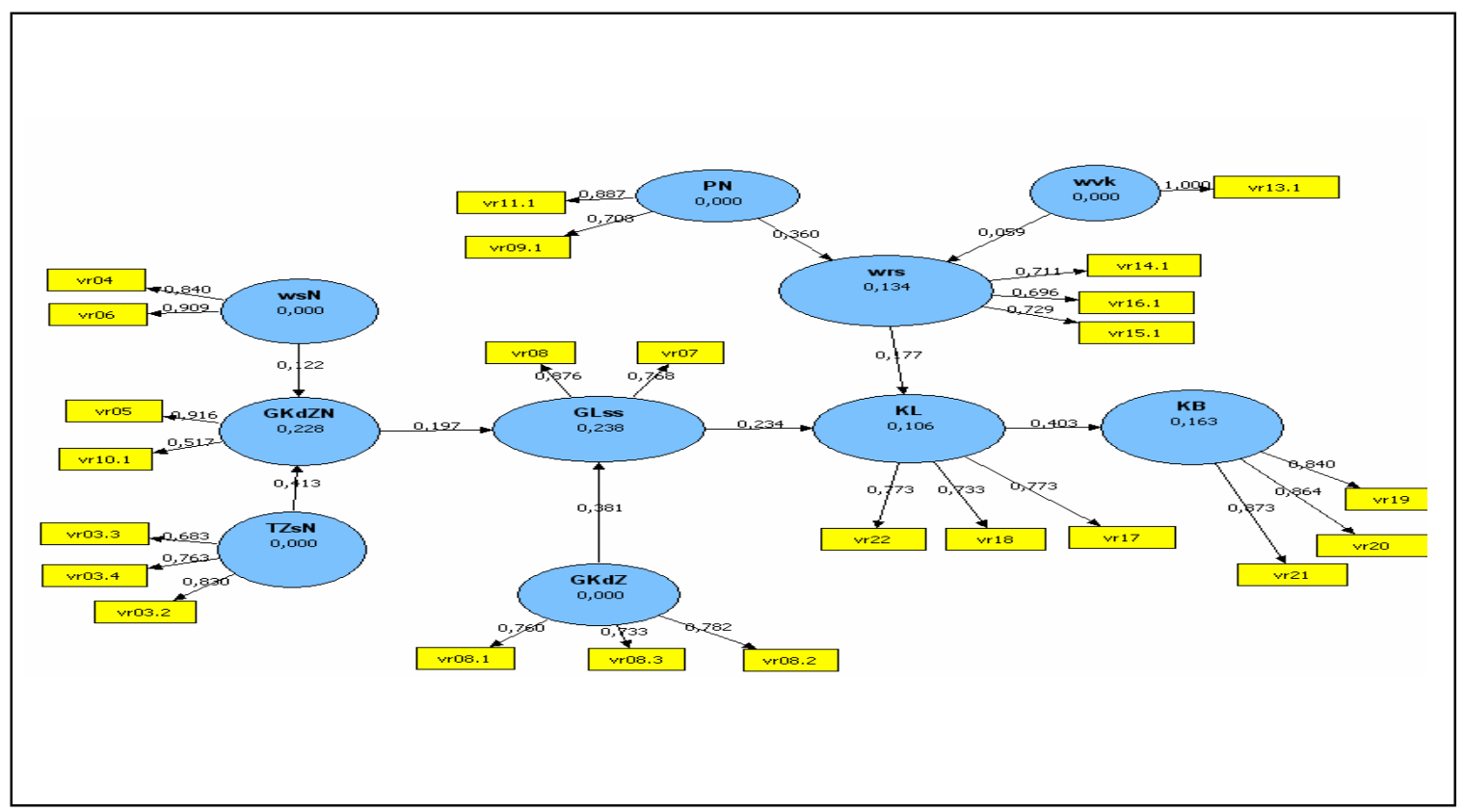

Abb.7.2: $\quad$ Befunde zum Bindungsmodell der deutschen Konsumenten

Zunächst werden die Befunde des Bindungsmodell der niederländischen Konsumenten evaluiert, anschließend die der deutschen Konsumenten. Danach werden beide Evaluationen miteinander verglichen. 


\subsubsection{Evaluation der Wirkungszusammenhänge und Modellbeurteilung der Kunden- bindung der niederländischen Konsumenten}

Zunächst werden die Qualitätskriterien für das Bindungsmodell betrachtet. Hieraus ergibt sich folgendes Bild:

\begin{tabular}{|c|c|c|c|c|}
\hline Quality Criteria & AVE & Composite Reliabiliy & Stone-Geiser-Test $\left(\mathrm{Q}^{2}\right)$ & R Square \\
\hline $\begin{array}{l}\text { Bedeutung der sozialen } \\
\text { Norm für den Konsumenten } \\
(\text { GKDZ SN) }\end{array}$ & 0,609 & 0,746 & 0,6131 & 0,065 \\
\hline $\begin{array}{l}\text { Wichtigkeit der sozialen } \\
\text { Norm (WSN) }\end{array}$ & 0,690 & 0,817 & 0,6899 & \\
\hline $\begin{array}{l}\text { Werturteile der sozialen } \\
\text { Gruppe (TZSN) }\end{array}$ & 0,652 & 0,849 & 0,6522 & \\
\hline $\begin{array}{l}\text { Gesamtzufriedenheit mit } \\
\text { dem Einkauf im benachbar- } \\
\text { tem Ausland (GLSS) }\end{array}$ & 0,610 & 0,758 & 0,6093 & 0,180 \\
\hline $\begin{array}{l}\text { Gesamtzufriedenheit mit } \\
\text { Stammgeschäft im Nachbar- } \\
\text { land (GKDZ Kauf) }\end{array}$ & 0,501 & 0,747 & 0,5014 & \\
\hline Kundenloyalität (KL) & 0,510 & 0,757 & 0,5064 & 0,114 \\
\hline Kundenbindung (KB) & 0,723 & 0,887 & 0,7227 & 0,158 \\
\hline $\begin{array}{l}\text { Wahrgenommene Selbstrele- } \\
\text { vanz (WRS) }\end{array}$ & 0,452 & 0,702 & 0,4659 & 0,077 \\
\hline Persönliche Norm (PN) & 0,601 & 0,745 & 0,6077 & \\
\hline $\begin{array}{l}\text { Wahrgenommene Verhal- } \\
\text { tenskontrolle (WVK) }\end{array}$ & $1,000^{*}$ & $1,000^{*}$ & $1,000^{*}$ & \\
\hline
\end{tabular}

Tab. 7.36: Quality Criteria der Daten der niederländischen Konsumenten (* es liegt kein Qualitätskriterium vor)

Die bisherigen Befunde zeigen, dass das Modell den in der Literatur vorgeschriebenen Qualitätskriterien genügt (siehe Kapitel 6.6). Die AVE Werte zeigen Werte nahe bzw. über 0,5 und die Composite Reliability zeigt ebenso in allen Bereichen einen Wert von über 0,6 auf. Die Composite Realibility Scale ist ähnlich dem Cronbachs Alpha und misst die interne Konsistenz, ,except that the latter presumes, a priori, that each indicator of a construct contributes equally (i.e., the loadings are set equal to one). Fornell and Larcker argued that their measure is superior to Cronbach`s alpha because it uses the actuel item loadings obtained within the nomological network to calculate internal consistency realibility. This measure, which is unaffected by scale length, is more general than Cronbach`s alpha, but the interpretation of the values abtained is simular and the guidelines offered by Nunnally can be adopted" (Howell, J.M., Avolio, B.J., 1993, S. 896). Werden zur Beurteilung der Modellgüte die R²-Werte des 
Strukturmodells betrachtet, so wird ersichtlich, dass die Konstrukte des Bindungsmodells nur einen akzeptablen Streuungsanteil der latenten endogenen Variablen erklären. Die moderate Höhe von $\mathrm{R}^{2}$ ist erklärbar durch die vielen Einflüsse auf die Kundenbindung im Grenzgebiet. Die Beurteilung des Strukturmodells erfolgt weiter anhand der Höhe der Pfadkoeffizienten, der Gewichte und Ladungen sowie der Höhe der t-Werte. Die unten stehende Tabelle 7.37 bildet die Höhe der Pfadkoeffizienten und deren t-Werte für das Strukturmodell ab. Alle Werte bis auf die Werte wvk und wsN übersteigen den geforderten Wert der Pfadkoeffizienten von 0,1 und haben einen t-Wert größer 2 .

\begin{tabular}{lllll}
\hline & Original Sam & Sample Mean & Standard Dev & T Statistics \\
\hline GKDZSN->GLSS & 0,203 & 0,201 & 0,053 & 3,821 \\
WSN->GKDZSN & 0,030 & 0,044 & 0,075 & 0,402 \\
TZSN->GKDZSN & 0,241 & 0,252 & 0,058 & 4,185 \\
GLSS->KL & 0,148 & 0,153 & 0,064 & 2,315 \\
GKDZKauf->GLSS & 0,342 & 0,356 & 0,061 & 5,604 \\
KL->KB & 0,397 & 0,402 & 0,057 & 6,914 \\
WRS->KL & 0,252 & 0,273 & 0,093 & 2,708 \\
PN->WRS & 0,271 & 0,282 & 0,064 & 4,245 \\
WVK->WRS & 0,042 & 0,011 & 0,138 & 0,307 \\
\hline
\end{tabular}

\section{Tab. 7.37: $\quad$ Befunde des Strukturmodells}

Im Modell übersteigen die Konstrukte Gesamtzufriedenheit mit dem Stammgeschäft im Nachbarland (GKdZ) und Kundenloyalität (KL) die geforderten Werte von 0,1 erheblich, doch auch die Konstrukte Werturteile der sozialen Gruppe (TZsN), wahrgenommene Selbstrelevanz (wrs), persönliche Norm (PN) und der Bedeutung der sozialen Norm für den Konsumenten (GKDZN) zeigen höhere Werte auf. Die Konstrukte Wichtigkeit der sozialen Norm und die wahrgenommene Verhaltenskontrolle tragen nicht weiter zur Erklärung der latenten Variablen bei. Alle anderen t-Werte sind größer als Eins, was bedeutet, dass alle Indikatoren zur Erklärung ihrer latenten Variablen beitragen. Auf die Gesamtzufriedenheit des Konsumenten mit dem Kauf in einer Einkaufsstätte im benachbartem Ausland hat die Bedeutung der sozialen Norm für den Konsumenten einen hohen Einfluß. Einen geringen Einfluß auf die Gesamtzufriedenheit haben dagegen die Werturteile der sozialen Gruppe. Auf die Kundenzufriedenheit hat wiederum die wahrgenommene Selbstrelevanz hohen Einfluß, die persönliche Norm sowie die Gesamtzufriedenheit mit der Einkaufsstätte dagegen nur geringen Einfluß. 
Die Realibilität hinsichtlich der Parameterschätzung wird anhand der t-Statistiken und den zugehörigen Signifikanzen überprüft. Die t-Statistiken werden mit Hilfe der Resampling Methode des Bootstrapping gewonnen. Die Jacknifing Methode wird vernachlässigt, da diese Methode eine Annäherung des Bootstrapping ist.

\begin{tabular}{|c|c|c|c|c|c|}
\hline & & Entire Sample estimate & Mean of Subsamples & Standard error & T-Statistic \\
\hline \multirow[t]{2}{*}{ GLSS } & vr07 & 0.5664 & 0.5456 & 0.0969 & 5.8478 \\
\hline & vr08 & 0.7079 & 0.7187 & 0.0862 & 8.2080 \\
\hline \multirow[t]{3}{*}{ KL } & vr17 & 0.4076 & 0.3900 & 0.0824 & 4.9466 \\
\hline & vr18 & 0.5154 & 0.5222 & 0.0699 & 7.3719 \\
\hline & vr22 & 0.4785 & 0.4782 & 0.0671 & 7.1346 \\
\hline \multirow[t]{3}{*}{$\mathrm{KB}$} & vr19 & 0.3725 & 0.3726 & 0.0369 & 10.0895 \\
\hline & vr20 & 0.4117 & 0.4076 & 0.0303 & 13.6005 \\
\hline & $\operatorname{vr} 21$ & 0.3911 & 0.3953 & 0.0346 & 11.3023 \\
\hline \multirow[t]{3}{*}{ WRS } & $\operatorname{vr} 14.1$ & 0.6433 & 0.5895 & 0.1427 & 4.5082 \\
\hline & $\operatorname{vr} 15.1$ & -0.1671 & -0.2693 & 0.2674 & -0.6249 \\
\hline & $\operatorname{vr} 16.1$ & 0.5278 & 0.4699 & 0.1320 & 3.9994 \\
\hline WVK & $\operatorname{vr} 13.1$ & 1.0000 & 1.0000 & 0.0000 & 0.0000 \\
\hline \multirow[t]{2}{*}{$\mathrm{PN}$} & vr09.1 & 0.5521 & 0.5434 & 0.1877 & 2.9409 \\
\hline & $\operatorname{vr} 11.1$ & 0.7209 & 0.7006 & 0.1841 & 3.9164 \\
\hline \multirow[t]{3}{*}{ GKDZ Kauf } & vr08.1 & 0.7107 & 0.7154 & 0.1131 & 6.2818 \\
\hline & vr08.2 & 0.3064 & 0.2993 & 0.1039 & 2.9492 \\
\hline & vr08.3 & 0.3358 & 0.3124 & 0.0931 & 3.6078 \\
\hline \multirow[t]{3}{*}{ TZSN } & vr03.2 & 0.4326 & 0.4382 & 0.1007 & 4.2976 \\
\hline & vr03.3 & 0.3308 & 0.3197 & 0.1006 & 3.2899 \\
\hline & vr03.4 & 0.4693 & 0.4655 & 0.0835 & 5.6212 \\
\hline \multirow[t]{2}{*}{ WSN } & vr04 & 0.5369 & 0.5150 & 0.2148 & 2.4994 \\
\hline & vr06 & 0.6625 & 0.6630 & 0.1986 & 3.3359 \\
\hline \multirow[t]{2}{*}{ GKdZ SN } & vr05 & 0.8397 & 0.8465 & 0.0860 & 9.7675 \\
\hline & vr10.1 & 0.3609 & 0.3380 & 0.1415 & 2.5509 \\
\hline
\end{tabular}

\section{Tab. 7.38: $\quad$ Niederländisches Structural Model-BootStrap}

Die Beurteilung der Realibilität der Pfadkoeffizienten kann anhand der festgestellten tStatistik als hinreichend betrachtet werden.

Anhand der Korrelationen lässt sich erkennen, ob Zusammenhänge zwischen Paaren von Variablen bzw. Konstrukten bestehen (Backhaus, 2003, S. 269). Insbesondere die wahrgenommene Verhaltenskontrolle korreliert schwach bzw. negativ mit den anderen Konstrukten. 


\begin{tabular}{|c|c|c|c|c|c|c|c|c|c|c|c|c|}
\hline \multicolumn{2}{|c|}{ Konstrukt } & Items & 1. & 2. & 3. & 4. & 5. & 6. & 7. & 8. & 9. & 10. \\
\hline 1. & $\begin{array}{l}\text { Bedeutung der sozia- } \\
\text { len Norm für den } \\
\text { Konsumenten (GKDZ } \\
\text { SN) }\end{array}$ & 2 & 1,000 & & & & & & & & & \\
\hline 2. & $\begin{array}{l}\text { Wichtigkeit der sozia- } \\
\text { len Norm (WSN) }\end{array}$ & 2 & 0,130 & 1,000 & & & & & & & & \\
\hline 3. & $\begin{array}{l}\text { Werturteile der sozia- } \\
\text { len Gruppe (TZSN) }\end{array}$ & 3 & $0,253^{*}$ & $0,417 *$ & 1,000 & & & & & & & \\
\hline 4. & $\begin{array}{l}\text { Gesamtzufriedenheit } \\
\text { mit dem Einkauf im } \\
\text { benachbartem Ausland } \\
\text { (GLSS) }\end{array}$ & 2 & $0,257^{*}$ & $0,247 *$ & $0,246^{*}$ & 1,000 & & & & & & \\
\hline 5. & $\begin{array}{l}\text { Gesamtzufriedenheit } \\
\text { mit Stammgeschäft im } \\
\text { Nachbarland (GKDZ } \\
\text { Kauf) }\end{array}$ & 3 & $0,158^{*}$ & $0,185^{*}$ & $0,419^{*}$ & $0,374 *$ & 1,000 & & & & & \\
\hline 6. & Kundenloyalität (KL) & 3 & $0,309^{*}$ & $-0,009$ & $0,152 *$ & $0,244^{*}$ & $0,158 *$ & 1,000 & & & & \\
\hline 7. & Kundenbindung (KB) & 3 & $0,219^{*}$ & $0,190 *$ & 0,107 & $0,138^{*}$ & 0,012 & $0,397^{*}$ & 1,000 & & & \\
\hline 8. & $\begin{array}{l}\text { Wahrgenommene } \\
\text { Selbstrelevanz (WRS) }\end{array}$ & 3 & $0,404 *$ & $0,147 *$ & 0,171 & $0,379 *$ & 0,253 & $0,308^{*}$ & $0,242 *$ & 1,000 & & \\
\hline 9. & $\begin{array}{l}\text { Persönliche Norm } \\
(\mathrm{PN})\end{array}$ & 2 & $0,387 *$ & $0,245^{*}$ & $0,383^{*}$ & $0,289 *$ & $0,178^{*}$ & $0,333^{*}$ & 0,209 & $0,274 *$ & 1,000 & \\
\hline 10 & $\begin{array}{l}\text { Wahrgenommene } \\
\text { Verhaltenskontrolle } \\
\text { (WVK) }\end{array}$ & 1 & $-0,044$ & 0,113 & 0,225 & 0,144 & 0,139 & $-0,170$ & $-0,101$ & 0,065 & 0,082 & 1,000 \\
\hline
\end{tabular}

Tab. 7.39: $\quad$ Korrelation der latenten Variablen $(N L * p<0.05)$

Diese Tabelle zeigt die Korrelationen der Konstrukte der Kundenbindung im grenzüberschreitenden Modell mit niederländischen Daten. Die Tabelle verdeutlich, dass einige Indikatoren stark miteinander korrelieren, andere dagegen sogar negativ. Hohe Werte des Korrelationskoeffizienten zwischen den Konstrukten Gesamtzufriedenheit mit dem Stammgeschäft im Nachbarland und Werturteile der sozialen Gruppe deuten auf das Vorliegen linearer Abhängigkeiten hin. Insgesamt ergeben sich für die niederländischen Konsumenten folgende starke lineare Abhängigkeiten:

1. Die Werturteile der sozialen Gruppe hat eine hohe lineare Abhängigkeit zur Wichtigkeit der sozialen Norm.

2. Die Gesamtzufriedenheit mit dem Stammgeschäft im Nachbarland hat eine hohe lineare Abhängigkeit zu den Werturteilen der sozialen Gruppe.

3. Die wahrgenommene Selbstrelevanz hat eine hohe lineare Abhängigkeit zur Bedeutung der sozialen Norm für den Konsumenten. 


\subsubsection{Evaluation der Wirkungszusammenhänge und Modellbeurteilung der Kundenbindung der deutschen Konsumenten}

Zunächst werden auch für das Bindungsmodell mit deutschen Daten die Qualitätskriterien betrachtet. Für das Bindungsmodell ergibt sich folgendes Bild:

\begin{tabular}{|c|c|c|c|c|}
\hline Quality Criteria & AVE & Composite Reliabiliy & Stone-Geiser-Test $\left(\mathrm{Q}^{2}\right)$ & R Square \\
\hline $\begin{array}{l}\text { Bedeutung der sozialen Norm für den } \\
\text { Konsumenten (GKDZ SN) }\end{array}$ & 0,553 & 0,696 & 0,556 & 0,228 \\
\hline Wichtigkeit der sozialen Norm (WSN) & 0,766 & 0,867 & 0,766 & \\
\hline $\begin{array}{l}\text { Werturteile der sozialen } \text { Gruppe } \\
\text { (TZSN) }\end{array}$ & 0,579 & 0,804 & 0,579 & \\
\hline $\begin{array}{l}\text { Gesamtzufriedenheit mit dem Einkauf } \\
\text { im benachbartem Ausland (GLSS) }\end{array}$ & 0,679 & 0,808 & 0,675 & 0,238 \\
\hline $\begin{array}{l}\text { Gesamtzufriedenheit mit Stammge- } \\
\text { schäft im Nachbarland (GKDZ Kauf) }\end{array}$ & 0,576 & 0,803 & 0,578 & \\
\hline Kundenloyalität (KL) & 0,577 & 0,804 & 0,576 & 0,106 \\
\hline Kundenbindung (KB) & 0,739 & 0,894 & 0,739 & 0,163 \\
\hline $\begin{array}{ll}\text { Wahrgenommene } & \text { Selbstrelevanz } \\
\text { (WRS) } & \end{array}$ & 0,507 & 0,755 & 0,510 & 0,134 \\
\hline Persönliche Norm (PN) & 0,644 & 0,781 & 0,645 & \\
\hline $\begin{array}{l}\text { Wahrgenommene Verhaltenskontrolle } \\
\text { (WVK) }\end{array}$ & $1,000^{*}$ & $1,000 *$ & $1,000 *$ & \\
\hline
\end{tabular}

Tab.7.40: Quality Criteria der Daten der deutschen Konsumenten (* es liegt kein Qualitätskriterium vor)

Auch die Daten der deutschen Konsumenten genügen den entsprechenden Qualitätskriterien mit einem AVE von über 0,5 und einer Composite Reliability von über 0,6. Werden zur Beurteilung der Modellgüte die $\mathrm{R}^{2}$-Werte des Strukturmodells betrachtet, so wird wie bei den niederländischen Daten ersichtlich, dass die Konstrukte des Bindungsmodells einen akzeptablen bis mittleren Streuungsanteil der latenten endogenen Variablen erklären. Die Beurteilung des Strukturmodells erfolgt weiter wie bei den niederländischen Daten anhand der Höhe der Pfadkoeffizienten, der Gewichte und Ladungen sowie der Höhe der durch das BootstrappingVerfahren erzeugten t-Werte. Die unten stehende Tabelle 7.41 bildet die Höhe der Pfadkoeffizienten und deren t-Werte für das Strukturmodell ab. Alle Werte bis auf den Wert wvk und übersteigen den geforderten Wert von 0,1. Alle t-Werte der Indikatoren außer WVK liegen über Eins, was bedeutet, dass alle Indikatoren zur Erklärung ihrer latenten Variablen beitragen. 
202 | Kapitel 7

\begin{tabular}{lllll}
\hline & Original Sam & Sample Mean & Standard Dev & T Statistics \\
\hline GKDZSN->GLSS & 0,197 & 0,203 & 0,063 & 3,122 \\
WSN->GKDZSN & 0,122 & 0,130 & 0,056 & 2,176 \\
TZSN->GKDZSN & 0,413 & 0,415 & 0,066 & 6,272 \\
GLSS->KL & 0,234 & 0,241 & 0,054 & 4,370 \\
GKDZKauf->GLSS & 0,403 & 0,407 & 0,060 & 6,767 \\
KL->KB & 0,177 & 0,182 & 0,065 & 2,728 \\
WRS->KL & 0,360 & 0,367 & 0,063 & 5,746 \\
PN->WRS & 0,381 & 0,379 & 0,066 & 5,796 \\
WVK->WRS & 0,059 & 0,059 & 0,069 & 0,848 \\
\hline
\end{tabular}

Tab. 7.41: $\quad$ Befunde des deutschen Strukturmodells

Wie im niederländischen Modell wird auch im deutschen Modell mit Hilfe des Bootstrapping die Reliabilität der einzelnen Pfadkoeffizienten mit Hilfe der t-Statistiken und den zugehörigen Signifikanzen überprüft. Die Güte wird anhand der vorliegenden t-Statistiken als gut betrachtet.

\begin{tabular}{|c|c|c|c|c|c|}
\hline & & Entire Sample estimate & Mean of Subsamples & Standard error & T-Statistic \\
\hline \multirow[t]{2}{*}{ GKdZSN } & vr05 & 0.8431 & 0.8478 & 0.0742 & 11.3591 \\
\hline & vr10.1 & 0.4399 & 0.4221 & 0.1110 & 3.9627 \\
\hline \multirow[t]{2}{*}{ GLSS } & vr07 & 0.5070 & 0.5116 & 0.0521 & 9.7322 \\
\hline & vr08 & 0.6969 & 0.6951 & 0.0546 & 12.7731 \\
\hline \multirow[t]{3}{*}{ KL } & vr17 & 0.4298 & 0.4420 & 0.0565 & 7.6026 \\
\hline & $\operatorname{vr} 18$ & 0.4634 & 0.4542 & 0.0645 & 7.1816 \\
\hline & vr22 & 0.4239 & 0.4248 & 0.0492 & 8.6102 \\
\hline \multirow{3}{*}{$\mathrm{KB}$} & vr19 & 0.4034 & 0.4048 & 0.0401 & 10.0682 \\
\hline & vr20 & 0.3850 & 0.3809 & 0.0348 & 11.0668 \\
\hline & vr21 & 0.3759 & 0.3756 & 0.0269 & 13.9765 \\
\hline \multirow{3}{*}{ WRS } & vr14.1 & 0.4196 & 0.4123 & 0.0823 & 5.0971 \\
\hline & vr15.1 & 0.5144 & 0.5159 & 0.0882 & 5.8326 \\
\hline & vr16.1 & 0.4649 & 0.4515 & 0.0890 & 5.2262 \\
\hline \multirow{2}{*}{ PN } & vr09.1 & 0.4925 & 0.5035 & 0.0891 & 5.5255 \\
\hline & vr11.1 & 0.7341 & 0.7197 & 0.0736 & 9.9694 \\
\hline WVK & vr13.1 & 1.0000 & 1.0000 & 0.0000 & 0.0000 \\
\hline \multirow{2}{*}{ GKDZ Kauf } & vr08.1 & 0.5888 & 0.5892 & 0.0704 & 8.3605 \\
\hline & vr08.2 & 0.3753 & 0.3749 & 0.0439 & 8.5464 \\
\hline \multirow{4}{*}{ TZSN } & vr08.3 & 0.3531 & 0.3564 & 0.0441 & 8.0052 \\
\hline & vr03.2 & 0.5175 & 0.5178 & 0.0571 & 9.0580 \\
\hline & vr03.3 & 0.3189 & 0.3202 & 0.0587 & 5.4373 \\
\hline & vr03.4 & 0.4619 & 0.4548 & 0.0607 & 7.6060 \\
\hline \multirow{2}{*}{ WSN } & vr04 & 0.4969 & 0.4852 & 0.0821 & 6.0539 \\
\hline & vr06 & 0.6411 & 0.6460 & 0.0750 & 8.5499 \\
\hline
\end{tabular}

Tab. 7.42: $\quad$ Deutsches Structural Model_BootStrap 
Für eine weitere inhaltliche Interpretation der Werte wurde der substanzielle Einfluss der Indikatoren auf die Gesamtzufriedenheit, Kundenloyalität und Kundenbindung getestet. Hinsichtlich der inhaltlichen Interpretation verdeutlicht ein Blick auf die direkten Wirkungsbeziehungen, welcher Indikator den größten und den niedrigsten, oder einen mittleren Einfluß auf die Gesamtzufriedenheit, Kundenloyalität und die Kundenbindung hat.

Als letzten Schritt soll wie bei den niederländischen Daten bei den deutschen Daten die Korrelationen der latenten Variablen untersucht werden. Auch hier zeigt sich, dass das Konstrukt wahrgenommene Verhaltenskontrolle nur schwach mit den anderen Konstrukten korreliert.

\begin{tabular}{|c|c|c|c|c|c|c|c|c|c|c|c|c|}
\hline Kons & trukte & Items & 1 & 2 & 3 & 4 & 5 & 6 & 7 & 8 & 9 & 10 \\
\hline 1. & $\begin{array}{l}\text { Bedeutung der } \\
\text { sozialen Norm für } \\
\text { den Konsumenten } \\
(\text { GKDZ SN) }\end{array}$ & 2 & 1,000 & & & & & & & & & \\
\hline 2. & $\begin{array}{lr}\text { Wichtigkeit } & \text { der } \\
\text { sozialen } & \text { Norm } \\
(\mathrm{WSN}) & \end{array}$ & 2 & $0,296^{*}$ & 1,000 & & & & & & & & \\
\hline 3. & $\begin{array}{lr}\text { Werturteile } & \text { der } \\
\text { sozialen } & \text { Gruppe } \\
(\mathrm{TZSN}) & \end{array}$ & 3 & $0,465^{*}$ & $0,421^{*}$ & 1,000 & & & & & & & \\
\hline 4 & $\begin{array}{l}\text { Gesamtzufriedenheit } \\
\text { mit dem Einkauf im } \\
\text { benachbartem Aus- } \\
\text { land (GLSS) }\end{array}$ & 2 & $0,333^{*}$ & $0,229 *$ & $0,293 *$ & 1,000 & & & & & & \\
\hline 5. & $\begin{array}{l}\text { Kundenloyalität } \\
(\mathrm{KL})\end{array}$ & 3 & $0,308^{*}$ & $0,230^{*}$ & $0,239 *$ & $0,276^{*}$ & 1,000 & & & & & \\
\hline 6. & $\begin{array}{l}\text { Kundenbindung } \\
(\mathrm{KB})\end{array}$ & 3 & $0,259^{*}$ & $0,317^{*}$ & $0,480 *$ & 0,159 & $0,403^{*}$ & 1,000 & & & & \\
\hline 7. & $\begin{array}{l}\text { Wahrgenommene } \\
\text { Selbstrelevanz } \\
\text { (WRS) }\end{array}$ & 3 & $0,364 *$ & 0,176 & $0,366^{*}$ & $0,239^{*}$ & $0,233^{*}$ & $0,319^{*}$ & 1,000 & & & \\
\hline 8. & $\begin{array}{l}\text { Persönliche Norm } \\
(\mathrm{PN})\end{array}$ & 2 & $0,314 *$ & $0,273^{*}$ & $0,438 *$ & $0,263^{*}$ & $0,198^{*}$ & $0,358^{*}$ & $0,361 *$ & 1,000 & & \\
\hline 9. & $\begin{array}{l}\text { Gesamtzufriedenheit } \\
\text { mit Stammgeschäft } \\
\text { im Nachbarland } \\
\text { (GKDZ Kauf) }\end{array}$ & 3 & $0,357^{*}$ & $0,257^{*}$ & $0,436^{*}$ & $0,451^{*}$ & $0,203^{*}$ & $0,280^{*}$ & 0,274 & $0,374 *$ & 1,000 & \\
\hline 10. & $\begin{array}{l}\text { Wahrgenommene } \\
\text { Verhaltenskontrolle } \\
\text { (WVK) }\end{array}$ & 1 & 0,054 & 0,099 & 0,169 & 0,045 & $-0,005$ & $-0,028$ & 0,066 & 0,021 & 0,024 & 1,000 \\
\hline
\end{tabular}

Tab. 7.43: $\quad$ Korrelation der latenten Variablen $(D, * p<0.05)$ 
Dagegen deutet das Vorliegen des hohen Korrelationskoeffizienten zwischen dem Konstrukt Kundenbindung und Werturteile der sozialen Gruppe auf das Vorliegen linearer Abhängigkeiten zwischen diesen Konstrukten bei deutschen Konsumenten hin.

Insgesamt ergeben sich für die deutschen Konsumenten folgende starke lineare Abhängigkeiten:

1. Die Kundenbindung hat eine hohe lineare Abhängigkeit zu den Werturteilen der sozialen Gruppe

2. Die Werturteile der sozialen Gruppe hat eine hohe lineare Abhängigkeit zur Bedeutung der sozialen Gruppe für den Konsumenten.

3. Die Gesamtzufriedenheit mit dem Stammgeschäft im Nachbarland hat eine hohe lineare Abhängigkeit zur Gesamtzufriedenheit mit dem Einkauf im Nachbarland.

\subsubsection{Vergleich der Evaluation der Wirkungszusammenhänge und Modellbeurteilung der Kundenbindung zwischen niederländischen und deutschen Konsumenten}

Werden die Pfadkoeffizienten der niederländischen und deutschen Konsumenten im erstellten Modell miteinander verglichen, so ist zu erkennen, dass für die niederländischen Konsumenten sieben der neun Hypothesen zu den direkten Wirkungsbeziehungen bestätigt werden, für die deutschen Konsumenten sind es acht Hypothesen. Alle Pfadkoeffizienten weisen hypothesenkonforme Vorzeichen auf. Allerdings sind für die niederländischen Konsumenten

\begin{tabular}{llllll|l|l|l|l|}
\hline & \multicolumn{2}{l}{ Original Sam } & \multicolumn{2}{l}{ Sample Mean } & \multicolumn{2}{l|}{ Standard Dev } & \multicolumn{2}{l|}{ T Statistics } \\
\cline { 2 - 10 } & NL & D & NL & D & NL & D & NL & D \\
\hline GKDZSN->GLSS & 0,203 & 0,197 & 0,201 & 0,203 & 0,053 & 0,063 & 3,821 & 3,122 \\
WSN->GKDZSN & 0,030 & 0,122 & 0,044 & 0,130 & 0,075 & 0,056 & 0,402 & 2,176 \\
TZSN->GKDZSN & 0,241 & 0,413 & 0,252 & 0,415 & 0,058 & 0,066 & 4,185 & 6,272 \\
GLSS->KL & 0,148 & 0,234 & 0,153 & 0,241 & 0,064 & 0,054 & 2,315 & 4,370 \\
GKDZKauf->GLSS & 0,342 & 0,403 & 0,356 & 0,407 & 0,061 & 0,060 & 5,604 & 6,767 \\
KL->KB & 0,397 & 0,177 & 0,402 & 0,182 & 0,057 & 0,065 & 6,914 & 2,728 \\
WRS->KL & 0,252 & 0,360 & 0,273 & 0,367 & 0,093 & 0,063 & 2,708 & 5,746 \\
PN->WRS & 0,271 & 0,381 & 0,282 & 0,379 & 0,064 & 0,066 & 4,245 & 5,796 \\
WVK->WRS & 0,042 & 0,059 & 0,011 & 0,059 & 0,138 & 0,069 & 0,307 & 0,848 \\
\hline
\end{tabular}

Tab. 7.44: $\quad$ Vergleich der Befunde der Höhe der Pfadkoeffizienten des Strukturmodells 
Wichtigkeit der sozialen Norm $(0,030)$ und die wahrgenommene Verhaltenskontrolle $(0,042)$ nur sehr schwach ausgeprägt. Das gleiche gilt die die wahrgenommene Verhaltenskontrolle $(0,059)$ auch für die deutschen Konsumenten. Dabei wird bei den niederländischen Konsumenten Kundenbindung, gemessen an der Höhe der Pfadkoeffizienten, durch Kundenloyalität $(0,397)$, gefolgt von Gesamtzufriedenheit mit Stammgeschäft im Nachbarland $(0,342)$ erklärt. Doch auch die Wirkungszusammenhänge der persönlichen Norm auf die wahrgenommene Selbstrelevanz $(0,271)$ und die Wirkung der wahrgenommenen Selbstrelevanz auf die Kundenloyalität $(0,252)$ fallen stark aus. Im Gegensatz dazu fällt die Wirkung der Zufriedenheit mit dem Kauf im benachbartem Ausland auf die Kundenloyalität $(0,148)$ schwächer aus. Einen mittleren Rang nehmen bei den niederländischen Konsumenten die Wirkungen der Bedeutung der sozialen Norm auf die Zufriedenheit mit dem Einkauf im benachbartem Ausland $(0,203)$ und die Werturteile der sozialen Gruppe auf die Bedeutung der sozialen Norm für den Konsumenten $(0,241)$ aus. Im Gegensatz dazu fällt die Wirkung der Wichtigkeit der sozialen Norm zur Bedeutung der sozialen Norm für den Konsumenten $(0,030)$ sowie die Wirkung der wahrgenommen Verhaltenskontrolle auf die wahrgenommene Selbstrelevanz $(0,042)$ interessanterweise schwach aus. Dieses Ergebnis erscheint vor dem Hintergrund der Untersuchung zum Einfluss der Kultur auf das Verhalten insofern plausibel, als das für niederländische Konsumenten die persönliche Norm eine höheren Wert hat als die Entscheidungen von Freunden und Bekannten.

Bei den deutschen Konsumenten haben dagegen die Werturteile der sozialen Gruppe, gemessen an der Höhe des Pfadkoeffizienten, eine hohe Wirkung auf die Bedeutung der sozialen Norm $(0,413)$. Aber auch die Gesamtzufriedenheit mit dem Stammgeschäft im Nachbarland auf die Gesamtzufriedenheit mit dem Kauf im Nachbarland (0,403), der Einfluss der persönlichen Norm auf die wahrgenommene Selbstrelevanz $(0,381)$ und wahrgenommene Selbstrelevanz und Kundenloyalität $(0,360)$ fallen in ihrer Wirkung nur geringfügig schwächer aus. Stark ist auch noch der Zusammenhang zwischen Gesamtzufriedenheit und Kundenloyalität (0,234), einen mittleren Rang nehmen dagegen die Beziehungen Bedeutung der sozialen Norm und Gesamtzufriedenheit (0,197), die Beziehung zwischen Kundenloyalität und Kundenbindung $(0,177)$ und Wichtigkeit der sozialen Norm und Bedeutung der sozialen Norm für den Konsumenten $(0,122)$ ein. Schwach wird auch bei den deutschen Konsumenten der Zusammenhang zwischen wahrgenommener Verhaltenskontrolle und wahrgenommener Selbstrelevanz erklärt. Auch hier erscheinen die Ergebnisse plausibel, als dass die deutschen Konsumenten stärkeren Wert auf die Meinung ihrer sozialen Gruppe legen und ihr Einkaufs- 
verhalten danach ausrichten. Allerdings muss sich die Meinung der sozialen Gruppe auch der persönlichen Norm annähern.

Bei beiden Kundengruppen ist allerdings nicht sicher, ob der Vorsatz des Wiederkaufs im Stammgeschäft im benachbartem Ausland auch wirklich umgesetzt wird. Starker Bindungstreiber ist für beide Kundengruppen die Gesamtzufriedenheit mit dem Einkauf im Stammgeschäft im Nachbarland.

Ein Vergleich der Außenladungen der beiden Kundengruppen zeigt den Einfluss der einzelnen Items auf die Indikatoren. Werden die Außenladungen der Items betrachtet, so zeigt sich insbesondere ein Unterschied in der Beurteilung des Stammgeschäftes zwischen niederländischen und deutschen Konsumenten. Sind niederländische Konsumenten insbesondere mit dem Sortiment ihrer deutschen Einkaufsstätte zufrieden $(0,831)$, so sind es bei den deutschen Konsumenten insbesondere der Service $(0,782)$ und die Beratungsleistung $(0,733)$ die den Unterschied darstellen. Auch die wahrgenommene Selbstrelevanz, verstanden als wertorientierte Konzeptualisierung des Involvementkonstruktes, zeigt Unterschiede zwischen niederländischen und deutschen Konsumenten. Ist es bei den deutschen Konsumenten der Einkaufsspass $(0,729)$ im Nachbarland, der zu Kundenloyalität führt, ist dies bei den niederländischen Kunden weniger stark ausgeprägt $(0,463)$. 


\begin{tabular}{|c|c|c|c|c|c|}
\hline & \multirow[t]{2}{*}{ Items } & \multicolumn{2}{|c|}{ NL } & \multicolumn{2}{|c|}{$\mathrm{D}$} \\
\hline & & $\begin{array}{l}\text { outer } \\
\text { Loadings }\end{array}$ & T-Test & $\begin{array}{l}\text { outer } \\
\text { Loadings }\end{array}$ & T-Test \\
\hline \multirow[t]{2}{*}{ GKDZN SN } & Im Nachbarland kaufe ich preiswerter. & 0,569 & 5.8478 & 0,517 & 11.3591 \\
\hline & $\begin{array}{l}\text { Freunde/Bekannte reagieren auf meinen Einkauf im Nachbarland } \\
\text { positiv. }\end{array}$ & 0,946 & 8.2080 & 0,916 & 3.9627 \\
\hline \multirow[t]{2}{*}{ WSN } & $\begin{array}{l}\text { Wenn Sie einmal an Ihren Freunde /Bekanntenkreis denken, in wie } \\
\text { vielen Fällen kaufen diese im Nachbarland ein? }\end{array}$ & 0,795 & 4.9466 & 0,840 & 9.7322 \\
\hline & $\begin{array}{l}\text { Wenn Sie einmal an die letzten Einkäufe zurückdenken, in wie vielen } \\
\text { Fällen haben Freunde, Bekannte Ihnen eine Empfehlung zum Ein- } \\
\text { kauf im Nachbarland gegeben? }\end{array}$ & 0,865 & 7.3719 & 0,909 & 12.7731 \\
\hline \multirow[t]{3}{*}{ TZSN } & $\begin{array}{l}\text { Mein Freundes/Bekanntenkreis findet, dass man im Nachbarland } \\
\text { qualitativ bessere Produkte einkaufen kann. }\end{array}$ & 0,801 & 7.1346 & 0,830 & 7.6026 \\
\hline & $\begin{array}{l}\text { Mein Freundes/Bekanntenkreis findet, dass man im Nachbarland eine } \\
\text { bessere Beratung erhält. }\end{array}$ & 0,848 & 10.0895 & 0,683 & 7.1816 \\
\hline & $\begin{array}{l}\text { Mein Freundes/Bekanntenkreis findet, dass man im Nachbarland } \\
\text { ausgefallenere (Design) Produkte erhält. }\end{array}$ & 0,772 & 13.6005 & 0,763 & 8.6102 \\
\hline \multirow[t]{2}{*}{ GLSS } & $\begin{array}{l}\text { Mit dem Einkauf in meinemStammgeschäft bin ich insgesamt zufrie- } \\
\text { den. }\end{array}$ & 0,814 & 11.3023 & 0,876 & 10.0682 \\
\hline & $\begin{array}{l}\text { Mit den Einkaufsmöglichkeiten in (Ort der Befragung) bin ich zu- } \\
\text { frieden. }\end{array}$ & 0,747 & 4.5082 & 0,768 & 11.0668 \\
\hline \multirow[t]{3}{*}{ GKDZ Kauf } & $\begin{array}{l}\text { Mit dem Einkauf in meinem Stammgeschäft bin ich zufrieden hin- } \\
\text { sichtlich Sortiment. }\end{array}$ & 0,831 & -0.6249 & 0,760 & 13.9765 \\
\hline & $\begin{array}{l}\text { Mit dem Einkauf in meinem Stammgeschäft bin ich zufrieden hin- } \\
\text { sichtlich Service. }\end{array}$ & 0,630 & 3.9994 & 0,782 & 5.0971 \\
\hline & $\begin{array}{l}\text { Mit dem Einkauf in meinem Stammgeschäft bin ich zufrieden hin- } \\
\text { sichtlich Beratungsleistung. }\end{array}$ & 0,644 & 0.0000 & 0,733 & 5.8326 \\
\hline \multirow[t]{3}{*}{ KL } & $\begin{array}{l}\text { Ich empfehle mein Geschäft (Stammgeschäft) im Nachbarland bei } \\
\text { meinen Freunden und Bekannten weiter. }\end{array}$ & 0,737 & 2.9409 & 0,773 & 5.2262 \\
\hline & $\begin{array}{l}\text { Ich möchte regelmäßig von meinem Geschäft (Stammgeschäft) im } \\
\text { Nachbarland über Angebote und besondere Dienstleistungen infor- } \\
\text { miert werden. }\end{array}$ & 0,721 & 3.9164 & 0,733 & 5.5255 \\
\hline & $\begin{array}{l}\text { Sofern mein Stammgeschäft in Zukunft auch andere Produktkatego- } \\
\text { rien anbietet, könnte ich mir vorstellen, auch dort zu kaufen. }\end{array}$ & 0,683 & 6.2818 & 0,773 & 9.9694 \\
\hline \multirow[t]{3}{*}{$\mathrm{KB}$} & $\begin{array}{l}\text { Mindestens einmal im Monat besuche ich mein Stammgeschäft im } \\
\text { Nachbarland }\end{array}$ & 0,805 & 2.9492 & 0,840 & 0.0000 \\
\hline & $\begin{array}{l}\text { In den vergangenen acht Wochen habe ich oft im Nachbarland } \\
\text { eingekauft }\end{array}$ & 0,889 & 3.6078 & 0,864 & 8.3605 \\
\hline & In den letzten Wochen habe ich oft im Stammgeschäft eingekauft & 0,854 & 4.2976 & 0,873 & 8.5464 \\
\hline \multirow[t]{3}{*}{ WRS } & $\begin{array}{l}\text { Einkaufen im Nachbarland macht mir Spass, weil ich eine angeneh- } \\
\text { me Umgebung mit Cafes und Unterhaltungsmöglichkeiten antreffe. }\end{array}$ & 0,463 & 3.2899 & 0,729 & 8.0052 \\
\hline & $\begin{array}{l}\text { Ich finde es schön, die Möglichkeit zu haben, schnell im Nachbar- } \\
\text { land einkaufen zu können. }\end{array}$ & 0,683 & 5.6212 & 0,696 & 9.0580 \\
\hline & Bei nächster Gelegenheit kaufe ich wieder im Nachbarland ein & 0,822 & 2.4994 & 0,711 & 5.4373 \\
\hline \multirow[t]{2}{*}{ PN } & $\begin{array}{l}\text { Im Nachbarland finde ich leichter die Produkte, die ich suche / die } \\
\text { ich benötige }\end{array}$ & 0,636 & 3.3359 & 0,887 & 7.6060 \\
\hline & $\begin{array}{l}\text { Im Nachbarland kann ich Produkte kaufen, die ich zuhause nicht } \\
\text { bekomme. }\end{array}$ & 0,893 & 9.7675 & 0,708 & 6.0539 \\
\hline WVK & Der Ort im Nachbarland in dem Einkäufe getätigt werden, sollte vom & 1,000 & 2.5509 & 1,000 & 8.5499 \\
\hline
\end{tabular}

Tab. 7.45: $\quad$ Außenladungen des PLS Modells

Dies zeigt auch die unterschiedliche Auffassung der persönlichen Norm. Finden deutsche Konsumenten, dass sie im Nachbarland leichter die Produkte finden, die sie suchen, ist es bei den niederländischen Konsumenten der Eindruck, dass sie in Deutschland die Produkte kaufen können, die im eigenen Land nicht angeboten werden (0,893). Die Gesamtzufriedenheit der Konsumenten mit dem Einkauf im Nachbarland ist bei beiden Konsumentengruppen ge- 
messen an den Außenladungen hoch $(\mathrm{NL}=0,814, \mathrm{D}=0,876)$, doch ist die Zufriedenheit der deutschen Konsumenten mit dem Einkauf im Nachbarland höher als bei den niederländischen Konsumenten. Die Wichtigkeit der sozialen Norm weist bei den deutschen Konsumenten deutlich höhere Werte auf als bei den niederländischen Konsumenten. Dies bestärkt die Vermutung, dass die deutschen Konsumenten stärker im Konsumverhalten vom Gruppenverhalten abhängig sind als niederländische Konsumenten.

\begin{tabular}{|c|c|c|c|c|c|c|c|c|}
\hline & \multicolumn{2}{|c|}{ Original Sam } & \multicolumn{2}{|c|}{ Sample Mean } & \multicolumn{2}{|c|}{ Standard Dev } & \multicolumn{2}{|c|}{ T Statistics } \\
\hline & NL & $\mathrm{D}$ & NL & $\mathrm{D}$ & NL & $\mathrm{D}$ & NL & $\mathrm{D}$ \\
\hline GKDZSN->GLSS & 0,203 & 0,197 & 0,201 & 0,203 & 0,053 & 0,063 & 3,821 & 3,122 \\
\hline GKDZSN->KL & 0,030 & 0,046 & 0,032 & 0,050 & 0,017 & 0,022 & 1,732 & 2,112 \\
\hline GKDZSN->KB & 0,012 & 0,019 & 0,013 & 0,020 & 0,007 & 0,009 & 1,636 & 1,995 \\
\hline WSN->GKDZSN & 0,030 & 0,122 & 0,044 & 0,130 & 0,075 & 0,056 & 0,402 & 2,176 \\
\hline WSN->GLSS & 0,006 & 0,024 & 0,009 & 0,027 & 0,015 & 0,015 & 0,401 & 1,560 \\
\hline WSN->KL & 0,001 & 0,006 & 0,002 & 0,007 & 0,003 & 0,004 & 0,344 & 1,276 \\
\hline WSN->KB & 0,000 & 0,002 & 0,001 & 0,00 & 0,001 & 0,002 & 0,322 & 1,223 \\
\hline TZSN->GKDZSN & 0,241 & 0,413 & 0,252 & 0,415 & 0,058 & 0,066 & 4,185 & 6,272 \\
\hline TZSN->GLSS & 0,049 & 0,082 & 0,051 & 0,084 & 0,019 & 0,030 & 2,614 & 2,702 \\
\hline TZSN->KL & 0,007 & 0,019 & 0,008 & 0,021 & 0,005 & 0,010 & 1,395 & 1,961 \\
\hline TZSN->KB & 0,003 & 0,008 & 0,003 & 0,008 & 0,002 & 0,004 & 1,371 & 1,836 \\
\hline GLSS->KL & 0,148 & 0,234 & 0,153 & 0,241 & 0,064 & 0,054 & 2,315 & 4,370 \\
\hline GLSS->KB & 0,059 & 0,095 & 0,062 & 0,098 & 0,028 & 0,026 & 2,120 & 3,660 \\
\hline GKDZKauf->GLSS & 0,342 & 0,381 & 0,356 & 0,379 & 0,061 & 0,066 & 5,604 & 5,796 \\
\hline GKDZKauf->KL & 0,051 & 0,089 & 0,054 & 0,090 & 0,025 & 0,021 & 2,034 & 4,223 \\
\hline GKDZKauf->KB & 0,020 & 0,036 & 0,022 & 0,037 & 0,011 & 0,010 & 1,859 & 3,550 \\
\hline $\mathrm{KL}->\mathrm{KB}$ & 0,397 & 0,403 & 0,402 & 0,407 & 0,057 & 0,060 & 6,914 & 6,770 \\
\hline WRS->KL & 0,252 & 0,177 & 0,273 & 0,182 & 0,093 & 0,065 & 2,708 & 2,728 \\
\hline WRS->KB & 0,100 & 0,071 & 0,110 & 0,074 & 0,041 & 0,030 & 2,441 & 2,361 \\
\hline $\mathrm{PN}->\mathrm{KL}$ & 0,068 & 0,064 & 0,078 & 0,067 & 0,034 & 0,028 & 2,031 & 2,280 \\
\hline $\mathrm{PN}->\mathrm{KB}$ & 0,027 & 0,026 & 0,031 & 0,028 & 0,014 & 0,013 & 1,911 & 2,010 \\
\hline PN->WRS & 0,271 & 0,360 & 0,282 & 0,367 & 0,064 & 0,063 & 4,245 & 5,746 \\
\hline WVK->KL & 0,011 & 0,010 & $-0,006$ & 0,010 & 0,045 & 0,013 & 0,235 & 0,795 \\
\hline WVK->KB & 0,004 & 0,004 & $-0,002$ & 0,004 & 0,019 & 0,006 & 0,226 & 0,767 \\
\hline WVK->WRS & 0,042 & 0,059 & 0,011 & 0,059 & 0,138 & 0,069 & 0,307 & 0,848 \\
\hline
\end{tabular}

Tab. 7.46: $\quad$ Ausprägungen der Effektgröße

In der Tabelle 7.44 finden sich die Pfadkoeffizienten der Wirkungsbeziehungen zwischen den Indikatoren sowie die zugehörigen t-Werte. Die deutschen Werte zeigen bis auf „wahrge- 
nommene Verhaltenskontrolle“ insgesamt signifikante Werte, die niederländischen Werte zeigen Signifikanz, ausgenommen in der „Wichtigkeit der sozialen Norm“ und der „wahrgenommen Verhaltenskontrolle“. Insgesamt wird eine hohe Übereinstimmung zwischen den beiden Konsumentengruppen erzielt.

Die Tabelle 7.46 stellt die Gegenüberstellung der Ladungskoeffizienten, die Gewichte sowie deren jeweiligen t-Werte und Signifikanzen des Messmodells der Gruppe der niederländischen und deutschen befragten Konsumenten dar. Die Betrachtung des Messmodells zeigt, dass die positiven Wirkungszusammenhänge der Gesamtzufriedenheit mit dem Einkauf im Nachbarland bei beiden Konsumentengruppen insbesondere auf die Zufriedenheit mit dem Einkauf im Stammgeschäft zurückzuführen sind. So zeigt der Indikator „Gesamtzufriedenheit mit Stammgeschäft im Nachbarland!“ für beide Kundengruppen ( $\mathrm{NL}=0,342$; $\mathrm{D}=0,381$ ) ein hohes Gewicht auf die Gesamtzufriedenheit. Für die Kundenloyalität hat die „wahrgenommene Selbstrelevanz“ (NL=0,252; $\mathrm{D}=0,177)$ und die „Gesamtzufriedenheit mit dem Einkauf im Nachbarland“ (NL=0,148; D=0,177) ein hohes Gewicht. Allerdings ist die Effktgröße „wahrgenommene Selbstrelevanz“ bei den niederländischen Konsumenten erheblich höher, bei „Gesamtzufriedenheit mit dem Einkauf im Nachbarland“ dagegen bei den deutschen Konsumenten. Für Kundenbindung zeigen die Effektgrößen bei „Kundenloyalität" $(\mathrm{NL}=0,397 ; \mathrm{D}=0,403)$ fast identische Werte auf. Alle anderen Indikatoren haben auf die Konstrukte nur mittleren, geringen oder keinen Einfluss. 


\begin{tabular}{|c|c|c|c|c|c|c|c|}
\hline \multirow[t]{2}{*}{ Konstrukt } & \multirow[t]{2}{*}{ Indikator } & \multicolumn{2}{|c|}{ Effektgröße } & \multicolumn{2}{|l|}{$\mathrm{t}$-Wert } & \multicolumn{2}{|c|}{ Substanzielle Einfluss } \\
\hline & & NL & $\mathrm{D}$ & NL & $\mathrm{D}$ & NL & $\mathrm{D}$ \\
\hline \multirow[t]{4}{*}{ GLSS } & GKDZSN & 0,203 & 0,197 & 3,821 & 3,122 & mittel & mittel \\
\hline & WSN & 0,006 & 0,024 & 0,401 & 1,560 & keiner & gering \\
\hline & TZSN & 0,049 & 0,082 & 2,614 & 2,702 & gering & gering \\
\hline & GKDZKauf & 0,342 & 0,381 & 5,604 & 5,796 & hoch & hoch \\
\hline \multirow[t]{8}{*}{$\mathrm{KL}$} & GKDZSN & 0,030 & 0,046 & 1,732 & 2,112 & gering & gering \\
\hline & WSN & 0,001 & 0,006 & 0,344 & 1,276 & keiner & keiner \\
\hline & TZSN & 0,007 & 0,019 & 1,395 & 1,961 & keiner & keiner \\
\hline & GKDZKauf & 0,051 & 0,089 & 2,034 & 4,223 & gering & gering \\
\hline & GLSS & 0,148 & 0,234 & 2,315 & 4,370 & mittel & mittel \\
\hline & WRS & 0,252 & 0,177 & 2,708 & 2,728 & mittel & mittel \\
\hline & PN & 0,068 & 0,064 & 2,031 & 2,280 & gering & gering \\
\hline & WVK & 0,011 & 0,010 & 0,235 & 0,795 & keiner & keiner \\
\hline \multirow[t]{9}{*}{$\mathrm{KB}$} & GKDZSN & 0,012 & 0,019 & 1,636 & 1,995 & keiner & keiner \\
\hline & WSN & 0,000 & 0,002 & 0,322 & 1,223 & keiner & keiner \\
\hline & TZSN & 0,003 & 0,008 & 1,371 & 1,836 & keiner & keiner \\
\hline & GLSS & 0,059 & 0,095 & 2,120 & 3,660 & gering & gering \\
\hline & GKDZKauf & 0,020 & 0,036 & 1,859 & 3,550 & keiner & gering \\
\hline & $\mathrm{KL}$ & 0,397 & 0,403 & 6,914 & 6,770 & hoch & hoch \\
\hline & WRS & 0,100 & 0,071 & 2,441 & 2,361 & gering & gering \\
\hline & PN & 0,027 & 0,026 & 1,911 & 2,010 & gering & gering \\
\hline & WVK & 0,004 & 0,004 & 0,226 & 0,767 & keiner & keiner \\
\hline
\end{tabular}

Tab. 7.47: $\quad$ Ausprägungen der Effektgröße und ihr substanzieller Einfluss

Hinsichtlich der „Kundenbindung“ ist bemerkenswert, dass bei den niederländischen Konsumenten die wahrgenommene Selbstrelevanz und die persönliche Norm einen höheren Einfluss auf Bindung haben als bei deutschen Konsumenten. Das gleiche gilt für „Kundenloyalität“. Hier hat insbesondere die „,wahrgenommene Selbstrelevanz“ $(0,100)$ und auch die ,persönliche Norm“ $(0,068)$ bei niederländischen Konsumenten eine höhere Effektgröße als bei deutschen Konsumenten. Dagegen weisen die „Werturteile der sozialen Gruppe“ eine höhere Effektgröße bei den deutschen Konsumenten $(0,019)$ hinsichtlich Kundenloyalität auf als bei den niederländischen Konsumenten (0,007). Hinsichtlich der Gesamtzufriedenheit mit dem Einkauf im Nachbarland zeigen die Effektgrößen „Wichtigkeit der sozialen Norm“ und „Werturteile der sozialen Gruppe“ bei den deutschen Konsumenten $(0,024 ; 0,082)$ höhere Werte auf als bei den niederländischen Konsumenten $(0,006 ; 0,049)$. Die Effektgrößen „Werturteile der sozialen Gruppe“ und Gesamtzufriedenheit mit Stammgeschäft im Nachbar- 
land“" sind ähnlich hoch und weisen einen positiven Totaleffekt auf Gesamtzufriedenheit mit dem Einkauf im Nachbarland auf.

\subsubsection{Fazit zu den Befunden und Hypothesenüberprüfung}

Mit Hilfe des PLS-Ansatzes wurden die Wirkungszusammenhänge zwischen den Teilkonstrukten der Kundenbindung und der Gesamtbindung zu einer Einkaufsstätte im Nachbarland untersucht. Bis auf den Wirkungszusammenhang „,wahrgenommene Verhaltenskontrolle“, der für beide Konsumentengruppen nicht nachgewiesen werden konnte und dem Wirkungszusammenhang „Wichtigkeit der sozialen Norm“ auf „Bedeutung der sozialen Norm“ für niederländische Konsumenten, konnten die angenommenen Wirkungszusammenhängge bestätigt werden. Die Tabelle 7.47 stellt die Befunde der Hypothesenüberprüfung übersichtlich dar.

\begin{tabular}{|c|c|c|c|c|c|c|c|c|}
\hline Hypothese & Beziehung & $\begin{array}{l}\text { Für niederländische } \\
\text { Konsumenten }\end{array}$ & Wirkung & $\begin{array}{l}\text { Pfad- } \\
\text { koeffizient }\end{array}$ & $\begin{array}{l}\text { Für deutsche } \\
\text { Konsumenten }\end{array}$ & Wirkung & $\begin{array}{l}\text { Pfad- } \\
\text { koeffizient }\end{array}$ & T-Test \\
\hline H1 & TZSN - GKDZ SN & nachgewiesen & + & 0,241 & nachgewiesen & + & 0,413 & 7,246 \\
\hline $\mathrm{H} 2$ & WSN - GKDZ SN & nicht nachgewiesen & + & 0,030 & nachgewiesen & + & 0,122 & $-0,480$ \\
\hline $\mathrm{H} 3$ & GKDZ SN- GLSS & nachgewiesen & + & 0,203 & nachgewiesen & + & 0,197 & 8,445 \\
\hline $\mathrm{H} 4$ & GKDZ Kauf - GLSS & nachgewiesen & + & 0,342 & nachgewiesen & + & 0,381 & 8,292 \\
\hline H5 & GLSS - KL & nachgewiesen & + & 0,148 & nachgewiesen & + & 0,234 & 9,137 \\
\hline H6 & WRS - KL & nachgewiesen & + & 0,252 & nachgewiesen & + & 0,177 & $-2,452$ \\
\hline $\mathrm{H} 7$ & PN - WRS & nachgewiesen & + & 0,271 & nachgewiesen & + & 0,360 & 13,224 \\
\hline H8 & WVK - WRS & nicht nachgewiesen & + & 0,042 & $\begin{array}{l}\text { nicht nachge- } \\
\text { wiesen }\end{array}$ & + & 0,059 & $-6,995$ \\
\hline H9 & $\mathrm{KL}-\mathrm{KB}$ & nachgewiesen & + & 0,397 & nachgewiesen & + & 0,403 & 6,295 \\
\hline
\end{tabular}

Tab. 7.48: $\quad$ Untersuchungsergebnisse zur Überprüfung der Hypothesen

Die Mehrzahl der aufgestellten Hypothesen wird somit bestätigt. Das Modell weist eine zufriedenstellende Güte auf und ist pronosefähig. Auch wird ein Großteil der hypothetischen Wirkungsbeziehungen zwischen den Konstrukten der Kundenbindung und den Teilkonstrukten bestätigt. Für die niederländischen Konsumenten weißt der Pfadkoeffizient Kundenloyali- 
tät und Kundenbindung den höchsten Wert auf, für die deutschen Kunden der Pfadkoeffizient Werturteile der sozialen Gruppe zu Bedeutung der sozialen Norm für den Konsumenten.

Aussagekräftiger als die reine Beurteilung der Hypothesenbestätigung ist die Analyse der Stärke der Wirkungszusammenhänge von den einzelnen Konstrukten und die Erfolgsmaße in den einzelnen Phasen. Damit eine Rangfolge der Stärke der Wirkungszusammenhänge zwischen den Konstrukten und den einzelnen Phasen der Kundenbindung aufgestellt werden kann, ist neben der Betrachtung der Stärke der Pfadkoeffizienten auch eine Betrachtung der jeweiligen Effektgröße vorzunehmen. Wird nun die Stärke der Pfadkoeffizienten mit der Effektgröße in Beziehung gesetzt, so ist aus der Übersicht (siehe Anlage 16) ersichtlich, ob ein Erfolgsfaktor mit einem hohen Pfadkoeffizienten auch einen starken Einfluss auf die Variable ausübt. Damit eine feinere Unterteilung der Effektgrößen möglich ist, wird von der Einteilung von Cohen (Cohen, 1992, S.157) abgewichen und Klassen von <0,02, 0,02 - 0,05, und $>0,05$ gebildet. Ferner wurden Klassen von Pfadkoeffizienten unterschiedlicher Stärke gebildet, wobei die in der Literatur geforderte Mindestgröße von 0,1 als kleinste Klasse beibehalten wurde.

Wie für die Phase „Bedeutung der sozialen Norm“ (siehe Anlage 16) zu erkennen, gibt es für die beiden betrachteten Konsumentengruppen Faktoren, die sowohl einen hohen Pfadkoeffizienten größer als 0,2, als auch eine relativ hohe Effektgröße mit größer 0,05 aufweisen. Zu diesen Faktoren gehören für beide Gruppen die Werturteile der sozialen Gruppe, bei den deutschen Konsumenten kommt die Wichtigkeit der sozialen Norm aber noch hinzu.

Ein Blick auf die Phase „Gesamtzufriedenheit mit dem Einkauf im Nachbarland“ zeigt, dass eine Veränderung in der Stärke der Wirkungszusammenhänge der unterschiedlichen Faktoren stattgefunden hat. Auffallend ist, dass für die deutschen Konsumenten die Werturteile der sozialen Gruppe als auch die soziale Norm eine größere Rolle spielt als für die niederländischen Konsumenten. Die „Wichtigkeit der sozialen Norm“ und die „Werturteile der sozialen Gruppe“ spielen für niederländische Konsumenten nur eine untergeordnete Rolle zur Bildung der „Gesamtzufriedenheit mit dem Einkauf im Nachbarland“. Dagegen hat für deutsche Konsumenten die „Bedeutung der soziale Norm“, „Wichtigkeit der sozialen Norm“ und „Werturteile der sozialen Gruppe“ Auswirkungen auf die „Gesamtzufriedenheit mit dem Einkauf im Nachbarland“. 
Unterschiede sind zwischen den beiden Konsumenten insbesondere in der Phase Kundenloyalität vorhanden. Für niederländische Konsumenten entsteht Kundenloyalität insbesondere durch die „persönliche Norm“ und die „wahrgenommene Selbstrelevanz“. Dagegen stehen bei den deutschen Konsumenten die ,persönliche Norm“ und die „Zufriedenheit mit dem Einkauf im Stammgeschäft im Nachbarland“" zentral zur Bildung von Loyalität. So stehen für die niederländischen Konsumenten - sofern Loyalität der niederländischen Konsumenten erreicht werden soll - die wertorientierte Konzeptualisierung des Involvmentkonstrutes zentral. Sofern die niederländischen Konsumenten überzeugt sind, dass die Werte die sie anstreben durch den Kauf in Deutschland realisiert werden, werden sie loyal. Dagegen ist es bei den deutschen Konsumenten erst der Fall, wenn sie durch den Gesamteindruck des Leistungsangebotes in einer konkreten Einkaufsstätte und einem konkreten Einkaufsort überzeugt werden (siehe Anlage 16).

Auch Kundenbindung wird bei den beiden Kundengruppen unterschiedlich gebildet. Niederländische Konsumenten werden erfolgreich gebunden, wenn das Stammgeschäft auf die“ persönliche Norm“ und die „wahrgenommene Selbstrelevanz“ der Konsumenten eingeht sowie „Loyalität“ bereits vorhanden ist. Deutsche Konsumenten lassen sich über „Kundenloyalität“, die insbesondere durch die Leistung der Einkaufsstätte, aber auch des Einkaufortes entsteht, binden (siehe Anlage 16). Die „wahrgenommene Selbstrelevanz“ wird dagegen von beiden Gruppen durch die „persönliche Norm“ gebildet (siehe Anlage 16). Nachdem nun die Pfadkoeffizienten und die Effektstärken gegenübergestellt wurden, wird in der Tabellen 7.47 und 7.48 zusammenfassend ein Überblick gegeben, welche Variable in den einzelnen Phasen welchen Einfluss ausübt. Die Variablen sind nach ihrer Wirkungsstärke (Effektstärke und Höhe des Pfadkoeffizienten, getrennt nach niederländischen und deutschen Konsumenten) geordnet. 


\begin{tabular}{|c|c|c|c|c|c|}
\hline \multirow[b]{2}{*}{$\begin{array}{l}\text { Rangfolge } \\
\text { der } \\
\text { Wirkungs- } \\
\text { stärke } \\
\text { NL }\end{array}$} & \multicolumn{5}{|c|}{ Konstrukte der Kundenbindung } \\
\hline & $\begin{array}{l}\text { Bedeutung der sozia- } \\
\text { len Norm für den } \\
\text { Konsumenten } \\
(\mathrm{GKdZN})\end{array}$ & $\begin{array}{l}\text { Gesamtzufriedenheit } \\
\text { mit Einkauf im Nach- } \\
\text { barland (GLSS) }\end{array}$ & $\begin{array}{l}\text { Wahrgenommene } \\
\text { Selbstrelevanz } \\
\text { (WRS) }\end{array}$ & $\begin{array}{l}\text { Kundenloyalität } \\
\text { (KL) }\end{array}$ & Kundenbindung (KB) \\
\hline 1 & $\begin{array}{l}\text { Werturteile } \quad \text { der } \\
\text { sozialen } \quad \text { Gruppe } \\
(\mathrm{TZSN})\end{array}$ & $\begin{array}{l}\text { Gesamtzufriedenheit } \\
\text { mit Stammgeschäft im } \\
\text { Nachbarland (GKDZ } \\
\text { Kauf) }\end{array}$ & $\begin{array}{l}\text { Persönliche Norm } \\
(\mathrm{PN})\end{array}$ & $\begin{array}{l}\text { Wahrgenommene } \\
\text { Selbstrelevanz } \\
\text { (WRS) }\end{array}$ & $\begin{array}{l}\text { Kundenloyalität } \\
\text { (KL) }\end{array}$ \\
\hline 2 & & $\begin{array}{l}\text { Bedeutung der sozialen } \\
\text { Norm für den Konsu- } \\
\text { menten (GKdZN) }\end{array}$ & & $\begin{array}{l}\text { Persönliche Norm } \\
(\mathrm{PN})\end{array}$ & $\begin{array}{l}\text { Wahrgenommene Selbstre- } \\
\text { levanz (WRS) }\end{array}$ \\
\hline 3 & & & & $\begin{array}{l}\text { Gesamtzufriedenheit } \\
\text { mit dem Einkauf im } \\
\text { Nachbarland } \\
\text { (GLSS) }\end{array}$ & $\begin{array}{l}\text { Gesamtzufriedenheit mit } \\
\text { dem Einkauf im Nachbar- } \\
\text { land (GSS) }\end{array}$ \\
\hline 4 & & & & $\begin{array}{l}\text { Gesamtzufriedenheit } \\
\text { mit Stammgeschäft } \\
\text { im Nachbarland } \\
\text { (GKDZ Kauf) }\end{array}$ & Persönliche Norm (PN) \\
\hline 5 & & & & $\begin{array}{l}\text { Bedeutung der } \\
\text { sozialen Norm für } \\
\text { den Konsumenten } \\
(\mathrm{GKdZN})\end{array}$ & $\begin{array}{l}\text { Gesamtzufriedenheit mit } \\
\text { Stammgeschäft im Nach- } \\
\text { barland (GKDZ Kauf) }\end{array}$ \\
\hline 6 & & & & & \\
\hline
\end{tabular}

Tab. 7.49: $\quad$ Überblick über die Konstrukte der Kundenbindung für niederländische Konsumenten

Deutlich erkennbar ist hier, dass insbesondere die Gesamtzufriedenheit mit dem Einkauf im Nachbarland unterschiedlich zwischen den beiden Kundengruppen gebildet wird. Für die deutschen Kunden wird die Gesamtzufriedenheit stark durch die soziale Gruppe und die soziale Norm geprägt. Zwar ist bei beiden Gruppen der Hauptfaktor die Gesamtzufriedenheit mit dem Einkauf im Stammgeschäft, doch insbesondere bei deutschen Konsumenten wird der Einfluss der sozialen Gruppe auf die Zufriedenheit deutlich. Dies ist ein starker Hinweis auf einen kulturellen Unterschied zwischen deutschen und niederländischen Konsumenten, da bei niederländischen Konsumenten die individuelle Entscheidung stärker ausgeprägt ist als bei deutschen Konsumenten. Auffallend ist ebenso, dass Kundenloyalität sich in den beiden betrachteten Gruppen unterschiedlich bildet. Sind die drei stärksten Wirkungszusammenhänge bei den niederländischen Konsumenten die wahrgenommene Selbstrelevanz, die persönliche Norm und die Gesamtzufriedenheit mit dem Einkauf im Nachbarland, sind es bei den deutschen Konsumenten die Gesamtzufriedenheit mit dem Einkauf im Nachbarland, die 
wahrgenommene Selbstrelevanz und die Gesamtzufriedenheit mit dem Stammgeschäft im Nachbarland.

\begin{tabular}{|c|c|c|c|c|c|}
\hline \multirow{2}{*}{$\begin{array}{l}\text { Rangfolge der } \\
\text { Wirkungsstärke } \\
\text { D }\end{array}$} & \multicolumn{5}{|c|}{ Konstrukte der Kundenbindung } \\
\hline & $\begin{array}{l}\text { Bedeutung der sozia- } \\
\text { len Norm für den } \\
\text { Konsumenten } \\
(\mathrm{GKdZN})\end{array}$ & $\begin{array}{l}\text { Gesamtzufriedenheit } \\
\text { mit Einkauf im Nach- } \\
\text { barland (GLSS) }\end{array}$ & $\begin{array}{l}\text { Wahrgenommene Selbst- } \\
\text { relevanz (WRS) }\end{array}$ & $\begin{array}{l}\text { Kundenloyalität } \\
\text { (KL) }\end{array}$ & $\begin{array}{l}\text { Kundenbindung } \\
\text { (KB) }\end{array}$ \\
\hline 1 & $\begin{array}{l}\text { Werturteile der sozia- } \\
\text { len Gruppe (TZSN) }\end{array}$ & $\begin{array}{l}\text { Gesamtzufriedenheit } \\
\text { mit Stammgeschäft im } \\
\text { Nachbarland (GKDZ } \\
\text { Kauf) }\end{array}$ & Persönliche Norm (PN) & $\begin{array}{l}\text { Gesamtzufriedenheit } \\
\text { mit dem Einkauf im } \\
\text { Nachbarland } \\
\text { (GLSS) }\end{array}$ & $\begin{array}{l}\text { Kundenloyalität } \\
\text { (KL) }\end{array}$ \\
\hline 2 & $\begin{array}{l}\text { Wichtigkeit der sozia- } \\
\text { len Norm (WSN) }\end{array}$ & $\begin{array}{l}\text { Bedeutung der sozia- } \\
\text { len Norm für den } \\
\text { Konsumenten } \\
(\mathrm{GKdZN})\end{array}$ & & $\begin{array}{l}\text { Wahrgenommene } \\
\text { Selbstrelevanz } \\
\text { (WRS) }\end{array}$ & $\begin{array}{l}\text { Gesamtzufriedenheit } \\
\text { mit Stammgeschäft } \\
\text { im Nachbarland } \\
\text { (GKDZ Kauf) }\end{array}$ \\
\hline 3 & & $\begin{array}{l}\text { Werturteile der sozia- } \\
\text { len Gruppe (TZSN) }\end{array}$ & & $\begin{array}{l}\text { Gesamtzufriedenheit } \\
\text { mit Stammgeschäft } \\
\text { im Nachbarland } \\
\text { (GKDZ Kauf) }\end{array}$ & $\begin{array}{l}\text { Wahrgenommene } \\
\text { Selbstrelevanz } \\
\text { (WRS) }\end{array}$ \\
\hline 4 & & $\begin{array}{l}\text { Wichtigkeit der sozia- } \\
\text { len Norm (WSN) }\end{array}$ & & $\begin{array}{l}\text { Persönliche Norm } \\
\text { (PN) }\end{array}$ & $\begin{array}{l}\text { Persönliche Norm } \\
\text { (PN) }\end{array}$ \\
\hline 5 & & & & & $\begin{array}{l}\text { Gesamtzufriedenheit } \\
\text { mit dem Einkauf im } \\
\text { Nachbarland } \\
\text { (GLSS) }\end{array}$ \\
\hline 6 & & & & $\begin{array}{l}\text { Bedeutung der } \\
\text { sozialen Norm für } \\
\text { den Konsumenten } \\
(\mathrm{GKdZN})\end{array}$ & \\
\hline
\end{tabular}

Tab. 7.50: $\quad$ Überblick über die Konstrukte der Kundenbindung für deutsche Konsumenten

Unterschiedlich ist ebenso die Reihenfolge der Wirkungsstärke der Variablen der Kundenbindung. Hat bei den niederländischen Konsumenten die Gesamtzufriedenheit mit dem Einkauf im Nachbarland eine hohe Wirkungsstärke, ist dies bei den deutschen Konsumenten die Gesamtzufriedenheit mit dem Stammgeschäft im Nachbarland. Auch hier ist ein Unterschied in der kulturellen Grundausrichtung der Konsumenten vorhanden. Deutsche Konsumenten lassen sich demnach an eine konkrete Einkaufsstätte im Nachbarland - sofern Loyalität vorhanden ist - einfacher binden als niederländische Konsumenten.

Für die Ableitung von Handlungsempfehlungen für Einkaufsstätten ist die Betrachtung der Wirkungsempfehlungen notwendig, um positive und negative Wirkungsmaßnahmen konkreter Maßnahmen zu identifizieren. Interessant ist die höhere Bedeutung der Wichtigkeit der sozialen Norm und die Werturteile der sozialen Gruppe hinsichtlich Bindung. Auch die Ge- 
samtzufriedenheit mit dem Einkauf im Stammgeschäft im Nachbarland hat für deutsche Konsumenten einen höheren Totaleffekt als bei niederländischen Konsumenten. Dagegen ist der Totaleffekt der wahrgenommenen Selbstrelevanz und der persönlichen Norm auf Bindung bei niederländischen Konsumenten höher als bei deutschen Konsumenten.

\begin{tabular}{|c|c|c|c|c|c|c|c|c|}
\hline & \multicolumn{2}{|c|}{$\begin{array}{l}\text { Bedeutung der sozia- } \\
\text { len Norm (GKdZN) }\end{array}$} & \multicolumn{2}{|c|}{$\begin{array}{l}\text { Gesamtzufriedenheit mit } \\
\text { Einkauf im Nachbarland } \\
\text { (GLSS) }\end{array}$} & \multicolumn{2}{|c|}{ Kundenloyalität (KL) } & \multicolumn{2}{|c|}{ Kundenbindung (KB) } \\
\hline & NL & $\mathrm{D}$ & $\mathrm{NL}$ & $\mathrm{D}$ & $\mathrm{NL}$ & $\mathrm{D}$ & $\mathrm{NL}$ & $\mathrm{D}$ \\
\hline $\begin{array}{l}\text { Wichtigkeit der sozialen } \\
\text { Norm (WSN) }\end{array}$ & 0,03 & 0,122 & 0,006 & 0,024 & 0,001 & 0,006 & 0,000 & 0,002 \\
\hline $\begin{array}{l}\text { Werturteile der sozialen } \\
\text { Gruppe (TZSN) }\end{array}$ & 0,241 & 0,413 & 0,049 & 0,081 & 0,007 & 0,019 & 0,003 & 0,008 \\
\hline Gesamtzufriedenheit mit & & & 0,342 & 0,381 & 0,051 & 0,089 & 0,020 & 0,036 \\
\hline Stammgeschäft $\quad$ im & & & & & & & & \\
\hline Nachbarland (GKDZ & & & & & & & & \\
\hline Kauf) & & & & & & & & \\
\hline Wahrgenommene & & & & & 0,252 & 0,234 & 0,100 & 0,095 \\
\hline Selbstrelevanz (WRS) & & & & & & & & \\
\hline Persönliche Norm (PN) & & & & & 0,068 & 0,064 & 0,027 & 0,026 \\
\hline
\end{tabular}

Tab. 7.51: Gegenüberstellung der Totaleffekte der deutschen und niederländischen Konstrukte zur Kundenbindung

Da alle Konstrukte fallende Totaleffekte aufweisen, bedeutet dies, dass der Einfluss der Werturteile der sozialen Gruppe und die Bedeutung der sozialen Norm im Zeitablauf abnehmen. Entscheidend zum Aufbau von Bindung ist dann für beide Gruppen die Gesamtzufriedenheit mit dem Stammgeschäft im Nachbarland sowie die wahrgenommene Selbstrelevanz. Die wahrgenommene Selbstrelevanz ist dann eine Assoziationskette, die dem Kunden hilft, eine Leistung des Stammgeschäftes im Nachbarland mit einem bestimmten Wert zu verknüpfen. Diese Werthaltung des Kunden ist dann kaufentscheidungsrelevant. Bei deutschen Konsumenten ist somit für niederländische Einkaufsstätten im ersten Schritt eine gemeinschaftliche Ansprache der deutschen Konsumenten Erfolg versprechender, da hierüber auch die soziale Gruppe der Konsumenten erreicht werden kann. Im zweiten Schritt hat eine gezielte Ansprache der Konsumenten über das jeweilige Stammgeschäft zu erfolgen, da über das Involvementkonstrukt die Werte, die der deutsche Konsument durch den Kauf anstrebt angesprochen werden können. Bei den niederländischen Konsumenten ist dagegen die direkte Ansprache über die persönliche Norm und ihre wahrgenommene Selbstrelevanz bindungsfördernd. 


\subsection{Zusammenfassung des Kapitels}

Folgende zwei Fragen bildeten den Ausgangspunkt dieses Kapitels:

1. Unterscheiden sich deutsche und niederländische Konsumenten hinsichtlich ihres Bindungsverhaltens zu einer Einkaufsstätte voneinander?

2. Unterscheiden sich die Bindungszustände der beiden Konsumentengruppen in ihrer verhaltenssteuernden Wirkung voneinander?

Erst wenn beide Fragen positiv beantwortet werden, gewinnt für einen Anbieter das Wissen um den inneren Bindungszustand seiner Kunden an Relevanz. Um diese Fragen empirisch beantworten zu können wurden die Hypothesen entwickelt und die Konstrukte getestet. Die Hypothesen wurden in Kausalmodelle umgesetzt und die Parameter getestet.

Die Ergebnisse des Modells zeigen, obwohl, beziehungsweise weil nicht alle Hypothesen nachgewiesen werden konnten, das deutsche und niederländische Konsumenten sich deutlich in ihrer Einstellung zum Einkauf im Nachbarland unterscheiden. Anerkennung in der Gruppe ist für niederländische Konsumenten nicht so entscheidend wie für deutsche Konsumenten um Kaufentscheidungen zu treffen. Die Ergebnisse zeigen, wodurch Bindung an eine ausländische Einkaufsstätte gebildet wird. Dies ist bei deutschen Konsumenten das soziale Umfeld des Konsumenten und die Einstellung des sozialen Umfeldes zum Einkauf im Nachbarland, unterstützt durch die wahrgenommene Selbstrelevanz des Konsumenten. Dagegen sind die niederländischen Konsumenten geprägt durch ihre persönliche Norm. Das Modell zeigt für beide Konsumentengruppen eine gute Erklärung der Kundenbindung und eine Bestätigung der Annahme, dass kulturelle Unterschiede, die sich im Kaufverhalten bemerkbar machen, zwischen niederländischen und deutschen Konsumenten vorhanden sind. Der Einfluss der wahrgenommenen Verhaltenskontrolle auf Bindung erweist sich allerdings als nicht signifikant. Ebenfalls lässt sich die Wirkung des vom Konsumenten gebildeten Vorsatzes zur Bindung an eine Einkaufsstätte im Nachbarland auf die tatsächlich vom Konsumenten gezeigte Kundenbindung für beide Konsumentengruppen nicht nachweisen.

Die befragten niederländischen Konsumenten wohnen zu einem hohen Anteil in zwei, drei und mehr Personen-Haushalten. Ferner haben sie zu einem großen Anteil ein Haushaltsnettoeinkommen von bis zu €2.000,- zur Verfügung. Sie wohnen in unmittelbarer Grenznähe und nutzen hauptsächlich den Samstag zum Einkauf im Nachbarland. Aachen wird als Einkaufsort niederländischer Konsumenten bevorzugt. Dabei werden zum Einkauf in Aachen auch größe- 
re Anreisewege in Kauf genommen. Die weiteren Einkaufsaktivitäten verteilen sich gleichmäßig auf Mönchengladbach, Viersen und Kaldenkirchen. Kaldenkirchen wird insbesondere zum Einkauf bei Discountern genutzt. Heinsberg spielt im Vergleich zu den anderen Städten nur eine untergeordnete Rolle als Einkaufsstadt. Als Verkehrsmittel wird über alle Altersgruppen insbesondere das Auto als Individualverkehrsmittel genutzt, doch auch Bus und Bahn werden insbesondere von jüngeren, als auch älteren Konsumenten zum Einkauf im Nachbarland genutzt. Die befragten niederländischen Konsumenten kaufen insbesondere Bekleidung und Schuhe in Deutschland ein, gefolgt von Elektroartikeln. Als Gründe für den Einkauf im Nachbarland werden von den niederländischen Konsumenten insbesondere der Preis, die Angebotsfläche und das große Sortiment, doch auch der Service genannt. Die Gruppe der zwischen 35 und 64jährigen kauft besonders häufig bei Discountern ein, wobei von der Altersgruppe der über 64jährigen das Fachgeschäft in Deutschland verstärkt gesucht wird.

Werden die subjektiven Eigenschaftsbeurteilungen zum Einkauf im Nachbarland auf Beurteilungsdimensionen verdichtet, so ergeben sich sieben Hauptfaktoren, anhand dessen niederländische Konsumenten ihre Bindung an deutsche Einkaufsstätten abhängig machen. Die zentralen Faktoren, die für niederländische Konsumenten für einen Einkauf im Nachbarland Bedeutung haben, sind insbesondere die soziale Norm, die Einstellung zum Einkauf im Nachbarland, der Aufbau von echter Bindung zu einer Einkaufsstätte, die Zufriedenheit mit der Einkaufsstätte, die generelle Einstellung zum Nachbarland, die persönliche Norm sowie die Wichtigkeit der sozialen Norm. Allerdings sind diese Faktoren nicht für alle niederländische Konsumenten gleichbedeutend. Nachweisbar ist allerdings nur der Einfluss der persönlichen Norm auf die Einstellung zum Einkauf im Nachbarland. Dieser Pfad ist signifikant. Bei den befragten niederländischen Konsumenten sind zwei kleinere Gruppen identifiziert worden. Dies ist einmal eine kleine Gruppe, die dem Einkauf im deutschen Nachbarland gegenüber sehr negativ gegenüber eingestellt ist. Diese Gruppe besteht aus zumeist jüngeren, weiblichen Konsumenten, die ein Haushaltsnettoeinkommen von bis zu $€ 2.000$,besitzen und deren Haushalt aus drei und mehr Personen besteht. Diese Gruppe sieht durch den Einkauf im Nachbarland für sich keinen Vorteil. Als Clustergruppe wurden diese Gruppe als die Minimalkunden bezeichnet.

Eine weitere kleine Gruppe von niederländischen Konsumenten gehören zu der Gruppe der preislastigen Schnellkäufer, die im Einkauf nur eine lästige Pflichtübung sehen und Preisunterschiede zwischen den beiden Ländern nutzen. Das Haushaltsnettoeinkommen dieser Gruppe liegt bei bis zu $€ 2.000$,-. Insbesondere in dieser Gruppe ist auch die soziale Gruppe der 
Meinung, dass man beim Einkauf im Nachbarland Preisvorteile hat und der Einkauf schnell und unkompliziert getätigt werden sollte. Diese Konsumenten benutzen zum Einkauf das Auto und sehen den Einkauf als Notwendigkeit an. Allerdings geben sie positive Erfahrungen an das soziale Umfeld weiter. Diese Gruppe kann als die pragmatische Gruppe bezeichnet werden, die grenznah einkaufen und durchaus informiert werden will über die Einkaufsmöglichkeiten im Nachbarland. Die größte Gruppe sind die jüngeren Konsumenten, die über ein Einkommen zwischen $€ 2.000$ und $€ 3.500$,- verfügen, in zwei Personenhaushalte leben und insbesondere in Filialbetrieben einkaufen.

Doch auch bei den niederländischen Konsumenten gibt es Konsumenten, die begeistert sind im Nachbarland einzukaufen. In dieser Gruppe ist sowohl in der Gruppe der bis zu 35jährigen, als auch in der Gruppe der über 64jährigen Konsumenten vertreten. Diese Gruppe (Single, Zwei-Personen Haushalte) hat ein Haushaltsnettoeinkommen von bis zu $€ 2.000$,und kauft gerne sowohl in kleinen Fachgeschäften als auch in Filialgeschäften ein. In dieser Gruppe ist insbesondere die soziale Gruppe dem Einkauf im Nachbarland gegenüber positiv eingestellt.

Abschließend ist mit PLS nachgewisen worden, welche Faktoren Auswirkungen auf das Bindungsverhalten niederländischer Konsumenten haben. Ein deutliches Ergebnis der Untersuchung ist, dass die persönliche Norm der entscheidende Faktor für niederländische Konsumenten zur Erklärung von Kundenbindung ist. Die persönliche Norm ist bei niederländischen Konsumenten für die Einstellung zum Einkauf im Nachbarland und der Bindung zu einer Einkaufsstätte im Nachbarland verantwortlich. Dies gilt sowohl für die Gruppe der Begeisterten, als auch für die Gruppe der pragmatischen Käufer. Beide Gruppen sind besonders offen für Informationen von Einkaufsstätten im Nachbarland. Die persönliche Norm beeinflusst hier sowohl die Gesamtzufriedenheit mit dem Kauf und die Reaktion des sozialen Umfeldes auf den Kauf, als auch die Kundenloyalität und die wahrgenommene Selbstrelevanz. Da die wahrgenommene Selbstrelevanz verstanden wird als die wertorientierte Konzeptualisierung des Involvementkonstruktes und das Ursache-Wirkungsgefüge des Handelns determiniert, ist die persönliche Norm der wesentliche Faktor, der Einfluss auf das Bindungsverhalten der niederländischen Konsumenten nimmt. Dies wird dadurch verstärkt, dass sowohl die persönliche Norm als auch die wahrgenommene Selbstrelevanz die Kundenloyalität der Konsumenten bestimmen und dadurch Bindung erklärt werden kann. Für die niederländischen Konsumenten konnten keine signifikanten Pfade zur sozialen Norm nachgewiesen werden. Die Umgebung des Konsumenten hat somit auch weniger Einfluss auf das Kaufverhalten des Einzelnen. Das 
Kaufverhalten des Einzelnen wird durch seine persönlichen Erfahrungen beeinflusst. In dieser Grundeinstellung unterschieden sich die niederländischen Konsumenten nicht voneinander, auch wenn die Konsumentengruppe hinsichtlich Geschlecht, Alter oder Einkommen segmentiert wurde. Um eine höhere Bindung niederländischer Konsumenten an eine deutsche Einkaufsstätte zu erreichen, ist insbesondere Ehrlichkeit in der Preisauszeichnung und Respekt gegenüber dem Konsumenten ein wesentlicher Aspekt. Diesen Aspekt scheinen große niederländische Konsumentengruppen bei deutschen Discountanbietern erfüllt zu sehen und zeigen sich deshalb diesem Betriebstyp gegenüber loyal. Unterstützt wird diese Aussage auch durch das cross buying Potenzial der Einkaufsstätten. Niederländische Konsumenten möchten Einkäufe gezielt tätigen und sind bereit auch andere (neue) Produkte in ihrem Stammgeschäft zu kaufen. Parkplatzangebot, Erreichbarkeit mit öffentlichen Verkehrsmitteln sind weitere Aspekte, die die Bindungsbereitschaft erhöhen. Da das Haushalteinkommen in Limburg im Landesvergleich relativ niedrig ist, suchen die Konsumenten ebenso Einkaufsstätten, die ein gutes Preis-Leistungsverhältnis bieten.

Die deutschen befragten Konsumenten wohnen zu einem hohen Anteil in zwei-, drei und mehr Personen-Haushalten und der Anteil der Haushaltsnettoeinkommen bis zu $€$ 3.500,überwiegt. Die befragten Konsumenten wohnen in den Regionen Mönchengladbach, Krefeld und Aachen. Weiter entfernte Wohnorte spielen für den Einkauf im Nachbarland nur eine untergeordnete Rolle. Die befragten Konsumenten kaufen insbesondere in Venlo, Roermond und Sittard ein. Heerlen und Maastricht sind insbesondere Einkaufsorte für Konsumenten mit Wohnorte im Postleitzahlgebiet Aachen. Die Erreichbarkeit der Städte im Nachbarland ist ein wesentliches Kriterium dafür, ob der Wille zur Bindung auch wirklich realisiert wird. Konsumenten aus Deutschland bevorzugen zum Einkauf im Nachbarland das Auto. Insbesondere die Altersgruppe der bis zu 64jährigen nutzt mit über $90 \%$ das Auto zum Einkauf. Allein die Gruppe der über 64jährigen nutzt zu einem größeren Anteil (31,3\%) die Bahn und den Bus (6,3\%). Die befragten Konsumenten kaufen zu einem hohen Anteil Bekleidung und Schuhe $(49,4 \%)$ und Gartenartikel (22,5\%) im Nachbarland, zu einem niedrigeren Anteil Möbel. Auch für die deutschen Konsumenten ist der Preis ein Kaufargument, aber auch das Sortiment und der Service. Dies ist auch nicht verwunderlich, da auch im deutschen Grenzgebiet zu den Niederlanden das Haushaltsnettoeinkommen im Verhältnis zum Landesdurchschnitt niedriger ist. Auffallend ist der hohe Anteil der deutschen Konsumenten, die gerne in kleinen Fachgeschäften $(45,5 \%)$ und bei Filialisten, die man aus anderen Einkaufsorten aus dem Nachbarland kennt (28,9\%), einkauft. Werden die Faktoren aus der Faktoranalyse herangezogen, so dienen insbesondere die Faktoren echte Bindung, Zufriedenheit mit der Einkaufsstätte, die persön- 
lche Norm, die Wichtigkeit der sozialen Norm, die Loyalität zur Einakufsstätte, die Einstellung zum Einkauf im Nachbarland und die soziale Norm als Erklärung der Kundenbindung. Allerdings konnten mit Hilfe der Faktorenanalyse nur der Einfluss der persönlichen Norm und die Einstellung zum Einkauf im Nachbarland auf die Kundenloyalität nachgewiesen werden. Die soziale Norm, die Wichtigkeit der sozialen Norm und die Zufriedenheit mit dem Einkauf selbst bilden die Gesamtzufriedenheit des Konsumenten mit dem Einkauf im Nachbarland ab. Die Einstellung zum Einkauf im Nachbarland nimmt im deutschen Modell Einfluss auf die Kundenloyalität. Mit Hilfe der Clusteranalyse sind insgesamt vier Cluster für deutsche Konsumenten identifiziert worden. Dies sind die Minimalkunden, die preisbewussten Kunden von Filialbetrieben, die Gelegenheitskunden und die begeisterten Fachgeschäftskunden. Die Gruppe der Minimalkunden ist gleichmäßig verteilt auf die Gruppe der jungen Konsumenten bis zu 35 Jahren und Konsumenten bis zu 64 Jahren. Diese Konsumenten stehen dem Einkauf im Nachbarland nicht positiv gegenüber - genau wie ihre soziale Gruppe nicht zum Einkauf ins Nachbarland fährt. Eine weiter Gruppe ist die Gruppe der preisbewussten Konsumenten, die bereits als Kind Erfahrungen mit dem Einkauf im Nachbarland gesammelt hat. Diese Konsumentengruppe kennt die preislichen Vorteile des Einkaufs im Nachbarland und nutzt die Unterschiede beim Einkauf aus. Die Konsumenten haben eine positive Grundeinstellung zum Einkauf im Nachbarland, haben ein Haushaltsnettoeinkommen von bis zu $€ 2.000,-$. Die Gruppe lebt in Haushalten mit mehr als drei Haushaltsmitgliedern und haben eine hohe $\mathrm{Zu}$ friedenheit mit ihrem Einkaufsort im benachbartem Ausland. Diese deutsche Konsumentengruppe kauft insbesondere in Einzelhandelsketten ein. Auch das soziale Umfeld dieser Gruppe ist dem Einkauf im Nachbarland positiv gegenüber eingestellt und unterstützt diese Entscheidung. Eine weitere Gruppe sind auch unter den deutschen Konsumenten die Gelegenheitskäufer, die dem Einkauf im Nachbarland keine große Bedeutung beimessen. Für sie gehört der Einkauf im Nachbarland nicht zur Lebensqualität und auch das soziale Umfeld ist dem Einkauf im Nachbarland nicht positiv gegenüber eingestellt. Die letzte identifizierte Gruppe ist die Gruppe der begeisterten Fachgeschäftskunden. Diese Kundengruppe verbindet mit dem Einkauf im Nachbarland Spass, findet es gut die Möglichkeit zu haben im Nachbarland einkaufen zu können. Auch die soziale Gruppe dieser Konsumenten ist dem Einkauf im Nachbarland gegenüber positiv eingestellt und sucht im Ausland ebenso nach Produkte die im eigenen Land gesucht, aber nicht gefunden werden. Die Kundengruppe besitzt eine hohe Zufriedenheit gegenüber dem Stammgeschäft und kauft insbesondere in kleinen Fachgeschäften ein. Mit den Faktoren aus der Faktoranalyse konnten nur die Indikatoren Zufriedenheit Einkauf Ausland, Kundenloyalität und Echte Bindung dargestellt werden. Ferner konnte nur der Pfad persönliche Norm zu Kundenloyalität als signifikant nachgewiesen werden. 
Im PLS-Modell wirkt die persönliche Norm der befragten Konsumenten auf die wahrgenommene Selbstrelevanz. Dies bedeutet, dass die Konsumenten in ihrem Involvement zu einer Einkaufsstätte im Nachbarland um so stärker beeinflusst werden, um so stärker sie ihre persönlichen Werte durch den Einkauf im Nachbarland bestätigt sehen. Die wahrgenommene Selbstrelevanz beeinflusst bei den deutschen Konsumenten sowohl die Kundenloyalität, als auch die Kundenbindung. Auf der anderen Seite ist aber die Gesamtzufriedenheit mit dem Einkauf im Ausland beeinflusst durch die Meinung der sozialen Gruppe und die Bedeutung dieser Meinung für den Konsumenten bindungsrelevant. Dies erklärt auch, dass je positiver die soziale Gruppe des Konsumenten dem Einkauf im Nachbarland gegenüber eingestellt ist, umso positiver ist auch der Konsument selbst dem Einkauf im Nachbarland gegenüber eingestellt. Bei der Gruppe der begeisterten Fachgeschäftskunden, die ein höheres Haushaltsnettoeinkommen haben als die übrigen Gruppen, überwiegt die Suche nach dem „Besonderem“ (Im Nachbarland erhalte ich Produkte, die ich im eigenen Land nicht bekomme), aber auch die Verbindung mit Einkauf und Spass. Dies wird von der sozialen Gruppe dieser Konsumenten unterstützt. Diese Gruppe verbindet den Einkauf im Nachbarland mit Gaststättenbesuchen, besucht das Nachbarland mit Freunden oder um Freunde im Nachbarland zu besuchen. Diese Gruppe ist auch insgesamt interessiert am Nachbarland und sucht bewusst die Atmosphäre des Nachbarlandes. Neben modischen Textilien werden von dieser Konsumentengruppe insbesondere Artikel aus Gartencentern oder auch Möbel gekauft. Hier sucht man insbesondere Produkte die einen Lebensstil charakterisieren, den man selbst verfolgt. 


\section{Kapitel 8: Empirische Ergebnisse und Schlussfolgerungen}

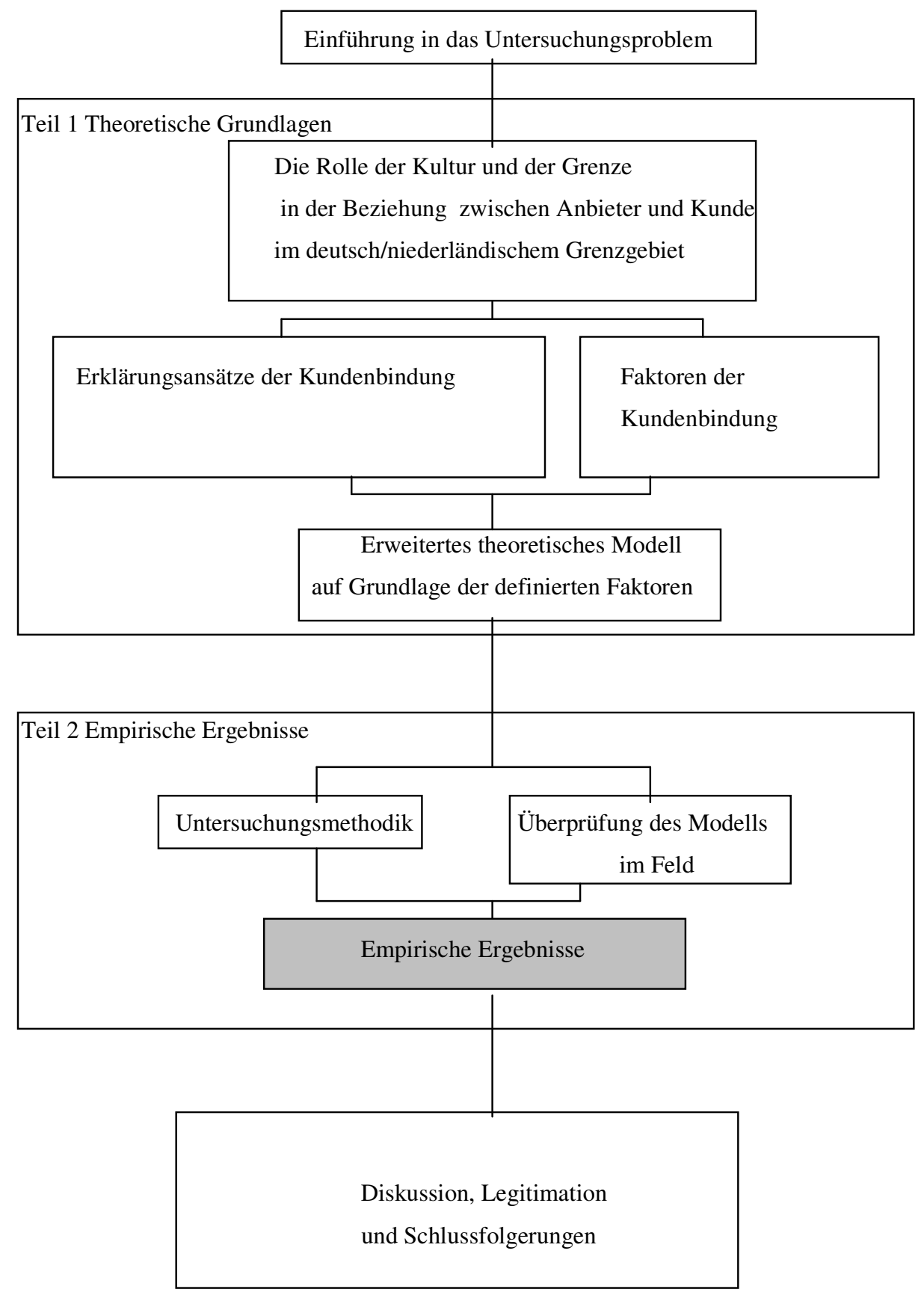




\subsection{Einleitung}

Der Ausgangspunkt der Forschungsarbeit war die Vermutung, die Kundenbindung in einem Grenzgebiet sei nicht konzeptionell durchdrungen. Die Konzeptualisierung der Kundenbindung in einem Grenzgebiet soll dazu beitragen, ein differenzierteres Verständnis des komplexen Marketingphänomens zu erhalten. Dazu sollten folgende Forschungsfragen beantwortet werden:

1. Welches Begriffsverständnis von Kundenbindung liegt in der Literatur vor?

2. Eignet sich die neuere Einstellungsforschung zur Erklärung der Kundenbindung in einem Grenzgebiet?

3. Wie kann Kundenbindung aus Kundensicht in einem Grenzgebiet durch Kundenbindungsfaktoren konzeptualisiert und operationalisiert werden?

4. Ist diese Konzeptualisierung aus Sicht der Grenzgebiete relevant?

In Kapitel 8 werden nun die gesamten Ergebnisse zusammengefasst, um die gestellten Forschungsfragen zu beantworten.

\subsection{Zusammenfassung der empirischen Ergebnisse der Untersuchung}

Der Begriff der Kundenbindung wird differenziert nach Gebundenheit und Verbundenheit. Für die vorliegende Arbeit wurde das Begriffsverständnis der Kundenbindung nach Eggert in Bezug auf Verbundenheit gewählt. Allerdings ist zu diesem Begriffsverständnis noch die Handlung des Wiederkaufs nach Diller hinzugefügt worden. Beide analysierte Konsumentengruppen werden durch unterschiedliche Konstrukte in ihrem Bindungsverhalten beeinflusst. Niederländische und deutsche Konsumenten reagieren somit auch im engen Grenzgebiet unterschiedlich. Der unterschiedliche Einfluss der sozialen Norm, Beeinflussung durch kulturelle Einflüsse, die unterschiedliche Bedeutung der wahrgenommenen Selbstrelevanz auf Loyalität und Bindung zu einer Einkaufsstätte im benachbartem Ausland gilt als nachgewiesen. Es ist allerdings zu berücksichtigen, dass bei den niederländischen Probanden ein höherer Anteil Frauen, ein höherer Anteil jüngerer Probanden und auch ein höherer Anteil niedrigerer Einkommensgruppen in die Befragung eingegangen sind. Allerdings sind diese Abweichungen nicht so hoch, dass grundsätzlich von unterschiedlichen Stichproben gesprochen werden muss. 


\subsubsection{Das modellierte Kundenbindungsmodell zur Erklärung der grenzüberschreiten- den Kundenbindung unter kulturellen Aspekten}

Die Bestandsaufnahme der wissenschaftlichen Marketingliteratur zeigte, dass die neuere Einstellungsforschung als Erklärungsmodell der Kundenbindung insbesondere in einem grenznahem Raum nicht den Anspruch erfüllt alle sozialen Verhaltensweisen vorherzusagen. Ajzen's Anspruch nicht nur willentlich kontrolliertes, sondern auch nicht willentlich kontrolliertes Handeln vorherzusagen und jegliche individuelle Kaufentscheidung durch die Theorie des geplanten Verhaltens vorherzusagen, kann nicht bestätigt werden. Für Konsumgüter werden vom Konsumenten nicht Informationen systematisch gesammelt und für Kaufentscheidungen genutzt. Allerdings neigen die Konsumenten zu einer Reduktion ihres kognitiven Aufwandes und zu habituellen Entscheidungen, die von der sozialen Gruppe des Konsumenten unterstützt werden. Aus diesem Grund eignet sich die Theorie des geplanten Verhaltens insbesondere bei erstmaliger Entscheidung oder bei Kaufentscheidungen mit einem hohem Involvement der Konsumenten. Kaufentscheidungen beruhen dann auf einem extensiven Kaufentscheidungsprozess.

Die Resultate der empirischen Untersuchung attestieren dem modellierten Kundenbindungsmodell eine beachtliche Erklärungskraft zur Kundenbindung in einem Grenzgebiet. Aus wissenschaftlicher Sicht ist von Interesse, dass auch in einem Grenzgebiet die Reaktion der Bezugspersonen eine wesentliche Stellgröße zur Kundenbindung ist. Die Berücksichtigung der Stellgröße erbringt eine höhere Erklärungsgüte des deutschen Kundenbindungsmodells. Für die wahrgenommene Verhaltenskontrolle konnten im Modell keine signifikanten Pfade nachgewiesen werden. Dies belegt, dass diese Größe branchenspezifisch zu deuten ist. Sofern Produkte keine sozialen Auffälligkeiten besitzen, sondern stärker situativen Faktoren (Textil, Schuhe) unterliegen, wird der Erwerb dieser Produkte auch nur mit einer geringen kognitiven Informationsverarbeitung begleitet. Das zentrale Forschungsanliegen bestand darin, zu klären, ob sich die Einstellung der Konsumenten zum Einkauf im Nachbarland in einem Grenzgebiet im Zeitablauf angleicht und gleiche Konstrukte in gleicher Intensität für Kundenbindung dieser Konsumenten verantwortlich ist. Dazu wurden die zu untersuchenden Fragen und Subfragen (siehe Kapitel 1.3) formuliert und zunächst ein einheitliches Bindungsmodell erstellt. Die in Kapitel 1.3 formulierten Fragen sollen zunächst beantwortet werden. Sofern der Einfluss durch die soziale Gruppe, geprägt durch die Kulturdimensionen und durch weitere Konstrukte (persönlichen Norm, wahrgenommene Selbstrelevanz) in den beiden Ländern unterschiedlich ist, wirken in einer Grenzregion wie dem deutsch-niederländischem Grenzgebiet unterschied- 
liche Faktoren mit unterschiedlicher Gewichtung auf die Kundenbindung zu einer Einkaufsstätte im Nachbarland auf die Einstellung der Konsumenten ein. Dies obwohl die Konsumenten beider Nationalitäten eng zusammenleben, verwandtschaftliche Beziehungen vorhanden sind und der Arbeitsmarkt transparent ist. Die Ergebnisse der empirischen Untersuchung dokumentieren eine hohe Erklärungskraft des linearen Strukturgleichungsmodells der Kundenbindung. Die Untersuchungen zeigen auch, dass das lineare Kundenbindungsmodell die Realisierung der Kundenbindung in der Realität abbildet. An dem Ursache-Wirkungsgefüge des Modells können sich somit die Einzelhandelsunternehmen orientieren, die auch Kunden aus dem Nachbarland an die Einkaufsstätte binden möchten. Entsprechende Maßnahmen können von den Einkaufsstätten durch die Betrachtung der einzelnen Komponenten des Modells abgeleitet werden. Die einzelnen Komponenten kann ein Einzelhandelsunternehmen individuell beeinflussen, sofern ein treuer, mit dem Unternehmen verbundener Kundenstamm aufgebaut werden soll. Einzelhandelsunternehmen können bei der Zusammenstellung von Kundenbindungsmaßnahmen darauf achten, dass sie solche Bündel zusammenstellen, die die einzelnen Zielkonstrukte am stärksten beeinflussen. Dies erscheint schon aus dem Aspekt der Kosten heraus sinnvoll, weil Kundenbindungsmaßnahmen erhebliche Kosten für die Unternehmen verursachen.

Die Einstellung der untersuchten Konsumenten zum Einkauf im Nachbarland ist abhängig davon, welcher Nationalität und welcher Kulturdimension sie angehören. Die beiden untersuchten Konsumentengruppen reagieren im Rahmen der Kundenbindung auf die Konstrukte unterschiedlich stark und auf Faktoren mit unterschiedlicher Intensität. Damit bestätigt sich auch Kasper's Cluster der europäischen Länder Kulturen. Niederländische und deutsche Konsumenten gehören auch im grenznahem Gebiet der deutsch-niederländischen Grenze unterschiedlichen Kulturdimensionen an. Dies erklärt auch die starke Bedeutung der persönlichen Norm der niederländischen Konsumenten. Somit kann vom deutsch-niederländischem Gebiet aus Sicht der Konsumenten nicht von einem gemeinsamen Konsumraum gesprochen werden. Auch der Einkauf im Nachbarland wird bei den Konsumenten durch unterschiedliche Konstrukte begründet. Dennoch hat die Landesgrenze für deutsche Konsumenten eine unterstützende Wirkung zum Aufbau von Bindung. Das Überschreiten einer Landesgrenze zur Erfüllung ihrer Bedürfnisse ist für deutsche Konsumenten ein Reiz, kein Hemmnis. Konkrete Produkterfahrungen verbessern allerdings nur dann die Einstellung eines Individuums, wenn es seine Erwartungen bestätigt sieht. Damit Einkaufsstätten ihre Kunden binden können, ist sicherzustellen, dass auch die Kunden mit dem Umfeld in dem die Produkte angeboten werden zufrieden sind. Einkaufsstätten sollten sich somit nicht nur an den Bedarf und den 
Bedürfnissen ihrer Kunden orientieren, sondern auch die Erwartungen des sozialen Umfeldes der Kunden berücksichtigen. Hinweise darauf wie die Zufriedenheit gesteigert werden kann, können aus den Konstrukten Werturteile der sozialen Gruppe und Wichtigkeit der sozialen Norm entnommen werden. Diese Aussagen gelten insbesondere für deutsche Konsumenten.

Die weitere Frage der Untersuchung, ob Konsumenten sich auch an ein Einzelhandelsgeschäft im Nachbarland binden lassen, lässt sich mit „Ja“ beantworten. Diese Frage kann insbesondere für die deutschen Konsumenten bejaht werden. Für niederländische Konsumenten ist diese Frage nicht einheitlich zu beantworten. Für diese Gruppe steht stark die persönliche Norm im Vordergrund. Die persönliche Norm hat bei dieser Konsumentengruppe Einfluss auf die Gesamtzufriedenheit mit dem Einkauf im benachbartem Ausland, der Kundenloyalität und der wahrgenommenen Selbstrelevanz. Die wahrgenommene Selbstrelevanz hat für beide Konsumentengruppen unterschiedliche Bedeutung. Während für die deutschen Konsumenten die wahrgenommene Selbstrelevanz Einfluss nimmt auf die Gesamtzufriedenheit mit dem Einkauf in einer Einkaufsstätte aber auch direkt Einfluss auf die Kundenbindung nimmt, hat sie bei den niederländischen Konsumenten direkten Einfluss auf das Loyalitätsverhalten und somit nur indirekt auf Bindung. Innerhalb der Untersuchung sollten aber auch folgende Teilziele untersucht werden:

- Identifizierung der Determinanten der Kundenbindung in ausgewählten Einzelhandelsbranchen in einer Grenzregion.

- Identifizierung relevanter Determinanten der Kundenbindung in einer Grenzregion.

- Identifizierung der Stimulanzen zum grenzüberschreitenden Einkauf und Einkaufsstättentreue.

Die Analyse des abgeleiteten Kundenbindungsmodells zeigt deutlich, dass Kundenbindung in einem grenznahen Raum wie dem deutsch-niederländischem Grenzgebiet zwischen Maastricht - Aachen und Venlo - Mönchengladbach in einem einheitlichen Kundenbindungsmodell darstellbar ist. Bindung bildet sich dennoch bei den beiden untersuchten Kundengruppen sehr unterschiedlich ab. Insbesondere der Einfluss des Umfeldes und die eigene Einstellung zum Einkauf im Nachbarland ist verschieden. Während deutsche Konsumenten ihre Bindung an eine Einkaufsstätte ausgewogen bilden durch Gesamtzufriedenheit mit dem Kauf insgesamt (GLSS), der wahrgenommenen Selbstrelevanz (WRS), und der Kundenloyalität (KL), wird die Bindung der niederländischen Konsumenten stark polarisierend gebildet durch die persönliche Norm und die wahrgenommene Selbstrelevanz. Die Kundenloyalität ist wiederum geprägt durch die persönliche Norm. Dies lässt den Schluss zu, dass Niederländer, wie auch von Hofstede bestätigt, eine ausgeprägte Neigung zum Individualismus, zur Unab- 
hängigkeit haben. Diese Grundeinstellung wird durch die Unterschiede der Kundengruppen im Modell bestätigt und erklärt das unterschiedliche Konsumverhalten von deutschen und niederländischen Konsumenten. Somit werden mit dem bestangepassten Modell die unterschiedlichen Werte im Rahmen der Kulturdimensionen bestätigt. Nicht bestätigt werden kann aufgrund des Kundenbindungsmodells für niederländische und deutsche Konsumenten die hypothetische Karte von Usunier zur Einteilung der sich ähnelnden und sich weniger ähnelnden europäischen Kulturen. Bestätigung findet dagegen durch das nun vorliegende Modell zur grenzüberschreitenden Kundenbindung die Einteilung von Kasper. Konsumenten aus unterschiedlichen Clustern besitzen unterschiedliche Werte und Normen und verhalten sich verschieden. Aus diesem Grund weicht auch, wie bestätigt, das Einkaufs- und Bindungsverhalten beider Konsumentengruppen voneinander ab. Nachbarschaftliche Beziehungen spielen insbesondere für deutsche Konsumenten eine besondere Rolle und beeinflussen das Bindungsverhalten. Selbst die grenznahen Gebiete zwischen den Niederlanden und Deutschland gehören demnach unterschiedlichen europäischen Kulturclustern an.

Verbundenheit mit vorhandenen Traditionen, auch Konsumtraditionen, sowie die Identifikation mit einer Region, hier der Grenzregion, sind für die deutschen Konsumenten ein wesentliches Bindungskriterium. Eng verbunden hiermit ist der Begriff der Vertrautheit (familiarity) für diese Konsumentengruppe. Diese Begriffe haben insbesondere für das Untersuchungsgebiet der grenznahen Regionen für deutsche Konsumenten eine herausragende Bedeutung, da in diesen Regionen der grenzüberschreitende Einkauf für sie traditionell Bestandteil des täglichen Lebens ist. Die Konsumenten der untersuchten Region finden nur dann Gefallen an Einkaufsstätten im benachbartem Grenzgebiet, wenn der Vorteil ihnen vertraut ist und entsprechend ihren Vorlieben die Einkaufsstätte sich präsentiert und vermarktet.

In dem bestangepassten Modell zur Erklärung der Kundenbindung kommt regionalisierten grenzüberschreitenden Zielgruppen keine Bedeutung zu. Ein Handelsunternehmen kann definierte Konsumentengruppen in der untersuchten Region grenzüberschreitend nicht $\mathrm{zu}-$ sammenfassen. Eine „grenzüberschreitende Zielgruppe“, bestehend aus Konsumenten von beiden Seiten der Grenze, kann nach den vorliegenden Daten von den Einkaufsstätten nicht gebildet werden. Beide Konsumentengruppen haben zwar ähnliche Interessen, können aber nicht als homogene Gruppe angesprochen werden. Die Meinungsbildung zur Kundenbindung gestaltet sich unterschiedlich. Insofern ist es für die untersuchten Einzelhandelsbereiche nicht möglich, deutsche und niederländische Konsumenten mit gleichen Kundenbindungskonzepten und gleichen Angeboten innerhalb der Bindungskonzepte an das Handelsunternehmen zu bin- 
den. Niederländische Konsumenten werden sehr stark durch ihre wahrgenommene Selbstrelevanz in ihrer Loyalität zu einer Einkaufsstätte geprägt. Zudem beeinflusst die persönliche Norm die Loyalität der niederländischen Konsumenten zu einer Einkaufsstätte. Werden die Ergebnisse der Untersuchung mit den Ergebnissen von Hofstede, Usunier und Kasper verglichen, wird der starke Einfluss von Erziehung, Bedeutung der sozialen Gruppe, allgemeine Grundwerte wie persönliche Freiheit auf Kauf- und Bindungsverhalten deutlich. Die Kultur nach in dieser Arbeit definierten Sinne - bestimmt damit das Kaufverhalten der Konsumenten. Diese Unterschiede wurden im Kapitel 7.1 dargestellt.

\subsubsection{Identifizierung der Determinanten der Kundenbindung in ausgewählten Einzelhandelsbranchen in einer Grenzregion}

Die Bereitschaft dem Rat einer Person zu folgen, wird über die soziale Norm erfasst. Gewichtungsfaktoren sind hierbei die Wichtigkeit der sozialen Norm für das Individuum sowie die Teilzufriedenheit des Individuums mit der Reaktion des sozialen Umfeldes auf seine Handlung. Für deutsche Konsumenten in der Grenzregion gehört es zum gelebten Alltag im Nachbarland einzukaufen. Die Befragung bestätigt, dass von deutschen Konsumenten - im Gegensatz zu niederländischen Konsumenten - im Nachbarland im hohen Maße Spontankäufe getätigt werden. Die individuellen Wertvorstellungen der deutschen Konsumenten haben einen hohen Einfluss auf ihr reales Verhalten. Diese Wertvorstellungen werden weiter verstärkt durch den Einfluss der Wertvorstellungen der deutschen Konsumenten gegenüber dem grenzüberschreitenden Einkauf. Die zunehmende Erlebnisorientierung der deutschen Konsumenten konnte in der vorliegenden Arbeit bestätigt werden.

In der Beantwortung der Frage nach dem bevorzugtestem Betriebstyp wird ein deutlicher Verhaltensunterschied deutscher und niederländischer Konsumenten erkennbar. Deutsche Konsumenten präferieren bei ihren grenzüberschreitenden Einkäufen insbesondere das Fachgeschäft, oder Filialunternehmen aus dem Nachbarland, niederländische Konsumenten kaufen verstärkt bei Filialisten, die sie aus dem eigenen Land kennen ein, oder bei Discountern. Allerdings können auch 20,4\% der befragten niederländischen Konsumenten als begeisterte Fachgeschäftskunden bezeichnet werden. Als Determinanten der Kundenbindung sind hierbei für deutsche Konsumenten folgende identifiziert worden:

- Der Preis.

- Die Andersartigkeit der Produkte. 
- Der Spaß in angenehmer Umgebung mit Cafés und Unterhaltungsmöglichkeiten einkaufen zu können.

Bei den niederländischen Konsumenten sind folgende Determinanten identifiziert worden:

- Der Preis.

- Die Einstellung der Bezugsgruppe zum Kauf im Nachbarland.

- Die eigene Einstellung zum Kauf im Nachbarland.

Deutsche Konsumenten können nach den vorliegenden Ergebnissen gezielt angesprochen werden, indem genau auf ihre Wertvorstellungen eingegangen wird. In den Ergebnissen der Untersuchung wird deutlich, dass Bindung an einer Einkaufsstätte im Nachbarland von deutschen Konsumenten insbesondere dann angestrebt wird, wenn sie zu der Gruppe der „Begeisterten Fachgeschäftskunden" und den „preisbewussten Kunden von Filialbetrieben“ gehören. Bei den niederländischen Konsumenten sind dies die beiden Gruppen der „Begeisterten für Stammgeschäfte" und die Gruppe der „schnellen, zielgerichteten preisorientierten Käufer". Auch aus diesen Ergebnissen wird deutlich, wie groß der Einfluss Dritter bei den Konsumentengruppen auf das Bindungsverhalten ist.

\subsubsection{Identifizierung der Stimulanzen zum grenzüberschreitenden Einkauf und Ein- kaufsstättentreue.}

Um die Stimulanzen des grenzüberschreitenden Einkaufs zu identifizieren, sollen noch einmal kurz die Einkaufsmotive der einzelnen Konsumentengruppen gegenübergestellt werden. Werden deutsche und niederländische Konsumenten miteinander verglichen, so kaufen deutsche Konsumenten häufiger und mit höheren Geldbeträgen ein als Niederländer. Deutsche suchen insbesondere die Abwechslung und verbinden Einkauf mit Freizeitspaß. Deutsche kaufen insbesondere modische Artikel (Textil, Schuhe). Niederländer suchen dagegen stärker nach Artikel des täglichen Lebens und decken sich stark auch bei deutschen Discountern ein. Dies gilt insbesondere für niederländische Konsumenten zwischen 30 und 60 Jahren. Jüngere niederländische Konsumenten fahren ins Nachbarland, weil sie Spaß am Einkaufen haben, werden allerdings auf Grund oft mangelnder Mobilität von ihrem Vorhaben abgehalten. Ältere niederländische Konsumenten kaufen dagegen sehr zielgerichtet im Nachbarland ein und fahren dann auch wieder schnell nach Hause. Ältere Konsumenten werden allgemein egal ob deutsche oder niederländische Konsumenten - durch rechtliche Restriktionen (Garan- 
tierecht, Umtauschrecht) vom Einkauf im Nachbarland abgehalten. Wird mangelnde Mobilität nicht durch öffentliche Verkehrsmittel aufgefangen, sind auch hier der Bereitschaft im Nachbarland einkaufen zu gehen Grenzen gesetzt. Sowohl deutsche als auch niederländische Konsumenten mit höherem Einkommen kaufen gerne im Nachbarland ein, um sich Artikel für besondere Ansprüche zu kaufen. Je höher das verfügbare Einkommen dieser Gruppe ist, umso öfter fährt man ins Nachbarland zum Einkaufen und besucht ebenfalls Restaurants und Cafés in der jeweiligen Einkaufsstadt. Doch insbesondere deutsche Konsumenten werden zum grenzüberschreitenden Einkauf stimuliert durch die Möglichkeit während und nach dem Einkauf Restaurants und Cafés besuchen zu können. Vielfach verbinden deutsche Konsumenten diese Möglichkeit mit einen Familienausflug oder den Besuch von Freunden. Die Stimulanzen für deutsche Konsumenten liegen somit auch verstärkt in diesem Bereich. Die Atmosphäre des Einkaufsortes ist sehr wichtig, da der deutsche Konsument für die gesamte Familie ein schönes Einkaufsevent schaffen möchte.

Niederländische Konsumenten dagegen legen großen Wert auf ein breites Sortimentangebot mit einem guten Preis/Leistungsverhältnis und suchen auch sehr gezielt nach bestimmten Artikeln. Stimuliert wird diese Konsumentengruppe somit insbesondere durch ein großes Angebot unterschiedlicher Anbieter und Vergleichsmöglichkeiten in einem kurzen Aktionsradius. Für Niederländer ist auch die Erreichbarkeit der Einkaufsstadt mit öffentlichem Verkehrsmittel eine wesentliche Stimulanz um grenzüberschreitend einzukaufen.

\subsubsection{Identifizierung relevanter Determinanten der Kundenbindung in einer Grenzregion.}

Sowohl niederländische als auch deutsche Konsumenten sehen als wesentlichste Determinante die Nutzung der unterschiedlichen Marktgegebenheiten in beiden Ländern an. Die Nutzung dieser für die Konsumenten vorteilhaften Unterschiede im Einkaufsangebot der Einkaufsstätten ist für beide Konsumentengruppen die wesentlichste Determinante der Kundenbindung. Allerdings unterscheiden sich die Bewohner der Grenzregion hierbei nicht von den Konsumenten außerhalb der Grenzregion. Für Bewohner der Grenzregion ist es aber auf Grund der Nähe zur Grenze einfacher die Vorteile der beiden unterschiedlichen Landesmärkte zu nutzen. Dazu zählen für niederländische Konsumenten der Einkauf bei deutschen Schuh-, Textil-, Elektro- und Lebensmitteldiscountern mit einem hohen Hardwareangebot aus Preisgesichtspunkten genauso, wie der Besuch von Fachhandelsgeschäften, die auf Grund ihrer 
Markenvielfalt eine Anziehungskraft auf niederländische Konsumenten ausüben. Ferner ist der Qualitätsgesichtspunkt der Produkte für niederländische Konsumenten eine wesentliche Determinante um in Deutschland einzukaufen. In allen Betriebstypen erwarten Niederländer von deutschen Einkaufsstätten Ehrlichkeit und Transparenz in der Preisauszeichnung und eine hohen Respekt gegenüber dem Kunden, der sich auch durch die Ansprache in der Landessprache dokumentiert. Obwohl die niederländischen Konsumenten sehr stark preisorientiert einkaufen, möchten sie aber nicht auf Service in der Einkaufsstätte verzichten. In der Befragung sprachen die befragten Niederländer von einer nicht so guten Atmosphäre und Ladenbaugestaltung in deutschen Einkaufsstätten.

Für die deutschen Konsumenten sind insbesondere das modische Warenbild und das PreisLeistungsverhältnis eine wichtige Determinante um sich an eine niederländische Einkaufsstätte zu binden. Hinzu kommen der unkomplizierte Einkauf ohne Sprachprobleme in niederländischen Einkaufsstätten sowie freundliche und hilfsbereite Mitarbeiter in einer der Ware angemessenen Einkaufsatmosphäre. Für deutsche Konsumenten ist aber auch das Umfeld des Einkaufs sehr wichtig, wie das Gesamtbild der Einkaufsstadt oder auch die Möglichkeit im Umfeld der Einkaufsstätte in Cafés oder Restaurants zu verweilen. Die Einkaufsmöglichkeit von Produkten, die man zuhause nicht erhält, ist eine weitere Determinante zur Bindung für deutsche Konsumenten. Dies ist eine weitere Begründung dafür, dass deutsche Konsumenten aus dem Grenzgebiet zwischen den Niederlanden und Deutschland verstärkt Produkte in den Niederlanden kaufen. Da niederländische Unternehmen nur in einem geringen Volumen niederländische Produkte in den deutschen Teil der Euregio exportieren, ist die Anziehungskraft niederländischer Einkaufsstädte auf deutsche Konsumenten zu erklären.

\subsection{Vergleich der Wirkungszusammenhänge und Modellbeurteilung}

Die wahrgenommene Selbstrelevanz und die persönliche Norm haben bei niederländischen Konsumenten einen höheren Einfluss auf Bindung als bei deutschen Konsumenten. Auch für die Bildung von Kundenloyalität haben die wahrgenommene Selbstrelevanz und die persönliche Norm einen hohen Einfluss bei niederländischen Konsumenten. Dagegen weisen die Werturteile der sozialen Gruppe einen hohen Einfluss auf das Loyalitäts- und Bindungsverhalten deutscher Konsumenten auf. Kundenbindung an eine Einkaufsstätte im Nachbarland entsteht für beide Kundengruppen erst, wenn Loyalität vorhanden ist. Dabei spielt die Ge- 
samtzufriedenheit mit dem Stammgeschäft für beide Kundengruppen eine entscheidende Rolle. Doch insbesondere bei deutschen Konsumenten wird der Einfluss der sozialen Gruppe auf die Zufriedenheit deutlich. Loyalität entsteht für niederländische Konsumenten insbesondere über die wahrgenommene Selbstrelevanz und über die persönliche Norm. Je positiver die persönliche Norm eines Konsumenten gegenüber dem grenzüberschreitendem Einkauf ist, um so stärker ist auch die Zufriedenheit des Konsumenten mit dem grenzüberschreitendem Kauferlebnis. Ein weiterer Faktor zur Bildung von Kundenloyalität ist für niederländische Kunden die Zufriedenheit mit dem Stammgeschäft im Nachbarland. Dagegen kommt zur Loyalitätsbildung der deutschen Konsumenten neben der Zufriedenheit mit dem Stammgeschäft die Gesamtzufriedenheit mit dem Einkauf im Nachbarland hinzu. Die Faktoren Freude und Spaß am Einkauf und die Bestätigung des Vorsatzes durch Freunde und Bekannte der Konsumenten sind entscheidende Faktoren zum Aufbau der Kundenloyalität und -bindung für deutsche Konsumenten.

Insgesamt konnten für niederländische Konsumenten sieben der neun Hypothesen bestätigt werden, für deutsche Konsumenten waren es acht Hypothesen. Entscheidend zum Aufbau von Bindung ist für beide Konsumentengruppen die Gesamtzufriedenheit mit dem Stammgeschäft im Nachbarland und die Erfüllung der wahrgenommenen Selbstrelevanz durch die entsprechende Einkaufsstätte. Leistungen des Stammgeschäftes im Nachbarland werden von beiden Konsumentengruppen mit einem bestimmten Wert verknüpft und werden bindungsentscheidend.

\subsection{Zusammenfassung}

Ausgangspunkt der Untersuchung bildeten die Defizite der Kundenbindungsforschung in einem Grenzgebiet. Zum einen verzichtete die Kundenbindungsforschung bislang auf die explizite Analyse des Zusammenhangs zwischen dem bekundeten Treueverhalten von Konsumenten in einem Grenzgebiet zweier benachbarter Länder und deren Kundenbindung als overtes Treueverhalten. Andererseits gelingt es mit den vorhandenen Kundenbindungsmodellen nicht, das Phänomen der grenzüberschreitenden Konsumentenbindung zu erklären.

An diesem Punkt setzt die Arbeit an. Dabei orientierten sich die Forschungsaktivitäten am theorie-monistischen Ansatz. Es galt eine Theorie zu finden, die offensichtlich eine Erklärung und Prognose der Kundenbindung leistet. Die neuere Einstellungstheorie genügt diesem Anspruch. Dabei wurde die Theorie des geplanten Verhaltens von Ajzen als Grundgerüst ge- 
nommen. Als weitere entscheidende Faktoren der Kundenbindung ließ sich die wahrgenommene Selbstrelevanz identifizieren. Es ergab sich ein nichtlineares Kundenbindungsmodell, das im niederländisch-deutschen Grenzgebiet überprüft wurde. Zur Modellschätzung leistete der Partial-Least Squares Ansatz (PLS) wertvolle Dienste. Die Evaluation der Schätzergebnisse des bestangepaßten linearen Kundenbindungsmodell zeigt, dass die theoretisch identifizierten Determinanten das Zielphänomen im Grenzgebiet erklären. Als entscheidende Bestimmungsfaktoren der Kundenbindung zeigten sich die Gesamtzufriedenheit mit dem Einkauf im Nachbarland, die Gesamtzufriedenheit mit dem Kauf im Stammgeschäft, die wahrgenommene Selbstrelevanz und die Kundenloyalität. Für deutsche Konsumenten zeigte sich die hohe Bedeutung der Werturteile der sozialen Gruppe und die Bedeutung der sozialen Norm für den Konsumenten. 


\section{Kapitel 9: Zusammenfassung}

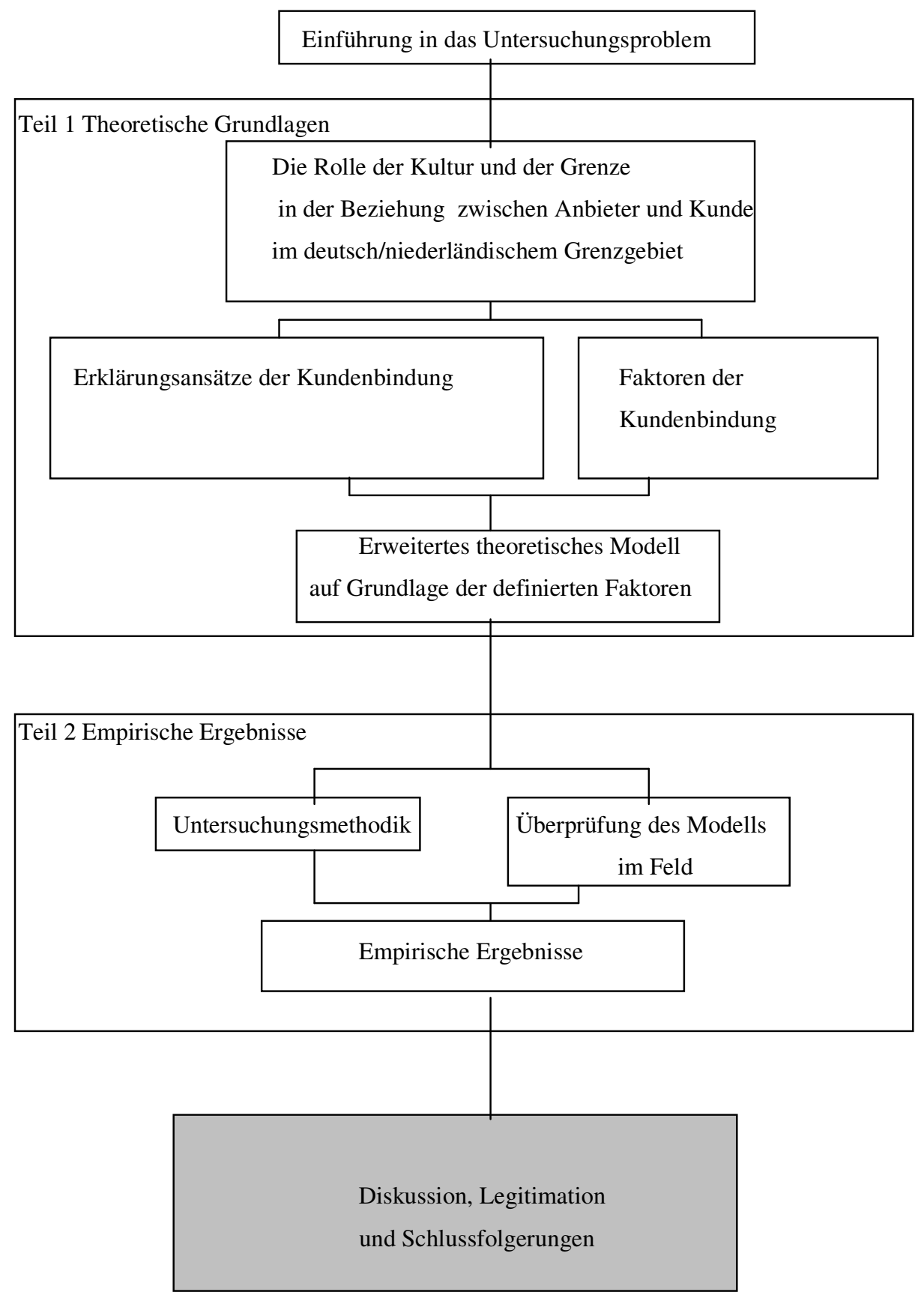




\subsection{Einleitung}

Im Rahmen der vorliegenden Untersuchung standen die Defizite der Kundenbindungsforschung in einem grenzüberschreitendem Raum im Mittelpunkt. Ferner sollte der Zusammenhang zwischen Kundenzufriedenheit-Kundenloyalität-Kundenbindung in einem kleinen geografischem Raum untersucht werden. Mit den in der Literatur vorliegenden Kundenbindungsmodellen gelingt es noch nicht Konsumentenbindung an eine Einkaufsstätte auch grenzüberschreitend zu erklären. Ansatz der vorliegenden Arbeit war es eine Erklärungstheorie zu finden, die in der Lage ist, eine Prognose zur Kundenbindung zu erstellen. Ausgangspunkt der vorliegenden Arbeit waren folgende Fragen:

- Ergeben sich für deutsche und niederländische Konsumenten unterschiedliche Kundenbindungsfaktoren?

- Welche Loyalitätseffekte entwickeln sich in Grenzregionen?

- Existieren grenzüberschreitend andere Kundenbindungsfaktoren als die, die bereits erforscht wurden?

- Gibt es ein gemeinsames Kundenbindungsmodell für deutsche und niederländische Konsumenten?

- Bestätigt sich dieses neue Modell in der Praxis?

Ferner sollte in der vorliegenden Arbeit die Bedeutung folgender Größen untersucht werden:

- Soziale Norm vor kulturellem Hintergrund.

- Einstellung gegenüber Handlung.

- Wahrgenommene Selbstrelevanz.

Im folgenden Kapitel wird auf die Ergebnisse der Untersuchung hinsichtlich dieser Punkte aus Kapitel 7 und 8 noch einmal zurückgegriffen und eine Antwort auf die in diesem Kapitel gestellten Fragen gegeben..

\subsection{Diskussion, Legitimation und Schlussfolgerungen}

Die vorhandene Literatur, insbesondere die Arbeiten von Odekerken-Schröder, Braunstein, Peter und Eggert haben die Grundlagen geschaffen, um die Motive des Konsumenten zur Kundenbindung darzulegen. Dabei hat Odekerken-Schröder einen Erklärungsansatz zur Kundenbindung im Rahmen eines Vergleichs von mehreren Ländern geliefert, Braunstein die Theorie des geplanten Verhaltens in die moderne Kundenbindungsforschung integriert. Die 
vorliegende Arbeit zeigt die Wirkungen der Kundenbindung in zwei benachbarten Ländern auf. Ferner liefert sie einen Erklärungsansatz, dass die Einstellung zum Einkauf im Nachbarland nicht nur abhängig ist von der Zufriedenheit mit dem Einkauf selbst, sondern auch vom sozialen Umfeld des Konsumenten. Die Kulturdimension des Konsumenten, seine Erziehung und seine Werte nehmen Einfluss auf seine Handlung. Obwohl die Handelsgrenzen im heutigen Europa nicht mehr existent sind, zeigt die Zugehörigkeit des Konsumenten zu einer bestimmten Kultur dennoch Handlungsgrenzen für ihn auf. Das Faktum der Bedeutung der sozialen Norm und der Kulturdimension ist durch diese Arbeit im grenznahem Raum zweier unterschiedlich großer Länder geschlossen worden. Auch im Grenzgebiet zwischen den Niederlanden und Deutschland haben sich beide Bevölkerungsgruppen ihre Eigenständigkeit bewahrt. Einkauf ist nicht nur abhängig von persönlichen Vorteilen, sondern auch von Einstellung der Konsumenten selbst und ihrer sozialen Gruppe. Der Einfluss der sozialen Gruppe ist wiederum abhängig von dem Einfluss der sozialen Norm und der Einfluss der sozialen Norm ist wiederum abhängig von der Kulturdimension aus der das Individuum entstammt. Diese Unterschiede sind auch bei sehr eng zusammenlebenden Konsumenten aus unterschiedlichen Ländern wie in einem Grenzgebiet erkennbar.

Um die Frage der unterschiedlichen oder gemeinsamen Faktoren der grenzüberschreitenden Kundenbindung zu beantworten, wurden verschiedene Analysemöglichkeiten angewendet. Zunächst wurde im Rahmen der Faktorenanalyse eine Hauptachsenanalyse durchgeführt. Das Ergebnis ist eine Gegenüberstellung der unterschiedlichen Faktoren und ein Hinweis darauf, das deutsche und niederländische Konsumenten sich hinsichtlich der Bildung von Kundenbindung im Grenzgebiet voneinander unterscheiden. So ist für niederländische Konsumenten ihre Einstellung zum Nachbarland eine die Kundenbindung beeinflussende Größe, Loyalität als Faktor dagegen nicht zu identifizieren. Im Gegensatz dazu ist für deutsche Konsumenten der Faktor Gesamtzufriedenheit mit dem Einkauf eine die Kundenbindung beeinflussende Größe, ferner ist auch der Faktor Loyalität zu identifizieren. Loyalität entwickelt sich bei niederländischen Konsumenten nicht durch die in der Faktoranalyse identifizierten Faktoren. Loyalität entsteht bei niederländischen aus anderen Faktoren. Aus diesem Grund reicht eine Faktorenanalyse alleine nicht aus um ein gemeinsames Kundenbindungsmodell für eine Grenzregion zu erstellen. 


\begin{tabular}{|c|c|c|c|c|c|c|c|c|c|}
\hline Modell & Faktor 1 & Faktor 2 & Faktor 3 & Faktor 4 & Faktor 5 & Faktor 6 & Faktor 7 & Faktor 8 & Faktor 9 \\
\hline NL & $\begin{array}{l}\text { Soziale } \\
\text { Norm NL }\end{array}$ & $\begin{array}{l}\text { Wichtigkeit } \\
\text { der sozialen } \\
\text { Norm NL }\end{array}$ & \begin{tabular}{|lr} 
Einstellung & zum \\
Einkauf & im \\
Nachbarland & NL
\end{tabular} & $\begin{array}{l}\text { Gesamtzu- } \\
\text { friedenheit } \\
\text { mit Ein- } \\
\text { kaufsstätte } \\
\text { NL }\end{array}$ & & $\begin{array}{l}\text { Einstel- } \\
\text { lung zum } \\
\text { Nachbar- } \\
\text { land NL }\end{array}$ & $\begin{array}{l}\text { Persönli- } \\
\text { che Norm } \\
\text { NL }\end{array}$ & & $\begin{array}{l}\text { Echte Kun- } \\
\text { denbindung } \\
\text { NL }\end{array}$ \\
\hline $\mathrm{D}$ & $\begin{array}{l}\text { Soziale } \\
\text { Norm D }\end{array}$ & $\begin{array}{l}\text { Wichtigkeit } \\
\text { der sozialen } \\
\text { Norm D }\end{array}$ & $\begin{array}{ll}\text { Einstellung } & \text { zum } \\
\text { Einkauf } & \text { im } \\
\text { Nachbarland D }\end{array}$ & $\begin{array}{l}\text { Gesamtzu- } \\
\text { friedenheit } \\
\text { mit Ein- } \\
\text { kaufsstätte } \\
\text { D }\end{array}$ & $\begin{array}{l}\text { Gesamtzu- } \\
\text { friedenheit } \\
\text { mit Einkauf } \\
\text { im Nachbar- } \\
\text { land D }\end{array}$ & & $\begin{array}{l}\text { Persönli- } \\
\text { che Norm } \\
\text { D }\end{array}$ & $\begin{array}{l}\text { Loyalität } \\
\text { zur Ein- } \\
\text { kaufsstätte } \\
\text { D }\end{array}$ & $\begin{array}{l}\text { Echte Kun- } \\
\text { denbindung } \\
\text { D }\end{array}$ \\
\hline
\end{tabular}

Diese Unterschiede zwischen niederländischen und deutschen Konsumenten werden auch durch die Ergebnisse der Clusteranalyse bestätigt. Auch hier ergeben sich Unterschiede zwischen den niederländischen und deutschen Konsumenten. Die Gegenüberstellung und der Vergleich der Cluster macht dies deutlich:

\begin{tabular}{|l|l|l|l|l|l|}
\hline \multicolumn{2}{|l|}{ Niederlande } & \multicolumn{2}{l|}{ Deutschland } \\
\hline $\mathrm{n}$ & Name & Anteil in \% & $\mathrm{n}$ & Name & Anteil in \% \\
\hline 36 & Die Minimalkunden & 14,7 & 53 & Die Minimalkunden & 21,0 \\
\hline $\begin{array}{l}11 \\
8\end{array}$ & Die Gelegenheitskäufer & 48,2 & 56 & Die Gelegenheitskäufer & 37,2 \\
\hline 50 & $\begin{array}{l}\text { Die Begeisterten für Stammge- } \\
\text { schäfte }\end{array}$ & 20,4 & 94 & Die begeisterten Fachgeschäftskunden & 19,8 \\
\hline $\begin{array}{l}\text { Die schnellen zielgerichteten, } \\
\text { preisorientierten Käufer }\end{array}$ & 16,7 & 50 & $\begin{array}{l}\text { Die preisbewussten Kunden von Fili- } \\
\text { albetrieben }\end{array}$ & 22,1 \\
\hline
\end{tabular}

Mit Hilfe der Software SmartPLS wurde dann ein gemeinsames Kundenbindungsmodell für niederländische und deutsche Konsumenten entwickelt. Die Ergebnisse zeigen deutlich, dass allein der Pfad der wahrgenommenen Verhaltenskontrolle im Grenzgebiet für beide Konsumentengruppen nicht nachgewiesen werden kann, die Wichtigkeit der sozialen Norm auf die Bedeutung der sozialen Norm kann allein für niederländische Konsumenten nicht nachgewiesen werden. Die einzelnen Pfadkoeffizienten zeigen unterschiedliche Effektstärken auf.

\begin{tabular}{|l|l|}
\hline Frage: & Antwort: \\
\hline $\begin{array}{l}\text { Ergeben sich für deutsche und niederländische Kon- } \\
\text { sumenten unterschiedliche Kundenbindungsfaktoren? }\end{array}$ & Ja \\
\hline
\end{tabular}

Die Ergebnisse aus Kapitel 7.4 zeigen auf, dass es unterschiedliche Loyalitätseffekte bei niederländischen und deutschen Konsumenten gibt. Die stärksten Unterschiede ergeben sich durch die wahrgenommene Selbstrelevanz und durch die persönliche Norm bei den niederländischen Konsumenten und die Gesamtzufriedenheit mit dem Einkauf im Nachbarland, 
der persönlichen Norm sowie der Gesamtzufriedenheit mit dem Stammgeschäft im Nachbarland bei den deutschen Konsumenten.

\begin{tabular}{|l|l|}
\hline \multirow{2}{*}{ Effektgröße NL } & \multicolumn{1}{c|}{ Pfadkoeffizient $>0,2$} \\
\hline$>0,05$ & $\begin{array}{l}\text { Wahrgenommene Selbstrelevanz } \\
\text { Persönliche Norm }\end{array}$ \\
\hline Effektgröße D & $\begin{array}{l}\text { Gesamtzufriedenheit mit dem Einkauf im Nachbarland } \\
\text { Persönliche Norm } \\
\text { Gesamtzufriedenheit mit Stammgeschäft im Nachbarland. }\end{array}$ \\
\hline$>0,05$ &
\end{tabular}

Loyalität bildet sich bei niederländischen Konsumenten somit durch andere Effekte als bei deutschen Konsumenten.

\begin{tabular}{|l|l|}
\hline Frage: & Antwort: \\
\hline $\begin{array}{l}\text { Welche Loyalitätseffekte entwickeln sich in Grenzre- } \\
\text { gionen? }\end{array}$ & $\begin{array}{l}\text { Die sich ergebenen Loyalitätseffekte sind abhängig } \\
\text { von der Konsumentengruppe. }\end{array}$ \\
\hline
\end{tabular}

Die Ergebnisse der Analyse der Kundenbindungsfaktoren in Kapitel 7 und 8 der Untersuchung konnten keinen Nachweis für die Existenz der wahrgenommenen Verhaltenskontrolle aufzeigen. Es existieren in einem Grenzgebiet somit keine anderen Kundenbindungsfaktoren als die bereits aus der Literatur bekannten, doch die Intensität der Wirkung der einzelnen Kundenbindungsfaktoren ist im Grenzgebiet unterschiedlich. Dies gilt im besonderen Maße für die persönliche Norm und die wahrgenommene Selbstrelevanz.

\begin{tabular}{|l|l|}
\hline Frage: & Antwort: \\
\hline $\begin{array}{l}\text { Existieren grenzüberschreitend andere Kundenbin- } \\
\text { dungsfaktoren als die, die bereits erforscht wurden? }\end{array}$ & Nein \\
\hline
\end{tabular}

In Kapitel 7.4 ist ein gemeinsames Kundenbindungsmodell für deutsche und niederländische Konsumenten entwickelt worden. Die Hypothesenüberprüfung erbrachte eine höchstmögliche Übereinstimmung der beiden Konsumentengruppen. Es ist somit ein gemeinsames Kundenbindungsmodell für beide Kundengruppen existent.

\begin{tabular}{|l|l|}
\hline Frage: & Antwort: \\
\hline $\begin{array}{l}\text { Gibt es ein gemeinsames Kundenbindungsmodell für } \\
\text { deutsche und niederländische Konsumenten? }\end{array}$ & Ja. \\
\hline
\end{tabular}


Die Überprüfung des Modells in der Praxis zeigte deutlich, dass das entwickelte Modell auch in der Praxis Bestand hat und als nachgewiesen angesehen werden kann. Somit ergibt sich für die letzte Frage:

\begin{tabular}{|l|l|}
\hline Frage: & Antwort: \\
\hline Bestätigt sich dieses neue Modell in der Praxis? & Ja. \\
\hline
\end{tabular}

Die Untersuchung der Größen soziale Norm vor kulturellem Hintergrund, Einstellung gegenüber Handlung und wahrgenommene Selbstrelevanz zeigen in der vorliegenden Untersuchung ein deutliches Bild über ihre Bedeutung zur Kundenbindung in einem Grenzgebiet. Sowohl für deutsche als auch niederländische Konsumenten hat die soziale Norm eine besondere Bedeutung für ihre Kaufentscheidungen. Allerdings ist die Wichtigkeit der sozialen Norm und die Reaktion der Umwelt auf Kaufentscheidungen für niederländische Konsumenten kein entscheidendes Kriterium um Kundenbindung zu einem konkreten Anbieter im Nachbarland zu entwickeln. Für niederländische Konsumenten ist die persönliche Norm der entscheidendere Faktor. Allerdings ist dies auch wiederum vor dem kulturellem Hintergrund zu sehen. Die Einstellung gegenüber der Handlung, dass heißt der Einfluss der sozialen Gruppe auf die Kaufentscheidung und die Einstellung zum Einkauf im Nachbarland selbst ist Ausdruck der Kultur eines Landes. Hier sind als Ergebnis Unterschiede zwischen Niederländern und Deutschen festzustellen - selbst in einer Grenzregion. Aus Hofstedes Ausführungen wäre ein aufgeschlossener niederländischer Konsument zu erwarten gewesen, der auch stark ins Ausland orientiert ist. Dies kann im untersuchten Grenzgebiet nicht bestätigt werden. Die wahrgenommene Selbstrelevanz trägt dazu bei, dass Kundenloyalität sich entwickelt und schließlich Kundenbindung entsteht. Die wahrgenommene Selbstrelevanz hat für beide Kundengruppen im Untersuchungsgebiet gleichermaßen Bedeutung. Allerdings hat die wahrgenommene Selbstrelevanz auf die Kundenloyalität der niederländischen Konsumenten eine stärkere Bedeutung als für deutsche Konsumenten. Die persönliche Norm hat für niederländische Konsumenten eine rationale Komponente, die die persönliche Norm prägt. Die analysierten Unterschiede der Einflüsse der sozialen Gruppen und der sozialen Norm auf das Kaufverhalten der niederländischen und deutschen Konsumenten sind deutlich auf unterschiedliche Positionierung der von Hofstede definierten Kulturdimensionen Individualismus und Kollektivismus zurückzuführen. Zwar sind sich niederländische und deutsche Konsumenten in vielen Verhaltensweisen ähnlich (dies ist auch auf die enge familiäre Bindung der Konsumentengruppen zurückzuführen), dennoch zeigen die niederländischen Konsumenten auch in der Grenzregion einen deutlicheren Hang zum Individualismus als deutsche Konsumenten. Dies erklärt auch, warum die soziale Gruppe auf die Kaufentscheidungen deutscher Konsumenten einen höheren 
Einfluss hat, als auf die Kaufentscheidungen niederländischer Konsumenten. Dennoch zeigte die Untersuchung, dass auch niederländische Konsumenten nicht frei von den Werturteilen der sozialen Gruppe sind. Der Unterschied zwischen beiden Gruppen liegt in der Bewertung der Wichtigkeit der sozialen Norm. Die Wichtigkeit der sozialen Norm ist bei deutschen Konsumenten erheblich höher als bei niederländischen Konsumenten und hat bei deutschen Konsumenten auch Auswirkungen auf die Gesamtzufriedenheit mit dem Stammgeschäft im Ausland. Die Wertunterschiede im Zusammenhang mit der Individualismus-KollektivismusDimension werden deshalb auch zwischen niederländischen und deutschen Konsumenten weiterhin bestehen, obwohl die Lebensumstände sich sehr angeglichen haben. Hofstede beschreibt diesen Trend als eine spürbare Annäherung der europäischen Länder und eine gemeinsame Verschiebung der Kulturen, so dass die Unterschiede zwischen ihnen bestehen bleiben (Hofstede, 2001, S. 108).

Für ausgewählte Einzelhandelsbrachen in der Grenzregion haben diese Erkenntnisse auch eine praktische Bedeutung um die Kundenzufriedenheit der ausländischen Kunden zu steigern, ihre Kundenloyalität $\mathrm{zu}$ fördern und Kundenbindung $\mathrm{zu}$ realisieren. Relevante Determinanten der Kundenzufriedenheit in diesen Einzelhandelsbranchen können unterteilt werden in gemeinsame Determinanten, Determinanten für Discounter, Determinanten für Textil- und Schuhanbieter und Determinanten für Elektrogeschäfte.

Gemeinsame Determinanten der Kundenzufriedenheit für alle ausgewählten Einzelhandelsbranchen sind insbesondere die in der Arbeit untersuchten Konstrukte der Teilzufriedenheiten mit der sozialen Norm sowie die Wichtigkeit der sozialen Norm. Diese Determinanten drücken für deutsche Konsumenten die Gesamtzufriedenheit mit dem Einkauf im benachbartem Ausland aus. Dazu kommt für deutsche Konsumenten die wahrgenommene Selbstrelevanz, die entscheidend durch die persönliche Norm der Konsumenten geprägt wird. Alle diese Determinanten haben Einfluss auf Zufriedenheit und bestimmen die Gesamtzufriedenheit mit dem Einkauf der deutschen Konsumenten im benachbarten Ausland. Bei den niederländischen Konsumenten wird die Gesamtzufriedenheit mit dem Einkauf im benachbartem Ausland insbesondere durch die persönliche Norm geprägt. Konkret bedeutet dies für die einzelnen Einkaufsstätten und Betriebstypen, dass die Konsumenten insbesondere von Discountern ein klares Profil erwarten, neben der Grundforderung nach einem sauberen und gepflegten Geschäft sowie gute Parkmöglichkeiten. Der Preis und das Preis-Leistungsverhältnis ist für diesen Betriebstyp sowohl für niederländische als auch für deutsche Konsumenten der wesentliche Zufriedenheitstreiber. Die Preisgestaltung und die Preisauszeichnung soll 
deutlich und transparent sein, ein eventuell notwendiger Umtausch unkompliziert und nach Möglichkeit grenzüberschreitend möglich sein.

Determinanten die die Kundenzufriedenheit in Textil- und Schuheinkaufsstätten fördern, sind sowohl für niederländische als auch deutsche Konsumenten eine gute Einkaufsatmosphäre in den Geschäften mit einem guten und dem Sortimente entsprechendem Ladenund Preisaufbau. Dies, gepaart mit einem der Zielgruppe entsprechendem Sortiment, definiert für den Konsumenten die Qualität einer Einkaufsstätte. Gestärkt und unterstützt wird dieser positive Eindruck durch das Umfeld der Einkaufsstätte. Hier sind es insbesondere die Atmosphäre des Einkaufsortes mit entsprechenden Freizeitmöglichkeiten und gastronomischen Angeboten. Insbesondere diese Einkaufsstätten benötigen zur Steigerung der Zufriedenheit ihrer Kunden eine gute Anbindung des Ortes ans Straßennetz ins Nachbarland, sowie gute innerörtliche Parkmöglichkeiten. Zufriedenheitstreibend sind hierbei auch eine gute Anbindung des öffentlichen Personennahverkehrs an den Einkaufsort. Insbesondre für den Textilund Schuhhandel ist zu differenzieren zwischen Determinanten, die innerhalb der eigenen Einkaufsstätte zu beeinflussen sind und Determinanten, die nur durch ein gemeinsames verbessertes Stadtmarketing veränderbar sind. Insbesondere für diese Branchen müssen diese Determinanten gemeinsam verbessert werden, soll es zu einer Verbesserung der Kundenzufriedenheit kommen. Dazu gehören die Qualität der Geschäfte hinsichtlich Erscheinungsbild, Service und Sortiment in der Einkaufsstadt. Ferner gehören dazu die Anzahl der Geschäfte, die für den Konsumenten ein Indiz der Angebotsvielfalt darstellt, sowie ein breites Sortimentsangebot innerhalb einer Stadt, um alle Wünsche hinsichtlich Mode und Preis zu erfüllen.

Bei den Elektrogeschäften sind die relevanten Determinanten zur Steigerung der Kundenzufriedenheit eine Kombination der identifizierten Determinanten für Discounter und Fachgeschäfte. Der Trend geht in dieser Branche auch beim grenzüberschreitenden Einkauf stark zu den großflächigen Anbietern, die nach Möglichkeit in beiden Ländern ansässig und bekannt sind. Elektrofachgeschäfte spielen beim grenzüberschreitenden Einkauf nur eine untergeordnete Rolle. Aus diesem Grund sind die Erfolgsdeterminanten zur Steigerung der Kundenzufriedenheit auch die Nutzung der unterschiedlichen Marktgegebenheiten (unterschiedlicher Preis für gleichen Artikel) bei entsprechendem Parkangebot, großes Sortiment, guter Service, Umtauschmöglichkeit im eigenen Land sofern Anbieter dort auch vertreten ist.

Für die Filialunternehmen sind im Rahmen der Untersuchung insbesondere als Determinanten zum Aufbau der Kundenzufriedenheit das einheitliche Sortiment, einheitlicher Ladenauf- 
bau und einheitliche Qualitätsstandards im Bereich Service identifiziert worden. Zufriedenheitstreiber sind insbesondere in Filialgeschäften der vom Kunden erwartete unkomplizierte Umtausch von Waren, die er im Nachbarland gekauft hat. Dadurch wird für die Konsumenten das Rechtsrisiko des Einkaufs reduziert. Gleichartige Garantieleistungen in allen Ländern, in denen die Filialisten vertreten sind, fördern ebenfalls die Kundenzufriedenheit und reduzieren die vorhandenen Einkaufsbarrieren.

Eine Anzahl von Einschränkungen ist allerdings für die Ergebnisse der vorliegende Arbeit einzuräumen. Erstens ist die vorliegende Untersuchung nicht in der Lage kausale Zusammenhänge zwischen den einzelnen der Untersuchung zugrunde liegenden Branchen aufzuzeigen. Eine weitere Einschränkung ist der Verzicht auf möglicherweise wichtige Variablen in der Untersuchung selbst. Dies musste allerdings in dieser Untersuchung aus Gründen der Vereinfachung der Analyse gemacht werden, um die Übersichtlichkeit zu wahren. Ferner sind in der vorliegenden Untersuchung mögliche Einflüsse durch die Interviewer nicht auszuschließen. Eine weitere mögliche Einschränkung ist, dass die Untersuchung nicht dem Merkmal der Normalverteilung unterliegt. Trotz der wesentlichen Ergebnisse dieser Arbeit zur Erklärung der Kundenbindung an eine Einkaufsstätte im Nachbarland bleibt noch erheblicher Diskussionsbedarf zur Beantwortung der Frage zur grenzüberschreitenden Kundenbindung in einem kleinen geografischem Gebiet. Durch die durchgeführte Untersuchung im deutschniederländischem Grenzgebiet konnte ein Kundenbindungsmodell erstellt werden, das aufzeigt, dass deutsche und niederländische Konsumenten, auch wenn sie geografisch nicht weit voneinander getrennt sind, aufgrund von Kulturunterschieden ein unterschiedliches Kauf- und Bindungsverhalten haben. Die reale Grenze spielt somit eine geringere Rolle als die Grenze des Denkens.

Die Ergebnisse der Arbeit zeigen deutlich, dass die Erkenntnisse der Einstellungs- und Verhaltensforschung allgemein einen hohen Beitrag zur Erklärung der Kundenbindung leisten. Die Teilzufriedenheit der Konsumenten mit der Reaktion des sozialen Umfeldes bilden bedeutsame Stellgrößen der Kundenbindung. Eine Vernachlässigung dieser Determinanten ist aufgrund der vorliegenden Ergebnisse ein wesentlicher Grund für die unbefriedigende Erklärungsgüte vieler Kundenbindungsmodelle insbesondere in einem Grenzgebiet. Zukünftig ist deshalb in dieser Art von Studien die soziale Norm in ihren unterschiedlichen Ausprägungen stärker zu berücksichtigen. Kulturelle Unterschiede, unterschiedliche Einstellungen zum grenzüberschreitenden Einkauf, die persönliche Norm und die wahrgenommene Selbstrelevanz als latente Größen spielen eine zentrale Rolle bei der Erklärung der Kundenbindung. 
Das erstellte Kundenbindungsmodell zeigt akzeptable bis sehr gute statistische Werte auf und ist valide. Das Ergebnis ist ein nichtlineares Kundenbindungsmodell, dessen Grundsätze auf den Erwartungs-Wert-Ansatz von Fishbein und Ajzen (Theorie des geplanten Verhaltens) beruht und in einem grenzüberschreitenden Wirtschaftsraum empirisch überprüft wurde. Die Überprüfung erfolgte mit Hilfe der Kausalanalyse und insbesondere mit dem PLS Modell. Die Überprüfung der Schätzergebnisse zeigt als Ergebnis ein gut angepasstes Kundenbindungsmodell, das die theoretisch identifizierten Determinanten im Grenzgebiet zwischen Deutschland und den Niederlanden gut erklärt. Als entscheidende Bestimmungsfaktoren der grenzüberschreitenden Abnehmerbindung zeigten sich die Kundenloyalität, die wahrgenommene Selbstrelevanz - beeinflusst durch die persönliche Norm, die Gesamtzufriedenheit mit dem grenzüberschreitendem Einkauf sowie die Teilzufriedenheiten mit der sozialen Norm. Allerdings konnten auf Grund der relativ kleinen Erhebung nicht alle Pfade als signifikant nachgewiesen werden. Es konnte aber nachweislich der Beweis erbracht werden, dass die wahrgenommene Selbstrelevanz eine hohe Wichtigkeit für das Entstehen von Loyalität und Bindung hat.

\subsection{Fazit}

Einheit und Vielfalt macht das typisch Relevante des deutsch-niederländischen Grenzgebietes aus. In dem ländlich geprägtem Grenzgebiet hält die Bevölkerung an ihrer (ähnlichen) heimischen Sprache, ihrem Lebensstil und an Kontakten auf beiden Seiten der Grenze fest. Dennoch darf nicht übersehen werden, dass eine Grenze infolge von Nationalisierung zu Unterschieden in der Mentalität führt. Niederländische und deutsche Konsumenten sind sich insbesondere in der Grenzregion sehr ähnlich, sehen aber insbesondere den grenzüberschreitenden Einkauf aus zwei unterschiedlichen Perspektiven. Diese Aussage wird durch die vorliegende Arbeit bestätigt. Somit ist ein kleiner Beitrag geleistet worden, um die unterschiedlichen Bindungsverhalten zu erklären. Durch die nun vorliegenden Modellansätze wird nachgewiesen, dass auch der grenzüberschreitende Konsument kein „homo oeconomicus“ ist, sondern teils spontan, teils habitualisiert, teils sich nach routinemäßigen Regeln verhält. Hierbei entstehen häufig Inkonsistenzen. Die erstellten Modelle sollen einen Beitrag dazu leisten diese Inkonsistenzen zu verstehen.

In theoretischer Hinsicht leistet diese Arbeit einen Beitrag zur theoretischen Fundierung des Konzepts der Erklärung und Prognose der Kundenbindung in einem Grenzgebiet zweier un- 
terschiedlich großer Länder. Damit sich die Theorie des geplanten Verhaltens zur Erklärung des Phänomens Kundenbindung eignet, bedurfte es einiger Anpassungsmaßnahmen des Modells. Betroffen waren hier die Einstellung vor dem kulturellen Hintergrund des Konsumenten sowie die soziale Norm und deren Erklärungsmodelle. Die Kombination aus theoretischen, konzeptionellen und empirischen Vorgehen ist in den meisten bisherigen Untersuchungen unterblieben. Allerdings hat sich insbesondere diese Kombination zur Erklärung der Kundenbindung in einem Grenzgebiet als zweckmäßig erwiesen.

Einen Forschungsbeitrag leistete diese Arbeit auch in methodischer Hinsicht. Im Bereich der Kundenbindungsforschung wurde in den bisherigen Studien fast ausnahmslos auf einfache regressionsanalytische Verfahren oder kovarianzbasierte Ansätze wie dem LISREL-Ansatz zurückgegriffen. Allerdings werden bei der Anwendung dieses Verfahrens nur sehr starke Zusammenhänge von latenten Faktoren sichtbar und es ist eine relativ große Anzahl von Stichproben notwendig. Aus diesem Grund wurde zur Analyse der Modelle das in der empirischen betriebswirtschaftlichen Forschung bisher wenig angewendete Analyseverfahren des Partial Least Square (PLS) verwendet. Dieses Analyseverfahren eignet sich insbesondere für kleine Stichproben und ermöglichst eine Modellierung von Messmodelle, die manifeste und latente Variablen miteinander verbinden. Mit Hilfe des Partial Least Square Verfahrens konnten auf Indikatorebene relevante Treiber in den jeweiligen Phasen „Bedeutung der sozialen Norm für den Konsumenten“, „Gesamtzufriedenheit mit dem Einkauf im Nachbarland“, „Wahrgenommene Selbstrelevanz“, „Kundenloyalität“ und „Kundenbindung“ jeweils für niederländische und deutsche Konsumenten identifiziert werden. Hiermit ist es möglich, neben der Betrachtung der Wirkungszusammenhänge auf Konstruktebene auch konkrete Gestaltungs- und Handlungsempfehlungen hinsichtlich einzelner Maßnahmen in den einzelnen Phasen zu geben. Die Empfehlungen an das Management der Einkaufsstätten, aber auch des Citymamagement können dabei genauer und detaillierter formuliert werden als es bisher möglich war.

Trotz der wesentlichen Ergebnisse dieser Arbeit zur Erklärung der Kundenbindung an eine Einkaufsstätte im Nachbarland, bleibt noch erheblicher Diskussionsbedarf zur Beantwortung der Frage zur grenzüberschreitenden Kundenbindung in einem kleinen geografischem Gebiet. Die persönliche Norm, die wahrgenommene Selbstrelevanz und die wahrgenommene Verhaltenskontrolle sollten in weiteren Untersuchungen des euregionalen Einkaufgebietes weiter untersucht werden, da sie in dieser Untersuchung sehr geringe Werte aufweisen. Ratsam wäre als nächster Schritt eine Langzeitstudie in der definierten Grenzregion mit einem erheblich 
höherem Datenvolumen. Eine Langzeitstudie gepaart mit einem höheren Datenvolumen wird dazu beitragen, eine Bestätigung der identifizierten Pfade statistisch zu belegen. Weitere Forschungsaktivitäten sollten die Validierung des Kundenbindungsmodells zentral stellen. Auch eine Überprüfung des Ansatzes mit Dienstleistungsangeboten steht noch aus. Eine weitere Ausdehnung der ohnehin schon sehr komplexen Untersuchung hätte den Rahmen dieser Arbeit allerdings gesprengt. Aus den nun identifizierten Konstrukten lassen sich eine Reihe von marketingpolitische Aktivitäten ableiten. Inwieweit diese Faktoren tatsächlich zur Steigerung der Kundenbindung beitragen, wird die Marketingpraxis zeigen. 


\section{Literaturverzeichnis:}

\section{$\underline{\mathbf{A}}$}

Ach, N., Über den Willensakt und das Temperament, Leipzig 1910

Adams, J.S., Inequity in Social Exchange, in : Advances in Experimental Social Psychology, 2,1965, S. 267-299

Ajzen, I., Attitudes, traits, and actions: Dispositional prediction of behavior in personality and social psychology, in: Advances in Experimental Social Psycholology, Vol. 20, S. 1-63, 1987 Ajzen, I., Fishbein, M., Understanding attidudes and predicting social behavior, New York 1980

Ajzen, I., Madden, T.J., Prediction of goal-directed behavior: Attitudes, intentions and perceived behavioral control, in: Journal of Experimental Social Pychology, 22, 1986, S. 453-474 Ajzen, I.: The theory of planned behavior, in: Organizational Behavior and Human Decision Prozesses, 50, 1991

Allport, G.D., The nature of prejudice, Reading, 1954

Anderson, E., Weitz, B.A.: Derminant of continuity in conventional industrial channel dyads, in: Marketing Science, 8,, 1989, S. 310-323 1989

Anderson, E.W., Sullivan, M., The antecedents and consequences of customer satisfaction for firms, in: Marketing Science, 12, 1993, S.125-143

Anderson, E.W., Sullivan, M.: The antecedents and consequences of customer satisfaction for firms, in: Marketing Science, 12, 1993, S. 125-143

Anderson, Eugene W., Cross-Category Variation in Customer Satisfaction and Retention, Marketing Letters, 5 (Winter), 1994, S. 19-30

Anderson, Eugene W., Sullivan Mary W.: The Antecedents and Consequences of Customer Satisfaction for Firms, Marketing Science, 12 (Spring) 1993, S. 125-143

Arndt, J. Toward a Concept of Domesticated Markets, in Journal of Marketing, Vol. 43, Oct. 1979, S. $69-75$

Atteslander, P.: Methoden der empirischen Sozialforschung, Berlin, New York, 1993, S. 169f.

Auh, S., Johnson, M.: The Complex Relationship between Customer Satisfaction and Loyalty for Automobiles. Arbeitspapier, University of Michigan Business School, Ann Arbor, 1997

Auh, S., Johnson, M.D.: The complex relationship netween customer satisfaction and loyalty for automobiles, in: Johnson, M.D., Herrmann, A., Huber, F., Gustafsson, A. (Hrsg.): Customer retention in the automotive industry, Wiesbaden, 1997, S. 141-166

Ausschuss für Begriffsdefinitionen aus der Handels- und Absatzwirtschaft (Hrsg.): Katalog E. 
Begriffsdefinitionen aus der Handels- und Absatzwirtschaft, 4. Ausgabe, Köln, 1995 Ausschuss für Begriffsdefinitionen aus der Handels- und Absatzwirtschaft (Hrsg.): Katalog E. Begriffsdefinitionen aus der Handels- und Absatzwirtschaft, 2. Ausgabe, Köln, 1975

\section{$\underline{\mathbf{B}}$}

Backhaus, K., Erichson, B., Plinke, W., Weiber, R.: Multivariate Analysemethoden, eine anwendungsorientierte Einführung 10. Aufl., Berlin, Heidelberg 2003

Backhaus, K., Erichson, B., Plinke, W., Weiber, R., Multivariate Analysemethoden: eine anwendungsorientierte Einführung, Berlin, Heidelberg, 8.Aufl. 1996

Backhaus, K., Industriegütermarketing, 7. Auflage, München 2003

Bagozzi, R.P., Baumgartner, H.: The Evaluation of Structural Equation Models and Hypothesis Testing, in: Bagozzi, R.P. (Hrsg.) Principles of Marketing Research, Cambridge, 1994

Bagozzi, R.P., Fornell, C.: Theoretical Concepts, Measurements and Meaning: in: Fornell, C. (ed.). A Second Generation of Multivariate Analysis, Vol.2, New York 1982, S.24-38

Bagozzi, R.P.: A Field Investigation of Causal Relations Among Cognitions, Affect, Intentions and Behavior, in : Journal of Marketing Research, 19, 1982, S. 562-583

Bagozzi, R.P.: Structural equation models in marketing research: Basic principles, in: Bagozzi, R.P. (Hrsg.), Principles of marketing research, Cambridge, 1994, S. 317-385

Bamberg, S., Allgemeine oder spezifische Einstellungen bei der Erklärung umweltschonenden Verhaltens? Eine Erweiterung der Theorie des geplanten Verhaltens um Einstellungen gegenüber Objekten, in: Zeitschrift für Sozialpsychologie, 27, 1996, S.47-60

Bamberg, S., Wie bekommt man den/die Autonutzer/-nutzerin in den Bus?, in: Zeitschrift für Sozialpsychologie, 1995, S. 243-262

Barnes, J., The Quality and Depth of Customer Relationships, 1995, S.1393-1402

Bauer, H.H., Herrmann, A., Huber, F.: Die Bestimmungsgrössen der Markentreue beim PKW-Kauf, Ergebnisse einer empirischen Untersuchung, in: der markt, 33. Jg, 1994, S. 165171

Bauer, H.H., Huber, F., Bräutigam, F.: Method supplied investigation of customer loyalty in the automotive industry - Results of a casual analytical study, in: Johnson, M.D., Herrmann, A., Huber, F., Gustafsson, A. (Hrsg.): Customer retention in the automotive industry: Quality, satisfaction, loyalty, Wiesbaden 1997, S. 167-213

Behrens, G. , Konsumentenverhalten, 2. Aufl., Heidelberg 1991

Bem, D.J., Self-perception theory, in: Berkowitz, L. (Hrsg.): Advances in experimental social psychology, 6, San Diego, 1972, S. 1- 62 
Bitner, M.J., Hubbert, A.R.: Encounter satisfaction versus overall satisfaction versus quality: The customer's voice, in: Rust, T.T., Oliver, R.L. (Hrsg.), Service Quality. New directions in theory and practice, Thousand Oaks, 1994, S. 72-95

Bloemer, J.M., Kasper, H.D, Lemmink, J.: The Relationship between overall dealer satisfaction, satisfaction with the attributes of dealer service, intended dealer loyalty and intended brand loyalty: A dutch automobile case, in: Consumer satisfaction, Dissatisfaction \& Complaint Behavior, 3, 1990, S. 42-47

Bloemer, J.M., Kasper, H.D.: The complex relationship between consumer satisfaction and brand loyalty, in: Journal of Economic Psychology, 16, 1995, S. 311-329

Bloemer, J.M., Poiesz, Th.B.: The illusion of consumer satisfaction. Journal of Consumer Satisfaction, Dissatisfaction and Complaining Behavior 2, S. 43-48

Bloemer, J.M., Ruyter, K. de: On the relationship between store image, store satisfaction and store loyalty, in: European Journal of Marketing, 32, 1998, S.499-513

Bolton, R., Drew, J., A multistage model of customers`assessments of service quality and value, in: Journal of Consumer Research, 17, 1991, S. 375-384

Boltz D.M., Leven, W. (Hrsg.), Effizienz der Markenführung, Hamburg 2004

Bortz, J.: Lehrbuch der empirischen Forschung für Sozialwissenschaftler, Berlin 1985

Böttcher, W.: Bilanz und Perspektive II, Übach-Palenberg 1995

Boulding, W., Kalra, A., Staelin, R., Zeithaml,V.: A dynamic process model of service quality, in: Journal of Marketing Research, 1993

Braunstein, Christine: Einstellungsforschung und Kundenbindung, Wiesbaden 2001

Brisoux, J.E., Laroche, M., A proposed Consumer Strategy of Simplification for Categorizing Brands, in: Summy, J.D., Taylor, R.D. (Hrsg.), Evolving Marketing Thought for 1980. Proceedings of the Annual Meeting of the Southern Marketing Association, Carbandale 1980, S. $112-114$

BRO: Monitor (Eu-)regiogrenzoverschrijdend Koopgedrag Zuid-Limburg e.o., 0-meting 2004, 2005

Brüne, G., Mit Meinungsführern erfolgreich arbeiten, in: absatzwirtschaft, Heft 6/1989, S. 7482

Buchanan, R., Gilles, C., Value managed relationships: The key to customer retention and profitability, in: European Management Journal ,8, 1990, S.523-526

Bühl, A., Zöfel, P., SPSS 11, München 2002

Burmann, C. , Konsumentenzufriedenheit als Determinante der Marken- und Händlerloyalität, Marketing, ZFP, Heft 4,1991, S. 249-258 
Büschken, J., von Thaden, Ch., Clusteranalyse, in: Herrmann, A., Homburg, Ch., Marktforschung, Wiesbaden 1999, S.339-380

Buttle, F., Relationship marketing. Theory and practice, London 1996

\section{$\underline{\mathbf{C}}$}

Cap Gemini Ernst \& Young: Searching for the Global Consumer: A European Study of Changing Lifestyles and Shopping Behaviour, 2003

Charng, H., Piliavin, J.A., Callero, P.L.: Role identity and reasoned action in the prediction of prepeated behavior, in: Social Psychological Quarterly, 1988, S. 303-317

Chin, W. W., The partial least squares approch to structural equation modeling in: Marcoulides, G.A. (Hrsg.), Modern methods for business research, Mahwah 1998, S. 258-358

Chin, W. W., Marcolin, B. L. und Newsted, P.R. A partial least squares latent variable modeling approach for measuring interaction effects: result from a monte carlo simulation study and voice mail emotion/adoption study, in: DeGross, J.I. et al. (Hrsg.), Proceeding of the Seventeenth International Conference on Information Systems, Cleveland 1996, S. 21ff

Chin, W. W. und Newsted, P.R., Structural equation modeling analysis with small samples using partial least squares, in: Hoyle, R.H. (Hrsg.): Statistical strategies for small sample research, Thousand Oaks et. Al., 1999 S. 307-342

Chin, W. W., Todd, P.A., On the Use, Usefulness, and Ease of Use of Structural Equation Modeling in MIS Research: A Note of Caution, in:MIS Quarterly, 19,2,S. 237-246

Churchill : A paradigm for developing better measures of marketing constructs, in: Journal of Marketing Research, 1979, S. 64-74

Churchill, Gilbert, A.: Marketing Research: Methodological Foundations, 1995

Cialdindi, R.B., Kallgren, C.A., Reno, R.R.: A focus theory of normative conduct, in: Zanna, M.P. (Hrsg.): Advances in experimental social pychology, New York 1991, 201-234

Clemones, D.S., Woodruff, R.B., Broadening the view of consumer (dis-)-satisfaction: A prosed means-end disconfirmation model of CS/D, in :Allen, C.T., Madden, T.J., Shimp, T.A., Howell, R.D., Zinkhank, G.M., Heiseley, D.D., Semenik, R.J., Dickson, P., Zeithaml, V., Jenk, R.L. (Hrsg.): Marketing theory and applications, Proceedings of the AMA Winter Conference, Chicago 1992, S. 209-216

Cohen, J., Statistical power and analysis for behavioral sciences, 2.Auflage, Hillsdale 1988

Conner, M., Armitage, C.J.: Extending the theory of planned behavior, 1998

Copeland, M., Relation of Consumer's Buying Habit to Marketing Methods, in : Harvard Business Review, Vol. 1, April, S. 282-289 
Corsten, H., Meier, B.: Konsumentenverhalten in unterschiedlichen Kaufsituationen. Entwurf eines Modells auf verhaltenswissenschaftlicher Grundlage, in: Jahrbuch der Absatz- und Verbraucherforschung, 28. Jg., Heft 2/1982, S. 112-136

Cortina, J.M.: What is Coefficient Alpha? An Examination of Theory and Applications, in Journal of Applied Psychology, Vol. 78, 1993

CPB: Challenging Neighbours, Berlin, Heidelberg, New York, 1997

Crocker, L.M., Algina, J.: Introduction to classical and modern test theheory, 1996, Orlando

Cunningham, R.M., Customer Loyalty to Store and Brand, Harvard Business Review, Vol. 39, Nov.Dez. 1961

Czepiel, J.A., Rosenberg, L.J.: The study of consumer satisfaction: Addressing the „So what question“", in: Hunt, 1977, H.K.: Conceptualization and measurement of consumer satisfaction and dissatisfaction, Cambridge, MA, 1979, S. 92-119

\section{$\underline{\mathbf{D}}$}

Day, G.: A Two-Dimensional Concept of Brand Loyalty, in: Journal of Advertising Research, 9. Jg. 1969, Nr. 3, S. 29-35

Day, R.: Modeling Choices Among Alternative Responses to Dissatisfaction, in: Kinnar, T. (Hrsg.) Advances in Consumer Research, Ann Arbor 1984, S. 496-499

Day, R.: The Next Step: Commonly Accepted Constructs for Satisfaction Research, in: Day, R./Hunt, H. (Hrsg.): International Fare in Consumer Satisfaction and Complaining Behavior. Boomington School of Business, Indiana University, 1982 a, S. 113-117

Day, R.: The Next Step: Commonly Accepted Constructs for Satisfaction Research. Arbeitspapier präsentiert bei der 7. Kundenzufriedenheitskonferenz, Knoxville 1882 b

Day, R.: Toward a Process Model of Consumer Satisfaction in: Hunt, H. (Hrsg.): Conceptualization and Measurement od Consumer Satisfaction and Dissatisfaction, Cambridge 1977, S. $153-183$

De Ruyter, K., Scholl, N., Kwalitatief Marktonderzoek: Theorie en Praktijkcases, Utrecht 1995

Dekimpe, M.G., Steenkamp, J.-B.E.M., Mellens, M., Vanden, Abeele, P.: Decline and variably in brand loyality, in: Intern. Journal of Research in Marketing, 14, 1997, S. 405-420

DeVellis, Robert, F.: Scale development, Theory and Applications. Applied Social Research Methods Series, 1991

Diamantopoulos, A., Winklhofer, H.M., Index Construction with Formative Indicators: An Alternative to Scale Development, in: Journal of Marketing Research, 38, 2, 2001, S. 269-277 
Dick, A.S., Basu, K.: Customer loyalty: Toward an integrated conceptual framework, in: Journal of the Academy of Marketing Science, 22, 1994, S. 99-113

Diller, H. , Kusterer, M.: Beziehungsmanagement. Theoretische Grundlagen und explorative Befunde, in: Marketing ZFP, 10.Jg. Heft 3/1988, S. 211-220

Diller, H., (Hrsg.) Vahlens Großes Marketing Lexikon, 2. Aufl. München 1992

Diller, H.: Beziehungsmanagement und Konsumentenforschung, Arbeitspapier Nr.32 des Lehrstuhls für Marketing an der Universität Erlangen, Nürnberg 1994

Diller, H.: Beziehungs-Marketing, in: WiSt, 24.Jg. 1995, S. 442-447

Diller, H.: Customer Loyalty: Fata Morgana or realistic goal? Managing relationships with customers, in: Henning-Thurau, T., Hansen, U. (Hrsg.): Relationship Marketing: Gaining competetive advantage through customer satisfaction and customer retention, Berlin et al. 2000, S. 29-48

Diller, H.: Die Marken- und Einkaufsstättentreue der Konsumenten als Bestimmungsfaktor der Markenführung im vertikalen Beziehungsmarketing, in: Esch, F.R. (Hrsg.): Moderne Markenführung: Grundlagen innovativer Ansätze praktischer Beispiele, München 1999, S.941-956

Diller, H.: Kundenbindung als Marketingziel, in: Marketing ZFP, 18, 1996, S. 81-94

Dillon, W., Madden, T., Firtle, N., Marketing Research in a Marketing Environment, second edition, Boston 1990

Dubois, B., Laurent, G.: Is there a Euro Consumer for Luxury Goods?, in: van Raaij, W.F., Bamossy, G. (Hrsg.): European Advances in Consumer Research, vol. 1 (Provo, UT: Association for Consumer Research 1993, S. 53- 69

Dunk, Hermann W. Von der, Holländer und Deutsche. Zwei politische Kulturen, in: Beiträge zur Konfliktforschung 16, 2 ,1986, S. 59-76

Dunk, V.d., H.: Die Sache mit der Grenze, in: Müller, B./Wielenga, F. (Hrsg.): Kannitverstaan?, Münster 1995, S. 47-62

\section{$\underline{\mathbf{E}}$}

Eagly, A.H., Chaiken, S., The psychology of attidudes, San Diego, Forth Worth, 1993

Earley, Ch., Mosakowski, E.: Creating Hybrid Team Cultures: An Emperical Test of Trenanational Team Functioning, Academy of Management Journal, 43 (1), 2000, S. 26-49

Eckert, S., Rentabilitätssteigerung durch Kundenbindung am Beispiel eines Buchclubs, St. Gallen, 1994 
Eggert, A., Kundenbindung aus Kundensicht, Wiesbaden 1999

\section{$\underline{\mathbf{F}}$}

Faison, E.: The neglected varity drive, in: Journal of Consumer Research, 4, 1977, S. 172-175

Farley, J.U.: Why does brand loyalty vary over products? in: Journal of Marketing Research, 1, 1964, S. 9-14

Fazio, J.R., Jackson, J.R., Dunton, B.C., Williams, Variability in automatic activation as an unobtrusive measure of racial attitudes: A bona fide pipeline? Journal of Personality and Social Psychology, 69, 6, S. 1013-1027

Fazio, R.H., Sanbonmatsu, D.M., Powell, M.C., Kardes, F.R., On the automatic activation of attitudes, Journal of Personality and Social Psychology, 50, S. 229-238

Fazio, R.H., Zanna, M.P.: Attitudinal qualities relation to the strength of the attitude behavior relationship, in: Journal of experimental Social Psychology, New York 1978

Fazio, R.H., Zanna, M.P.: Direct experience and attitude -behavior consistancy, in: Berkowitz, L. (Hrsg.): Advances in experimental social psychology, New York, 1981

Fazio, R.H.: Multiple processes by which attitudes guide behavior, in: Advances in Experimental Social Psychology, 1990, S. 75-109

Fazio, R.H.: Multiple processes by which attitudes guide behavior: The mode model an an integrative framework, in: Journal of Experimental Social Psychology, 14, 1978, S. 398-408 Festinger, Leon: A Theory of Cognitive Dissonance, Standford 1957

Fishbein, M., Ajzen, I., Attitudes and Opinions, in: Annual Review of Psychology, Vol. 23, 1972, S. 487-544

Fishbein, M., Attitude and the prediction of behavior, in: Fishbein, M. (Hrsg.): Readings in Attitude theory and measurement, New York et al. , 1967, S.477-493

Fornell, C., Johnson, M.D., Anderson, E.W., Cha, J., Bryant, B.E.: The American Customer Satisfaction Index: Nature, purpose and findings, in: Journal of Marketing, 60, 1996, S. 7-18

Fornell, C.: A National Customer Satisfaction Barometer: The Swedish Experience, in: Journal of Marketing, 56. Jg., 1992, January, S. 6-21

Fowler, H.W., Fowler, F.G.: The Oxford Dictionary, Oxford 1964

Franzoi, Stephem, L.: Social psychology, Dubuque, 1996

Frey, D., Benning, E.: Informationssuche von Konsumenten nach Entscheidungen, in: Marketing ZFP, Heft 2,1984, S. 107-113

Frey, D., Stahlberg, D., Gollwitzer, P.M., Einstellung und Verhalten: Die Theorie des über- 
legten Handelns und die Theorie des geplanten Handelns, in: Frey, D., Irle, M. (Hrsg.), Theorien der Sozialpsychologie, Bd. 1, Kognitive Theorien, 2. Aufl., Bern, 1993, S. 363-398

\section{$\underline{\mathbf{G}}$}

Gerpott, T.J. Kundenbindung - Konzepteinordnung und Bestandsaufnahme der neueren empirischen Forschung, in: Die Unternehmung, 54, 2000, S. 23-42

Geyskens, I.: Trust, Satisfaction, and Equity in Marketing Channel Relationships. KUL Dissertation Series, 122. Leuven: Catholic University Leuven, 1998

Gefen, D., Straub, D.W. und Bourdreau, M.-C., Structual equation modeling and regression: guidelines for research practice, Communications of the Association for Information Systems, Jg. 4, H.7, S. 1-78

Gilligan, S.G.; Bower, G.H.: Cognitive consequences of emotional arousal. In Izard, C.;Kagen, J.; Zajonc, R. (Eds.): Emotions, cognition, and behavior (S. 547 - 588). New York: Cambridge University Press 1988, S.547-588

Gilly, M.C., Enis, B.M.: Recycling the Family Life Cycle: A Proposal for Definition, in: Advances in Consumer Research, 9, S. 271-276

Gollwitzer, P.M., Malzacher, J.: Absichten und Vorsätze, in: Kuhl, J., Heckhausen, H. (Hrsg.): Motivation, Volition und Handlungen, Enzyklopädie der Psychologie, Bd. 4, 1996, S. $427-468$

Green, Paul. E., Tull, Donald, S., Albaum, G., Research for Marketing Decisions, 1988

Green, Paul. E., Tull, Donald, S., Methoden und Techniken der Marketingforschung, 4. Auflage, 1982

Greve, G., Erfolgsfaktoren von Customer-Relationship-Management-Implementierungen, Wiesbaden 2006

Guest, L.: Last Versus Usual Purchase Questions. In: Journal of Applied Psychology, 26.Jg. 1942, S. $180-186$

\section{$\underline{\mathbf{H}}$}

Hair, J.F., Anderson, R.E., Tatham, R.L., Black, W.C.: Multivariate data analysis, 5th. Edition, Upper Saddle River, 1998

Hansen, U., Jeschke, K., Nachkaufmarketing. Ein neuer Trend im Konsumgütermarketing?, Marketing ZFP, Feb. 1992, S. 88-97 
Hausknecht, D.R.: Measurement scales in consumer disatisfaction/dissatisfaction, in: Journal of Consumer Satisfaction, Dissatisfaction and Complaining Behavior, 3, 1990, S. 1-11

Heckhausen, H., Motivation und Handeln, Berlin 1983

Heinemann, M., Einkaufsstättenwahl und Firmentreue der Konsumenten. Verhaltenswissenschaftliche Erklärungsmodelle und ihr Aussagewert für das Handelsmarketing, Münster 1976 Helfferich, E., Hinfelaar, M., Kasper, H.: Towards a clear terminology on international retailing, in: Reader Retail Management, 5010K, Block4.1, Maastricht 2001

Henning-Thurau, T., Gwinner, K., Gremler, W.: Wy customers build relationships with companies - and why not, in: Henning-Thurau, T., Hansen, U. (Hrsg.) Relationship marketing: Gaining competitive advanges through customer satisfaction and customer rentention, Berlin et al., 2000, S. 3-27

Henning-Thurau, T., Hansen, U. (Hrsg.) Relationship marketing: Gaining competitive advanges through customer satisfaction and customer rentention, Berlin et al., 2000

Henning-Thurau, T., Klee, A.: The impact of customer satisfaction and relationship quality on customer retention: A critecal reassessment and model development, in: Psychology \& Marketing, 14, 1997, S. 737-764

Herkner, W.W.: Sozialpsychologie, 5. Aufl., Bern u.a. 1991

Hermans, Baeyens, F., Eelen, P., Odours as affective-processing context for word evaluation: A case of cross-modal affective priming. Ecognition and Emotion, 12, 4, S. 601-613

Herrmann, A., Homburg, C. (Hrsg.): Marktforschung: Methoden, Anwendungen , Praxisbeispiele, Wiesbaden 1999,

Herrmann, A., Homburg, C.: Marktforschung: Ziele, Vorgehensweisen und Methoden, in: Herrmann, A., Homburg, C. (Hrsg.): Marktforschung: Methoden, Anwendungen, Praxisbeispiele , 2. Aufl. , Wiesbaden 2000, S.13-32

Herrmann, A.: Nachfrageorientierte Produktgestaltung: Ein Ansatz auf Basis der „means end"-Theorie, Wiesbaden 1996

Herrmann, A.: Produktmanagement, München 1998

Herzberg, F., Mausner, B., Snyderman, B.B., : The Motivation to work, 2nd. Ed., 1959, New York/London/Sydney

Hildebrandt, L.: Hypothesenbildung und empirische Überprüfung, in: Herrmann, A., Homburg, C. (Hrsg.): Marktforschung, 2. Aufl., Wiesbaden, 2000, S. 33-57

Hofstede, F., Audenaert, A., Steenkamp, J.-B.E.M., Wedel, M.: An investigation into the association pattern technique as a quantitative approach to measuring meansend Chains, in: International Journal of Research in Marketing, 15, 1998, S. 132-140 
Hofstede, G.: Culture's Consequences: International Differences in Work-Related Values, Beverly Hills, 1980

Hofstede, Geert, Bond, M.H., Hofstede's culture dimensions: an independent validation using Rokeach`s Value Survey, in: Journal of cross-cultural Psychology, 15, 1984

Hofstede, Geert: Lokales Denken, globales Handeln, 2. Auflage, München 2001

Holland/Heeg, Erfolgreiche Strategien für die Kundenbindung, Wiesbaden 1998

Homburg, C., Fassnacht, M., Kundennähe, Kundenzufriedenheit und Kundenbindung bei Dienstleistungsunternehmen, in: Bruhn, M., Meffert, H. (Hrsg.), Dienstleistungsmanagement: Grundlagen, Konzepte, Erfahrungen, Wiesbaden 1998, S. 405-428

Homburg, C., Giering, A., Menon, A.: Relationship charakteristics as moderators of satisfaction-loyalty link: Findings in a business-to business-context, Arbeitspapier des Instituts für Marktorientierte Unternehmensführung, Nr. 30, Mannheim 1999

Homburg, C., Giering, A.: Konzeptualisierung und Operationalisierung komplexer Konstrukte, in: Hildebrandt, L., Homburg, C. (Hrsg.): Die Kausalanalyse: Ein Instrument der empirischen betriebswirtschaftlichen Forschung, Stuttgart, 1998, S. 111-147

Homburg, C., Rudolph, B., Theoretische Perspektiven zur Kundenzufriedenheit in: Simon, H./Homburg, C. (Hrsg.): Kundenzufriedenheit, 3. Auflage, Wiesbaden 1998, S. 33-55

Homburg, Ch., Bruhn, M., Kundenbindungsmanagement, in: Bruhn, M., Homburg, Ch. (Hrsg.): Handbuch Kundenbindungsmanagement, Wiesbaden 1998, S. 3-35

Homburg, Ch., Giering, A., Hentschel, F.: Der Zusammenhang zwischen Kundenzufriedenheit und Kundenbindung, Koblenz 1998, S.8

Homburg, Ch., Kundennähe von Industriegüterunternehmen, 3. Aufl., Wiesbaden 2000

Homburg, Ch.: Single Sourcing, Double Sourcing, Multiple Sourcing...? In ZfB, 65. Jg. 1995, S. 813-834

Homburg, C., Baumgartner, H.: Beurteilung von Kausalmodellen: Bestandsaufnahme und Anwendungsempfehlungen, in: Hildebrand, L., Homurg, C. (Hrsg.): Die Kausalanalyse: ein Instrument der betriebswirtschaftlichen Forschung, Stuttgart, S. $343 \mathrm{ff}$ Homburg/Bruhn: Kundenbindungsmanagement, in: Homburg/Bruhn (Hrsg.): Handbuch Kundenbindungsmanagement, 4. Auflage, Wiesbaden 2003, S. 3-30

Hossinger, H.P.: Pretests in der Marketingforschung. Die Validität von Pretestverfahren in der Marketingforschung unter besonderer Berücksichtigung der Tachistoskopie, Würzburg u.a. 1982

Housten, M.B., Walker, B.A.: Self-relevance and purchase goals: Mapping a consumer decision, in: Journal of the Academy of Marketing Science, 24, 1996, S. 232-254 
Hoveland, C.I., Rosenberg, M.J., Summery and further theoretical issus, in: Hoveland, C.I., Rosenberg, M.J. (Hrsg.): Attitude organization and change: An analysis of consistency among attitude components, New Haven, 1960, S. 198-232

Hulland, J., Use of Partial Least Squares (PLS) In Strategic Management Research: A Review of Four Recent Studies, in: Strategic Management Journal, 20,1, S. 195-204

Howell, J.M., Avolio, B.J., Transformational Leadership, Tranactional Leadership, Locus of Control, and Support for Information: Key Predictors of Consolidated-Business-Unit Performance, in: Journal of Applied Psychology; December 1993, 78, 6, S. 891-902

\section{$\underline{\mathbf{J}}$}

Jacoby , J., A Model of Multi-Brand Loyalty, in: Journal of Advertising Research, Jg. 3, June 1971, S. 25-26

Jacoby, J., Chestnut, R., Brand Loyalty Measurement and Management, New York 1978

Jacoby, J., Kyner, D., Brand Loyalty versus Repeat Purchasing Behavior, in: Journal of Marketing Research, Vol. 10, February 1973, S. 1-9

Jacoby, J., Olson, J., An Attitude Model of Brand Loyalty: Conceptual Underpinnings and Instrumentation Research. Arbeitspapier präsentiert auf der Konferenz für Einstellungsforschung und Konsumentenverhalten. Urbana, Illinois 1970

Jank, W., Meyer, H.: Didaktische Modelle, Berlin 2000

Jansen, Lutsen, B.: Bekannt und ungeliebt, in:Müller, B., Wielenga, F.(Hrsg.): Kannisverstan?

Deutschlandbilder aus den Niederlanden, Münster 1995

Johnsons, M.D.: Customer orientation and market action, Upper Saddle River, 1998

Jones, E. E., Davis, K.E. (1965), From acts to dispositions : the attribution process in person perception. In: Berkowitz,L. (ed.) .Advances in experimental social psychology. New York., S. $223-235$

Jöreskog, K., A general method for estimating a linear structural equation system, in: Goldberger, A.S., Duncan, O.D. (Hrsg.), Structual equation models in the social sciences, New York, London 1973, S.265-287

Jungwirth, G., Geschäftstreue im Einzelhandel, Wiesbaden 1997

$\underline{\mathbf{K}}$

Kashima, Y., Kashima, E.: Individual differences in the prediction of behavioral intentions, in: The Journal of Social Psychology, 128, S. 711-720 
Kasper, J.D.P.: On problem perception, dissatisfaction, brand loyalty, in: Journal of Economic Psychology, 9, 1988, S. 387-397

Kasper, H., Bloemer, J.: Cultural closeness and it's impact on internationalization strategies, Procceedings $26^{\text {th }}$ EMAC Conference, Budapest 1995, pp. 1805-1815

Katz, D., The Functional Approach to the Study of Attitude, in: Fishbein, M. (Hrsg.): Readings in Attitude theory and measurement, New York et al. , 1967, S.457-468

Katz, Daniel, The Functional Approach to the Study of Attitude, Public Opinion Quarterly 24, 1960, S. 163-204

Kepper, G.: Methoden der Qualitativen Marktforschung, in:Herrmann, A., Homburg, Ch. (Hrsg.), Marktforschung, Wiesbaden 1999

Klimsa, P.: Neue Medien und Weiterbildung: Anwendung und Nutzung in Lernprozessen der Weiterbildung. Deutscher Studien Verlag; Weinheim; 1993

Kokkinaki, F.: Predicting product purchase and usage intentions with the theory of planned behavior, in: IAREP-Proceedings 1996, Paris 1996, S. 354-365

Kotler, P. Bliemel, F.: Marketing Management, 10. Auflage, Stuttgart 1995

Kotler, P., Armstrong, G., Saunders, J., Wong, V.: Principles of Marketing, Second European Edition, London et al. 1999

Kotler, P., Marketing Management, 11. Aufl. 2003

Kotler, Ph., Armstrong, G., Saunders, J., Wong, V, Principles of Marketing, New Jersey 1999

KPMG, Regiovisie Noord-en Midden-Limburg 2030, 1998

Kroeber-Riel, W., Weinberg, P.: Konsumentenverhalten, 2000

Kroeber-Riel, W.: Konsumentenverhalten, 1990

Kroeber-Riel,W., Weinberg, P., Konsumentenverhalten, 8.Auflage, München 2003

Krummer, R.: Innere Struktur, Reichweite und Attraktivität des Einzelhandels in den Innenstädten von Düren, Jülich und Eschweiler, Aachen 1993

Kuhl, J., Motivation, Konflikt und Handlungskontrolle, Berlin 1983

Kuhl, J.: A theory of action and state orientation, in: Kuhl, J., Beckmann, J. (Hrsg.), Volition and personality, Göttingen et al., 1994, S. 9-45

Kuhl, J.: Handlungs- und Lageorientierung, Forschungsbericht aus dem Fachbereich Psychologie der Universität Osnabrück, Osnabrück 1995

\section{$\underline{\mathbf{L}}$}

LaBarbera, P.A., Mazursky, D.: A longitudinal assessment of Customer Satisfaction/Dissatisfaction: the dynamic aspect of the cognitive process, in: Journal of Marketing 
Research, 20, 1983, S. 393-404

LaPiere, R.T., Attitudes vs. Actions, in: Social Focus, 13, 1934, S. 230-236

Lessig, V.P., Consumer Store Images and Store Loyalties, Journal of Marketing, 37/1973

Levitt, T., After the Sale is over, in : Sviokla, J. J./Shapiro, B.P. /Sds.), Keeping Customers, Boston, MA 1993, S. 39-52

Litwin, M.S.,: How to measure survey reliability and validity, The survey kit , 1995

Luhmann, N.: Die Realität der Massenmedien, Opladen 1996

\section{$\underline{\mathbf{M}}$}

Malhotra, N.K., Modeling Store Choice based on Censored Preference Data, in: Journal of Retailing, Heft 2/1986, S. 129

Malhotra, Naresh, K.: Marketing Research, An Applied Orientation. 2. Edit., Upper Saddle River, New Jersey 1996

Manstead, A.S.R., Parker, D.: Evaluating and extending the theory of planned behaviour, in: European Review of Social Psychology, 6, 1995, S. 65-95

Mayer, H., van Eimeren, B., Einstellungen als Prädikatoren von (Kauf-) Verhalten, in: Jahrbuch der Absatz und Verbrauchsforschung, Heft 3/1985, S. 207-229

McAlister, L., Pessemier, E.: Varity Seeking behavior: An interdisciplinary review, in: Journal of Consumer Research, 9, 1982, S. 311-322

Meffert, H. Marketing, 8. Aufl. , Wiesbaden1998

Meffert, H., Marketingforschung und Kaufverhalten, 2. Auflage, Wiesbaden 1992

Meffert, Heribert, Marketing, 9. Auflage, Wiesbaden 2000

Middendorp, van, A., Velde, van der, B.M.R., Vergossen, Th.W.M.: Kauf- und Besuchsströme im Einzugsgebiet der Euregio Rhein-Waal, Nijmegen, Duisburg, 1999

Mittal, Vikas, Kamakura , Wagner: Satisfaction, Repurchase Intent, and Repurchase Behavior: Investigation the Moderating Effect of Customer Characteristics, in: Journal of Marketing Research, 38 (1), 2001, S. 131-142

Mittal, Vikas, Kumar, Pankaj, Tsiros, Michael: Attribute-Level Performance, Satisfaction and Behavioral Intentions over Time: A Consumption-System Approach, in: Journal of Marketing, 63 (April) ,1999,S. 88-101

Mittal, Vikas, Ross,William T., Baldasare Patrick M. The Assymetric Impact of Negative and Positive Attribut-Level Performance on Overall Satisfaction and Repurchase Intentions, in: Journal of Marketing, 62 (January) 1998, S. 33-47 
Mohme, J., Der Einsatz von Kundenkarten im Einzelhandel, Frankfurt a.M. 1993

Morgan, R.M., Hunt, S.D., The commitment-trust theory of relationship marketing, in: Journal of Marketing, 58, 1994, S. 20-38

Morgan, R.M., Hunt, S.D.: The commitment-trust theory of relationship marketing, in: Journal of Marketing, 58, 1994, S. 20-38

Müller, S.: Die Psyche des Managers als Determinante des Exporterfolges, Stuttgart 1991

Müller, W.,Riesenbeck , H.J.: Wie aus zufriedenen Kunden auch anhängliche Kunden werden, Harvard Business Manager, März 1991, S.67-79

Myers, J.H., Alpert, M.I., : Determinant Attributes: Meaning and Measurement, in: Journal of Marketing, October, 32, 1968, S. 13-20

\section{$\underline{\mathbf{N}}$}

Narayandas, N.: The link between customer satisfaction and customer loyalty: An empirical investigation, Working Paper No. 97-017, Harvard Business School, Cambridge 1996

Narayandes, N.: Measuring and managing the consequences of consumer loyalty:An empirical investigation, Working Paper No. 98-003, Harvard Business School, 1997

Nieschlag, Dichtl, Hörschgen: Marketing, 19. Aufl., Berlin 2002

Nunnally, J., Psychometric Theory, 2. Edition, New York, 1978

\section{$\underline{\mathbf{0}}$}

O`Brien, L., Jones, C., Do rewards really create loyalty?, in Harvard Business Review, 4, 1995, S. $75-82$

Odekerken-Schröder, G., The Role of the Buyer in Affecting Buyer-Seller Relationships, Maastricht 1999

Ohlwein, M., Märkte für gebrauchte Güter, Wiesbaden 1999

Oliva, Terence; Oliver, Richard L; MacMillan, Ian C.: A Catastrophe Model For Developing Service Satisfaction Strategies, in: Journal of Marketing, 56 (July) 1992, S. 83-95

Oliver, R.L., Cognitive, affective, and attribute basis of the satisfaction responses, in: Journal of Consumer Research, 20, 1993, S. 418-430

Oliver, R.L.,: Satisfaction: A behavioral perspective on the consumer, New York 1997

Oliver, R.L.: Processing of the satisfaction consumption: A suggested framework and research response propositions, in: Journal of Consumer Satisfaction/Dissatisfaction and Com- 
plaining Behavior,2, 1989, S. 1-16

Oliver, R.L.: Whence consumer loyalty?, in: Journal of Marketing, 63, 1999, S. 33-44

Ouellette, J.A., Wood, W.: Habit and intention in everyday life, in: Psychological Bulletin, 2, 1998

$\underline{\mathbf{P}}$

Parasuraman, A., Zeithaml, V.A., Berry, L.L.,: Moving Forward in Service - Quality Research: Measuring Different Customer Expectation Levels, Comparing Alternative Scales and Examining the Performance-Behavioral-Intentions Link, MSI Report, Rep.Nr.: 94-114, 1994, Cambridge, USA

Peter, J.P., Olson, J.C., Grunert, K.G. Consumer behavior and marketing strategy, London 1999

Peter, S., Kundenbindung als Marketingziel, 2. Auflage,Wiesbaden 1999

Peter, S.: Kundenbindung als Marketingziel: Identifikation und Analyse zentraler Determinanten, Wiesbaden 1997

Peterson, T.J., A Meta-Analysis of Cronbach's Coefficient Alpha, in: Journal of Consumer Research, Vol. 21, 1994

Plinke, W.: Die Geschäftsbeziehung als Investition, in: Specht, G. , Silberer, G., Engelhardt, W.H. (Hrsg.): Marketing-Schnittstellen. Herausforderungen für das Management, Stuttgart 1989, S. 305-321

Popper, K.R.: Objektive Erkenntnis, Hamburg 1973, S.213

\section{$\underline{\mathbf{R}}$}

Raaij, W.F. van, Ester, P., Bronner, A.E., Olivier, A.J.: Recente ontwikklingen in het marktonderzoek, Haarlem: Uitgeverij De Vrieseborgh, 1997

Reber, R., Haeter, A., Sollberger, B., Unbewusstes affektives Priming: Zwei neue experimentelle Paradigmen. Vortrag auf der 7. Tagung der Fachgruppe Sozialpsychologie in Kassel 1999

Reichheld, F., Loyalty-Based-Management, Harvard Business Review, 1993, S. 64-73

Reichheld, F.F., Sasser, W.E., Zero defections: Qualtiy comes to services, in: Harvard Business Review, 68, 1990, S. 105-111

Reichheld, F.F., The loyalty effect, Boston, 1996

Reichheld, Frederick, F., Mundpropaganda als Maßstab für den Erfolg, in: Harvard Business 
manager, März 2004

Ringle, Ch., Gütemaße für den Partial Least Squares-Ansatz zur Bestimmung von Kausalmodellen, in: Hansmann, K.W. (Hrsg.), Industrielles Management, Arbeitspapier Nr.16, Hamburg 2004

Rokeach, M., The nature of human Values, New York, 1973

Russell, H. Fazio, Lenn, T.M., Effrein, E.A., Spontaneous Attitude Formation, Social Cognition 2, 1984, S. 214-234

\section{$\underline{\mathbf{S}}$}

Sageder, J.: Didaktische Aspekte des Einsatzes von Computern für Lehren und Lernen. in: Seidel, C. (Hrsg.): Computer Based Training: Erfahrungen mit interaktivem Computerlernen; Verlag für Angewandte Psychologie; Stuttgart; 1993; S. 59-86

Sauerwein, Elmar: Das Kano-Modell der Kundenzufriedenheit, Wiesbaden 2000

Schäfer, W.: Wirtschaftswörterbuch, München 1979

Schelten, A.: Grundlagen der Testbeurteilung und Testerstellung, 1980, Heidelberg

Schlegel, R.P., Multidimensional measurement of attitude towards smoking marijuana, in: Canadian Journal of Behavioral Science, 7, 1975, S. 387-396

Schröder, Hartwig: Grundwortschatz Erziehungswissenschaft. 2., überarb. Aufl. München: Ehrenwirth 1992.

Schwartz, S.H., Tessler, R.C.: A test of a model for reducing measured attitude-behavior relationship, in: Journal of Personality and Social Psychology, 24, 1972, S. 225-236

Schweiger, W., Die Optimierung der Kundenzufriedenheit, Loyalität und Profitabilität. Neue Methoden effizienter Markenstrategien, Vortrag am CASH Handelsforum am 30. März bis 1. April 1993

Seggev, E., Brand Assortment and Consumer Brand Choice, Journal of Marketing, Vol. 34, Oct. 1970, S. 18-24

Seidel, Christoph; Lipsmeier, Antonius: Computerunterstütztes Lernen. Stuttgart: Verlag für Angewandte Psychologie 1989

Sellien, R, Sellien, H. (Hrsg.): Dr. Gablers Wirtschaftslexikon, 9. Auflage, Wiesbaden 1977

Seyffert, Rudolf: Wirtschaftslehre des Handels, 5. Auflage, Opladen 1972

Simon, H./Homburg, Ch., Kundenzufriedenheit, 3. Auflage, 1998

Simon/Homburg, Kundenzufriedenheit, 3. Auflage, Wiesbaden 1998

Singh, Jagdip, Sirdeshmukh, Deepak: Agency and Trust Mechanisms in Consumer Satisfac- 
tion and Loyalty Judgements, in: Journal of the Academy of Marketing Science, 2000,Vol. 28, No., S. 150

Snyder, M., Swann, W.B., When action reflects attitudes, in: Journal of Personality and Social Psychology, 1998

Snyder, M.: Self-monitoring of expressive behavior, in: Journal of Personality and Social Psychology, 1974

Söllner, A., Asymmetrical Commitment in Business Relationships, Journal of Business, Research 46, 199, S. 219-233

Solomon, M., Bamossy, G., Askegaard, Konsumentenverhalten, München 2001

Sparks, P., Guthrie, C.A.: Self-identity and the theory of planned behavior, in: Journal of the Applied Social Psychology, 1998

Sparks, P., Shepard, R., Wieringa, N., Zimmermann, N.: Perceived behavioral control, unrealistic optimism and dietary change, in: Appetite, 1995

Spencer, H., First principles, New York, 1862

Stahlberg, D., Frey D., Einstellung: Struktur, Messung und Funktion, in Stroebe, W., Hewstone, M., Stephenson, G. (Hrsg.), Sozialpsychologie: Eine Einführung, 3. Aufl. , Berlin et al., 1996, S. 219-252

Stauss, B., Henschel, B., Messung von Kundenzufriedenheit in: Marktforschung und Management, 36. Jg. 1992, Nr. 3, S. 115-122

Strüver, A., Getuigen van een „Gewone Grens“: Mentale Constructies van het NederlandsDuitse Grensland, Diss. University Nijmegen, 2004

Swan, J.E., Combs, L.J.: Product Performance and Consumer Satisfaction: A new Concept, in Journal of Marketing, April, (40), 1976, S. 25-33

Swift, J., Cultural Closeness as a Fact of Cultural Affinity: A Contribution to the Theory of Psychic Distance, International Marketing Review, 16, (3), S. 182-201

\section{$\underline{\mathbf{T}}$}

Thorndike, E.L., Educational Psychology-Three Volumes in One, New York Tourangeau, R., Rasinski, K.A., D’Andrade, R., Attitude structure and belief accessibility, Journal of Experimental Social Psychology, 27, 1991, S. 48-74

Trommsdorf, V., Konsumentenverhalten, 2. Aufl., Stuttgart 1993

Trommsdorff, V.: Konsumentenverhalten, 2. Aufl., Stuttgart, 1993 
264 | Literaturverzeichnis

$\underline{\mathbf{U}}$

Usunier, J.C.: International Marketing: A Cultural Approach, New York 1993

\section{$\underline{\mathbf{V}}$}

Völckner, F.: Empirische Analyse zum Markentransfererfolg bei kurzlebigen Konsumgütern, Diss. Universität Hamburg, Hamburg 2003

\section{$\underline{\mathbf{W}}$}

Wagner, R.: Kulturschock Deutschland, 3. Aufl., Hamburg 1997

Weinberg, P., Verhaltenswissenschaftliche Aspekte der Kundenbindung, in: Bruhn, M., Meffert, H. (Hrsg.), Dienstleistungsmanagement: Grundlagen, Konzepte, Erfahrungen, 1998, S. $39-53$

Wentura, D., Dräger, D., Brandstädter, J., Altersstereotype im frühen und höheren Erwachsenenalter: Analyse akkomodativer Veränderungen anhand einer Satzpriming-Technik, Zeitschrift für Sozialpsychologie, 28, 1997, S.109-128

Wold, H., Model Construction and Evaluation when Theoretical Knowledge is Scare: Theory and Application of Partial Least Squares, in: Kmenta, J. und Ramsey, J.B. (Hrsg.): Evaluation of Econometric Models, New York, S. 47-74

\section{Sonstige Quellen:}

Landesamt für Datenverarbeitung und Statistik NRW: Volkswirtschaftliche Gesamtrechnung Nordrhein-Westfalen, 2004

CBS, E,til BV, 2002

E`til: Konsumentenbefragung 2003

\section{Internetquellen:}

www.Euregio.de Abfrage 30.6.2005

www.omg-online.de/markenklima-index_q1_2005.ppt Abfrage 28.7.2005

www.auswaertiges-amt.de Abfrage 21.8.200

http://euregio.etil.nl

www.deutschland.de Abfrage 21.8.2005 


\section{Anhang:}

\section{Anhang 1:}

\section{Fragebogen niederländische Konsumenten:}

\section{Donderdag $\square \quad$ Zatertag}

Bent u naar Duitland/Nederland gekomen om te winkelen?
Koopt u vaker in
dezelfde winkel?

Ik lees $u$ nu enkele uitspraken voor. $U$ vertelt, of $u$ het hiermee eens of oneens bent.

$1=$ volledig eens $; 5=$ oneens $; 6=$ niet van toepassing (n.v.t.)

01. Als kind al ging ik met mijn ouders winkelen over de grens

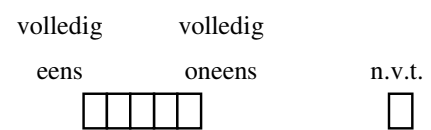

02. Voor mij, is winkelen over de grens een stuk toegevoegde waarde in mijn leven $\square \square \square \square$

03. Mijn vrienden-/bekendenkring vindt, dat je in het buurland

- goedkoper kan kopen

- kwalitatief betere producten kan kopen

- $\quad$ beter advies/hulp krijgt

- meer aparte producten, qua design, kan krijgen

04. Wanneer u aan uw vrienden-/bekendenkring denkt, hoeveel \% wordt in het buurland ingekocht

- minder dan $20 \%$ van de totale inkopen

- tussen 20 - $50 \%$ van de totale inkopen

- meer dan $50 \%$ van de totale inkopen

\section{Voor volgende uitspraken hoor ik graag, in hoeverre u het hiermee eens bent.}

$1=$ volledig eens; $5=$ oneens; $6=$ niet van toepassing

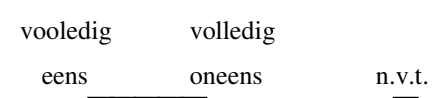

05. Vrienden/bekenden reageren positief wanneer ik ga winkelen in het buurland

\begin{tabular}{|l|l|l|}
\hline$口$ &
\end{tabular}

06. Wanneer $\mathrm{u}$ nu even terugdenkt aan de laatste keer dat $\mathrm{u}$ bent gaan winkelen.

In hoeveel gevallen hebben vrienden, bekenden u een tip/aanbeveling van een winkel gegeven?

- minder dan 2 gevallen

- $\quad$ in 2 - 5 gevallen

- $\quad$ in meer dan 5 gevallen 
Voor de volgende uitspraken hoor ik graag, in hoeverre u tevreden bent.

$1=$ zeer tevreden $; 5$ zeer ontevreden ; $6=$ niet van toepassing

07. Met de winkelmogelijkheden in deze stad ben ik

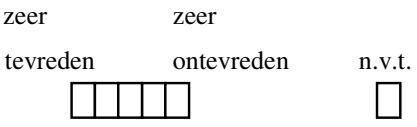

08. Bij het winkelen bij mijn favoriete / vaste winkeladres ben ik

Naam favoriete / vaste winkeladres:

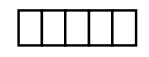

- Hulpvaardigheid / advies

- Assortiment

- Service

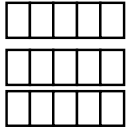

- Inrichting van de winkel

Voor volgende uitspraken hoor ik graag in hoeverre u het ermee eens bent. Ten tweede; hoe belangrijk de reaktie van uw omgeving is.

$1=$ volledig eens $; 5$ oneens; 6 = niet van toepassing

$1=$ zeer belangrijk ; $5=$ helemaal onbelangrijk

\begin{tabular}{lllrl} 
volledig & volledig & \multicolumn{2}{c}{ zeer } & helemaal \\
eens & onens & n.v.t. & belangrijk & onbelangrijk
\end{tabular}

09. In mijn buurland kan ik producten kopen, die ik thuis niet kan krijgen

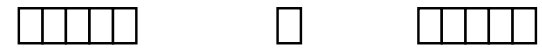

10. In mijn buurland koop ik goedkoper

11. In mijn buurland vind ik gemakkelijker de produkten die ik zoek / nodig heb.

12. a. In mijn buurland koop ik liever bij de grote ketens.

b. In mijn buurland koop in liever bij de kleinere vakhandel / specialist

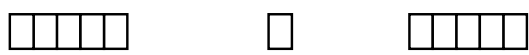

13. De plaats waar ik ga winkelen in mijn buurland, moet goed bereikbaar zijn met het openbaar vervoer.

14. De volgende keer ga ik weer in mijn buurland winkelen

15. Winkelen in mijn buurland vindt ik leuk, omdat ik in een mooie omgeving ben en er café's, entertainment is.

16. Ik ben blij, dat ik de mogelijkheid heb, dat ik snel in mijn buurland kan gaan winkelen
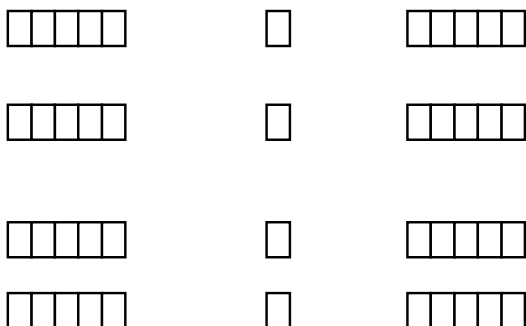
nt $u$ het eens met volgende uitspraken?

17. Mijn vaste / favoriete winkeladres in mijn buurland zal ik, bij vrienden en bekenden, aanbevelen.

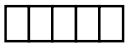

18. Ik wil regelmatig geinformeerd worden over aanbiedingen en bijzondere service, van mijn vaste / favoriete winkeladres.

19. Minstens 1 keer per maand bezoek ik mijn vaste / favoriete winkeladres in mijn buurland

20. In de laatste 8 weken heb ik vaker in mijn buurland gewinkeld

21 . In de laatste 8 weken heb ik vaker produkten gekocht bij mijn vaste / favoriete winkel
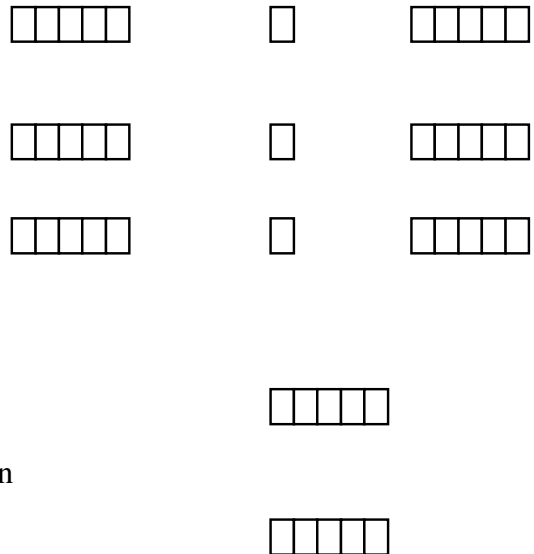

2. Wanneer mijn vast / favoriete winkel in de toekomst ook andere 
produktencategoriën aanbiedt, kan ik me voorstellen,

deze daar te kopen.

\section{Nu nog een paar vragen, die ons helpen om uw antwoorden juist te rangschikken.}

23. Wanneer $u$ denkt aan de volgende keer dat $u$ gaat

winkelen, naar wat voor soort winkels in uw buurland

gaat u als eerste? (voor één groep beslissen)

Prijs

- Vakhandel/specialist met bediening

Groter assortiment

- Filiaalbedrijven zoals HEMA, die ik van meer winkelcentra in het buurland ken

Grootte van de winkel

- Filiaalbedrijven zoals H\&M, Van Haren , die ik van meer winkelcentra in het buurland ken

Parkeerplaatsen

- Discounters (prijsvechters) zoals ALDI, LIDL

Hulpvaardigheid/advies

- Warenhuis/Supermarkt zoals Wal-Mart, Allkauf

Bereikb. per openb.vervoer $\square$

- Warenhuis zoals Bijenkorf / V\&D

Service

- Andere aanbieders

Anders

Welke producten koopt u hoofdzakelijk naast levensmiddelen?

- Tuinartikelen

- $\quad$ Kleding

- Schoenen

(max 2 noemen) 1. eerst genoemd

- Electronica

- anders

\section{Allgemeiner Teil:}

24.

$\begin{array}{lrrr}\text { Nationaliteit } & \text { NL } \square & \mathrm{D} \square & \text { anders } \square \\ \text { Geslacht } & \mathrm{m} \square & \mathrm{w} \square & \\ \text { Leeftijd } & \text { tot } 35 \square & 35-64 \square & \text { ouder dan } 64 \square\end{array}$

Uw postcode

Hoeveel personen wonen in uw huishouden 1 Pers.

\section{$\square 2$ Pers.}

tot 3.500,-

Netto inkomen voor uw huishouden tot 2.000,-

Met welk vervoersmiddel komt $\mathrm{u}$ in de regel naar uw buurland ?
Auto
Bus
Trein
Fiets
Lopend 


\section{Anhang 2:}

\section{Fragebogen deutsche Konsumenten:}

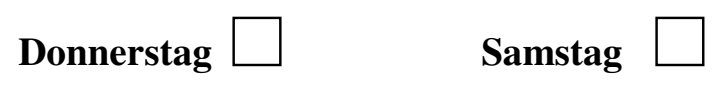
Sind Sie zum Einkauf nach Deutschland/in die Niederlande gekommen?
Ja Nein
Kaufen Sie öfter in.......................im gleichen Geschäft ein?

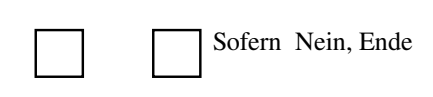
Sofern Nein, Ende

Ich lese Ihnen jetzt einige Aussagen vor. Sagen Sie mir bitte, ob diese stimmen oder nicht stimmen.

$1=$ stimme ich voll $\mathrm{zu} ; 5=$ stimme ich nicht $\mathrm{zu} ; 6=$ trifft nicht $\mathrm{zu}$

$\begin{array}{rlr}\text { stimme ich voll } & \text { stimme ich } & \text { trifft } \\ \mathrm{zu} & \text { nicht zu } & \text { nicht zu }\end{array}$

01. Bereits als Kind fuhr ich mit meinen Eltern zum Einkauf über die Grenze

$\mathrm{zu}$

nicht zu

nicht zu

02. Für mich ist der Einkauf im Nachbarland ein Stück Lebensqualität

03. Mein Freundes/Bekanntenkreis findet, dass man im Nachbarland

- preislich besser einkaufen kann

- qualitativ bessere Produkte einkaufen kann

- $\quad$ eine bessere Beratung erhält

- $\quad$ ausgefallenere (Design) Produkte erhält

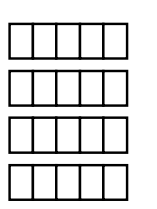

04. Wenn Sie einmal an Ihren Freunde /Bekanntenkreis denken,

in wie vielen Fällen kaufen diese im Nachbarland ein?

- weniger als $20 \%$ aller Einkäufe

- zwischen 20 - 50\% aller Einkäufe

- mehr als $50 \%$ aller Einkäufe

Bei den nächsten Aussagen sagen Sie mir bitte, ob Sie diesen Aussagen zustimmen.

$1=$ stimme ich voll $\mathrm{zu} ; 5=$ stimme ich nicht $\mathrm{zu} ; 6=$ trifft nicht $\mathrm{zu}$

$\begin{array}{rrr}\text { stimme ich voll } & \text { stimme ich } & \text { trifft } \\ \mathrm{zu} & \text { nicht zu } & \text { nicht zu }\end{array}$

05. Freunde/Bekannte reagieren auf meinen Einkauf im Nachbarland positiv.

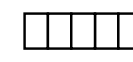

06. Wenn Sie einmal an die letzten Einkäufe zurückdenken, in wie vielen Fällen

haben Freunde, Bekannte Ihnen eine Empfehlung zum Einkauf im

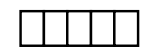

Nachbarland gegeben?

- $\quad$ in weniger als zwei Fällen

- $\quad$ in zwei bis fünf Fällen

- $\quad$ in mehr als fünf Fällen 


\section{Bei den nächsten Aussagen sagen Sie mir bitte wie zufrieden Sie sind}

$1=$ sehr zufrieden ; 5 sehr unzufrieden ; $6=$ trifft nicht $\mathrm{zu}$

07. Mit den Einkaufsmöglichkeiten in (Ort der Befragung) bin ich

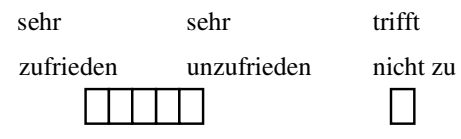

08. Mit dem Einkauf in Geschäft

(Name Stammgeschäft)

bin ich insgesamt

- $\quad$ Beratungsleistung

- $\quad$ Sortiment

- $\quad$ Service

- $\quad$ Einrichtung des Geschäfts

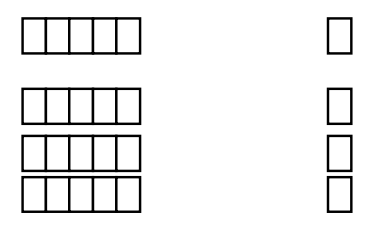

Bei den nächsten Aussagen sagen Sie mir bitte, ob Sie diesen Aussagen zustimmen und wie wichtig Ihnen die Reaktion Ihrer Umwelt ist.

$1=$ stimme ich voll zu; $5=$ stimme ich nicht $\mathrm{zu} ; 6=$ trifft nicht $\mathrm{zu}$ $1=$ sehr wichtig; $5=$ nicht wichtig

09. Im Nachbarland kann ich Produkte kaufen,

\begin{tabular}{cllll} 
stimme ich & stimme ich & trifft & sehr & nicht \\
voll zu & nicht zu & nicht zu & wichtig & wichtig \\
\hline & 0 & 0 & $\square$
\end{tabular}

die ich Zuhause nicht bekomme.

10. Im Nachbarland kaufe ich preiswerter
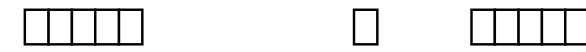

11. Im Nachbarland finde ich leichter die Produkte, die ich suche / die ich benötige.
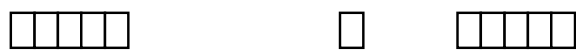

12. a. Im Nachbarland kaufe ich lieber bei großen Einzelhandelsketten ein

b. Im Nachbarland kaufe ich lieber in kleinen Fachgeschäften ein

13. Der Ort im Nachbarland in dem Einkäufe getätigt werden, sollte vom Heimatort mit öffentlichem Verkehrsmitteln gut erreichbar sein.
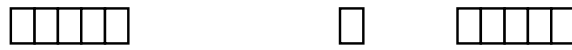

14. Bei nächster Gelegenheit kaufe ich wieder im Nachbarland ein
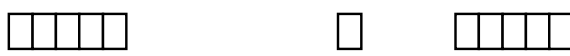

15. Einkaufen im Nachbarland macht mir Spaß, weil ich eine angenehme Umgebung mit Cafes und Unterhaltungsmöglichkeiten antreffe

16. Ich finde es schön, die Möglichkeit zu haben, schnell im Nachbarland einkaufen zu können (Wohlbefinden) 


\section{Stimmen Sie diesen Aussagen zu?}

17. Ich empfehle mein Geschäft (Stammgeschäft) im Nachbarland bei meinen Freunden und Bekannten weiter.

18. Ich möchte regelmäßig von meinem Geschäft (Stammgeschäft) im Nachbarland über Angebote und besondere Dienstleistungen informiert werden.

19. Mindestens 1mal im Monat besuche ich mein Stammgeschäft im Nachbarland.

20. In den vergangenen acht Wochen habe ich oft im Nachbarland eingekauft

21. In den vergangenen acht Wochen habe ich häufig in meinem Stammgeschäft eingekauft.

22. Sofern mein Stammgeschäft in Zukunft auch andere Produktkategorien anbietet, könnte ich mir vorstellen, auch dort zu kaufen.

\section{Zusatzfragen: Jetzt noch ein paar Fragen, die uns helfen, Ihre Antworten richtig einzuordnen.}

23. Wenn Sie an die nächsten Einkäufe denken,

in welchen Geschäften kaufen Sie im Nachbarland in erster Linie ein?

(für einen Typ entscheiden)

- Kleines Fachgeschäft mit Bedienung

- Filialisten wie HEMA, die ich von mehreren Einkaufsorten im Nachbarland kenne

- Filialisten wie H\&M, Deichmann (van Haren), die ich von mehreren Einkaufsorten im Heimatland kenne

- Discounter wie ALDI, LIDL

- Verbrauchermärkte wie Wal-Mart,Allkauf

- Warenhäuser wie Kaufhof

- $\quad$ sonstige Anbieter

Welche Produkte kaufen Sie dort hauptsächlich außer Lebensmittel?

- Gartenartikel

- $\quad$ Bekleidung

- Schuhe

- $\quad$ Elektroartikel

- $\quad$ sonstiges
2Gründe: 1. Nennung=1

2.Nennung $=2$

Preis

großes Sortiment

Größe des Geschäftes

Parkplätze

Beratung

Erreichb. Öffentl. Verkehrsmittel

Service

sonstiges

max. 2 nennen 1. Nennung 1

2.Nennung 2 
Allgemeiner Teil:

24. Frage

Nationalität

NL $\square$

D

Geschlecht

$\mathrm{m} \square$

w

Alter

bis 35

35-64

über 64

Ihr Wohnort PLZ

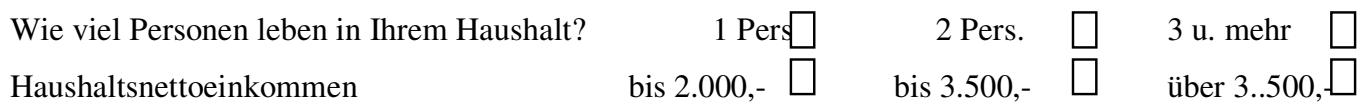

Mit welchem Verkehrsmittel kommen Sie in der Regel hier ins Nachbarland?

PKW $\square$ Bus $\square$ Bahn $\square$ Fahrrad $\square$ zu Fuß

Vielen Dank für Ihre Mitarbeit! 


\section{Anhang 3:}

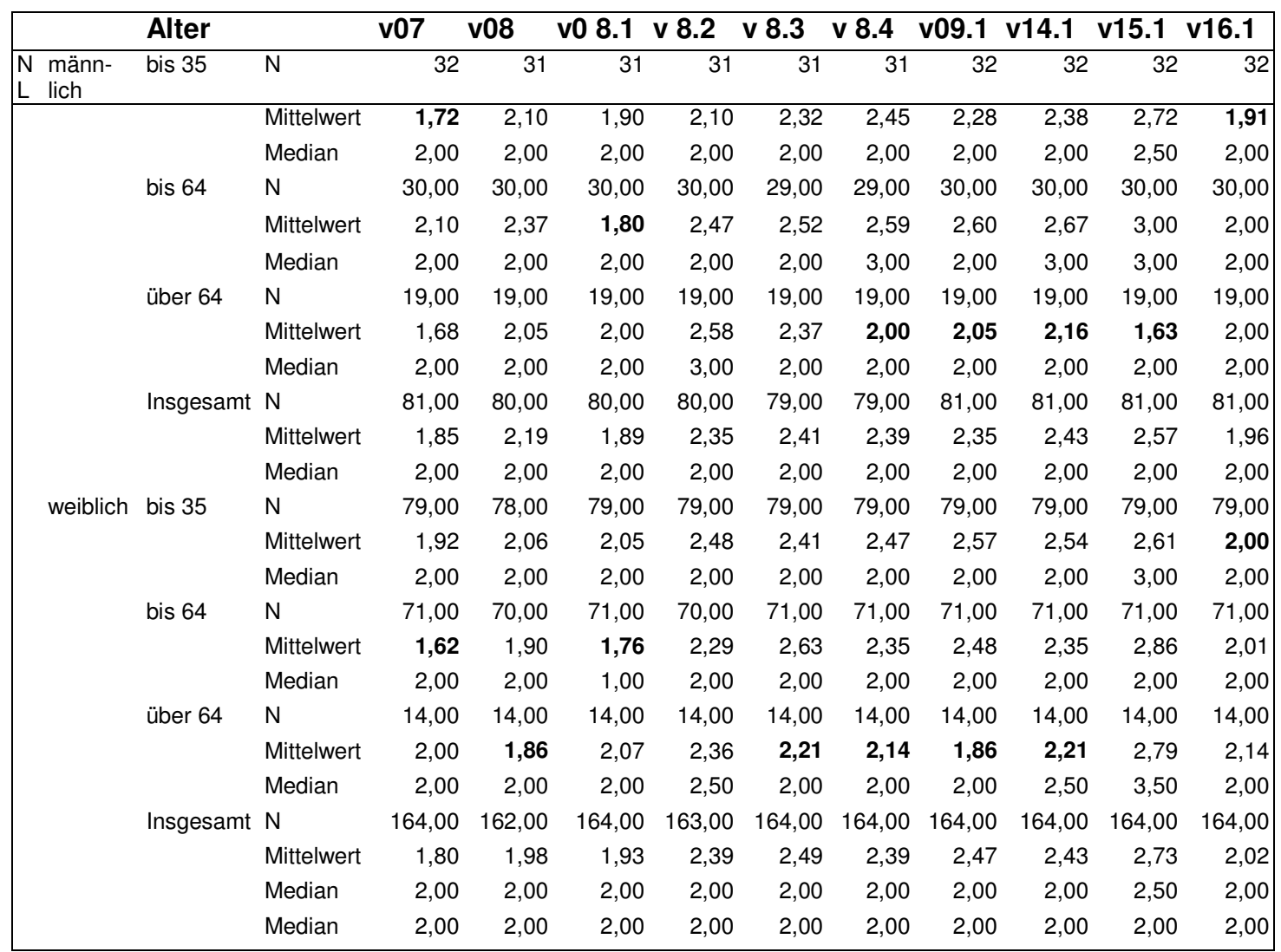

Einstellung der niederländischen Konsumenten zum Einkaufsort und zur Einkaufsstätte. 


\section{Anhang 4:}

\begin{tabular}{|c|c|c|c|c|c|c|c|c|c|c|c|c|}
\hline & Alter & & v07 & v08 & v8.1 & v8.2 & v8.3 & v8.4 & v09.1 & v14.1 & v15.1 & v16.1 \\
\hline $\begin{array}{l}\text { männ- } \\
\text { lich }\end{array}$ & bis 35 & $\bar{N}$ & 42,00 & 42,00 & 42,00 & 42,00 & 42,00 & 42,00 & 42,00 & 42,00 & 42,00 & 42,00 \\
\hline \multirow{23}{*}{ weiblich } & \multirow{5}{*}{ bis 64} & Mittelwert & 2,29 & 1,98 & 1,71 & 2,48 & 2,79 & 2,29 & 1,90 & 1,86 & 2,24 & 1,74 \\
\hline & & Median & 2,00 & 2,00 & 2,00 & 2,00 & 3,00 & 2,00 & 2,00 & 2,00 & 2,00 & 1,00 \\
\hline & & $\mathrm{N}$ & 45,00 & 45,00 & 45,00 & 45,00 & 45,00 & 45,00 & 45,00 & 45,00 & 45,00 & 45,00 \\
\hline & & Mittelwert & 1,84 & 1,80 & 1,69 & 2,09 & 2,31 & 2,04 & 2,00 & 1,96 & 2,13 & 1,80 \\
\hline & & Median & 2,00 & 2,00 & 2,00 & 2,00 & 2,00 & 2,00 & 2,00 & 2,00 & 2,00 & 2,00 \\
\hline & \multirow[t]{3}{*}{ über 64} & $\mathrm{~N}$ & 15,00 & 15,00 & 15,00 & 15,00 & 14,00 & 14,00 & 15,00 & 15,00 & 15,00 & 15,00 \\
\hline & & Mittelwert & 1,87 & 1,67 & 1,53 & 2,13 & 2,36 & 1,64 & 2,07 & 1,73 & 2,33 & 1,67 \\
\hline & & Median & 2,00 & 2,00 & 1,00 & 2,00 & 2,50 & 1,50 & 2,00 & 2,00 & 2,00 & 2,00 \\
\hline & \multirow[t]{3}{*}{ Insgesamt } & $N$ & 102,00 & 102,00 & 102,00 & 102,00 & 101,00 & 101,00 & 102,00 & 102,00 & 102,00 & 102,00 \\
\hline & & Mittelwert & 2,03 & 1,85 & 1,68 & 2,25 & 2,51 & 2,09 & 1,97 & 1,88 & 2,21 & 1,75 \\
\hline & & Median & 2,00 & 2,00 & 2,00 & 2,00 & 3,00 & 2,00 & 2,00 & 2,00 & 2,00 & 2,00 \\
\hline & \multirow[t]{3}{*}{ bis 35} & $\mathrm{~N}$ & 62,00 & 62,00 & 62,00 & 62,00 & 62,00 & 62,00 & 61,00 & 62,00 & 61,00 & 62,00 \\
\hline & & Mittelwert & 1,92 & 1,81 & 1,68 & 2,39 & 2,40 & 2,16 & 1,93 & 1,92 & 1,64 & 1,71 \\
\hline & & Median & 2,00 & 2,00 & 1,50 & 2,00 & 2,00 & 2,00 & 2,00 & 2,00 & 1,00 & 1,50 \\
\hline & \multirow[t]{3}{*}{ bis 64} & $\mathrm{~N}$ & 72,00 & 72,00 & 72,00 & 72,00 & 72,00 & 72,00 & 72,00 & 72,00 & 72,00 & 72,00 \\
\hline & & Mittelwert & 1,75 & 1,58 & 1,60 & 2,19 & 2,24 & 1,88 & 1,88 & 1,74 & 1,64 & 1,50 \\
\hline & & Median & 2,00 & 1,50 & 1,00 & 2,00 & 2,00 & 2,00 & 1,00 & 2,00 & 1,00 & 1,00 \\
\hline & \multirow[t]{3}{*}{ über 64} & $\mathrm{~N}$ & 17,00 & 17,00 & 17,00 & 17,00 & 17,00 & 17,00 & 17,00 & 17,00 & 17,00 & 17,00 \\
\hline & & Mittelwert & 1,94 & 1,53 & 1,65 & 1,94 & 1,82 & 1,53 & 1,71 & 2,00 & 1,82 & 1,88 \\
\hline & & Median & 2,00 & 1,00 & 2,00 & 1,00 & 2,00 & 1,00 & 2,00 & 1,00 & 2,00 & 2,00 \\
\hline & \multirow[t]{3}{*}{ Insgesamt } & $N$ & 151,00 & 151,00 & 151,00 & 151,00 & 151,00 & 151,00 & 150,00 & 151,00 & 150,00 & 151,00 \\
\hline & & Mitte & 1,84 & 1,67 & 1,64 & 2,25 & 2,26 & 1,95 & 1,88 & 1,84 & 1,66 & 1,63 \\
\hline & & Median & 2,00 & 2,00 & 1,00 & 2,00 & 2,00 & 2,00 & 2,00 & 2,00 & 1,00 & 1,0 \\
\hline
\end{tabular}

Einstellung der deutschen Konsumenten zum Einkaufsort und zur Einkaufsstätte. 


\section{Anhang 5:}

\begin{tabular}{|c|c|c|}
\hline Konstrukt & Indikatoren & Bedeutung \\
\hline TZSN & v032 v033 v034 & Werturteile der sozialen Gruppe \\
\hline WSN & v04 v06 & $\begin{array}{l}\text { Wichtigkeit der sozialen Norm, d.h. Freunde } \\
\text { und Bekannte kaufen viel im Nachbarland } \\
\text { und empfehlen es oft }\end{array}$ \\
\hline GKDZ SN & Vo1 V05 & $\begin{array}{l}\text { Bedeutung der sozialen Norm für den Kon- } \\
\text { sumenten }\end{array}$ \\
\hline GKDZ Kauf & v08 v082 v083 v084 & $\begin{array}{l}\text { Gesamtkundenzufriedenheit mit Stammge- } \\
\text { schäft im Nachbarland }\end{array}$ \\
\hline GLSS & 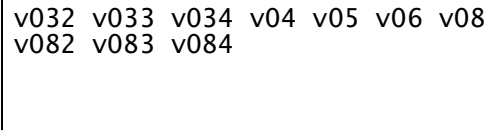 & $\begin{array}{l}\text { Die Zufriedenheit des Konsumenten mit dem } \\
\text { Kauf in der Einkaufsstätte und seine Zufrie- } \\
\text { denheit mit der Reaktion seines sozialen } \\
\text { Umfeldes auf den Kauf }\end{array}$ \\
\hline $\mathrm{KL}$ & v17 v18 v22 & Kundenloyalität (Treue zum Stammgeschäft) \\
\hline WRS & v141 v151 v161 & $\begin{array}{l}\text { Wahrgenommene Selbstrelevanz (Lust auf } \\
\text { Einkaufen im Nachbarland) }\end{array}$ \\
\hline BINDUNG & v19 v20 v21 & $\begin{array}{l}\text { (tatsächliches häufiges Einkaufen im Nach- } \\
\text { barland) }\end{array}$ \\
\hline
\end{tabular}

Konstrukte, Indikatoren und ihre Bedeutung im Rahmen des Strukturgleichungsmodells 


\section{Anlage 6:}

\begin{tabular}{|c|c|c|c|c|c|c|c|c|}
\hline \multirow{3}{*}{$\mathrm{N}$} & \multicolumn{2}{|c|}{ Cluster 1} & \multicolumn{2}{|c|}{ Cluster 2} & \multicolumn{2}{|c|}{ Cluster 3} & \multicolumn{2}{|l|}{ Cluster 4} \\
\hline & \multicolumn{2}{|l|}{53} & \multicolumn{2}{|c|}{56} & \multicolumn{2}{|c|}{94} & \multicolumn{2}{|c|}{50} \\
\hline & $\begin{array}{c}\text { Mittel- } \\
\text { wert }\end{array}$ & $\begin{array}{l}\text { Mittel- } \\
\text { wert } \\
\text { Gesamt }\end{array}$ & $\begin{array}{c}\text { Mittel- } \\
\text { wert }\end{array}$ & $\begin{array}{l}\text { Mittel- } \\
\text { wert } \\
\text { Gesamt }\end{array}$ & $\begin{array}{c}\text { Mittel- } \\
\text { wert }\end{array}$ & $\begin{array}{l}\text { Mittel- } \\
\text { wert } \\
\text { Gesamt }\end{array}$ & $\begin{array}{l}\text { Mittel- } \\
\text { wert }\end{array}$ & $\begin{array}{l}\text { Mittelwer } \\
\text { Gesamt }\end{array}$ \\
\hline $\begin{array}{l}\text { Bereits als Kind fuhr ich mit meinen } \\
\text { Eltern zum Einkauf über die Grenze }\end{array}$ & 2,17 & 2,45 & 2,93 & 2,45 & 2,72 & 2,45 & 1,70 & 2,45 \\
\hline $\begin{array}{l}\text { Für mich ist der Einkauf Nachbarland } \\
\text { ein Stück Lebensqualität }\end{array}$ & 2,57 & 2,32 & 1,95 & 2,32 & 2,74 & 2,32 & 1,66 & 2,32 \\
\hline $\begin{array}{l}\text { Mein Freundes/Bekanntenkreis } \\
\text { findet, dass man im Nachbarland } \\
\text { preislich besser einkaufen kann }\end{array}$ & 3,23 & 2,54 & 2,11 & 2,54 & 2,53 & 2,54 & 2,32 & 2,54 \\
\hline $\begin{array}{l}\text { Mein Freundes/Bekanntenkreis } \\
\text { findet, dass man im Nachbarland } \\
\text { qualitativ besser einkaufen kann }\end{array}$ & 3,94 & 2,74 & 2,38 & 2,74 & 2,80 & 2,74 & 1,78 & 2,74 \\
\hline $\begin{array}{l}\text { Mein Freundes/Bekanntenkreis } \\
\text { findet, dass man im Nachbarland } \\
\text { bessere Beratung erhält }\end{array}$ & 3,68 & 2,96 & 3,07 & 2,96 & 3,07 & 2,96 & 1,84 & 2,96 \\
\hline $\begin{array}{l}\text { Mein Freundes/Bekanntenkreis } \\
\text { findet, dass man im Nachbarland } \\
\text { ausgefallenere Design/Produkte } \\
\text { erhält }\end{array}$ & 3,34 & 2,08 & 2,04 & 2,08 & 1,78 & 2,08 & 1,34 & 2,08 \\
\hline $\begin{array}{l}\text { Wie oft kaufen Freunde im Nachbar- } \\
\text { land? }\end{array}$ & 2,64 & 2,33 & 2,21 & 2,33 & 2,39 & 2,33 & 2,02 & 2,33 \\
\hline $\begin{array}{l}\text { Freunde/Bekannte reagieren auf } \\
\text { meinen Einkauf im Nachbarland } \\
\text { positiv }\end{array}$ & 2,70 & 2,06 & 1,80 & 2,06 & 2,10 & 2,06 & 1,58 & 2,06 \\
\hline $\begin{array}{l}\text { Freunde haben Einkauf im Nachbar- } \\
\text { land empfohlen }\end{array}$ & 2,51 & 2,22 & 2,18 & 2,22 & 2,24 & 2,22 & 1,90 & 2,22 \\
\hline $\begin{array}{l}\text { Mit den Einkaufsmöglichkeiten } \\
\text { Ort/Befragung bin ich zufrieden }\end{array}$ & 2,49 & 1,92 & 1,68 & 1,92 & 1,80 & 1,92 & 1,80 & 1,92 \\
\hline $\begin{array}{l}\text { Mit dem Einkauf in meinem Stamm- } \\
\text { geschäft bin ich zufrieden }\end{array}$ & 2,08 & 1,74 & 1,59 & 1,74 & 1,80 & 1,74 & 1,46 & 1,74 \\
\hline $\begin{array}{l}\text { Zufriedenheit Sortiment } \\
\text { Stammgeschäft }\end{array}$ & 2,04 & 1,65 & 1,50 & 1,65 & 1,68 & 1,65 & 1,36 & 1,65 \\
\hline Zufriedenheit Service Stammgeschäft & 2,72 & 2,25 & 2,25 & 2,25 & 2,32 & 2,25 & 1,62 & 2,25 \\
\hline $\begin{array}{l}\text { Zufriedenehit Beratung } \\
\text { Stammgeschäft }\end{array}$ & 2,83 & 2,36 & 2,50 & 2,36 & 2,51 & 2,36 & 1,42 & 2,36 \\
\hline $\begin{array}{l}\text { Zufriedenheit Einrichtung } \\
\text { Stammgeschäft }\end{array}$ & 2,40 & 2,01 & 2,00 & 2,01 & 2,09 & 2,01 & 1,46 & 2,01 \\
\hline $\begin{array}{l}\text { Im Nachbarland kann ich Produkte } \\
\text { kaufen, die ich Zuhause nicht be- } \\
\text { komme }\end{array}$ & 2,83 & 1,92 & 1,89 & 1,92 & 1,72 & 1,92 & 1,34 & 1,92 \\
\hline $\begin{array}{l}\text { Im Nachbarland kaufe ich preiswerter } \\
\text { Zustimmung }\end{array}$ & 3,36 & 2,69 & 2,39 & 2,69 & 2,65 & 2,69 & 2,40 & 2,69 \\
\hline $\begin{array}{l}\text { Im Nachbarland finde ich Produkte } \\
\text { leichter die ich suche Zustimmung }\end{array}$ & 3,19 & 2,46 & 2,21 & 2,46 & 2,61 & 2,46 & 1,70 & 2,46 \\
\hline $\begin{array}{l}\text { Im Nachbarland kaufe ich lieber bei } \\
\text { Einzelhandelsketten Zustimmung }\end{array}$ & 3,28 & 2,92 & 2,43 & 2,92 & 2,95 & 2,92 & 3,02 & 2,92 \\
\hline $\begin{array}{l}\text { Im Nachbarland kaufe ich lieber in } \\
\text { kleinen Einzelhandelsgeschäften ein } \\
\text { Zustimmung }\end{array}$ & 2,75 & 2,28 & 2,73 & 2,28 & 2,20 & 2,28 & 1,44 & 2,28 \\
\hline $\begin{array}{l}\text { Der Ort im Nachbarland in dem } \\
\text { Einkäufe getätigt werden, sollte vom } \\
\text { Heimatort mit öffentlichen Verkehrs- } \\
\text { mitteln gut erreichbar Zustimmung }\end{array}$ & 3,32 & 2,65 & 3,07 & 2,65 & 2,16 & 2,65 & 2,40 & 2,65 \\
\hline $\begin{array}{l}\text { Bei nächster Gelegenheit kaufe ich } \\
\text { wieder im Nachbarland Zustimmung }\end{array}$ & 2,32 & 1,86 & 1,75 & 1,86 & 1,72 & 1,86 & 1,74 & 1,86 \\
\hline $\begin{array}{l}\text { Einkaufen im Ausland macht } \\
\text { mirSpass, weil ich eine angenehme } \\
\text { Umgebung mit Cafes und Unterhal- } \\
\text { tungsmöglichkeiten antreffe Zustim- } \\
\text { mung }\end{array}$ & 2,55 & 1,88 & 2,00 & 1,88 & 1,81 & 1,88 & 1,18 & 1,88 \\
\hline $\begin{array}{l}\text { Ich finde es schön, die Möglichkeit zu } \\
\text { haben, schnell im Nachbarland ein- } \\
\text { kaufen zu können Wohlbefinden } \\
\text { Zustimmung }\end{array}$ & 2,04 & 1,68 & 1,64 & 1,68 & 1,71 & 1,68 & 1,28 & 1,68 \\
\hline
\end{tabular}




\begin{tabular}{|l|l|l|l|l|l|l|l|l|}
\hline $\begin{array}{l}\text { Ich empfehle mein Stammgeschäft } \\
\text { im Nachbarland bei meinen Freun- } \\
\text { den und Bekannten weiter }\end{array}$ & 2,43 & 2,04 & 1,68 & 2,04 & 2,33 & 2,04 & 1,46 & 2,04 \\
\hline $\begin{array}{l}\text { Ich möchte regelmäßig von meinem } \\
\text { Stammgeschäft im Nachbarland über } \\
\text { Angebote und besondere Dienstleis- } \\
\text { tungen informiert }\end{array}$ & 2,89 & 2,66 & 1,84 & 2,66 & 3,57 & 2,66 & 1,60 & 2,66 \\
\hline $\begin{array}{l}\text { Mindestens einmal im Monat besu- } \\
\text { che ich mein Stammgeschäft im } \\
\text { Nachbarland }\end{array}$ & 3,47 & 2,81 & 2,16 & 2,81 & 3,48 & 2,81 & 1,56 & 2,81 \\
\hline $\begin{array}{l}\text { In den vergangenen acht Wochen } \\
\text { habe ich oft im Nachbarland einge- } \\
\text { kauft }\end{array}$ & 3,38 & 2,48 & 1,79 & 2,48 & 2,90 & 2,48 & 1,50 & 2,48 \\
\hline $\begin{array}{l}\text { in den letzten acht Wochen habe ich } \\
\text { oft im Stammgeschäft eingekauft }\end{array}$ & 3,79 & 2,65 & 1,96 & 2,65 & 3,07 & 2,65 & 1,40 & 2,65 \\
\hline $\begin{array}{l}\text { Sofern mein Stammgeschäft in Zu- } \\
\text { kunft auch andere Produkte anbietet, } \\
\text { könnte ich mir vorstellen, diese auch } \\
\text { dort zu kaufen Cross buying }\end{array}$ & 2,42 & 2,15 & 1,66 & 2,15 & 2,63 & 2,15 & 1,52 & 2,15 \\
\hline
\end{tabular}

\section{Clusteranalyse der Fälle für deutsche Konsumenten}




\section{Anhang 7:}

\section{Geschlecht}

\begin{tabular}{|ll|r|r|r|r|}
\hline & Häufigkeit & \multicolumn{1}{c|}{$\begin{array}{c}\text { Pro- } \\
\text { zent }\end{array}$} & $\begin{array}{c}\text { Gültige } \\
\text { Prozente }\end{array}$ & $\begin{array}{c}\text { Kumulierte } \\
\text { Prozente }\end{array}$ \\
\hline Gültig & männlich & 25 & 47,2 & 47,2 & 47,2 \\
& weiblich & 28 & 52,8 & 52,8 & 100,0 \\
& Gesamt & 53 & 100,0 & 100,0 & \\
\hline
\end{tabular}

Alter

\begin{tabular}{|ll|r|r|r|r|}
\hline & Häufigkeit & Prozent & $\begin{array}{c}\text { Gültige } \\
\text { Prozente }\end{array}$ & $\begin{array}{c}\text { Kumulierte } \\
\text { Prozente }\end{array}$ \\
\hline Gültig & Bis 35 & 23 & 43,4 & 43,4 & 43,4 \\
& 35 bis & 26 & 49,1 & 49,1 & 92,5 \\
64 & 4 & 7,5 & 7,5 & 100,0 \\
& Über 64 & 53 & 100,0 & 100,0 & \\
Gesamt & &
\end{tabular}

Anzahl Personen im Haushalt

\begin{tabular}{|rl|r|r|r|r|}
\hline & Häufigkeit & Prozent & $\begin{array}{c}\text { Gültige } \\
\text { Prozente }\end{array}$ & $\begin{array}{c}\text { Kumulierte } \\
\text { Prozente }\end{array}$ \\
\hline Gültig & 1 & 10 & 18,9 & 18,9 & 18,9 \\
& 2 & 28 & 52,8 & 52,8 & 71,7 \\
& 3 und & 15 & 28,3 & 28,3 & 100,0 \\
mehr & 53 & 100,0 & 100,0 & \\
Gesamt & &
\end{tabular}

Haushaltsnettoeinkommen

\begin{tabular}{|c|c|c|c|c|c|}
\hline & & Häufigkeit & Prozent & $\begin{array}{l}\text { Gültige } \\
\text { Prozente }\end{array}$ & $\begin{array}{l}\text { Kumulierte } \\
\text { Prozente }\end{array}$ \\
\hline Gültig & $\begin{array}{l}\text { Bis } \\
2.000,- \\
\text { Bis } \\
3.500,- \\
\text { Über } \\
\text { 3.500,- } \\
\text { Gesamt }\end{array}$ & $\begin{array}{r}28 \\
16 \\
9 \\
53\end{array}$ & $\begin{array}{r}52,8 \\
30,2 \\
17,0 \\
100,0\end{array}$ & $\begin{array}{r}52,8 \\
30,2 \\
17,0 \\
100,0\end{array}$ & $\begin{array}{r}52,8 \\
83,0 \\
100,0\end{array}$ \\
\hline
\end{tabular}

Verkehrsmittel

\begin{tabular}{|rl|r|r|r|r|}
\hline & Häufigkeit & Prozent & $\begin{array}{c}\text { Gültige } \\
\text { Prozente }\end{array}$ & $\begin{array}{c}\text { Kumulierte } \\
\text { Prozente }\end{array}$ \\
\hline Gültig & Auto & 52 & 98,1 & 98,1 & 98,1 \\
& Bus & 1 & 1,9 & 1,9 & 100,0 \\
& Gesamt & 53 & 100,0 & 100,0 & \\
\hline
\end{tabular}


„Die Minimalkunden“ (Cluster 1) deutsche Konsumenten

\section{Anhang 8:}

Geschlecht

\begin{tabular}{|ll|r|r|r|r|}
\hline & Häufigkeit & Prozent & $\begin{array}{c}\text { Gültige } \\
\text { Prozente }\end{array}$ & $\begin{array}{c}\text { Kumulierte } \\
\text { Prozente }\end{array}$ \\
\hline Gültig & männlich & 24 & 42,9 & 42,9 & 42,9 \\
& weiblich & 32 & 57,1 & 57,1 & 100,0 \\
& Gesamt & 56 & 100,0 & 100,0 & \\
\hline
\end{tabular}

Alter

\begin{tabular}{|rl|r|r|r|r|}
\hline & Häufigkeit & Prozent & $\begin{array}{c}\text { Gültige } \\
\text { Prozente }\end{array}$ & $\begin{array}{c}\text { Kumulierte } \\
\text { Prozente }\end{array}$ \\
\hline Gültig & Bis 35 & 20 & 35,7 & 35,7 & 35,7 \\
& 35 bis & 26 & 46,4 & 46,4 & 82,1 \\
64 & 10 & 17,9 & 17,9 & 100,0 \\
& Über 64 & 56 & 100,0 & 100,0 & \\
& Gesamt & &
\end{tabular}

Anzahl Personen im Haushalt

\begin{tabular}{|rl|r|r|r|r|}
\hline & Häufigkeit & Prozent & $\begin{array}{c}\text { Gültige } \\
\text { Prozente }\end{array}$ & $\begin{array}{c}\text { Kumulierte } \\
\text { Prozente }\end{array}$ \\
\hline Gültig & 1 & 4 & 7,1 & 7,1 & 7,1 \\
& 2 & 25 & 44,6 & 44,6 & 51,8 \\
& 3 und & 27 & 48,2 & 48,2 & 100,0 \\
mehr & 56 & 100,0 & 100,0 & \\
\hline
\end{tabular}

Haushaltsnettoeinkommen

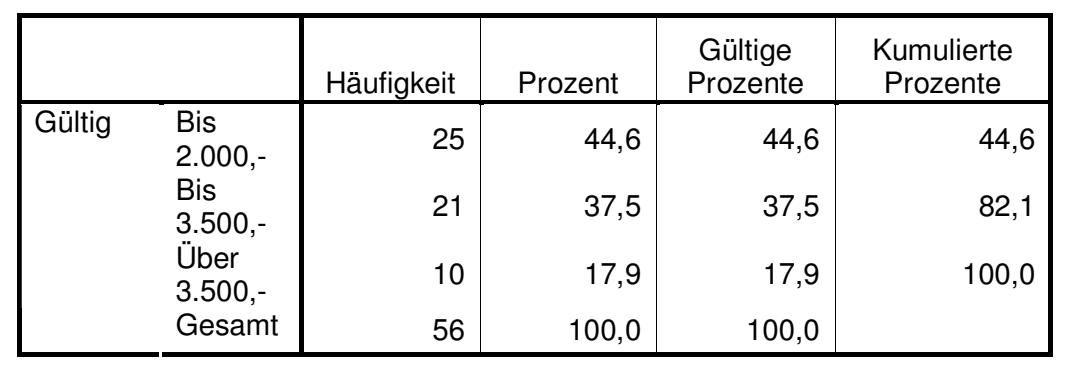

\section{Verkehrsmittel}

\begin{tabular}{|rl|r|r|r|r|}
\hline & Häufigkeit & Prozent & $\begin{array}{c}\text { Gültige } \\
\text { Prozente }\end{array}$ & $\begin{array}{c}\text { Kumulierte } \\
\text { Prozente }\end{array}$ \\
\hline Gültig & Auto & 49 & 87,5 & 87,5 & 87,5 \\
& Bus & 2 & 3,6 & 3,6 & 91,1 \\
& Bahn & 5 & 8,9 & 8,9 & 100,0 \\
& Gesamt & 56 & 100,0 & 100,0 & \\
\hline
\end{tabular}




\section{Anhang 9}

\section{Geschlecht}

\begin{tabular}{|ll|r|r|r|r|}
\hline & Häufigkeit & Prozent & $\begin{array}{c}\text { Gültige } \\
\text { Prozente }\end{array}$ & $\begin{array}{c}\text { Kumulierte } \\
\text { Prozente }\end{array}$ \\
\hline Gültig & männlich & 43 & 45,7 & 45,7 & 45,7 \\
& weiblich & 51 & 54,3 & 54,3 & 100,0 \\
& Gesamt & 94 & 100,0 & 100,0 & \\
\hline
\end{tabular}

Alter

\begin{tabular}{|rl|r|r|r|r|}
\hline & Häufigkeit & Prozent & $\begin{array}{c}\text { Gültige } \\
\text { Prozente }\end{array}$ & $\begin{array}{c}\text { Kumulierte } \\
\text { Prozente }\end{array}$ \\
\hline Gültig & Bis 35 & 47 & 50,0 & 50,0 & 50,0 \\
& 35 bis & 37 & 39,4 & 39,4 & 89,4 \\
64 & 10 & 10,6 & 10,6 & 100,0 \\
& Über 64 & 94 & 100,0 & 100,0 & \\
& Gesamt & 9 &
\end{tabular}

Anzahl Personen im Haushalt

\begin{tabular}{|rl|r|r|r|r|}
\hline & Häufigkeit & Prozent & $\begin{array}{c}\text { Gültige } \\
\text { Prozente }\end{array}$ & $\begin{array}{c}\text { Kumulierte } \\
\text { Prozente }\end{array}$ \\
\hline Gültig & 1 & 23 & 24,5 & 24,5 & 24,5 \\
& 2 & 38 & 40,4 & 40,4 & 64,9 \\
& 3 und & 33 & 35,1 & 35,1 & 100,0 \\
mehr & 94 & 100,0 & 100,0 & \\
Gesamt & &
\end{tabular}

Haushaltsnettoeinkommen

\begin{tabular}{|c|c|c|c|c|c|}
\hline & & Häufigkeit & Prozent & $\begin{array}{l}\text { Gültige } \\
\text { Prozente }\end{array}$ & $\begin{array}{l}\text { Kumulierte } \\
\text { Prozente }\end{array}$ \\
\hline \multirow[t]{4}{*}{ Gültig } & \multirow{4}{*}{$\begin{array}{l}\text { Bis } \\
2.000,- \\
\text { Bis } \\
3.500,- \\
\text { Über } \\
\text { 3.500,-- } \\
\text { Gesamt }\end{array}$} & 41 & 43,6 & 43,6 & 43,6 \\
\hline & & 38 & 40,4 & 40,4 & 84,0 \\
\hline & & 15 & 16,0 & 16,0 & 100,0 \\
\hline & & 94 & 100,0 & 100,0 & \\
\hline
\end{tabular}

Verkehrsmittel

\begin{tabular}{|ll|r|r|r|r|}
\hline & Häufigkeit & Prozent & $\begin{array}{c}\text { Gültige } \\
\text { Prozente }\end{array}$ & $\begin{array}{c}\text { Kumulierte } \\
\text { Prozente }\end{array}$ \\
\hline Gültig & Auto & 77 & 81,9 & 81,9 & 81,9 \\
& Bus & 8 & 8,5 & 8,5 & 90,4 \\
Bahn & 8 & 8,5 & 8,5 & 98,9 \\
& Fahrrad & 1 & 1,1 & 1,1 & 100,0 \\
Gesamt & 94 & 100,0 & 100,0 & \\
\hline
\end{tabular}

„Die Gelegenheitskunden“ (Cluster3) deutsche Konsumenten 


\section{Anhang 10:}

Geschlecht

\begin{tabular}{|ll|r|r|r|r|}
\hline & Häufigkeit & Prozent & $\begin{array}{c}\text { Gültige } \\
\text { Prozente }\end{array}$ & $\begin{array}{c}\text { Kumulierte } \\
\text { Prozente }\end{array}$ \\
\hline Gültig & männlich & 10 & 20,0 & 20,0 & 20,0 \\
& weiblich & 40 & 80,0 & 80,0 & 100,0 \\
& Gesamt & 50 & 100,0 & 100,0 & \\
\hline
\end{tabular}

Alter

\begin{tabular}{|rl|r|r|r|r|}
\hline & Häufigkeit & Prozent & $\begin{array}{c}\text { Gültige } \\
\text { Prozente }\end{array}$ & $\begin{array}{c}\text { Kumulierte } \\
\text { Prozente }\end{array}$ \\
\hline Gültig & Bis 35 & 14 & 28,0 & 28,0 & 28,0 \\
& 35 bis & 28 & 56,0 & 56,0 & 84,0 \\
64 & 8 & 16,0 & 16,0 & 100,0 \\
& Über 64 & 50 & 100,0 & 100,0 & \\
Gesamt & &
\end{tabular}

Anzahl Personen im Haushalt

\begin{tabular}{|rl|r|r|r|r|}
\hline & Häufigkeit & Prozent & $\begin{array}{c}\text { Gültige } \\
\text { Prozente }\end{array}$ & $\begin{array}{c}\text { Kumulierte } \\
\text { Prozente }\end{array}$ \\
\hline Gültig & 1 & 6 & 12,0 & 12,0 & 12,0 \\
& 2 & 21 & 42,0 & 42,0 & 54,0 \\
& 3 und & 23 & 46,0 & 46,0 & 100,0 \\
mehr & 50 & 100,0 & 100,0 & \\
\hline
\end{tabular}

Haushaltsnettoeinkommen

\begin{tabular}{|rl|r|r|r|r|}
\hline & Häufigkeit & Prozent & \multicolumn{1}{c|}{$\begin{array}{c}\text { Gültige } \\
\text { Prozente }\end{array}$} & $\begin{array}{c}\text { Kumulierte } \\
\text { Prozente }\end{array}$ \\
\hline Gültig & Bis & 14 & 28,0 & 28,0 & 28,0 \\
& $\begin{array}{r}\text { 2.000,- } \\
\text { Bis }\end{array}$ & 21 & 42,0 & 42,0 & 70,0 \\
& $\begin{array}{r}\text { 3.500,- } \\
\text { Über } \\
\text { 3.500,- } \\
\text { Gesamt }\end{array}$ & 15 & 30,0 & 30,0 & 100,0 \\
\hline
\end{tabular}

Verkehrsmittel

\begin{tabular}{|ll|r|r|r|r|}
\hline & Häufigkeit & Prozent & $\begin{array}{c}\text { Gültige } \\
\text { Prozente }\end{array}$ & $\begin{array}{c}\text { Kumulierte } \\
\text { Prozente }\end{array}$ \\
\hline Gültig & Auto & 44 & 88,0 & 88,0 & 88,0 \\
& Bahn & 6 & 12,0 & 12,0 & 100,0 \\
& Gesamt & 50 & 100,0 & 100,0 & \\
\hline
\end{tabular}

„Die begeisterten Fachgeschäftskunden“(Cluster 4) deutsche Konsumenten 


\section{Anlage 11:}

\begin{tabular}{|c|c|c|c|c|c|c|c|c|}
\hline \multirow{3}{*}{$\mathrm{N}$} & \multicolumn{2}{|c|}{ Cluster 1} & \multicolumn{2}{|c|}{ Cluster 2} & \multicolumn{2}{|c|}{ Cluster 3} & \multicolumn{2}{|l|}{ Cluster 4} \\
\hline & \multicolumn{2}{|l|}{36} & \multicolumn{2}{|c|}{118} & \multicolumn{2}{|c|}{50} & \multicolumn{2}{|c|}{41} \\
\hline & $\begin{array}{c}\text { Mit- } \\
\text { tel- } \\
\text { wert }\end{array}$ & $\begin{array}{c}\text { Mittel- } \\
\text { wert } \\
\text { Gesamt }\end{array}$ & $\begin{array}{l}\text { Mittel- } \\
\text { wert }\end{array}$ & $\begin{array}{l}\text { Mittel- } \\
\text { wert } \\
\text { Gesamt }\end{array}$ & $\begin{array}{l}\text { Mittel- } \\
\text { wert }\end{array}$ & $\begin{array}{l}\text { Mittel- } \\
\text { wert } \\
\text { Gesamt }\end{array}$ & $\begin{array}{l}\text { Mittel- } \\
\text { wert }\end{array}$ & $\begin{array}{c}\text { Mittel- } \\
\text { wert } \\
\text { Gesamt }\end{array}$ \\
\hline $\begin{array}{l}\text { Bereits als Kind fuhr ich mit meinen Eltern } \\
\text { zum Einkauf über die Grenze }\end{array}$ & 3,03 & 2,66 & 2,73 & 2,66 & 2,42 & 2,66 & 2,44 & 2,66 \\
\hline $\begin{array}{l}\text { Für mich ist der Einkauf Nachbarland ein } \\
\text { Stück Lebensqualität }\end{array}$ & 3,47 & 2,91 & 2,87 & 2,91 & 2,22 & 2,91 & 3,37 & 2,91 \\
\hline $\begin{array}{l}\text { Mein Freundes/Bekanntenkreis findet, dass } \\
\text { man im Nachbarland preislich besser einkau- } \\
\text { fen kann }\end{array}$ & 2,61 & 2,29 & 2,48 & 2,29 & 2,14 & 2,29 & 1,63 & 2,29 \\
\hline $\begin{array}{l}\text { Mein Freundes/Bekanntenkreis findet, dass } \\
\text { man im Nachbarland qualitativ besser einkau- } \\
\text { fen kann }\end{array}$ & 3,56 & 2,66 & 2,74 & 2,66 & 1,66 & 2,66 & 2,88 & 2,66 \\
\hline $\begin{array}{l}\text { Mein Freundes/Bekanntenkreis findet, dass } \\
\text { man im Nachbarland bessere Beratung erhält }\end{array}$ & 3,86 & 2,98 & 2,90 & 2,98 & 1,94 & 2,98 & 3,68 & 2,98 \\
\hline $\begin{array}{l}\text { Mein Freundes/Bekanntenkreis findet, dass } \\
\text { man im Nachbarland ausgefallenere De- } \\
\text { sign/Produkte erhält }\end{array}$ & 3,28 & 2,67 & 2,80 & 2,67 & 1,76 & 2,67 & 2,85 & 2,67 \\
\hline Wie oft kaufen Freunde im Nachbarland? & 2,81 & 2,47 & 2,43 & 2,47 & 2,32 & 2,47 & 2,44 & 2,47 \\
\hline $\begin{array}{l}\text { Freunde/Bekannte reagieren auf meinen } \\
\text { Einkauf im Nachbarland positiv }\end{array}$ & 2,97 & 2,46 & 2,70 & 2,46 & 1,90 & 2,46 & 2,00 & 2,46 \\
\hline $\begin{array}{l}\text { Freunde haben Einkauf im Nachbarland emp- } \\
\text { fohlen }\end{array}$ & 2,89 & 2,40 & 2,34 & 2,40 & 2,10 & 2,40 & 2,49 & 2,40 \\
\hline $\begin{array}{l}\text { Mit den Einkaufsmöglichkeiten Ort/Befragung } \\
\text { bin ich zufrieden }\end{array}$ & 2,22 & 1,82 & 1,93 & 1,82 & 1,44 & 1,82 & 1,59 & 1,82 \\
\hline $\begin{array}{l}\text { Mit dem Einkauf in meinem Stammgeschäft } \\
\text { bin ich zufrieden }\end{array}$ & 2,03 & 2,04 & 2,36 & 2,04 & 1,36 & 2,04 & 1,98 & 2,04 \\
\hline Zufriedenheit Sortiment Stammgeschäft & 2,22 & 1,91 & 2,21 & 1,91 & 1,28 & 1,91 & 1,56 & 1,91 \\
\hline Zufriedenheit Service Stammgeschäft & 2,69 & 2,37 & 2,50 & 2,37 & 1,48 & 2,37 & 2,80 & 2,37 \\
\hline Zufriedenehit Beratung Stammgeschäft & 2,81 & 2,46 & 2,54 & 2,46 & 1,42 & 2,46 & 3,17 & 2,46 \\
\hline Zufriedenheit Einrichtung Stammgeschäft & 2,86 & 2,39 & 2,41 & 2,39 & 1,52 & 2,39 & 2,98 & 2,39 \\
\hline $\begin{array}{l}\text { Im Nachbarland kann ich Produkte kaufen, die } \\
\text { ich Zuhause nicht bekomme }\end{array}$ & 2,69 & 2,43 & 2,74 & 2,43 & 2,00 & 2,43 & 1,83 & 2,43 \\
\hline $\begin{array}{l}\text { Im Nachbarland kaufe ich preiswerter Zu- } \\
\text { stimmung }\end{array}$ & 2,67 & 2,46 & 2,71 & 2,46 & 2,10 & 2,46 & 2,00 & 2,46 \\
\hline $\begin{array}{l}\text { Im Nachbarland finde ich Produkte leichter die } \\
\text { ich suche Zustimmung }\end{array}$ & 3,75 & 2,68 & 2,69 & 2,68 & 1,86 & 2,68 & 2,68 & 2,68 \\
\hline $\begin{array}{l}\text { Im Nachbarland kaufe ich lieber bei Einzel- } \\
\text { handelsketten Zustimmung }\end{array}$ & 2,56 & 2,55 & 2,58 & 2,55 & 2,32 & 2,55 & 2,73 & 2,55 \\
\hline $\begin{array}{l}\text { Im Nachbarland kaufe ich lieber in kleinen } \\
\text { Einzelhandelsgeschäften ein Zustimmung }\end{array}$ & 3,33 & 3,12 & 3,12 & 3,12 & 2,62 & 3,12 & 3,56 & 3,12 \\
\hline $\begin{array}{l}\text { Der Ort im Nachbarland in dem Einkäufe } \\
\text { getätigt werden, sollte vom Heimatort mit } \\
\text { offentlichen Verkehrsmitteln gut erreichbar } \\
\text { Zustimmung }\end{array}$ & 2,39 & 2,96 & 2,98 & 2,96 & 1,94 & 2,96 & 4,66 & 2,96 \\
\hline $\begin{array}{l}\text { Bei nächster Gelegenheit kaufe ich wieder im } \\
\text { Nachbarland Zustimmung }\end{array}$ & 2,81 & 2,43 & 2,77 & 2,43 & 1,92 & 2,43 & 1,76 & 2,43 \\
\hline $\begin{array}{l}\text { Einkaufen im Ausland macht mirSpass, weil } \\
\text { ich eine angenehme Umgebung mit Cafes und } \\
\text { Unterhaltungsmöglichkeiten antreffe Zustim- } \\
\text { mung }\end{array}$ & 3,00 & 2,68 & 2,49 & 2,68 & 2,00 & 2,68 & 3,76 & 2,68 \\
\hline $\begin{array}{l}\text { Ich finde es schön, die Möglichkeit zu haben, } \\
\text { schnell im Nachbarland einkaufen zu können } \\
\text { Wohlbefinden Zustimmung }\end{array}$ & 2,25 & 2,00 & 2,23 & 2,00 & 1,76 & 2,00 & 1,41 & 2,00 \\
\hline $\begin{array}{l}\text { Ich empfehle mein Stammgeschäft im Nach- } \\
\text { barland bei meinen Freunden und Bekannten } \\
\text { weiter }\end{array}$ & 2,69 & 2,22 & 2,56 & 2,22 & 1,62 & 2,22 & 1,54 & 2,22 \\
\hline
\end{tabular}




\begin{tabular}{|l|l|l|l|l|l|l|l|l|}
\hline $\begin{array}{l}\text { Ich mö̈chte regelmäßig von meinem Stamm- } \\
\text { geschäft im Nachbarland über Angebote und } \\
\text { besondere Dienstleistungen informiert }\end{array}$ & 4,08 & 2,81 & 2,98 & 2,81 & 2,50 & 2,81 & 1,59 & 2,81 \\
\hline $\begin{array}{l}\text { Mindestens einmal im Monat besuche ich } \\
\text { mein Stammgeschäft im Nachbarland }\end{array}$ & 4,81 & 2,93 & 2,67 & 2,93 & 2,72 & 2,93 & 2,29 & 2,93 \\
\hline $\begin{array}{l}\text { In den vergangenen acht Wochen habe ich oft } \\
\text { im Nachbarland eingekauft }\end{array}$ & 4,64 & 2,96 & 2,77 & 2,96 & 2,68 & 2,96 & 2,39 & 2,96 \\
\hline $\begin{array}{l}\text { in den letzten acht Wochen habe ich oft im } \\
\text { Stammgeschäft eingekauft }\end{array}$ & 4,58 & 3,09 & 3,03 & 3,09 & 2,94 & 3,09 & 2,15 & 3,09 \\
\hline $\begin{array}{l}\text { Sofern mein Stammgeschäft in Zukunft auch } \\
\text { andere Produkte anbietet, könnte ich mir } \\
\text { vorstellen, diese auch dort zu kaufen Cross } \\
\text { buying }\end{array}$ & 3,47 & 2,55 & 2,69 & 2,55 & 2,20 & 2,55 & 1,76 & 2,55 \\
\hline
\end{tabular}

\section{Clusteranalyse der Fälle für niederländische Konsumenten}




\section{Anhang 12:}

\section{Geschlecht}

\begin{tabular}{|ll|r|r|r|r|}
\hline & Häufigkeit & Prozent & $\begin{array}{c}\text { Gültige } \\
\text { Prozente }\end{array}$ & $\begin{array}{c}\text { Kumulierte } \\
\text { Prozente }\end{array}$ \\
\hline Gültig & männlich & 6 & 16,7 & 16,7 & 16,7 \\
& weiblich & 30 & 83,3 & 83,3 & 100,0 \\
& Gesamt & 36 & 100,0 & 100,0 & \\
\hline
\end{tabular}

Alter

\begin{tabular}{|ll|r|r|r|r|}
\hline & Häufigkeit & Prozent & $\begin{array}{c}\text { Gültige } \\
\text { Prozente }\end{array}$ & $\begin{array}{c}\text { Kumulierte } \\
\text { Prozente }\end{array}$ \\
\hline Gültig & Bis 35 & 21 & 58,3 & 58,3 & 58,3 \\
& 35 bis & 12 & 33,3 & 33,3 & 91,7 \\
64 & 3 & 8,3 & 8,3 & 100,0 \\
Über 64 & 36 & 100,0 & 100,0 & \\
Gesamt &
\end{tabular}

Anzahl Personen im Haushalt

\begin{tabular}{|rl|r|r|r|r|}
\hline & Häufigkeit & Prozent & $\begin{array}{c}\text { Gültige } \\
\text { Prozente }\end{array}$ & $\begin{array}{c}\text { Kumulierte } \\
\text { Prozente }\end{array}$ \\
\hline Gültig & 1 & 4 & 11,1 & 11,1 & 11,1 \\
& 2 & 10 & 27,8 & 27,8 & 38,9 \\
& 3 und & 22 & 61,1 & 61,1 & 100,0 \\
mehr & 36 & 100,0 & 100,0 & \\
\hline
\end{tabular}

Haushaltsnettoeinkommen

\begin{tabular}{|rl|r|r|r|r|}
\hline & Häufigkeit & \multicolumn{1}{c|}{ Prozent } & $\begin{array}{c}\text { Gültige } \\
\text { Prozente }\end{array}$ & \multicolumn{1}{c|}{$\begin{array}{c}\text { Kumulierte } \\
\text { Prozente }\end{array}$} \\
\hline Gültig & Bis & 20 & 55,6 & 55,6 & 55,6 \\
& $\begin{array}{r}\text { 2.000,- } \\
\text { Bis }\end{array}$ & 11 & 30,6 & 30,6 & 86,1 \\
& $\begin{array}{l}3.500,- \\
\text { Über } \\
\text { 3.500,- } \\
\text { Gesamt }\end{array}$ & 5 & 13,9 & 13,9 & 100,0 \\
\hline
\end{tabular}

Verkehrsmittel

\begin{tabular}{|rl|r|r|r|r|}
\hline & Häufigkeit & Prozent & $\begin{array}{c}\text { Gültige } \\
\text { Prozente }\end{array}$ & $\begin{array}{c}\text { Kumulierte } \\
\text { Prozente }\end{array}$ \\
\hline Gültig & Auto & 26 & 72,2 & 72,2 & 72,2 \\
& Bahn & 10 & 27,8 & 27,8 & 100,0 \\
& Gesamt & 36 & 100,0 & 100,0 & \\
\hline
\end{tabular}

„Die Minimalkunden“ (Cluster 1) niederländische Konsumenten 


\section{Anhang 13:}

Geschlecht

\begin{tabular}{|ll|r|r|r|r|}
\hline & Häufigkeit & Prozent & $\begin{array}{c}\text { Gültige } \\
\text { Prozente }\end{array}$ & $\begin{array}{c}\text { Kumulierte } \\
\text { Prozente }\end{array}$ \\
\hline Gültig & männlich & 45 & 38,1 & 38,1 & 38,1 \\
& weiblich & 73 & 61,9 & 61,9 & 100,0 \\
& Gesamt & 118 & 100,0 & 100,0 & \\
\hline
\end{tabular}

Alter

\begin{tabular}{|rl|r|r|r|r|}
\hline & Häufigkeit & Prozent & $\begin{array}{c}\text { Gültige } \\
\text { Prozente }\end{array}$ & $\begin{array}{c}\text { Kumulierte } \\
\text { Prozente }\end{array}$ \\
\hline Gültig & Bis 35 & 54 & 45,8 & 45,8 & 45,8 \\
& 35 bis & 44 & 37,3 & 37,3 & 83,1 \\
64 & 20 & 16,9 & 16,9 & 100,0 \\
& Über 64 & 118 & 100,0 & 100,0 & \\
Gesamt & &
\end{tabular}

Anzahl Personen im Haushalt

\begin{tabular}{|rl|r|r|r|r|}
\hline & Häufigkeit & Prozent & $\begin{array}{c}\text { Gültige } \\
\text { Prozente }\end{array}$ & $\begin{array}{c}\text { Kumulierte } \\
\text { Prozente }\end{array}$ \\
\hline Gültig & 1 & 34 & 28,8 & 28,8 & 28,8 \\
& 2 & 56 & 47,5 & 47,5 & 76,3 \\
& 3 und & 28 & 23,7 & 23,7 & 100,0 \\
mehr & 118 & 100,0 & 100,0 & \\
\hline
\end{tabular}

Haushaltsnettoeinkommen

\begin{tabular}{|c|c|c|c|c|c|}
\hline & & Häufigkeit & Prozent & $\begin{array}{c}\text { Gültige } \\
\text { Prozente }\end{array}$ & $\begin{array}{c}\text { Kumulierte } \\
\text { Prozente }\end{array}$ \\
\hline Gültig & $\begin{array}{l}\text { Bis } \\
2.000,- \\
\text { Bis } \\
3.500,- \\
\text { Über } \\
3.500,- \\
\text { Gesamt }\end{array}$ & $\begin{array}{r}51 \\
45 \\
22 \\
118\end{array}$ & $\begin{array}{r}43,2 \\
38,1 \\
18,6 \\
100,0\end{array}$ & $\begin{array}{r}43,2 \\
38,1 \\
18,6 \\
100,0\end{array}$ & $\begin{array}{r}43,2 \\
81,4 \\
100,0\end{array}$ \\
\hline
\end{tabular}

Verkehrsmittel

\begin{tabular}{|ll|r|r|r|r|}
\hline & Häufigkeit & Prozent & $\begin{array}{c}\text { Gültige } \\
\text { Prozente }\end{array}$ & $\begin{array}{c}\text { Kumulierte } \\
\text { Prozente }\end{array}$ \\
\hline Gültig & Auto & 96 & 81,4 & 81,4 & 81,4 \\
& Bus & 13 & 11,0 & 11,0 & 92,4 \\
& Bahn & 5 & 5,1 & 5,1 & 97,5 \\
Fahrrad & 3 & 2,5 & 2,5 & 100,0 \\
& Gesamt & 118 & 100,0 & 100,0 & \\
\hline
\end{tabular}

„Die Gelegenheitskäufer“ (Cluster 2) niederländische Kunden 


\section{Anhang 14:}

Geschlecht

\begin{tabular}{|ll|r|r|r|r|}
\hline & Häufigkeit & Prozent & $\begin{array}{c}\text { Gültige } \\
\text { Prozente }\end{array}$ & $\begin{array}{c}\text { Kumulierte } \\
\text { Prozente }\end{array}$ \\
\hline Gültig & männlich & 13 & 26,0 & 26,0 & 26,0 \\
& weiblich & 37 & 74,0 & 74,0 & 100,0 \\
& Gesamt & 50 & 100,0 & 100,0 & \\
\hline
\end{tabular}

Alter

\begin{tabular}{|ll|r|r|r|r|}
\hline & Häufigkeit & Prozent & $\begin{array}{c}\text { Gültige } \\
\text { Prozente }\end{array}$ & $\begin{array}{c}\text { Kumulierte } \\
\text { Prozente }\end{array}$ \\
\hline Gültig & Bis 35 & 21 & 42,0 & 42,0 & 42,0 \\
& 35 bis & 19 & 38,0 & 38,0 & 80,0 \\
64 & 10 & 20,0 & 20,0 & 100,0 \\
& Über 64 & 50 & 100,0 & 100,0 & \\
Gesamt & &
\end{tabular}

Anzahl Personen im Haushalt

\begin{tabular}{|rl|r|r|r|r|}
\hline & Häufigkeit & Prozent & $\begin{array}{c}\text { Gültige } \\
\text { Prozente }\end{array}$ & $\begin{array}{c}\text { Kumulierte } \\
\text { Prozente }\end{array}$ \\
\hline Gültig & 1 & 11 & 22,0 & 22,0 & 22,0 \\
& 2 & 21 & 42,0 & 42,0 & 64,0 \\
& 3 und & 18 & 36,0 & 36,0 & 100,0 \\
mehr & 50 & 100,0 & 100,0 & \\
\hline
\end{tabular}

Haushaltsnettoeinkommen

\begin{tabular}{|rl|r|r|r|r|}
\hline & Häufigkeit & Prozent & $\begin{array}{c}\text { Gültige } \\
\text { Prozente }\end{array}$ & $\begin{array}{c}\text { Kumulierte } \\
\text { Prozente }\end{array}$ \\
\hline Gültig & Bis & 23 & 46,0 & 46,0 & 46,0 \\
& $2.000,-$ & & & 72,0 \\
& Bis & 13 & 26,0 & 26,0 & 100,0 \\
& $\begin{array}{r}\text { 3.500,- } \\
\text { Über }\end{array}$ & 14 & 28,0 & 28,0 & \\
& 3.500,- \\
Gesamt & 50 & 100,0 & 100,0 & \\
\hline
\end{tabular}

Verkehrsmittel

\begin{tabular}{|ll|r|r|r|r|}
\hline & Häufigkeit & Prozent & $\begin{array}{c}\text { Gültige } \\
\text { Prozente }\end{array}$ & $\begin{array}{c}\text { Kumulierte } \\
\text { Prozente }\end{array}$ \\
\hline Gültig & Auto & 22 & 44,0 & 44,0 & 44,0 \\
& Bus & 6 & 12,0 & 12,0 & 56,0 \\
Bahn & 21 & 42,0 & 42,0 & 98,0 \\
Fahrrad & 1 & 2,0 & 2,0 & 100,0 \\
& Gesamt & 50 & 100,0 & 100,0 & \\
\hline
\end{tabular}

„Die begeisterten für Stammgeschäfte“(Cluster 3) niederländische Konsumenten 


\section{Anhang 15:}

Geschlecht

\begin{tabular}{|rl|r|r|r|r|}
\hline & Häufigkeit & Prozent & $\begin{array}{c}\text { Gültige } \\
\text { Prozente }\end{array}$ & $\begin{array}{c}\text { Kumulierte } \\
\text { Prozente }\end{array}$ \\
\hline Gültig & männlich & 17 & 41,5 & 41,5 & 41,5 \\
& weiblich & 24 & 58,5 & 58,5 & 100,0 \\
& Gesamt & 41 & 100,0 & 100,0 & \\
\hline
\end{tabular}

Alter

\begin{tabular}{|rl|r|r|r|r|}
\hline & Häufigkeit & Prozent & $\begin{array}{c}\text { Gültige } \\
\text { Prozente }\end{array}$ & $\begin{array}{c}\text { Kumulierte } \\
\text { Prozente }\end{array}$ \\
\hline Gültig & Bis 35 & 15 & 36,6 & 36,6 & 36,6 \\
& 35 bis & 26 & 63,4 & 63,4 & 100,0 \\
& 64 & 41 & 100,0 & 100,0 & \\
\hline
\end{tabular}

Anzahl Personen im Haushalt

\begin{tabular}{|rl|r|r|r|r|}
\hline & Häufigkeit & Prozent & $\begin{array}{c}\text { Gültige } \\
\text { Prozente }\end{array}$ & $\begin{array}{c}\text { Kumulierte } \\
\text { Prozente }\end{array}$ \\
\hline Gültig & 2 & 19 & 46,3 & 46,3 & 46,3 \\
& 3 und & 22 & 53,7 & 53,7 & 100,0 \\
mehr & 41 & 100,0 & 100,0 & \\
\hline
\end{tabular}

Haushaltsnettoeinkommen

\begin{tabular}{|c|c|c|c|c|c|}
\hline & & Häufigkeit & Prozent & $\begin{array}{c}\text { Gültige } \\
\text { Prozente }\end{array}$ & $\begin{array}{c}\text { Kumulierte } \\
\text { Prozente }\end{array}$ \\
\hline Gültig & $\begin{array}{l}\text { Bis } \\
2.000,- \\
\text { Bis } \\
\text { 3.500,- } \\
\text { Über } \\
\text { 3.500,- } \\
\text { Gesamt }\end{array}$ & $\begin{array}{r}29 \\
11 \\
1 \\
41\end{array}$ & $\begin{array}{r}70,7 \\
26,8 \\
2,4 \\
100,0\end{array}$ & $\begin{array}{r}70,7 \\
26,8 \\
2,4 \\
100,0\end{array}$ & $\begin{array}{r}70,7 \\
97,6 \\
100,0\end{array}$ \\
\hline
\end{tabular}

Verkehrsmittel

\begin{tabular}{|ll|r|r|r|r|}
\hline & & & \multicolumn{1}{c|}{$\begin{array}{c}\text { Gültige } \\
\text { Prozente }\end{array}$} & $\begin{array}{c}\text { Kumulierte } \\
\text { Prozente }\end{array}$ \\
\hline Gültig & Auto & 40 & 97,6 & 97,6 & 97,6 \\
& Bahn & 1 & 2,4 & 2,4 & 100,0 \\
& Gesamt & 41 & 100,0 & 100,0 & \\
\hline
\end{tabular}

„Die schnellen zielgerichteten, preisorientierten Käufer“ (Cluster 4) niederländische Konsumenten 


\section{Anhang 16}

\begin{tabular}{|l|l|l|l|}
\hline \multirow{2}{*}{$\begin{array}{l}\text { Effektgröße } \\
\text { NL }\end{array}$} & \multicolumn{2}{|c|}{ Pfadkoeffizient } \\
\cline { 2 - 4 } & \multicolumn{1}{|c|}{$<0,1$} & $0,1-0,2$ & \multicolumn{1}{|c|}{$>0,2$} \\
\hline$>0,05$ & & & $\begin{array}{l}\text { Werturteile der sozialen Gruppe } \\
\text { (TZSN) }\end{array}$ \\
\hline $0,02-0,05$ & & & \\
\hline$<0,02$ & & & $\begin{array}{l}\text { Wichtigkeit der sozialen Norm } \\
\text { (WSN) } \\
\text { Werturteile der sozialen Gruppe } \\
\text { (TZSN) }\end{array}$ \\
\hline $\mathrm{D}$ & & & \\
\hline$>0,05$ & & & \\
\hline $0,02-0,05$ & & & \\
\hline$<0,02$ & & & \\
\hline
\end{tabular}

Gegenüberstellung von Pfadkoeffizienten und Effektstärke in dem Konstrukt Bedeutung der sozialen Norm für den Konsumenten

\begin{tabular}{|c|c|c|c|}
\hline \multirow{2}{*}{$\begin{array}{l}\text { Effektgröße } \\
\text { NL }\end{array}$} & \multicolumn{3}{|c|}{ Pfadkoeffizient } \\
\hline & $<0,1$ & $0,1-0,2$ & $>0,2$ \\
\hline$>0,05$ & & & $\begin{array}{l}\text { Bedeutung der sozialen Norm } \\
\text { für den Konsumenten } \\
\text { (GKDZN) } \\
\text { Gesamtzufriedenheit mit } \\
\text { Stammgeschäft im Nachbarland } \\
\text { (GKDZ Kauf) }\end{array}$ \\
\hline $0,02-0,05$ & $\begin{array}{l}\text { Werturteile der sozialen Gruppe } \\
\text { (TZSN) }\end{array}$ & & \\
\hline$<0,02$ & & & \\
\hline $\mathrm{D}$ & & & \\
\hline$>0,05$ & & $\begin{array}{l}\text { Bedeutung der sozialen Norm } \\
\text { für den Konsumenten. (GKdZ) }\end{array}$ & $\begin{array}{l}\text { Gesamtzufriedenheit mit } \\
\text { Stammgeschäft im Nachbarland } \\
\text { (GLSS) }\end{array}$ \\
\hline $0,02-0,05$ & & $\begin{array}{l}\text { Wichtigkeit der sozialen Norm } \\
\text { (WSN) }\end{array}$ & $\begin{array}{l}\text { Werturteile der sozialen Gruppe } \\
\text { (TZSN) }\end{array}$ \\
\hline$<0,02$ & & & \\
\hline
\end{tabular}

Gegenüberstellung von Pfadkoeffizienten und Effektstärke in dem Konstrukt Gesamtzufriedenheit mit dem Einkauf im Nachbarland

\begin{tabular}{|c|c|c|c|}
\hline \multirow{2}{*}{$\begin{array}{l}\text { Effektgröße } \\
\text { NL }\end{array}$} & \multicolumn{3}{|c|}{ Pfadkoeffizient } \\
\hline & $<0,1$ & $0,1-0,2$ & $>0,2$ \\
\hline$>0,05$ & & $\begin{array}{l}\text { Gesamtzufriedenheit mit dem } \\
\text { Einkauf im Nachbarland } \\
\text { (GLSS) }\end{array}$ & $\begin{array}{l}\text { Wahrgenommene Selbstrele- } \\
\text { vanz (WRS) } \\
\text { Persönliche Norm (PN) }\end{array}$ \\
\hline $0,02-0,05$ & & & $\begin{array}{l}\text { Bedeutung der sozialen Norm } \\
\text { für den Konsumenten } \\
\text { (GKDZN) } \\
\text { Gesamtzufriedenheit mit } \\
\text { Stammgeschäft im Nachbarland } \\
\text { (GKDZ Kauf) }\end{array}$ \\
\hline$<0,02$ & $\begin{array}{l}\text { Werturteile der sozialen Gruppe (TZSN) } \\
\text { Wahrgenommene Verhaltenskontrolle (WvK) }\end{array}$ & & \\
\hline $\mathrm{D}$ & & & \\
\hline$>0,05$ & & $\begin{array}{l}\text { Wahrgenommene Selbstrele- } \\
\text { vanz (WRS) }\end{array}$ & $\begin{array}{l}\text { Gesamtzufriedenheit mit dem } \\
\text { Einkauf im Nachbarland } \\
\text { (GLSS) } \\
\text { Persönliche Norm } \\
\text { Gesamtzufriedenheit mit } \\
\text { Stammgeschäft im Nachbarland } \\
\text { (GKDZ Kauf) } \\
\end{array}$ \\
\hline $0,02-0,05$ & & & \\
\hline$<0,02$ & $\begin{array}{l}\text { Bedeutung der sozialen Norm für den Konsu- } \\
\text { menten (GKDZN) }\end{array}$ & & \\
\hline
\end{tabular}


Wahrgenommene Verhaltenskontrolle (WvK)

Gegenüberstellung von Pfadkoeffizienten und Effektstärke in dem Konstrukt Kundenloyalität

\begin{tabular}{|c|c|c|c|}
\hline \multirow{2}{*}{$\begin{array}{l}\text { Effektgröße } \\
\text { NL }\end{array}$} & \multicolumn{3}{|c|}{ Pfadkoeffizient } \\
\hline & $<0,1$ & $0,1-0,2$ & $>0,2$ \\
\hline$>0,05$ & & $\begin{array}{l}\text { Gesamtzufriedenheit mit dem } \\
\text { Einkauf im Nachbarland } \\
\text { (GLSS) }\end{array}$ & $\begin{array}{l}\text { Kundenloyalität (KL) } \\
\text { Wahrgenommene Selbstrele- } \\
\text { vanz (WRS) }\end{array}$ \\
\hline $0,02-0,05$ & & & $\begin{array}{l}\text { Gesamtzufriedenheit mit } \\
\text { Stammgeschäft im Nachbar- } \\
\text { land. (GKDZ Kauf) } \\
\text { Persönliche Norm (PN) }\end{array}$ \\
\hline$<0,02$ & Werturteile der sozialen Gruppe (TZSN) & $\begin{array}{l}\text { Bedeutung der sozialen Norm } \\
\text { für den Konsumenten } \\
(\text { GKDZN) }\end{array}$ & \\
\hline $\mathrm{D}$ & & & \\
\hline$>0,05$ & $\begin{array}{l}\text { Gesamtzufriedenheit mit dem Einkauf im Nach- } \\
\text { barland (GLSS) }\end{array}$ & $\begin{array}{l}\text { Wahrgenommene Selbstrele- } \\
\text { vanz (WRS) }\end{array}$ & Kundenloyalität (KL) \\
\hline $0,02-0,05$ & & & $\begin{array}{l}\text { Persönliche Norm (PN) } \\
\text { Gesamtzufriedenheit mit dem } \\
\text { Stammgeschäft im Nachbarland } \\
\text { (GKDZ Kauf) }\end{array}$ \\
\hline$<0,02$ & $\begin{array}{l}\text { Bedeutung der sozialen Norm für den Konsu- } \\
\text { menten (GKDZN) } \\
\text { Wichtigkeit soziale Norm (WSN) } \\
\text { Werturteile der sozialen Gruppe(TZSN) }\end{array}$ & & \\
\hline
\end{tabular}

Gegenüberstellung von Pfadkoeffizienten und Effektstärke in dem Konstrukt Kundenbindung

\begin{tabular}{|l|c|c|c|}
\hline \multirow{2}{*}{$\begin{array}{l}\text { Effektgröße } \\
\text { NL }\end{array}$} & \multicolumn{2}{|c|}{ Pfadkoeffizient } \\
\hline$>0,05$ & & $0,1-0,2$ & $>0,2$ \\
\hline $0,02-0,05$ & & & Persönliche Norm (PN) \\
\hline$<0,02$ & & & \\
\hline $\mathrm{D}$ & & & \\
\hline$>0,05$ & & & Persönliche Norm (PN) \\
\hline $0,02-0,05$ & & & \\
\hline$<0,02$ & & & \\
\hline
\end{tabular}

Gegenüberstellung von Pfadkoeffizienten und Effektstärke in dem Konstrukt wahrgenommene Selbstrelevanz 
\begin{tabular}{l|l} 
Anhang & 289
\end{tabular} 

Samenvatting

\section{Grensoverschrijdende klantenbinding in de detailhandel \\ - trouw gedrag van Duitse en Nederlandse consumenten in het grensgebied van het buurland}

\section{Introductie in de problematiek}

Bedrijven in een markteconomisch systeem hebben als doel om een gepaste rentabiliteit op het geïnvesteerde kapitaal te vergaren en een profitabele groei te bereiken. Dit is echter in een grensgebied tussen twee buurlanden voor de detailhandel alleen bereikbaar als begrip voor en kennis van het gedrag van consumenten uit de buurlanden aanwezig is. Wat zijn dus de motieven en wat is de motivatie van consumenten om producten voor eigen behoeften in het buurland te kopen ofschoon veel producten ook in het eigen land verkrijgbaar zijn? Bestudering van de literatuur toont aan dat nauwelijks onderzoek is gedaan naar de motieven van consumenten om in het buurland inkopen te doen. De hier gepresenteerde studie is bedoeld om een bijdrage te leveren aan de uitleg van de motivatie van consumenten om in het buitenland inkopen te doen, om dit gat in het onderzoeksgebied te sluiten.

Als gevolg van deze doelstellingen worden de volgende onderzoeksvragen geformuleerd:

- Zijn er voor Duitse en Nederlandse consumenten verschillende factoren voor klantenbinding?

- Welke loyaliteitseffecten ontwikkelen zich in grensregio`s?

- Bestaan grensoverschrijdend andere factoren voor klantenbinding dan de bekende?

- Is er een gezamenlijk model voor klantenbinding van Duitse en Nederlandse consumenten?

- Wordt dit nieuwe model in de praktijk bevestigd?

Winkelspecifieke determinanten van grensoverschrijdende zakelijke loyaliteit

In hoofdstuk twee worden de winkelspecifieke determinanten van de grensoverschrijdende zakelijke loyaliteit onderzocht. Hierbij wordt op de rol van de landsgrens als promotor van klantenbinding of als handelsbarrière ingegaan. Verder wordt er onderzocht welke invloed de sociale omgeving en de individuele typering door de cultuur op het winkelgedrag van de consumenten in Nederland en Duitsland hebben. In deze samenhang worden de cultuurdimensies van Hofstede gepresenteerd, alsook Usunier's cultuurzones van Europa en 
Kasper's cluster van Europese landsculturen. Achteraf worden de culturen van Nederland en Duitsland met elkaar vergeleken, met het oog op de invloed op het winkelgedrag. Als er echter cultuurverschillen tussen deze twee landen zijn en ook de houding tot het doen van inkopen verschillend is, betekent dit niet dat deze uitspraken ook in het grensgebied van de twee landen geldig zijn. Deze verschillen en hun gevolgen worden in dit hoofdstuk uitgewerkt. Er wordt aangetoond welke verschillende stijlen van leven en koopgedrag tussen Nederlandse en Duitse consumenten aanwezig zijn en welke gevolgen deze op het koopgedrag in de grensregio hebben.

\section{Stand van zaken met betrekking tot onderzoek naar klantenbinding}

In hoofdstuk drie worden in het kader van het onderzoek verschillende begrippen zoals klanttevredenheid, klantenloyaliteit en klantenbinding gedefinieerd en aan de Engelse terminologie getoetst. Een verdere punt van dit hoofdstuk zijn de determinanten van klantenbinding. Hierbij wordt een verschil gemaakt tussen prestatieafhankelijke determinanten, zoals de houding van de vrager tot het product, de handelaar of in het bijzonder ook de kwaliteit van de prestatie, en de prestatieonafhankelijke determinanten van klantenbinding, welke door Faison als "variety seeking" gedefinieerd zijn. Als verdere determinant van klantenbinding wordt in dit onderzoek de sociale, situatieve en sociodemografische situatie van de consumenten als factoren voor klantenbinding vastgelegd. De neiging van consumenten om zichzelf aan een winkel te binden - in het bijzonder als deze ook nog in het buurland ligt - is afhankelijk van economische en buiteneconomische redenen. Economische en buiteneconomische redenen tot promotie van klantenbinding van consumenten wordt echter ook door de winkels zelf bedreven. Doel van de winkels is het om in de 'evoked set' van de consumenten te komen. Daarbij zijn kopers veel sneller bereid om bij ontevredenheid met de inkoop van winkel te veranderen dan van merk. Op basis van intensieve literatuurstudie wordt voor het onderzoek vastgelegd dat klanttevredenheid het resultaat van een psychisch vergelijkingsproces is, klantenbinding uit perspectief van de klant zich, zoals Eggert het definieert, als "interne toestand van de klant" manifesteert en klantenbinding uit perspectief van de klant altijd aanwezig is, "als de klant een binding constateert". Echter moet klantenbinding door herhaalde inkoop in een winkel door de consumenten verstevigd worden. Een bijzondere betekenis komt hierbij toe aan de neiging van de consument, zich op een nauwere relatie met een specifieke detailhandelwinkel in te laten. Resultaat van de analyse in dit hoofdstuk is dat klantenbinding van een consument die in het buurland inkopen doet zich in de loyaliteit van de consument met de inkoopplek, de 
winkel, en door de sociale en persoonlijke norm manifesteert welke tot uiting brengt hoe de consument zich aan het buurland verbonden voelt. De verbondenheid van de consument aan de cultuur en het aanbod van de grenssteden is voor handelsbedrijven wederom een beslissende mogelijkheid om consumenten die grensoverschrijdend inkopen doen doelgerichter aan te kunnen spreken.

\section{Houdingstheorie als uitlegbeginsel van klantenbinding}

In hoofdstuk vier wordt onderzocht hoe onderzoek naar de houding en in het bijzonder de theorie van het geplande gedrag tot uitleg van klantenbinding in het grensgebied van twee landen kan bijdragen. Er wordt geconstateerd dat de theorie van het geplande gedrag een bijdrage kan leveren aan uitleg over hoe de beslissingsvinding bij de consument plaatsvindt. Bouwend op deze constatering wordt de uitgebreide theorie van het geplande gedrag met het doel onderzocht om de mogelijkheid tot prognose van klantenbinding te verbeteren. Als resultaat van het literatuuronderzoek wordt geconstateerd dat naast de factoren grens en cultuur de aanknoping van houdingstheoretische componenten met het huidige weten van klantenbinding een beslissende rol speelt om de mogelijkheid tot prognose van klantenbinding te verhogen en de motieven van consumenten tot inkoop in het buurland uit te leggen. Het aspect van zelfidentiteit is beslissend in het onmiddellijke buurland. Voor de sociale en persoonlijke norm van de consument is hier een bijzondere rol weggelegd. Uiteindelijk leidt dit tot individuele ideeën van de consument over waarde. Het individuele idee van de consument over waarde heeft een sterke invloed op het winkelgedrag.

\section{Onderzoeksmodel, -hypothesen en onderzoeksmethodologie}

In hoofdstukken vijf en zes wordt onderbouwd hoe het model van klantenbinding tot uitleg van klantenbinding van de consumenten aan een winkel in het buurland in elkaar zit. Er worden eerst hypothesen gevormd welke de klantenbinding moeten verduidelijken, en uiteindelijk worden deze hypothesen in een model samengevoegd. In tegenstelling tot de tot nu toe in de literatuur bediscussieerde modellen van klantenbinding ligt in dit onderzoek de nadruk op de invloed van en de sociale en de persoonlijke norm en de geconstateerde zelfrelevantie op de grensoverschrijdende binding aan een winkel. De geconstateerde zelfrelevantie bepaalt in deze samenhang het stelsel van oorzaak en gevolg en heeft hierdoor een onmiddellijke invloed op de klantenloyaliteit en de klantenbinding zelf. Op het construct van klantenbinding werken dus direct en indirect drie factoren. Dit zijn klantenloyaliteit, 
totale tevredenheid van het individu met de aankoop in het buitenland en geconstateerde zelfrelevantie. Klantenbinding is volgens het ontwikkelde model afhankelijk van en de sociale norm van de subjecten, de reactie van de sociale omgeving op het gedrag, en de totale tevredenheid van de koop op zich en de omstandigheden waaronder de koop plaatsvindt. Volgens het ontwikkelde model heeft de sociale norm niet alleen een belangrijkere invloed de totale tevredenheid met de koop, maar ook op het bindingsgedrag van de consumenten zelf. De gedefinieerde hypothesen worden in uitlegclusters samengevoegd. Hierbij wordt met behulp van de hypothesen uitgelegd wat klanttevredenheid met een winkel in het buurland uitmaakt; verder wordt de vraag beantwoord of Nederlandse consumenten hetzelfde of anders moeten worden aangesproken dan Duitse consumenten. Uiteindelijk wordt uitgelegd waardoor bij de twee doelgroepen klantenbinding ontstaat. De geldigheid van het gepresenteerde model voor klantenbinding is in eerste instantie afhankelijk van de onderliggende theorie van het geplande gedrag en het voldoen aan de volgende voorwaarden:

1. De sociale norm en de geconstateerde zelfrelevantie beïnvloeden het gedrag van de consument die in het buurland inkopen doet.

2. De toestanden van binding van de consumenten die in het buurland inkopen doen zijn generisch bepaald en hebben verschillende effecten die het gedrag sturen.

In hoofdstuk zes wordt de keuze voor een cross-sectoraal, niet experimenteel onderzoek onderbouwd, dat gebaseerd is op persoonlijke ondervraging in centrums van steden in de grensstreek. Daarnaast wordt het proces en het gebruik voor het genereren en voor een gedeelte de enquête omschreven. Voor het genereren van de enquête wordt literatuur geraadpleegd en vervolgens worden de vragen met focusgroepen van klanten uit beide landen getest. De hieruit ontstane vragen zijn onderhevig aan een pretest en worden wederom in beide landen kwalitatief getest. Met de ervaringen van de pretest worden de vragen nog beter aan de doelgroep aangepast. Vervolgens wordt de kwalitatieve test in Nederland en Duitsland in het gedefinieerde grensgebied uitgevoerd. Uiteindelijk wordt de toepasselijkheid van multivariate analysemethodes voor de schatting van het model bediscussieerd, om een geldig toetsschema op te stellen. Hierbij worden drie stappen gedaan. Eerst wordt het exploratie sample met behulp van de exploratieve factoranalyse getoetst, vervolgens worden de hypothetische constructen geanalyseerd. Aan het einde worden de betrouwbaarheid en validiteit getoetst. 
Met behulp van het PLS beginsel worden de samenhangen van effecten tussen de deelconstructen van klantenbinding en de totale binding tot een winkel in het buurland onderzocht. Met uitzondering van een effectsamenhang "geconstateerde gedragscontrole", welke voor beide consumentengroepen niet kon worden bewezen, en de effectsamenhang "belang van de sociale norm" op "betekenis van de sociale norm" voor Nederlandse consumenten, kunnen de veronderstelde effectsamenhangen worden bevestigd. De meeste opgestelde hypothesen worden bevestigd. Het model toont voldoende kwaliteit en is geschikt voor prognose. Ook wordt een groot gedeelte van de hypothetische effectrelaties tussen de constructen van klantenbinding en de deelconstructen bevestigd. Voor de Nederlandse consumenten toont de padcoëfficiënt klantenloyaliteit en klantenbinding de hoogste waarde, voor de Duitse klanten de padcoëfficiënt waardeoordeel van de sociale groep ten opzichte van de betekenis van de sociale norm voor de consumenten.

\section{Bijdrage van het werkstuk tot onderzoek naar klantenbinding}

In theoretisch opzicht levert dit werkstuk een bijdrage tot de theoretische fundering van het concept voor uitleg en prognose van klantenbinding in een grensgebied van twee landen van verschillende grootte. Sommige aanpassingsmaatregelen aan het model waren nodig om de theorie van het geplande gedrag voor uitleg van het fenomeen klantenbinding geschikt te maken. Dit betreft de houding van de consument tegen de culturele achtergrond en de sociale norm en modellen voor haar uitleg. De combinatie van theoretische, conceptuele en empirische aanpak is in onderzoeken in het verleden vaak niet gedaan. Juist deze combinatie is echter voor uitleg van klantenbinding in een grensgebied als doelmatig gebleken.

Dit werkstuk levert ook in methodisch opzicht een bijdrage aan onderzoek. Met behulp van de Partial Least Square methode kunnen op het niveau van indicatoren relevante drivers in de respectievelijke fases "betekenis van de sociale norm voor de consument", "totale tevredenheid met de inkoop in het buurland", "geconstateerde zelfrelevantie", "klantenloyaliteit" en "klantenbinding", elk voor Nederlandse en Duitse consumenten worden geïdentificeerd. Hiermee is het mogelijk om naast het bekijken van de effectsamenhangen op niveau van de constructen ook concrete aanbevelingen voor vormgeving en maatregels in de aparte fases te geven. De aanbevelingen voor het management van de winkels, maar ook voor het stadsmanagement, kunnen hierbij nauwkeuriger en gedetailleerder worden geformuleerd dan dit tot nu toe mogelijk was. 



\section{Lebenslauf}

Ulrich Scholz wurde am 8. Oktober 1955 in Essen (Deutschland) geboren. Nach dem Abitur und dem Grundwehrdienst studierte er an der Universität Köln Betriebswirtschaft mit den Schwerpunkten Handel und Absatz, Marketing und Informatik. Nach dem Studium arbeitete er in der Verkaufsorganisation des Unternehmen Procter\&Gamble, zum Schluss als KeyAccount-Manager. Anschließend war er als Geschäftsführer eines Handelsunternehmens tätig. Im Jahre 1996 wechselte er zur RAG BILDUNG, im Jahre 1998 zur Fontys Internationale Hogeschool Economie als Dozent für Marketing und Unternehmensführung. Im Jahre 1999 schloss er das Lehramtstudium der Sek. II mit dem Staatsexamen an der RWTH Aachen ab. Im Jahre 2002 startete er seine Doktorarbeit an der Universität Maastricht. Seine Untersuchungsschwerpunkte liegen im Bereich Projektmanagement und Customer Relationship Management, insbesondere customer loyalty und consumer behavior. Seine bisherigen Arbeiten sind veröffentlicht worden durch die GPM (Gesellschaft für Projektmanagement) und Oeconomische Forschung und Beratung. 